\title{
Enantioselective Formal Total Synthesis of the Antitumor Macrolide Bryostatin 7
}

\author{
Soraya Manaviazar, ${ }^{\star}$ Mark Frigerio, \\ Gurpreet S. Bhatia, Marc G. Hummersone, \\ Abil E. Aliev, and Karl J. Hale* \\ The Christopher Ingold Laboratories, \\ The Chemistry Department, \\ University College London, \\ 20 Gordon Street, \\ London WC1HOAJ, UK.
}

Supporting Information

\section{(282 Pages Inclusive of Title Pages)}

\begin{abstract}
Index
Part 1. Experimental Procedures for the UCL Synthesis of the Masamune Bryostatin 7 AB Intermediate 1

Part 2. Copies of the Spectra for the Intermediates Used in the UCL Synthesis of the Masamune Bryostatin 7 AB Intermediate 1

Part 3. Proof of Structure of the UCL Version of the Masamune Bryostatin $7 \mathrm{AB}$ Intermediate 1

Part 4. Experimental Procedures for the Synthesis of Compounds 32, 33, 35, and $\mathbf{3 6}$ and their Characterisation Data

Part 5. Detailed Tabulated Spectral Comparisons of Compounds 34, 37, and 36 Prepared Via Our Route with the Spectral data Reported for the Same Intermediates Independently Prepared by Hoffmann and Masamune
\end{abstract}

Pages 2-22

Pages 23-206

Pages 207-211

Pages 212-218

Pages 219-223

Part 6. Spectra of the Intermediates Used in the Various Correlations Performed with the Hoffmann and Masamune Bryostatin Intermediates

Pages 224-282 


\section{Enantioselective Formal Total Synthesis of the Antitumor Macrolide Bryostatin 7}

Soraya Manaviazar, ${ }^{*}$ Mark Frigerio, Gurpreet S. Bhatia, Marc G. Hummersone, Abil E. Aliev, and Karl J. Hale*

The Christopher Ingold Laboratories, The Chemistry Department, University College London, 20 Gordon Street, London WC1H OAJ, UK.

Supporting Information

Part 1. Experimental Procedures for the UCL Synthesis of the Masamune Bryostatin 7 AB Intermediate 1 


\section{Experimental Procedures for the Synthesis of Masamune's Bryostatin 7 AB Intermediate 1}

Please Note: For our NMR assignments, we have used bryostatin numbering throughout.

\section{Ketone 9 (SM-3-192)}

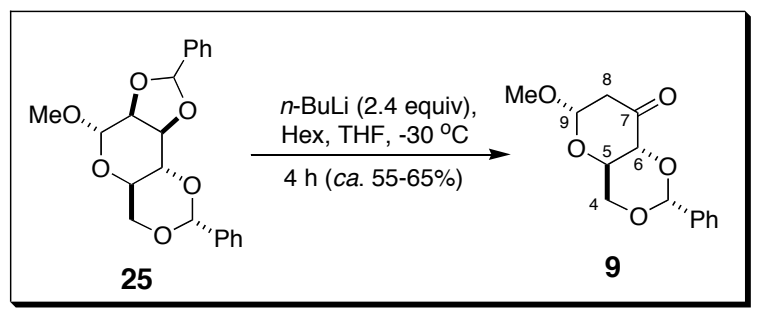

To a stirred solution of recrystallized benzylidene acetal $25(70 \mathrm{~g}, 188.99 \mathrm{mmol})$ in dry THF $(980 \mathrm{~mL})$ at $30{ }^{\circ} \mathrm{C}$ under $\mathrm{N}_{2}$ was added $n$-BuLi (2.5 M solution in hexanes, $181.4 \mathrm{~mL}, 453.6 \mathrm{mmol}, 2.4$ equiv) dropwise over $10 \mathrm{~min}$. The dark purple solution so obtained was stirred at $-30{ }^{\circ} \mathrm{C}$ for a further $4 \mathrm{~h}$, whereafter EtOAc $(500 \mathrm{~mL})$ was added followed by saturated aqueous $\mathrm{NH}_{4} \mathrm{Cl}(500 \mathrm{~mL})$. After separation of the organic layer, the aqueous phase was extracted with EtOAc $(3 \times 1 \mathrm{~L})$. The combined organic layers were dried over $\mathrm{MgSO}_{4}$, filtered, and concentrated in vacuo. Two successive recrystallisations of the orange residue with $\mathrm{EtOH}$ (absolute-GPR) gave ketone $9(32.6 \mathrm{~g}, 65 \%)$ as fluffy fine white crystals; this material was still, however, contaminated with a small quantity of phenylpentan-2-ol (5-10\%). Hereafter, this material is referred to as "technical grade" 9. An analytically pure sample of ketone $\mathbf{9}$ was obtained by further recrystallisation from EtOH-petrol.

Data for 9: m.p. 170-173 º; IR (KBr) 3068 (w), 3038 (w), 2972 (s), 2935 (s), 2914 (s), 2871 (s), 2833 (m), 1731 (s), 1499 (w), 1450 (s), 1407 (s), 1378 (s), 1332 (w), 1313 (w), 1296 (s), 1273 (s), 1233 (s), 1273 (s), 1219 (s), 1194 (s), 1135 (s), 1094 (s), 1053 (s), 1017 (s), 982 (s), 910 (s), 862 (w), 826 (m), 748 (s), 696 (s), 655 (s), 600 (m), 551 (w), 515 (m), 407 (m). ${ }^{1} \mathrm{H}$ NMR (500 MHz, $\left.\mathrm{CDCl}_{3}, 298 \mathrm{~K}\right) \delta 7.51$ -

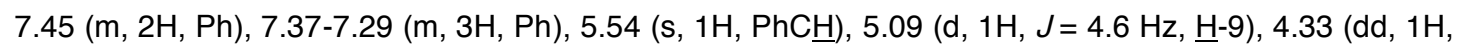
$J=4.8,-10.3 \mathrm{~Hz}, \underline{\mathrm{H}}-4 \mathrm{a}), 4.26(\mathrm{~d}, 1 \mathrm{H}, J=9.9 \mathrm{~Hz}, \underline{\mathrm{H}}-6), 4.10$ (ddd, $1 \mathrm{H}, J=4.8,10.0,10.0 \mathrm{~Hz}, \underline{\mathrm{H}}-5$ ), 3.88

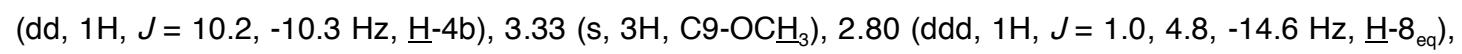
$2.62\left(\mathrm{~d}, 1 \mathrm{H}, J=-14.6 \mathrm{~Hz}, \underline{\mathrm{H}}-8_{\mathrm{ax}}\right) \mathrm{ppm} ;{ }^{13} \mathrm{C} \mathrm{NMR}\left(125 \mathrm{MHz}, \mathrm{CDCl}_{3}, 298 \mathrm{~K}\right) \delta 197.53$ (ㄷ-7 C=O), 136.50

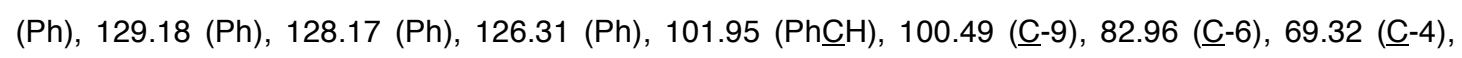
64.99 (ㄷ-5), $54.87\left(\mathrm{C}-9-\mathrm{OCH}_{3}\right), 46.31$ (ㄷ-8) ppm; VG 70-SE Positive Cl-Methane HRMS: Calcd. for $\mathrm{C}_{14} \mathrm{H}_{17} \mathrm{O}_{5}(\mathrm{M}+\mathrm{H})^{+}:$265.10759. Found: 265.10661.

\section{Ketone $11^{14}(\mathrm{SM}-4-14)$}

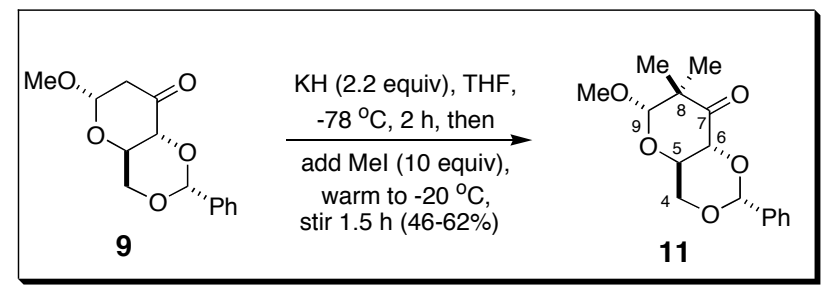


$\mathrm{KH}$ (30\% wt suspension in mineral oil, $19.48 \mathrm{~g}, 145.70 \mathrm{mmol}, 2.2$ equiv) was weighed into a 2-necked $1 \mathrm{~L}$ round-bottomed flask containing a magnetic stirring bar. After evacuation and introduction of a $\mathrm{N}_{2}$ atmosphere, the oil was removed from the $\mathrm{KH}$ by adding freshly distilled dry petrol $(3 \times 10 \mathrm{~mL})$ and removing the petrol/oil mixture by syringe after brief stirring and allowing the solid $\mathrm{KH}$ to settle to the bottom of the flask on each occasion. The flask, containing the petrol-washed $\mathrm{KH}$, was re-evacuated and a $\mathrm{N}_{2}$ atmosphere was introduced followed by dry THF $(150 \mathrm{~mL})$. The resulting suspension was cooled to $-78^{\circ} \mathrm{C}$ and a solution of the "technical grade" ketone 9 (17.5 g, $\left.66.22 \mathrm{mmol}\right)$ in dry THF (580 $\mathrm{mL})$ was added by cannula over $15 \mathrm{~min}$. The reaction mixture was stirred at $-78^{\circ} \mathrm{C}$ for $2 \mathrm{~h}$ and Mel $(41.2$ $\mathrm{mL}, 662.2 \mathrm{mmol}, 10$ equiv) was added dropwise over $5 \mathrm{~min}$. After the addition was complete the reaction mixture was stirred at $-78^{\circ} \mathrm{C}$ for $10 \mathrm{~min}$ and then slowly warmed to $-20^{\circ} \mathrm{C}$ over $1.5 \mathrm{~h}$. The mixture was diluted with EtOAc $(500 \mathrm{~mL})$ and saturated aqueous $\mathrm{NH}_{4} \mathrm{Cl}(500 \mathrm{~mL})$ was added. The organic layer was separated, and the aqueous phase was extracted with EtOAc ( $3 \times 500 \mathrm{~mL})$. The combined organic extracts were washed with brine $(1 \times 500 \mathrm{~mL})$, dried over $\mathrm{MgSO}_{4}$, filtered and concentrated in vacuo. Purification of the residue by $\mathrm{SiO}_{2}$ flash chromatography with (14:1 to 13:1) petrol-EtOAc as eluent furnished known ketone $11^{14}(8.87 \mathrm{~g}, 46 \%)$ as a colorless oil.

(N.B. Using totally pure starting ketone 9 , the above procedure gives a $62 \%$ yield of ketone 11.$)$

Data for 11: IR (neat film) 3067 (w), 2936 (s), 2868 (s), 2835 (s), 1705 (s), 1673 (w), 1600 (w), 1454 (s), 1378 (s), 1336 (w), 1313 (w), 1296 (w), 1278 (w), 1240 (m), 1209 (s), 1135 (s), 1098 (s), 1061 (s), 1032 (s), 976 (s), 915 (m), 753 (s), 699 (s), 664 (m). ${ }^{1} \mathrm{H}$ NMR (500 MHz, CDCl $\left.{ }_{3}, 298 \mathrm{~K}\right) \delta$ 7.51-7.44 (m, 2H, $\mathrm{Ph})$, 7.35-7.26 (m, 3H, Ph), 5.51 (s, $1 \mathrm{H}, \mathrm{PhC} \underline{\mathrm{H}}), 4.51$ (d, $1 \mathrm{H}, J=10.0 \mathrm{~Hz}, \underline{\mathrm{H}}-6)$ superimposed upon 4.50 (s, $1 \mathrm{H}, \underline{\mathrm{H}}-9$ ), 4.27 (dd, $1 \mathrm{H}, J=4.6,-10.2 \mathrm{~Hz}, \underline{\mathrm{H}}-4 \mathrm{a}$ ), 4.04 (ddd, $1 \mathrm{H}, J=4.7,10.1,10.0 \mathrm{~Hz}, \underline{\mathrm{H}}-5$ ), 3.88 (dd,

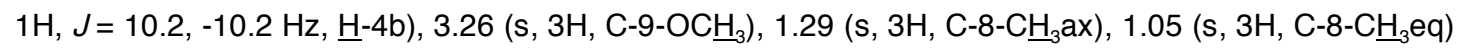
ppm; ${ }^{13} \mathrm{C}$ NMR (125 MHz, $\left.\mathrm{CDCl}_{3}, 298 \mathrm{~K}\right) \delta 202.64$ (ㄸ-7 C=O), $136.54(\mathrm{Ph}), 128.82(\mathrm{Ph}), 127.85(\mathrm{Ph})$,

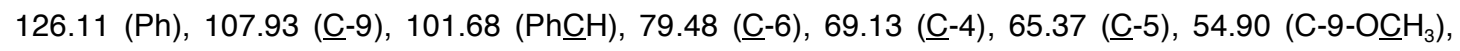
50.98 (ㄷ-8), 25.04 (C-8- $\left.\underline{C H}_{3} \mathrm{ax}\right), 18.72\left(\mathrm{C}-8-\underline{\mathrm{CH}}_{3} \mathrm{eq}\right)$ ppm. VG 70-SE Positive CI-Methane HRMS: Calcd. for $\mathrm{C}_{16} \mathrm{H}_{21} \mathrm{O}_{5}(\mathrm{M}+\mathrm{H})^{+}:$293.13889. Found: 293.13853.

\section{Diol 12 (SM-4-17)}

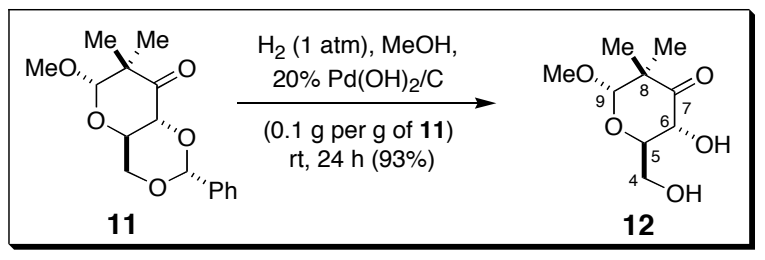

To a solution of the benzylidene acetal 11 (7.81 g, $26.72 \mathrm{mmol})$ in Analar $\mathrm{MeOH}(100 \mathrm{~mL})$ was added $20 \% \mathrm{Pd}(\mathrm{OH})_{2}$ on $\mathrm{C}(0.78 \mathrm{~g})$. The reaction flask was multiply evacuated and purged with $\mathrm{H}_{2}$ gas and thereafter vigorously stirred at $\mathrm{rt}$ and atmospheric pressure for $24 \mathrm{~h}$. The catalyst was then removed by filtration through a Celite pad, and the filter pad was washed exhaustively with $\mathrm{MeOH}$. The solvent was 
removed in vacuo and the residue was purified by $\mathrm{SiO}_{2}$ flash chromatography using petrol-EtOAc (3:1 to 2:1) as eluent. The pure diol 12 (5.09 g, 93\%) was obtained as a colorless oil.

Data for 12: $[\alpha]_{\mathrm{D}}+67.1^{\circ}\left(c 1.146, \mathrm{CH}_{2} \mathrm{Cl}_{2}\right)$; IR (neat film) $3433(\mathrm{~s}), 2912(\mathrm{~s}), 2837(\mathrm{~m}), 1719(\mathrm{~s}), 1638(\mathrm{w})$, 1470 (s), 1393 (s), 1354 (m), 1259 (s), 1232 (m), 1196 (s), 1111 (s), 1050 (s), 989 (s), 922 (m), 833 (m), 551 (m). ${ }^{1} \mathrm{H}$ NMR $\left(500 \mathrm{MHz}, \mathrm{CDCl}_{3}, 298 \mathrm{~K}\right) \delta 4.54$ (s, $1 \mathrm{H}, \underline{\mathrm{H}}-9$ ), 4.45 (d, $\left.1 \mathrm{H}, J=9.7 \mathrm{~Hz}, \underline{\mathrm{H}}-6\right), 3.93$ (dd, $1 \mathrm{H}, J=2.7,-12.0 \mathrm{~Hz}, \underline{\mathrm{H}}-4 \mathrm{a}), 3.87$ (dd, $1 \mathrm{H}, J=4.1,-12.0 \mathrm{~Hz}, \underline{\mathrm{H}}-4 \mathrm{~b}), 3.66$ (ddd, $1 \mathrm{H}, J=3.0,3.7,9.5 \mathrm{~Hz}$,

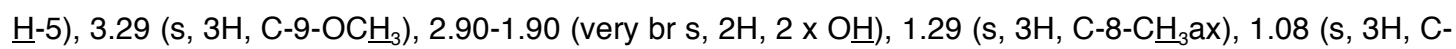

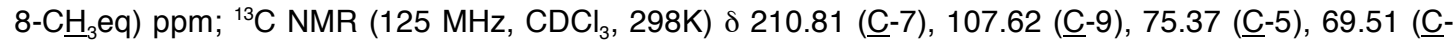
6), 62.52 (ㄷ-4), $55.23\left(\mathrm{C}-9-\mathrm{O} \underline{\mathrm{C}} \mathrm{H}_{3}\right), 49.90(\underline{\mathrm{C}}-8), 24.18\left(\mathrm{C}-8-\underline{\mathrm{CH}}_{3} \mathrm{ax}\right), 18.62\left(\mathrm{C}-8-\underline{\mathrm{CH}}_{3} \mathrm{eq}\right) \mathrm{ppm}$. VG 70-SE Positive Cl-Isobutane HRMS: Calcd. for $\mathrm{C}_{9} \mathrm{H}_{17} \mathrm{O}_{5}(\mathrm{M}+\mathrm{H})^{+}:$205.10759. Found: 205.10847.

\section{a-Hydroxyketone 13 (SM-4-21)}

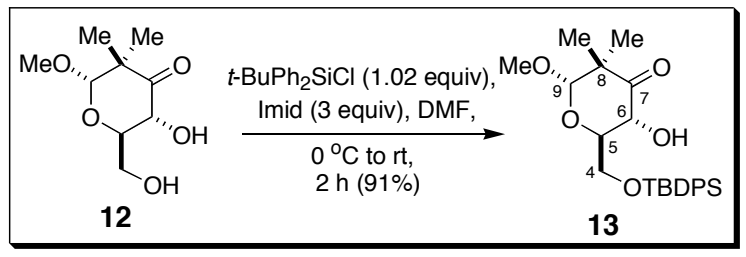

To a stirred solution of diol $12(25.5 \mathrm{~g}, 124.87 \mathrm{mmol})$ and imidazole $(25.5 \mathrm{~g}, 374.5 \mathrm{mmol}, 3.0$ equiv) in dry $N, N$-dimethylformamide $(540 \mathrm{~mL})$ was added tert-butylchlorodiphenylsilane $(33 \mathrm{~mL}, 126.89 \mathrm{mmol}$, 1.02 equiv) over $3 \mathrm{~min}$ at $0{ }^{\circ} \mathrm{C}$. After the addition was complete, the reactants were warmed to $\mathrm{rt}$ and stirred for $2 \mathrm{~h}$. Excess solid $\mathrm{NaHCO}_{3}$ was added and the reaction mixture was diluted with $\mathrm{Et}_{2} \mathrm{O}(1 \mathrm{~L})$ before saturated aqueous $\mathrm{NaHCO}_{3}(500 \mathrm{~mL})$ was added. The organic layer was separated, and the aqueous layer was extracted with $\mathrm{Et}_{2} \mathrm{O}(3 \times 500 \mathrm{~mL})$. The combined organic extracts were washed with $\mathrm{H}_{2} \mathrm{O}(2 \times 500 \mathrm{~mL})$, dried over $\mathrm{MgSO}_{4}$, filtered, and concentrated in vacuo. The crude residue was purified by $\mathrm{SiO}_{2}$ flash chromatography (gradient elution with 30:1 then 20:1 petrol-EtOAc as eluent); alcohol 13 (50.3 g, 91\%) was obtained as shiny white crystals.

Data for 13: $[\alpha]_{\mathrm{D}}+43.2^{\circ}\left(c 0.854, \mathrm{CH}_{2} \mathrm{Cl}_{2}\right)$; m.p. 102-106 ${ }^{\circ} \mathrm{C}$; IR $(\mathrm{KBr}) 3482(\mathrm{~m}), 3071(\mathrm{~m}), 3049(\mathrm{~m})$, $2932(\mathrm{~s}), 2893(\mathrm{~m}), 2857(\mathrm{~m}), 1719(\mathrm{~s}), 1472(\mathrm{~m}), 1463(\mathrm{~m}), 1428(\mathrm{~s}), 1391(\mathrm{~m}), 1268(\mathrm{~m}), 1195(\mathrm{~m})$, 1113 (s), 1094 (m), 1059 (s), 1041 (s), 1000 (s), 824 (m), 791 (m), 742 (m), 702 (s), 688 (m), 505 (s), 490 (m). ${ }^{1} \mathrm{H}$ NMR (500 MHz, $\left.\mathrm{CDCl}_{3}, 298 \mathrm{~K}\right) \delta$ 7.80-7.69 (m, 4H, Ph), 7.46-7.34 (m, 6H, Ph), $4.62(\mathrm{dd}, 1 \mathrm{H}$, $J=3.6,9.6 \mathrm{~Hz}, \underline{\mathrm{H}}-6), 4.59(\mathrm{~s}, 1 \mathrm{H}, \underline{\mathrm{H}}-9), 4.01(\mathrm{dd}, 1 \mathrm{H}, J=3.7,-11.2 \mathrm{~Hz}, \underline{\mathrm{H}}-4 \mathrm{a})$ superimposed upon 3.98 (dd, $1 \mathrm{H}, J=2.3,-11.3 \mathrm{~Hz}, \underline{\mathrm{H}}-4 \mathrm{~b}$ ), 3.68 (ddd, $1 \mathrm{H}, J=2.4,3.5,9.6 \mathrm{~Hz}, \underline{\mathrm{H}}-5$ ), 3.52 (d, $1 \mathrm{H}, J=3.9 \mathrm{~Hz}, \mathrm{C}-6$ $\mathrm{OH}), 3.31\left(\mathrm{~s}, 3 \mathrm{H}, \mathrm{C}-9-\mathrm{OC}_{3}\right), 1.36\left(\mathrm{~s}, 3 \mathrm{H}, \mathrm{C}-8-\underline{\mathrm{H}}_{3}\right), 1.13\left(\mathrm{~s}, 3 \mathrm{H}, \mathrm{C}-8-\mathrm{C}_{3}\right), 1.08\left(\mathrm{~s}, 9 \mathrm{H},-\mathrm{C}\left(\mathrm{CH}_{3}\right)_{3}\right) \mathrm{ppm}$;

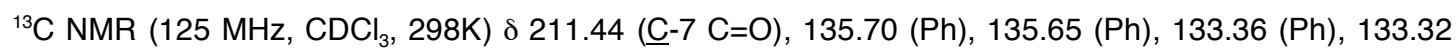

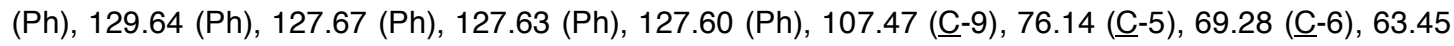
(ㅁ-4), $55.03\left(\mathrm{C}-9-\mathrm{O} \underline{\mathrm{C}} \mathrm{H}_{3}\right), 49.92(\underline{\mathrm{C}}-8), 26.75\left(-\mathrm{C}\left(\underline{\mathrm{CH}}_{3}\right)_{3}\right), 24.16\left(\mathrm{C}-8-\underline{\mathrm{C}} \mathrm{H}_{3}\right), 19.32\left(-\underline{\mathrm{C}}\left(\mathrm{CH}_{3}\right)_{3}\right) 18.65(\mathrm{C}-8-$ $\mathrm{CH}_{3}$ ) ppm. VG 70-SE Positive Ion FAB HRMS: Calcd. for $\mathrm{C}_{25} \mathrm{H}_{34} \mathrm{NaO}_{5} \mathrm{Si}(\mathrm{M}+\mathrm{Na})^{+}:$465.20731. Found: 465.20627. 


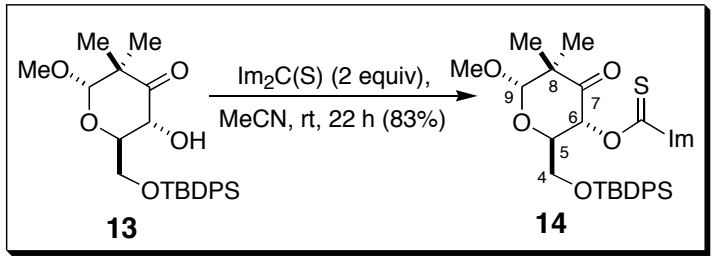

To a stirred solution of alcohol $13(18.64 \mathrm{~g}, 42.11 \mathrm{mmol})$ in dry MeCN (260 mL) at rt was added 1,1'thiocarbonyldiimidazole (15.0 g, $84.17 \mathrm{mmol}, 2.0$ equiv) in one portion. The reactants were stirred at rt for $22 \mathrm{~h}$ before being diluted with $\mathrm{Et}_{2} \mathrm{O}(200 \mathrm{~mL})$ and $\mathrm{H}_{2} \mathrm{O}(200 \mathrm{~mL})$. The organic layer was separated off and the aqueous phase was extracted with $\mathrm{Et}_{2} \mathrm{O}(2 \times 500 \mathrm{~mL})$. The combined organic extracts were dried over $\mathrm{MgSO}_{4}$, filtered, and concentrated in vacuo. Purification of the crude residue by $\mathrm{SiO}_{2}$ flash chromatography (gradient elution with 10:1 then 5:1 petrol-EtOAc) furnished $14(19.39 \mathrm{~g}, 83 \%)$ as shiny white crystals.

Data for 14: $[\alpha]_{\mathrm{D}}+91.0^{\circ}\left(c\right.$ 0.51, $\left.\mathrm{CH}_{2} \mathrm{Cl}_{2}\right)$; m.p. 163-166 ${ }^{\circ} \mathrm{C}$; IR (KBr) $3142(\mathrm{w}), 3072(\mathrm{~m}), 3044(\mathrm{w}), 2965$ (s), 2935 (s), 2900 (s), 2858 (s), 2832 (m), 1734 (s), 1703 (w), 1533 (m), 1488 (s), 1463 (s), 1429 (s), 1397 (s), 1350 (s), 1326 (s), 1250 (s), 1232 (s), 1191 (m), 1151 (s), 1117 (s), 1063 (s), 1025 (s), 942 (m), $920(\mathrm{~s}), 889(\mathrm{w}), 870(\mathrm{~m}), 853(\mathrm{w}), 828(\mathrm{~s}), 783(\mathrm{~s}), 740(\mathrm{~s}), 700(\mathrm{~s}), 686(\mathrm{~s}), 649(\mathrm{~m}), 621(\mathrm{~m}), 601$ (m), $583(\mathrm{~m}), 556(\mathrm{w}), 515$ (s), 488 (s), 427 (m). ${ }^{1} \mathrm{H}$ NMR $\left(500 \mathrm{MHz}, \mathrm{CDCl}_{3}, 298 \mathrm{~K}\right) \delta 8.23(\mathrm{~s}, 1 \mathrm{H}, \mathrm{N}-\mathrm{C} \underline{\mathrm{H}}-$ N), $7.70(\mathrm{~m}, 2 \mathrm{H}, \mathrm{Ph}), 7.56(\mathrm{~m}, 2 \mathrm{H}, \mathrm{Ph}), 7.53$ (m, 1H, Imid-CH), 7.48-7.14 (m, 6H, Ph), 7.02 (m, 1H, ImidCㅡㅏ), $6.56(\mathrm{~d}, 1 \mathrm{H}, J=10.0 \mathrm{~Hz}, \underline{\mathrm{H}}-6), 4.64$ (s, 1H, $\underline{\mathrm{H}}-9$ ), 4.19 (ddd, $1 \mathrm{H}, J=1.5,2.6,10.0 \mathrm{~Hz}, \underline{\mathrm{H}}-5$ ), 3.94 (dd, $1 \mathrm{H}, J=1.6,-11.7 \mathrm{~Hz}, \underline{\mathrm{H}}-4 \mathrm{a}), 3.79$ (dd, $1 \mathrm{H}, J=3.2,-11.7 \mathrm{~Hz}, \underline{\mathrm{H}}-4 \mathrm{~b}), 3.35$ (s, 3H, C-9-OC年), 1.47 (s, $\left.3 \mathrm{H}, \mathrm{C}-8-\underline{\mathrm{C}}_{3} \mathrm{ax}\right), 1.10$ (s, 3H, C-8- $\left.\underline{\mathrm{H}}_{3} \mathrm{eq}\right), 1.06$ (s, 9H, $\left.-\mathrm{C}\left(\mathrm{C}_{3}\right)_{3}\right) \mathrm{ppm} ;{ }^{13} \mathrm{C} \mathrm{NMR}(125 \mathrm{MHz}, \mathrm{CDCl} 3,298 \mathrm{~K})$

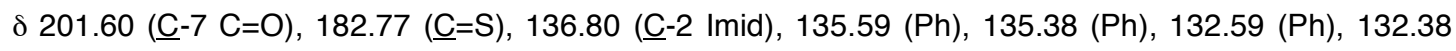

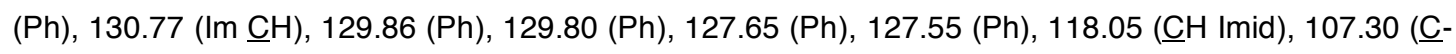

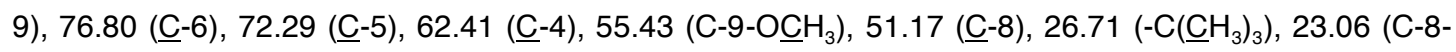
$\left.\underline{\mathrm{C}}_{3} \mathrm{ax}\right), 19.06\left(-\underline{\mathrm{C}}\left(\mathrm{CH}_{3}\right)_{3}\right), 18.49\left(\mathrm{C}-8-\underline{\mathrm{C}}_{3} \mathrm{eq}\right)$ ppm. VG 70-SE Positive Ion FAB HRMS: Calcd. for $\mathrm{C}_{29} \mathrm{H}_{36} \mathrm{~N}_{2} \mathrm{NaO}_{5} \mathrm{SSi}(\mathrm{M}+\mathrm{Na})^{+}:$575.20118. Found: 575.20162.

\section{Ketone 8 (SM-4-62)}

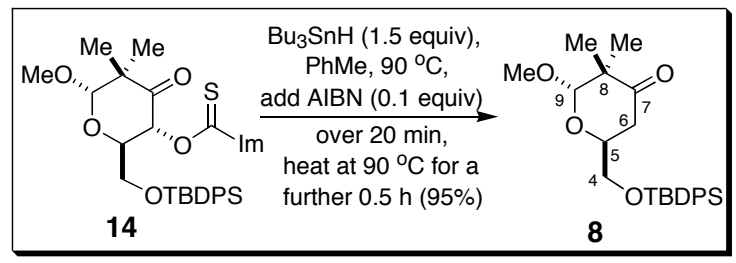

To a stirred solution of the O-thiocarbonylimidazolide $14(20.34 \mathrm{~g}, 36.80 \mathrm{mmol})$ in dry PhMe $(150 \mathrm{~mL})$ heated to $90{ }^{\circ} \mathrm{C}$ was added $\mathrm{Bu}_{3} \mathrm{SnH}$ (14.63 mL, $55.20 \mathrm{mmol}$, ca. 1.5 equiv). $\alpha$-Azobisisobutyronitrile (AIBN) (0.60 g, $3.68 \mathrm{mmol}, 0.1$ equiv) was then added in 4 equal portions at 5 min intervals. Following heating of the reaction mixture at $90{ }^{\circ} \mathrm{C}$ for $0.5 \mathrm{~h}$, and cooling to $\mathrm{rt}$, the solvent was removed in vacuo. 
Purification of the crude residue by $\mathrm{SiO}_{2}$ flash chromatography (gradient elution with 30:1 then 20:1 petrol-EtOAc as eluent) provided ketone 8 (14.9 g, 95\%) as a colorless oil.

Data for 8: $[\alpha]_{D}+26.8^{\circ}\left(c 0.658, \mathrm{CH}_{2} \mathrm{Cl}_{2}\right)$; IR (neat film) $3071(\mathrm{~m}), 3048(\mathrm{~m}), 2997(\mathrm{~m}), 2960(\mathrm{~s}), 2931(\mathrm{~s})$, 2892 (s), 2858 (s), 2832 (m), 1735 (m), 1719 (s), 1589 (m), 1474 (s), 1466 (s), 1462 (s), 1428 (s), 1391 (s), $1364(\mathrm{~s}), 1284(\mathrm{~m}), 1259(\mathrm{~m}), 1193(\mathrm{~m}), 1149$ (s), 1131 (s), 1114 (s), 1093 (s), 1047 (s), $1023(\mathrm{~m})$, 1003 (s), 978 (m), 824 (s), 797 (m), 742 (s), 703 (s), 690 (m), 623 (m), $616(\mathrm{~m}), 505$ (s), 490 (m). ${ }^{1} \mathrm{H}$

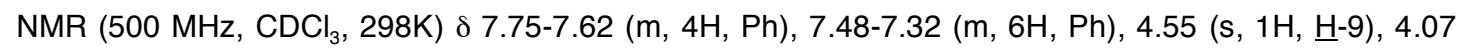
(ddd, $1 \mathrm{H}, J=3.8,7.8,11.6 \mathrm{~Hz}, \underline{\mathrm{H}}-5), 3.79$ (dd, $1 \mathrm{H}, J=3.8,10.8 \mathrm{~Hz}, \underline{\mathrm{H}}-4 \mathrm{a}), 3.73$ (dd, $1 \mathrm{H}, J=4.6,10.9$ $\mathrm{Hz}, \underline{\mathrm{H}}-4 \mathrm{~b}), 3.30$ (s, 3H, C-9-OC $\underline{H}_{3}$ ), 2.81 (dd, $1 \mathrm{H}, J=11.4,-14.9 \mathrm{~Hz}, \underline{\mathrm{H}}-6_{\mathrm{ax}}$ ), 2.20 (dd, $1 \mathrm{H}, J=3.4,-14.9$ $\left.\mathrm{Hz}, \underline{\mathrm{H}}-6_{\mathrm{eq}}\right), 1.26$ (s, 3H, C-8- $\left.\mathrm{C}_{3} \mathrm{ax}\right), 1.07$ (s, 9H, $\left.-\mathrm{C}\left(\mathrm{C}_{3}\right)_{3}\right), 1.05$ (s, 3H, C-8- $\left.\underline{\mathrm{H}}_{3} \mathrm{eq}\right) \mathrm{ppm} ;{ }^{13} \mathrm{C}$ NMR $(125$ $\left.\mathrm{MHz}, \mathrm{CDCl}_{3}, 298 \mathrm{~K}\right) \delta 210.42(\underline{\mathrm{C}}-7 \mathrm{C}=\mathrm{O}), 135.61(\mathrm{Ph}), 135.57(\mathrm{Ph}), 134.77(\mathrm{Ph}), 133.21(\mathrm{Ph}), 133.18$

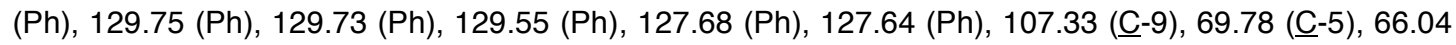
$(\underline{\mathrm{C}}-4), 55.02\left(\mathrm{C}-9-\mathrm{O}_{\mathrm{C}} \mathrm{H}_{3}\right), 49.83(\underline{\mathrm{C}}-8), 39.44(\underline{\mathrm{C}}-6), 26.75\left(-\mathrm{C}\left(\underline{\mathrm{C}} \mathrm{H}_{3}\right)_{3}\right), 24.35\left(\mathrm{C}-8-\underline{\mathrm{C}}_{3} \mathrm{ax}\right), 19.22$ ($\left.\underline{\mathrm{C}}\left(\mathrm{CH}_{3}\right)_{3}\right), 18.67\left(\mathrm{C}-8-\mathrm{CH}_{3} \mathrm{eq}\right)$ ppm. VG 70-SE Positive Ion FAB HRMS: Calcd. for $\mathrm{C}_{25} \mathrm{H}_{34} \mathrm{NaO}_{4} \mathrm{Si}$ $(\mathrm{M}+\mathrm{Na})^{+}:$449.21239. Found: 449.21304 .

\section{Alcohols 15 and 16 (SM-4-50A \& SM-4-50B)}

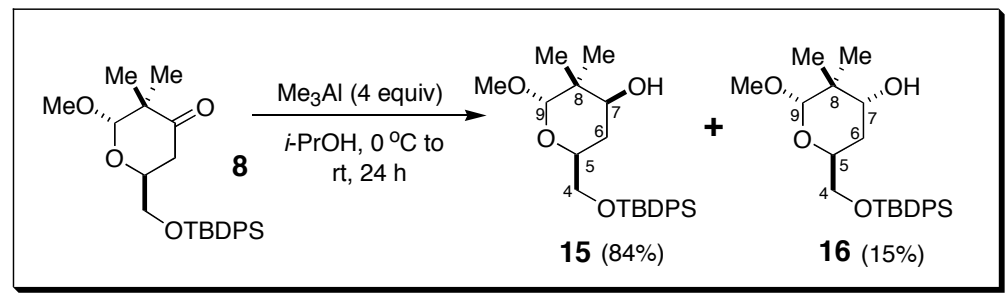

To a vigorously stirred solution of $i-\mathrm{PrOH}(700 \mathrm{~mL})$ at $0{ }^{\circ} \mathrm{C}$ was very cautiously added $\mathrm{Me}_{3} \mathrm{Al}(2.0 \mathrm{M}$ solution in hexanes, $100 \mathrm{~mL}, 200 \mathrm{mmol}, 4.0$ equiv) dropwise over $1 \mathrm{~h}$. To this chilled solution of $\mathrm{Al}(\mathrm{OPr}$ $I_{3}$ in $i-\mathrm{PrOH}$ was added a steady stream of a solution of the ketone $8(21.34 \mathrm{~g}, 50.02 \mathrm{mmol})$ in $i-\mathrm{PrOH}$ (555 mL) over approx. 30-40 min via cannula. Once the addition was complete, the reactants were warmed to $\mathrm{rt}$ where they were stirred for a further $24 \mathrm{~h}$. TLC analysis at this time revealed that two reduction products 15 and $\mathbf{1 6}$ had formed where the former predominated. The reaction mixture was recooled to $0{ }^{\circ} \mathrm{C}$, diluted with EtOAc $(500 \mathrm{~mL})$ and then carefully quenched with $10 \%$ aqueous Rochelle salt solution $(1.2 \mathrm{~L})$. The resulting mixture was stirred at $\mathrm{rt}$ for $1 \mathrm{~h}$ and then extracted with EtOAc $(3 \times 1$ $\mathrm{L})$. The combined organic layers were dried over $\mathrm{MgSO}_{4}$, filtered, and concentrated in vacuo. Purification of the residue by $\mathrm{SiO}_{2}$ flash chromatography with petrol-EtOAc (25:1) as eluent initially gave the undesired alcohol 16 (3.20 g, 15\%) as a very pale yellow oil; this was followed by desired alcohol 15 (18.08 $\mathrm{g}, 84 \%)$ which was obtained as a colorless oil. The undesired isomer could be recycled back into the synthesis by TPAP/NMO oxidation in $\mathrm{CH}_{2} \mathrm{Cl}_{2}$.

Data for 16: $[\alpha]_{\mathrm{D}}+37.8^{\circ}\left(c 0.506, \mathrm{CH}_{2} \mathrm{Cl}_{2}\right)$; IR (neat film) $3531(\mathrm{~m}), 3071(\mathrm{~m}), 3049(\mathrm{~m}), 2996(\mathrm{~m}), 2957$ (s), $2931(\mathrm{~s}), 2892(\mathrm{~s}), 2858(\mathrm{~s}), 1474(\mathrm{~m}), 1463(\mathrm{~m}), 1428(\mathrm{~s}), 1411(\mathrm{~m}), 1389(\mathrm{~m}), 1362(\mathrm{~m}), 1195(\mathrm{~m})$, 
1138 (s), 1111 (s), 1041 (s), 1014 (s), 973 (m), $824(\mathrm{~m}), 807$ (m), 742 (m), 704 (s), 612 (m), 506 (s), 491 (m). ${ }^{1} \mathrm{H}$ NMR (500 MHz, $\left.\mathrm{CDCl}_{3}, 298 \mathrm{~K}\right) \delta$ 7.75-7.64 (m, 4H, Ph), 7.46-7.31 (m, 6H, Ph), 4.33 (s, 1H, $\left.\underline{\mathrm{H}}-9\right)$, 3.99 (br m, $1 \mathrm{H}, \underline{\mathrm{H}}-5), 3.78-3.66(\mathrm{~m}, 2 \mathrm{H}, \underline{\mathrm{H}}-4 \mathrm{a}$ and $\underline{\mathrm{H}}-4 \mathrm{~b}), 3.53$ (narrow br s, $1 \mathrm{H}, \underline{\mathrm{H}}-7$ superimposed upon on a very br s, $1 \mathrm{H}, \mathrm{OH}), 3.36\left(\mathrm{~s}, 3 \mathrm{H}, \mathrm{C}-9-\mathrm{OCH}_{3}\right), 1.95$ (partially overlapped and broadened ddd, $1 \mathrm{H}, \underline{\mathrm{H}}-$ $\left.6_{\mathrm{ax}}\right), 1.60\left(\mathrm{~m}, 1 \mathrm{H}, \mathrm{J}_{6 \mathrm{ax}, 6 \mathrm{eq}}=13.9 \mathrm{~Hz}, \underline{\mathrm{H}}-6_{\mathrm{eq}}\right), 1.09\left(\mathrm{~s}, 3 \mathrm{H}, \mathrm{C}-8-\mathrm{C}_{3} \mathrm{ax}\right), 1.07\left(\mathrm{~s}, 9 \mathrm{H},-\mathrm{C}\left(\mathrm{C}_{3}\right)_{3}\right), 1.01(\mathrm{~s}, 3 \mathrm{H}$, $\mathrm{C}-8-\mathrm{CH}_{3}$ eq) ppm; ${ }^{13} \mathrm{C}$ NMR (125 MHz, $\left.\mathrm{CDCl}_{3}, 298 \mathrm{~K}\right) \delta 135.63(\mathrm{Ph}), 135.62(\mathrm{Ph}), 133.54(\mathrm{Ph}), 133.51$

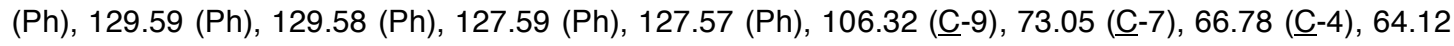

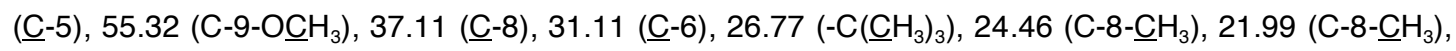
$19.20\left(\underline{\mathrm{C}}\left(\mathrm{CH}_{3}\right)_{3}\right)$ ppm. VG 70-SE Positive Ion FAB HRMS: Calcd. for $\mathrm{C}_{25} \mathrm{H}_{36} \mathrm{NaO}_{4} \mathrm{Si}(\mathrm{M}+\mathrm{Na})^{+}: 451.22804$. Found: 451.22622.

Data for 15: $[\alpha]_{\mathrm{D}}+44.4^{\circ}\left(c 0.54, \mathrm{CH}_{2} \mathrm{Cl}_{2}\right)$; IR (neat oil) $3424(\mathrm{~m}), 3071(\mathrm{~m}), 3049(\mathrm{~m}), 2932(\mathrm{~s}), 2879(\mathrm{~s})$, $2859(\mathrm{~s}), 1473(\mathrm{~m}), 1428(\mathrm{~m}), 1389(\mathrm{~m}), 1362(\mathrm{~m}), 1261(\mathrm{~m}), 1187(\mathrm{~m}), 1113(\mathrm{~s}), 1052(\mathrm{~s}), 1029(\mathrm{~s}), 987$ (m), $977(\mathrm{~m}), 938(\mathrm{~m}), 824(\mathrm{~m}), 803(\mathrm{~m}), 758(\mathrm{~m}), 742(\mathrm{~m}), 704(\mathrm{~s}), 615(\mathrm{~m}), 505(\mathrm{~s}), 490(\mathrm{~m}) .{ }^{1} \mathrm{H}$ NMR $\left(500 \mathrm{MHz}, \mathrm{CDCl}_{3}, 298 \mathrm{~K}\right) \delta$ 7.75-7.62 (m, 4H, Ph), 7.46-7.32 (m, 6H, Ph), 4.25 (s, $\left.1 \mathrm{H}, \underline{\mathrm{H}}-9\right), 3.88$ (dd, $1 \mathrm{H}$, $J=5.0,11.7 \mathrm{~Hz}, \underline{\mathrm{H}}-7), 3.82(\mathrm{~m}, 1 \mathrm{H}, \underline{\mathrm{H}}-5), 3.72(\mathrm{dd}, 1 \mathrm{H}, J=5.4,-10.5 \mathrm{~Hz}, \underline{\mathrm{H}}-4 \mathrm{a}), 3.65$ (dd, $1 \mathrm{H}, J=4.7$, -

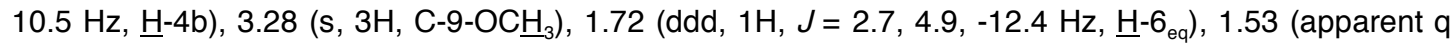
but actually an overlapped ddd, $\left.1 \mathrm{H}, J=12.0,12.1,-12.0 \mathrm{~Hz}, \underline{\mathrm{H}}-6_{\mathrm{ax}}\right), 1.46(\mathrm{~s}, 1 \mathrm{H}, \mathrm{OH}), 1.06(\mathrm{~s}, 9 \mathrm{H},-$ $\left.\mathrm{C}\left(\mathrm{CH}_{3}\right)_{3}\right), 1.02\left(\mathrm{~s}, 3 \mathrm{H}, \mathrm{C}-8-\mathrm{CH}_{3}\right), 0.94$ (s, 3H, C-8- $\left.\mathrm{CH}_{3}\right)$ ppm; ${ }^{33} \mathrm{C}$ NMR $\left(125 \mathrm{MHz}, \mathrm{CDCl}_{3}, 298 \mathrm{~K}\right) \delta 135.63$

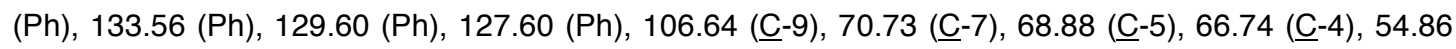

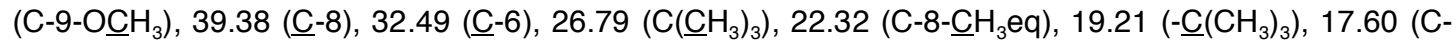
8- $\underline{\mathrm{CH}}_{3} \mathrm{ax}$ ) ppm. VG 70-SE Positive Ion FAB HRMS: Calcd. for $\mathrm{C}_{25} \mathrm{H}_{36} \mathrm{NaO}_{4} \mathrm{Si}(\mathrm{M}+\mathrm{Na})^{+}: 451.22804$. Found: 451.22740 .

\section{PMB Ether 26 (SM-8-62)}

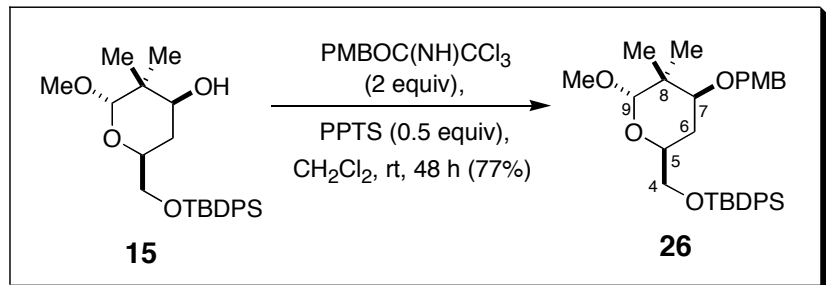

To a solution of alcohol $15(14.73 \mathrm{~g}, 34.36 \mathrm{mmol})$ in dry $\mathrm{CH}_{2} \mathrm{Cl}_{2}(150 \mathrm{~mL})$ at rt, under $\mathrm{N}_{2}$ was added a solution of $p$-methoxybenzyltrichloroacetimidate (freshly prepared) (19.42 g, $68.73 \mathrm{mmol}, 2.0$ equiv) in dry $\mathrm{CH}_{2} \mathrm{Cl}_{2}(22 \mathrm{~mL})$ as a slow stream via cannula. After $5 \mathrm{~min}$, pyridinium $p$-toluenesulfonate (PPTS) (4.32 g, $17.19 \mathrm{mmol}, 0.5$ equiv) was then added in one portion. The reactants were stirred at rt for $48 \mathrm{~h}$ and then quenched with solid $\mathrm{NaHCO}_{3}(3.0 \mathrm{~g})$ followed by saturated aqueous $\mathrm{NaHCO}_{3}$ solution (150 $\mathrm{mL})$. The resulting mixture was diluted with $\mathrm{CH}_{2} \mathrm{Cl}_{2}(500 \mathrm{~mL})$. The organic layer was separated, and the aqueous phase was extracted with $\mathrm{CH}_{2} \mathrm{Cl}_{2}(3 \times 500 \mathrm{~mL})$. The combined organic extracts were dried over $\mathrm{MgSO}_{4}$, filtered, and concentrated in vacuo. The crude residue was purified by $\mathrm{SiO}_{2}$ flash chromatography with petrol:EtOAc (15:1) as eluent, to give the p-methoxybenzyl ether 26 (14.5 g, 77\%) as a colorless oil. Further elution with petrol:EtOAc $(10: 1$ then $5: 1)$ as eluent, allowed the unconsumed starting material $15(2.83 \mathrm{~g}, 19 \%)$ to be recovered which was thereafter recycled into the synthesis. 
Data for 26: $[\alpha]_{\mathrm{D}}+65.2^{\circ}\left(c 0.63, \mathrm{CH}_{2} \mathrm{Cl}_{2}\right)$; IR (neat film) $3071(\mathrm{w}), 3048(\mathrm{w}), 2931(\mathrm{~s}), 2858(\mathrm{~s}), 2833(\mathrm{~m})$, $1612(\mathrm{~m}), 1587(\mathrm{w}), 1513(\mathrm{~s}), 1474(\mathrm{~m}), 1465(\mathrm{~m}), 1428(\mathrm{~m}), 1388(\mathrm{~m}), 1361(\mathrm{~m}), 1302(\mathrm{w}), 1248(\mathrm{~s})$, $1186(\mathrm{~m}), 1172(\mathrm{~m}), 1113$ (s), 1106 (s), 1082 (s), 1057 (s), 1035 (s), $1009(\mathrm{~m}), 998(\mathrm{~m}), 976(\mathrm{~m}), 934$ (m), $824(\mathrm{~m}), 809(\mathrm{~m}), 742(\mathrm{~m}), 703(\mathrm{~s}), 690(\mathrm{~m}), 615(\mathrm{~m}), 504(\mathrm{~s}), 492(\mathrm{~m}) .{ }^{1} \mathrm{H}$ NMR $\left(500 \mathrm{MHz}, \mathrm{CDCl}_{3}\right.$, 298K) $\delta$ 7.77-7.64 (m, 4H, Ph), 7.48-7.32 (m, 6H, Ph), 7.26 (d, 2H, J=8.6 Hz, Ph of PMP group), 6.88 (d, $2 \mathrm{H}, J=8.6 \mathrm{~Hz}$, Ph of PMP group), $4.54\left(\mathrm{~d}, 1 \mathrm{H}, J=-11.5 \mathrm{~Hz}, \underline{\mathrm{H}}_{2} \mathrm{PMP}\right), 4.40$ (d, $1 \mathrm{H}, J=-11.5 \mathrm{~Hz}$, $\mathrm{C}_{2} \mathrm{PMP}$ ), 4.23 (s, $\left.1 \mathrm{H}, \underline{\mathrm{H}}-9\right), 3.80$ (s, 3H, $-\mathrm{OC}_{\mathrm{H}_{3}}$ of PMP group), $3.78(\mathrm{~m}, 1 \mathrm{H}, \underline{\mathrm{H}}-5), 3.75$ (dd, $1 \mathrm{H}, J=5.4$, $-10.2 \mathrm{~Hz}, \underline{\mathrm{H}}-4 \mathrm{a}), 3.66$ (dd, $1 \mathrm{H}, J=4.4,-10.1 \mathrm{~Hz}, \underline{\mathrm{H}}-4 \mathrm{~b}), 3.61$ (dd, $1 \mathrm{H}, J=4.8,11.7 \mathrm{~Hz}, \underline{\mathrm{H}}-7$ ), 3.29 (s, 3H, $\mathrm{C}-9-\mathrm{OCH}_{3}$ ), 1.81 (overlapped ddd, $1 \mathrm{H}, J=2.4,4.7,-12.6 \mathrm{~Hz}, \underline{\mathrm{H}}-6_{\mathrm{eq}}$ ), 1.48 (apparent q but actually an overlapped ddd, $\left.1 \mathrm{H}, J=11.8,11.9,-12.1 \mathrm{~Hz}, \underline{\mathrm{H}}-6_{\mathrm{ax}}\right), 1.08$ (s, 9H, C( $\left.\left.\mathrm{C}_{3}\right)_{3}\right), 1.04$ (s, 3H, C-8-C $\left.\underline{H}_{3} \mathrm{ax}\right)$, 1.00 (s, 3H, C-8- $\underline{H}_{3}$ eq) ppm; ${ }^{13} \mathrm{C}$ NMR (125 MHz, $\left.\mathrm{CDCl}_{3}, 298 \mathrm{~K}\right) \delta 158.93(\mathrm{Ph}), 135.65(\mathrm{Ph}), 133.62$

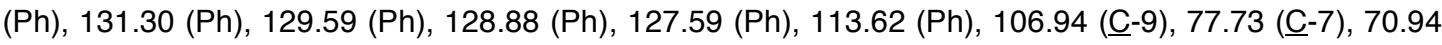

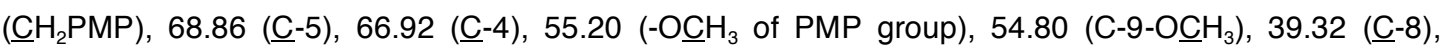
$29.38(\underline{\mathrm{C}}-6), 26.81\left(-\mathrm{C}\left(\underline{\mathrm{CH}}_{3}\right)_{3}\right), 22.80\left(\mathrm{C}-8-\underline{\mathrm{CH}}_{3}\right), 19.22\left(\underline{\mathrm{C}}\left(\mathrm{CH}_{3}\right)_{3}\right), 18.71\left(\mathrm{C}-8-\underline{\mathrm{CH}}_{3}\right)$ ppm. VG 70-SE Positive lon FAB HRMS: Calcd. for $\mathrm{C}_{33} \mathrm{H}_{44} \mathrm{NaO}_{5} \mathrm{Si}(\mathrm{M}+\mathrm{Na})^{+}: 571.28556$. Found: 571.28591.

\section{Alcohol 17 (SM-8-67)}

$\underset{26}{\frac{\substack{(1.2 \text { equiv), THF, } \\ \mathrm{rt}, 2.5 \mathrm{~h}(98 \%)}}{\text { OTBDPS }}}$

To a well-stirred a solution of the p-methoxybenzyl ether $26(14.5 \mathrm{~g}, 26.42 \mathrm{mmol})$ in dry THF $(88 \mathrm{~mL})$ was added tetra- $n$-butylammonium fluoride (1.0 M solution in THF, $31.7 \mathrm{~mL}, 31.7 \mathrm{mmol}, 1.2$ equiv) over $3 \mathrm{~min}$. The reactants were stirred for $2.5 \mathrm{~h}$ at $\mathrm{rt}$ whereafter the solvent was removed in vacuo. The crude residue was purified by $\mathrm{SiO}_{2}$ flash chromatography using petrol:EtOAc (5:1 to 1:1) as eluent. The pure alcohol $17(8.01 \mathrm{~g}, 98 \%)$ was isolated as a colorless oil.

Data for 17: $[\alpha]_{D}+122.1^{\circ}\left(c 0.516, \mathrm{CH}_{2} \mathrm{Cl}_{2}\right)$; IR (neat film) 3452 (m br), $2936(\mathrm{~s}), 2911$ (s), 2877 (s), 2834 (m), 1612 (s), $1586(\mathrm{w}), 1514(\mathrm{~s}), 1465(\mathrm{~m}), 1388(\mathrm{~m}), 1362(\mathrm{~m}), 1302(\mathrm{~m}), 1248$ (s), $1185(\mathrm{~m}), 1173$ (m), 1159 (w), 1112 (s), 1057 (s), 1033 (s), 974 (m), 934 (m), 821 (m), 757 (m), 583 (w). ${ }^{1} \mathrm{H}$ NMR (500 $\mathrm{MHz}_{\mathrm{CDCl}}, 298 \mathrm{~K}$ ) $\delta 7.22$ (m, 2H, PMP), 6.84 (d, 2H, PMP), 4.52 (d, $1 \mathrm{H}, J=-11.4 \mathrm{~Hz}, \mathrm{CH}_{2} \mathrm{PMP}$ ), 4.36 (d, $\left.1 \mathrm{H}, J=-11.4 \mathrm{~Hz}, \underline{\mathrm{H}}_{2} \mathrm{PMP}\right), 4.21$ (s, $\left.1 \mathrm{H}, \underline{\mathrm{H}}-9\right), 3.78(\mathrm{~m}, 1 \mathrm{H}, \underline{\mathrm{H}}-7), 3.77$ (s, 3H, OC$\underline{H}_{3}$ of PMB group), 3.66-3.52 (m, 3H, $\underline{H}-5, \underline{H}-4 a$ and $\underline{H}-4 b), 3.28$ (s, 3H, C-9-OC $\left.\underline{H}_{3}\right), 2.22$ (dd, $\left.1 \mathrm{H}, J=5.4,7.4 \mathrm{~Hz}, \mathrm{OH}\right), 1.73$ (ddd, $1 \mathrm{H}, J=2.8,4.8,-12.5 \mathrm{~Hz}, \underline{\mathrm{H}}-6_{\text {eq }}$ ) 1.49 (apparent q that is actually an overlapped ddd, $1 \mathrm{H}, J=$ $11.9,12.2,-12.3 \mathrm{~Hz}, \underline{\mathrm{H}}-\mathrm{G}_{\mathrm{ax}}$ ), 1.00 (s, 3H, C-8- $\underline{\mathrm{H}}_{3} \mathrm{eq}$ ), 0.96 (s, 3H, C-8- $\left.\underline{\mathrm{H}}_{3} \mathrm{ax}\right) \mathrm{ppm} ;{ }^{13} \mathrm{C} \mathrm{NMR}(125 \mathrm{MHz}$,

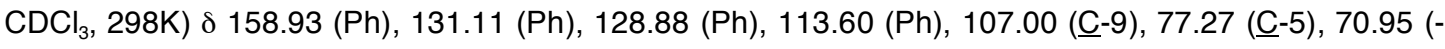

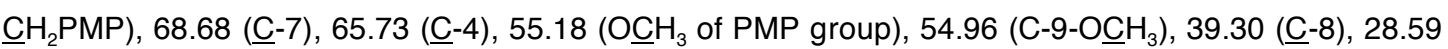
(ㅡ-6), $22.73\left(\mathrm{C}-8-\underline{\mathrm{CH}}_{3} \mathrm{eq}\right), 18.61\left(\mathrm{C}-8-\underline{\mathrm{CH}}_{3} \mathrm{ax}\right)$ ppm. VG 70-SE Positive Ion FAB HRMS: Calcd. for $\mathrm{C}_{17} \mathrm{H}_{26} \mathrm{NaO}_{5}(\mathrm{M}+\mathrm{Na})^{+}:$333.16778. Found: 333.16742. 
Alkene 20 (SM-8-72)

\section{Part 1. Synthesis of Aldehyde 18}

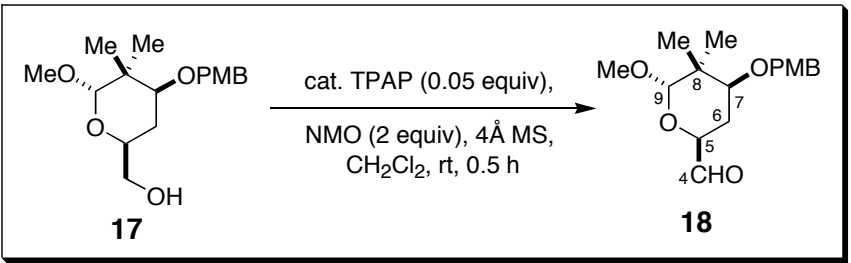

To a stirred solution of alcohol $17(8.01 \mathrm{~g}, 25.81 \mathrm{mmol})$, powdered $4 \AA$ molecular sieves $(10.0 \mathrm{~g})$ and $N$ methyl morpholine $\mathrm{N}$-oxide $\left(6.06 \mathrm{~g}, 51.73 \mathrm{mmol}, 2.0\right.$ equiv) in dry $\mathrm{CH}_{2} \mathrm{Cl}_{2}(200 \mathrm{~mL})$ at rt was added tetra$n$-propylammonium perruthenate (TPAP) $(0.45 \mathrm{~g}, 1.28 \mathrm{mmol}, 0.05$ equiv) in one portion. After $30 \mathrm{~min}$ of stirring at $\mathrm{rt}$, the reaction mixture was filtered through a pad of Celite and the filtrate concentrated in vacuo. The oily black residue 18 (8.0 g, crude) was then used without further purification directly for the next step.

Part 2. The Kocienski-Julia Olefination of Aldehyde 18 with 19 to Obtain Alkene 20

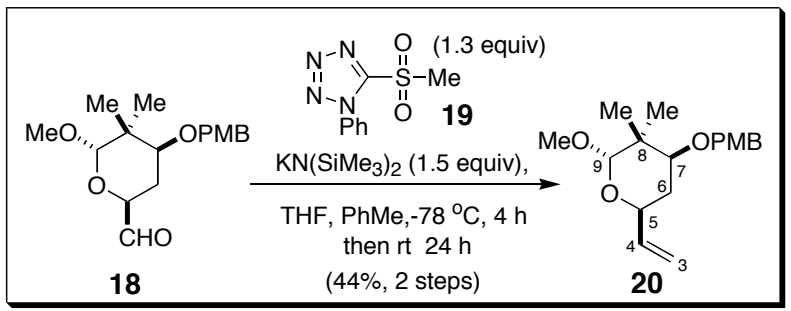

To a stirred $-78{ }^{\circ} \mathrm{C}$ solution of the unpurified aldehyde 18 (ca. $8.0 \mathrm{~g}, 25.81 \mathrm{mmol}$ ) and $\mathrm{N}$ phenyltetrazolylmethylsulfone $19(7.56 \mathrm{~g}, 33.71 \mathrm{mmol}, 1.3$ equiv) in dry THF (80 $\mathrm{mL})$ was added potassium bis(trimethylsilyl)amide $(0.5 \mathrm{M}$ solution in PhMe, $77.8 \mathrm{~mL}, 38.9 \mathrm{mmol}, 1.5$ equiv) dropwise over $10 \mathrm{~min}$. The reactants were stirred at $-78^{\circ} \mathrm{C}$ for $4 \mathrm{~h}$ and then allowed to warm to $\mathrm{rt}$ where they were maintained for $24 \mathrm{~h}$. The reaction mixture was then diluted with $\mathrm{Et}_{2} \mathrm{O}(200 \mathrm{~mL})$ and $\mathrm{H}_{2} \mathrm{O}(200 \mathrm{~mL})$, transferred to a separatory funnel and shaken. After the organic layer was separated, the aqueous layer was further extracted with $\mathrm{Et}_{2} \mathrm{O}(3 \times 200 \mathrm{~mL})$, and the combined ethereal extracts were dried over $\mathrm{MgSO}_{4}$, filtered, and concentrated in vacuo. Purification of the crude residue by $\mathrm{SiO}_{2}$ flash chromatography using petrol:EtOAc (20:1) as eluent, provided alkene 20 (3.48 g, 44\% for the 2 steps) as colorless runny oil.

Data for 20: $[\alpha]_{D}+122.4^{\circ}\left(c 0.5, \mathrm{CH}_{2} \mathrm{Cl}_{2}\right)$; IR (neat film) 2987 (m), 2947 (s), 2935 (s), 2909 (s), 2877 (s), $2833(\mathrm{~m}), 1613(\mathrm{~m}), 1586(\mathrm{w}), 1514(\mathrm{~s}), 1465(\mathrm{~m}), 1390(\mathrm{w}), 1361(\mathrm{~m}), 1301(\mathrm{~m}), 1248(\mathrm{~s}), 1224(\mathrm{w})$, 1184 (m), 1175 (m), 1105 (s), 1076 (s), 1041 (s), 1001 (w), 929 (m), 822 (m). ${ }^{1} \mathrm{H}$ NMR (500 MHz, CDCl ${ }_{3}$,

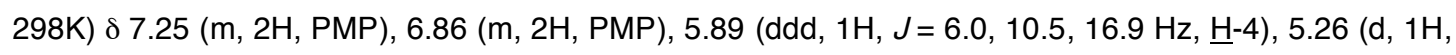
$J=17.3 \mathrm{~Hz}, \underline{\mathrm{H}}-3 \mathrm{a}), 5.12$ (d, $1 \mathrm{H}, J=10.5 \mathrm{~Hz}, \underline{\mathrm{H}}-3 \mathrm{~b}), 4.55$ (d, 1H, $\left.J=-11.5 \mathrm{~Hz}, \underline{\mathrm{H}}_{2} \mathrm{PMP}\right), 4.38(\mathrm{~d}, 1 \mathrm{H}, J=$ $\left.-11.5 \mathrm{~Hz}, \mathrm{CH}_{2} \mathrm{PMP}\right), 4.23(\mathrm{~s}, 1 \mathrm{H}, \underline{\mathrm{H}}-9), 4.16(\mathrm{~m}, 1 \mathrm{H}, \underline{\mathrm{H}}-5), 3.79\left(\mathrm{~s}, 3 \mathrm{H}, \mathrm{OCH}_{3}\right.$ of PMP group), $3.62(\mathrm{~d}, 1 \mathrm{H}$, 
$J=4.8,11.5 \mathrm{~Hz}, \underline{\mathrm{H}}-7), 3.30\left(\mathrm{~s}, 3 \mathrm{H}, \mathrm{C}-9-\mathrm{OC}_{3}\right), 1.90$ (ddd, $\left.1 \mathrm{H}, J=2.9,4.7,-12.7 \mathrm{~Hz}, \underline{\mathrm{H}}-6_{\mathrm{eq}}\right), 1.51$

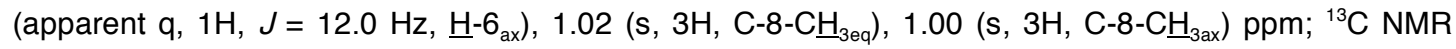

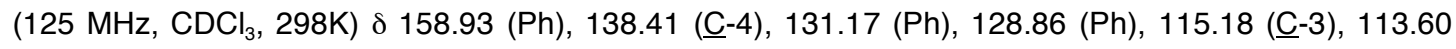

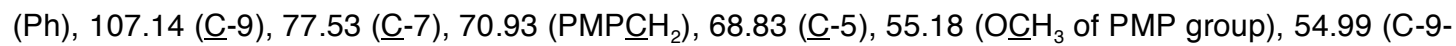

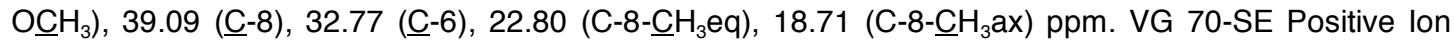
FAB HRMS: Calcd. for $\mathrm{C}_{18} \mathrm{H}_{26} \mathrm{NaO}_{4}(\mathrm{M}+\mathrm{Na})^{+}$: 329.17287. Found: 329.17238 .

\section{Alcohol 7 (SM-8-73)}

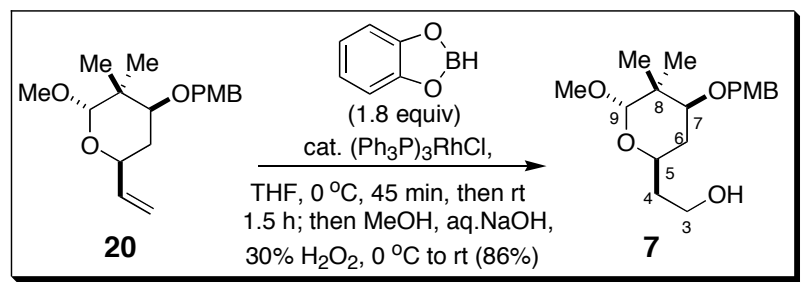

To a stirred solution of alkene $20(1.0 \mathrm{~g}, 3.26 \mathrm{mmol})$ in dry THF $(27 \mathrm{~mL})$ under $\mathrm{N}_{2}$ at $\mathrm{rt}$ was added Wilkinson's catalyst $\left(\mathrm{Ph}_{3} \mathrm{P}\right)_{3} \mathrm{RhCl}(30.2 \mathrm{mg}, 0.033 \mathrm{mmol}, 0.01$ equiv) in one portion. The reaction mixture was cooled to $0{ }^{\circ} \mathrm{C}$ and catecholborane $(1.0 \mathrm{M}$ solution in THF, $5.88 \mathrm{~mL}, 5.88 \mathrm{mmol}, 1.8$ equiv) was added dropwise over $1 \mathrm{~min}$. The contents of the flask were stirred at $0{ }^{\circ} \mathrm{C}$ for $45 \mathrm{~min}$ and then warmed to rt where they were stirred for a further $1.5 \mathrm{~h}$. After this time, TLC analysis indicated that a slowermoving product had formed. The reaction mixture was cooled to $0{ }^{\circ} \mathrm{C}$ and quenched by slow addition of $\mathrm{MeOH}(25 \mathrm{~mL}), 2 \mathrm{M}$ aqueous $\mathrm{NaOH}(25 \mathrm{~mL})$ and $\mathrm{H}_{2} \mathrm{O}_{2}\left(30 \%\right.$ in $\left.\mathrm{H}_{2} \mathrm{O}, 25 \mathrm{~mL}\right)$. The resulting mixture was stirred at $\mathrm{rt}$ for $1 \mathrm{~h}$ and then extracted with EtOAc $(3 \times 100 \mathrm{~mL})$. The combined organic extracts were washed with saturated aqueous $\mathrm{NaHCO}_{3}$ solution $(2 \times 100 \mathrm{~mL})$, dried over $\mathrm{MgSO}_{4}$, filtered, and concentrated in vacuo. The crude residue was purified by $\mathrm{SiO}_{2}$ flash chromatography (gradient elution with $5: 1$ then $4: 1$ petrol-EtOAc as eluent) with alcohol $7(0.90 \mathrm{~g}, 86 \%)$ being obtained as a colorless oil.

Data for 7: $[\alpha]_{\mathrm{D}}+121.3^{\circ}\left(c\right.$ 0.642, $\mathrm{CH}_{2} \mathrm{Cl}_{2}$ ); IR (neat film) 3440 (br s), 2944 (s), 2876 (s), 2834 (s), 1613 (s), 1586 (m), 1514 (s), 1466 (s), 1451 (s), 1391 (s), 1360 (s), 1302 (s), 1248 (s), 1184 (s), 1175 (s), $1153(\mathrm{~m}), 1107$ (s), 1087 (s), 1058 (s), 1037 (s), 976 (s), 932 (s), 822 (s), 734 (m), 629 (m), 581 (m), $517(\mathrm{~m}) .{ }^{1} \mathrm{H}$ NMR $\left(500 \mathrm{MHz}, \mathrm{CDCl}_{3}, 298 \mathrm{~K}\right) \delta 7.21$ (d, 2H, J=8.7 Hz, PMP), 6.83 (d, 2H, J = 8.7 Hz,

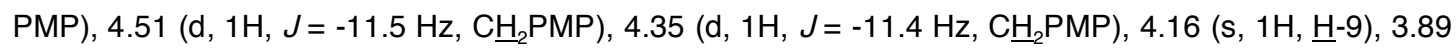
(m, $1 \mathrm{H}, \underline{\mathrm{H}}-5), 3.76$ (s, 3H, OC$\underline{\mathrm{H}}_{3}$ of PMP group) superimposed upon 3.75 (m, 2H, $\underline{\mathrm{H}}-3 \mathrm{a}$ and $\left.\underline{\mathrm{H}}-3 \mathrm{~b}\right), 3.56$

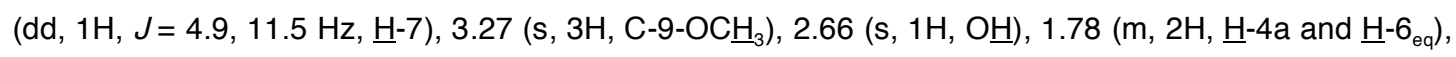
$1.69(\mathrm{~m}, 1 \mathrm{H}, \underline{\mathrm{H}}-4 \mathrm{~b}), 1.47$ (apparent q that actually is an overlapped ddd, $1 \mathrm{H}, J=11.9,12.0,-12.0 \mathrm{~Hz}, \underline{\mathrm{H}}-$ $\left.6_{\mathrm{ax}}\right), 0.99$ (s, 3H, C-8- $\left.\mathrm{CH}_{3} \mathrm{eq}\right), 0.96$ (s, 3H, C-8- $\left.\underline{\mathrm{H}}_{3} \mathrm{ax}\right) \mathrm{ppm} ;{ }^{13} \mathrm{C} \mathrm{NMR}\left(125 \mathrm{MHz}, \mathrm{CDCl}_{3}, 298 \mathrm{~K}\right) \delta 158.87$

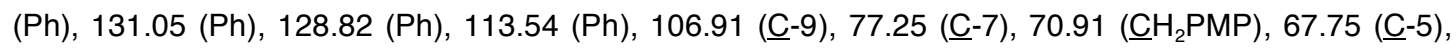

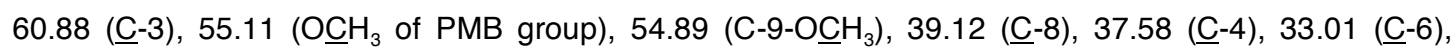
$22.69\left(\mathrm{C}-8-\underline{\mathrm{C}} \mathrm{H}_{3}\right), 18.65\left(\mathrm{C}-8-\underline{\mathrm{CH}}_{3}\right)$ ppm. VG 70-SE Positive Ion FAB HRMS: Calcd. for $\mathrm{C}_{18} \mathrm{H}_{27} \mathrm{NaO}_{5}(\mathrm{M}-$ $\mathrm{H}+\mathrm{Na})^{+}:$346.17561. Found: 346.17690 . 
Triol 21 (SM-8-105)

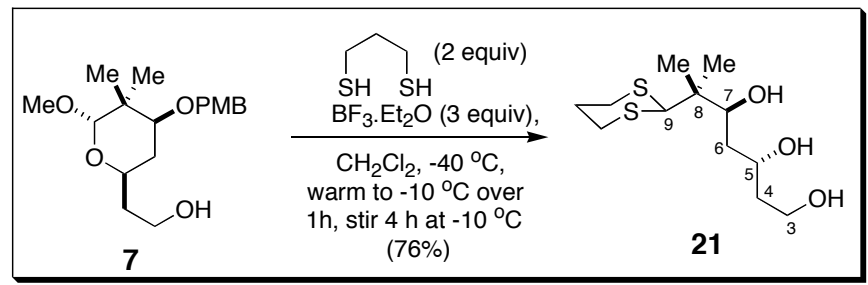

To a stirred solution of alcohol $7(2.86 \mathrm{~g}, 8.82 \mathrm{mmol})$ in dry $\mathrm{CH}_{2} \mathrm{Cl}_{2}(10 \mathrm{~mL})$ under $\mathrm{N}_{2}$ at rt was added 1,3-propanedithiol (1.77 mL, $17.63 \mathrm{mmol}, 2.0$ equiv). The reaction mixture was cooled to $-40{ }^{\circ} \mathrm{C}$ before $\mathrm{BF}_{3} . \mathrm{Et}_{2} \mathrm{O}$ (3.32 mL, $26.20 \mathrm{mmol}$, ca. 3.0 equiv) was added dropwise over $2 \mathrm{~min}$. The mixture was then held between $-40^{\circ} \mathrm{C}$ and $-10^{\circ} \mathrm{C}$ for $1 \mathrm{~h}$ and kept at $-10{ }^{\circ} \mathrm{C}$ for a further $4 \mathrm{~h}$. At the end of this time, TLC analysis indicated that the reaction was complete. The cooling bath was removed and the reaction mixture was quenched by adding solid $\mathrm{NaHCO}_{3}(2.86 \mathrm{~g})$ and saturated aqueous $\mathrm{NaHCO}_{3}(10 \mathrm{~mL})$. After extraction with $\mathrm{CH}_{2} \mathrm{Cl}_{2}(3 \times 20 \mathrm{~mL})$, the combined organic extracts were dried over $\mathrm{MgSO}_{4}$, filtered and evaporated in vacuo. Purification of the crude residue by $\mathrm{SiO}_{2}$ flash chromatography, initially with petrolEtOAc (1:1) to remove faster-moving impurities, and then $(3: 1$ to $5: 1)$ EtOAc-petrol furnished the desired triol $21(1.88 \mathrm{~g}, 76 \%)$ as a white crystalline solid.

Data for 21: $[\alpha]_{D}-35.6^{\circ}$ (c 0.542, MeOH); m.p. 100-102 ㅇ; IR (KBr) 3260 (s), 2953 (s), 2924 (s), 2904 (s), $2881(\mathrm{~s}), 1467(\mathrm{~m}), 1418(\mathrm{~m}), 1391(\mathrm{~m}), 1368(\mathrm{~m}), 1327(\mathrm{w}), 1297(\mathrm{w}), 1274(\mathrm{w}), 1237(\mathrm{w}), 1180$ (w), 1096 (s), 1082 (s), 1067 (s), 1045 (s), 1031 (m), 997 (m), 960 (m), 907 (w), 891 (w), $834(w), 780$ (m), 665 (w), 575 (w). ${ }^{1} \mathrm{H}$ NMR (500 MHz, $\left.\mathrm{CD}_{3} \mathrm{OD}, 298 \mathrm{~K}\right) \delta 4.34$ (s, 1H, $\left.\underline{\mathrm{H}}-9\right), 4.06$ (dd, $1 \mathrm{H}, J=1.7,10.5$ $\mathrm{Hz}, \underline{\mathrm{H}}-7), 3.94$ (m 1H, $\underline{\mathrm{H}}-5), 3.70$ (t, 2H, J=6.5 Hz, $\underline{\mathrm{H}}-3 \mathrm{a}, \underline{\mathrm{H}}-3 \mathrm{~b}), 2.95$ (2 x dd, each $1 \mathrm{H}$, superimposed upon one another, $\left.2 \times-\underline{C}_{2 a x} \mathrm{~S}-\right), 2.83\left(\mathrm{~m}, 2 \mathrm{H}, 2 \times-\underline{\mathrm{H}}_{2 \mathrm{eq}} \mathrm{S}-\right), 2.08\left(\mathrm{~m}, 1 \mathrm{H},-\mathrm{SCH}_{2}-\mathrm{C}_{2 \mathrm{eq}}-\mathrm{CH}_{2} \mathrm{~S}-\right), 1.76(\mathrm{~m}$, $1 \mathrm{H},-\mathrm{SCH}_{2}-\mathrm{CH}_{2 a x}-\mathrm{CH}_{2} \mathrm{~S}-$ ), 1.69 (apparent q, $2 \mathrm{H}, J=6.5 \mathrm{~Hz}, \underline{\mathrm{H}}-4 \mathrm{a}$ and $\left.\underline{\mathrm{H}}-4 \mathrm{~b}\right), 1.55$ (ddd, $1 \mathrm{H}, J=1.8,9.3$, $14.1 \mathrm{~Hz}, \underline{\mathrm{H}}-6 \mathrm{a}), 1.46$ (ddd, $1 \mathrm{H}, J=2.8,10.6,-13.3 \mathrm{~Hz}, \underline{\mathrm{H}}-6 \mathrm{~b}), 1.03$ (s, 3H, C-8-C $\underline{H}_{3}$ ), 0.97 (s, 3H, C-8-

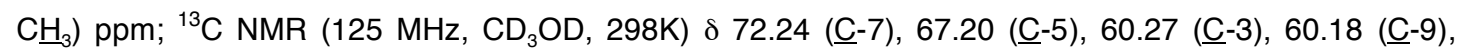

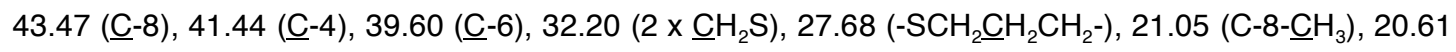
$\left(\mathrm{C}-8-\underline{\mathrm{C}} \mathrm{H}_{3}\right)$ ppm. VG 70-SE Positive Ion FAB HRMS: Calcd. for $\mathrm{C}_{12} \mathrm{H}_{24} \mathrm{NaO}_{3} \mathrm{~S}_{2}(\mathrm{M}+\mathrm{Na})^{+}$: 303.10645 . Found: 303.10802 .

\section{Diol 22 (SM-8-106)}

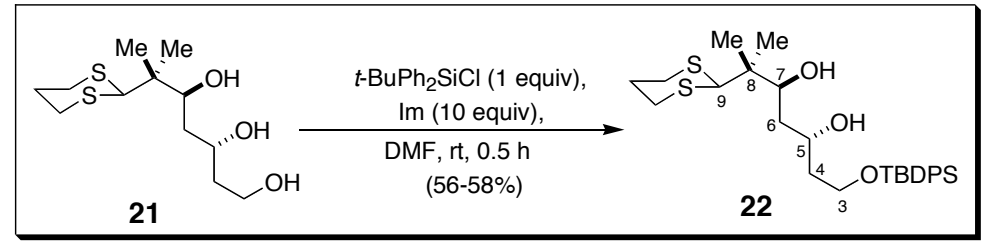

To a stirred solution of triol 21 (1.09 g, $3.89 \mathrm{mmol})$, imidazole (2.65 g, $38.87 \mathrm{mmol}, 10.0 \mathrm{equiv})$ in dry DMF $(22 \mathrm{~mL})$ was added tert-butylchlorodiphenylsilane $(1.01 \mathrm{ml}, 3.89 \mathrm{mmol}, 1.0$ equiv) slowly and dropwise. After $0.5 \mathrm{~h}$, the reactants were quenched with solid $\mathrm{NaHCO}_{3}$ followed by a saturated aqueous $\mathrm{NaHCO}_{3}$. The resulting mixture was extracted with $\mathrm{Et}_{2} \mathrm{O}(3 \times 20 \mathrm{~mL})$. The combined organic extracts were washed with $\mathrm{H}_{2} \mathrm{O}(2 \times 50 \mathrm{~mL})$, dried over $\mathrm{MgSO}_{4}$, filtered and concentrated in vacuo. Purification of 
the organic residue by $\mathrm{SiO}_{2}$ flash chromatography using petrol-EtOAc (7:1 to 5:1) as eluent, afforded diol $22(1.17 \mathrm{~g}, 58 \%)$ as a colorless oil that foamed when placed in vacuo.

Data for 22: $[\alpha]_{D}-18.2^{\circ}\left(c 0.358, \mathrm{CH}_{2} \mathrm{Cl}_{2}\right)$; IR (neat film) $3452(\mathrm{~s}), 3071$ (w), $3049(\mathrm{w}), 2958(\mathrm{~s}), 2933$ (s), 2896 (s), 2858 (s), 1468 (m), 1426 (s), 1389 (m), 1366 (w), 1278 (w), 1244 (w), 1217 (w), 1190 (w), 1110 (s), 1081 (s), 1001 (w), 939 (w), 898 (w), $824(\mathrm{~m}), 756$ (s), 705 (s), $688(\mathrm{~m}), 666$ (w), $615(\mathrm{~m}), 506$ (s), 490 (m). ${ }^{1} \mathrm{H}$ NMR (500 MHz, $\left.\mathrm{CDCl}_{3}, 298 \mathrm{~K}\right) \delta$ 7.72-7.61 (m, 4H, Ph), 7.46-7.33 (m, 6H, Ph), 4.27 (s, $1 \mathrm{H}, \underline{\mathrm{H}}-9), 4.24(\mathrm{~m}, 1 \mathrm{H}, \underline{\mathrm{H}}-5), 4.12$ (dd, $1 \mathrm{H}, J=2.2,10.3 \mathrm{~Hz}, \underline{\mathrm{H}}-7), 3.92-3.80$ (m, 2H, $\underline{\mathrm{H}}-3 \mathrm{a}, \underline{\mathrm{H}}-3 \mathrm{~b}), 3.40-$ 3.00 (very br s, 2H, C-5-Oㅡㅡ, C-7-Oㅡ), 2.95-2.78 (m, 4H, 2 x - $\left.\underline{\mathrm{H}}_{2} \mathrm{~S}-\right), 2.07$ (m, 1H, $-\mathrm{SCH}_{2} \mathrm{CH}_{2(\mathrm{eq})} \mathrm{CH}_{2} \mathrm{~S}-$ ), $1.92(\mathrm{~m}, 1 \mathrm{H}, \underline{\mathrm{H}}-4 \mathrm{a}), 1.82\left(\mathrm{~m}, 1 \mathrm{H},-\mathrm{SCH}_{2} \mathrm{CH}_{2(\mathrm{ax})} \mathrm{CH}_{2} \mathrm{~S}-\right), 1.65(\mathrm{~m}, 1 \mathrm{H}, \underline{\mathrm{H}}-4 \mathrm{~b}), 1.62(\mathrm{~m}, 1 \mathrm{H}, \underline{\mathrm{H}}-6 \mathrm{a}), 1.55$ (ddd, $1 \mathrm{H}, J=2.2,6.9,-14.1 \mathrm{~Hz}, \underline{\mathrm{H}}-6 \mathrm{~b}), 1.08\left(\mathrm{~s}, 3 \mathrm{H}, \mathrm{C}-8-\mathrm{C}_{3}\right), 1.03\left(\mathrm{~s}, 9 \mathrm{H},-\mathrm{C}\left(\mathrm{C}_{3}\right)_{3}\right), 1.01\left(\mathrm{~s}, 3 \mathrm{H}, \mathrm{C}-8-\underline{\mathrm{H}}_{3}\right)$ ppm; ${ }^{13} \mathrm{C}$ NMR (125 MHz, $\left.\mathrm{CDCl}_{3}, 298 \mathrm{~K}\right) \delta 135.46(\mathrm{Ph}), 132.82(\mathrm{Ph}), 132.69(\mathrm{Ph}), 129.80(\mathrm{Ph}), 129.78$

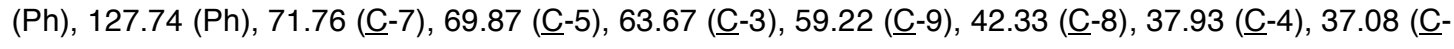
6), 31.48 and $31.35\left(2 \times-\underline{\mathrm{C}} \mathrm{H}_{2} \mathrm{~S}\right), 26.74\left(-\mathrm{C}\left(\mathrm{CH}_{3}\right)_{3}\right), 26.32\left(-\mathrm{SCH}_{2} \underline{\mathrm{CH}}_{2} \mathrm{CH}_{2} \mathrm{~S}-\right), 20.96\left(\mathrm{C}-8-\underline{\mathrm{CH}}_{3}\right), 19.86$ ($\left.\underline{\mathrm{C}}\left(\mathrm{CH}_{3}\right)_{3}\right), 18.93\left(\mathrm{C}-8-\underline{\mathrm{C}} \mathrm{H}_{3}\right)$ ppm. VG 70-SE Positive Ion FAB HRMS: Calcd. for $\mathrm{C}_{28} \mathrm{H}_{42} \mathrm{NaO}_{3} \mathrm{~S}_{2} \mathrm{Si}(\mathrm{M}+\mathrm{Na})^{+}$: 541.22422. Found: 541.22489.

\section{Dithiane 6 (SM-8-60)}

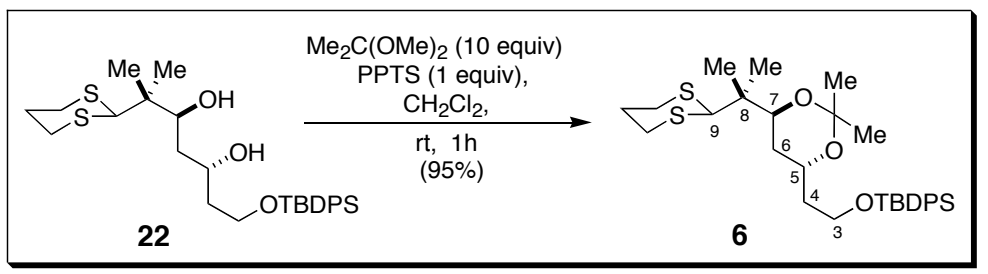

To a solution of diol $22(0.48 \mathrm{~g}, 0.925 \mathrm{mmol})$ and 2,2-dimethoxypropane (1.14 mL, $9.27 \mathrm{mmol}, 10.0$ equiv) in $\mathrm{CH}_{2} \mathrm{Cl}_{2}$ (7.4 mL) was added pyridinium $p$-toluenesulfonate $(0.23 \mathrm{~g}, 0.915 \mathrm{mmol}$, ca. 1 equiv) in one portion. The reactants were stirred at $\mathrm{rt}$ for $1 \mathrm{~h}$ and then quenched by adding solid $\mathrm{NaHCO}_{3}(0.5 \mathrm{~g})$. The resulting mixture was evaporated to dryness in vacuo. The residue was then purified by $\mathrm{SiO}_{2}$ flash chromatography with petrol-EtOAc $(50: 1)$ as eluent; dithiane $6(0.49 \mathrm{~g}, 95 \%)$ was obtained as a colorless oil.

Data for 6: $[\alpha]_{D}-9.6^{\circ}\left(c 0.522, \mathrm{CH}_{2} \mathrm{Cl}_{2}\right)$; IR (neat film) $3071(\mathrm{~m}), 3048(\mathrm{~m}), 2983(\mathrm{~s}), 2933(\mathrm{~s}), 2896(\mathrm{~s})$, 2857 (s), 1468 (m), 1427 (s), 1378 (s), 1276 (m), 1223 (s), 1171 (s), 1111 (s), 1092 (s), 1039 (m), 1028 (m), 995 (m), 959 (m), 908 (w), $824(\mathrm{~m}), 739$ (s), 704 (s), 682 (s), 612 (m), 506 (s), 490 (m). ${ }^{1} \mathrm{H}$ NMR $\left(500 \mathrm{MHz}, \mathrm{C}_{6} \mathrm{D}_{6}, 298 \mathrm{~K}\right) \delta$ 7.83-7.72 (m, 4H, Ph), 7.29-7.18 (m, 6H, Ph), $4.36(\mathrm{~s}, 1 \mathrm{H}, \underline{\mathrm{H}}-9), 4.31$ (dd, $1 \mathrm{H}, J$ $=6.4,9.9 \mathrm{~Hz}, \underline{\mathrm{H}}-7$ ), 4.06 (ddd, $1 \mathrm{H}, \underline{\mathrm{H}}-5$ ), 3.90 (overlapped ddd, $1 \mathrm{H}, J=5.4,8.0,-10.1 \mathrm{~Hz}, \underline{\mathrm{H}}-3 \mathrm{a}$ ), 3.80 (ddd, $1 \mathrm{H}, J=5.5,5.6,-10.9 \mathrm{~Hz}, \underline{\mathrm{H}}-3 \mathrm{~b}), 2.50-2.36$ (m, 4H, 2 x - $\left.\underline{\mathrm{H}}_{2} \mathrm{~S}-\right)$, 1.87-1.77 (m, $\left.1 \mathrm{H}, \underline{\mathrm{H}}-4 \mathrm{a}\right), 1.77-$ $1.69(\mathrm{~m}, 1 \mathrm{H}, \underline{\mathrm{H}}-4 \mathrm{~b}), 1.69-1.51\left(\mathrm{~m}, 2 \mathrm{H}, \underline{\mathrm{H}}-6 \mathrm{a}\right.$, and $\left.-\mathrm{SCH}_{2} \mathrm{CH}_{2} \mathrm{CH}_{2} \mathrm{~S}-\right), 1.49$ (s, 3H, isopropylidene- $\left.\underline{\mathrm{H}}_{3}\right)$, $1.40\left(\mathrm{~m}, 2 \mathrm{H}, \underline{\mathrm{H}}-6 \mathrm{~b}\right.$, and $\left.-\mathrm{SCH}_{2} \underline{\mathrm{H}}_{2} \mathrm{CH}_{2} \mathrm{~S}-\right), 1.40$ (s, 3H, isopropylidene- $\left.\mathrm{C}_{3}\right), 1.32$ (s, 3H, C-8- $\left.\underline{\mathrm{H}}_{3}\right), 1.17$ $\left(\mathrm{s}, 9 \mathrm{H},-\mathrm{C}\left(\mathrm{CH}_{3}\right)_{3}\right), 1.10\left(\mathrm{~s}, 3 \mathrm{H}, \mathrm{C}-8-\mathrm{CH}_{3}\right) \mathrm{ppm} ;{ }^{13} \mathrm{C} \mathrm{NMR}\left(125 \mathrm{MHz}, \mathrm{C}_{6} \mathrm{D}_{6}, 298 \mathrm{~K}\right) \delta 135.96(\mathrm{Ph}), 135.95$ 
(Ph), $134.28(\mathrm{Ph}), 134.27(\mathrm{Ph}), 129.94(\mathrm{Ph}), 128.53(\mathrm{Ph}), 128.05(\mathrm{Ph}), 128.04(\mathrm{Ph}), 100.73(-$

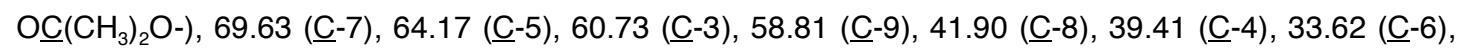
31.38 (- $\left.\underline{\mathrm{C}}_{2} \mathrm{~S}-\right), 31.31$ (- $\left.\underline{\mathrm{CH}}_{2} \mathrm{~S}-\right), 27.09\left(\mathrm{C}\left(\underline{\mathrm{C}} \mathrm{H}_{3}\right)_{3}\right), 26.62\left(-\mathrm{SCH}_{2} \underline{\mathrm{C}} \mathrm{H}_{2} \mathrm{CH}_{2} \mathrm{~S}-\right)$, 25.03 and $24.97\left(-\mathrm{O}-\mathrm{C}\left(\underline{\mathrm{C}} \mathrm{H}_{3}\right)_{2}{ }^{-}\right.$ O-), $20.42\left(\mathrm{C}-8-\underline{\mathrm{C}} \mathrm{H}_{3}\right), 19.75\left(\mathrm{C}-8-\underline{\mathrm{CH}_{3}}\right), 19.43\left(\underline{\mathrm{C}}\left(\mathrm{CH}_{3}\right)_{3}\right)$ ppm. VG 70-SE Positive lon FAB HRMS: Calcd. for $\mathrm{C}_{31} \mathrm{H}_{46} \mathrm{NaO}_{3} \mathrm{~S}_{2} \mathrm{Si}(\mathrm{M}+\mathrm{Na})^{+}: 581.25552$. Found: 581.25458 .

\section{O-Tosylate 27 (SM-8-75)}

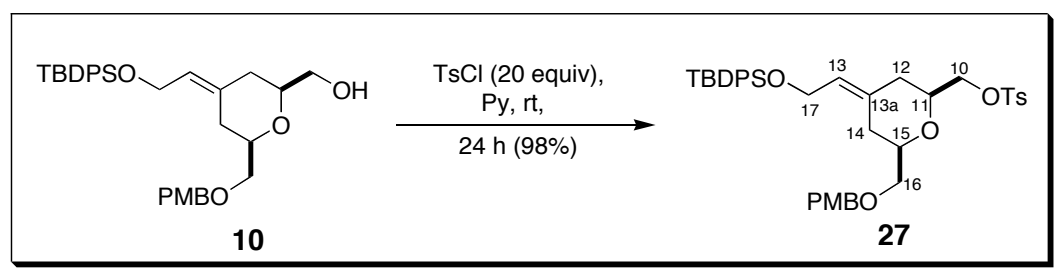

To a stirred rt solution of alcohol $1^{10 \mathrm{~d}}(0.46 \mathrm{~g}, 0.84 \mathrm{mmol})$ in dry pyridine $(14 \mathrm{~mL})$ under $\mathrm{N}_{2}$ was added p-toluenesulfonyl chloride $(3.21 \mathrm{~g}, 16.83 \mathrm{mmol}, 20$ equiv). After stirring for $24 \mathrm{~h}$ at $\mathrm{rt}$, the reaction mixture was quenched by successively adding solid $\mathrm{NaHCO}_{3}(3.0 \mathrm{~g})$ and saturated aqueous $\mathrm{NaHCO}_{3}(50 \mathrm{~mL})$ followed by EtOAc $(100 \mathrm{~mL})$. The organic layer was separated and the aqueous layer was further extracted with EtOAc $(3 \times 100 \mathrm{~mL})$. The combined organic extracts were washed with $\mathrm{H}_{2} \mathrm{O}(2 \times 50 \mathrm{~mL})$, dried over $\mathrm{MgSO}_{4}$, filtered, and concentrated in vacuo. The residue was co-evaporated with PhMe ( $1 \mathrm{x}$ $100 \mathrm{~mL}$ ) and then purified by $\mathrm{SiO}_{2}$ flash chromatography with petrol-EtOAc (6:1) as eluent to provide the O-tosylate $27(0.58 \mathrm{~g}, 98 \%)$ as a colorless oil.

Data for 27: $[\alpha]_{D}+4.32^{\circ}\left(c 0.718, \mathrm{CH}_{2} \mathrm{Cl}_{2}\right)$; IR (neat film) $3070(w), 3047(w), 3032(w), 2998(w), 2956$ (s), 2932 (s), 2892 (s), 2857 (s), 1612 (m), 1599 (w), 1587 (w), 1513 (s), 1469 (m), 1462 (m), 1428 (m), 1362 (s), 1304 (m), 1248 (s), 1209 (w), 1177 (s), 1110 (s), 1064 (s), 1038 (s), 983 (s), 937 (m), 824 (s), $780(\mathrm{~m}), 743(\mathrm{~m}), 705$ (s), 665 (m), 612 (w), 577 (w), 665 (s), 556 (s), 505 (s), 492 (m). ${ }^{1} \mathrm{H}$ NMR (500 $\left.\mathrm{MHz}, \mathrm{CDCl}_{3}, 298 \mathrm{~K}\right) \delta 7.80\left(\mathrm{~d}, 2 \mathrm{H}, J=8.3 \mathrm{~Hz}, \mathrm{C}_{6} \mathrm{H}_{4}\right.$ of Ts), 7.72-7.64 (m, 4H, Ph), 7.44-7.33 (m, 6H, Ph), 7.31 (d, 2H, J=7.9 Hz, C $\mathrm{H}_{4}$ of Ts), 7.22 (m, 2H, PMP), 6.87 (m, 2H, PMP), 5.47 (t, 1H, J=6.5 Hz, $\underline{\mathrm{H}}-$ 13), 4.46 (d, $\left.1 \mathrm{H}, J=-11.8 \mathrm{~Hz}, \mathrm{C}_{2} \mathrm{PMP}\right), 4.42$ (d, $\left.1 \mathrm{H}, J=-11.8 \mathrm{~Hz}, \mathrm{C}_{2} \mathrm{PMP}\right) 4.23$ (dd, $1 \mathrm{H}, J=6.7 \mathrm{~Hz}$, $12.7 \mathrm{~Hz}, \underline{\mathrm{H}}-17 \mathrm{a})$ superimposed upon 4.19 (dd, $1 \mathrm{H}, J=6.5,-12.7 \mathrm{~Hz}, \underline{\mathrm{H}}-17 \mathrm{~b}), 4.05$ (dd, $1 \mathrm{H}, J=5.7,-10.2$

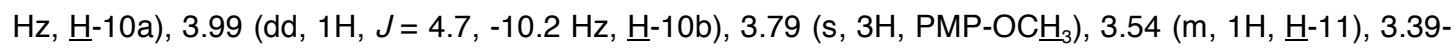
$3.26(\mathrm{~m}, 3 \mathrm{H}, \underline{\mathrm{H}}-16 \mathrm{a}, \underline{\mathrm{H}}-16 \mathrm{~b}, \underline{\mathrm{H}}-15), 2.42$ (s, 3H, $\mathrm{CH}_{3}$ of Ts), 2.20 (d, 1H, J = -13.7 Hz, $\left.\underline{\mathrm{H}}-14_{\mathrm{eq}}\right), 2.10$ (d, $1 \mathrm{H}, J=-13.1 \mathrm{~Hz}, \underline{\mathrm{H}}-12_{\mathrm{eq}}$ ), $1.96\left(\mathrm{dd}, 1 \mathrm{H}, J=12.1,-12.7 \mathrm{~Hz}, \underline{\mathrm{H}}-12_{\mathrm{ax}}\right.$ ), 1.62 (dd, $1 \mathrm{H}, J=12.3,-12.3 \mathrm{~Hz}, \underline{\mathrm{H}}-$

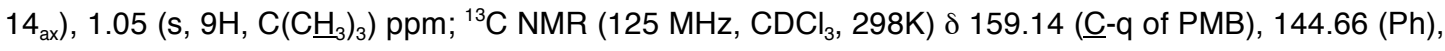
$135.48(\mathrm{Ph}), 135.47(\mathrm{Ph}), 134.01(\mathrm{Ph}), 133.67(\mathrm{Ph}), 132.88(\mathrm{Ph}), 130.13(\mathrm{Ph}), 129.71(\mathrm{Ph}), 129.55(\mathrm{Ph})$,

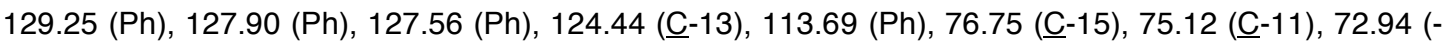

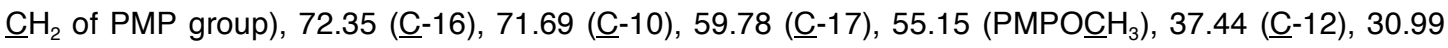
(-14), $26.74\left(-\mathrm{C}\left(\underline{\mathrm{CH}}_{3}\right)_{3}\right), 21.51\left(\underline{\mathrm{CH}}_{3}\right.$ of Ts), $19.03\left(-\underline{\mathrm{C}}\left(\mathrm{CH}_{3}\right)_{3}\right)$ ppm (N.B. C-13a lies amongst the Ph resonances and has not been assigned). VG 70-SE Positive Ion FAB HRMS: Calcd. for $\mathrm{C}_{40} \mathrm{H}_{48} \mathrm{NaO}_{7} \mathrm{SSi}$ $(\mathrm{M}+\mathrm{Na})^{+}:$723.27876. Found: 723.27827. 


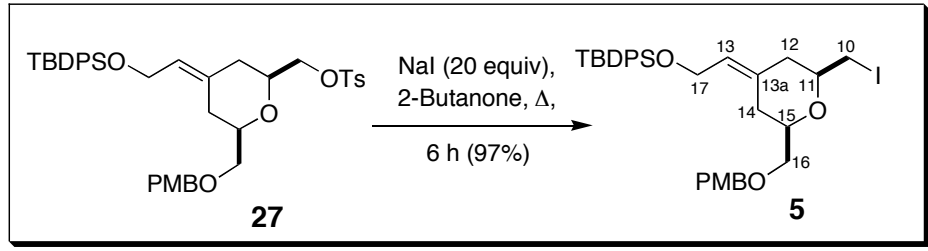

To a rt solution of the O-tosylate $27(0.707 \mathrm{~g}, 1.01 \mathrm{mmol})$ in 2-butanone $(14 \mathrm{~mL})$ was added Nal $(3.02 \mathrm{~g}$, $20.17 \mathrm{mmol}, 20.0$ equiv). The reaction flask was placed inside an oil-bath and the reactants were heated at $90-100^{\circ} \mathrm{C}$ for $6 \mathrm{~h}$, whereafter TLC analysis indicated that the reaction was complete. After cooling to $\mathrm{rt}$, the mixture was diluted with EtOAc $(100 \mathrm{~mL})$ and saturated aqueous $\mathrm{NaHCO}_{3}(50 \mathrm{~mL})$ was added. The organic layer was separated, and the aqueous layer was extracted with EtOAc $(3 \times 50 \mathrm{~mL})$. The combined organic extracts were dried over $\mathrm{MgSO}_{4}$, filtered and concentrated in vacuo. The crude residue was purified by $\mathrm{SiO}_{2}$ flash chromatography with petrol-EtOAc (10:1) as eluent; iodide 5 (0.64 g, $97 \%$ ) was obtained as a colorless oil.

Data for 5: $[\alpha]_{\mathrm{D}}+7.49^{\circ}\left(c 0.694, \mathrm{CH}_{2} \mathrm{Cl}_{2}\right)$; IR (neat film) $3070(\mathrm{w}), 3047$ (w), $3001(\mathrm{w}), 2956(\mathrm{~s}), 2932$ (s), 2891 (s), 2856 (s), 1612 (m), 1587 (w), 1513 (s), 1467 (m), 1427 (s), 1386 (w), 1360 (w), 1302 (w), 1248 (s), 1215 (w), 1177 (m), 1109 (s), 1040 (s), 1004 (w), 823 (m), 758 (s), 705 (s), 667 (w), 613 (w), 505 (m). ${ }^{1} \mathrm{H}$ NMR $\left(500 \mathrm{MHz}, \mathrm{CDCl}_{3}, 298 \mathrm{~K}\right) \delta$ 7.73-7.63 (m, 4H, Ph), 7.44-7.32 (m, 6H, Ph), 7.26 (m, 2H, PMP), 6.87 (m, 2H, PMP), 5.48 (t, $1 \mathrm{H}, J=6.4 \mathrm{~Hz}, \underline{\mathrm{H}}-13), 4.53$ (d, $\left.1 \mathrm{H}, J=-11.9 \mathrm{~Hz},-\underline{\mathrm{H}}_{2} \mathrm{PMP}\right), 4.49$ (d, $\left.1 \mathrm{H}, J=-11.9 \mathrm{~Hz}, \underline{\mathrm{H}}_{2} \mathrm{PMP}\right), 4.23(\mathrm{dd}, 1 \mathrm{H}, J=6.7,-12.6 \mathrm{~Hz}, \underline{\mathrm{H}}-17 \mathrm{a})$ superimposed upon $4.20(\mathrm{dd}, 1 \mathrm{H}, J$ $=6.4,-12.7 \mathrm{~Hz}, \underline{\mathrm{H}}-17 \mathrm{~b}), 3.79\left(\mathrm{~s}, 3 \mathrm{H}, \mathrm{PMP}-\mathrm{OC}_{\vec{H}}\right), 3.42(\mathrm{~m}, 1 \mathrm{H}, \underline{\mathrm{H}}-16 \mathrm{a}), 3.41(\mathrm{~m}, 1 \mathrm{H}, \underline{\mathrm{H}}-15), 3.38(\mathrm{~m}, 1 \mathrm{H}$, ㅂ- $-16 \mathrm{~b}), 3.32(\mathrm{~m}, 1 \mathrm{H}, \underline{\mathrm{H}}-11), 3.24(\mathrm{dd}, 1 \mathrm{H}, J=5.5,-10.1 \mathrm{~Hz}, \underline{\mathrm{H}}-10 \mathrm{a}), 3.21$ (dd, $1 \mathrm{H}, J=5.8,-10.1 \mathrm{~Hz}, \underline{\mathrm{H}}-$ 10b), 2.36 (d, 1H, J = -13.2 Hz, $\underline{\mathrm{H}}-12_{\text {eq }}$ ), 2.18 (d, 1H, J = -13.7 Hz, 브-14 eq), 1.95 (dd, 1H, J = 11.7, -12.6 $\left.\mathrm{Hz}, \underline{\mathrm{H}}-12_{\mathrm{ax}}\right), 1.64$ (dd, $\left.1 \mathrm{H}, J=12.3,-12.8 \mathrm{~Hz}, \underline{\mathrm{H}}-14_{\mathrm{ax}}\right), 1.04\left(\mathrm{~s}, 9 \mathrm{H},-\mathrm{C}\left(\mathrm{C}_{3}\right)_{3}\right) \mathrm{ppm} ;{ }^{13} \mathrm{C} \mathrm{NMR}(125 \mathrm{MHz}$, $\left.\mathrm{CDCl}_{3}, 298 \mathrm{~K}\right) \delta 159.15$ (ㄷ-q of PMP group), $135.55(\mathrm{Ph}), 134.67(\mathrm{Ph}), 133.78(\mathrm{Ph}), 133.75(\mathrm{Ph}), 130.35$

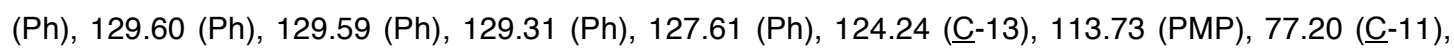

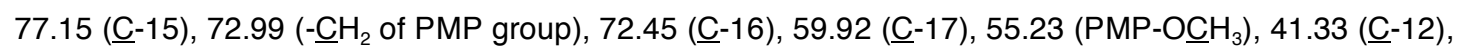

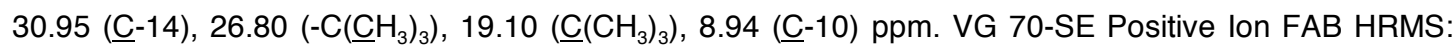
Calcd. for $\mathrm{C}_{33} \mathrm{H}_{41} \mathrm{INaO}_{4} \mathrm{Si}(\mathrm{M}+\mathrm{Na})^{+}: 679.17164$. Found: 679.17089 . 


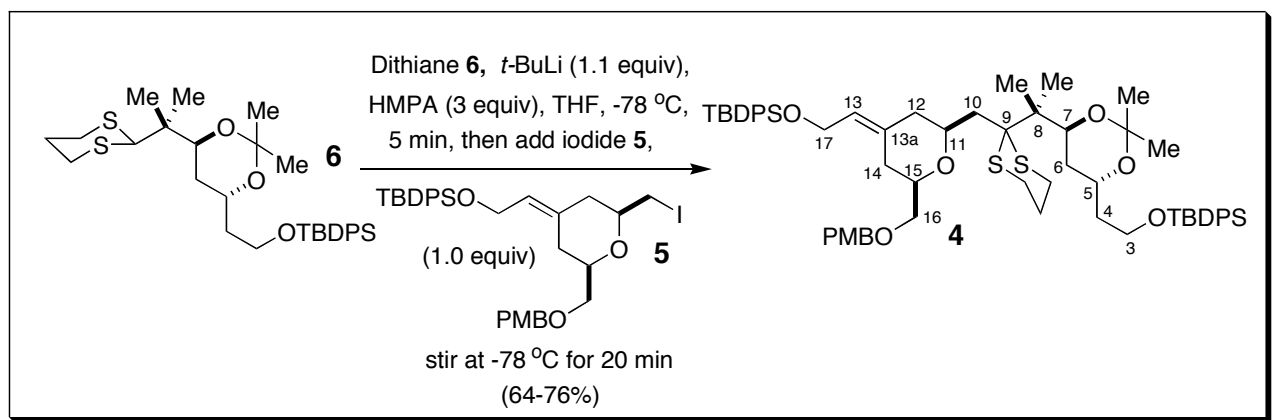

To a stirred solution of dithiane $6(1.55 \mathrm{~g}, 2.77 \mathrm{mmol}, 1.0$ equiv) in dry THF (12 mL) and HMPA (1.45 $\mathrm{mL}, 8.32 \mathrm{mmol}, 3.0$ equiv) at $-78{ }^{\circ} \mathrm{C}$ under $\mathrm{N}_{2}$ was added $t$-BuLi (1.7 M solution in pentane, $1.8 \mathrm{~mL}, 3.05$ mmol, 1.1 equiv) dropwise over $1 \mathrm{~min}$. The reaction mixture was stirred at $-78{ }^{\circ} \mathrm{C}$ for 5 min after which a dark red-coloured anion was formed. A solution of iodide 5 (1.82 g, 2.77 mmol, 1.0 equiv) in dry THF (6 $\mathrm{mL}$ ) was then added dropwise over $2 \mathrm{~min}$. The flask containing the iodide $\mathbf{5}$ was then washed with dry THF ( $2 \mathrm{~mL})$ and the washings were added dropwise to the reaction mixture over $1 \mathrm{~min}$. After a further 20 min at $-78{ }^{\circ} \mathrm{C}$, the pale yellow reaction mixture was quenched with saturated aqueous $\mathrm{NaHCO}_{3}(50 \mathrm{~mL})$ and extracted with EtOAc $(3 \times 100 \mathrm{~mL})$. The combined organic extracts were washed with $\mathrm{H}_{2} \mathrm{O}(1 \times 50$ $\mathrm{mL}$ ), dried over $\mathrm{MgSO}_{4}$, filtered, and concentrated in vacuo. The crude residue was purified by gradient elution $\mathrm{SiO}_{2}$ flash chromatography as follows:

i) with petrol-EtOAc $(40: 1$ to $30: 1)$ as eluent, to recover the unreacted dithiane $6(0.11 \mathrm{~g})$;

ii) with petrol-EtOAc $(20: 1)$ as eluent, to recover the starting iodide $5(79.1 \mathrm{mg})$ and;

iii) with petrol-EtOAc (15:1 to $10: 1)$ as eluent to give the desired coupling product 4 (2.29 g, $76 \%)$ as a colorless oil.

Data for 4: $[\alpha]_{D}-0.34^{\circ}\left(c 0.59, \mathrm{CH}_{2} \mathrm{Cl}_{2}\right)$; IR (neat film) $3070(\mathrm{w}), 3047(\mathrm{w}), 2932(\mathrm{~s}), 2892(\mathrm{~m}), 2857(\mathrm{~s})$, $1612(w), 1587(w), 1513(m), 1469(m), 1427(m), 1383(m), 1362(w), 1301(w), 1247(m), 1224(m)$, $1171(\mathrm{~m}), 1109$ (s), 1038 (m), $823(\mathrm{~m}), 739$ (m), 702 (s). ${ }^{1} \mathrm{H}$ NMR spectrum (500 MHz, $\left.\mathrm{C}_{6} \mathrm{D}_{6}, 298 \mathrm{~K}\right)$ without resolution enhancement (N.B. There is significant broadening associated with many of the proton resonances in 4 due to restricted rotation about the C8-C9 bond which links two adjacent quaternary C-atoms): $\delta$ 7.86-7.75 (m, 8H, Ph), 7.30-7.18 (m, 14H, Ph and PMP), $6.79(\mathrm{~m}, 2 \mathrm{H}, \mathrm{PMP})$, $5.69(\mathrm{t}, 1 \mathrm{H}, J=6.4 \mathrm{~Hz}, \underline{\mathrm{H}}-13), 4.57(\mathrm{dd}, 1 \mathrm{H}, J=6.0,10.1 \mathrm{~Hz}, \underline{\mathrm{H}}-7), 4.38\left(\mathrm{~d}, 1 \mathrm{H}, J=-11.7, \underline{H}_{2} \mathrm{PMP}\right)$, $4.35\left(\mathrm{~d}, 1 \mathrm{H}, J=-11.7 \mathrm{~Hz},-\underline{\mathrm{H}}_{2} \mathrm{PMP}\right)$ superimposed upon 4.36 and $4.34(\mathrm{~m}, 2 \mathrm{H}, \underline{\mathrm{H}}-17 \mathrm{a}, \underline{\mathrm{H}}-17 \mathrm{~b}), 4.17-$ $4.04(\mathrm{~m}, 2 \mathrm{H}, \underline{\mathrm{H}}-5$ and $\underline{\mathrm{H}}-11), 3.92(\mathrm{~m}, 1 \mathrm{H}, \underline{\mathrm{H}}-3 \mathrm{a}), 3.82$ (ddd, $1 \mathrm{H}, J=5.1,6.0,-10.3 \mathrm{~Hz}, \underline{\mathrm{H}}-3 \mathrm{~b}), 3.52(\mathrm{~m}$, $1 \mathrm{H}, \underline{\mathrm{H}}-15), 3.47$ (dd, $1 \mathrm{H}, J=5.6,-9.9 \mathrm{~Hz}, \underline{\mathrm{H}}-16 \mathrm{a}), 3.33$ (dd, $1 \mathrm{H}, J=4.3,-9.8 \mathrm{~Hz}, \underline{\mathrm{H}}-16 \mathrm{~b}), 3.31$ (s, $3 \mathrm{H}$, PMP-OC $\underline{H}_{3}$ ), 2.76 (partially overlapped ddd, $1 \mathrm{H}$, axial $-\underline{\mathrm{C}}_{2} \mathrm{~S}-$ ), 2.68-2.57 (m, $\left.2 \mathrm{H}, \underline{\mathrm{H}}-10 \mathrm{a}, \underline{\mathrm{H}}-10 \mathrm{~b}\right), 2.53$ (partially overlapped ddd, $1 \mathrm{H}, J=3.9,9.4,-13.9 \mathrm{~Hz}$, axial $-\mathrm{C}_{2} \mathrm{~S}-$ ), $2.41\left(\mathrm{~d}, 1 \mathrm{H}, J=-12.9 \mathrm{~Hz}, \underline{\mathrm{H}}-12_{\text {eq }}\right.$ ), $2.34\left(\mathrm{~d}, 1 \mathrm{H}, J=-13.5 \mathrm{~Hz}, \underline{\mathrm{H}}-14{ }_{\text {eq }}\right.$ superimposed upon $\mathrm{m}, 2 \mathrm{H}$, equatorial $\left.-\mathrm{C}_{2} \mathrm{~S}-\right), 2.15$ (dd, $1 \mathrm{H}, J=12.0$, $\left.12.3 \mathrm{~Hz}, \underline{\mathrm{H}}-12_{\mathrm{ax}}\right), 1.92(\mathrm{~m}, 1 \mathrm{H}, \underline{\mathrm{H}}-6 \mathrm{a}) 1.86(\mathrm{~m}, 1 \mathrm{H}, \underline{\mathrm{H}}-4 \mathrm{a}), 1.79(\mathrm{~m}, 1 \mathrm{H}, \mathrm{H}-4 \mathrm{~b}), 1.73(\mathrm{dd}, 1 \mathrm{H}, J=12.7,-$ $\left.12.8 \mathrm{~Hz}, \underline{\mathrm{H}}-14_{\mathrm{ax}}\right), 1.67$ (br m, $\left.1 \mathrm{H}, \underline{\mathrm{H}}-6 \mathrm{~b}\right), 1.56$ (s, 3H, isopropylidene- $\underline{\mathrm{H}}_{3}$ cis to $\mathrm{H}-7$ ) superimposed upon $1.53\left(\mathrm{~m}, 1 \mathrm{H},-\mathrm{SCH}_{2} \mathrm{CH}_{2} \mathrm{CH}_{2} \mathrm{~S}-\right), 1.48\left(\mathrm{~m}, 1 \mathrm{H},-\mathrm{SCH}_{2} \mathrm{CH}_{2} \mathrm{CH}_{2} \mathrm{~S}-\right), 1.43\left(\mathrm{~s}, 3 \mathrm{H}, \mathrm{C}-8-\mathrm{CH}_{3}\right), 1.41$ (s, 3H, 
isopropylidene $\mathrm{CH}_{3}$ cis to $\left.\mathrm{H}-5\right), 1.30\left(\mathrm{~s}, 3 \mathrm{H}, \mathrm{C}-8-\mathrm{CH}_{3}\right), 1.18\left(\mathrm{~s}, 9 \mathrm{H}, \mathrm{C}\left(\mathrm{CH}_{3}\right)_{3}\right), 1.17\left(\mathrm{~s}, 9 \mathrm{H}, \mathrm{C}\left(\mathrm{CH}_{3}\right)_{3}\right)$ ppm;

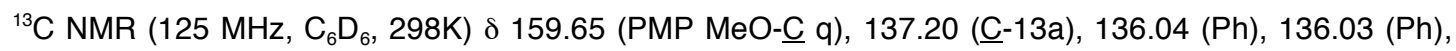
$135.96(\mathrm{Ph}), 135.95(\mathrm{Ph}), 134.33(\mathrm{Ph} \underline{\mathrm{C}} \mathrm{q}), 134.30$ (Ph $\underline{\mathrm{C}} \mathrm{q}), 131.11$ ( $\left.\mathrm{H}_{2} \mathrm{C}-\underline{\mathrm{C}} \mathrm{PMP} q\right), 129.94(\mathrm{Ph})$, $129.92(\mathrm{Ph}), 129.90(\mathrm{Ph}), 129.40(\mathrm{Ph}), 128.29(\mathrm{Ph}), 128.07(\mathrm{Ph}), 128.06(\mathrm{Ph}), 128.05(\mathrm{Ph}), 123.66(\underline{\mathrm{C}}-$

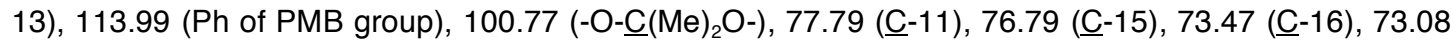

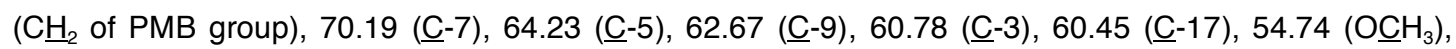

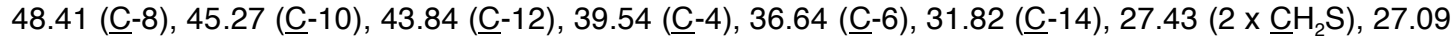
$\left(-\mathrm{C}\left(\mathrm{CH}_{3}\right)_{3}\right), 27.06\left(-\mathrm{C}\left(\mathrm{CH}_{3}\right)_{3}\right), 25.08$ (isopropylidene- $\mathrm{CH}_{3}$ cis to $\left.\mathrm{H}-5\right), 25.05$ (isopropylidene- $\mathrm{CH}_{3}$ cis to $\mathrm{H}$ 7), $24.72\left(-\mathrm{SCH}_{2} \underline{\mathrm{C}} \mathrm{H}_{2} \mathrm{CH}_{2} \mathrm{~S}-\right), 21.31$ (very br C-8- $\left.\underline{\mathrm{C}} \mathrm{H}_{3}\right), 19.43$ and $19.40\left(2 \times \underline{\mathrm{C}}\left(\mathrm{CH}_{3}\right)_{3}\right), 18.98\left(\right.$ br C-8- $\left.\underline{\mathrm{C}} \mathrm{H}_{3}\right)$ ppm. VG 70-SE Positive Ion FAB HRMS: Calcd. for $\mathrm{C}_{64} \mathrm{H}_{86} \mathrm{NaO}_{7} \mathrm{~S}_{2} \mathrm{Si}_{2}(\mathrm{M}+\mathrm{Na})^{+}$: 1109.52509. Fond: 1109.52236

\section{Ketone 23 (SM-8-113)}

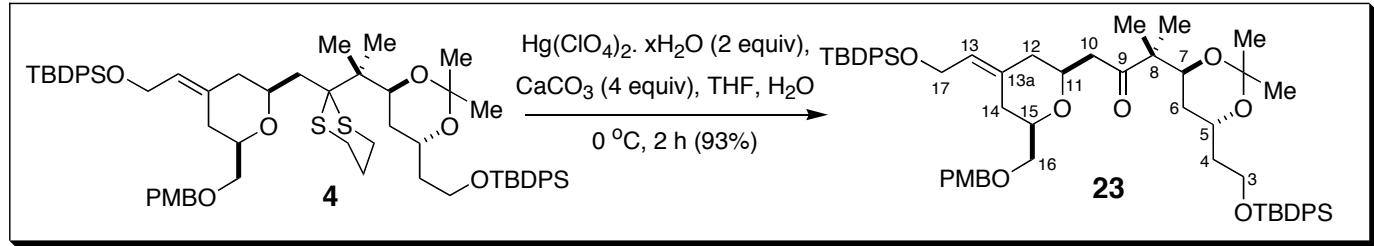

To the coupled product $4(2.12 \mathrm{~g}, 1.95 \mathrm{mmol})$ in THF $(42 \mathrm{~mL})$ and $\mathrm{H}_{2} \mathrm{O}(21 \mathrm{~mL})$ at $0{ }^{\circ} \mathrm{C}$ was added $\mathrm{CaCO}_{3}(0.78 \mathrm{~g}, 7.80 \mathrm{mmol}, 4.0$ equiv) and mercury (II) perchlorate hydrate $(1.56 \mathrm{~g}, 3.90 \mathrm{mmol}, 2.0$ equiv). After $2 \mathrm{~h}$ stirring at $0{ }^{\circ} \mathrm{C}$, the reaction mixture was diluted with EtOAc $(200 \mathrm{~mL})$ and saturated aqueous $\mathrm{NaHCO}_{3}(50 \mathrm{~mL})$. The organic layer was separated, and the aqueous phase was further extracted with EtOAc $(3 \times 100 \mathrm{~mL})$. The combined organic extracts were washed with brine $(1 \times 100$ $\mathrm{mL}$ ), dried over $\mathrm{MgSO}_{4}$, filtered and concentrated in vacuo. The residue was purified by $\mathrm{SiO}_{2}$ flash chromatography with petrol-EtOAc (10:1) as eluent, to give ketone $23(1.8 \mathrm{~g}, 93 \%)$ as a colorless oil.

Data for 23: $[\alpha]_{\mathrm{D}}+25.5^{\circ}\left(c 0.42, \mathrm{CH}_{2} \mathrm{Cl}_{2}\right)$; IR (neat film) $3071(\mathrm{~m}), 3048(\mathrm{~m}), 2957(\mathrm{~s}), 2933(\mathrm{~s}), 2890(\mathrm{~s})$, $2857(\mathrm{~s}), 1704(\mathrm{~m}), 1612(\mathrm{w}), 1587(\mathrm{w}), 1513(\mathrm{~m}), 1469(\mathrm{~m}), 1427(\mathrm{~m}), 1383(\mathrm{~m}), 1364(\mathrm{~m}), 1328(\mathrm{w})$, $1302(\mathrm{w}), 1248(\mathrm{~s}), 1224(\mathrm{~m}), 1173(\mathrm{~m}), 1110(\mathrm{~s}), 1039(\mathrm{~m}), 1003(\mathrm{w}), 823(\mathrm{~m}), 740(\mathrm{~m}), 704(\mathrm{~s}) .{ }^{1} \mathrm{H}$ NMR (500 MHz, $\left.\mathrm{C}_{6} \mathrm{D}_{6}, 298 \mathrm{~K}\right)$ d 7.86-7.72 (m, 8H, Ph), 7.32-7.20 (m, 12H, Ph), 7.20 (m, 2H, PMP), 6.79 (m, 2H, PMP), 5.61 (t, $1 \mathrm{H}, J=6.4 \mathrm{~Hz}, \underline{\mathrm{H}}-13), 4.36$ (d, $1 \mathrm{H}, J=11.8 \mathrm{~Hz},-\underline{\mathrm{H}}_{2} \mathrm{PMP}$ ), 4.33 (d, $1 \mathrm{H}, J=-11.8$ $\left.\mathrm{Hz},-\underline{\mathrm{H}}_{2} \mathrm{PMP}\right), 4.32(\mathrm{dd}, 1 \mathrm{H}, J=6.5,-15.8 \mathrm{~Hz}, \underline{\mathrm{H}}-17 \mathrm{a})$ superimposed upon $4.29(\mathrm{dd}, 1 \mathrm{H}, J=6.5,-15.8$ $\mathrm{Hz}, \underline{\mathrm{H}}-1 \mathrm{bb}), 4.04(\mathrm{dd}, 1 \mathrm{H}, J=6.4,9.8 \mathrm{~Hz}, \underline{\mathrm{H}}-7)$ superimposed upon $4.05(\mathrm{~m}, 1 \mathrm{H}, \underline{\mathrm{H}}-11)$ and $4.01(\mathrm{~m}, 1 \mathrm{H}$, $\underline{\mathrm{H}}-5$ ), 3.87 (partially overlapped ddd, $1 \mathrm{H}, J=5.4,8.0,-10.3 \mathrm{~Hz}, \underline{\mathrm{H}}-3 \mathrm{a}$ ), 3.77 (partially overlapped ddd, $1 \mathrm{H}, J=5.1,5.9,-10.3 \mathrm{~Hz}, \underline{\mathrm{H}}-3 \mathrm{~b}), 3.43\left(\mathrm{~m}, 1 \mathrm{H}, \underline{\mathrm{H}}-15_{\mathrm{ax}}\right.$ ), 3.39 (dd, $\left.1 \mathrm{H}, J=5.4,-10.0 \mathrm{~Hz}, \underline{\mathrm{H}}-16 \mathrm{a}\right), 3.31$ (s, $3 \mathrm{H}, \mathrm{PMP}-\mathrm{OC} \underline{\mathrm{H}}_{3}$ ), 3.29 (dd, $1 \mathrm{H}, J=4.1,-10.0 \mathrm{~Hz}, \underline{\mathrm{H}}-16 \mathrm{~b}$ ), 2.90 (dd, $\left.1 \mathrm{H}, J=5.8,-17.1 \mathrm{~Hz}, \underline{\mathrm{H}}-10 \mathrm{a}\right), 2.42$ (dd, $1 \mathrm{H}, J=6.4,-17.2 \mathrm{~Hz}, \underline{\mathrm{H}}-10 \mathrm{~b}), 2.29\left(\mathrm{~d}, 1 \mathrm{H}, J=-13.4 \mathrm{~Hz}, \underline{\mathrm{H}}-14_{\text {eq }}\right.$ ), 2.23 (d, $1 \mathrm{H}, J=-13.0 \mathrm{~Hz}, \underline{\mathrm{H}}-12_{\text {eq }}$ ), $1.87\left(\mathrm{t}, 1 \mathrm{H}, J=12.1 \mathrm{~Hz}, \underline{\mathrm{H}}-12_{\mathrm{ax}}\right), 1.75(\mathrm{~m}, 1 \mathrm{H}, \underline{\mathrm{H}}-4 \mathrm{a})$ superimposed upon $1.69(\mathrm{~m}, 1 \mathrm{H}, \underline{\mathrm{H}}-4 \mathrm{~b})$ and $1.69(\mathrm{t}$, $1 \mathrm{H}, J=12.8 \mathrm{~Hz}, \underline{\mathrm{H}}-14_{\mathrm{ax}}$ ), 1.62 (ddd, $\left.1 \mathrm{H}, J=5.9,9.8,-12.7 \mathrm{~Hz}, \underline{\mathrm{H}}-6 \mathrm{a}\right), 1.33$ (m, $\left.1 \mathrm{H}, \underline{\mathrm{H}}-6 \mathrm{~b}\right), 1.31$ (s, 3H, isopropylidene- $\mathrm{CH}_{3}$ cis to $\left.\mathrm{H}-7\right), 1.29\left(\mathrm{~s}, 3 \mathrm{H}\right.$, isopropylidene- $\mathrm{CH}_{3}$ cis to $\left.\mathrm{H}-5\right), 1.16\left(\mathrm{~s}, 18 \mathrm{H},-\mathrm{C}\left(\mathrm{CH}_{3}\right)_{3}\right), 1.10$ 


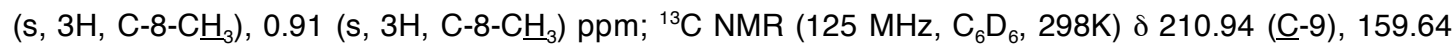
(PMP $\underline{\mathrm{C}}-\mathrm{OMe}$ quaternary), 136.26 (ㄸ-13a), 136.04 (o-Ph), 136.02 (o-Ph), 135.94 (o-Ph), 135.92 (o-Ph), 134.31 (Ph quaternary), 134.24 (Ph quaternary), 134.23 (Ph quaternary), 131.09 (PMP $\underline{\mathrm{C}}-\mathrm{CH}_{2}$ quaternary), 129.97 (p-Ph), 129.96 (p-Ph), 129.95 (p-Ph), 129.92 (p-Ph), 129.42 (o-PMP), 128.07 (m$\mathrm{Ph}), 128.06$ (m-Ph), 128.05 (m-Ph), 128.04 (m-Ph), 123.97 (ㄷ-13), 114.00 (m-PMP), 100.51 (-

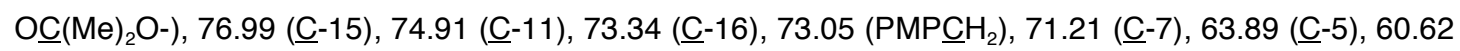

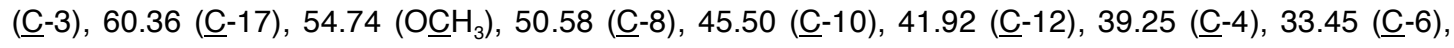

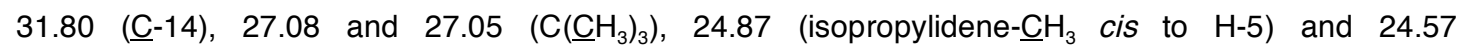
(isopropylidene- $\underline{\mathrm{C}} \mathrm{H}_{3}$ cis to $\left.\mathrm{H}-7\right), 20.41\left(\mathrm{C} 8-\underline{\mathrm{C}} \mathrm{H}_{3}\right), 19.41$ and $19.38\left(\underline{\mathrm{C}}\left(\mathrm{CH}_{3}\right)_{3}\right), 19.08\left(\mathrm{C} 8-\underline{\mathrm{C}} \mathrm{H}_{3}\right)$ ppm. VG 70-SE Positive Ion FAB HRMS: Calcd. for $\mathrm{C}_{61} \mathrm{H}_{80} \mathrm{NaO}_{8} \mathrm{Si}_{2}(\mathrm{M}+\mathrm{Na})^{+}:$1019.52891. Found: 1019.52644.

\section{Methyl Glycoside 3 (SM-7-146)}

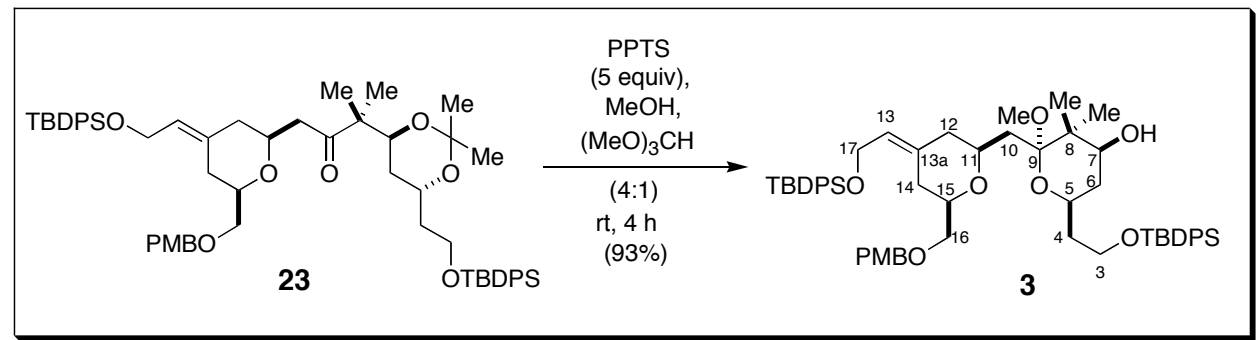

To a rt solution of ketone $23(1.72 \mathrm{~g}, 1.72 \mathrm{mmol}$ ) in a 4:1 mixture of $\mathrm{MeOH}$ (Analar, $138 \mathrm{~mL}$ ) and trimethylorthoformate $(34.5 \mathrm{~mL})$ was added pyridinium $p$-toluenesulfonate $(2.17 \mathrm{~g}, 8.63 \mathrm{mmol}, 5.0$ equiv) in one portion. The reaction mixture was stirred at $\mathrm{rt}$ for $4 \mathrm{~h}$ and then quenched by adding solid $\mathrm{NaHCO}_{3}$ $(2.0 \mathrm{~g})$ and diluting with EtOAc $(500 \mathrm{~mL})$ followed by saturated aqueous $\mathrm{NaHCO}_{3}(50 \mathrm{~mL})$. The organic layer was separated, and the aqueous phase extracted with EtOAc $(3 \times 100 \mathrm{~mL})$. The combined organic extracts were washed with brine $(1 \times 100 \mathrm{~mL})$, dried over $\mathrm{MgSO}_{4}$, filtered and concentrated in vacuo. The residue was purified by $\mathrm{SiO}_{2}$ flash chromatography (gradient elution with 6:1 to 5:1 with petrolEtOAc) as eluent, to give alcohol $3(1.55 \mathrm{~g}, 93 \%)$ as a colorless oil.

Data for 3: $[\alpha]_{D}+31.3^{\circ}\left(c 0.584, \mathrm{CH}_{2} \mathrm{Cl}_{2}\right)$; IR (neat film) $3444(\mathrm{br} \mathrm{m}), 3071(\mathrm{~m}), 3048(\mathrm{~m}), 2934(\mathrm{~s}), 2892$ (s), 2857 (s), 1612 (w), 1587 (w), $1513(\mathrm{~m}), 1469$ (m), 1427 (m), $1386(\mathrm{~m}), 1362$ (w), 1301 (w), 1249 (m), 1175 (w), 1110 (s), $1072(\mathrm{~m}), 1040$ (m), 964 (w), 938 (w), 823 (m), $740(\mathrm{~m}), 705$ (s), 611 (w), 505 (m), $491(\mathrm{w}) .{ }^{1} \mathrm{H}$ NMR $\left(500 \mathrm{MHz}, \mathrm{C}_{6} \mathrm{D}_{6}\right.$, 298K) $\delta$ 7.88-7.71 (m, 8H, Ph), 7.33-7.15 (m, 14H, Ph), $6.79(\mathrm{~m}$, 2H, PMP), 5.59 (t, $1 \mathrm{H}, J=6.4 \mathrm{~Hz}, \underline{\mathrm{H}}-13), 4.40$ (d, $\left.1 \mathrm{H}, J=-11.8 \mathrm{~Hz}, \underline{\mathrm{H}}_{2} \mathrm{PMP}\right), 4.36(\mathrm{~d}, 1 \mathrm{H}, J=-11.8 \mathrm{~Hz}$, $\mathrm{C}_{2} \mathrm{PMP}$ ), 4.34 (d, $2 \mathrm{H}, J=6.9 \mathrm{~Hz}, \underline{\mathrm{H}}-17 \mathrm{a}$ and $\underline{\mathrm{H}}-17 \mathrm{~b}$ ), 3.96 (ddd, $1 \mathrm{H}, J=5.1,6.2,-10.2 \mathrm{~Hz}, \underline{\mathrm{H}}-3 \mathrm{a}$ ), 3.94 (dd, $\left.1 \mathrm{H}, J=4.8,11.4 \mathrm{~Hz}, \underline{\mathrm{H}}-7_{\mathrm{ax}}\right), 3.84\left(\mathrm{~m}, 1 \mathrm{H}, \underline{\mathrm{H}}-5_{\mathrm{ax}}\right), 3.80$ (dt, $\left.1 \mathrm{H}, J=5.4(\mathrm{t}),-10.2 \mathrm{~Hz}, \underline{\mathrm{H}}-3 \mathrm{~b}\right), 3.68$ (m, $\left.1 \mathrm{H}, \underline{\mathrm{H}}-11_{\mathrm{ax}}\right), 3.44$ (dd, $\left.1 \mathrm{H}, J=5.4,-9.6 \mathrm{~Hz}, \underline{\mathrm{H}}-16 \mathrm{a}\right), 3.39\left(\mathrm{~m}, 1 \mathrm{H}, \underline{\mathrm{H}}-15_{\mathrm{ax}}\right), 3.31$ (dd, $1 \mathrm{H}, J=4.1 \mathrm{~Hz}, \underline{\mathrm{H}}-$ 16b), 3.31 (s, 3H, PMP - $\mathrm{OCH}_{3}$ ), 3.17 (s, 3H, C9-OC $\left.\underline{H}_{3}\right), 2.30\left(\mathrm{~d}, 2 \mathrm{H}, J=-13.3 \mathrm{~Hz}, \underline{\mathrm{H}}-12_{\text {eq }}\right.$ superimposed upon $\underline{\mathrm{H}}-14_{\text {eq }}$; the two signals coincide), 2.25 (dd, $\left.1 \mathrm{H}, J=4.9,-15.8 \mathrm{~Hz}, \underline{\mathrm{H}}-10 \mathrm{a}\right), 1.94(\mathrm{dd}, 1 \mathrm{H}, J=12.1$, $12.1 \mathrm{~Hz}, \underline{\mathrm{H}}-12_{\mathrm{ax}}$ ), 1.80 (dd, $1 \mathrm{H}, J=5.2,-15.8 \mathrm{~Hz}, \underline{\mathrm{H}}-10 \mathrm{~b}$ ), 1.76-1.54 (complex m, 3H, $\underline{\mathrm{H}}-4 \mathrm{a}, \underline{\mathrm{H}}-4 \mathrm{~b}$ and $\underline{\mathrm{H}}-$ $14_{\text {eq }}$ ), 1.41 (ddd, $1 \mathrm{H}, J=2.9,4.8,-12.5 \mathrm{~Hz}, \underline{\mathrm{H}}-6_{\text {eq }}$ ), 1.30 (apparent q, $1 \mathrm{H}, J=12.0 \mathrm{~Hz}, \underline{\mathrm{H}}-6_{\mathrm{ax}}$ ), 1.18 (s, 
$12 \mathrm{H}, \mathrm{CH}_{3}$ and $\left.\mathrm{C}\left(\mathrm{CH}_{3}\right)_{3}\right), 1.17$ (s, 9H, $\left.\mathrm{C}\left(\mathrm{CH}_{3}\right)_{3}\right), 1.07$ (s, 3H, $\left.\underline{\mathrm{CH}}_{3}\right)$ ppm (N.B. C-7-OH resonance hidden); ${ }^{13} \mathrm{C}$ NMR (125 MHz, $\left.\mathrm{C}_{6} \mathrm{D}_{6}, 298 \mathrm{~K}\right) \delta 159.62$ (PMP $\underline{\mathrm{C}}$-OMe quaternary), 137.19 (ㄸ-13a), 136.03 (o-Ph), 136.02 (o-Ph), 135.96 (o-Ph), 135.93 (o-Ph), 134.31 (Ph quaternary), 134.24 and 134.22 (Ph quaternary), 131.22 (PMP $\underline{\mathrm{C}}-\mathrm{CH}_{2}$ quaternary), 129.99 (p-Ph), 2 x 129.94 (p-Ph), 129.32 (o-Ph), 128.09

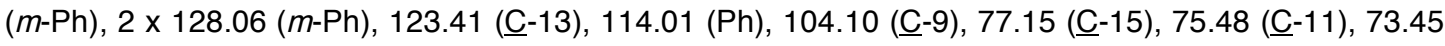

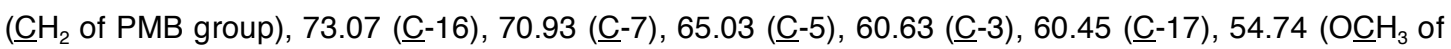

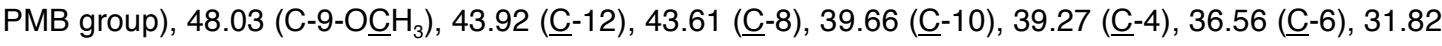

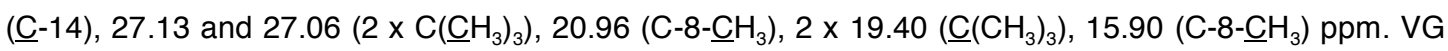
70-SE Positive Ion FAB HRMS: Calcd. for $\mathrm{C}_{59} \mathrm{H}_{78} \mathrm{NaO}_{8} \mathrm{Si}_{2}(\mathrm{M}+\mathrm{Na})^{+}:$993.51326. Found: 993.51553.

\section{Acetate 28 (SM-8-103)}

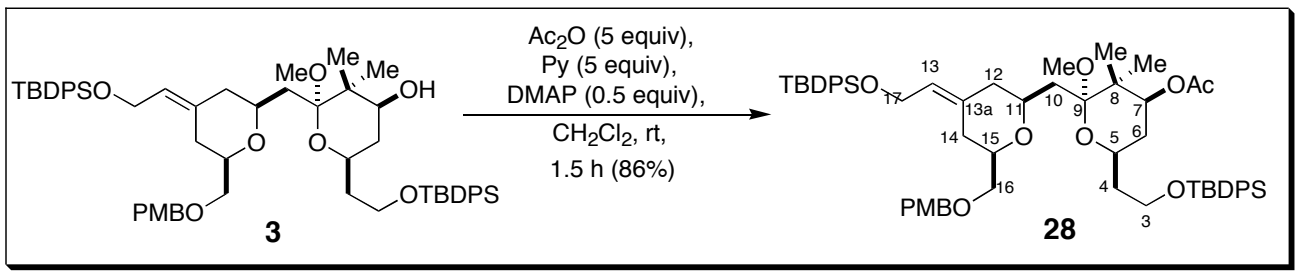

To a solution of alcohol $3(0.29 \mathrm{~g}, 0.299 \mathrm{mmol})$ in pyridine $\left(0.12 \mathrm{~mL}, 1.49 \mathrm{mmol}, 5\right.$ equiv) and $\mathrm{CH}_{2} \mathrm{Cl}_{2}$ $(3.4 \mathrm{~mL})$ at $\mathrm{rt}$ was added acetic anhydride $(0.14 \mathrm{~mL}, 1.49 \mathrm{mmol}, \mathrm{ca} .5$ equiv) and 4dimethylaminopyridine (DMAP) (18.24 mg, $0.15 \mathrm{mmol}, 0.5$ equiv). After stirring at $\mathrm{rt}$ for $1.5 \mathrm{~h}$, the reaction mixture was co-evaporated to dryness with PhMe $(2 \times 20 \mathrm{~mL})$ in vacuo. Purification of the residue by $\mathrm{SiO}_{2}$ flash chromatography with petrol-EtOAc (10:1) as eluent afforded $O$-acetate 28 (0.26 g, $86 \%)$ as a colorless oil.

Data for 28: $[\alpha]_{\mathrm{D}}+54.7^{\circ}\left(c 0.3, \mathrm{CH}_{2} \mathrm{Cl}_{2}\right)$; IR (neat film) $3071(\mathrm{w}), 3048(\mathrm{w}), 2956(\mathrm{~s}), 2933(\mathrm{~s}), 2892(\mathrm{~m})$, 2857 (s), 1734 (m), 1612 (w), 1587 (w), $1513(\mathrm{~m}), 1470(\mathrm{~m}), 1444$ (w), $1428(\mathrm{~m}), 1387(\mathrm{~m}), 1365(\mathrm{~m})$, 1301 (w), 1247 (s), 1175 (w), 1109 (s), 1037 (m), 936 (w), 823 (m), 740 (m), 705 (s), 611 (w), 505 (s). ${ }^{1} \mathrm{H}$ NMR (500 MHz, $\left.\mathrm{C}_{6} \mathrm{D}_{6}, 298 \mathrm{~K}\right) \delta$ 7.87-7.70 (m, 8H, Ph), 7.35-7.15 (m, 14H, Ph), $6.79(\mathrm{~m}, 2 \mathrm{H}, \mathrm{PMP}), 5.60$ (t, $1 \mathrm{H}, J=6.5 \mathrm{~Hz}, \underline{\mathrm{H}}-13)$ superimposed upon $5.58\left(\mathrm{dd}, 1 \mathrm{H}, J=4.9,11.9 \mathrm{~Hz}, \underline{\mathrm{H}}-7_{\mathrm{ax}}\right), 4.39(\mathrm{~d}, 1 \mathrm{H}, J=-11.7$ $\mathrm{Hz}, \underline{\mathrm{C}}_{2}$ of PMB group), $4.36\left(\mathrm{~d}, 1 \mathrm{H}, J=-11.7 \mathrm{~Hz}, \mathrm{C}_{2}\right.$ of $\mathrm{PMB}$ group) superimposed upon $4.34(\mathrm{~d}, 2 \mathrm{H}, J$ $=6.9 \mathrm{~Hz}, \underline{\mathrm{H}}-17 \mathrm{a}, \underline{\mathrm{H}}-17 \mathrm{~b}), 3.89(\mathrm{~m}, 1 \mathrm{H}, \underline{\mathrm{H}}-3 \mathrm{a})$ superimposed upon $3.86\left(\mathrm{~m}, 1 \mathrm{H}, \underline{\mathrm{H}}-5_{\mathrm{ax}}\right), 3.78(\mathrm{dt}, 1 \mathrm{H}, J=$ 5.4 (t), $-10.5 \mathrm{~Hz}, \underline{\mathrm{H}}-3 \mathrm{~b}), 3.65\left(\mathrm{~m}, 1 \mathrm{H}, \underline{\mathrm{H}}-11_{\mathrm{ax}}\right), 3.42$ (dd, $\left.1 \mathrm{H}, J=5.5,-9.7 \mathrm{~Hz}, \underline{\mathrm{H}}-16 \mathrm{a}\right), 3.37(\mathrm{~m}, 1 \mathrm{H}, \underline{\mathrm{H}}-$

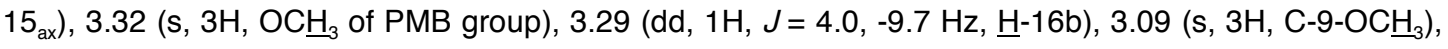
$2.29\left(\mathrm{~d}, 1 \mathrm{H}, J=-13.8 \mathrm{~Hz}, \underline{\mathrm{H}}-14_{\text {eq }}\right), 2.26\left(\mathrm{~d}, 1 \mathrm{H}, J=-13.5 \mathrm{~Hz}, \underline{\mathrm{H}}-12_{\text {eq }}\right), 2.20$ (dd, $1 \mathrm{H}, J=5.0,-15.9 \mathrm{~Hz}, \underline{\mathrm{H}}-$ 10a), 1.92 (t, $1 \mathrm{H}, J=11.8,-12.2 \mathrm{~Hz}, \underline{\mathrm{H}}-12_{\mathrm{ax}}$ ), 1.83 (ddd, $1 \mathrm{H}, J=2.9,4.6,-12.1 \mathrm{~Hz}, \underline{\mathrm{H}}-6_{\text {eq }}$ ), 1.77 (dd, $1 \mathrm{H}$, $J=5.0,-15.9 \mathrm{~Hz}, \underline{\mathrm{H}}-10 \mathrm{~b}), 1.72(\mathrm{~m}, 1 \mathrm{H}, \underline{\mathrm{H}}-4 \mathrm{a}), 1.70\left(\mathrm{~s}, 3 \mathrm{H}, \mathrm{OCOC} \underline{H}_{3}\right), 1.66\left(\mathrm{~m}, 1 \mathrm{H}, \underline{\mathrm{H}}-14_{\mathrm{ax}}\right)$ superimposed upon 1.59 (m, 1H, $\underline{\mathrm{H}}-4 \mathrm{~b}), 1.42$ (apparent q, $1 \mathrm{H}, J=12.0 \mathrm{~Hz}, \underline{\mathrm{H}}-6_{\mathrm{ax}}$ ), 1.17 (s, 3H, C-8$\left.\mathrm{CH}_{3} \mathrm{ax}\right), 1.16\left(\mathrm{~s}, 9 \mathrm{H}, \mathrm{C}\left(\mathrm{C}_{3}\right)_{3}\right), 1.15\left(\mathrm{~s}, 9 \mathrm{H}, \mathrm{C}\left(\mathrm{C}_{3}\right)_{3}\right), 1.12$ (s, 3H, C-8- $\left.\underline{H}_{3} \mathrm{eq}\right) \mathrm{ppm} ;{ }^{13} \mathrm{C} \mathrm{NMR}(125 \mathrm{MHz}$, $\left.\mathrm{C}_{6} \mathrm{D}_{6}, 298 \mathrm{~K}\right) \delta 169.68$ ( $\underline{\mathrm{C}}=\mathrm{O}$ OAc), 159.63 (q $\underline{\mathrm{C}}$ of PMP group), 137.07 ($\left.-13 \mathrm{a}\right), 136.02$ (o-Ph), 136.01 (o-Ph), 135.96 (o-Ph), 135.93 (o-Ph), 134.28 (q Ph), 134.27 (q Ph), 134.17 (q Ph), 134.11 (q Ph), 131.17 (quaternary $\underline{\mathrm{C}}-\mathrm{CH}_{2}$ of PMP group), 130.00 (p-Ph), 129.99 (p-Ph), 129.95 (2 x p-Ph), 129.31 (o-

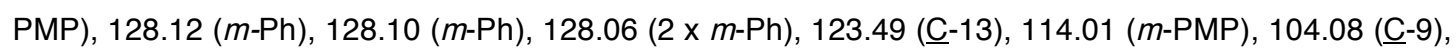




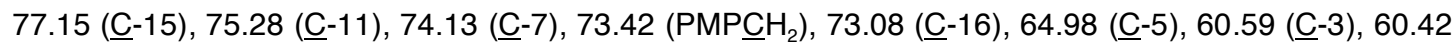

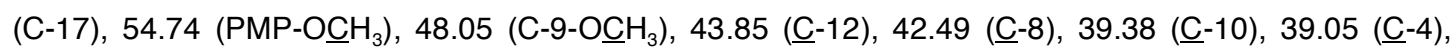

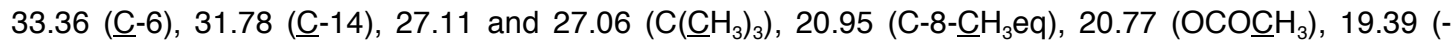
$\left.\underline{\mathrm{C}}\left(\mathrm{CH}_{3}\right)_{3}\right), 19.37\left(\underline{-} \underline{\mathrm{C}}\left(\mathrm{CH}_{3}\right)_{3}\right), 17.17\left(\mathrm{C} 8-\underline{\mathrm{C}} \mathrm{H}_{3} \mathrm{ax}\right)$ ppm. VG 70-SE Positive Ion FAB HRMS: Calcd. for $\mathrm{C}_{61} \mathrm{H}_{80} \mathrm{NaO}_{9} \mathrm{Si}_{2}(\mathrm{M}+\mathrm{Na})^{+}:$1035.52383. Found: 1035.52669 .

\section{Alcohol 24 (SM-8-118)}

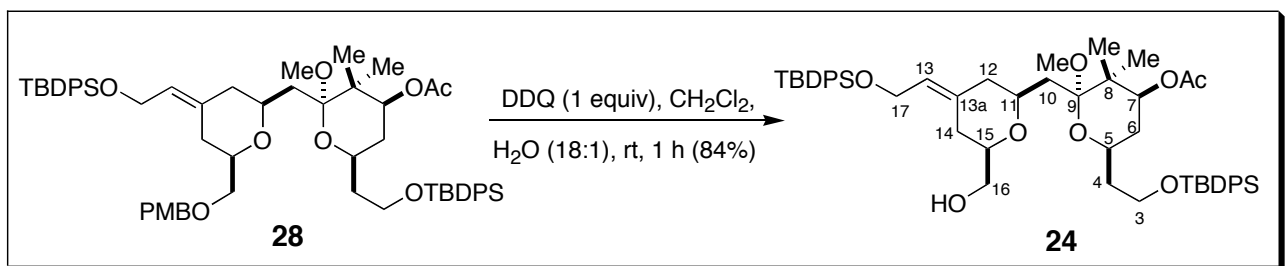

To a solution of acetate $28(1.54 \mathrm{~g}, 1.52 \mathrm{mmol})$ in a (18:1) mixture of $\mathrm{CH}_{2} \mathrm{Cl}_{2}(54 \mathrm{~mL})$ and $\mathrm{H}_{2} \mathrm{O}(3 \mathrm{~mL})$ at rt was added 2,3-dichloro-5,6-dicyanobenzoquinone (DDQ) (345 mg, $1.52 \mathrm{mmol}, 1.0$ equiv) in one portion. The reactants were stirred at $\mathrm{rt}$ for $1 \mathrm{~h}$ and then quenched with solid $\mathrm{NaHCO}_{3}(1 \mathrm{~g})$ followed by saturated aqueous $\mathrm{NaHCO}_{3}(50 \mathrm{~mL})$. The resulting mixture was diluted with $\mathrm{CH}_{2} \mathrm{Cl}_{2}(100 \mathrm{~mL})$ and the organic layer was separated. The aqueous phase was extracted with $\mathrm{CH}_{2} \mathrm{Cl}_{2}(3 \times 100 \mathrm{~mL})$ and the combined organic extracts were washed with saturated aqueous $\mathrm{NaHCO}_{3}(2 \times 100 \mathrm{~mL})$, dried over $\mathrm{MgSO}_{4}$, filtered, and concentrated in vacuo. Purification of the crude residue by $\mathrm{SiO}_{2}$ flash chromatography (gradient elution with $10: 1$ to $8: 1$ petrol-EtOAc) gave alcohol $24(1.14 \mathrm{~g}, 84 \%$ ) as a colorless syrup.

Data for 24: $[\alpha]_{\mathrm{D}}+41.6^{\circ}\left(c\right.$ 0.414, $\left.\mathrm{CH}_{2} \mathrm{Cl}_{2}\right)$; IR (neat film) $3473(\mathrm{~m}, \mathrm{br}), 3071(\mathrm{~m}), 3049(\mathrm{~m}), 2956(\mathrm{~s})$, 2933 (s), 2889 (s), 2857 (s), 1740 (s), 1589 (w), 1471 (s), 1428 (s), 1388 (s), 1365 (s), 1331 (w), 1245 (s), 1191 (w), 1110 (s), 1073 (s), 1026 (s), 931 (w), 823 (m), 741 (m), 705 (s), 611 (m), 503 (s). ${ }^{1} \mathrm{H}$ NMR (500 MHz, $\left.\mathrm{C}_{6} \mathrm{D}_{6}, 298 \mathrm{~K}\right) \delta$ 7.85-7.70 (m, 8H, Ph), 7.32-7.16 (m, 12H, Ph), $5.58(\mathrm{t}, 1 \mathrm{H}, J=6.5 \mathrm{~Hz}, \underline{\mathrm{H}}-13)$, $5.54(\mathrm{dd}, 1 \mathrm{H}, J=4.8,11.8 \mathrm{~Hz}, \underline{\mathrm{H}}-7 \mathrm{ax}), 4.33(\mathrm{dd}, 1 \mathrm{H}, J=6.6,-16.7 \mathrm{~Hz}, \underline{\mathrm{H}}-17 \mathrm{a})$ superimposed upon 4.29 (dd, $1 \mathrm{H}, J=6.5,-16.7 \mathrm{~Hz}, \underline{\mathrm{H}}-17 \mathrm{~b}), 3.89(\mathrm{~m}, 1 \mathrm{H}, \underline{\mathrm{H}}-3 \mathrm{a})$ superimposed upon $3.86(\mathrm{~m}, 1 \mathrm{H}, \underline{\mathrm{H}}-5), 3.78$ (ddd, $1 \mathrm{H}, J=5.2,5.5,-10.4 \mathrm{~Hz}, \underline{\mathrm{H}}-3 \mathrm{~b}), 3.57\left(\mathrm{~m}, 1 \mathrm{H}, \underline{\mathrm{H}}-11_{\mathrm{ax}}\right), 3.32(\mathrm{~m}, 1 \mathrm{H}, \underline{\mathrm{H}}-16 \mathrm{a})$ superimposed upon $3.31(\mathrm{~m}$, $1 \mathrm{H}, \underline{\mathrm{H}}-16 \mathrm{~b}), 3.03\left(\mathrm{~m}, 1 \mathrm{H}, \underline{\mathrm{H}}-15_{\mathrm{ax}}\right)$ superimposed upon $3.01\left(\mathrm{~s}, 3 \mathrm{H}, \mathrm{C}-9-\mathrm{OCH}_{3}\right), 2.11(\mathrm{t}, 1 \mathrm{H}, J=6.1 \mathrm{~Hz}$, $\mathrm{OH}$ ) superimposed upon $2.10\left(\mathrm{~d}, 1 \mathrm{H}, J=-13.6 \mathrm{~Hz}, \underline{\mathrm{H}}-12_{\mathrm{eq}}\right), 2.06(\mathrm{dd}, 1 \mathrm{H}, J=6.8,-15.9 \mathrm{~Hz}, \underline{\mathrm{H}}-10 \mathrm{a})$, $1.89\left(\mathrm{~d}, 1 \mathrm{H}, J=-13.3 \mathrm{~Hz}, \underline{\mathrm{H}}-14_{\mathrm{eq}}\right), 1.81\left(\mathrm{~m}, 2 \mathrm{H}, \underline{\mathrm{H}}-12_{\mathrm{ax}}\right.$ and $\left.\underline{\mathrm{H}}-6_{\text {eq }}\right), 1.71(\mathrm{~m}, 1 \mathrm{H}, \underline{\mathrm{H}}-4 \mathrm{a})$ superimposed upon $1.70\left(\mathrm{~s}, 3 \mathrm{H}, \mathrm{OCOC} \underline{H}_{3}\right), 1.64(\mathrm{dd}, 1 \mathrm{H}, J=4.0,-15.9 \mathrm{~Hz}, \underline{\mathrm{H}}-10 \mathrm{~b})$ superimposed upon $1.61(\mathrm{~m}, 1 \mathrm{H}, \underline{\mathrm{H}}-$ 4b), 1.44 (broadened t, $1 \mathrm{H}, J=12.5 \mathrm{~Hz}, \underline{\mathrm{H}}-14_{\mathrm{ax}}$ ), 1.36 (apparent q, $\left.1 \mathrm{H}, J=12.0 \mathrm{~Hz}, \underline{\mathrm{H}}-6_{\mathrm{ax}}\right), 1.16(\mathrm{~s}, 9 \mathrm{H}$, $\left.\mathrm{C}\left(\mathrm{CH}_{3}\right)_{3}\right), 1.15$ (s, 9H, C( $\left.\left.\mathrm{CH}_{3}\right)_{3}\right), 1.09$ (s, 3H, C-8- $\left.\underline{\mathrm{H}}_{3} \mathrm{ax}\right), 1.08$ (s, 3H, C-8- $\left.\underline{\mathrm{H}}_{3} \mathrm{eq}\right) \mathrm{ppm} ;{ }^{13} \mathrm{C}$ NMR (125 $\left.\mathrm{MHz}, \mathrm{C}_{6} \mathrm{D}_{6}, 298 \mathrm{~K}\right) \delta 169.68$ ( $\underline{\mathrm{C}}=\mathrm{O} \mathrm{OAc}$ ), 136.21 (드-13a), 136.01 (o-Ph), 136.00 (o-Ph), 135.98 (o-Ph), 135.94 (o-Ph), 134.21 (q Ph), 134.18 (q Ph), 134.17 (q Ph), 134.05 (q Ph), 130.05 (p-Ph), 130.03 ( $p$ -

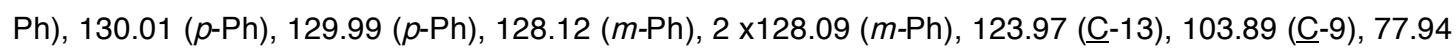




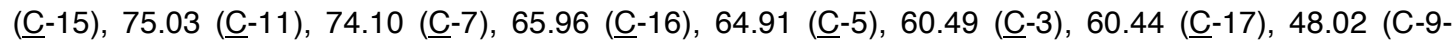

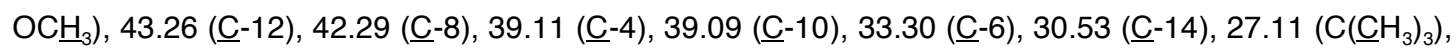
$27.04\left(\mathrm{C}\left(\underline{\mathrm{CH}}_{3}\right)_{3}\right), 20.91\left(\mathrm{C}-8-\underline{\mathrm{C}}_{3} \mathrm{eq}\right), 20.78\left(\mathrm{OCO} \underline{\mathrm{CH}}_{3}\right), 19.38$ and $19.35\left(\underline{\mathrm{C}}\left(\mathrm{CH}_{3}\right)_{3}\right), 17.31\left(\mathrm{C}-8-\underline{\mathrm{C}} \mathrm{H}_{3} \mathrm{ax}\right)$ ppm. VG 70-SE Positive FAB HRMS: Calcd. for $\mathrm{C}_{53} \mathrm{H}_{72} \mathrm{O}_{8} \mathrm{Si}_{2} \mathrm{Na}(\mathrm{M}+\mathrm{Na})^{+}:$: 915.46632. Found: 915.46882.

\section{Masamune's AB Fragment 1 (SM-8-119)}

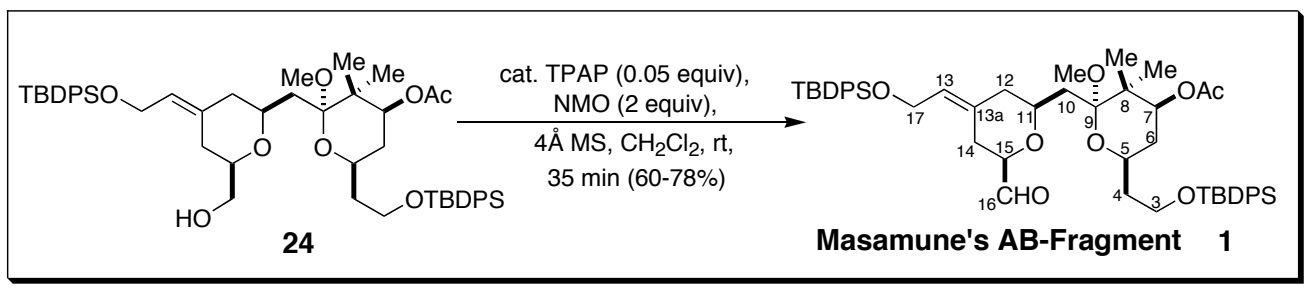

To a stirred solution of alcohol $24(0.2 \mathrm{~g}, 0.22 \mathrm{mmol})$, powdered $4 \AA$ molecular sieves (freshly flame dried under high vacuum) $(0.5 \mathrm{~g})$ and $N$-methylmorpholine $N$-oxide $(52.61 \mathrm{mg}, 0.45 \mathrm{mmol}, 2.0$ equiv) in dry $\mathrm{CH}_{2} \mathrm{Cl}_{2}(5 \mathrm{~mL})$ at rt was added tetra- $n$-propylammonium perruthenate (TPAP) (3.93 mg, $0.01 \mathrm{mmol}, 0.05$ equiv) in one portion. After $35 \mathrm{~min}$, the reaction mixture was filtered through a pad of Celite and the filtrate concentrated in vacuo. Purification of the crude black residue by $\mathrm{SiO}_{2}$ flash chromatography using petrol-EtOAc (10:1) as eluent furnished aldehyde $1(0.12 \mathrm{~g}, 60 \%)$ as a colorless oil.

Data for 1: $[\alpha]_{\mathrm{D}}+52.1^{\circ}\left(\mathrm{c} 0.384, \mathrm{CH}_{2} \mathrm{Cl}_{2}\right)$; IR (neat film) $3035(\mathrm{w}), 3071(\mathrm{w}), 2956(\mathrm{~s}), 2933(\mathrm{~s}), 2892(\mathrm{~m})$, 2857 (s), 1740 (s), $1474(\mathrm{~m}), 1428(\mathrm{~m}), 1387$ (m), 1366 (m), 1244 (s), 1110 (s), $1070(\mathrm{~m}), 1027(\mathrm{~m}), 740$ (m), 704 (s), 680 (m), 504 (m). ${ }^{1} \mathrm{H}$ NMR $\left(500 \mathrm{MHz}, \mathrm{C}_{6} \mathrm{D}_{6}, 298 \mathrm{~K}\right) \delta 9.45$ (d, 1H, J = $\left.0.6 \mathrm{~Hz}, \mathrm{C}-16-\mathrm{C} \underline{\mathrm{HO}}\right)$, 7.85-7.72 (m, 8H, Ph), 7.32-7.17 (m, 12H, Ph), $5.57(\mathrm{dd}, 1 \mathrm{H}, J=4.8,11.8 \mathrm{~Hz}, \underline{\mathrm{H}}-7)$ partially superimposed upon $5.54(\mathrm{t}, 1 \mathrm{H}, J=6.4 \mathrm{~Hz}, \underline{\mathrm{H}}-13), 4.28(\mathrm{dd}, 1 \mathrm{H}, J=6.4,-17.0 \mathrm{~Hz}, \underline{\mathrm{H}}-17 \mathrm{a})$ overlapped onto 4.25 (dd, $1 \mathrm{H}, J=6.4,-17.0 \mathrm{~Hz}, \underline{\mathrm{H}}-17 \mathrm{~b}), 3.93-3.78(\mathrm{~m}, 2 \mathrm{H}, \underline{\mathrm{H}}-3 \mathrm{a}$ and $\underline{\mathrm{H}}-5), 3.77$ (ddd, $1 \mathrm{H}, J=5.7$, 6.0, -10.3 Hz, $\underline{\mathrm{H}}-3 \mathrm{~b}), 3.54(\mathrm{~m}, 1 \mathrm{H}, \underline{\mathrm{H}}-11), 3.21$ (dd, $1 \mathrm{H}, J=2.9,12.0 \mathrm{~Hz}, \underline{\mathrm{H}}-15_{\mathrm{ax}}$ ), 3.01 (s, 3H, C-9-OC $\left.\underline{H}_{3}\right)$, $2.42\left(\mathrm{br} \mathrm{d}, 1 \mathrm{H}, J=-14.0 \mathrm{~Hz}, \underline{\mathrm{H}}-14_{\text {eq }}\right.$ ), $2.17\left(\mathrm{br} \mathrm{d}, 1 \mathrm{H}, J=-13.6 \mathrm{~Hz}, \underline{\mathrm{H}}-12_{\text {eq }}\right.$ ) superimposed upon 2.15 (dd, $1 \mathrm{H}, J=5.1,-16.0 \mathrm{~Hz}, \underline{\mathrm{H}}-10 \mathrm{a}), 1.84$ (ddd, $1 \mathrm{H}, J=2.8,4.8,-12.3 \mathrm{~Hz}, \underline{\mathrm{H}}-6_{\text {eq }}$ ), 1.76 (br d, $1 \mathrm{H}, J=-13.6 \mathrm{~Hz}$, $\left.\underline{\mathrm{H}}-12_{\mathrm{ax}}\right), 1.72$ (s, 3H, C-7-OCOC$\left.H_{3}\right), 1.71$ (m, 1H, $\left.\underline{\mathrm{H}}-10 \mathrm{~b}\right), 1.67$ (m, 1H, $\left.\underline{\mathrm{H}}-4 \mathrm{a}\right), 1.60(\mathrm{~m}, 1 \mathrm{H}, \underline{\mathrm{H}}-4 \mathrm{~b}), 1.51$ (br dd, $\left.1 \mathrm{H}, J=12.8,-13.2 \mathrm{~Hz}, \underline{\mathrm{H}}-14_{\mathrm{ax}}\right), 1.42$ (apparent q, $\left.1 \mathrm{H}, J=12.0 \mathrm{~Hz}, \underline{\mathrm{H}}-6_{\mathrm{ax}}\right), 1.162\left(\mathrm{~s}, 9 \mathrm{H}, \mathrm{C}\left(\mathrm{CH}_{3}\right)_{3}\right)$, $1.156\left(\mathrm{~s}, 9 \mathrm{H}, \mathrm{C}\left(\mathrm{CH}_{3}\right)_{3}\right), 1.12$ (s, 3H, C-8- $\left.\underline{\mathrm{H}}_{3} \mathrm{ax}\right), 1.10$ (s, 3H, C-8-C $\left.\underline{\mathrm{H}}_{3} \mathrm{eq}\right) \mathrm{ppm} ;{ }^{13} \mathrm{C} \mathrm{NMR}(125 \mathrm{MHz}$, $\left.\mathrm{C}_{6} \mathrm{D}_{6}, 298 \mathrm{~K}\right) \delta 199.82(\mathrm{C}-16-\underline{\mathrm{C}} \mathrm{HO}), 169.68\left(\mathrm{O}_{\underline{C} O \mathrm{CH}_{3}}\right), 136.02(o-\mathrm{Ph}), 136.00(o-\mathrm{Ph}), 135.96(o-\mathrm{Ph})$, 135.93 (o-Ph), 134.65 (ㄸ-13a), 134.14 (quaternary $\underline{\mathrm{C}}, \mathrm{Ph}), 134.09$ (quaternary $\underline{\mathrm{C}}, \mathrm{Ph}), 134.06$ (quaternary $\underline{\mathrm{C}}, \mathrm{Ph}), 134.01$ (quaternary $\underline{\mathrm{C}}, \mathrm{Ph}), 130.07$ (p-Ph), 130.06 (p-Ph), 2 x 130.05 (p-Ph), 128.15

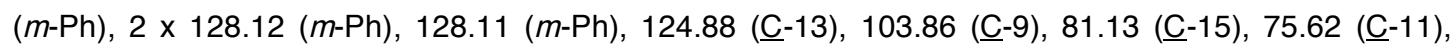

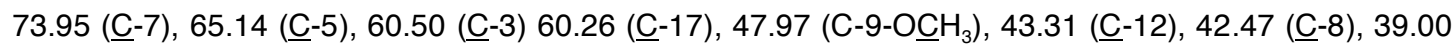

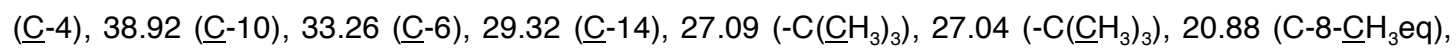
$20.76\left(-\mathrm{OCO}^{\mathrm{C}} \mathrm{H}_{3}\right), 19.37$ and $19.35\left(-\underline{\mathrm{C}}\left(\mathrm{CH}_{3}\right)_{3}\right), 17.15\left(\mathrm{C}-8-\underline{\mathrm{C}} \mathrm{H}_{3} \mathrm{ax}\right) \mathrm{ppm}$. VG 70-SE Positive Ion FAB HRMS: Calcd. for $\mathrm{C}_{53} \mathrm{H}_{70} \mathrm{NaO}_{8} \mathrm{Si}_{2}(\mathrm{M}+\mathrm{Na})^{+}:$913.45067. Found: 913.44719 . 
Important Notes: (1) The $500 \mathrm{MHz}{ }^{1} \mathrm{H}$ NMR $J$ values reported above are derived from the normal ${ }^{1} \mathrm{H}$ NMR spectrum without the aid of resolution enhancement; the fully assigned $J$ values obtained via resolution enhancement are reported in Parts 3 and 4 of this SI.

(2) We have found that the Masamune AB-fragment 1 is very acid-labile and readily undergoes rapid decomposition in commercially available $\mathrm{CDCl}_{3}$. As a consequence, we specifically recommend that other workers wishing to record the NMR spectra of this compound do so in $\mathrm{C}_{6} \mathrm{D}_{6}$ or some other nonacidic solvent.

(3) For the accurate, fully-assigned, ${ }^{1} \mathrm{H}$ and ${ }^{13} \mathrm{C}$ NMR chemical shifts and $J$ values for $\mathbf{1}$ in $\mathrm{CDCl}_{3}$, see the new data and spectra that we report, in Parts 3 and 6 of this $\mathrm{SI}$, which corrects the previous ${ }^{1} \mathrm{H}$ NMR spectral data that was published for 1 in $\mathrm{CDCl}_{3}$ by: Kageyama, M.; Tamura, T.; Nantz, M.H.; Roberts, J.C.; Somfai, P.; Whritenour, D.C.; Masamune, S. J. Am. Chem. Soc. 1990, 112, 7407. Part 3 also reports important chemical and NMR correlations that we have made on several advanced A- and B-ring fragments used in our synthesis of 1, with identical compounds prepared by Masamune (Ref. 7a) and H.M.R Hoffmann (Ref. 13a) in their respective syntheses of their bryostatin AB fragments. 


\title{
Enantioselective Formal Total Synthesis of the Antitumor Macrolide Bryostatin 7
}

\author{
Soraya Manaviazar, ${ }^{*}$ Mark Frigerio, \\ Gurpreet S. Bhatia, Marc G. Hummersone, \\ Abil E. Aliev, and Karl J. Hale* \\ The Christopher Ingold Laboratories, \\ The Chemistry Department, \\ University College London, \\ 20 Gordon Street, \\ London WC1H OAJ, UK. \\ Supporting Information
}

Part 2. Copies of the Spectra for the Intermediates Used in the UCL Synthesis of the Masamune Bryostatin 7 AB Intermediate 1 


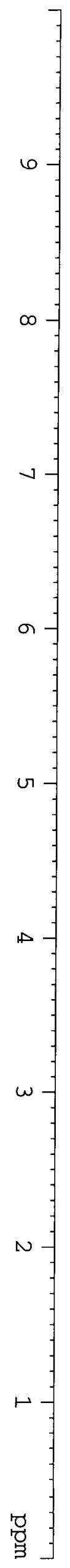
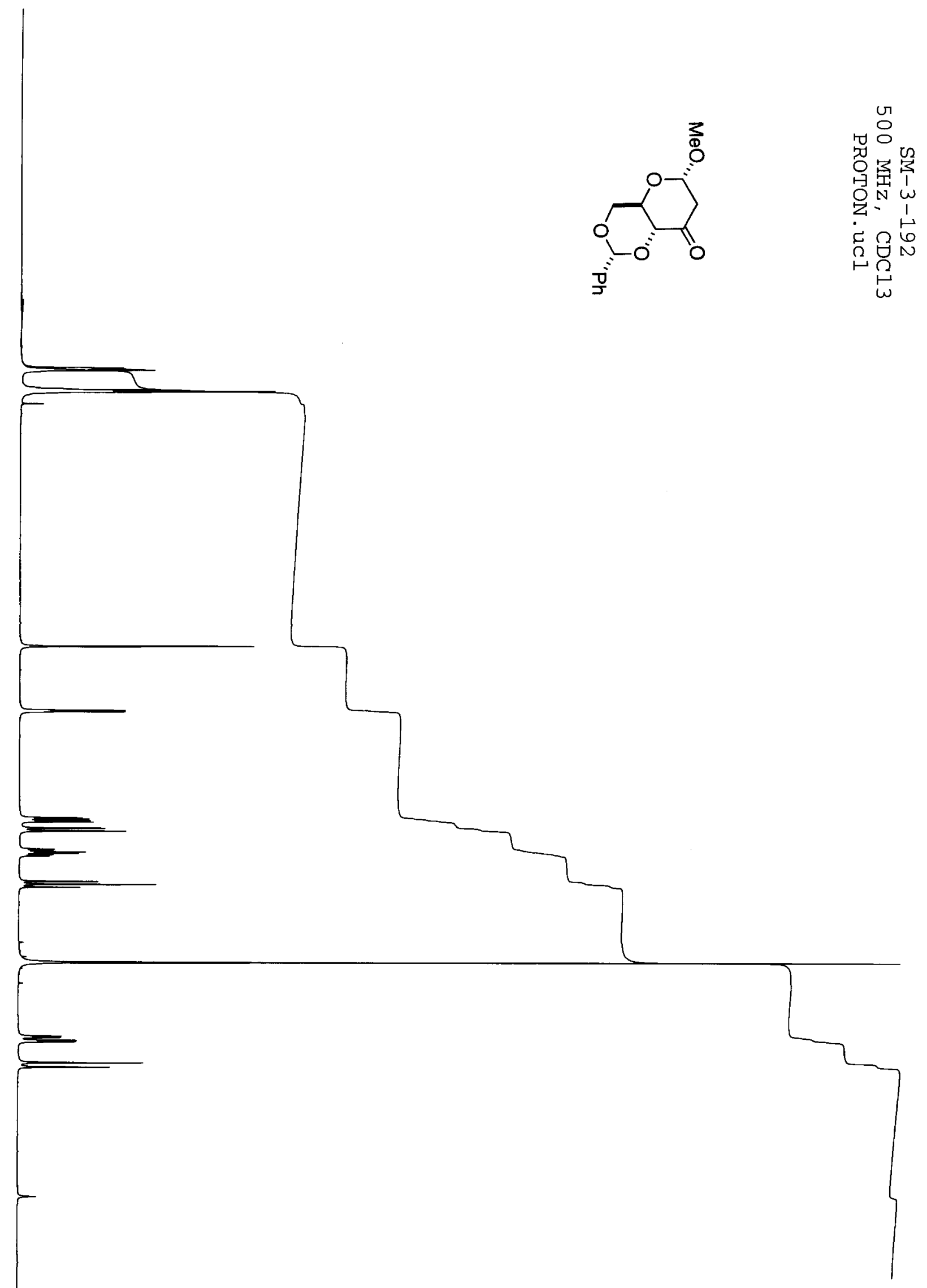

24 

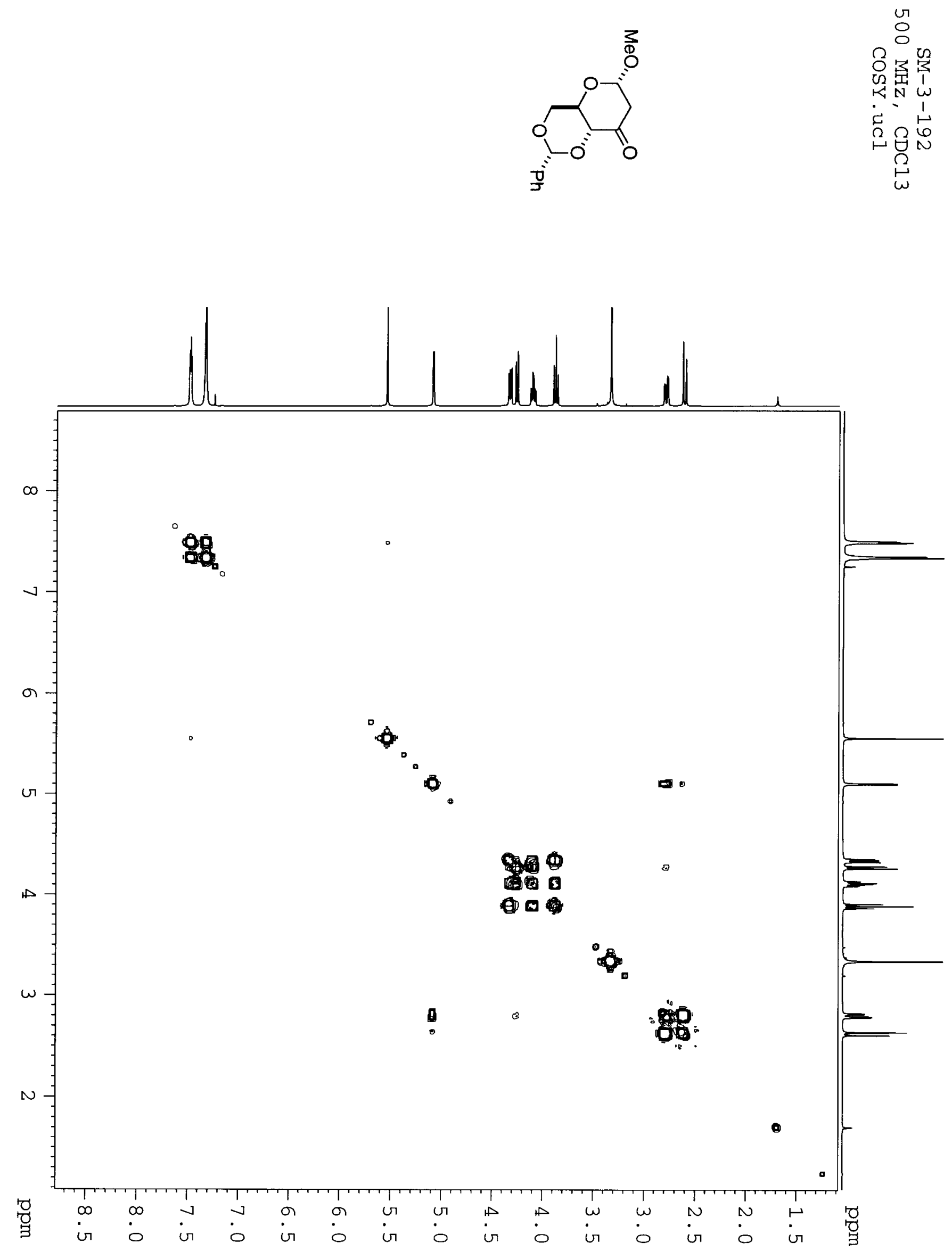


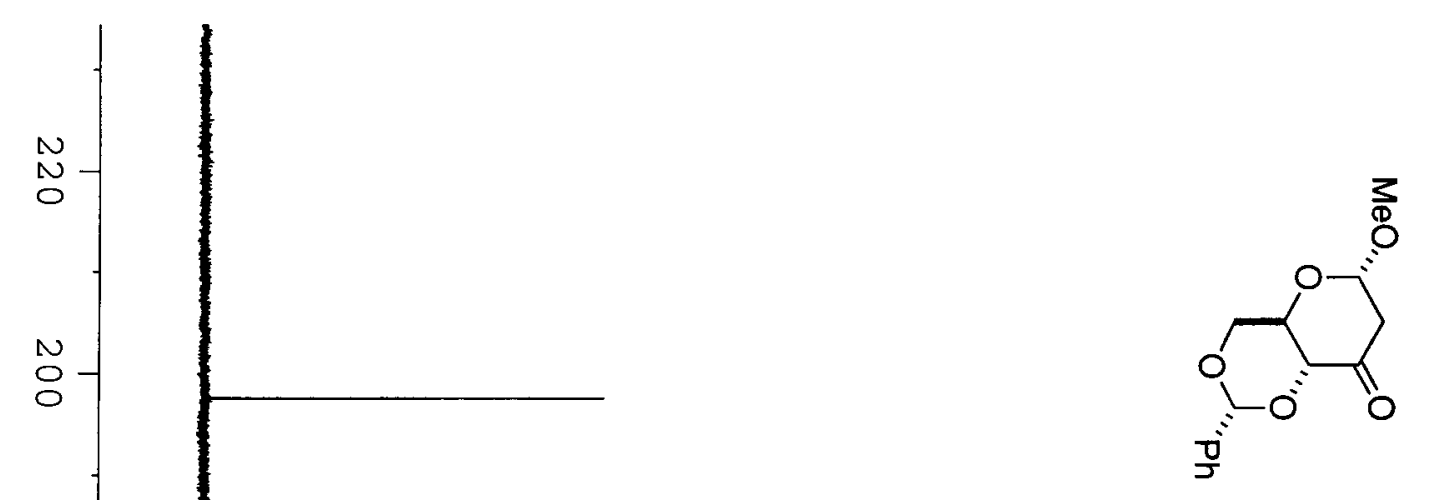

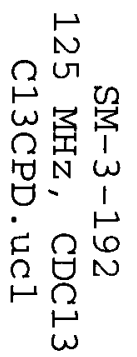

$\bullet$
$\infty$
0

몽

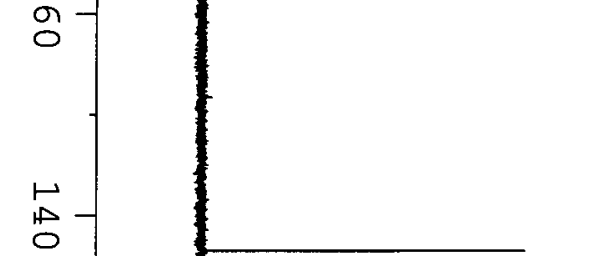

号

$\stackrel{\bullet}{N}$

뭉

$\infty$

a

o

$\stackrel{1}{0}$

N

$N$

o-

㕷 


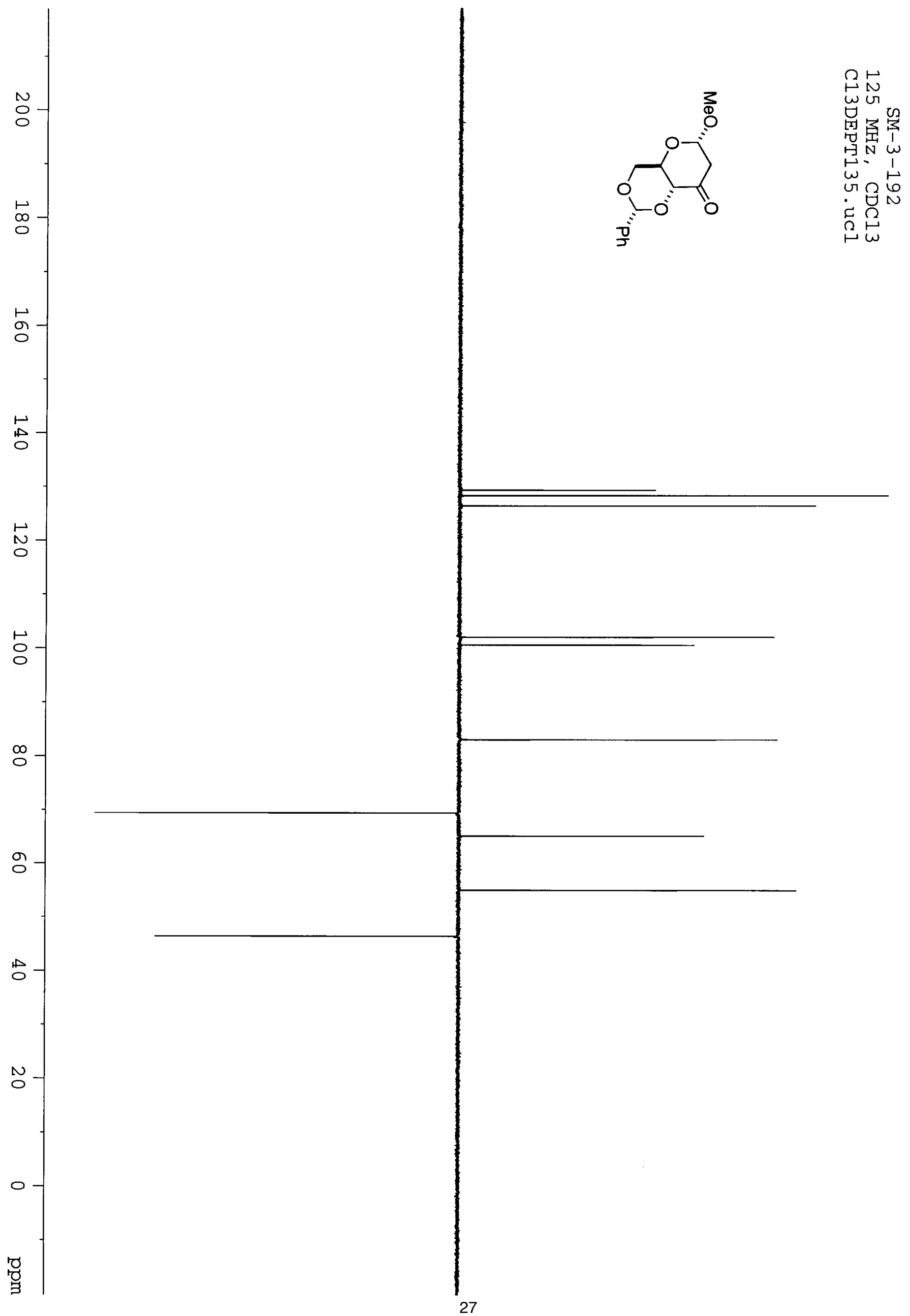



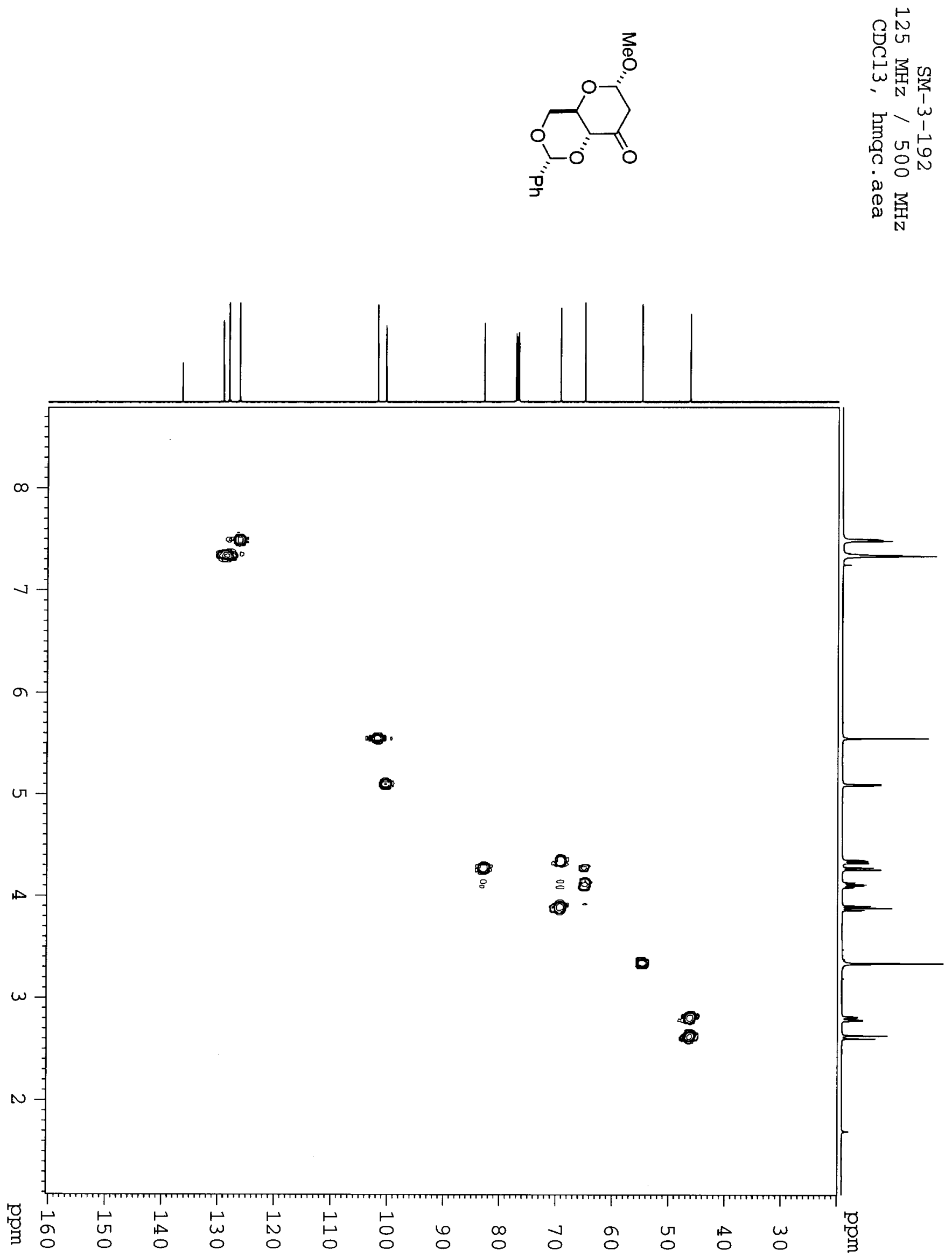


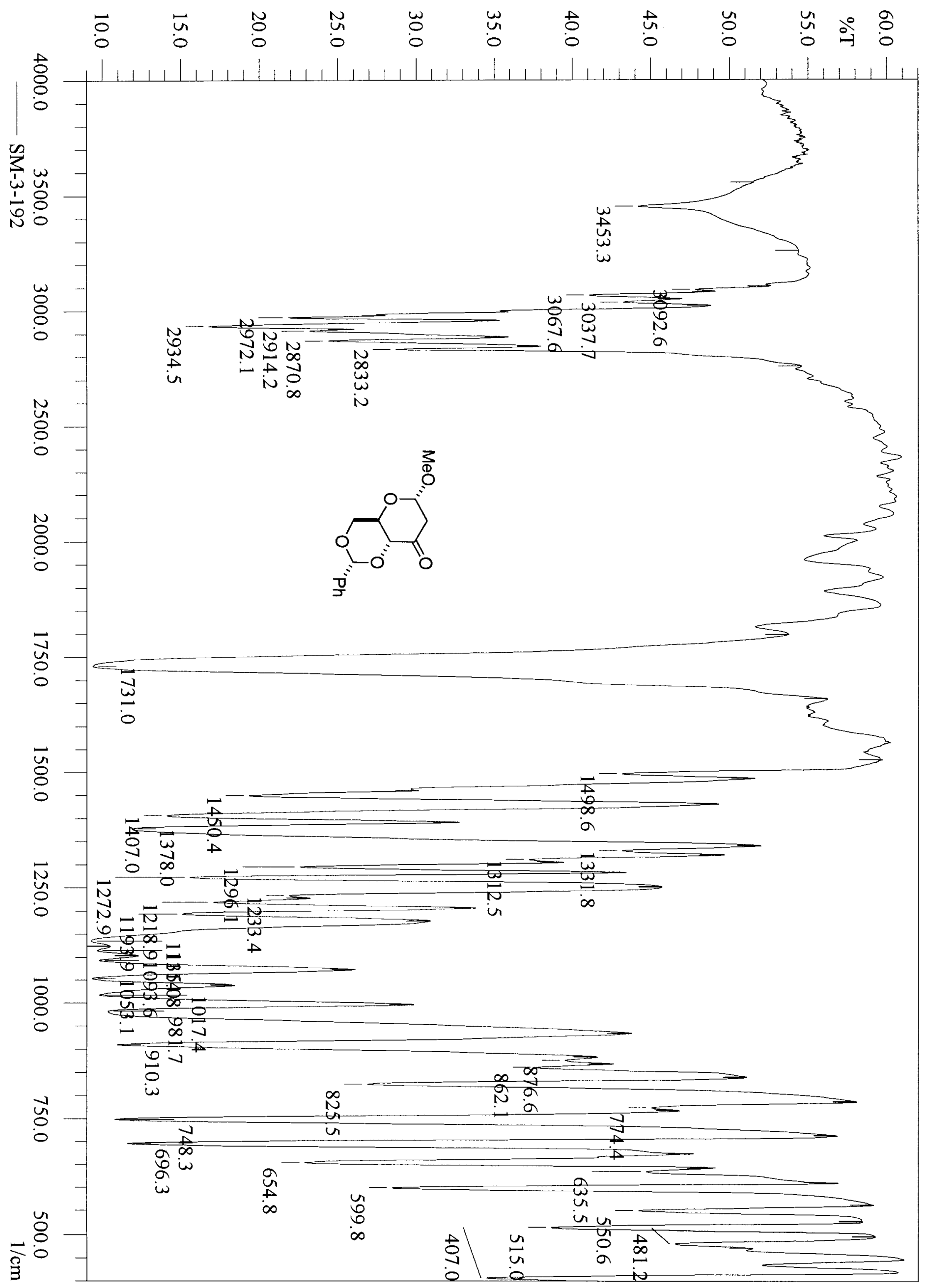




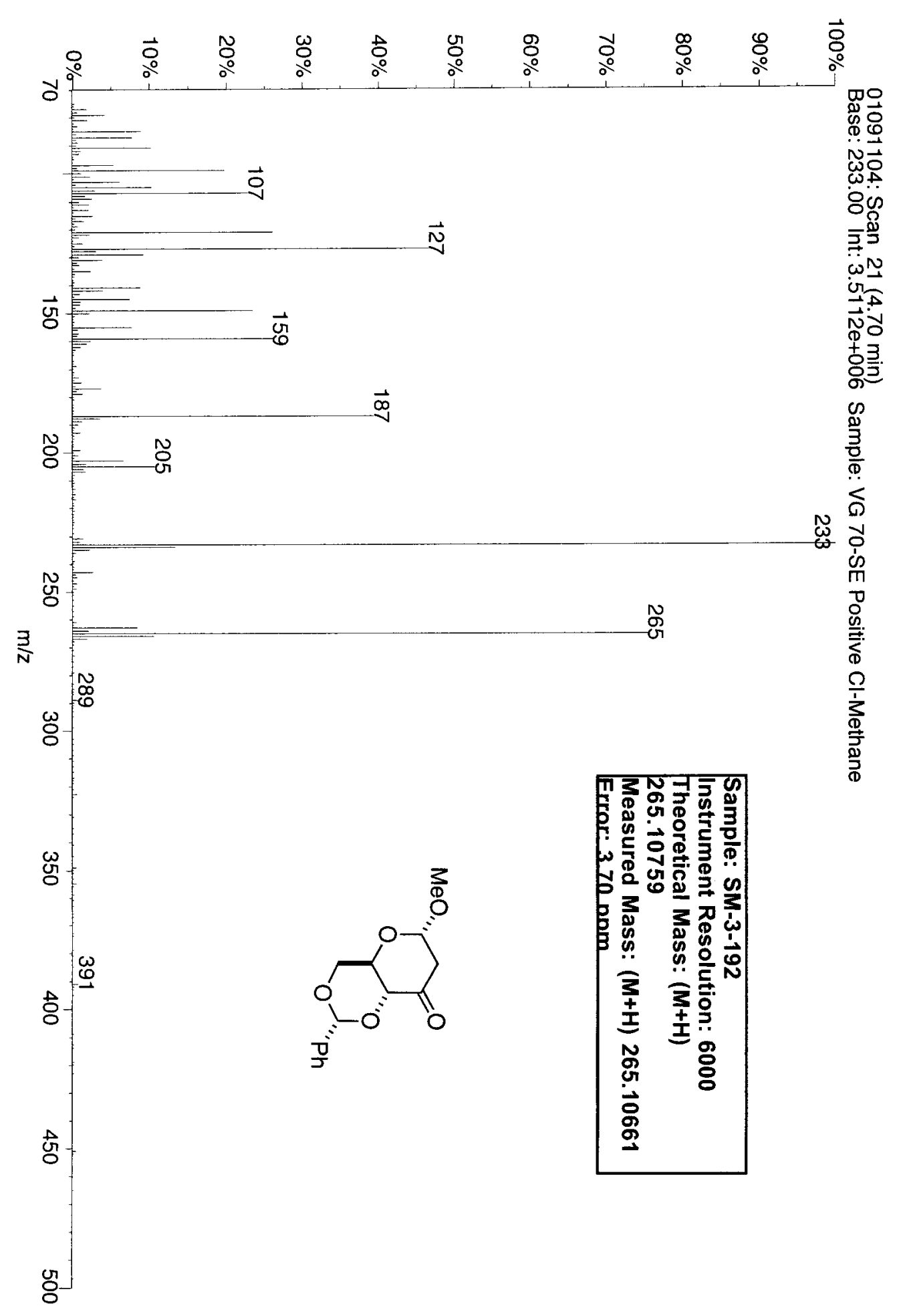



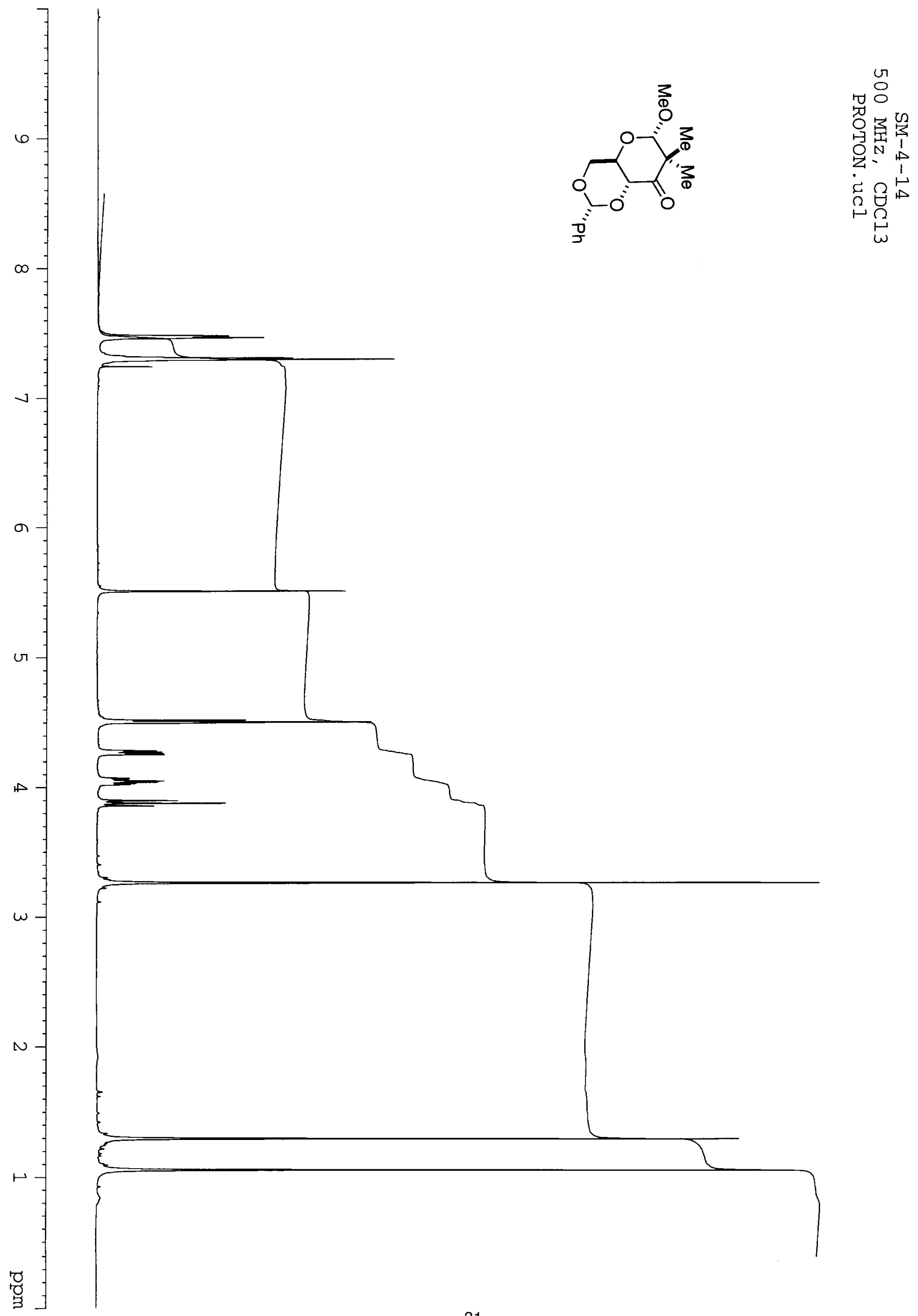

31 

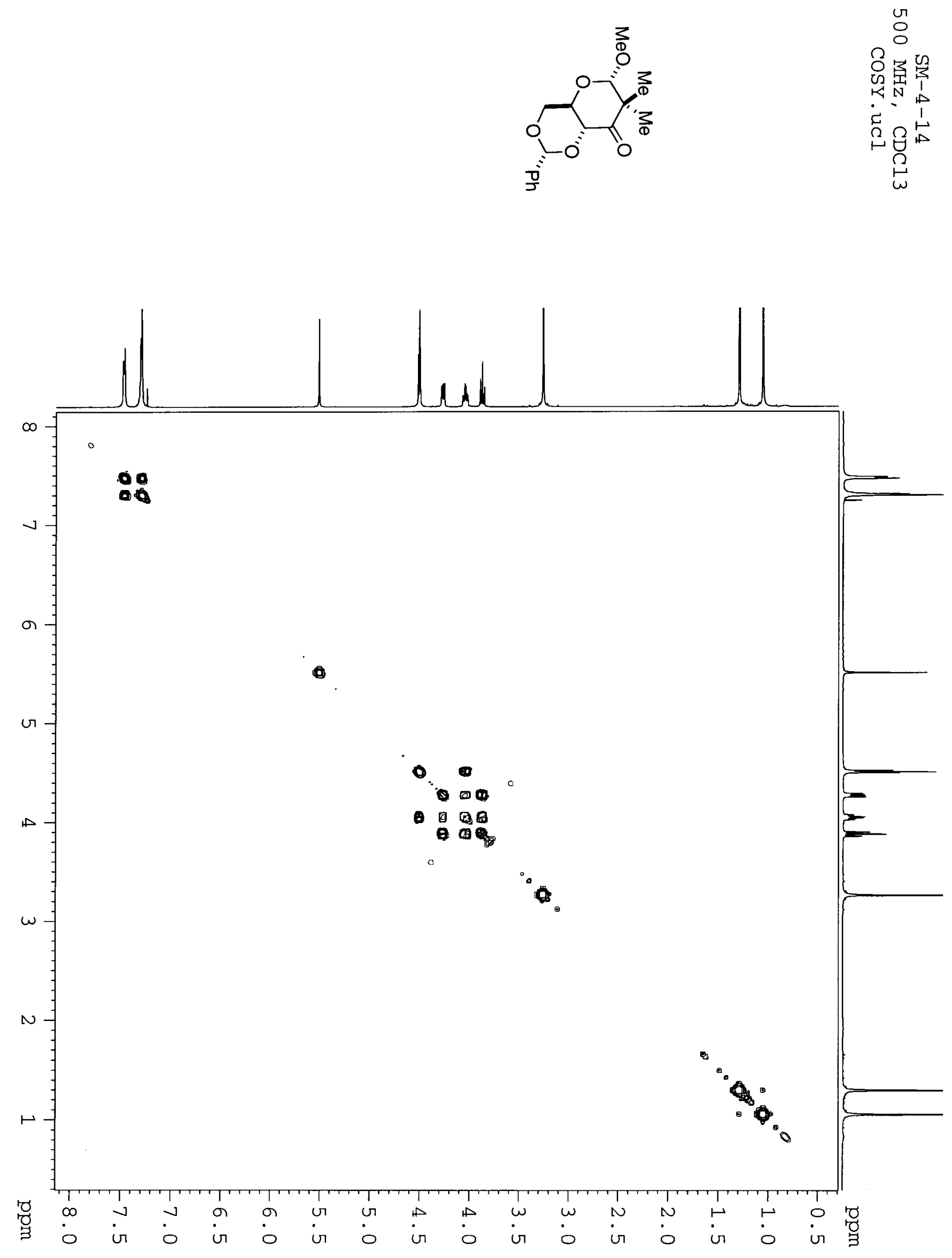

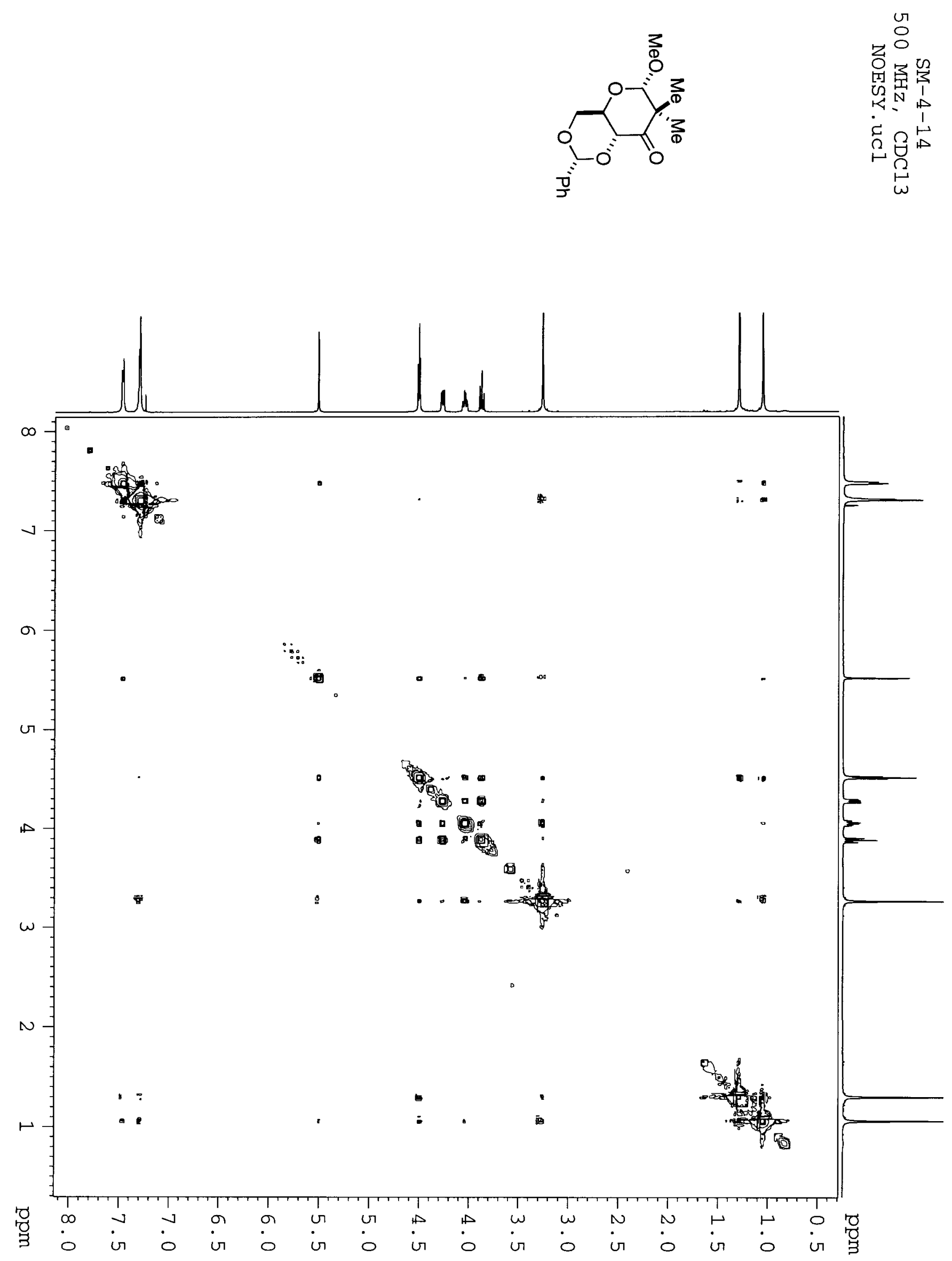

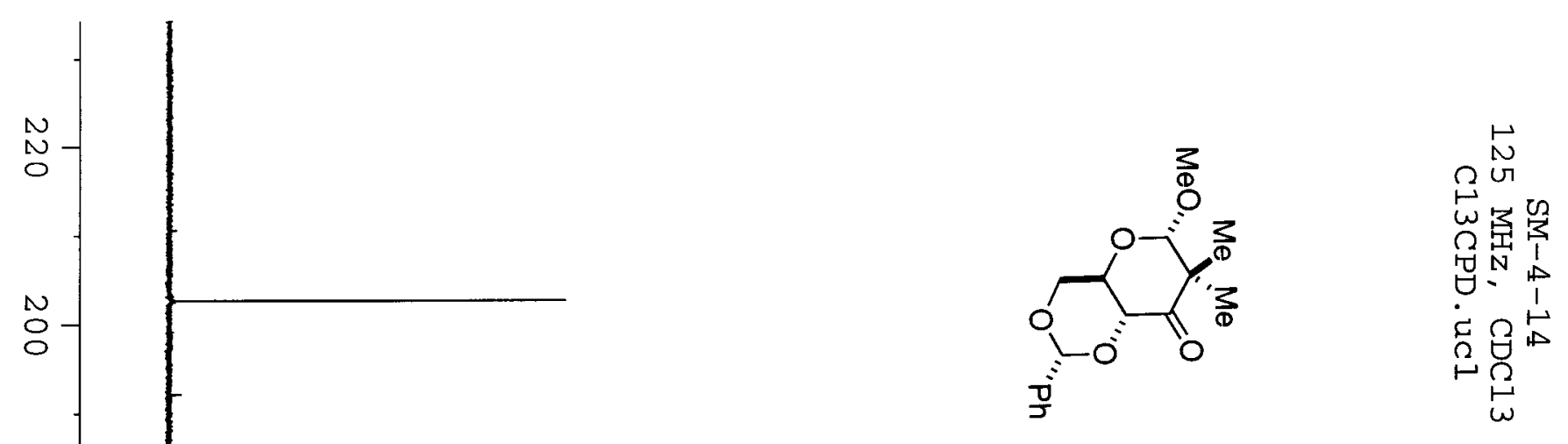

$\stackrel{\infty}{\infty}$

ڤ

$\stackrel{\oplus}{0}$

$\stackrel{1}{0}$

$\stackrel{N}{\circ}$

品

$\infty$

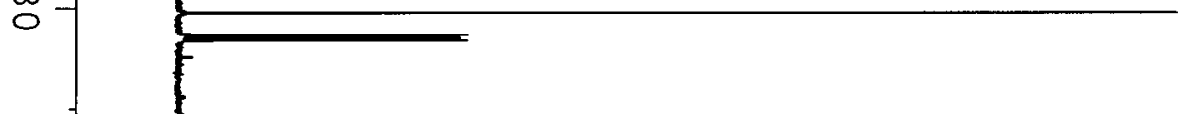

잉

g

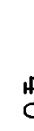

$\stackrel{p}{\circ}$

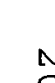

N

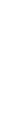

'

盟 


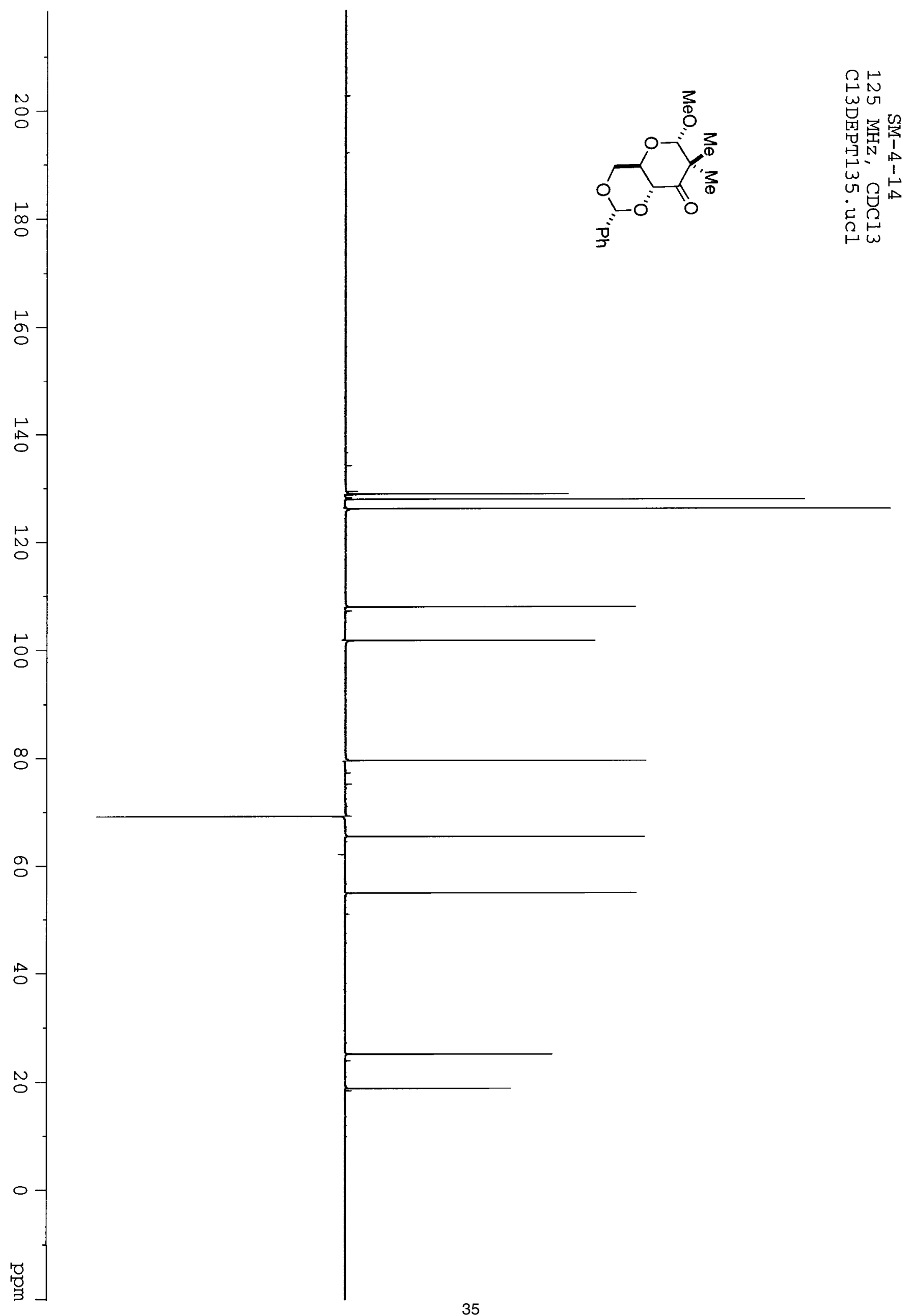



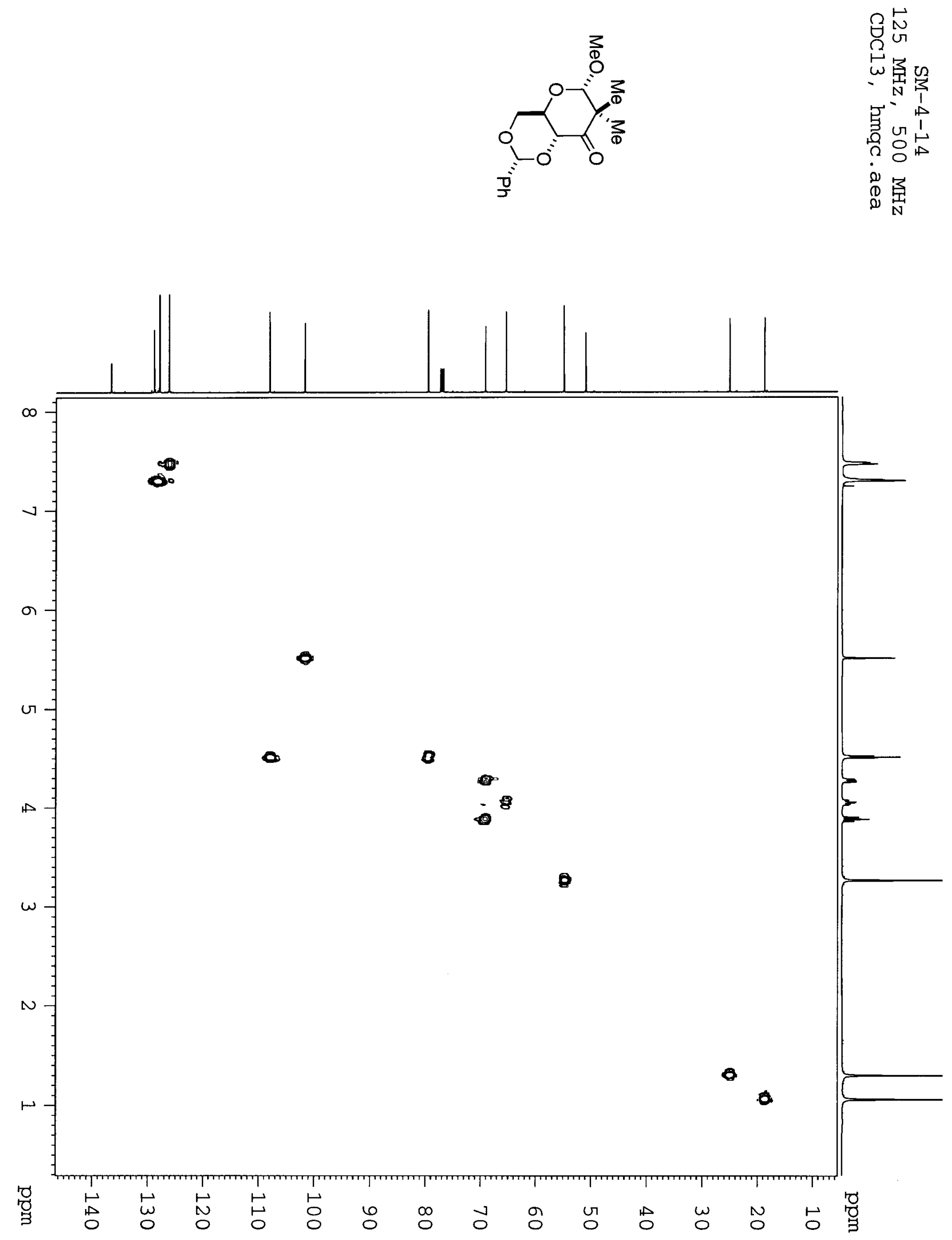


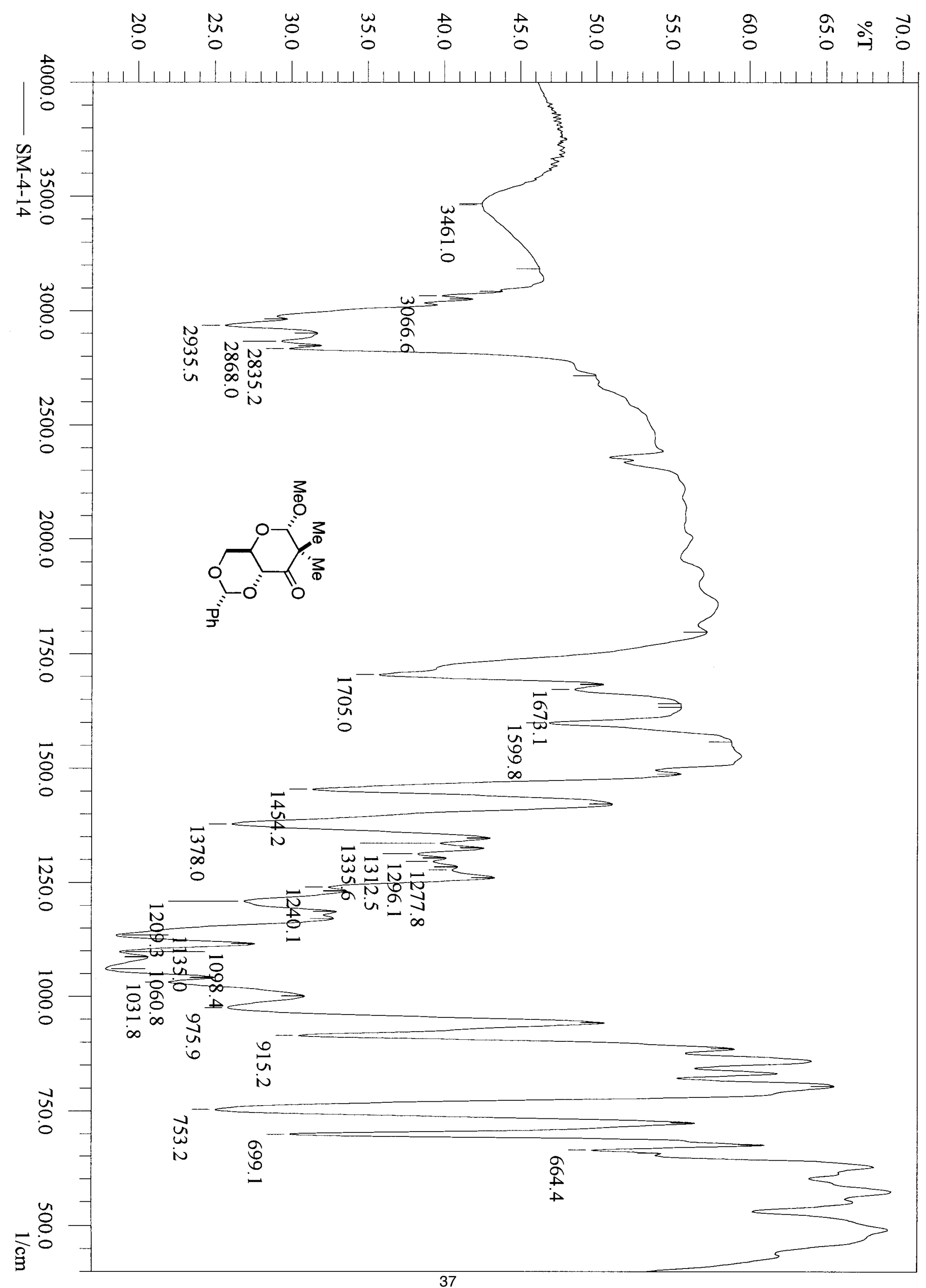




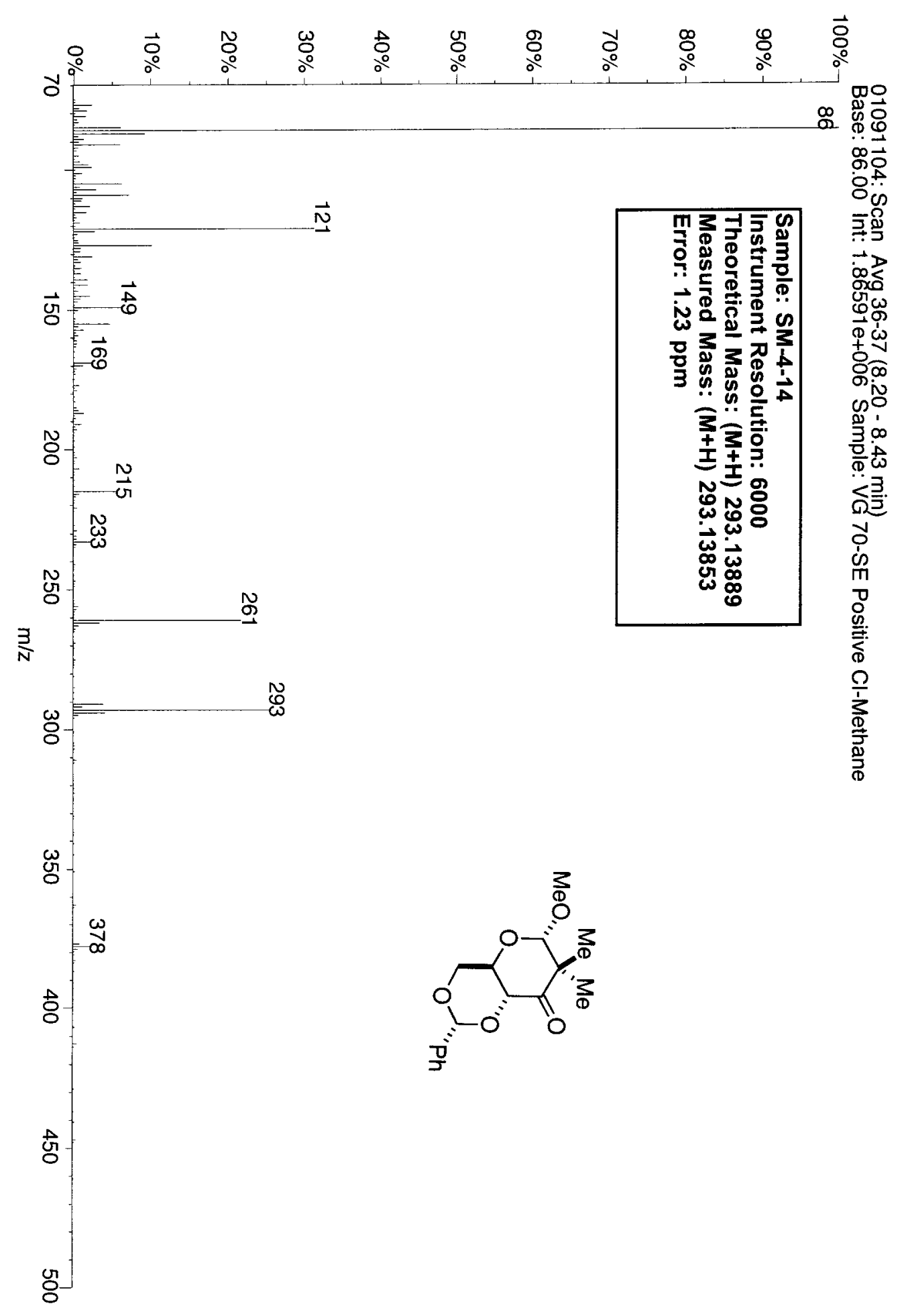




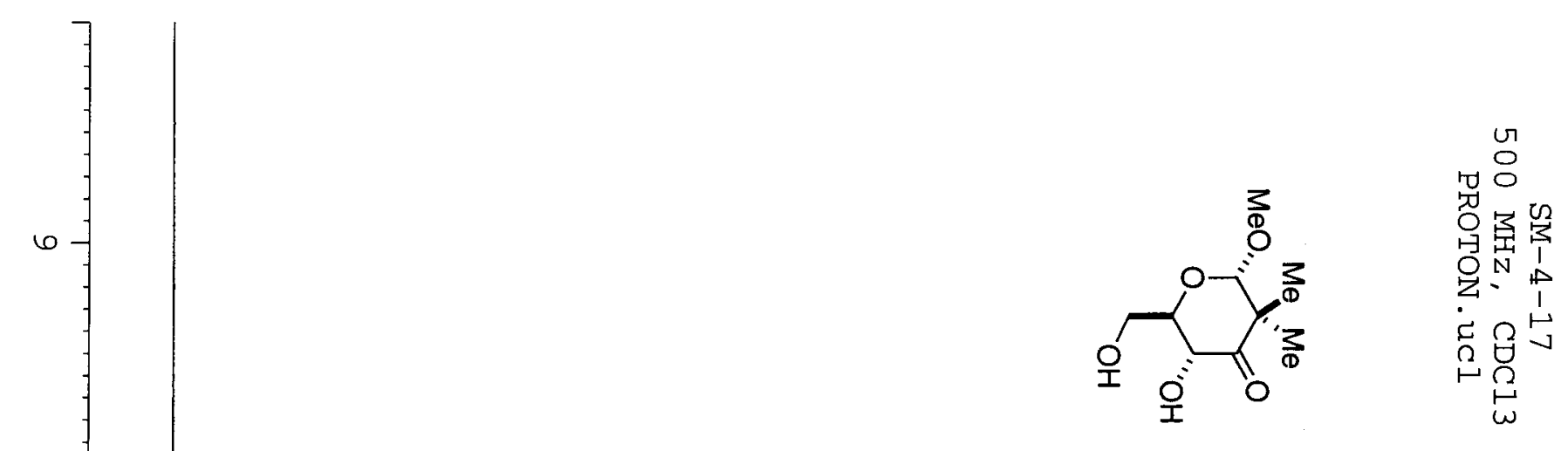

1

$\checkmark$

1

a

$\mathrm{v}$

$\triangleright$

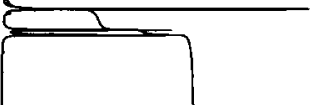

w

N-

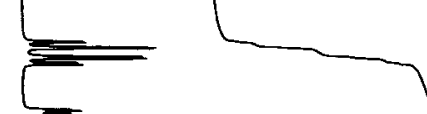



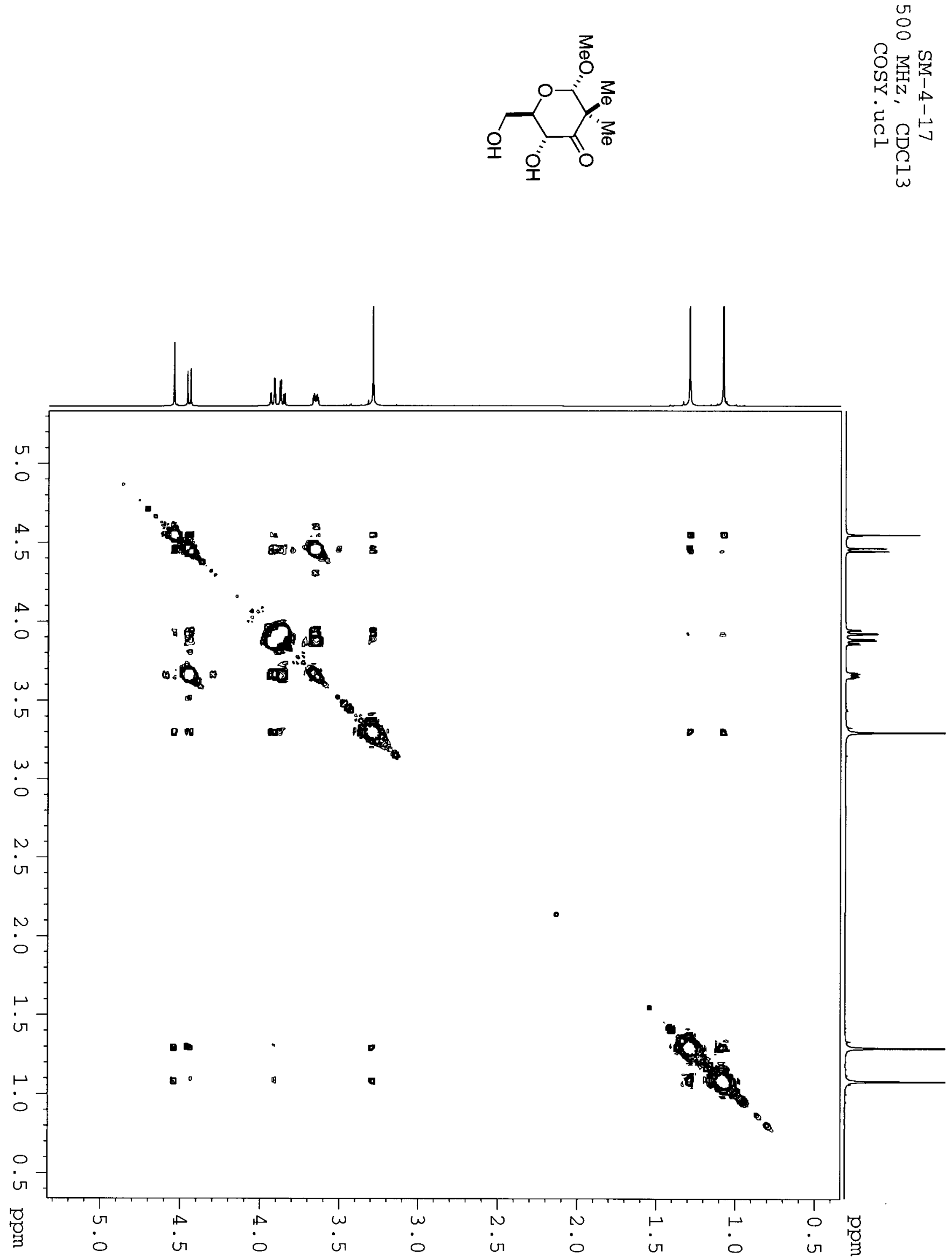

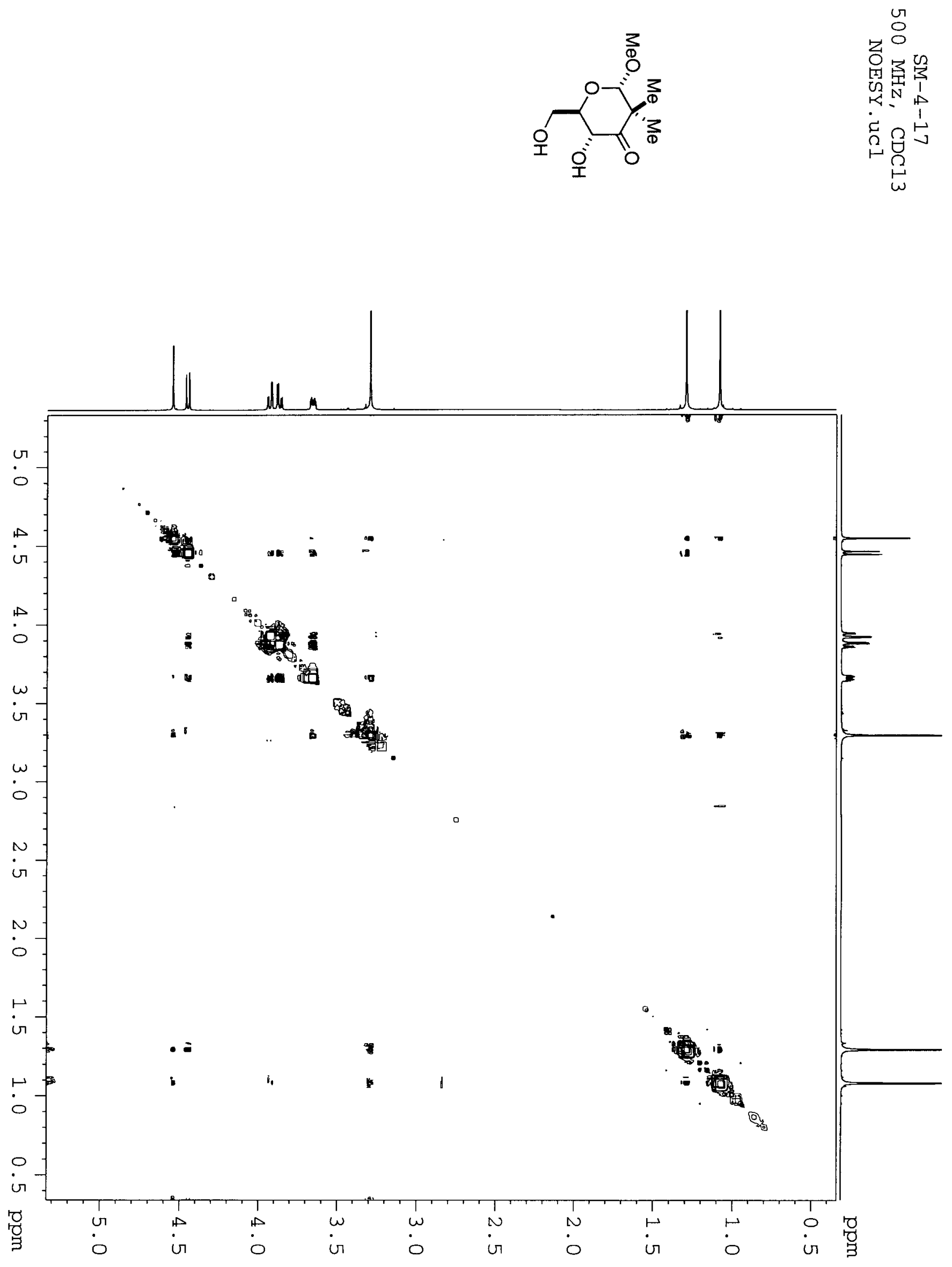


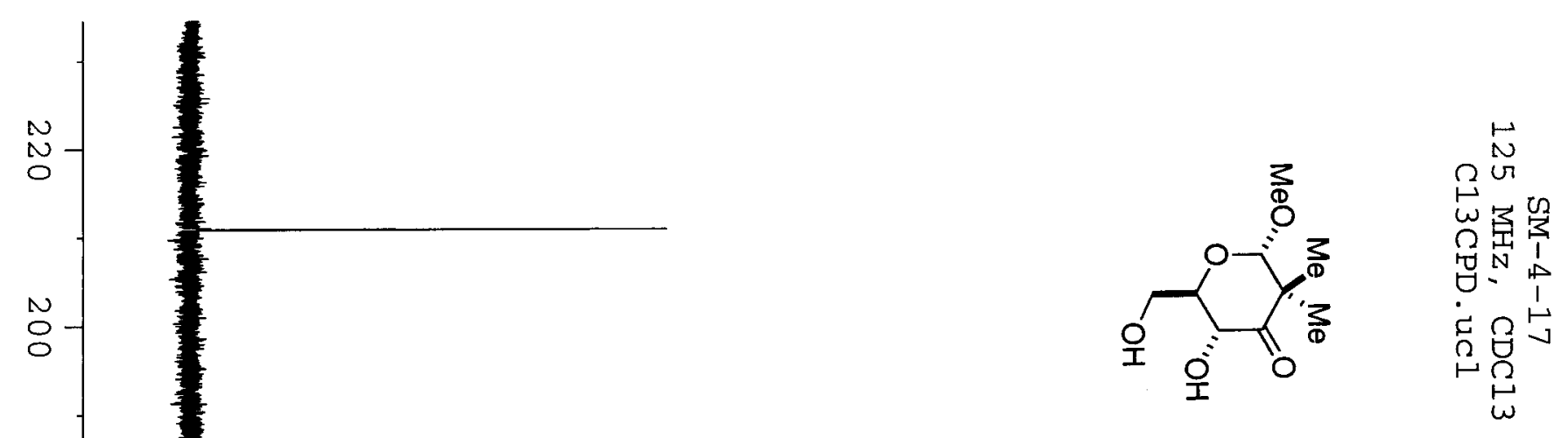

.

品

点

崩

홍

$\stackrel{\infty}{\circ}$

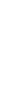

a

$\stackrel{\circ}{\circ}$

i) 


$$
H^{\prime \prime}
$$




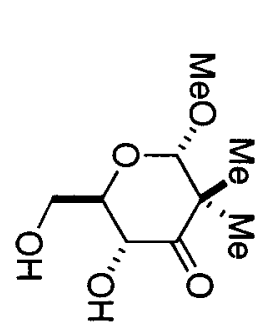

원

刍

虫空

它乎

僉 ง

?잉

点

$\mathrm{N}$

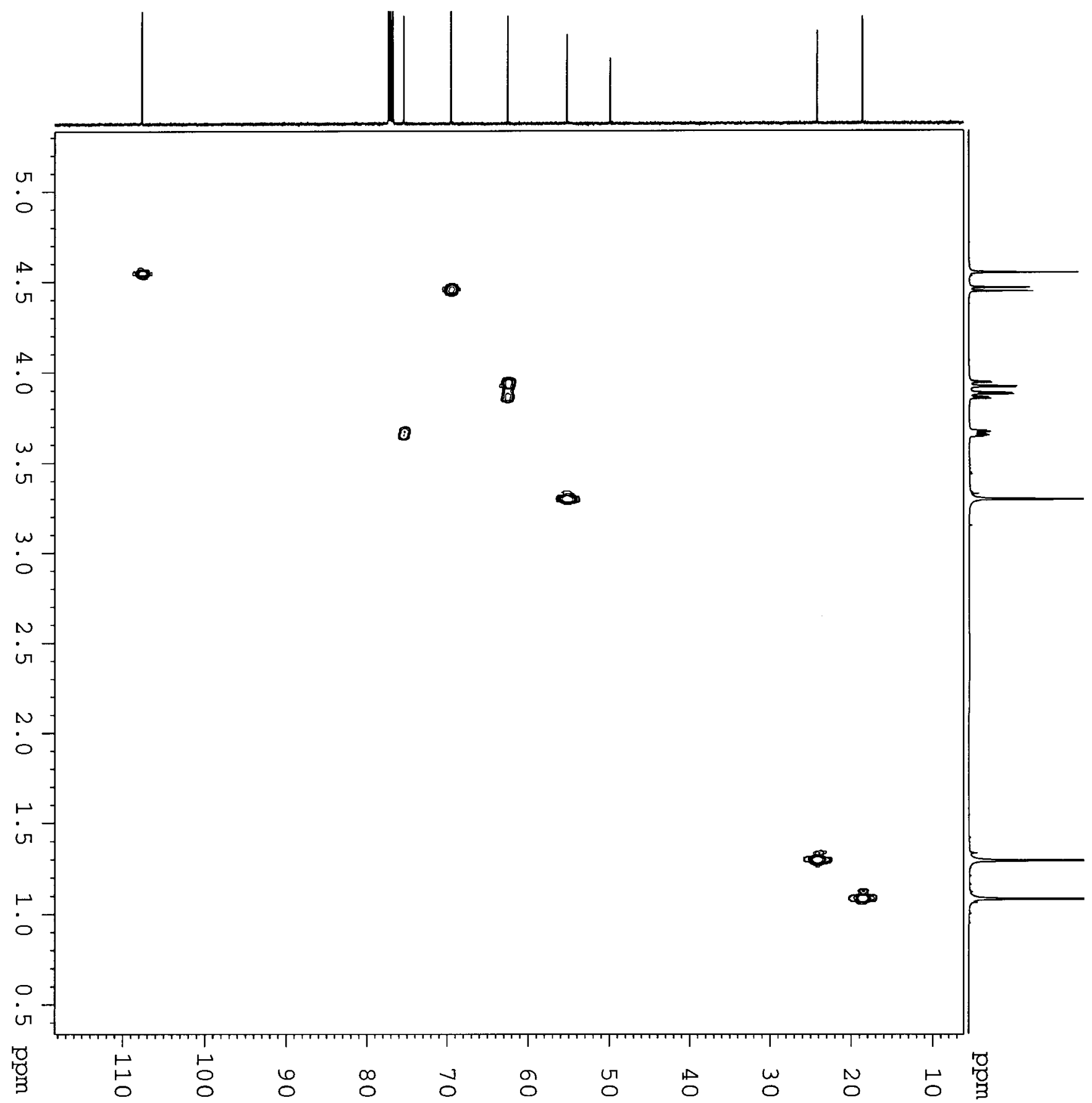




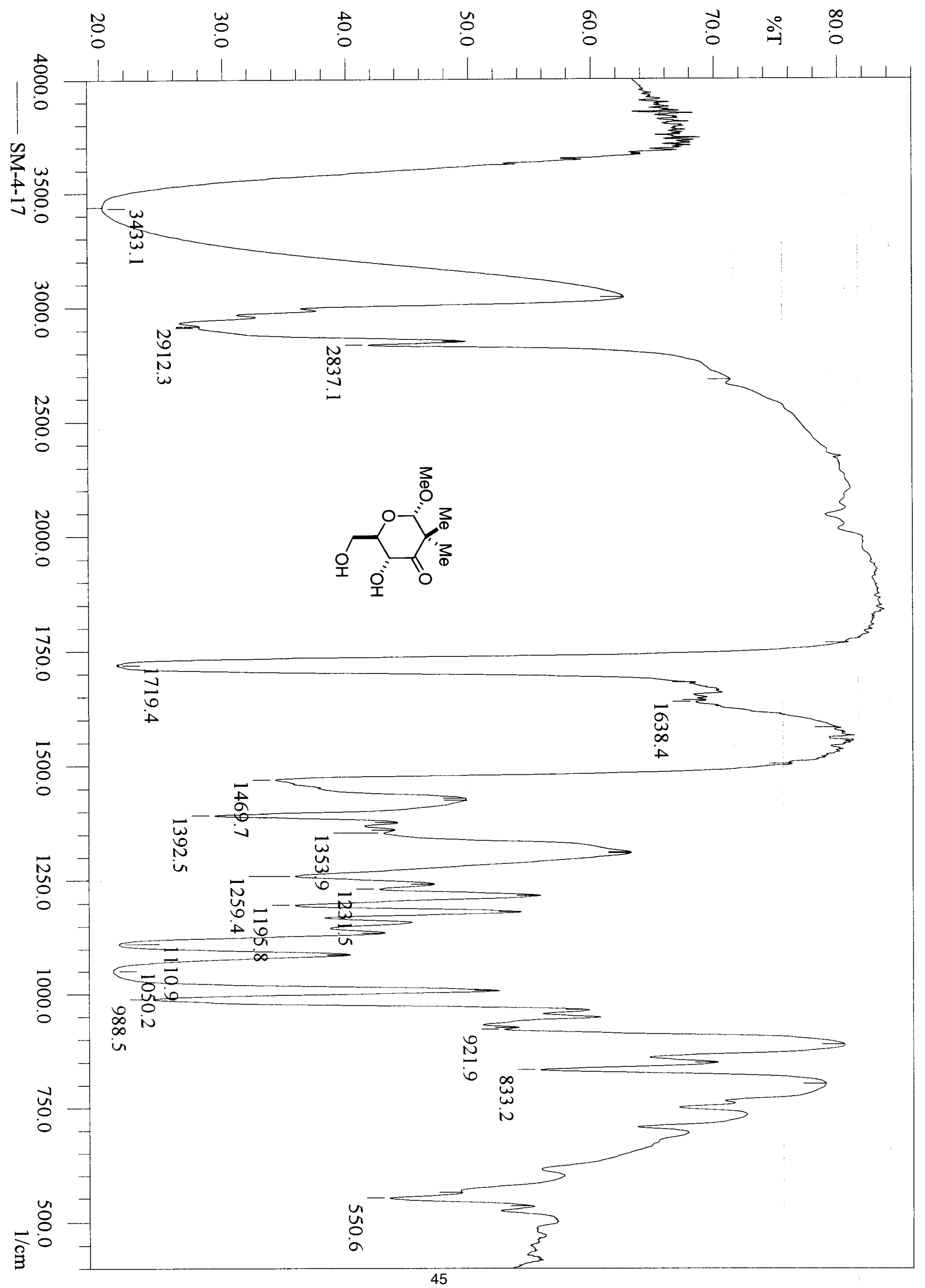




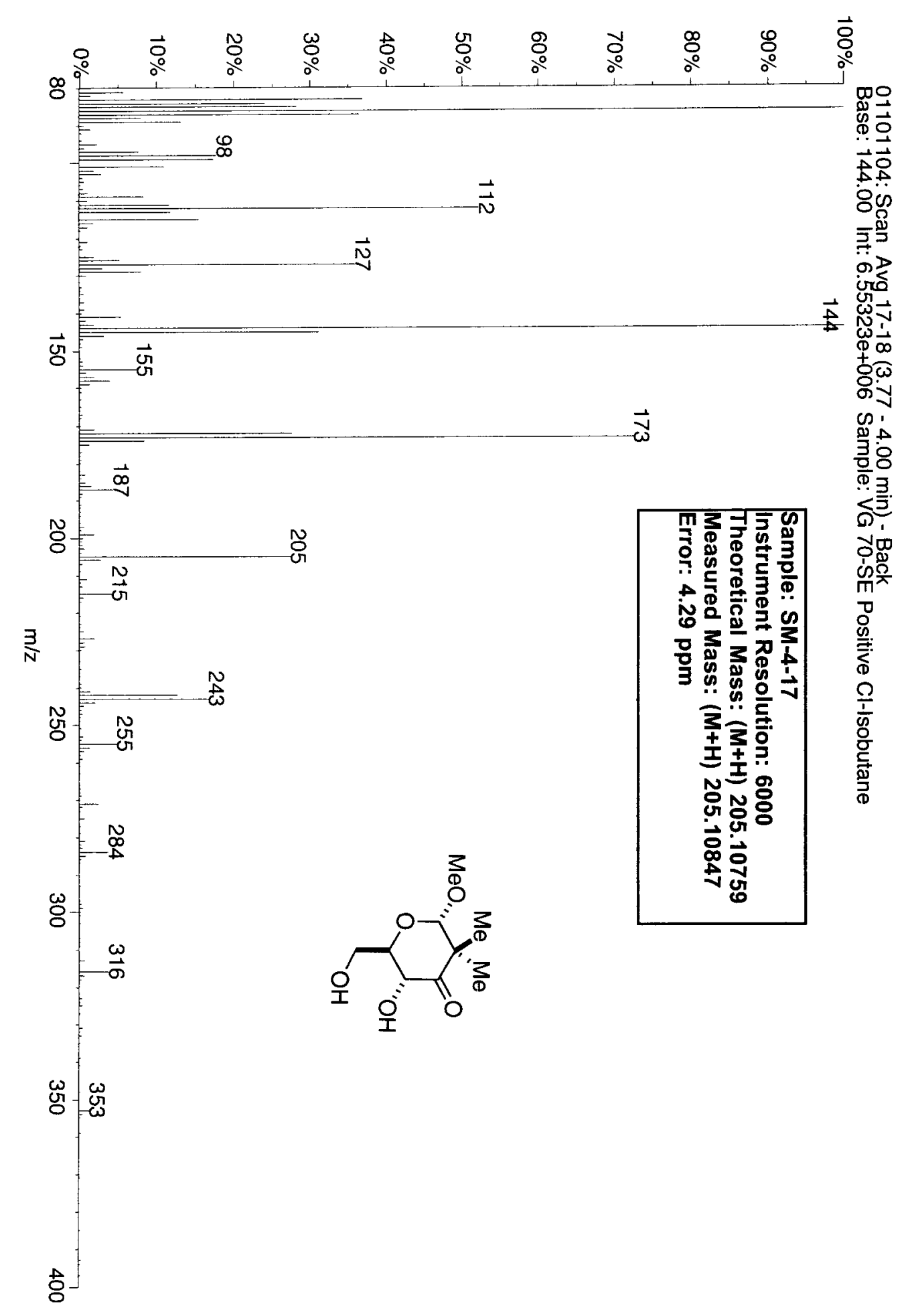




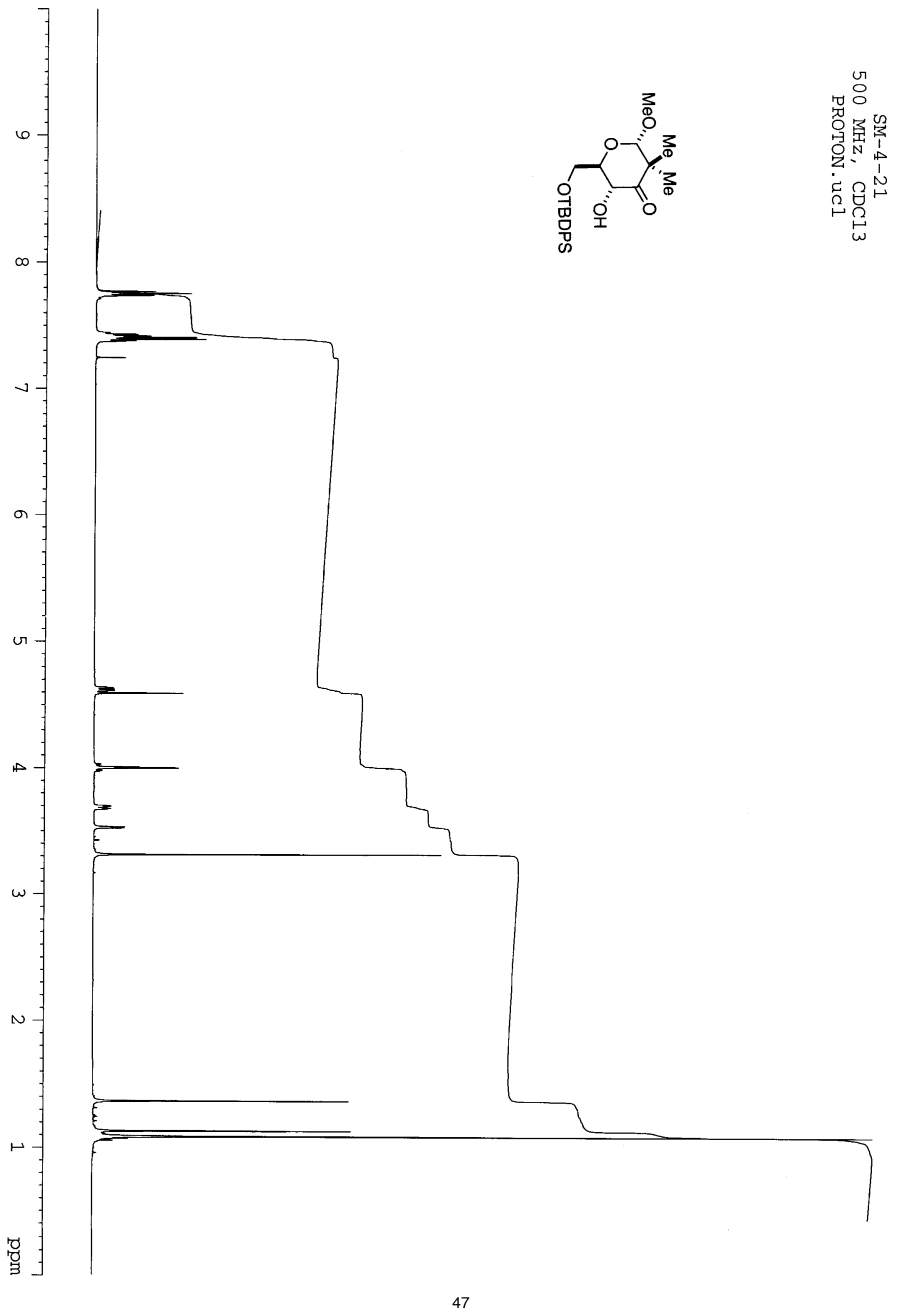



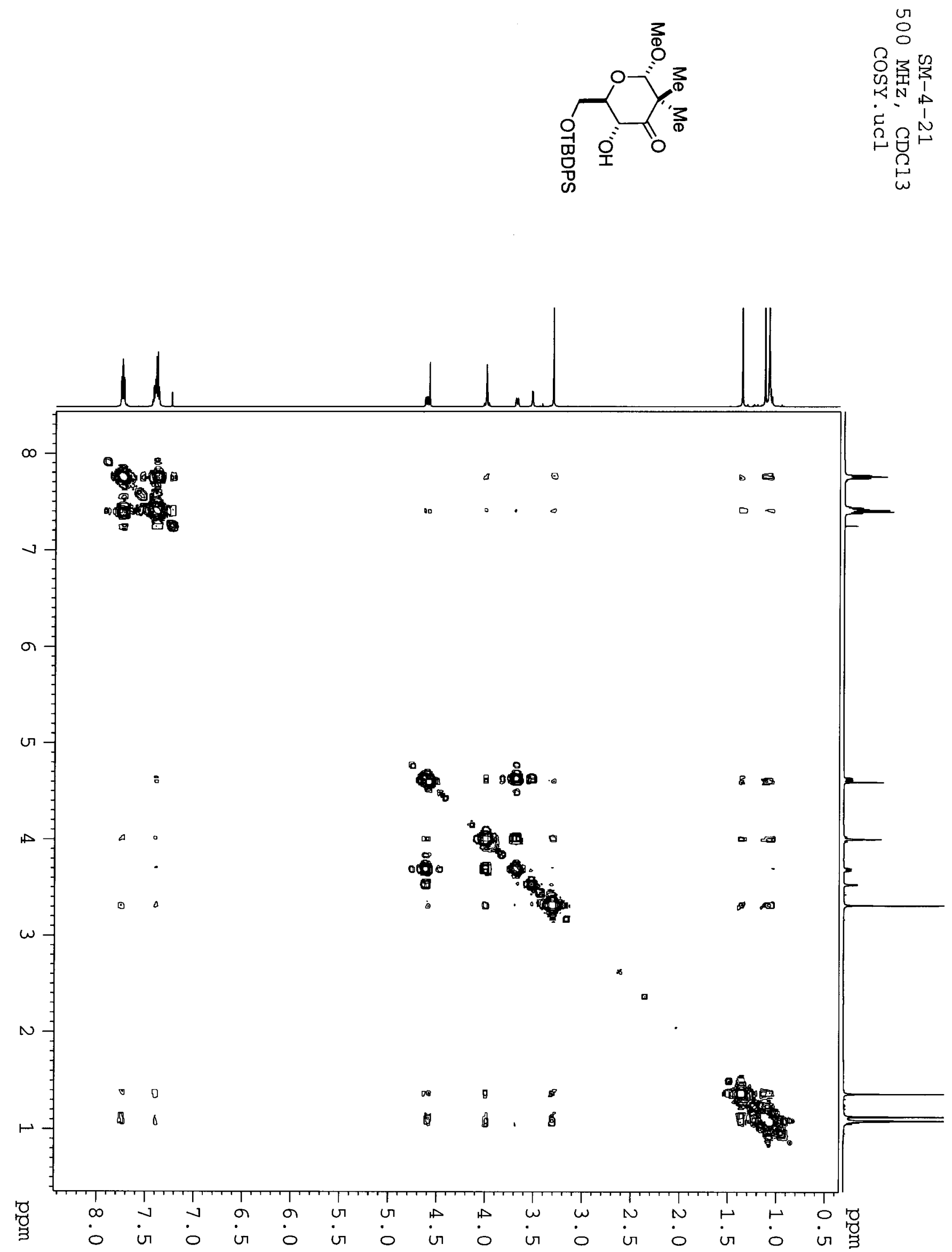

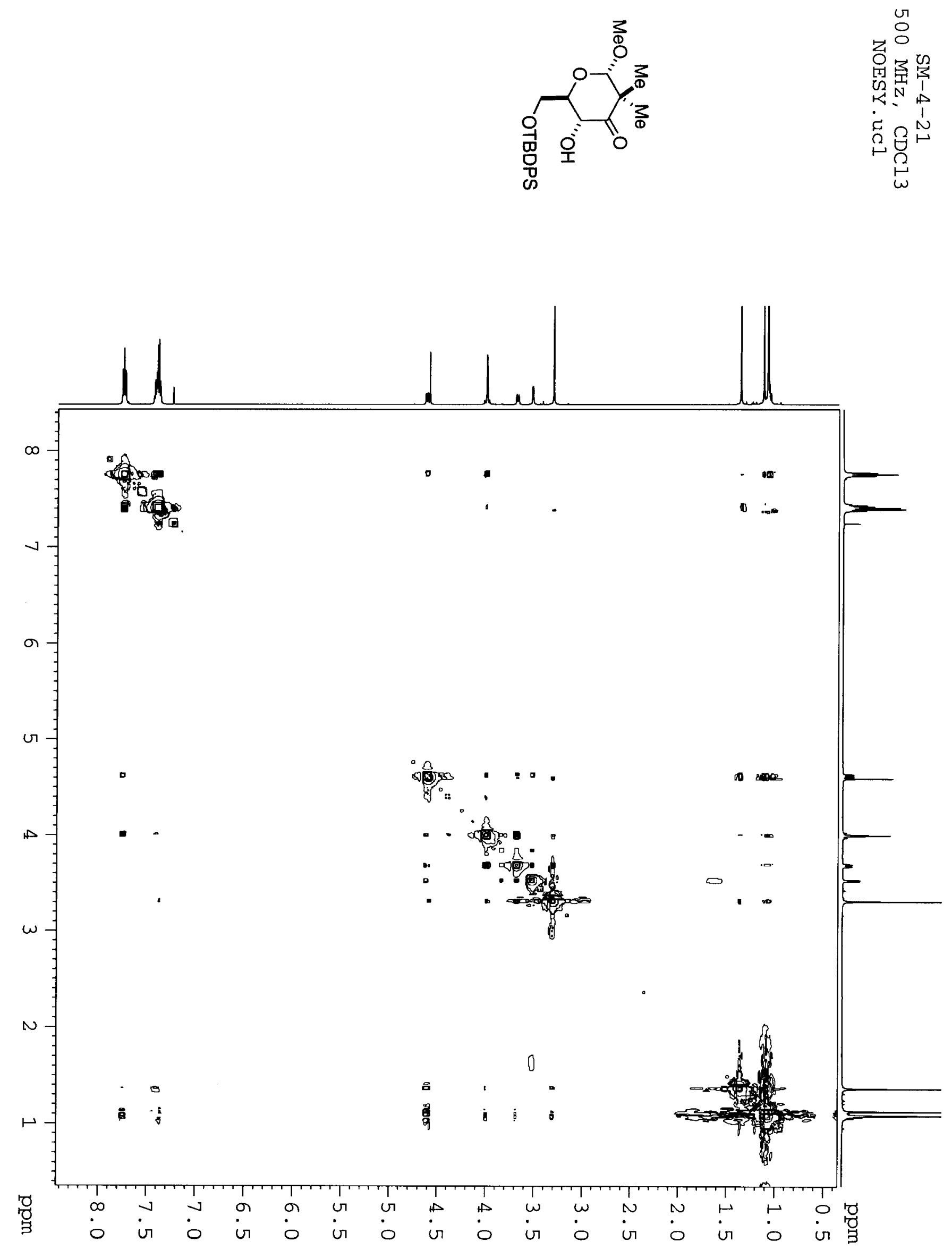

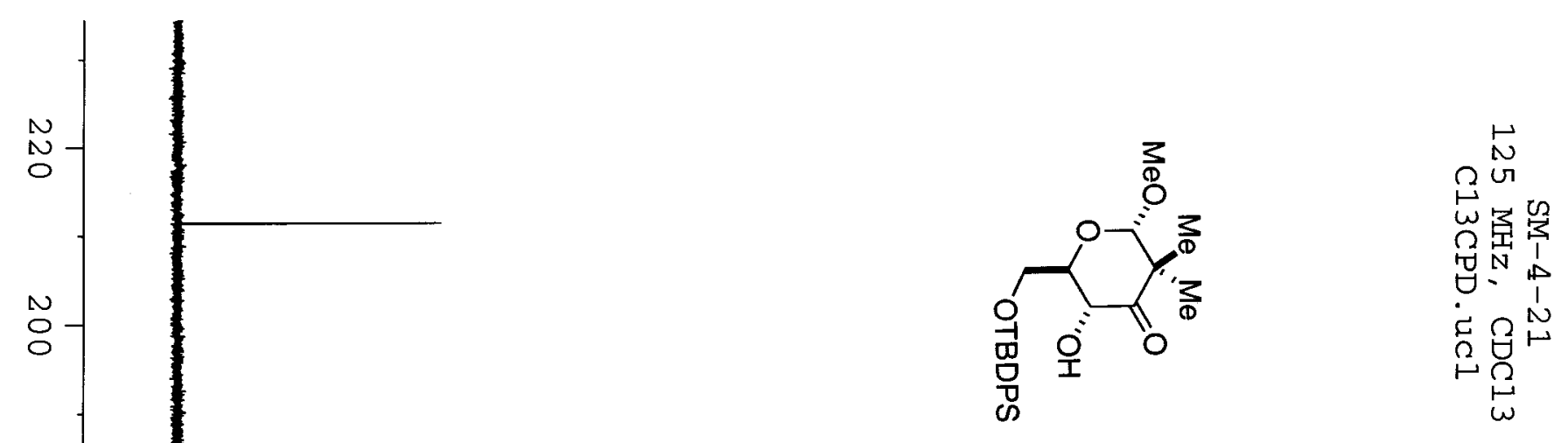

$\stackrel{\vdash}{\infty}$

คั

$\stackrel{\bullet}{\circ}$

N

5

$\infty$

$\infty$

g

a

我 


$$
\|^{\|}
$$




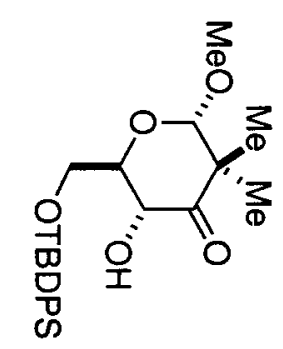

ชิํํำ

卓

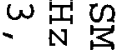

它-

量

?앙

i

点

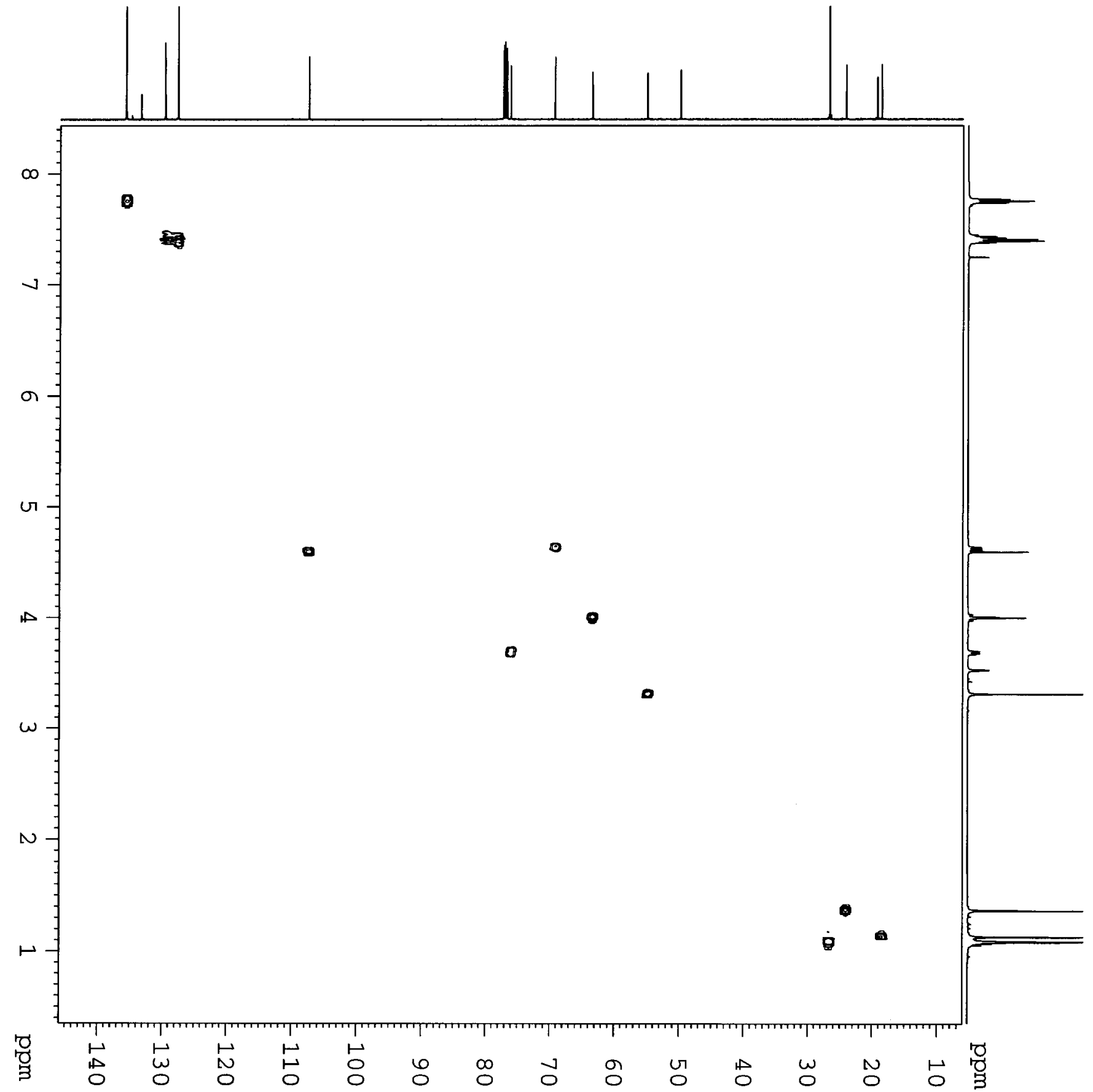




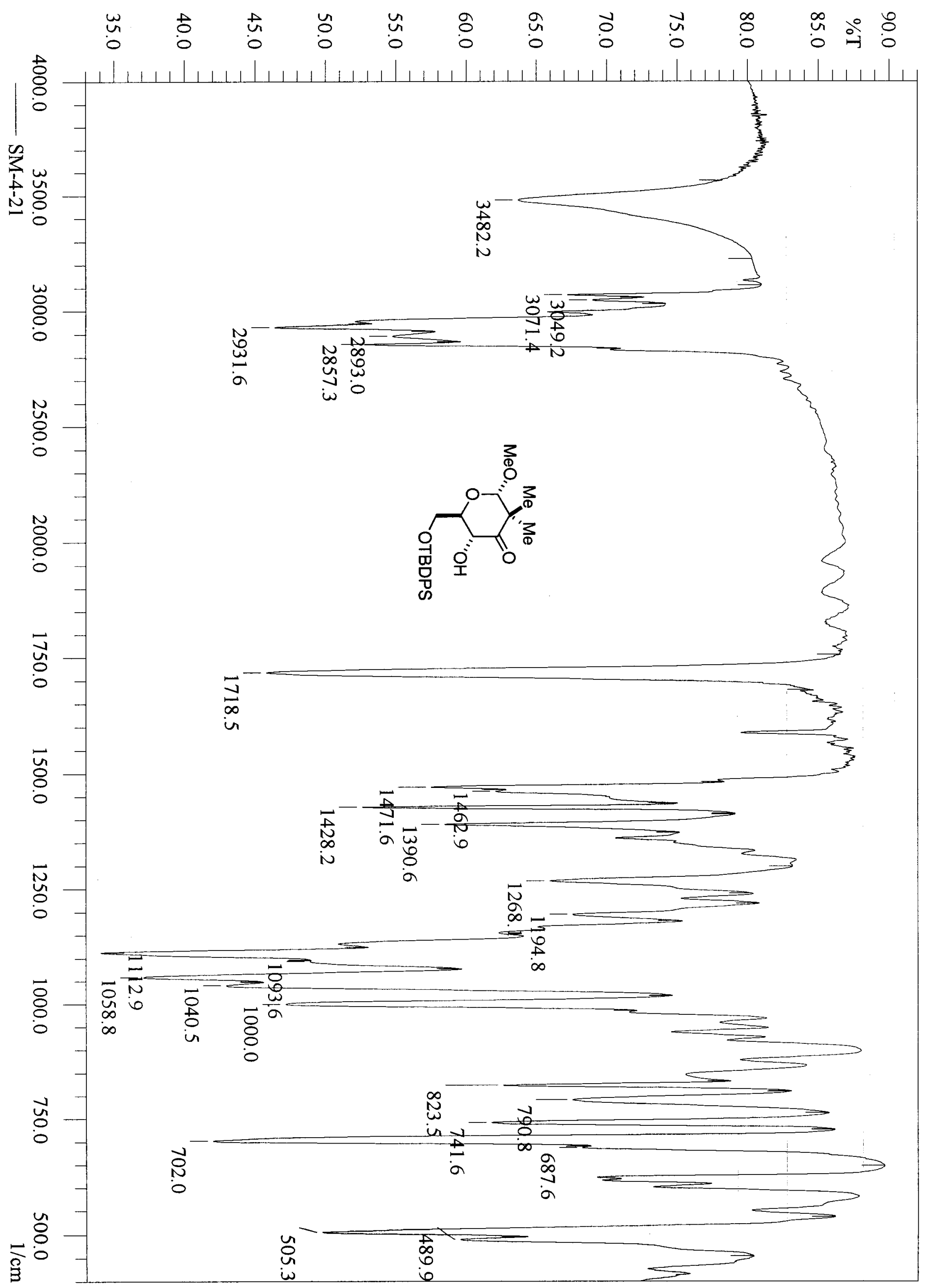




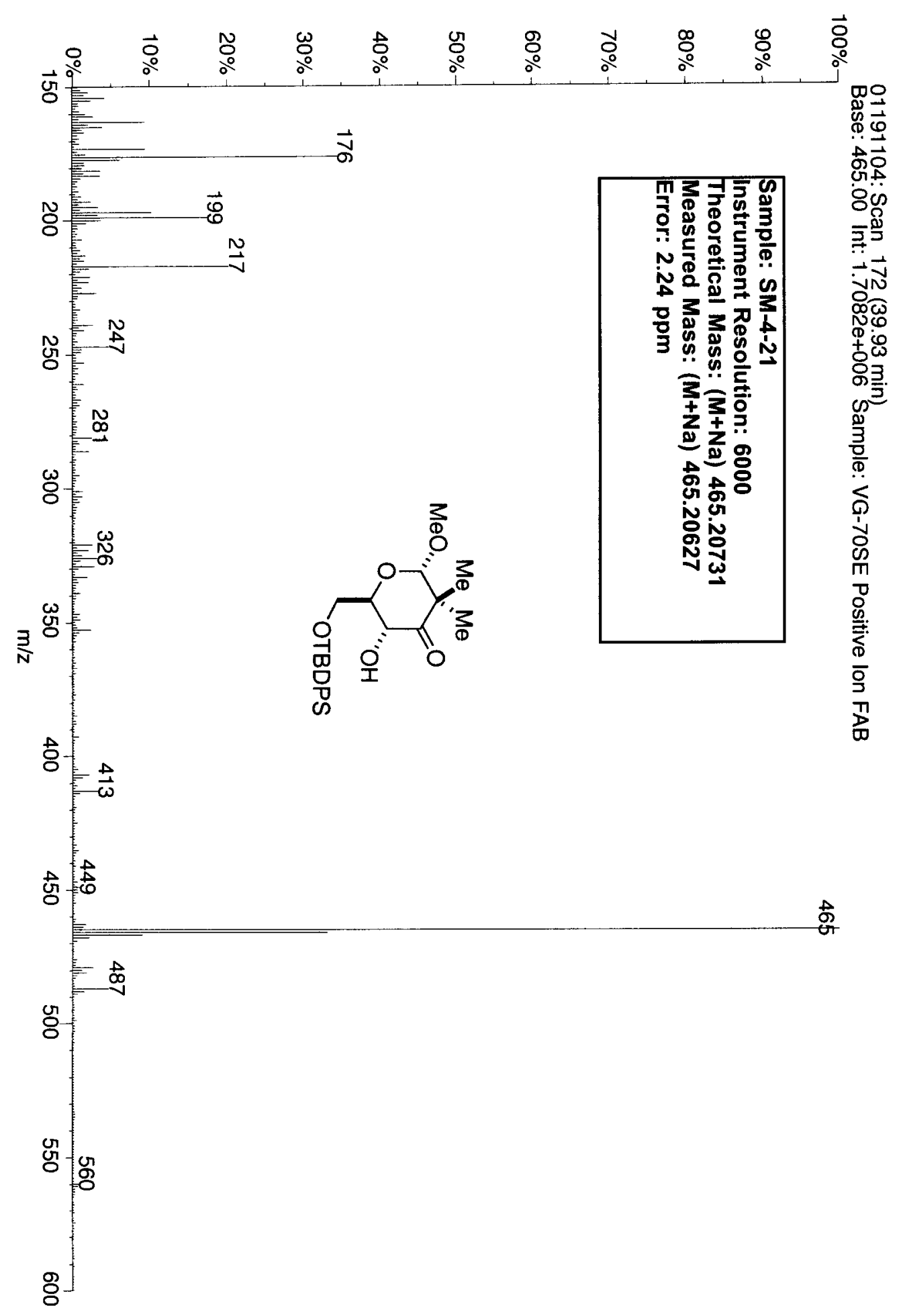




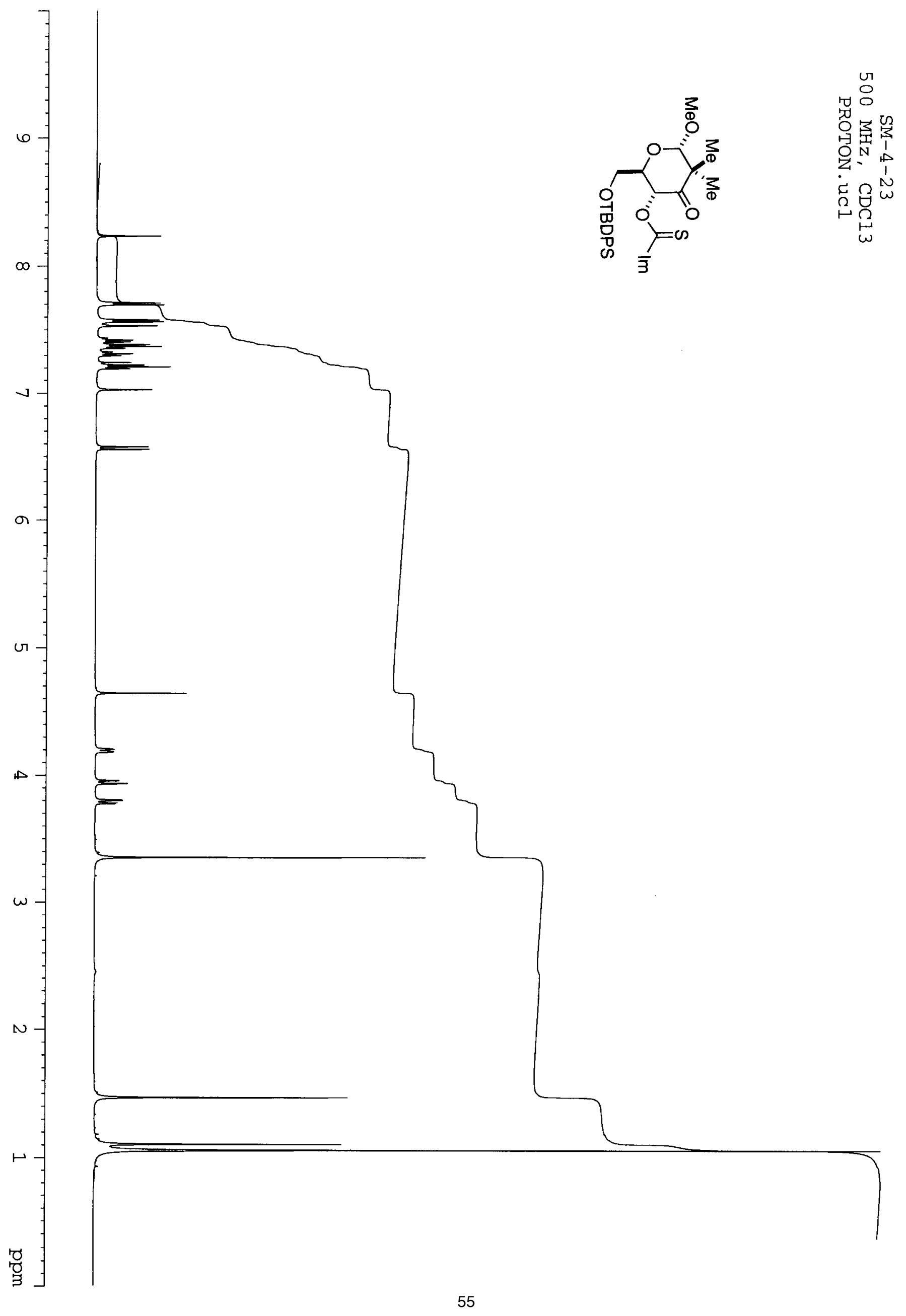



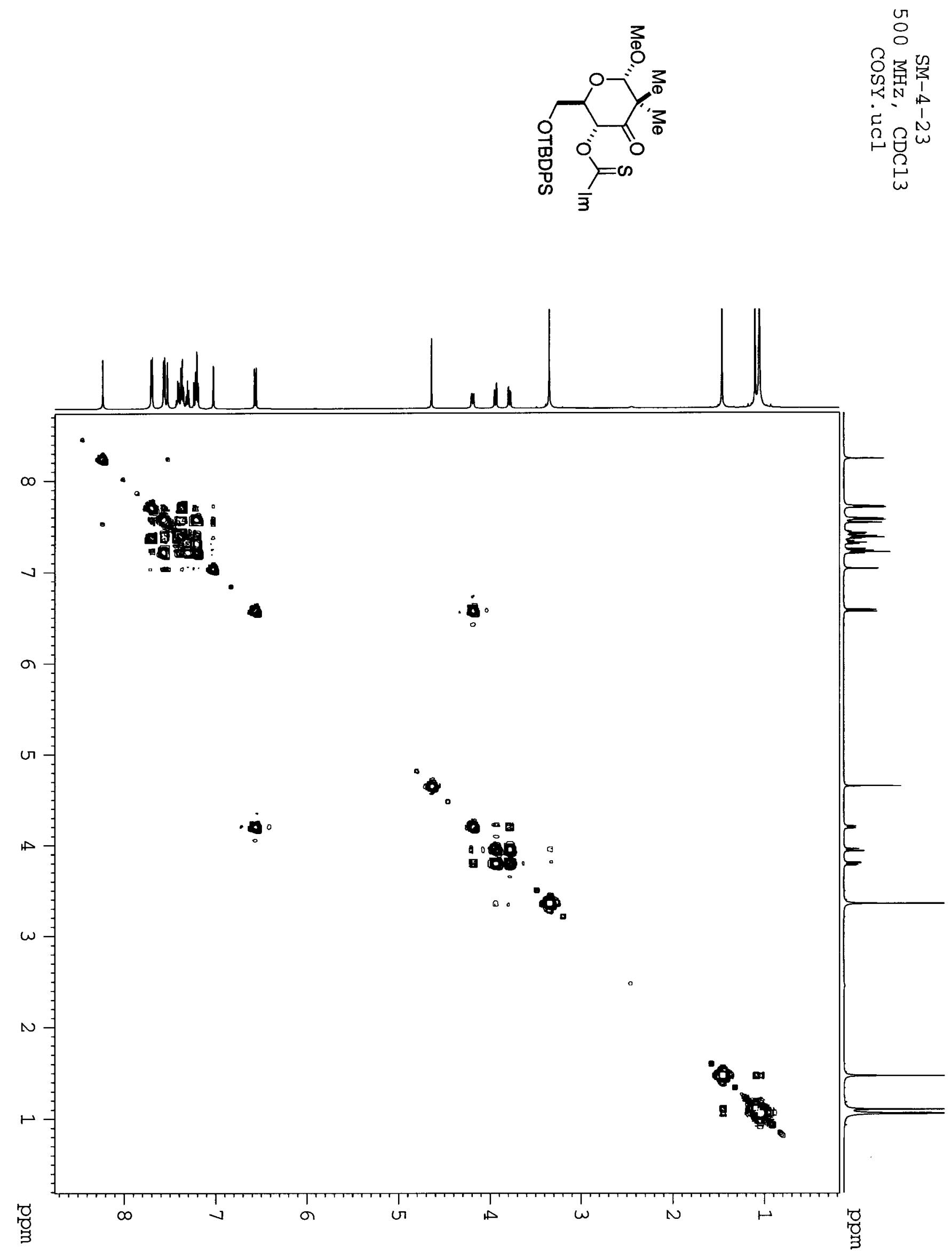

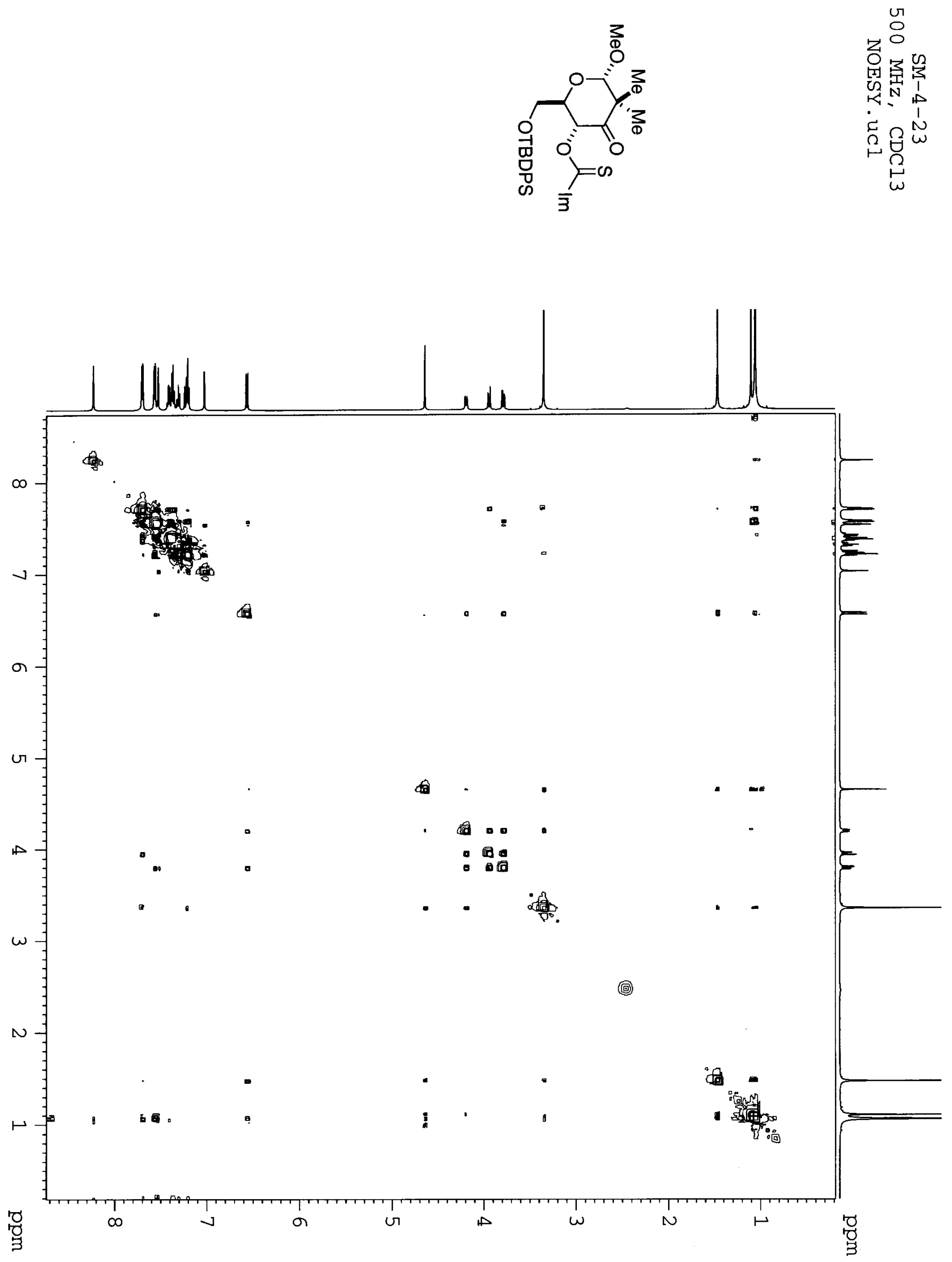

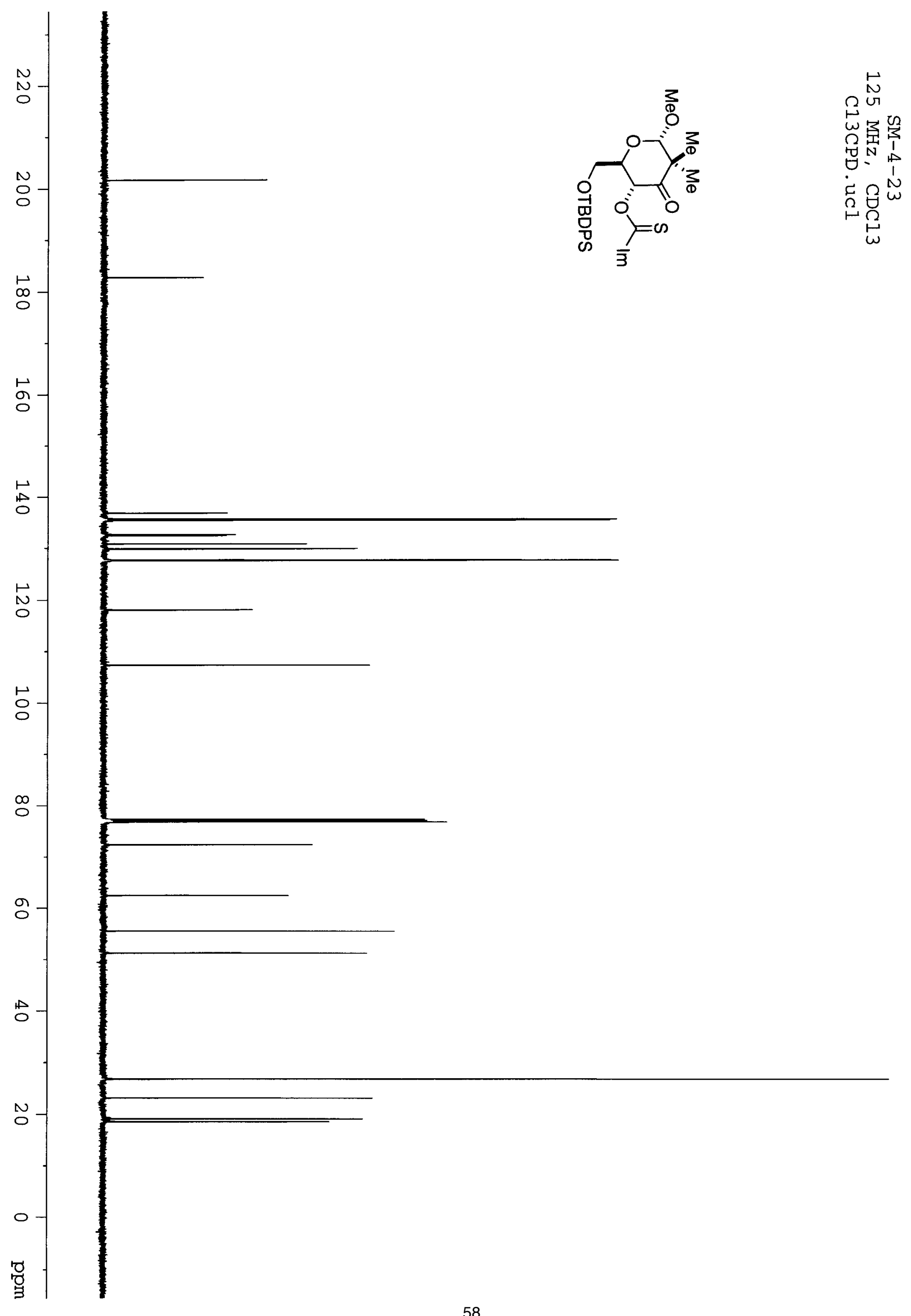

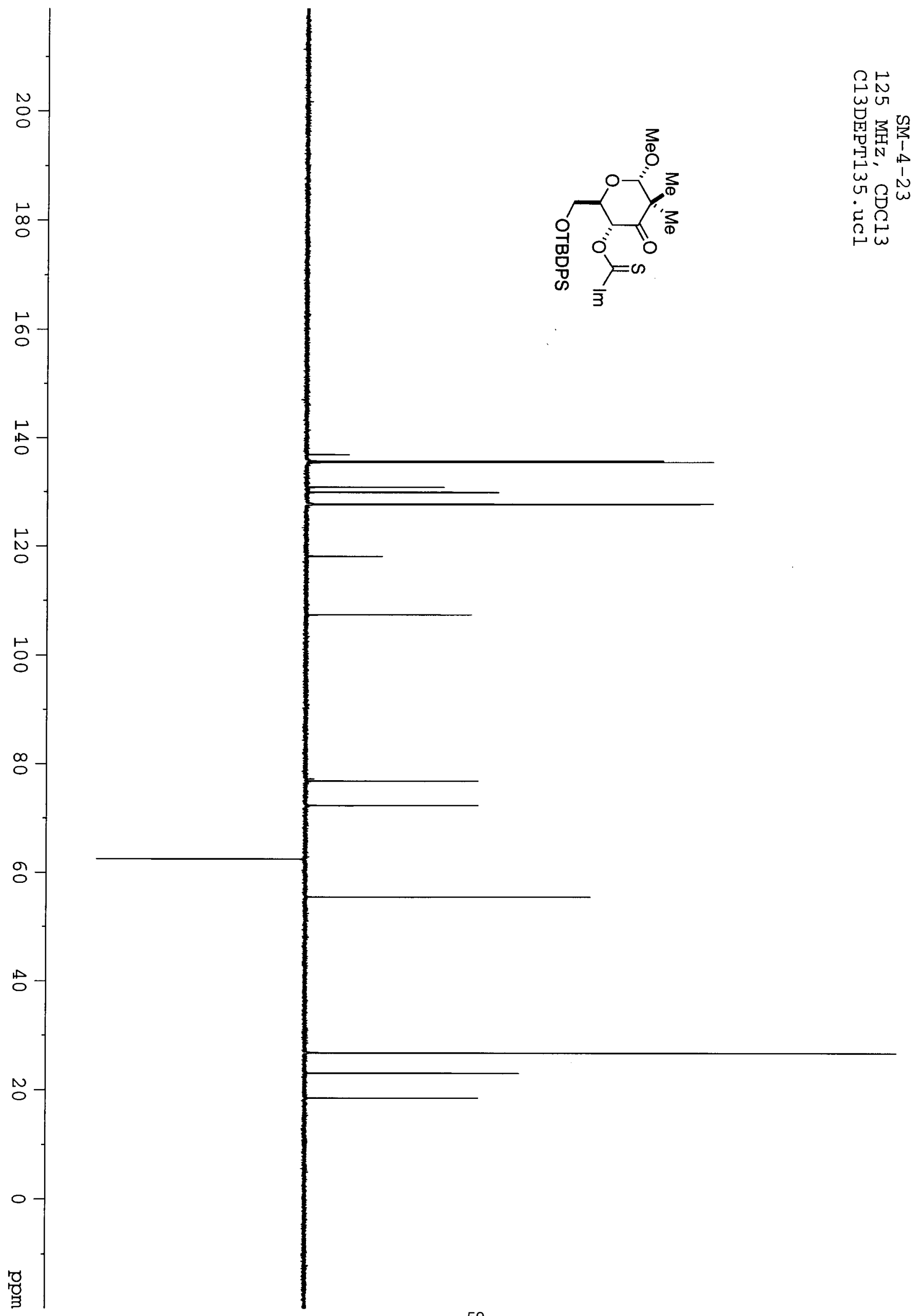

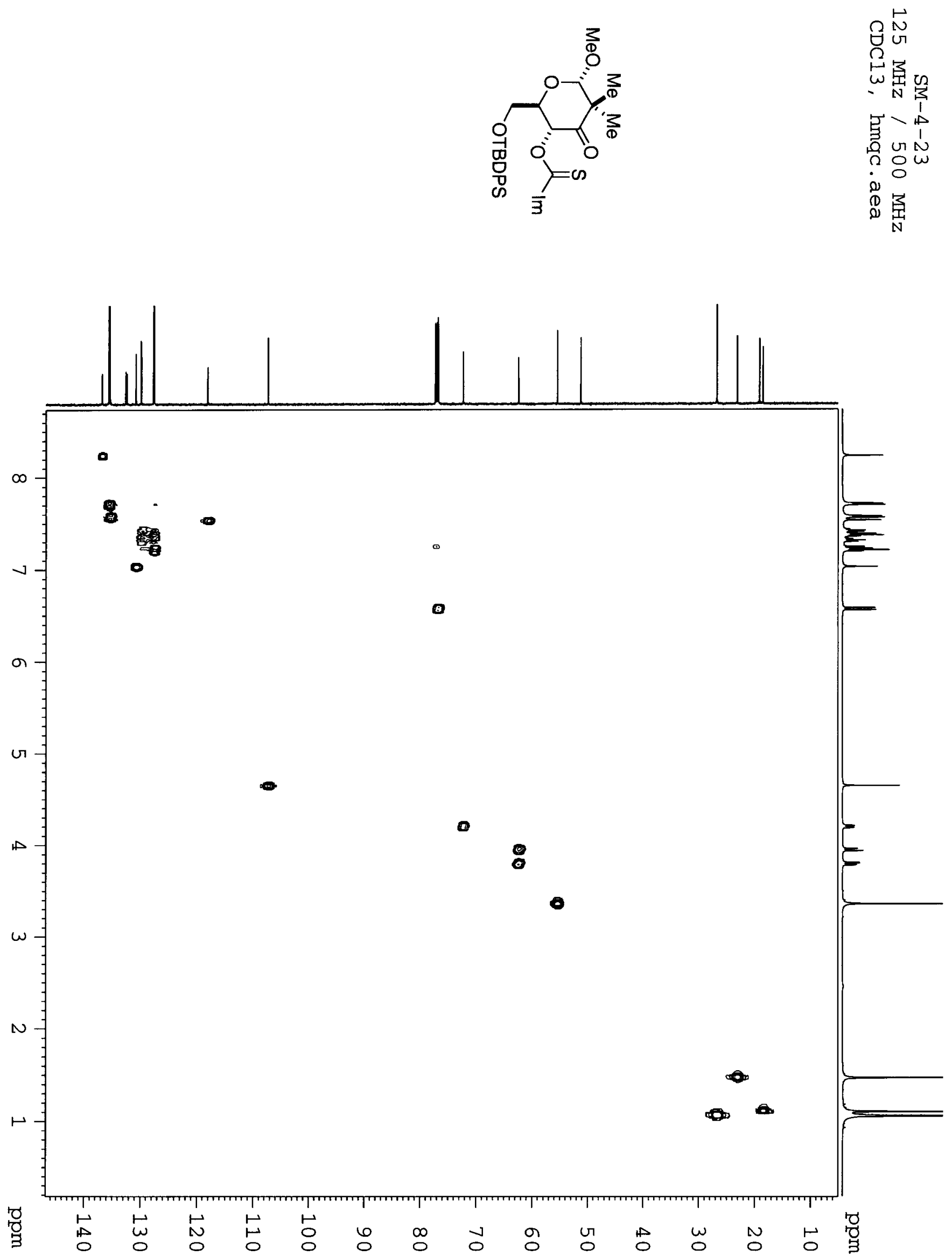


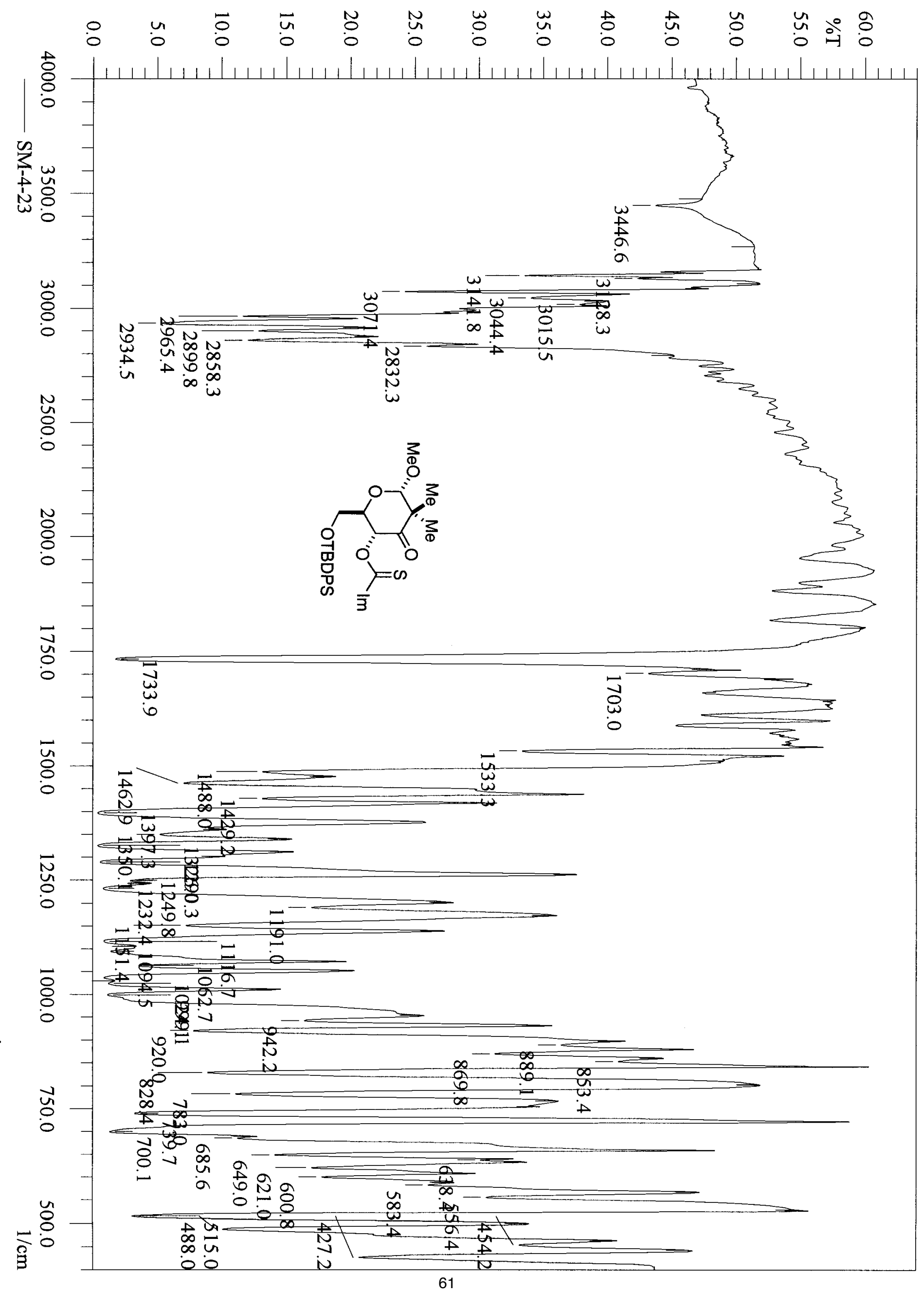




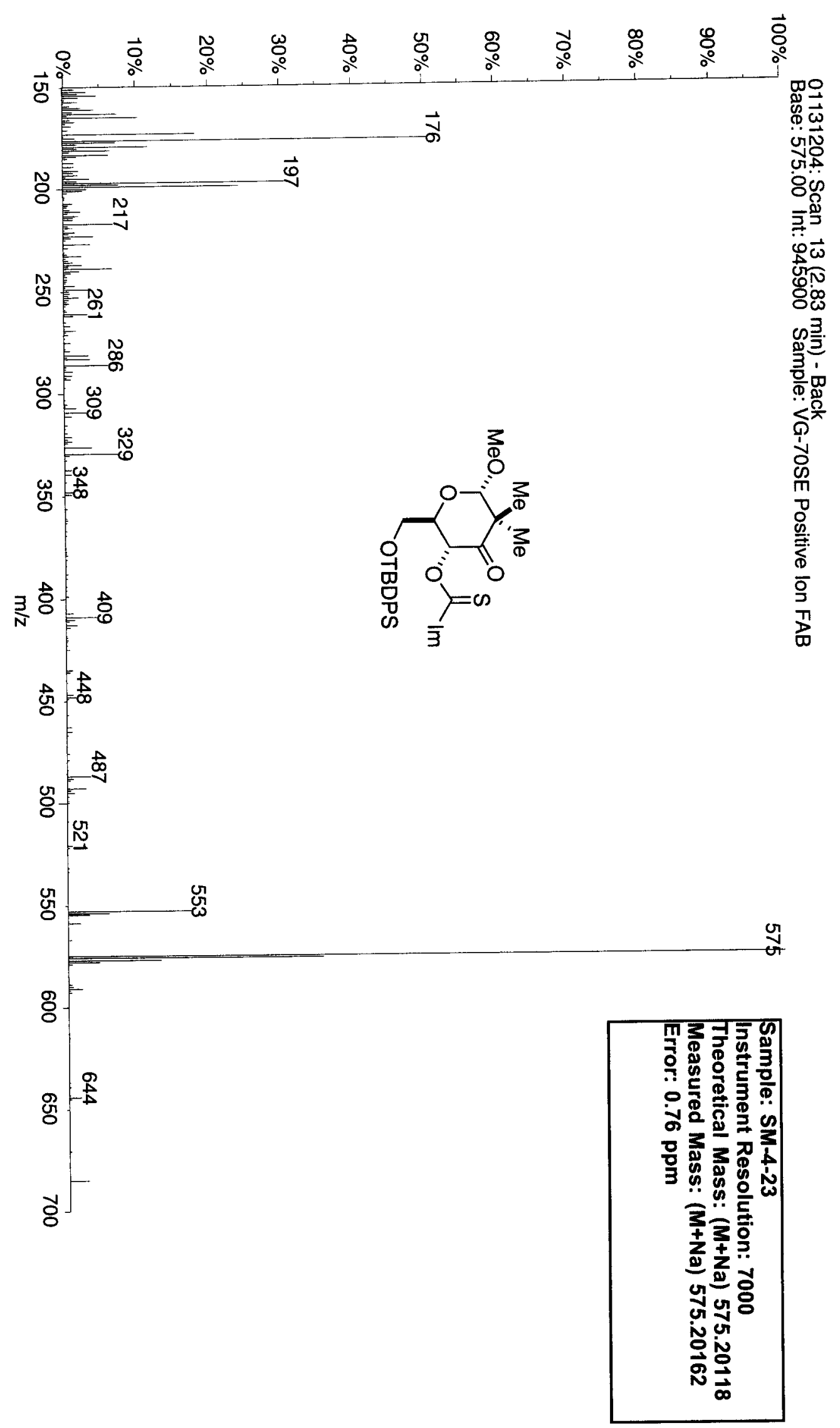




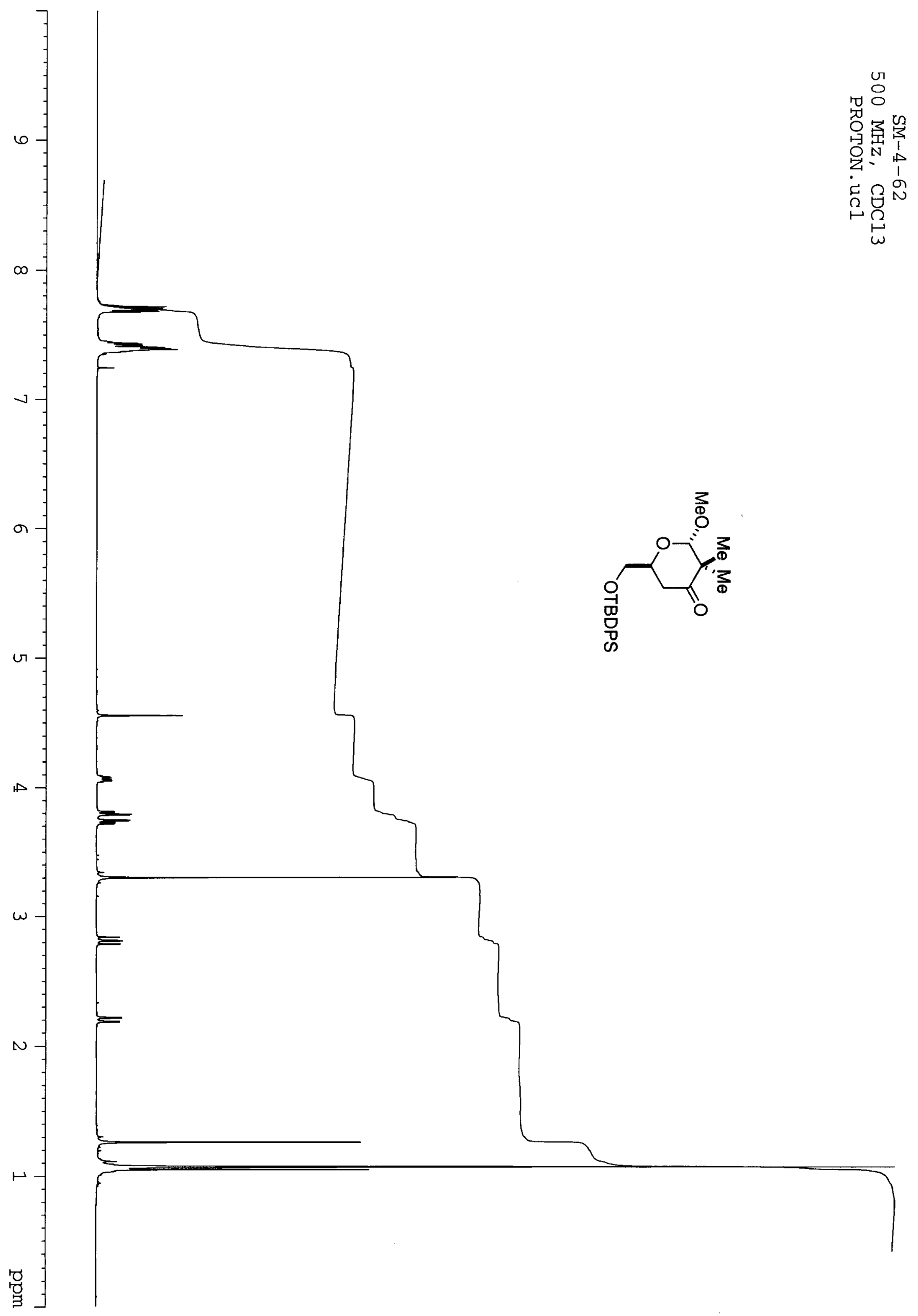



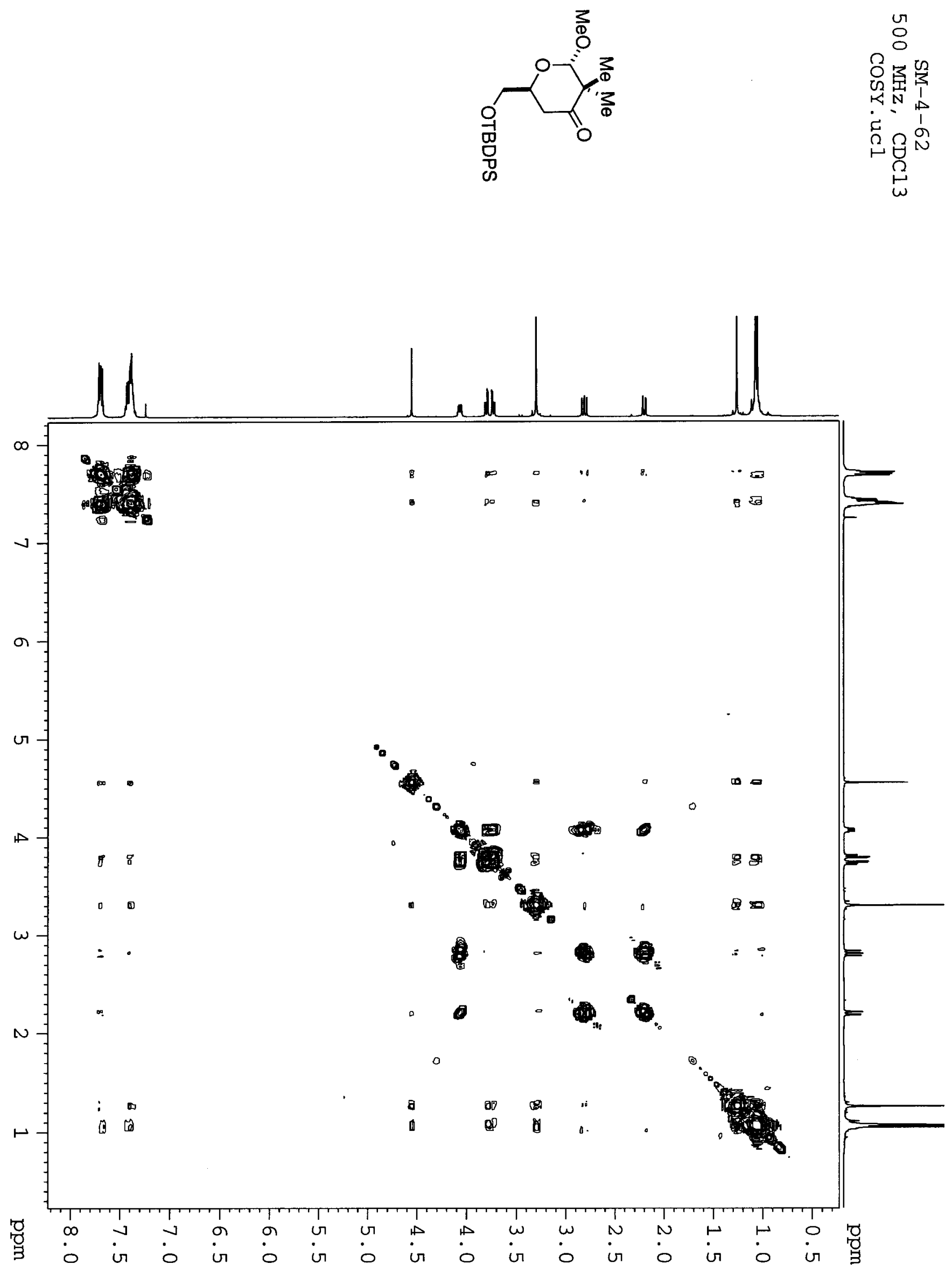

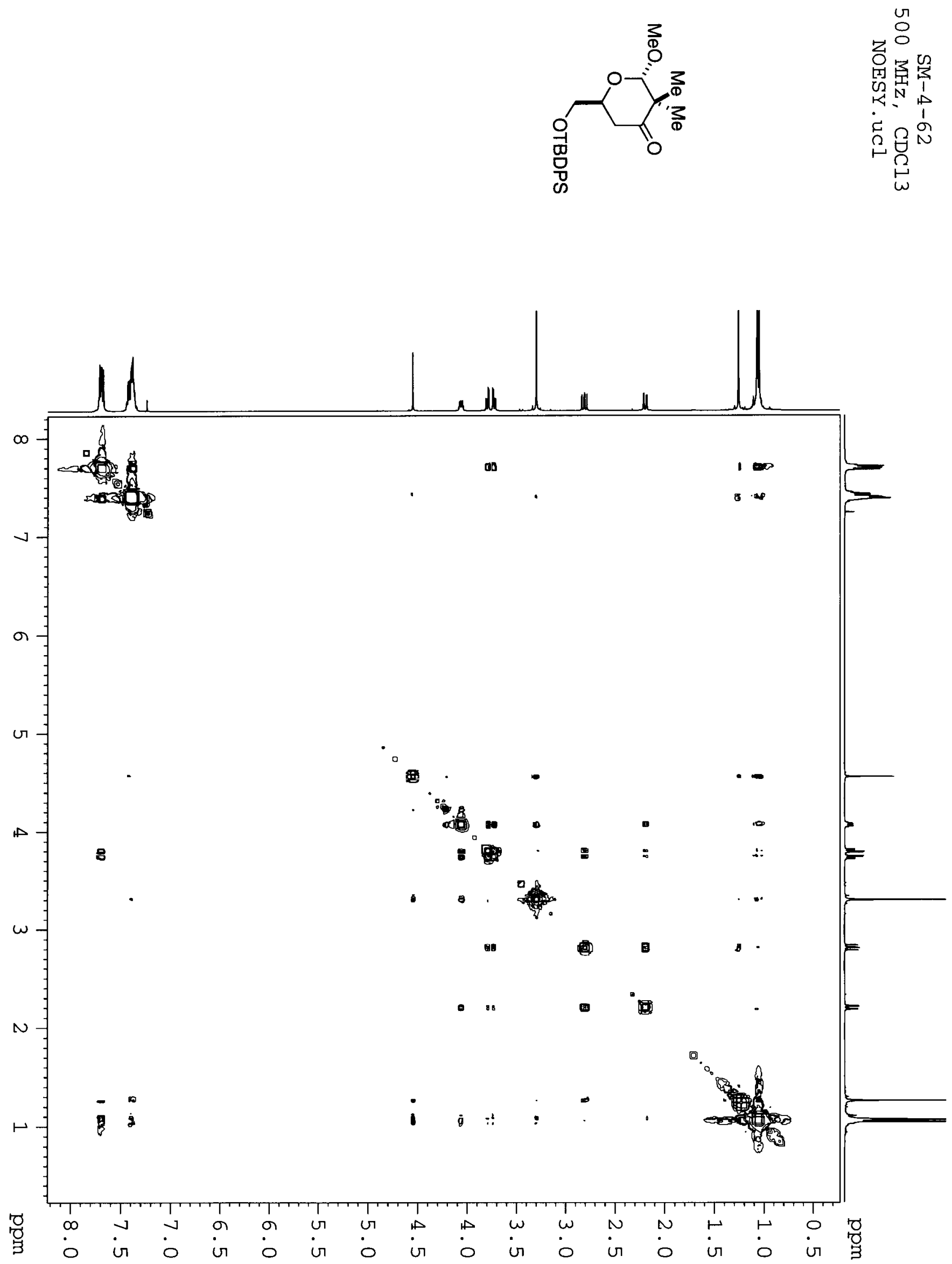


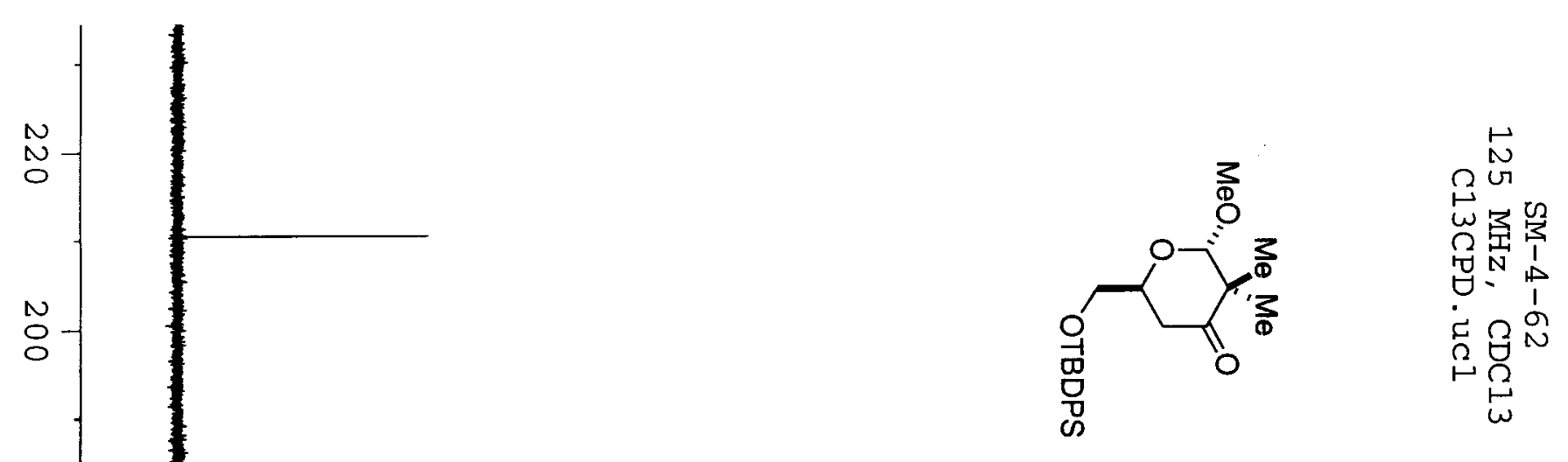

$\stackrel{+}{\infty}$

홍

$\stackrel{\bullet}{0}$

$\stackrel{\mapsto}{\circ}$

๑

$\infty$

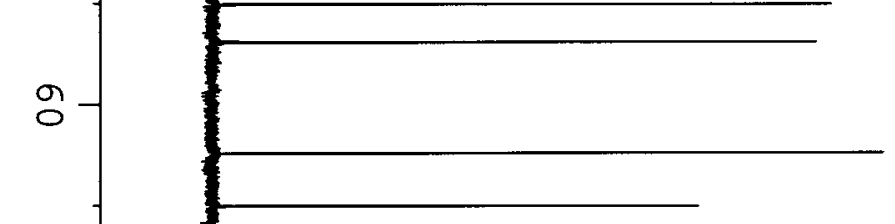

号

O 


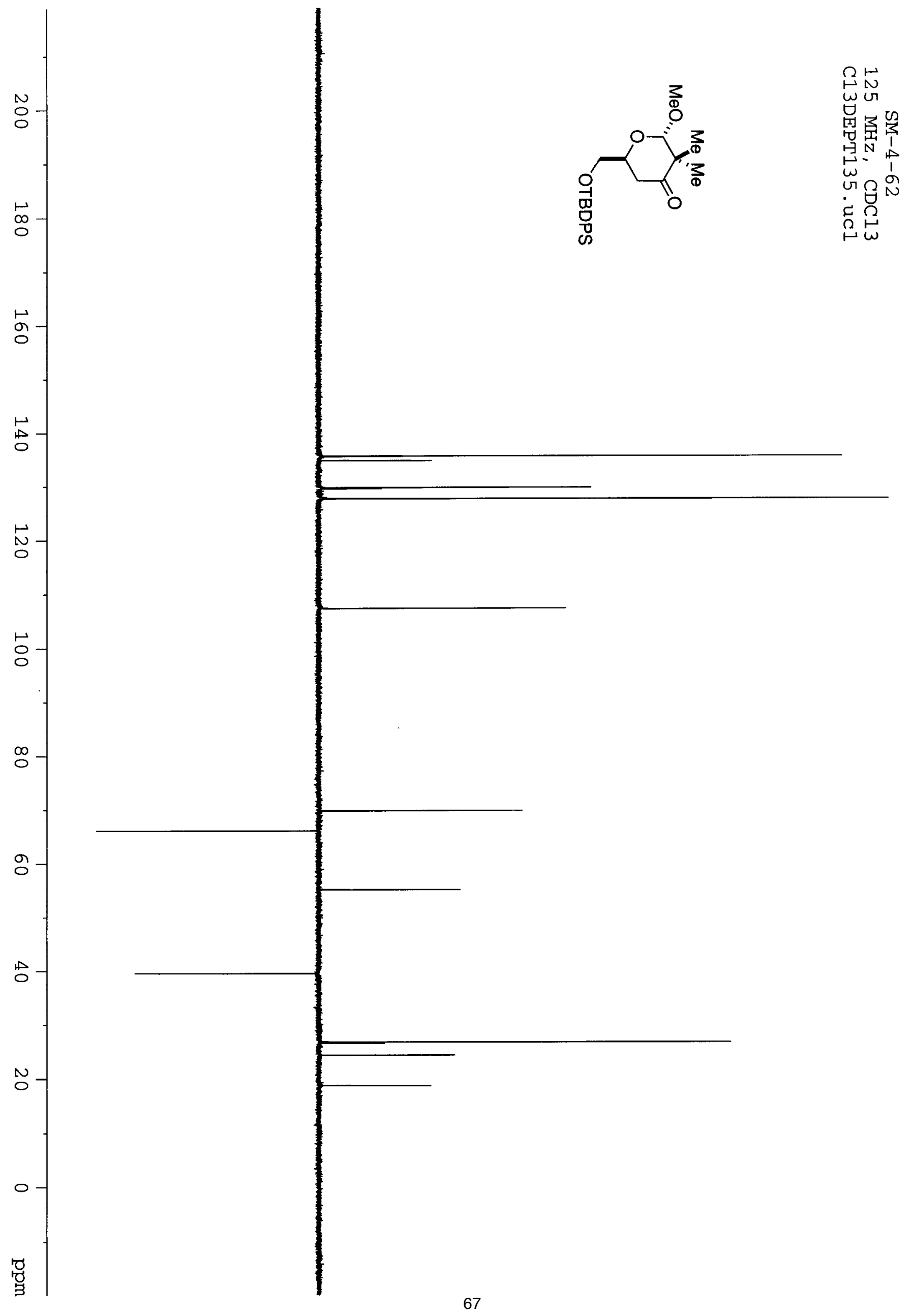



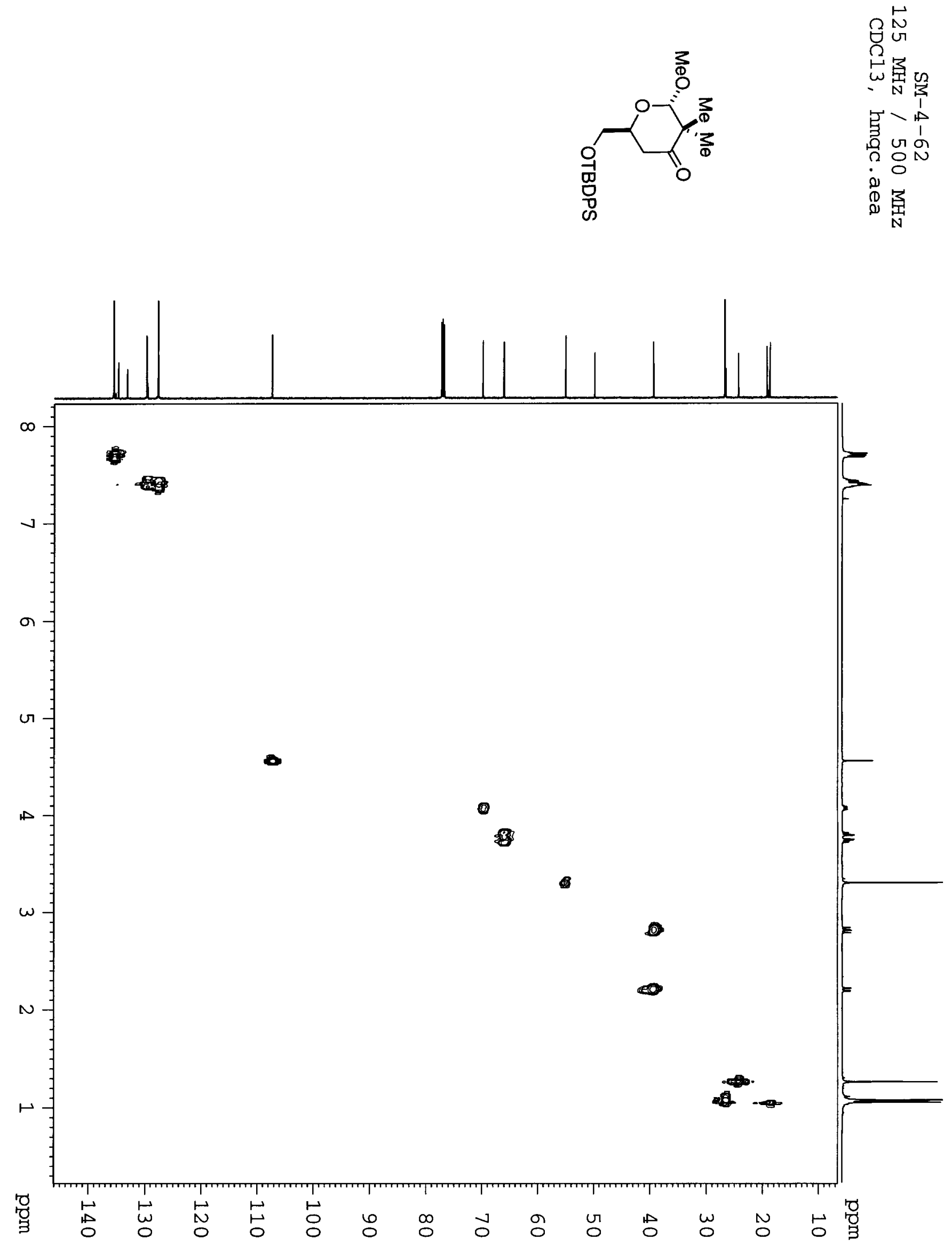


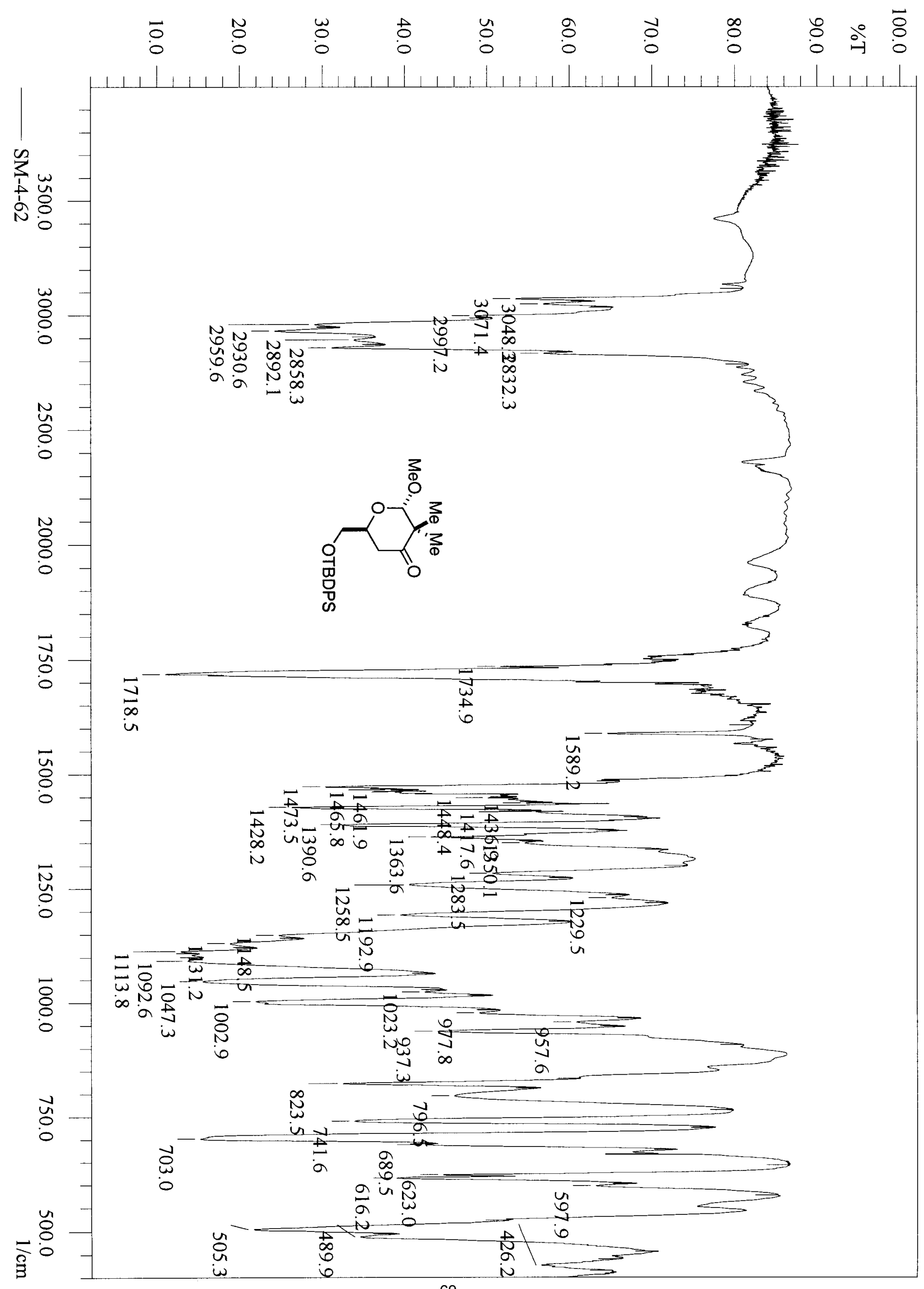




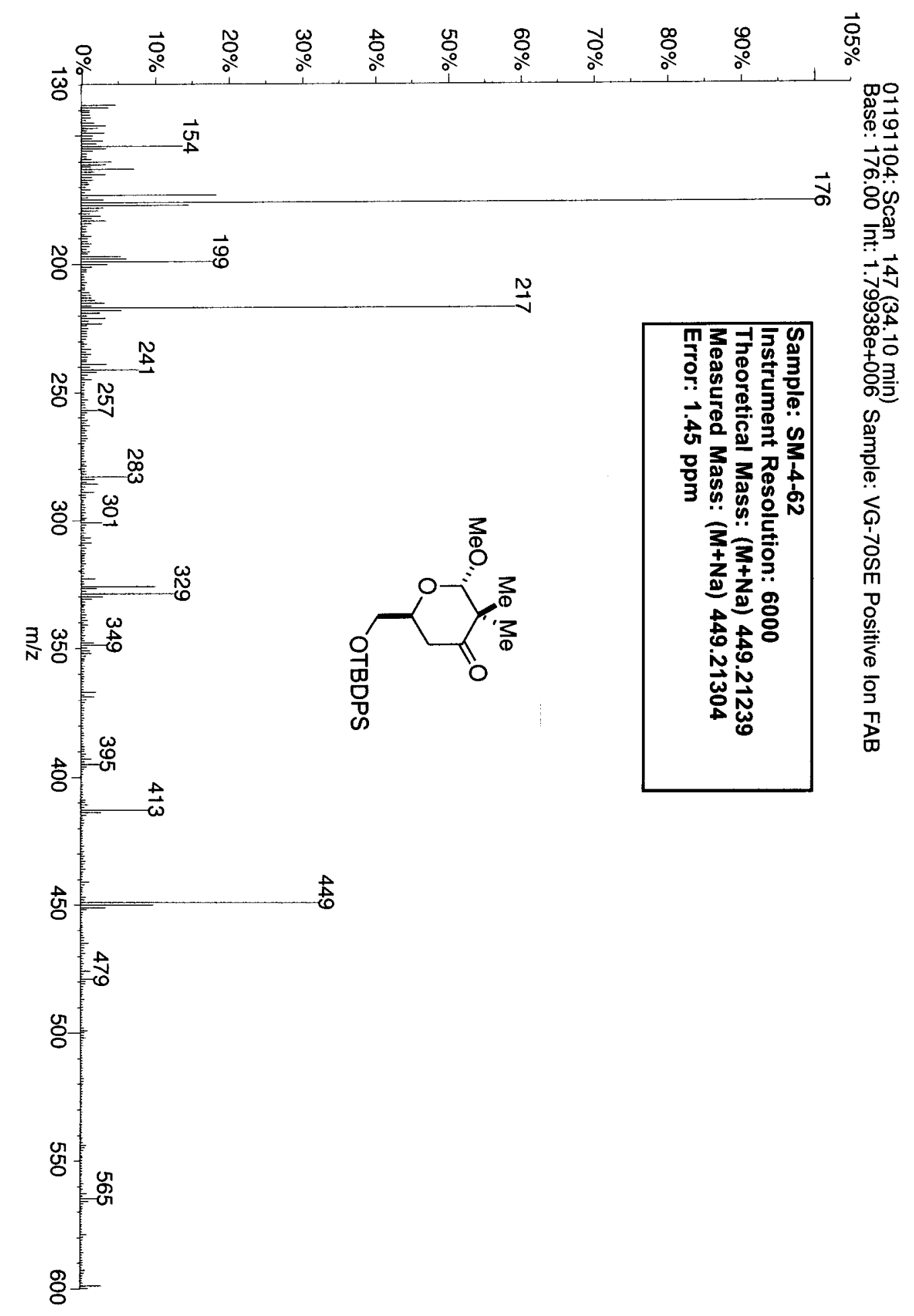




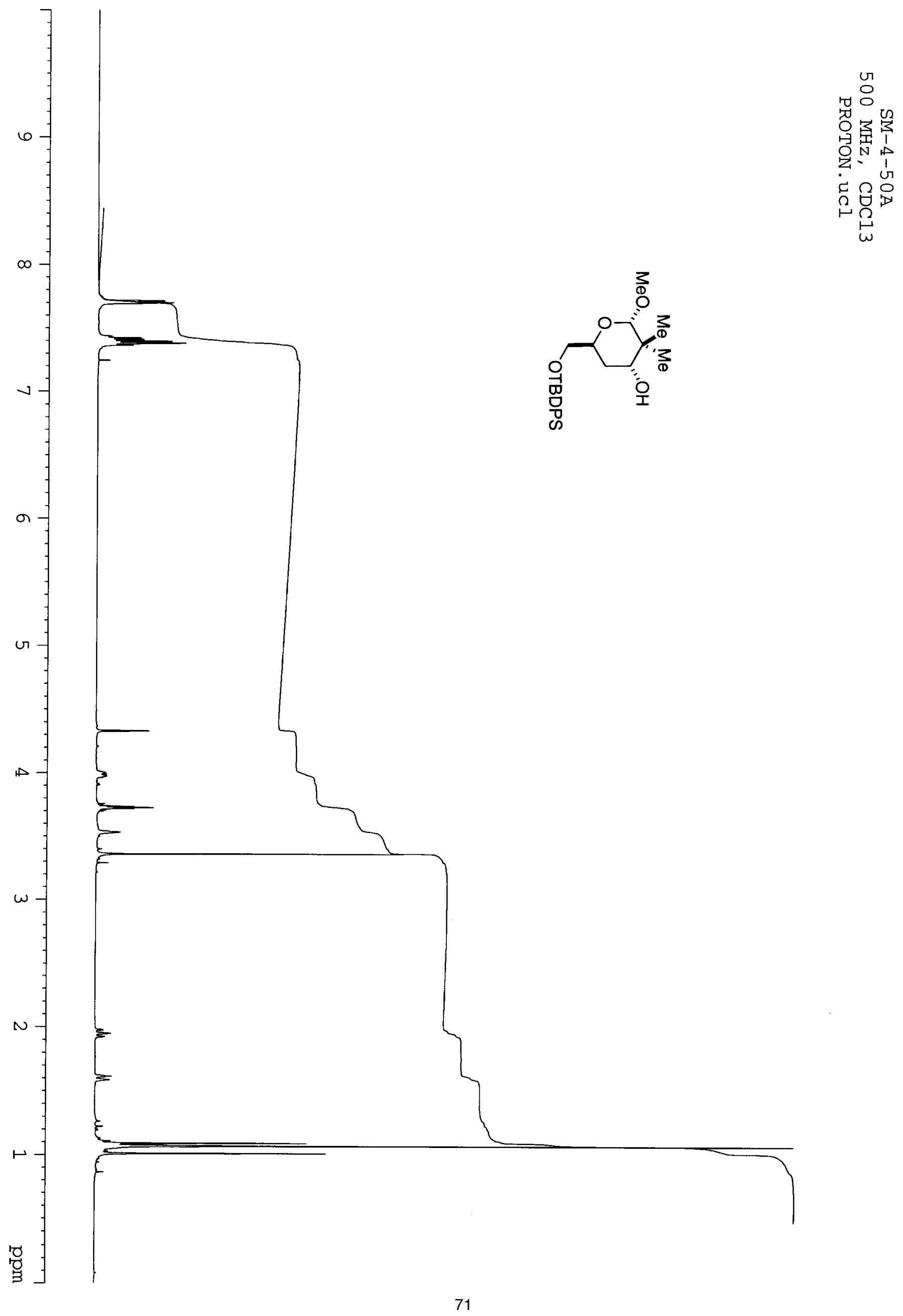




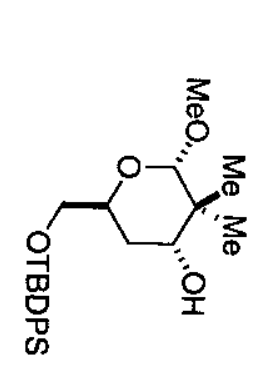

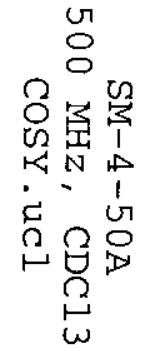

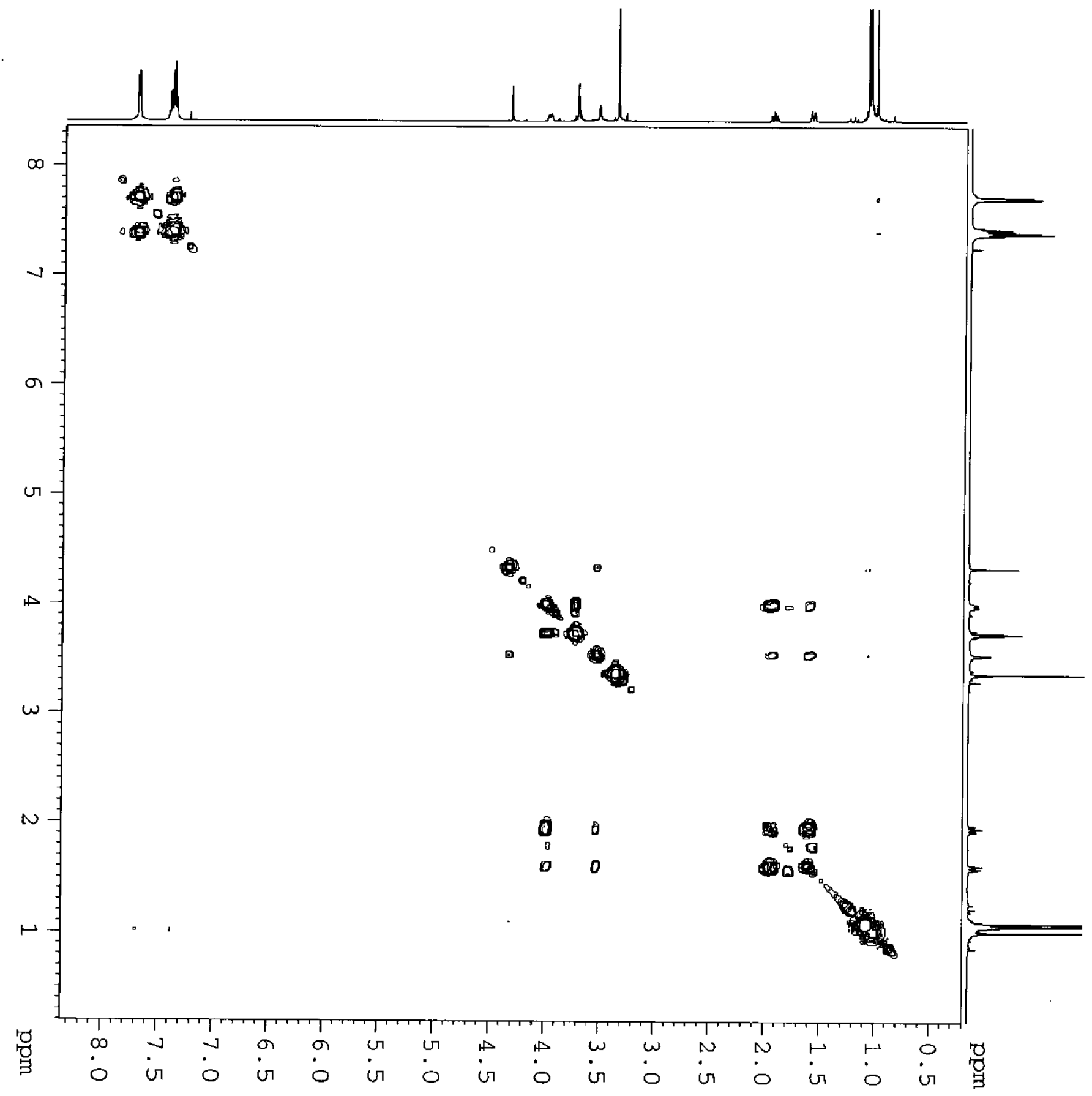



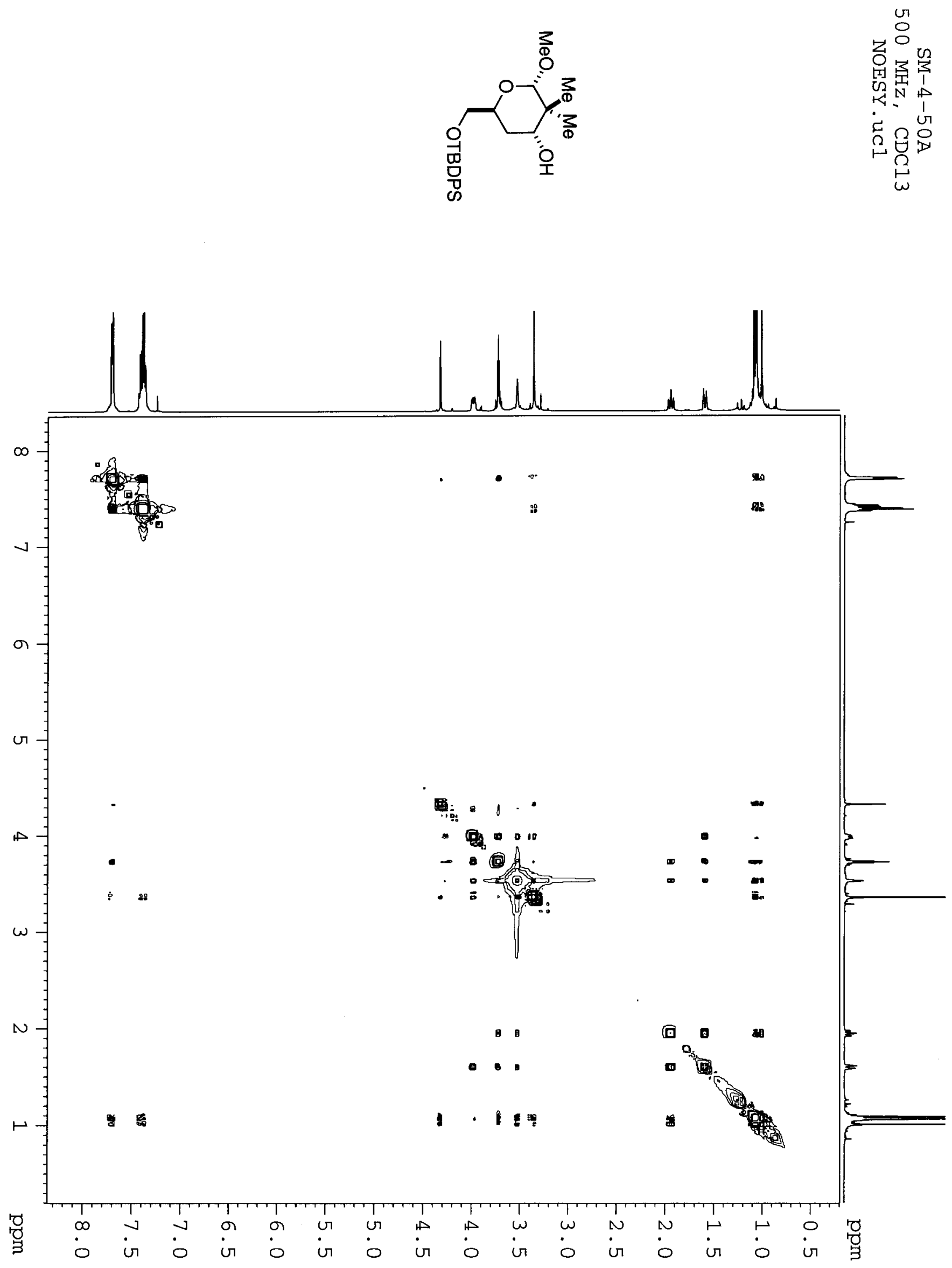

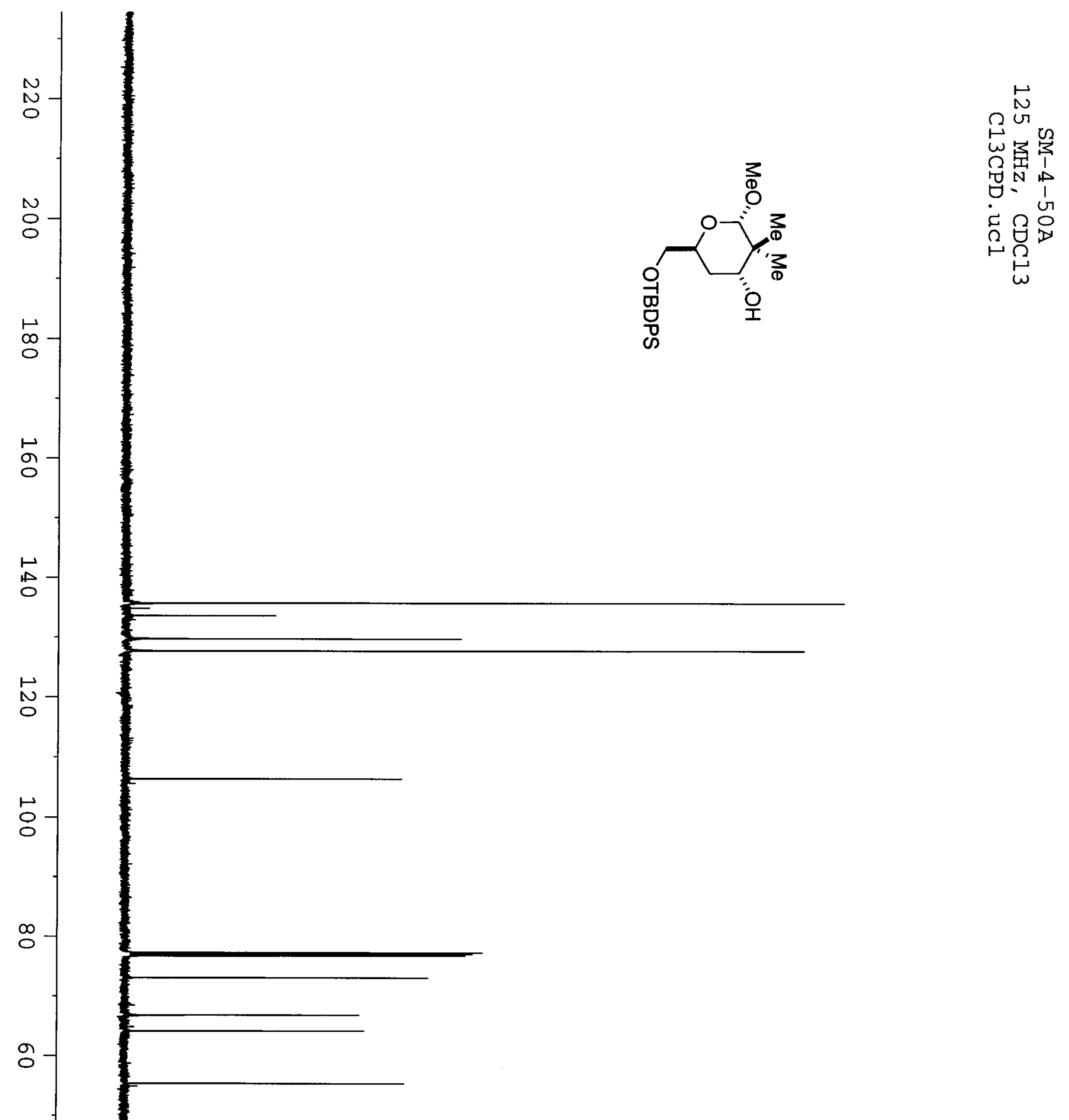

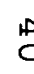

ס्: 


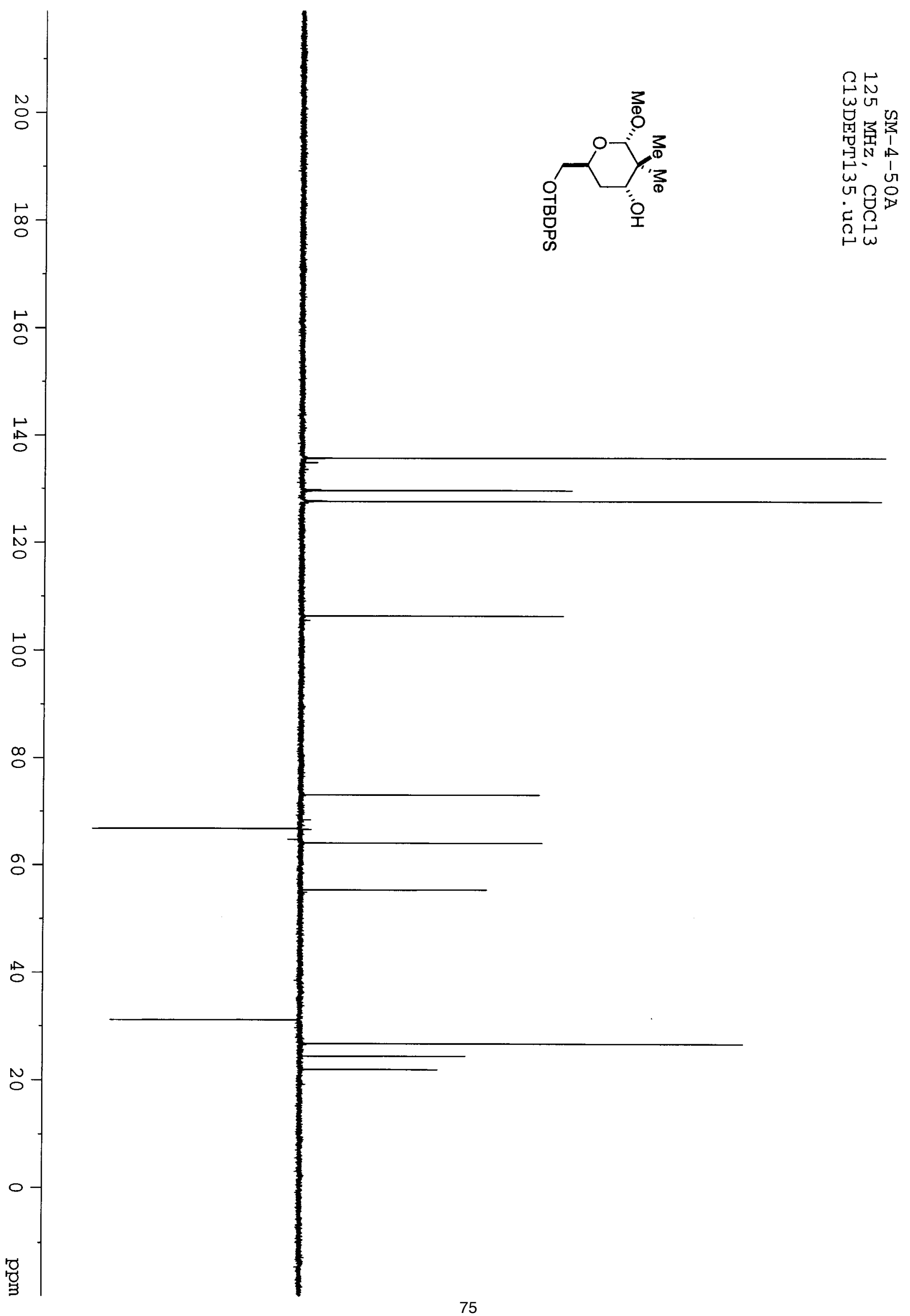



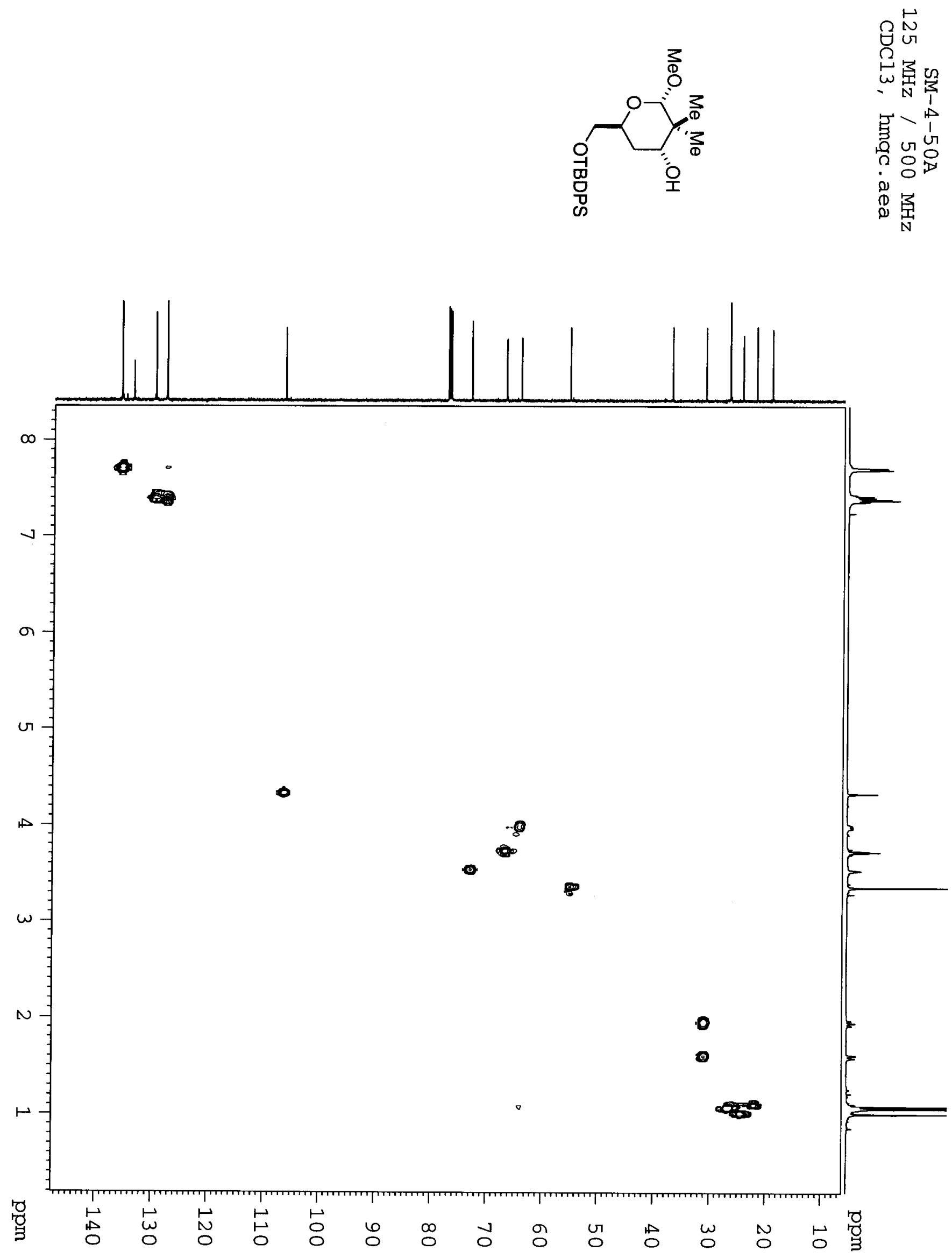


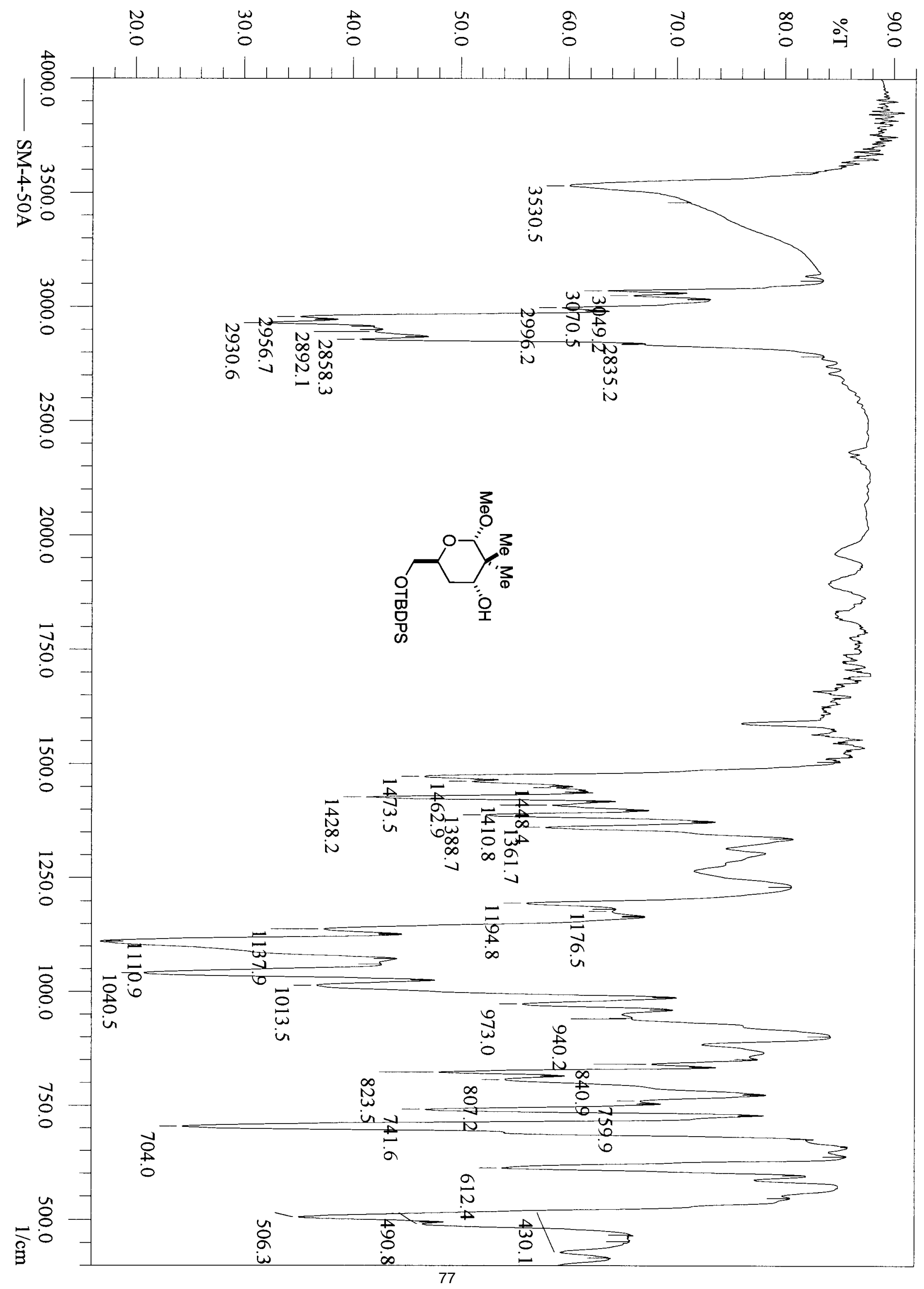




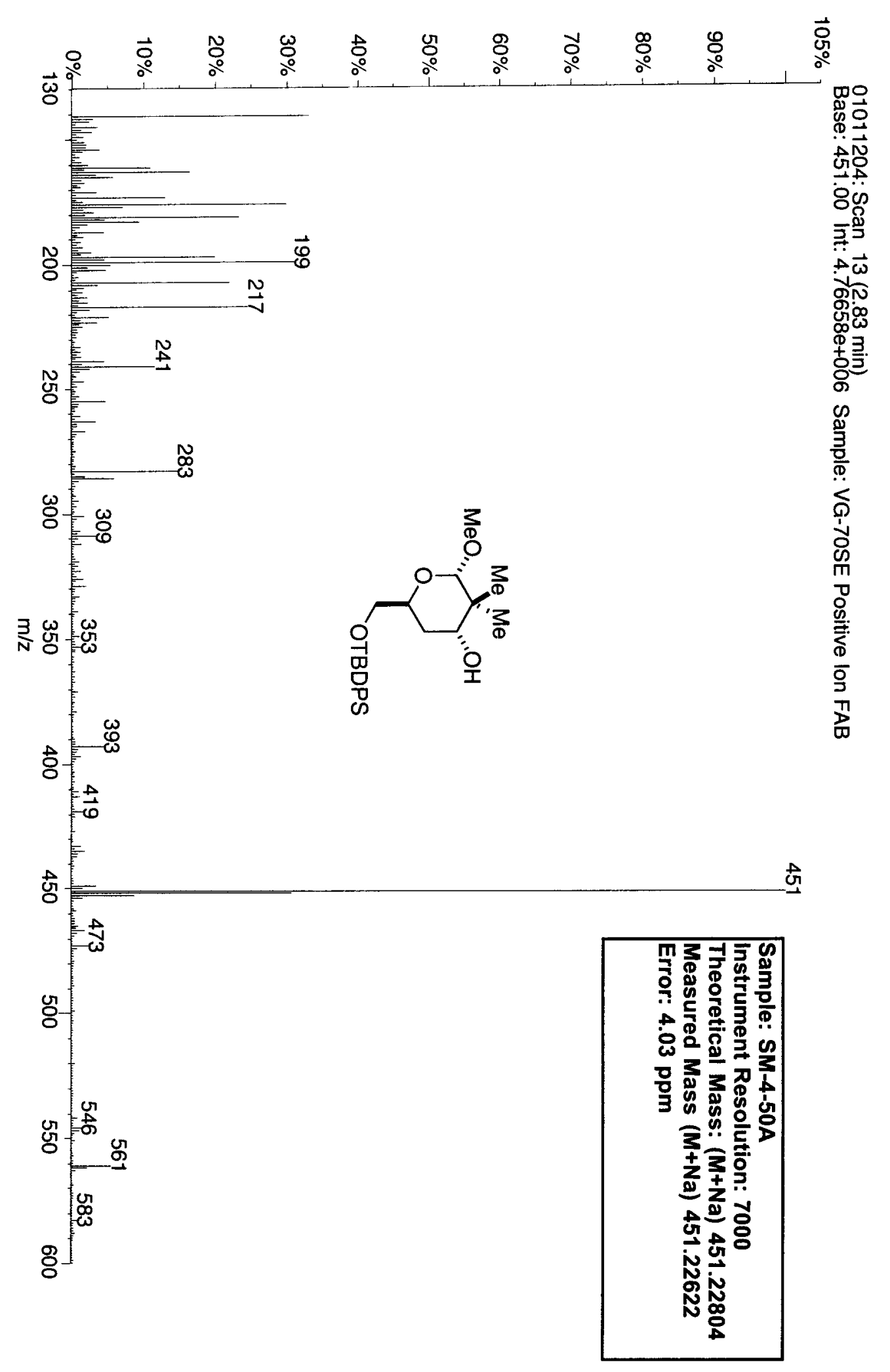




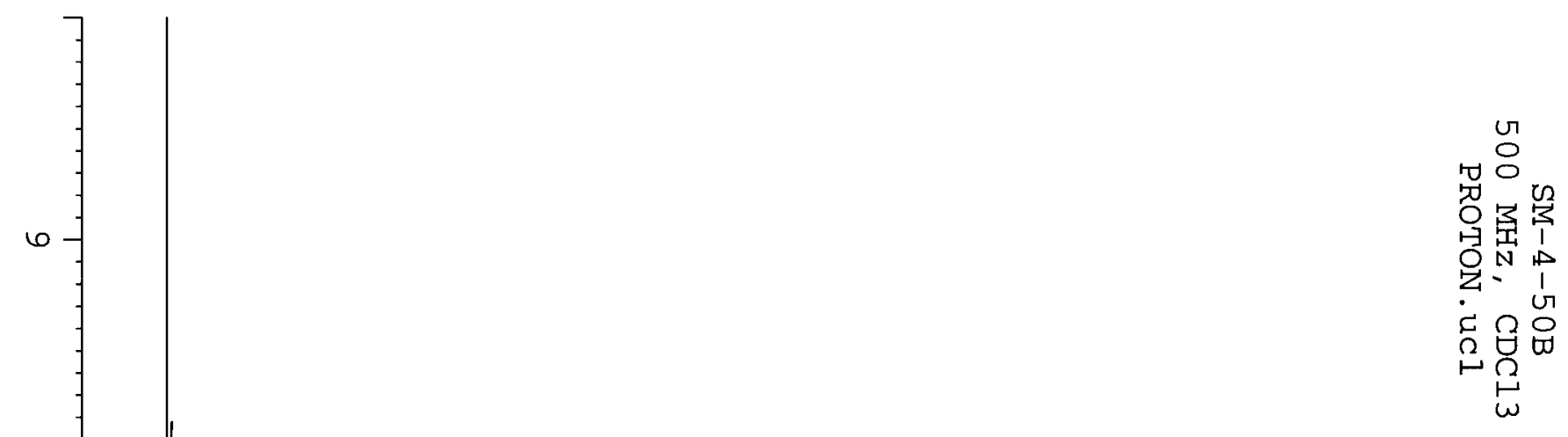

$\infty$

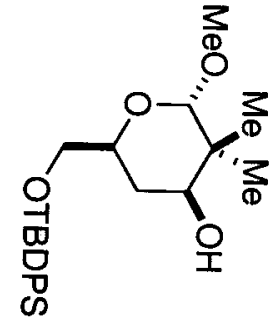

u

I

$\Delta-1$

$E$

$\omega$

.

N

N-

$=$
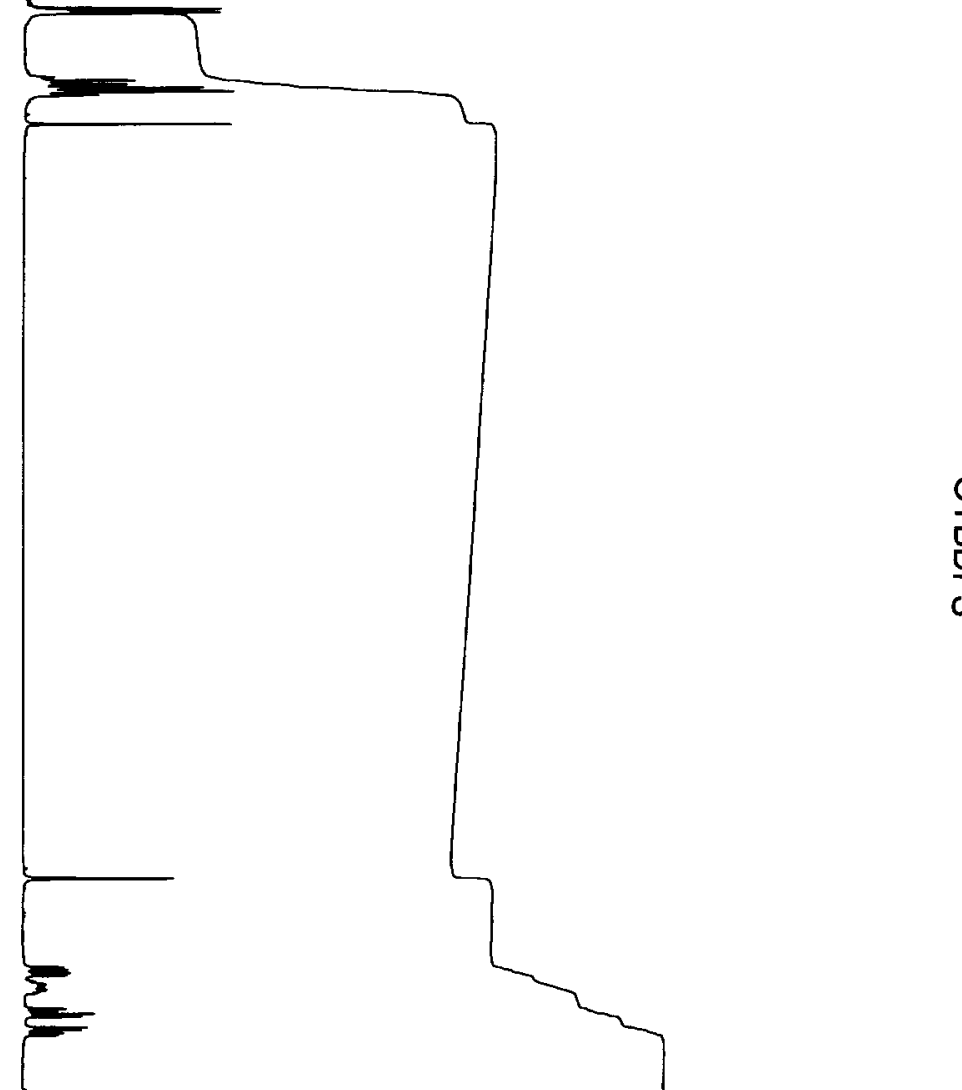

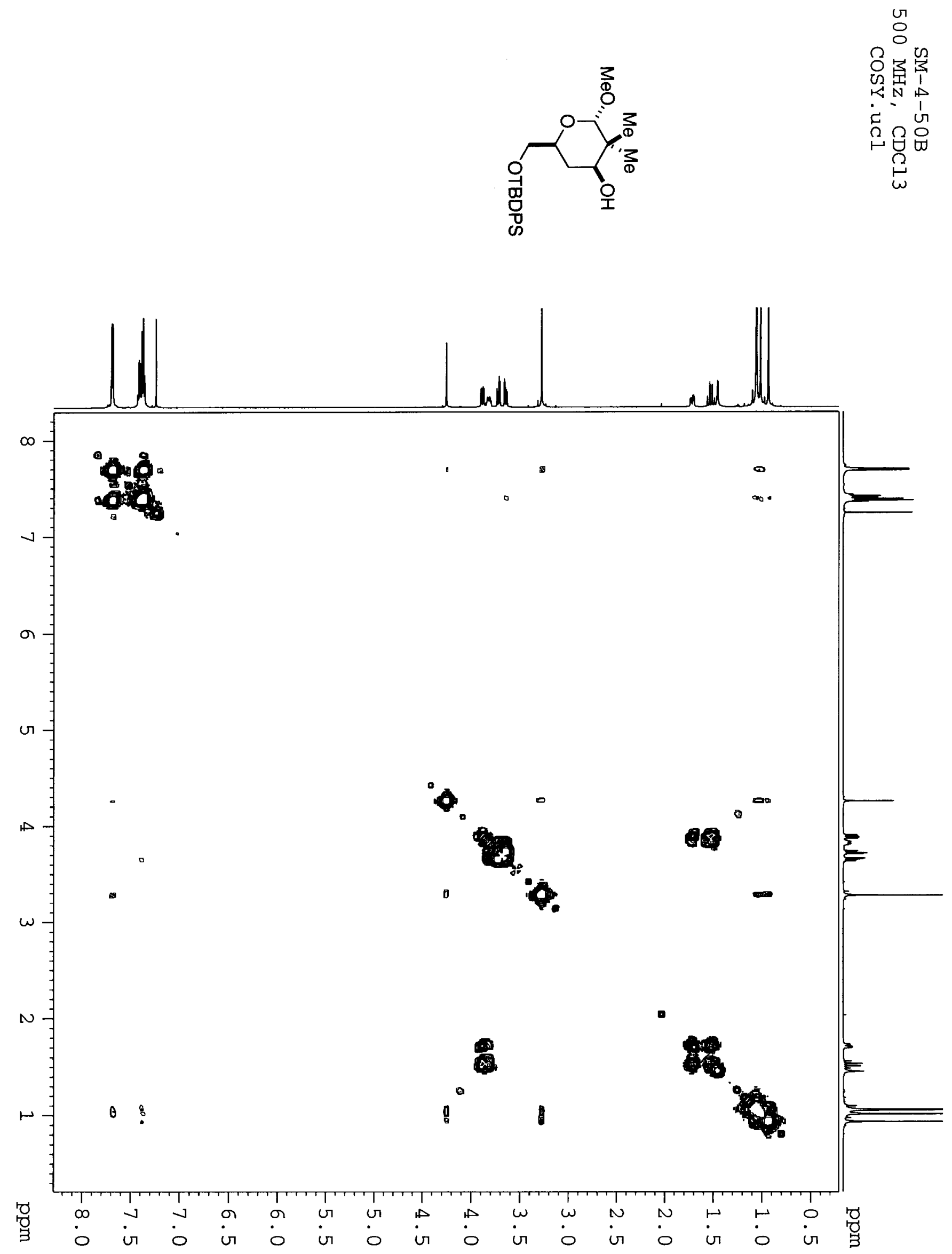

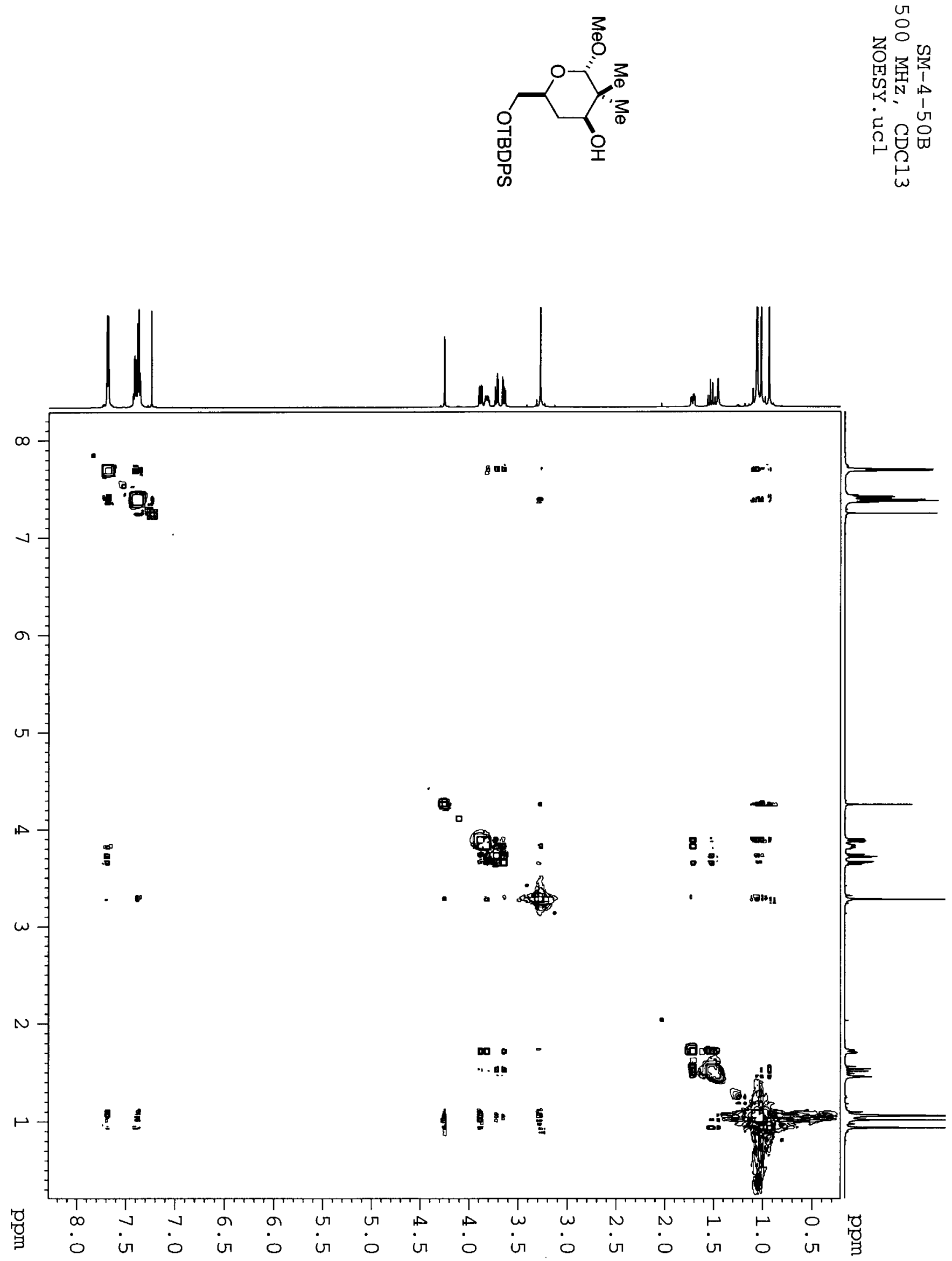


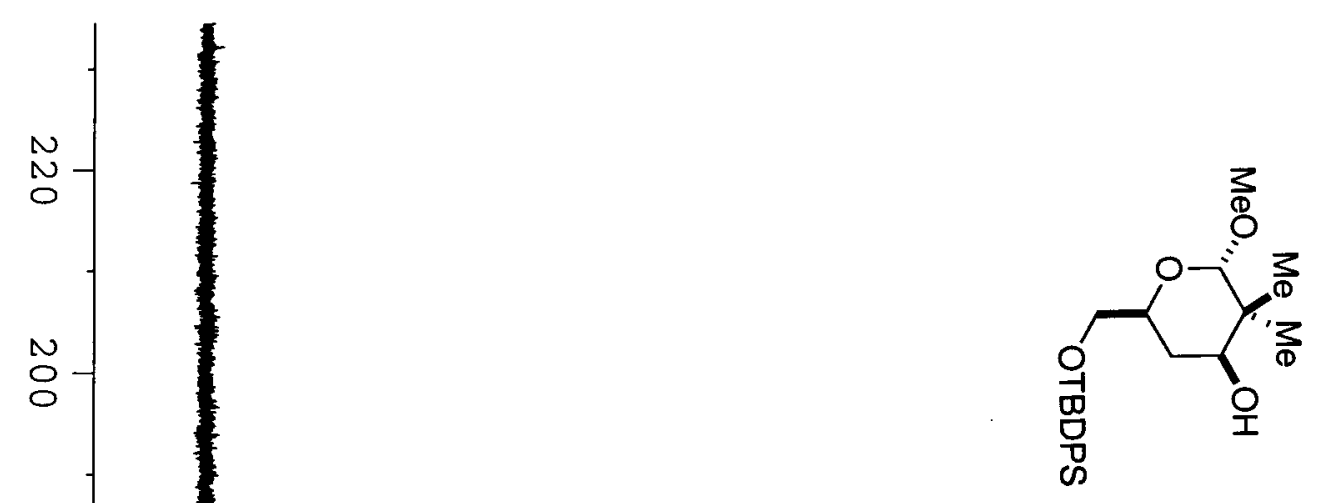

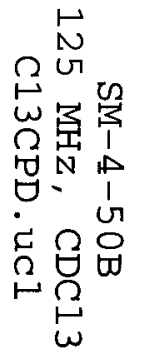

$\stackrel{\bullet}{\infty}$

뭉

$\stackrel{\bullet}{\circ}$

N

둥-

$\infty$

o

D



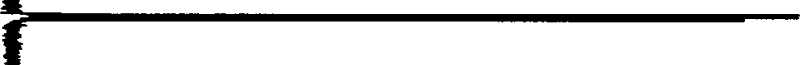




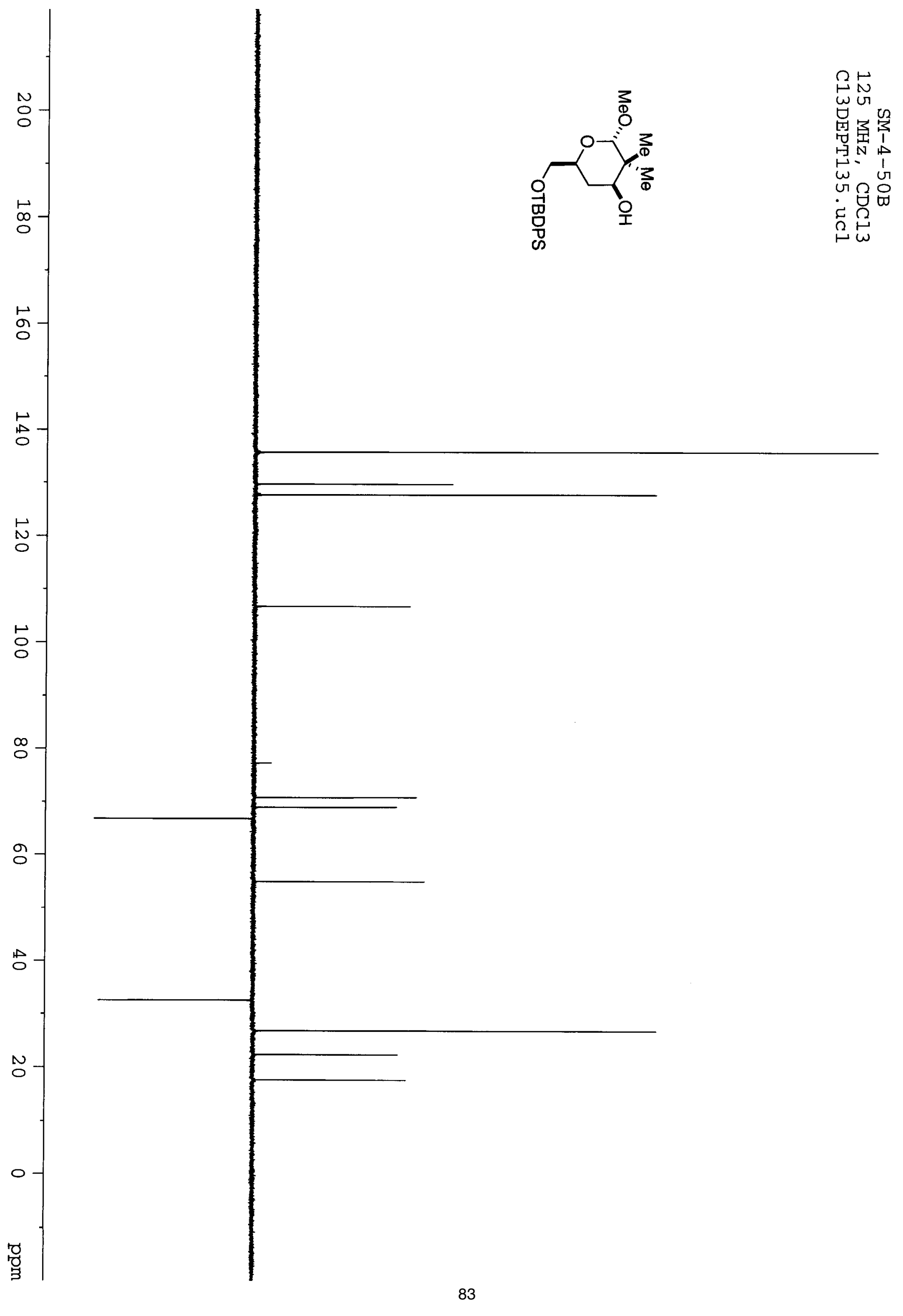



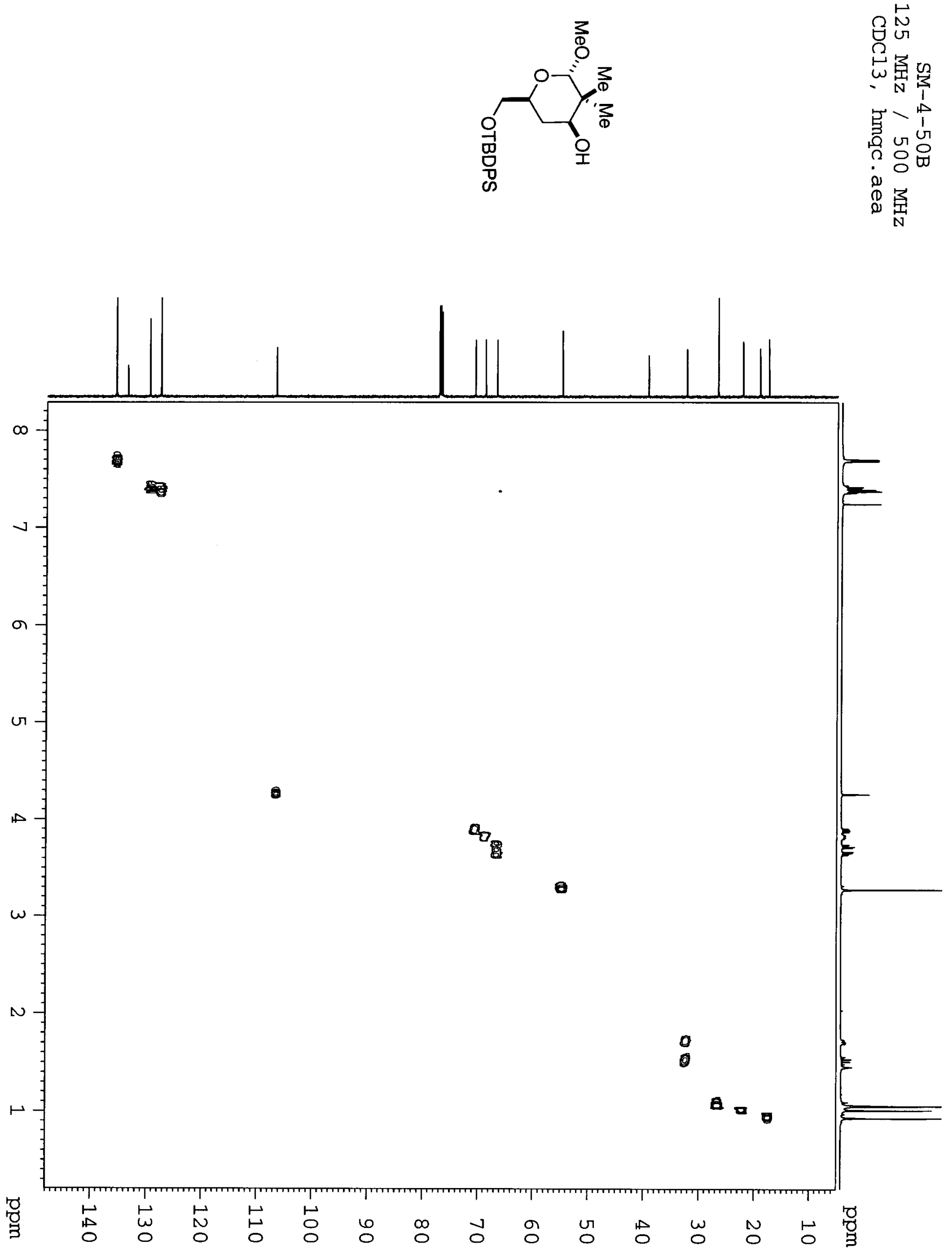


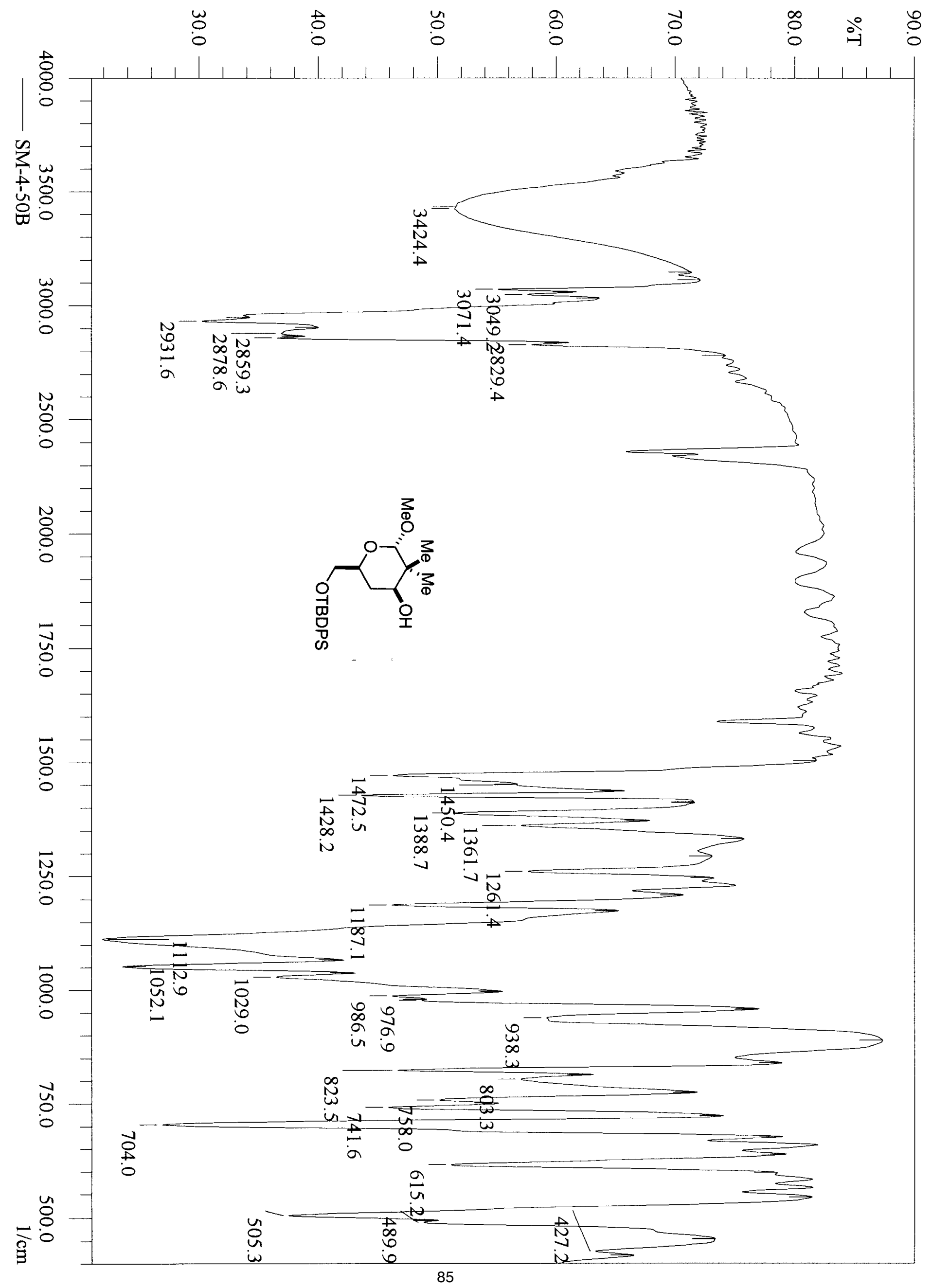




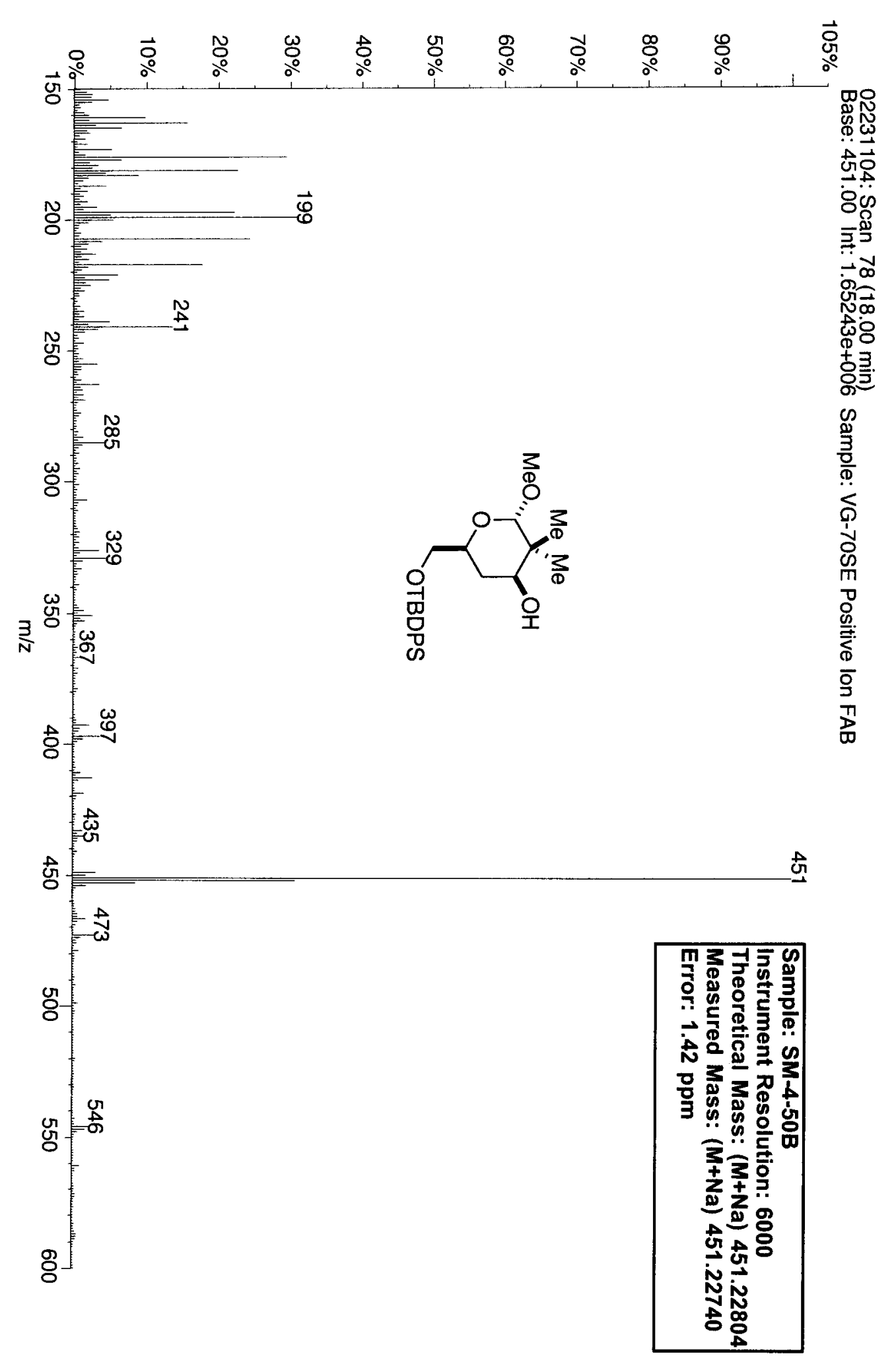




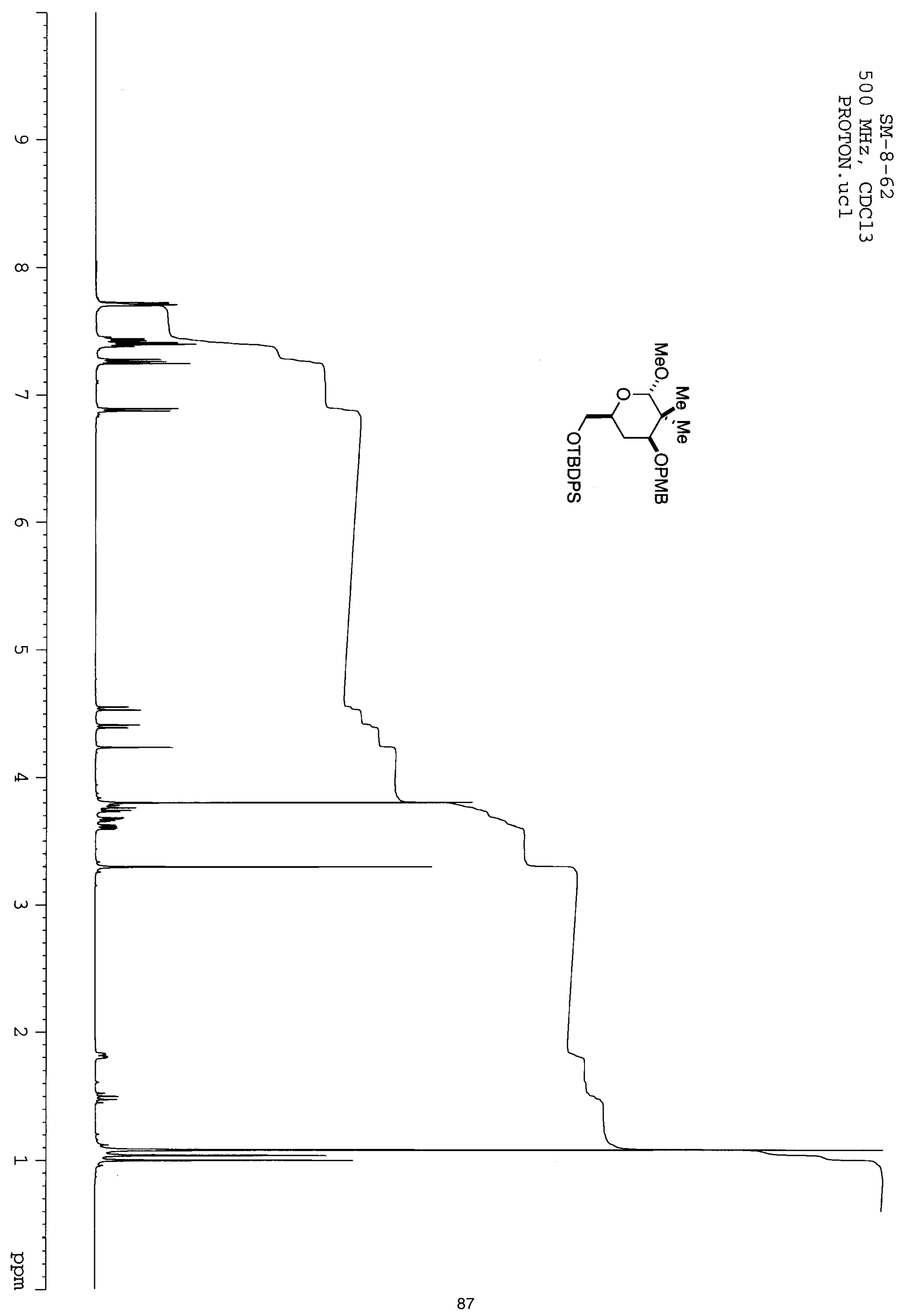



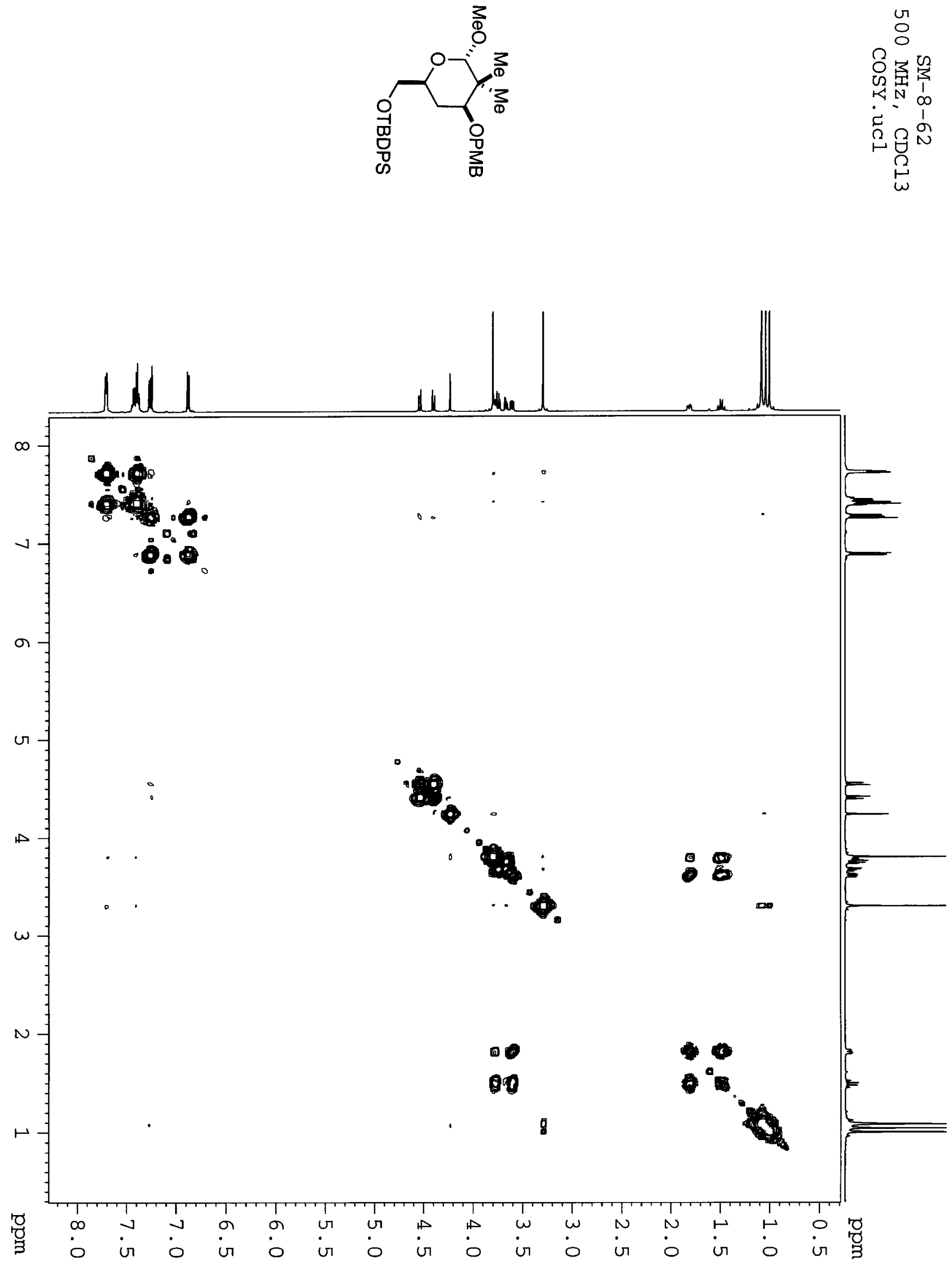

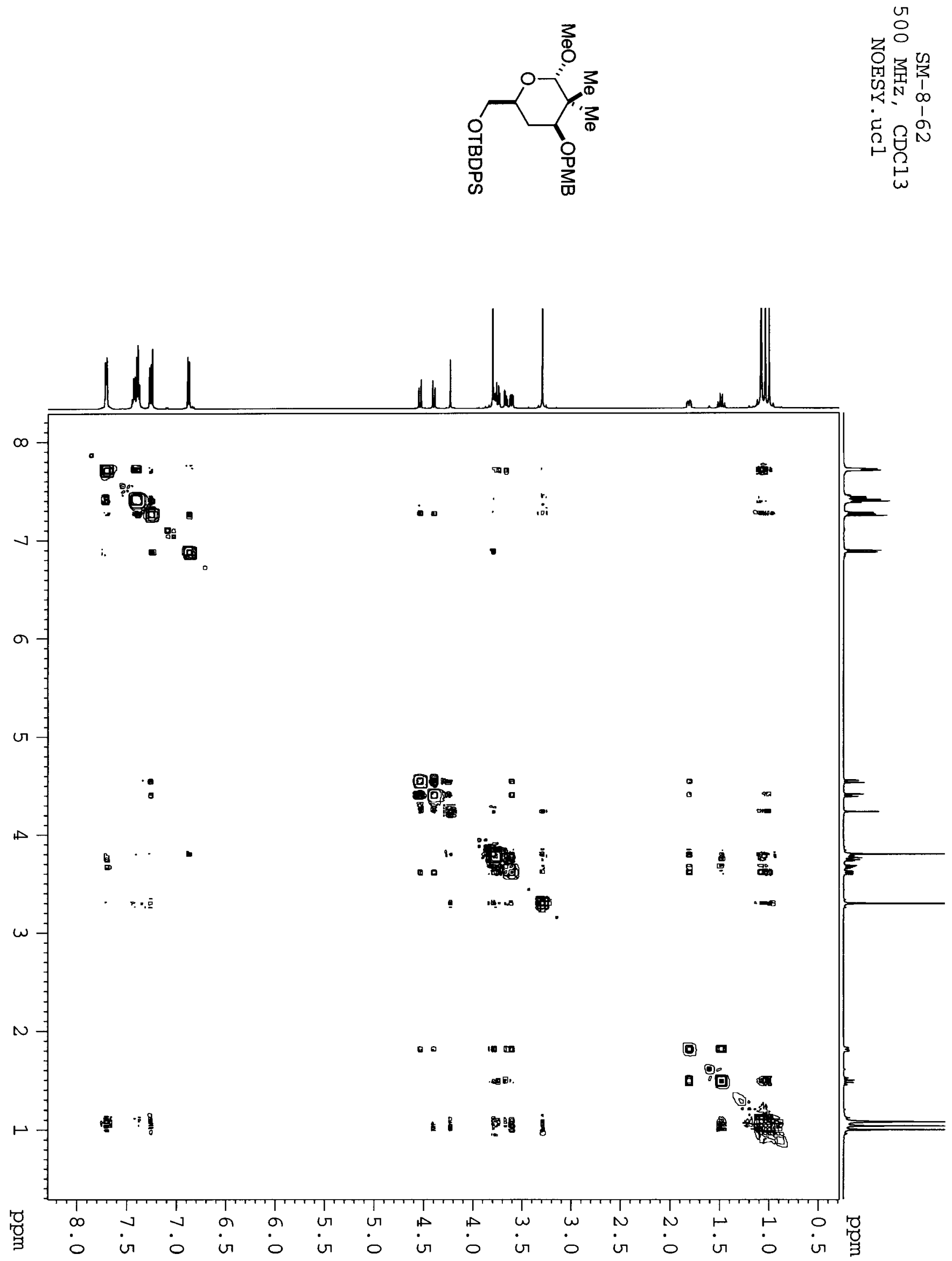


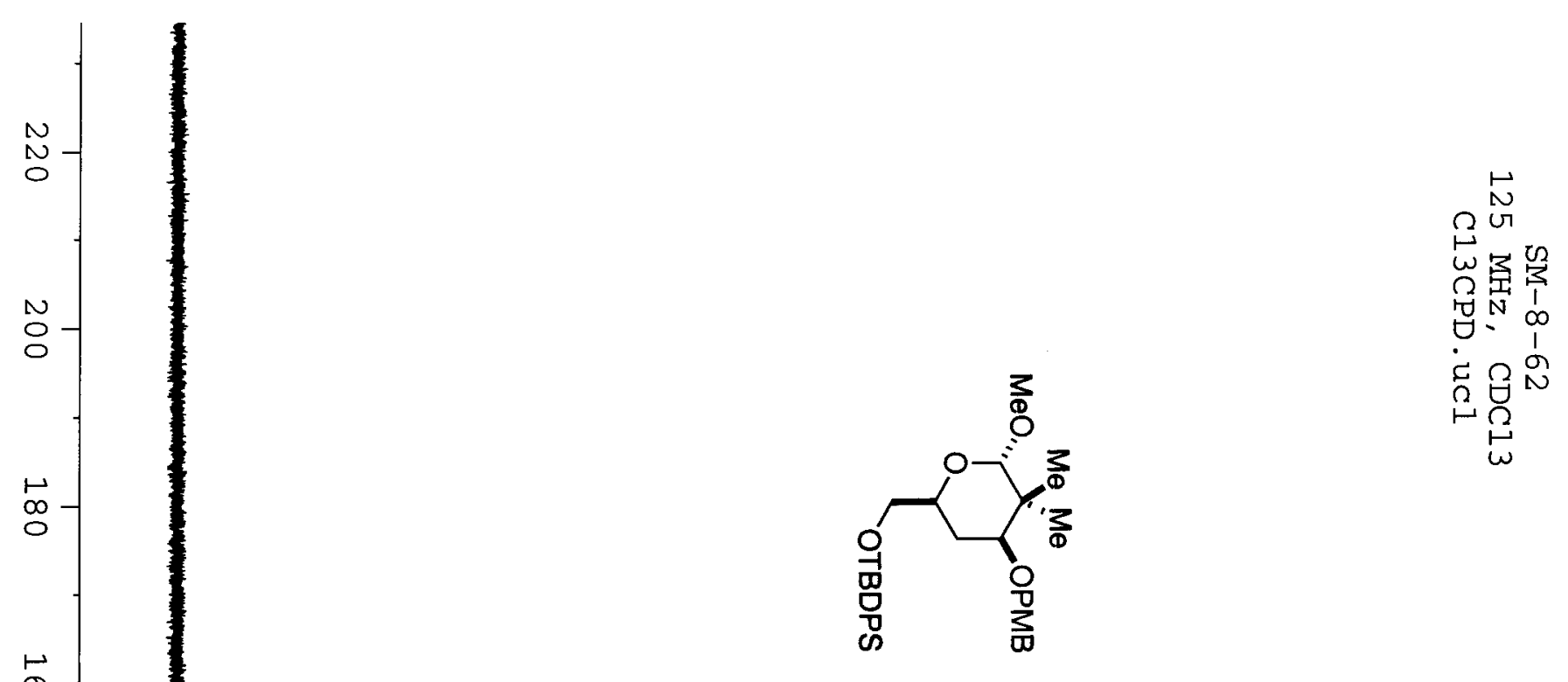

\%

$\stackrel{\bullet}{\circ}$
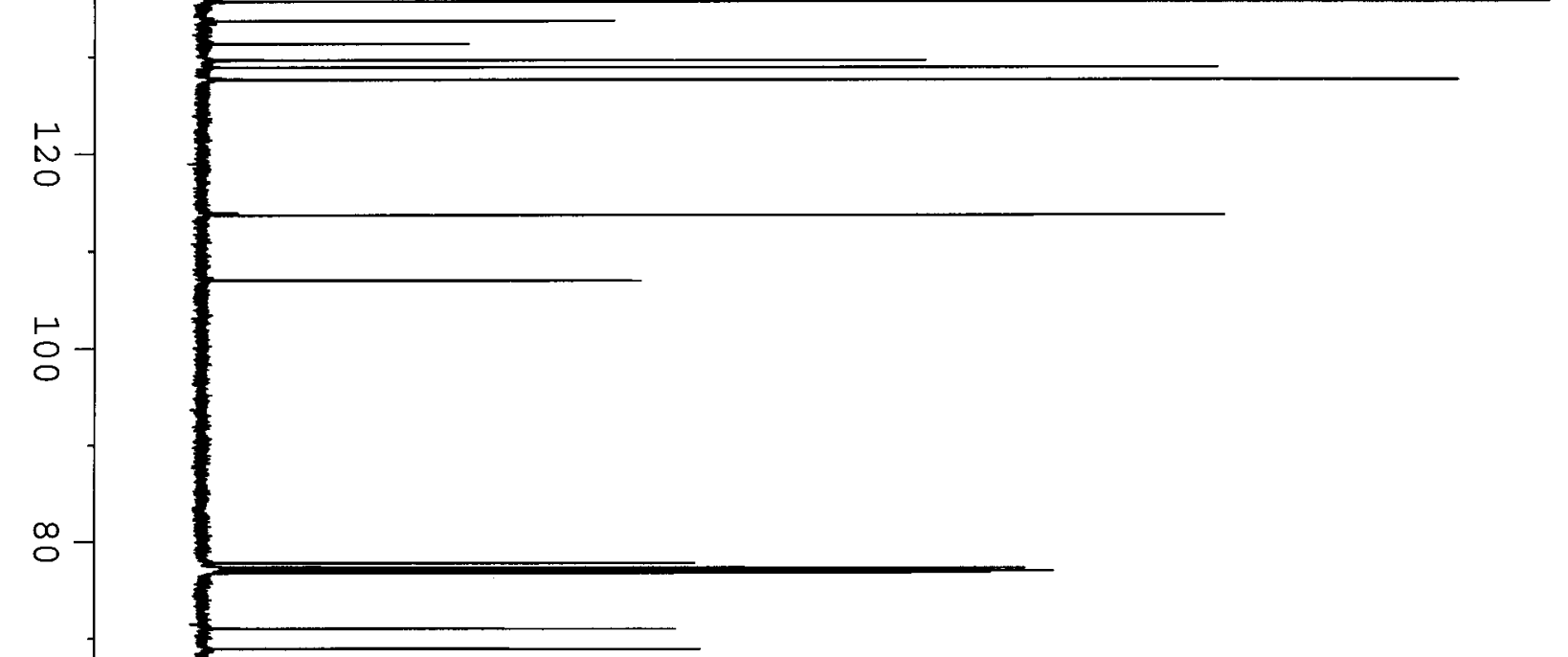

o

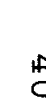

|

- 


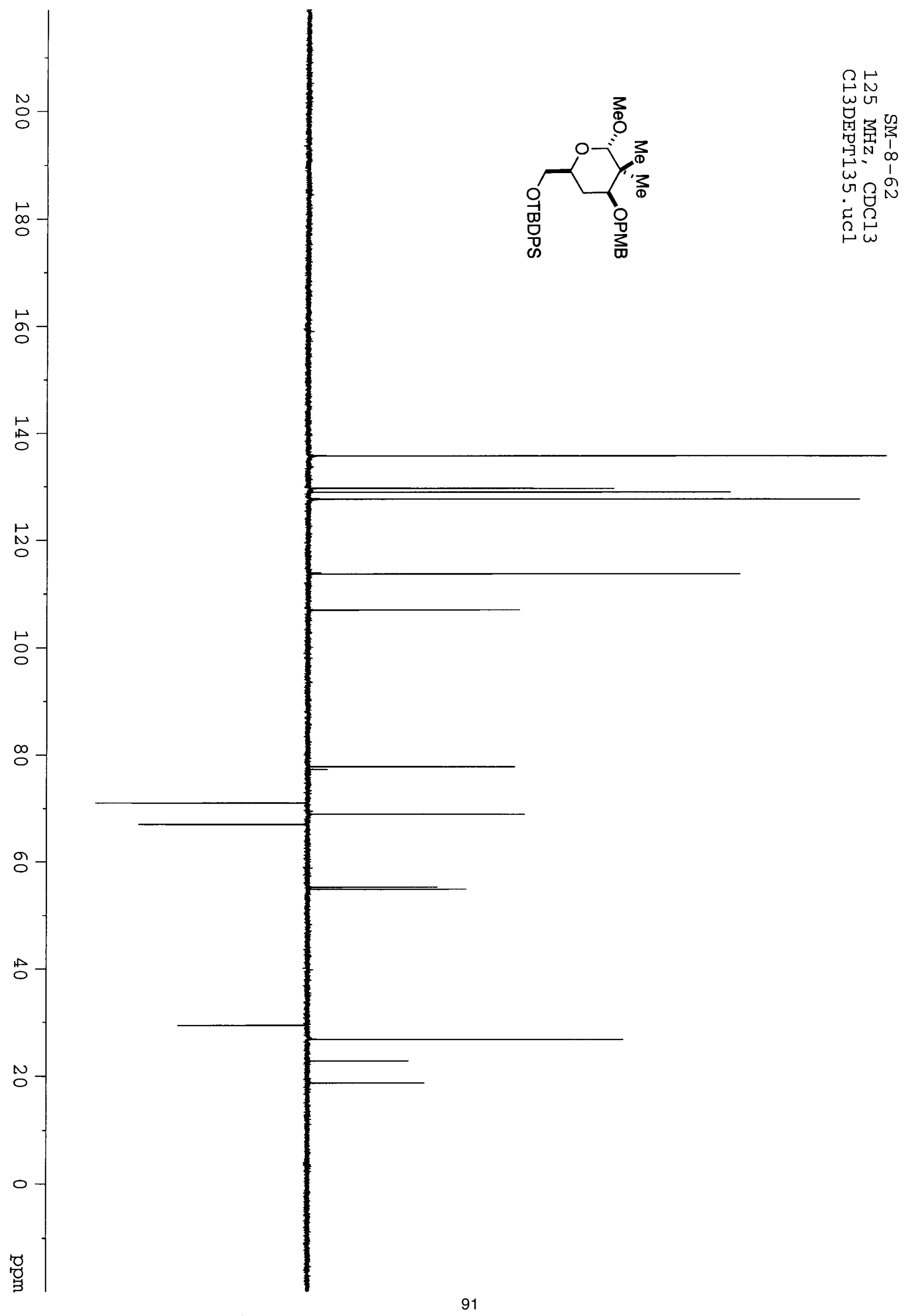



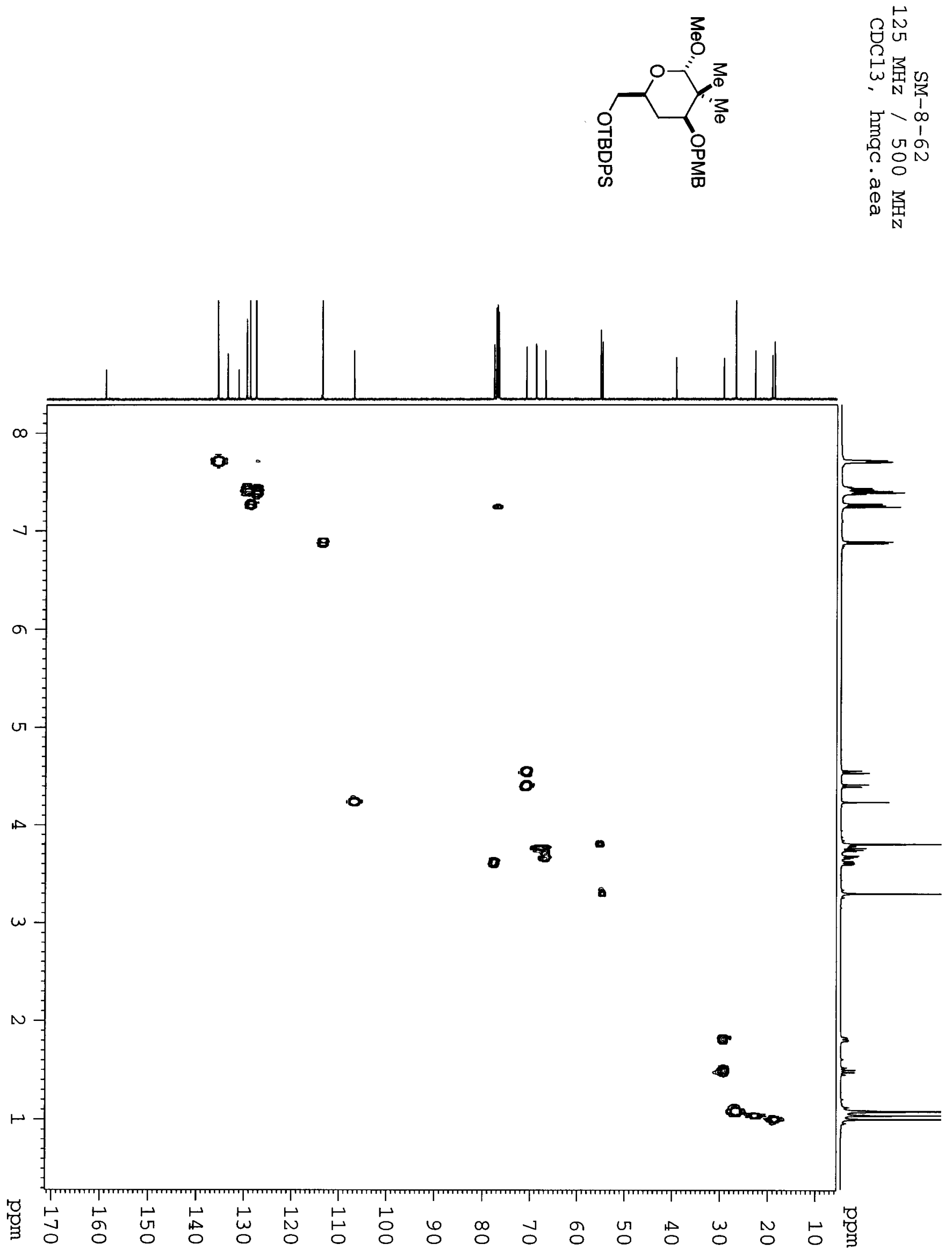


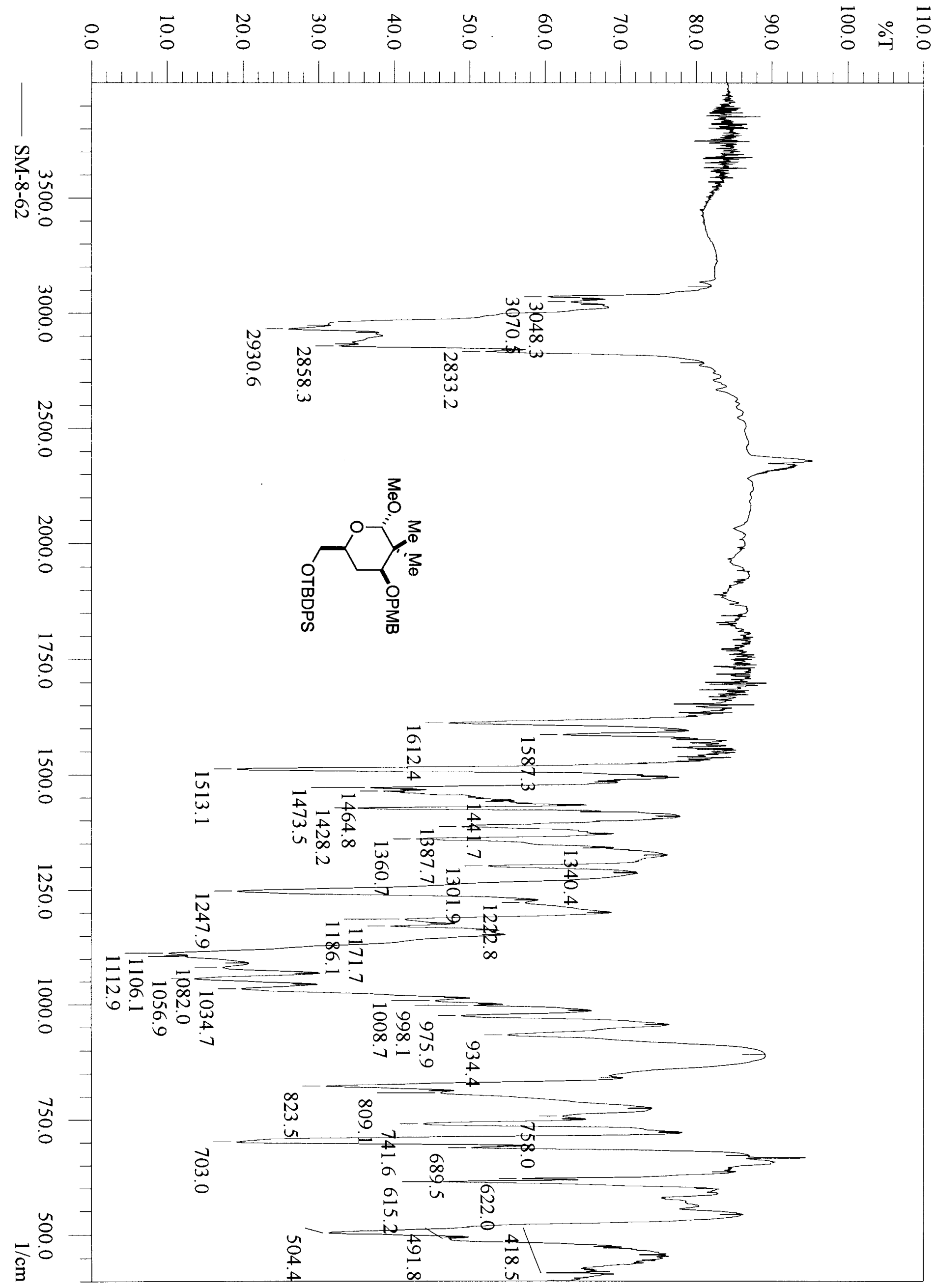




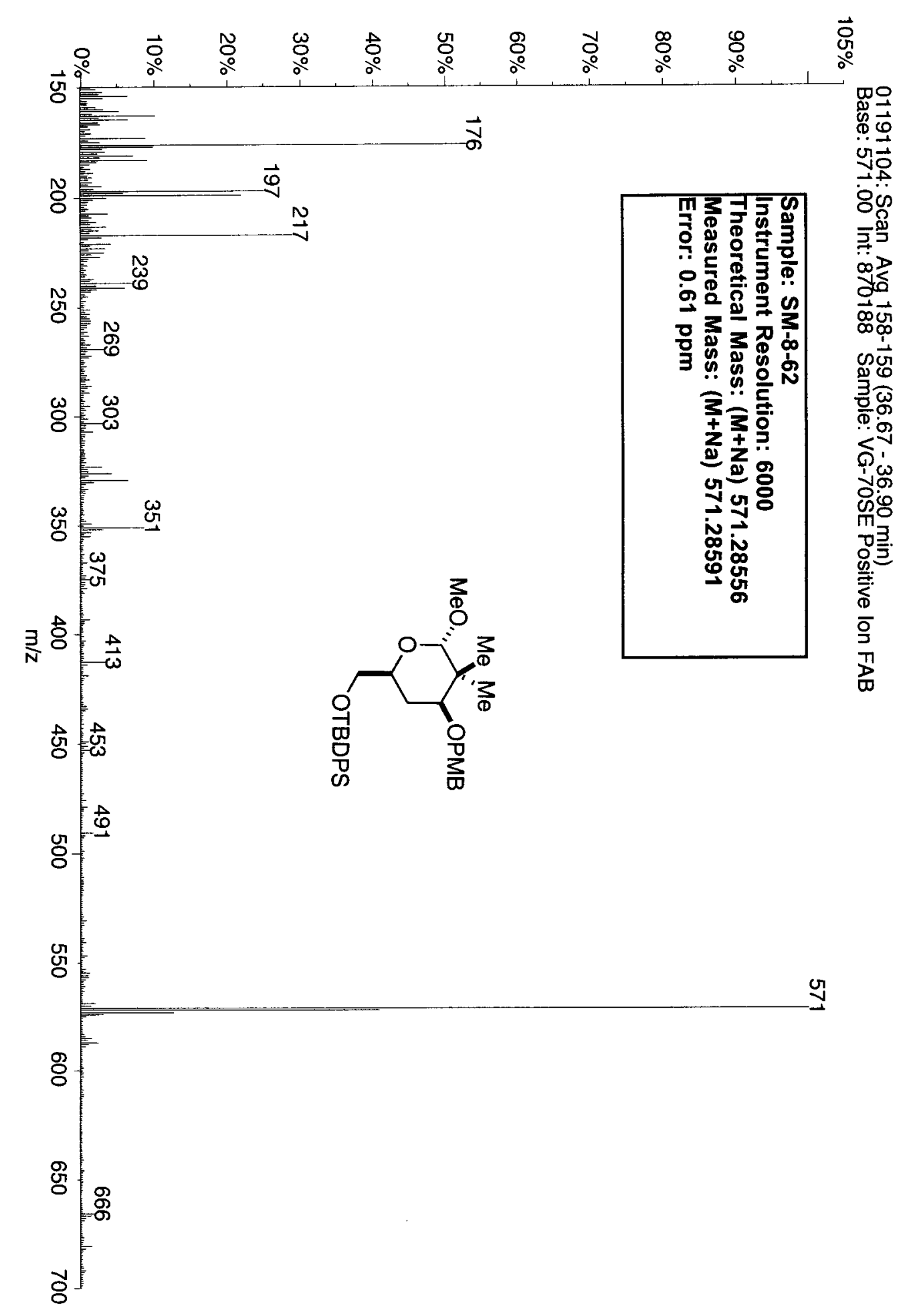



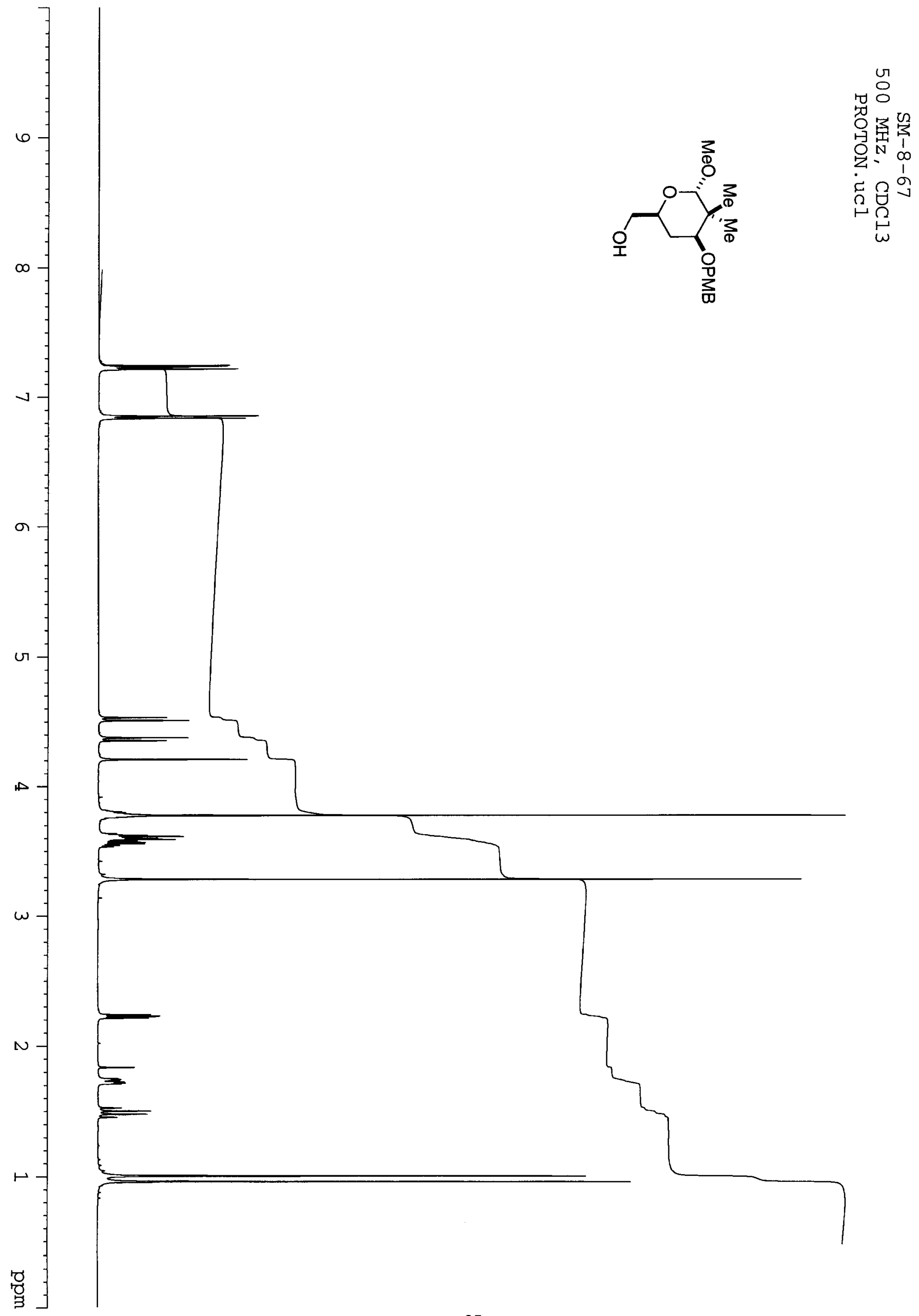

95 

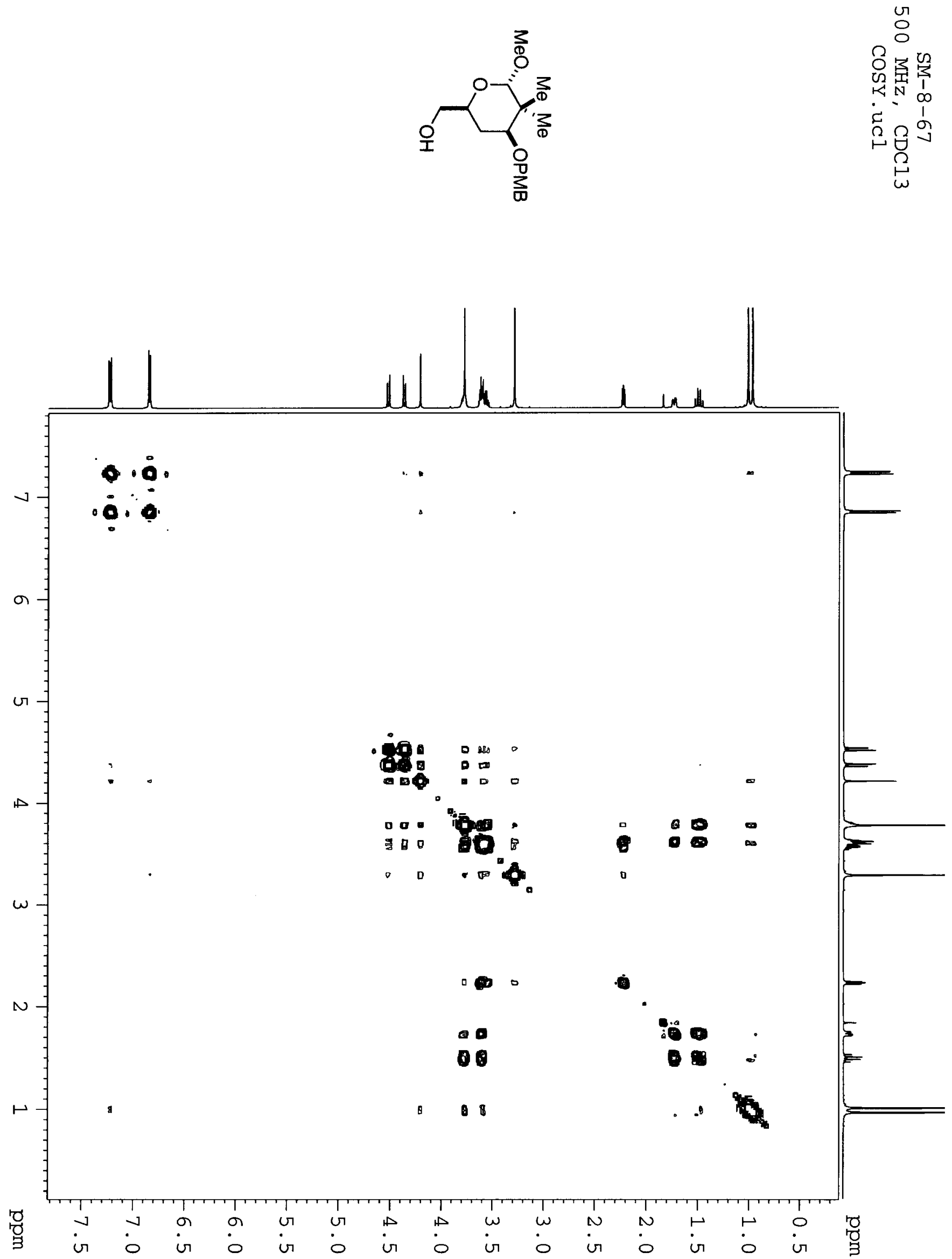

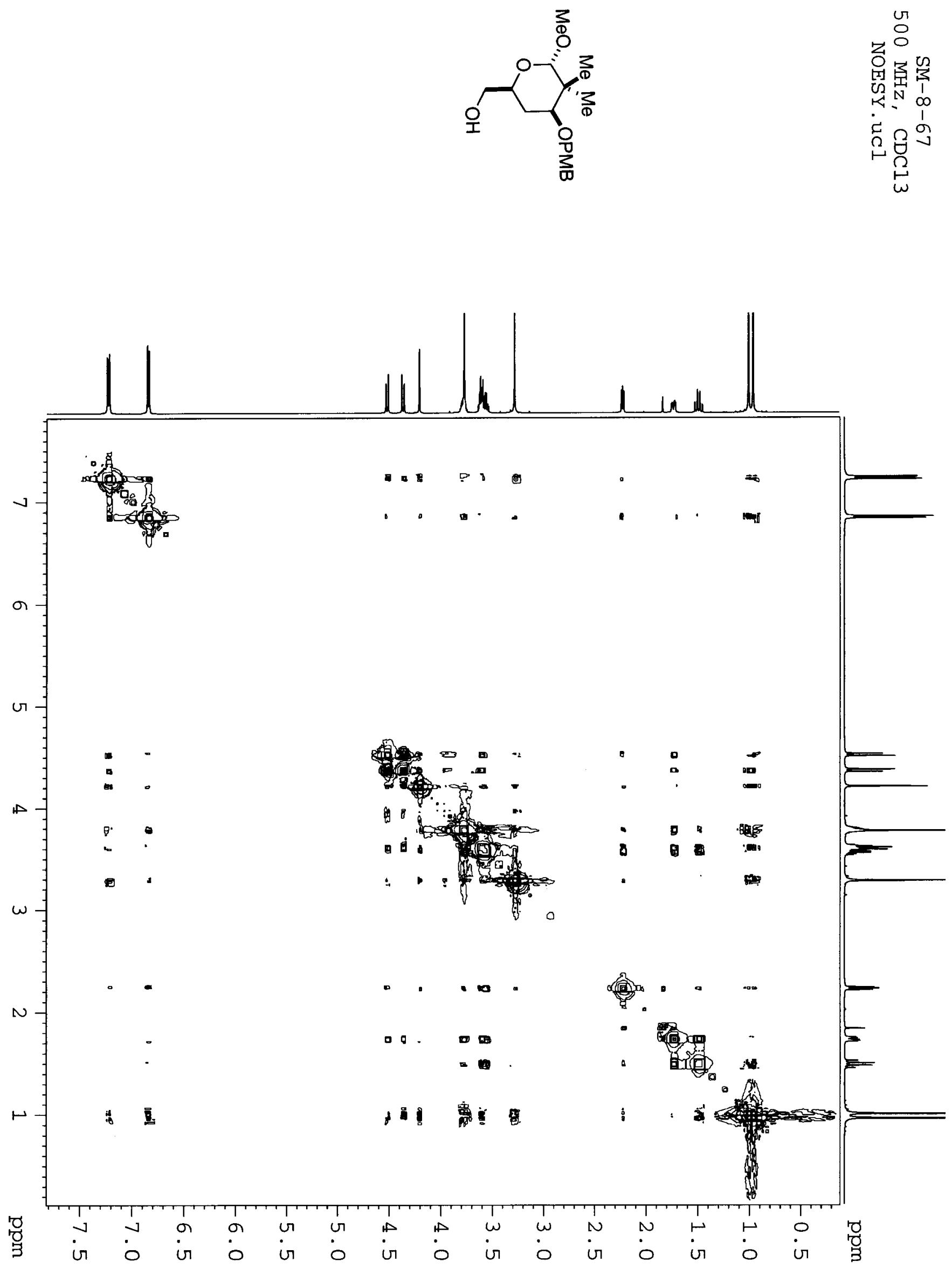


$$
|l| "^{*}
$$




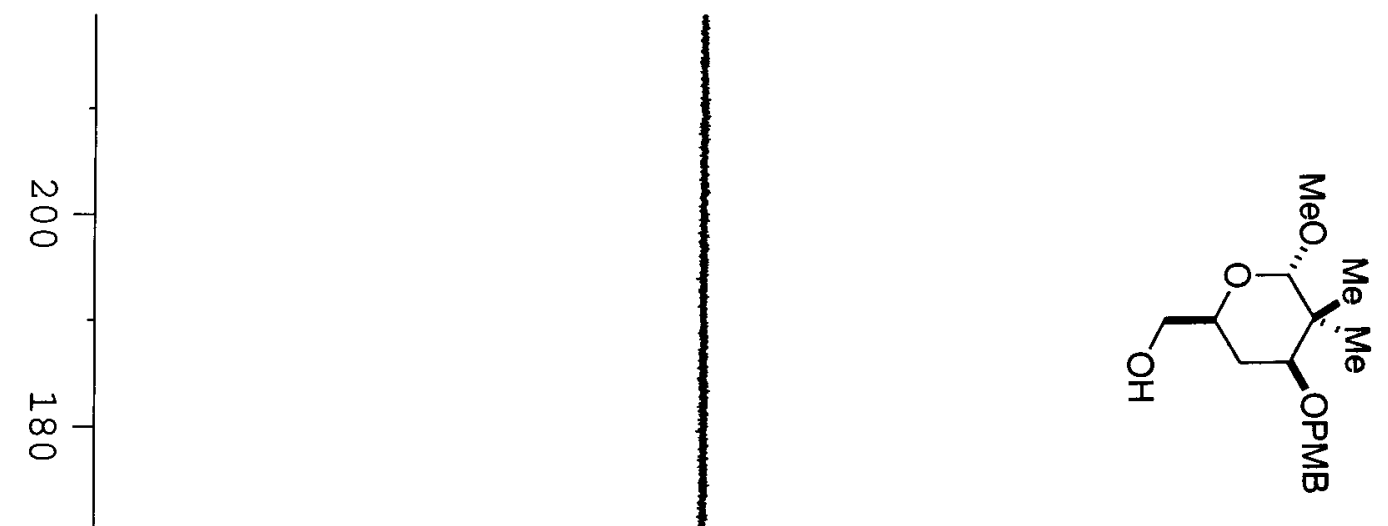

ค

u

琵寔

鼠 1

$\omega \Omega$ o

苗苜

\[ \]
$\circ$
0
0
10
0

N

๖

$\infty$

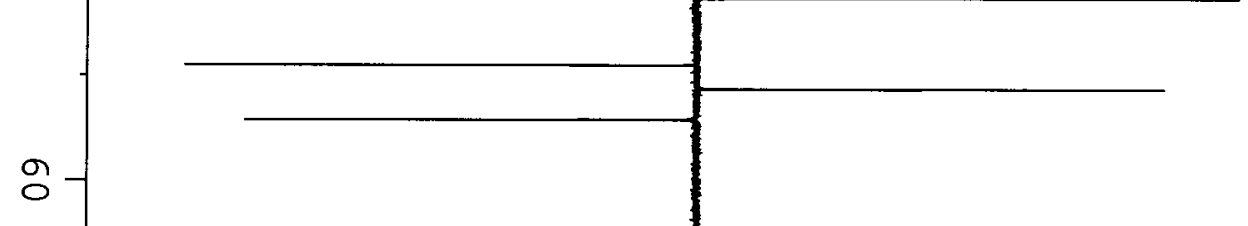

No

$\stackrel{\oplus}{\circ}$

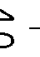

o

N

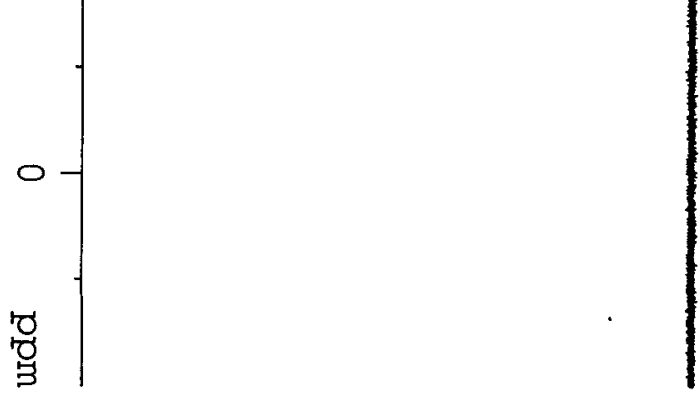

읍 


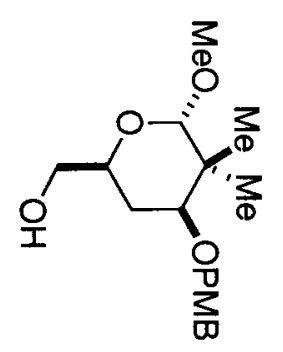

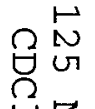

3

焉尽

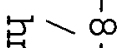

ด ง

ก 0

(i)

芯

N

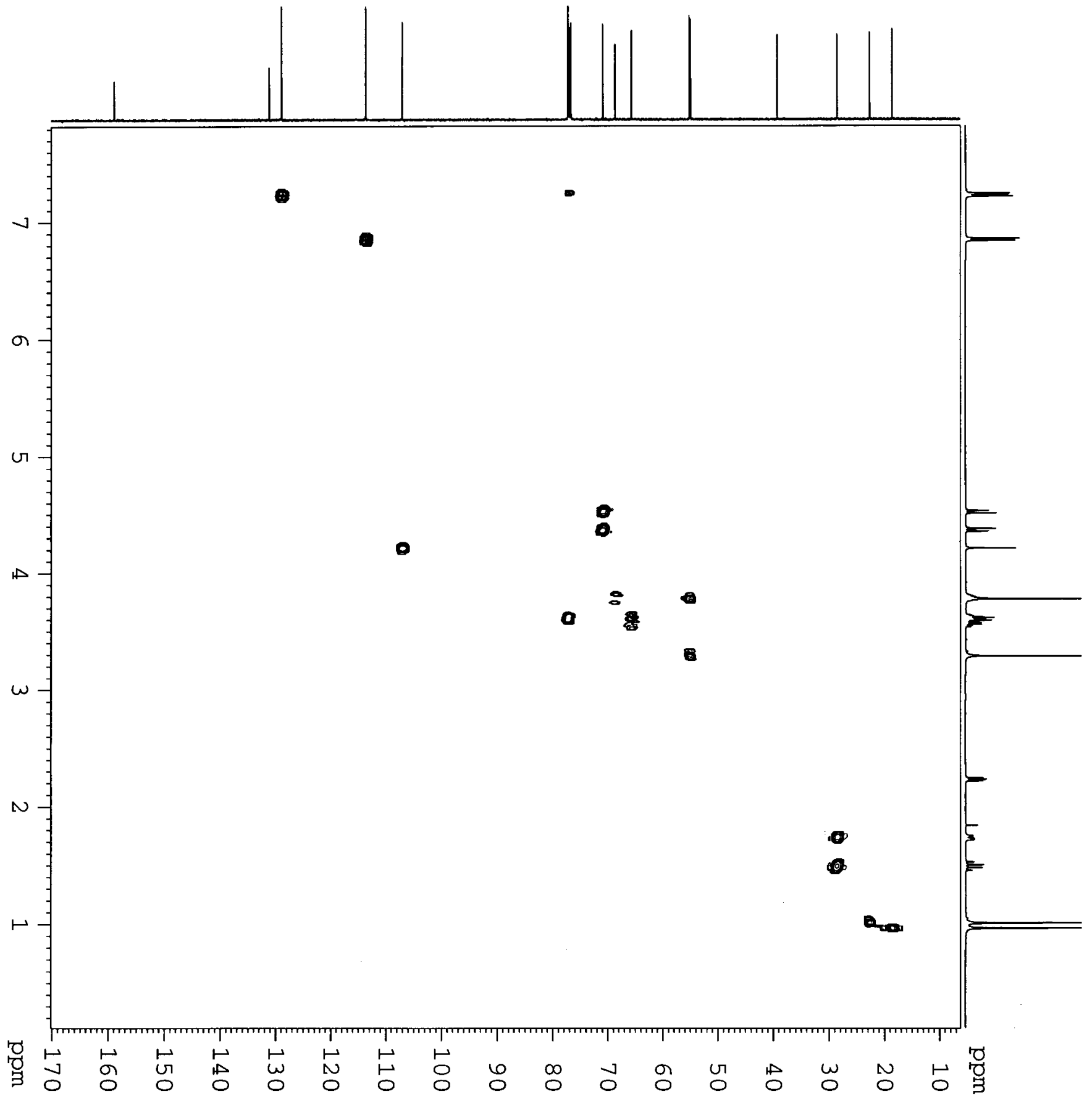




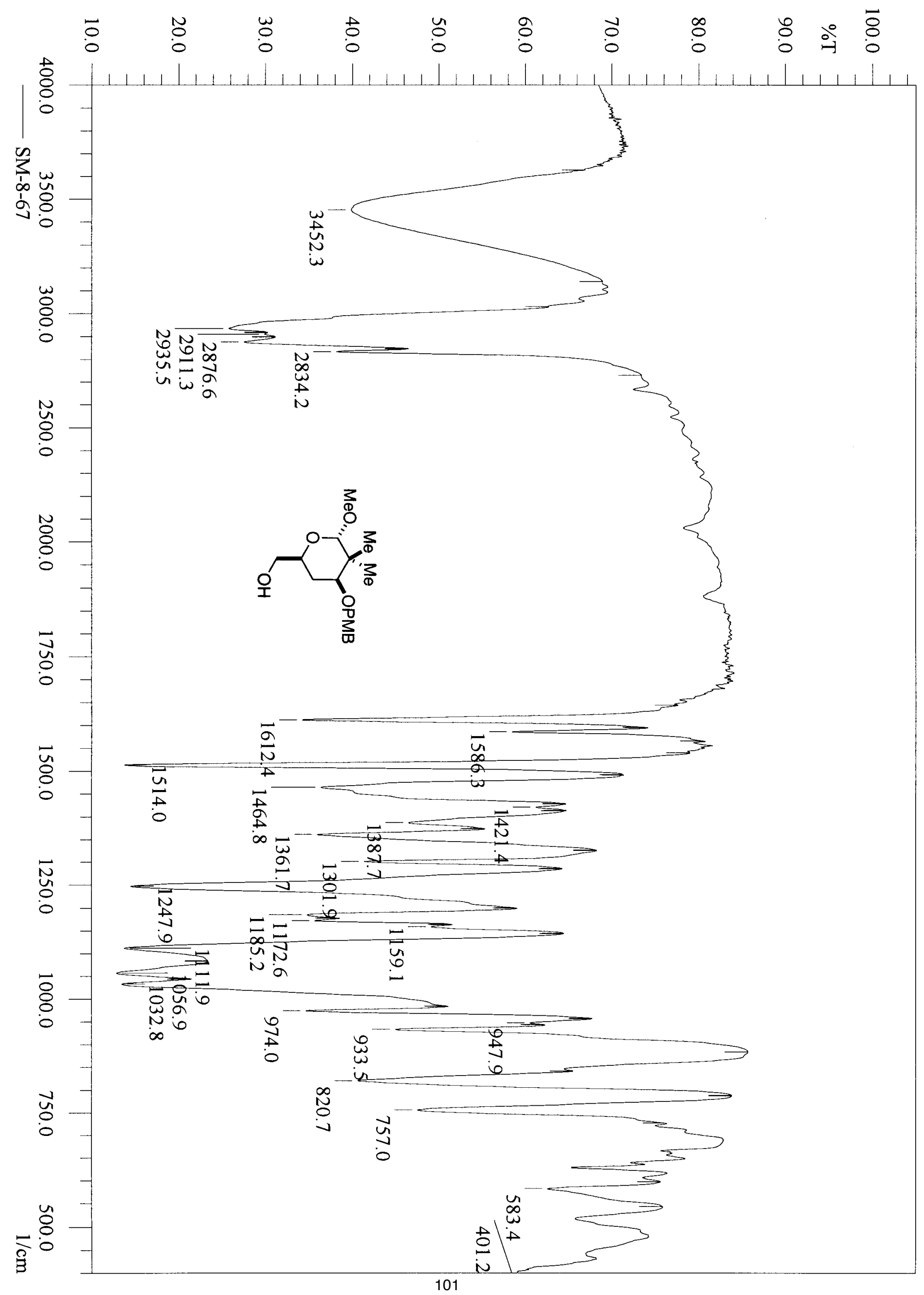




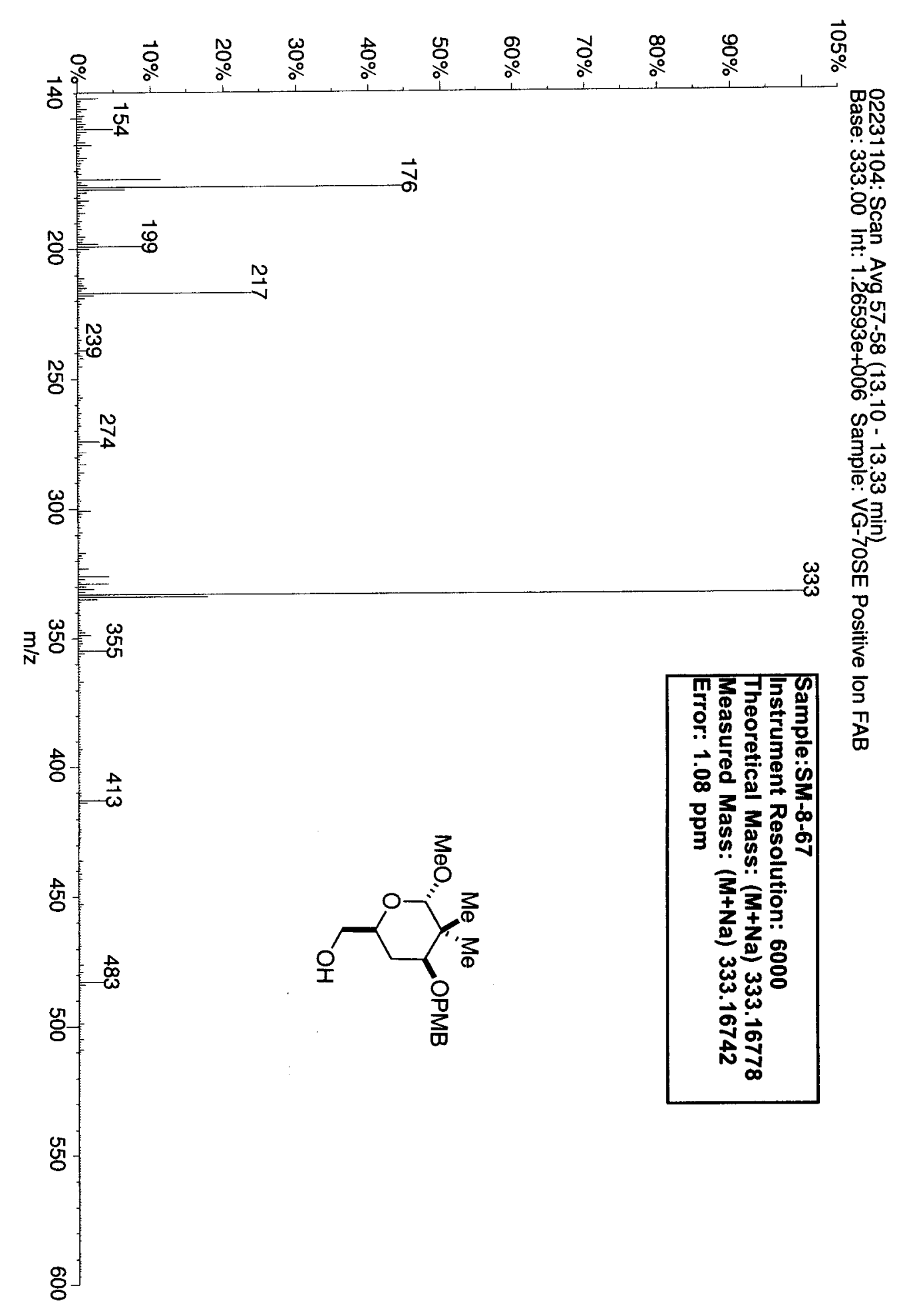




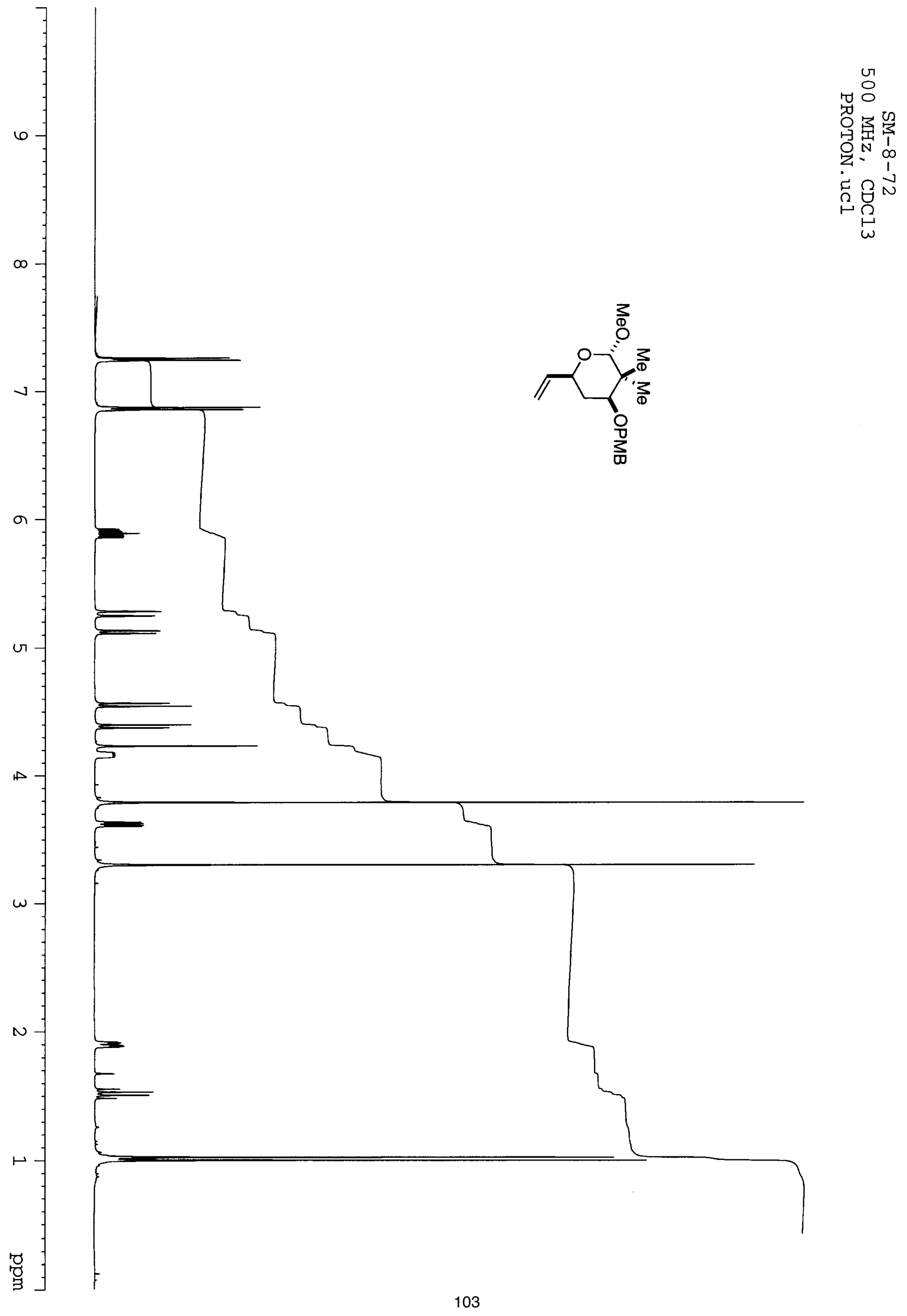



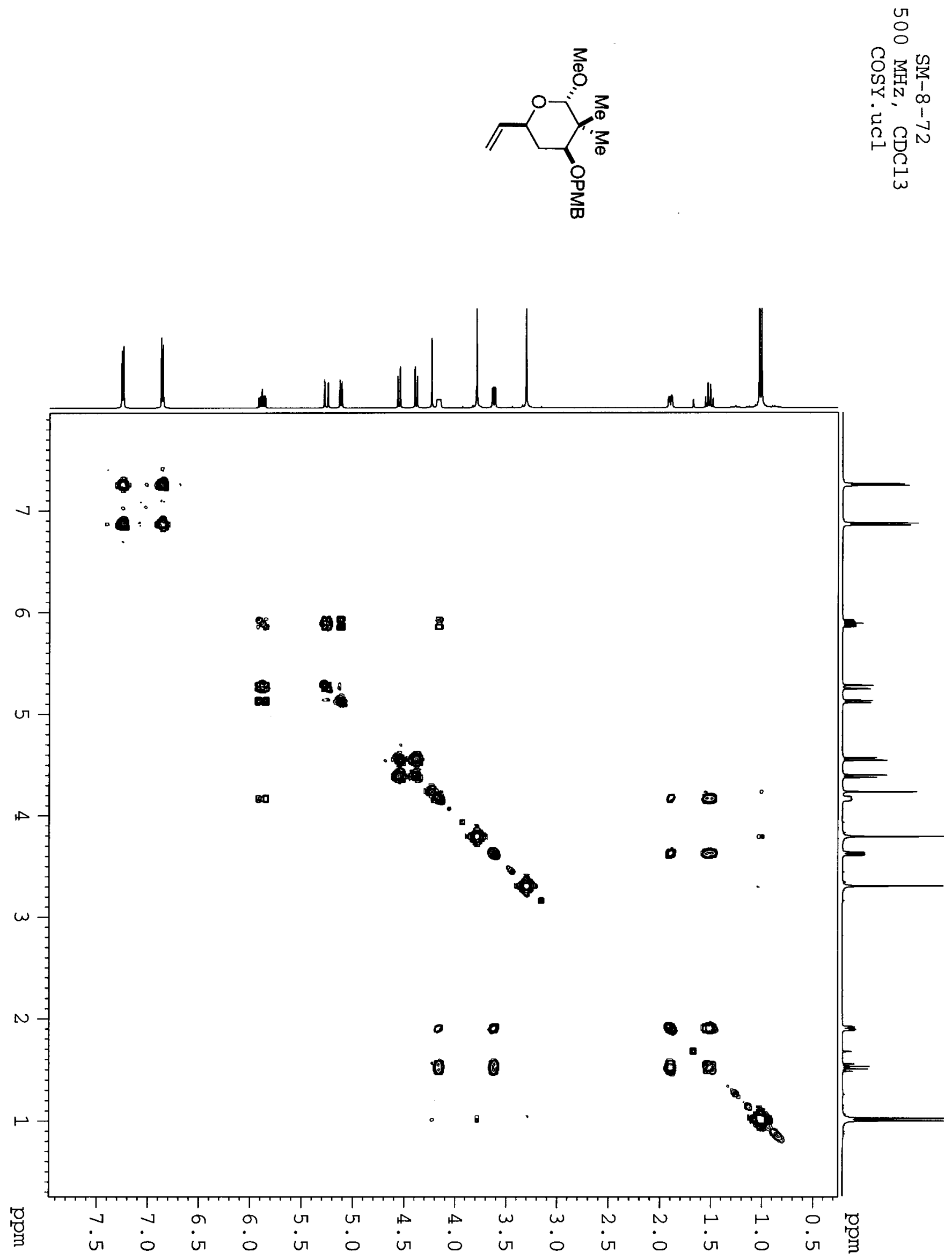

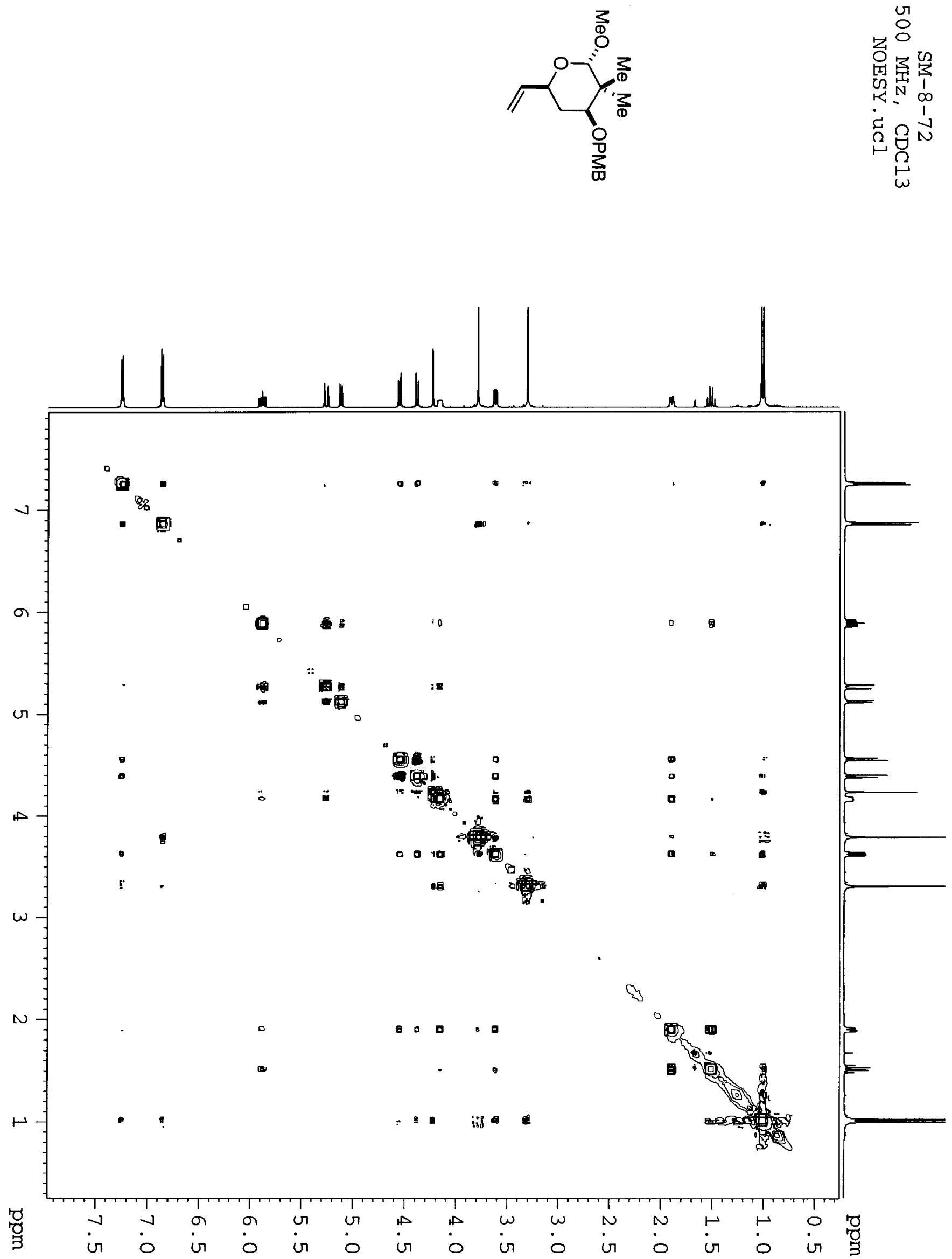

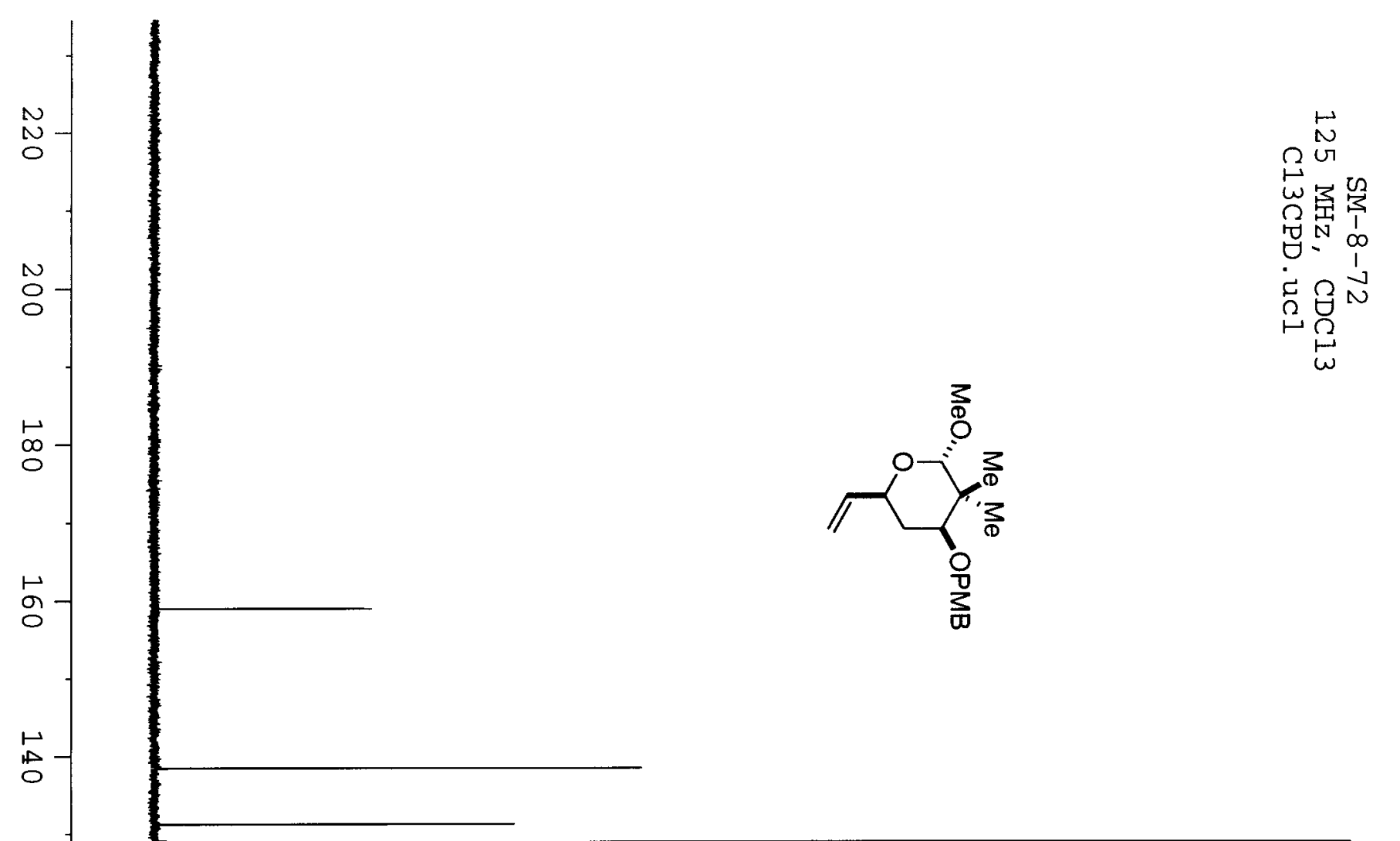

$\stackrel{\circ}{0}$
$\bullet$
0

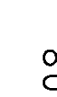

$\stackrel{\infty}{\circ}$

용

o

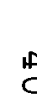

$\stackrel{\circ}{0}$

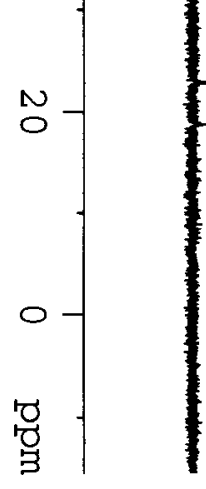




$$
\text { ll" }
$$




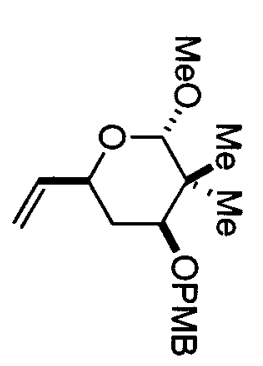

ํํㄴ

争

点思

- $\infty$

ق ज

口N

i)

点

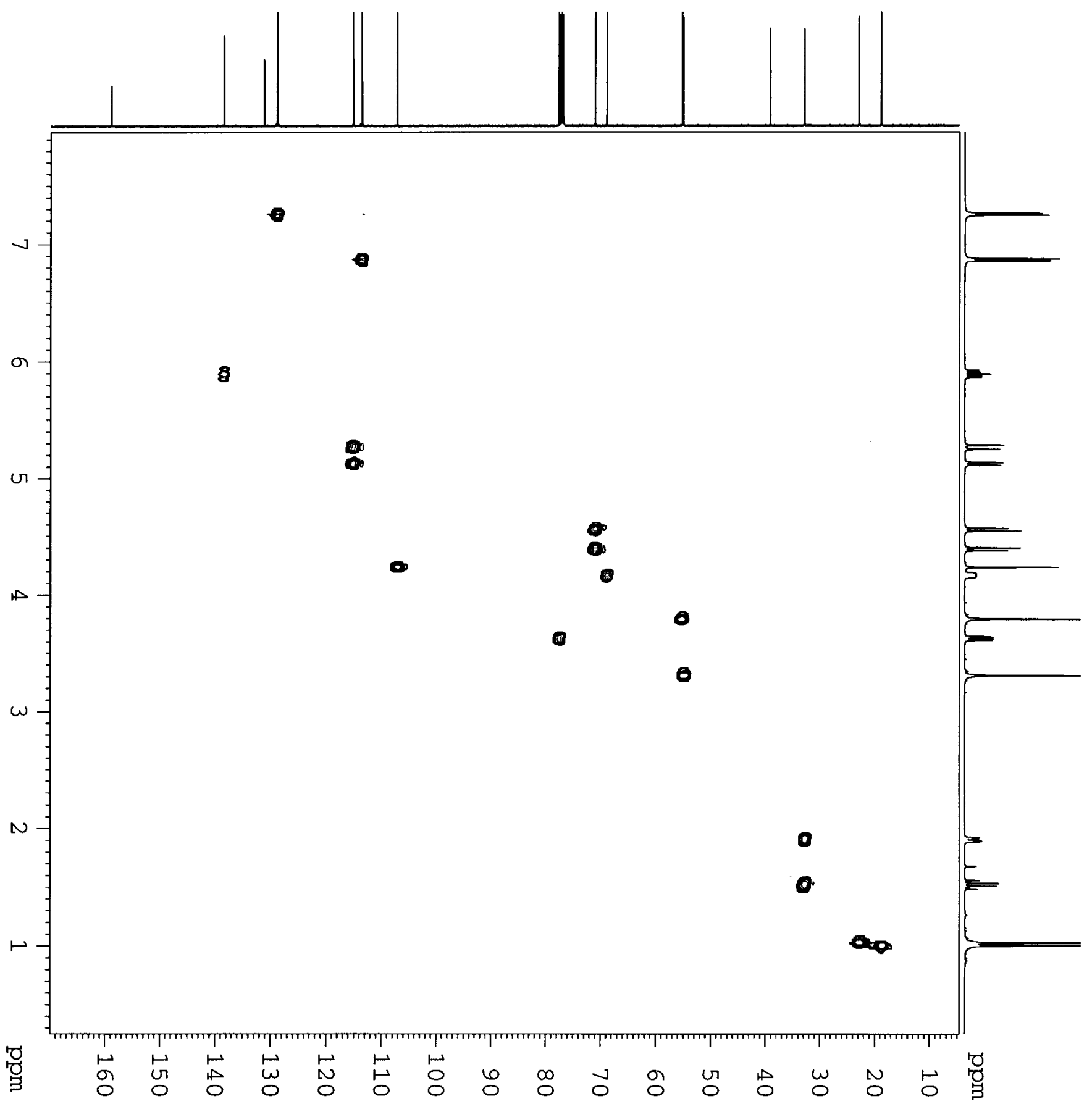




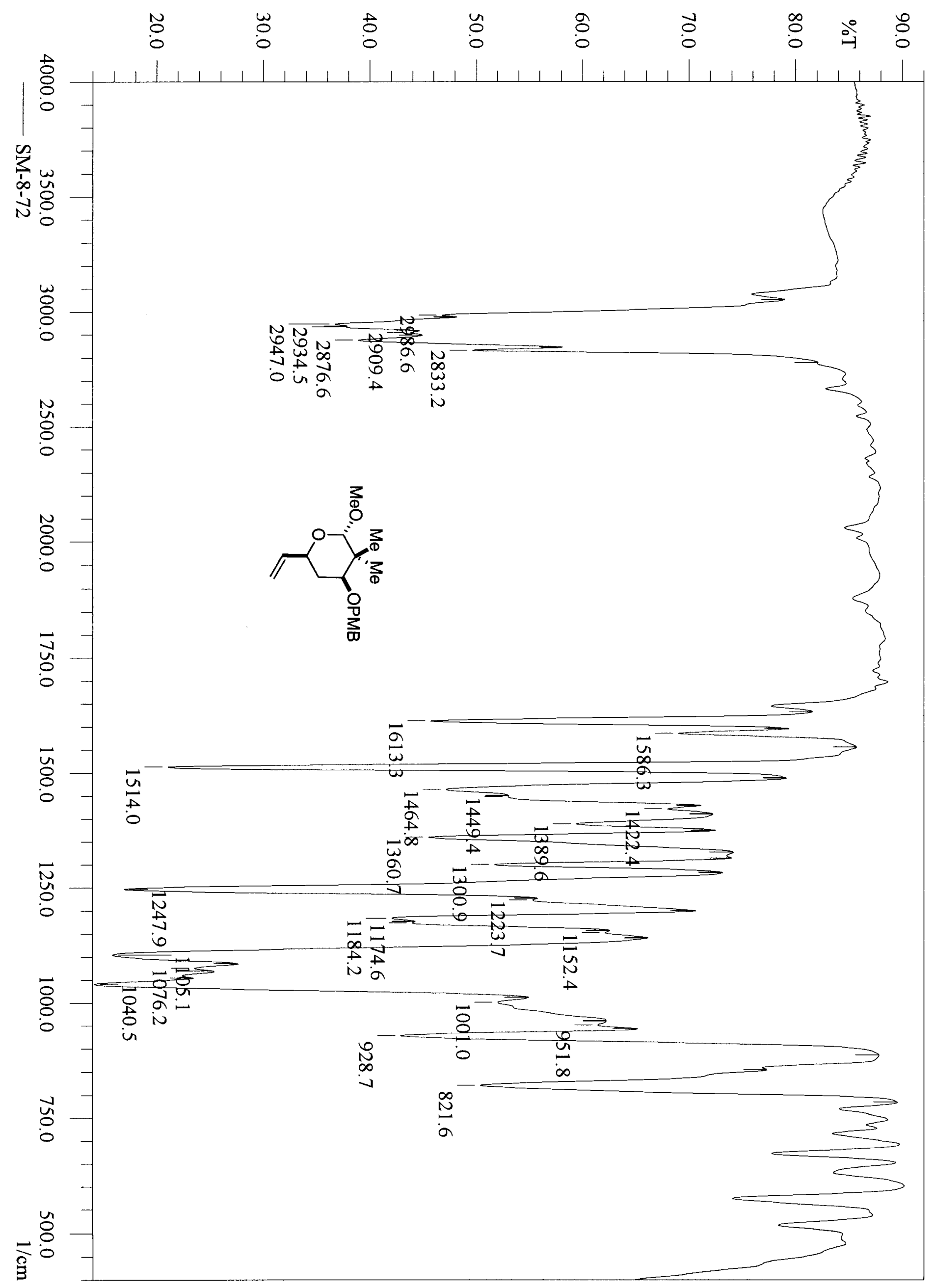




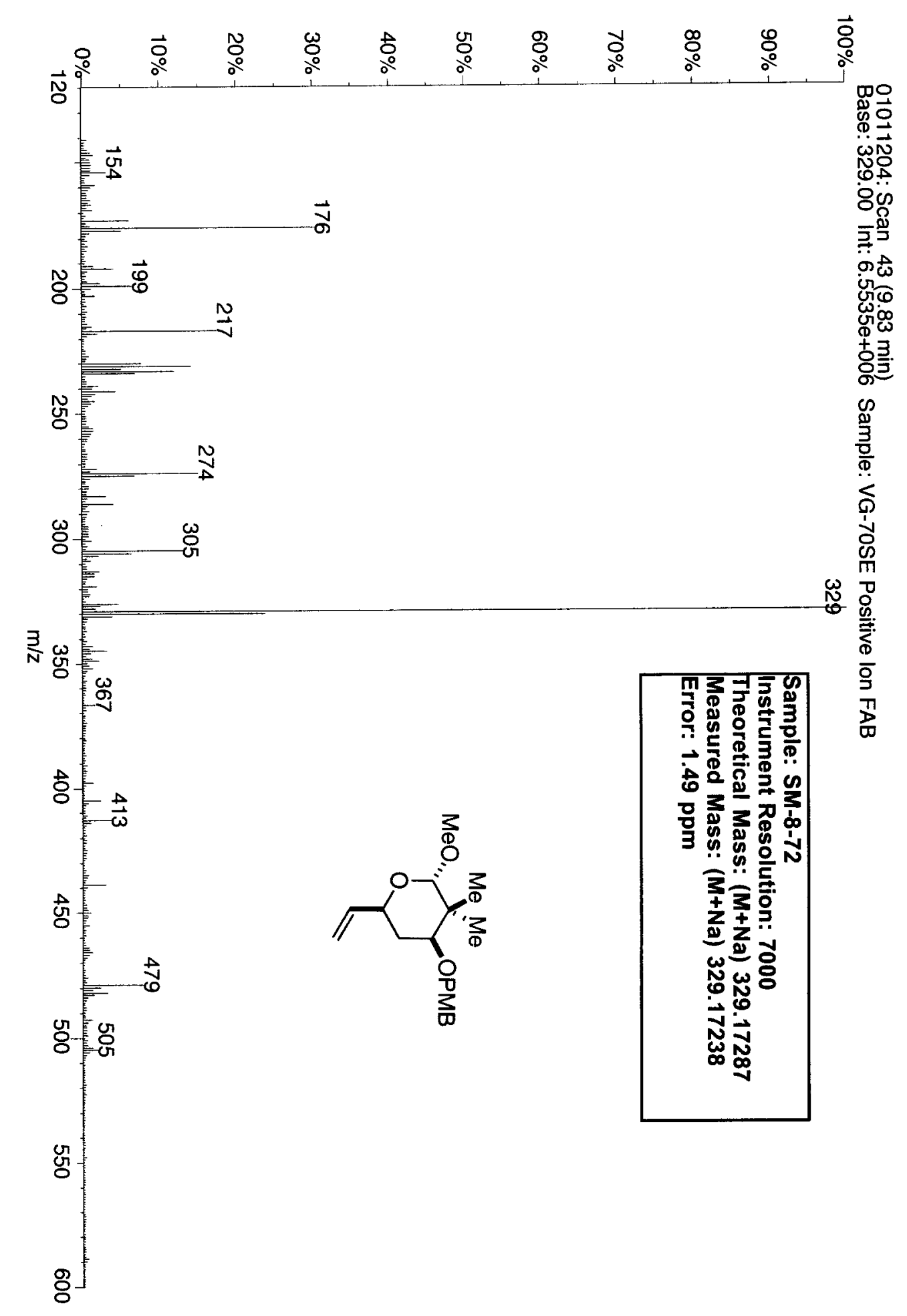



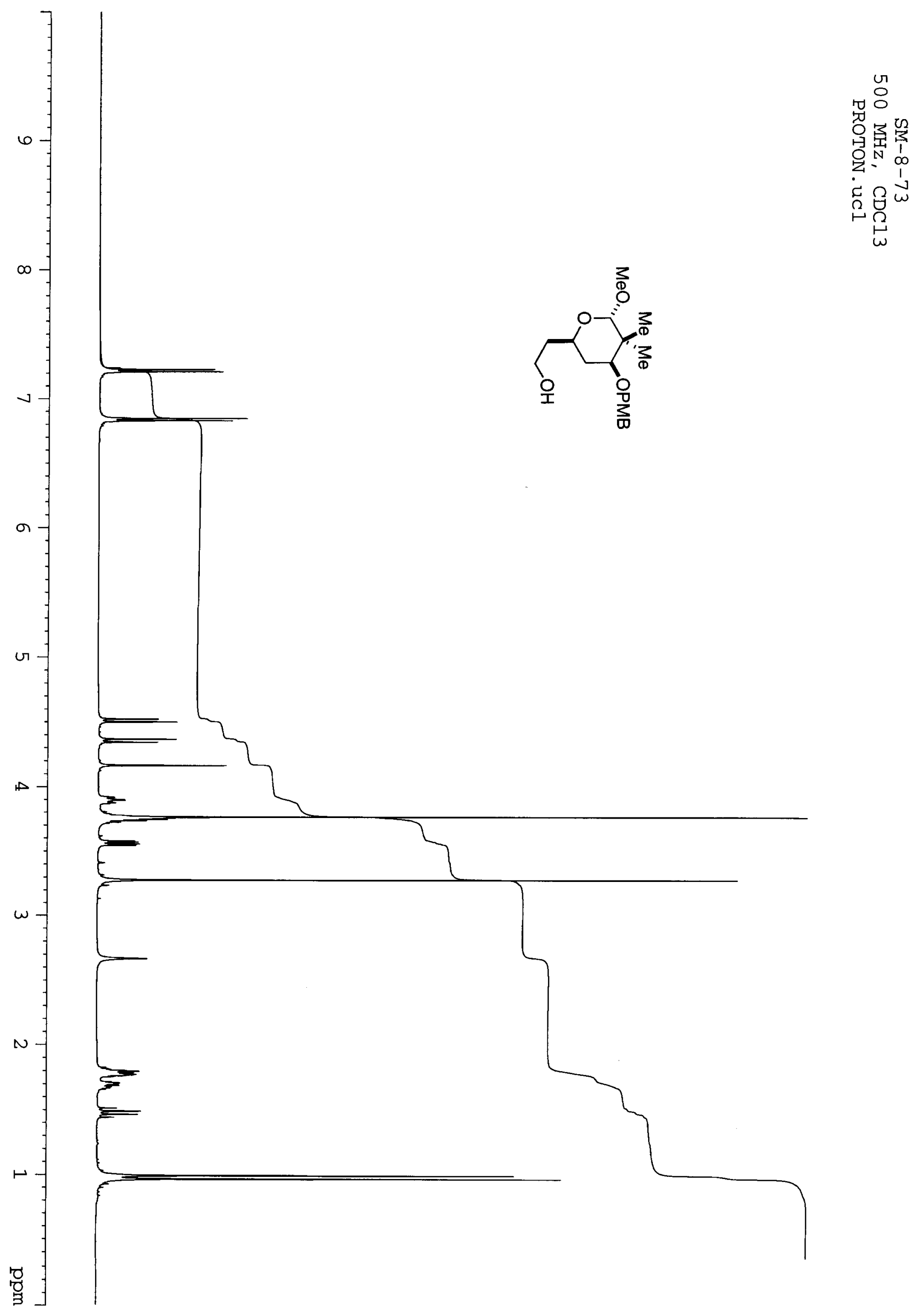

111 

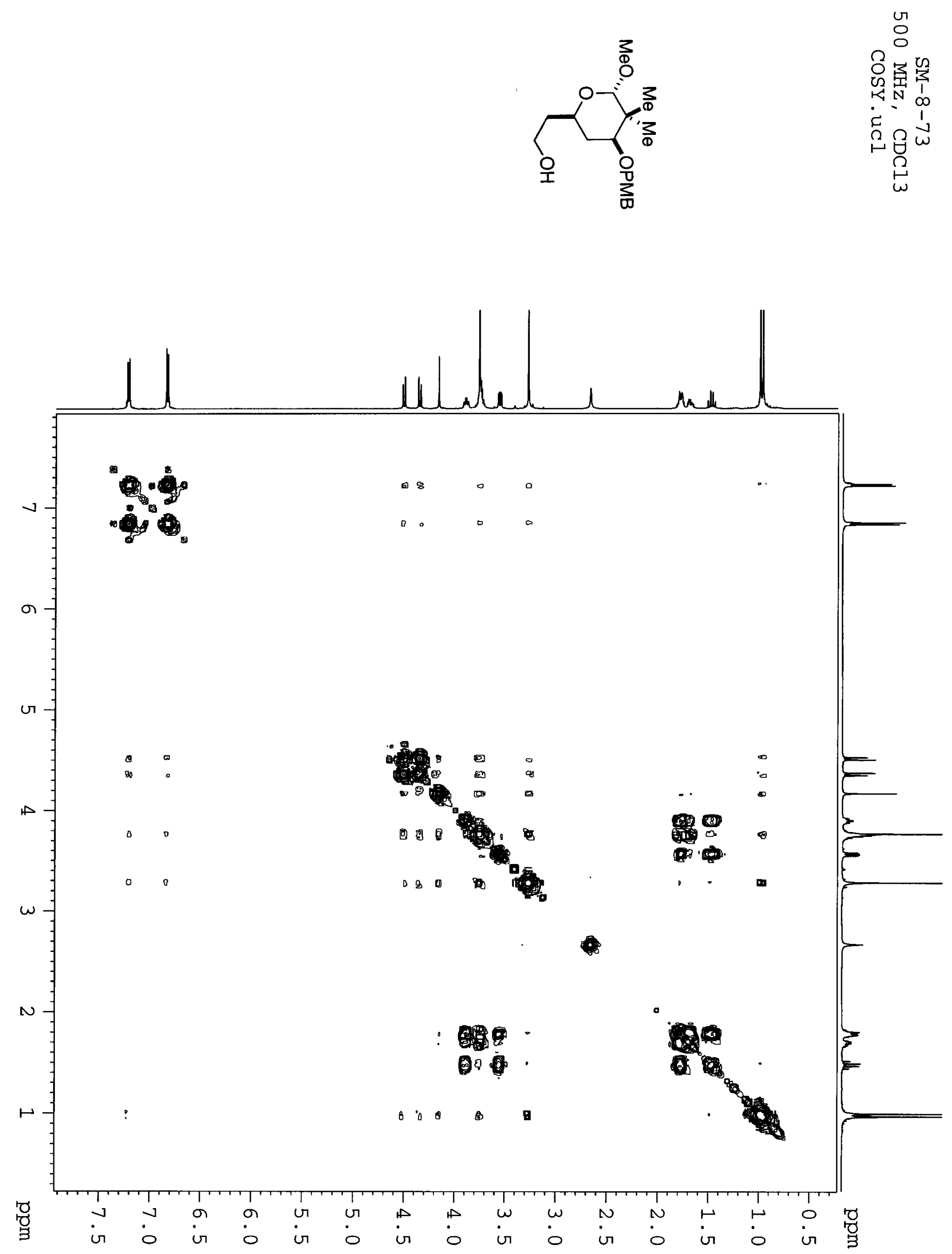

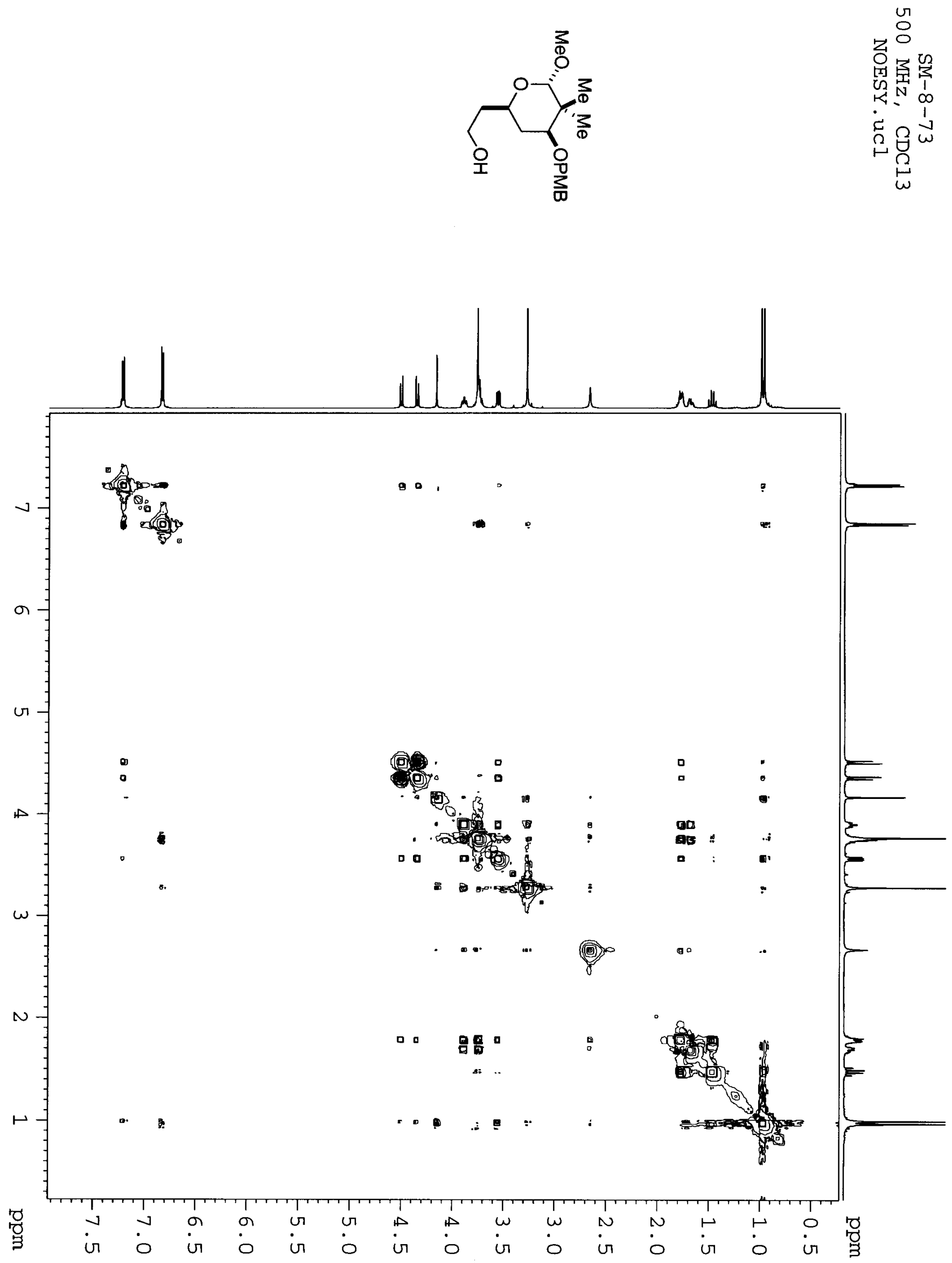


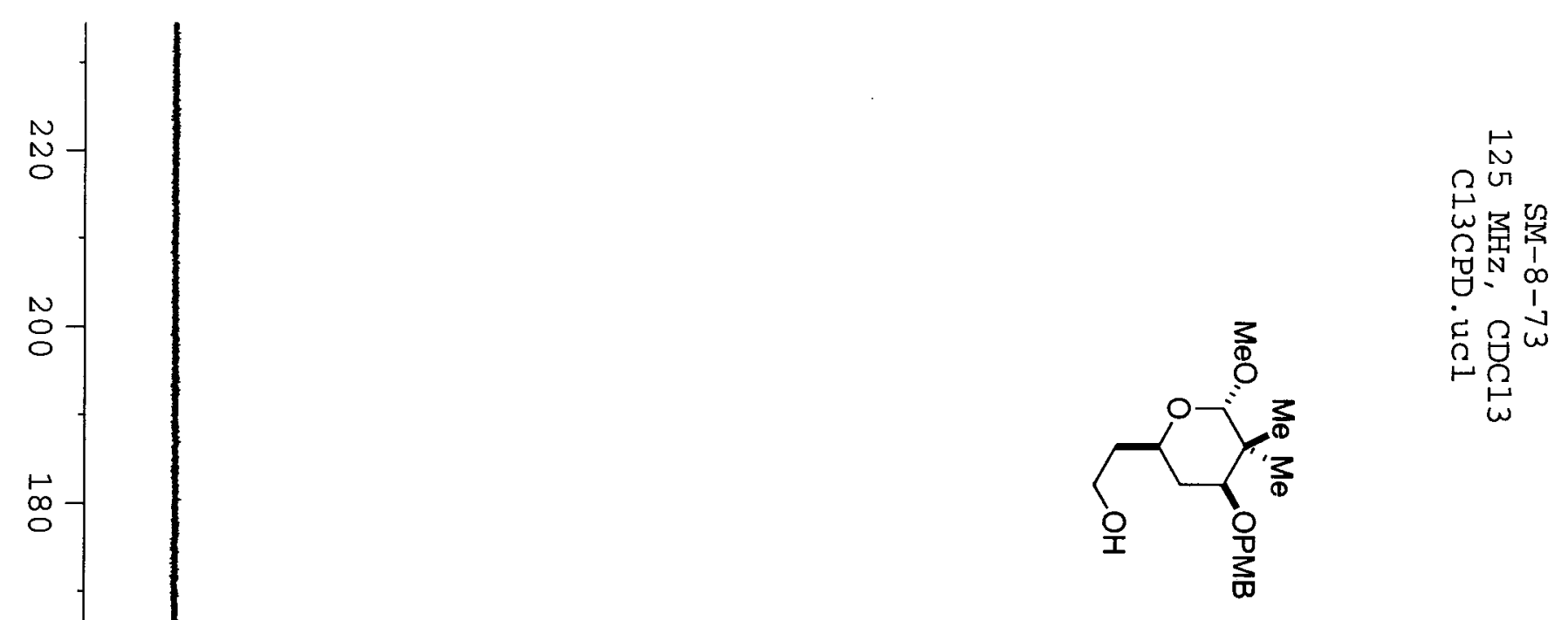

홍

$\stackrel{\bullet}{\mathbb{D}}$

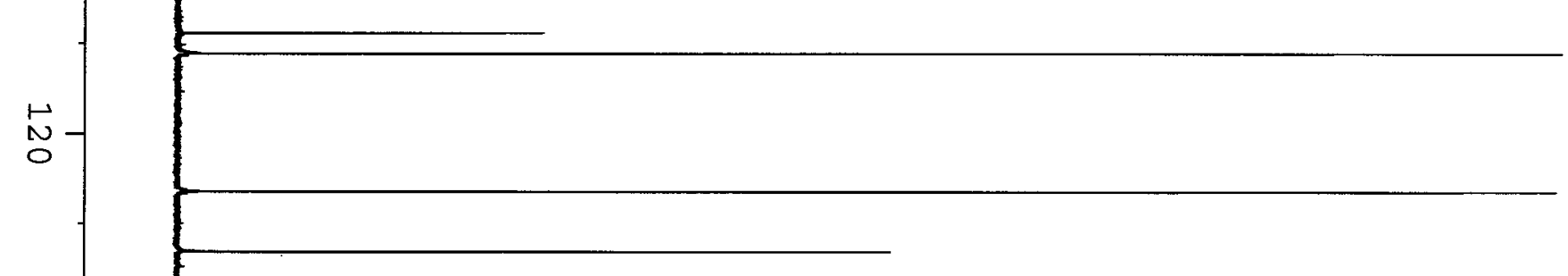

웅

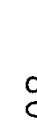

$\infty$

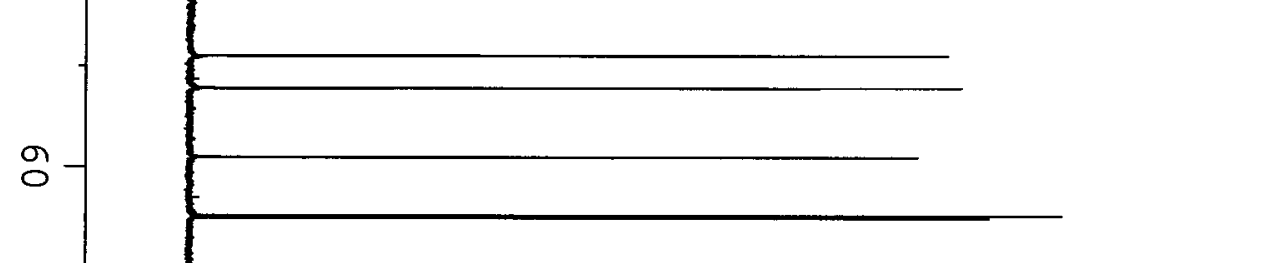

뭉

品

N

0
$0-1$
0
9
9 


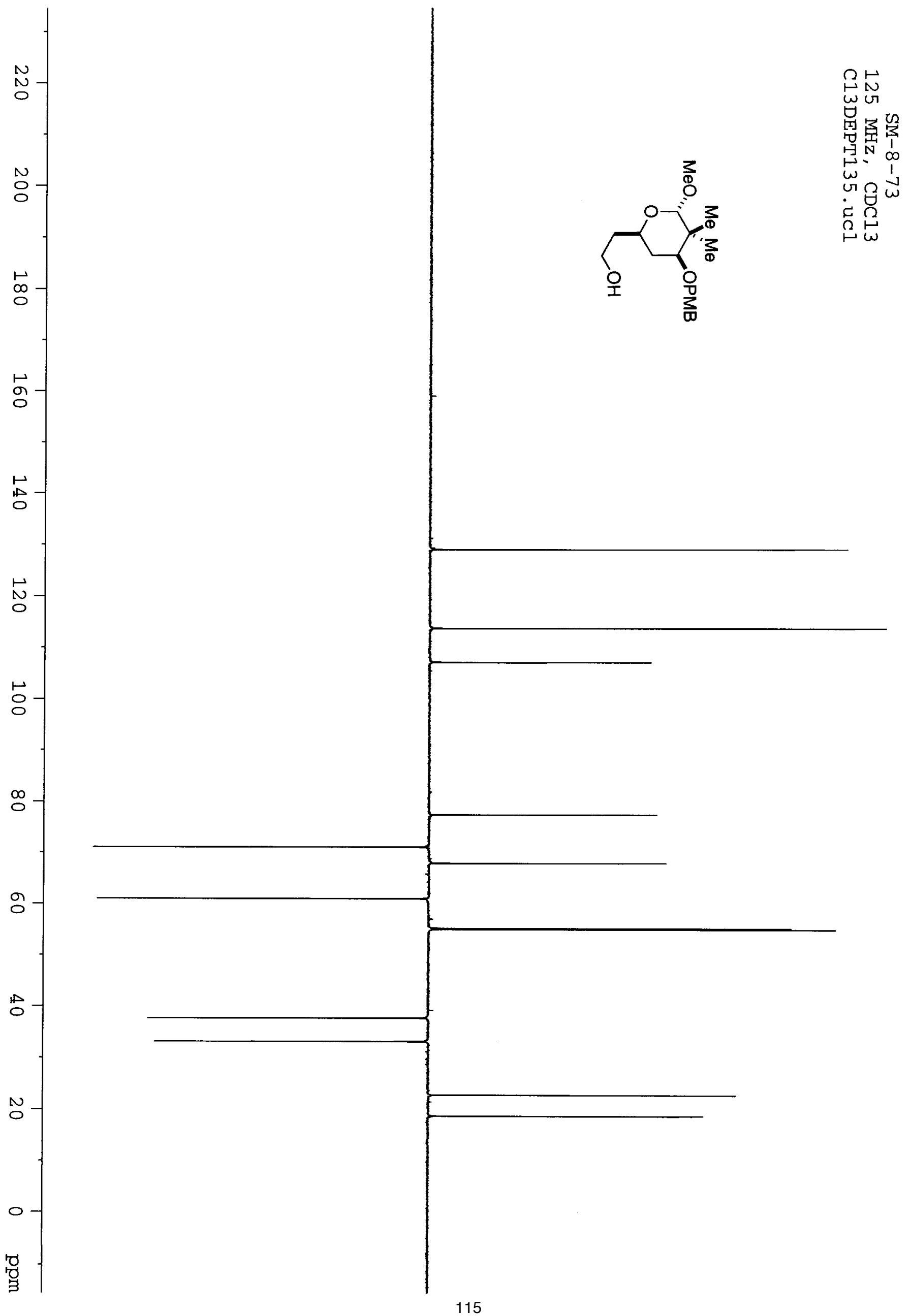




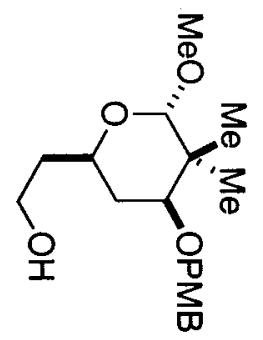

○ิ

写

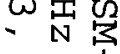

$5-\infty$

ติ ज

○

\%

责

采

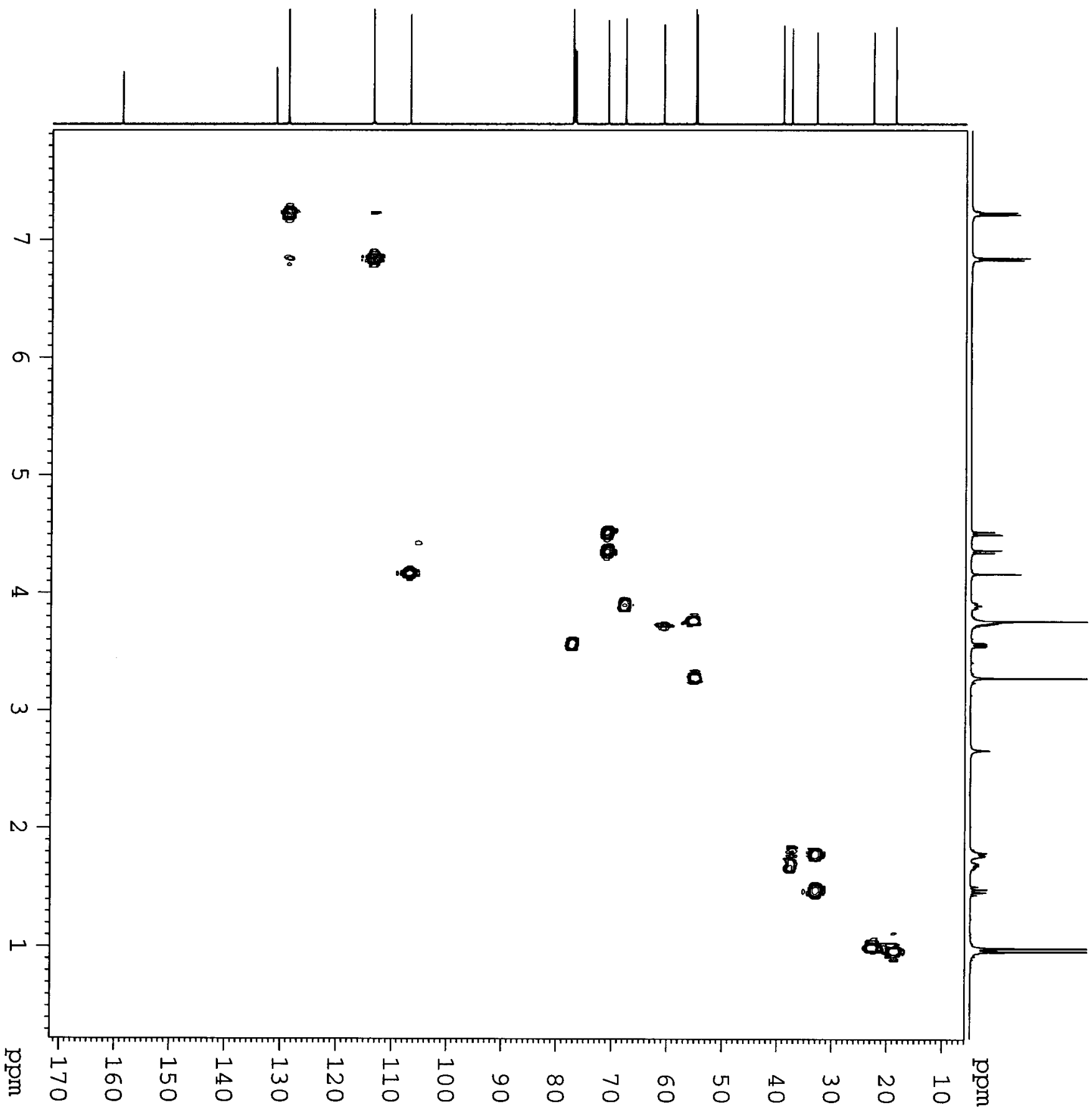




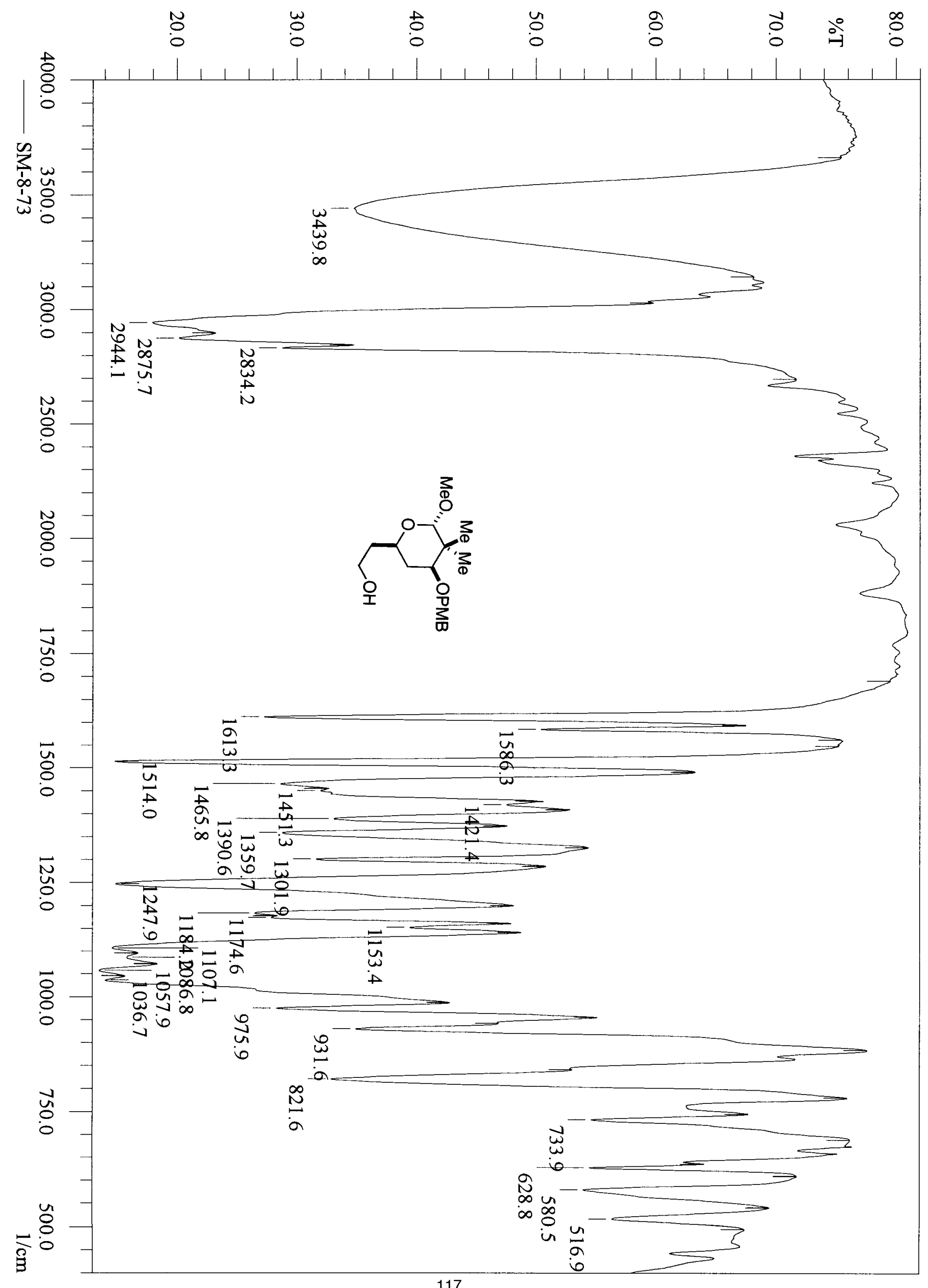




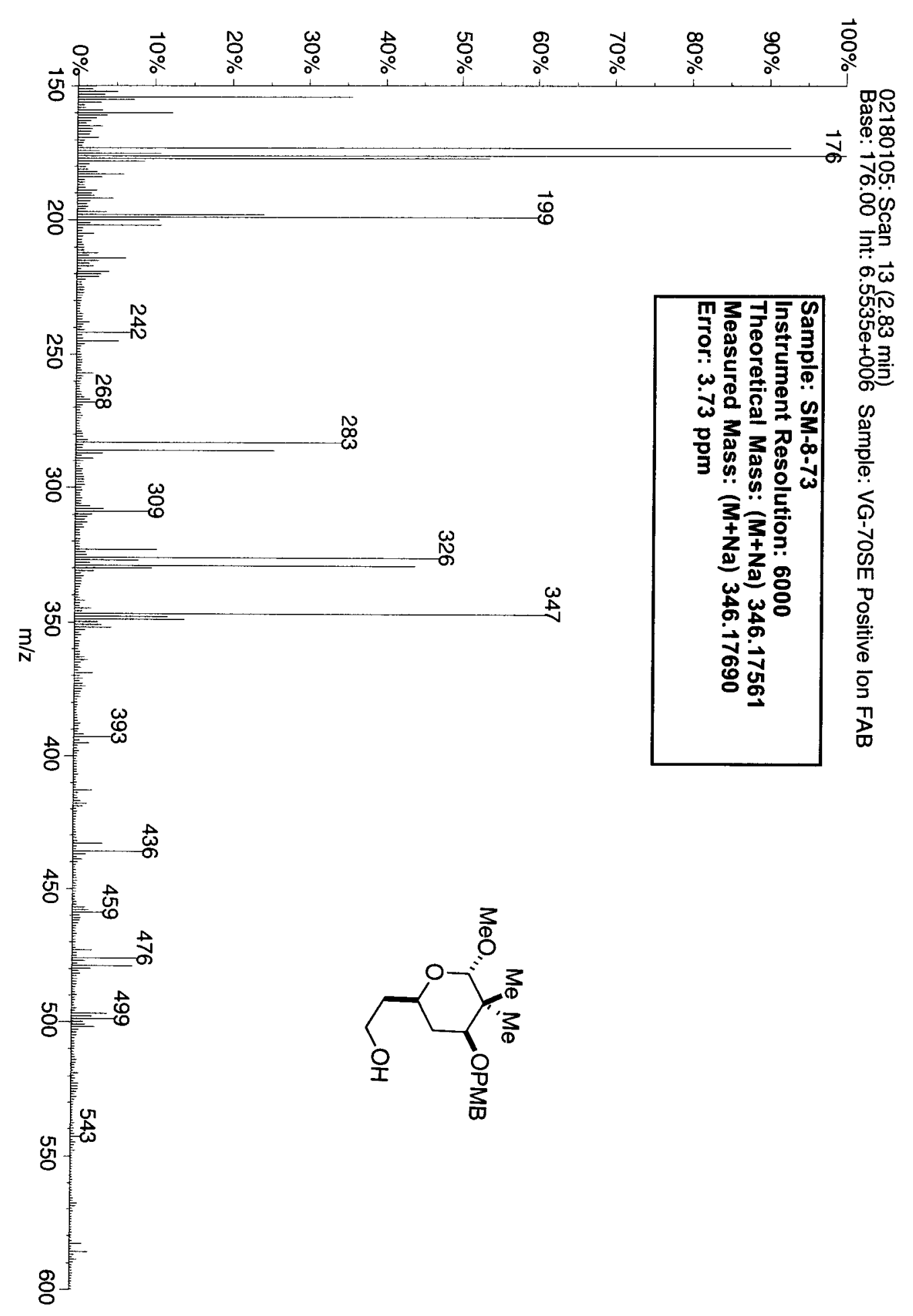




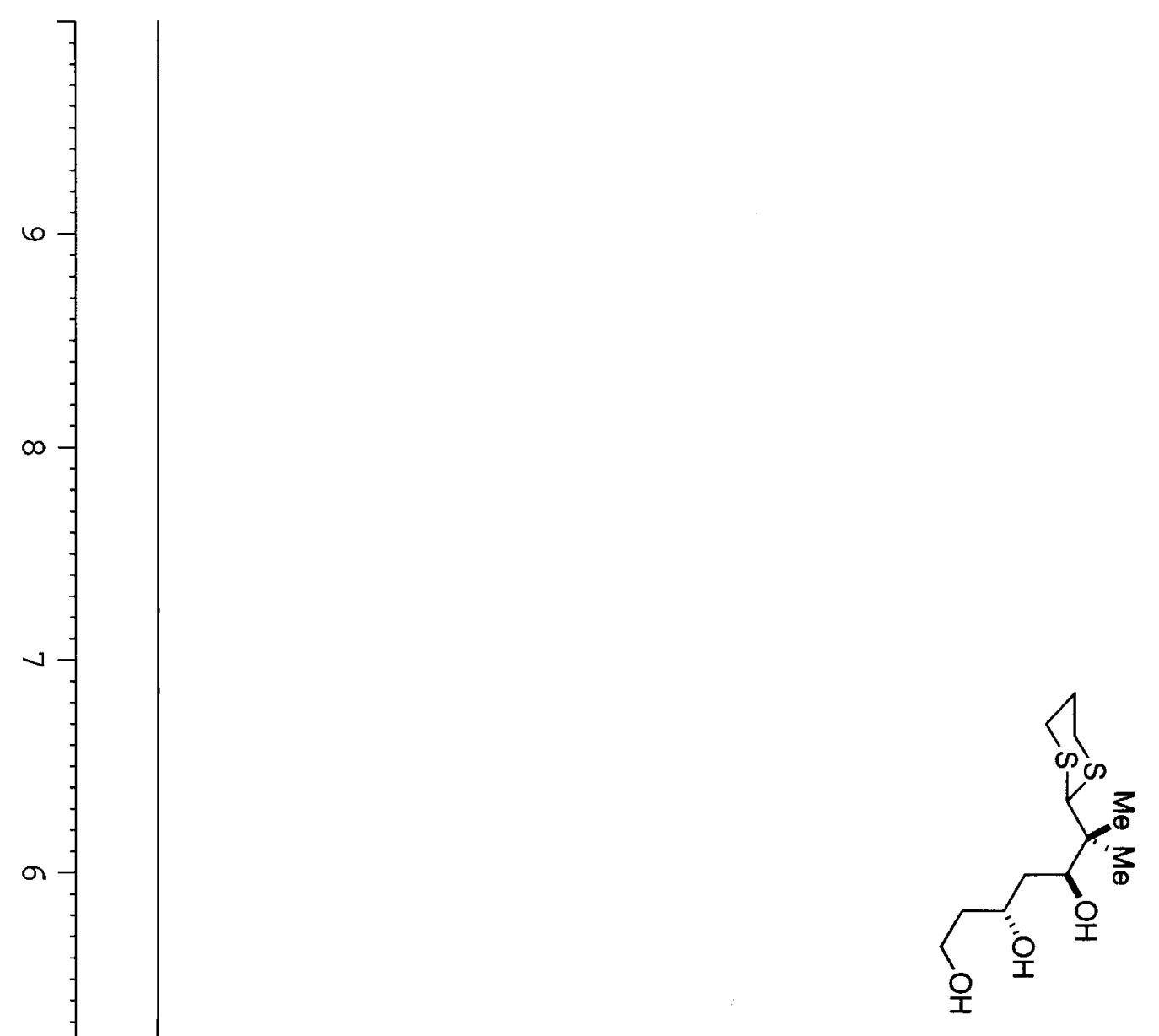

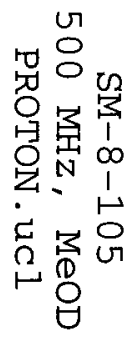

G

$\triangleright$

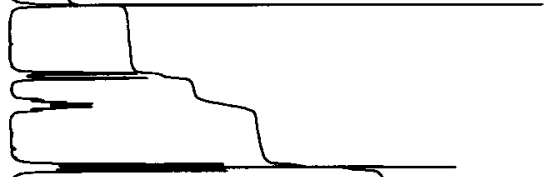

$\omega$

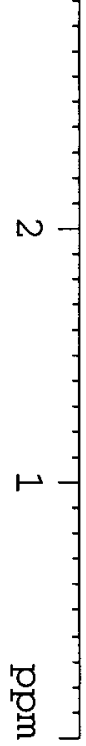



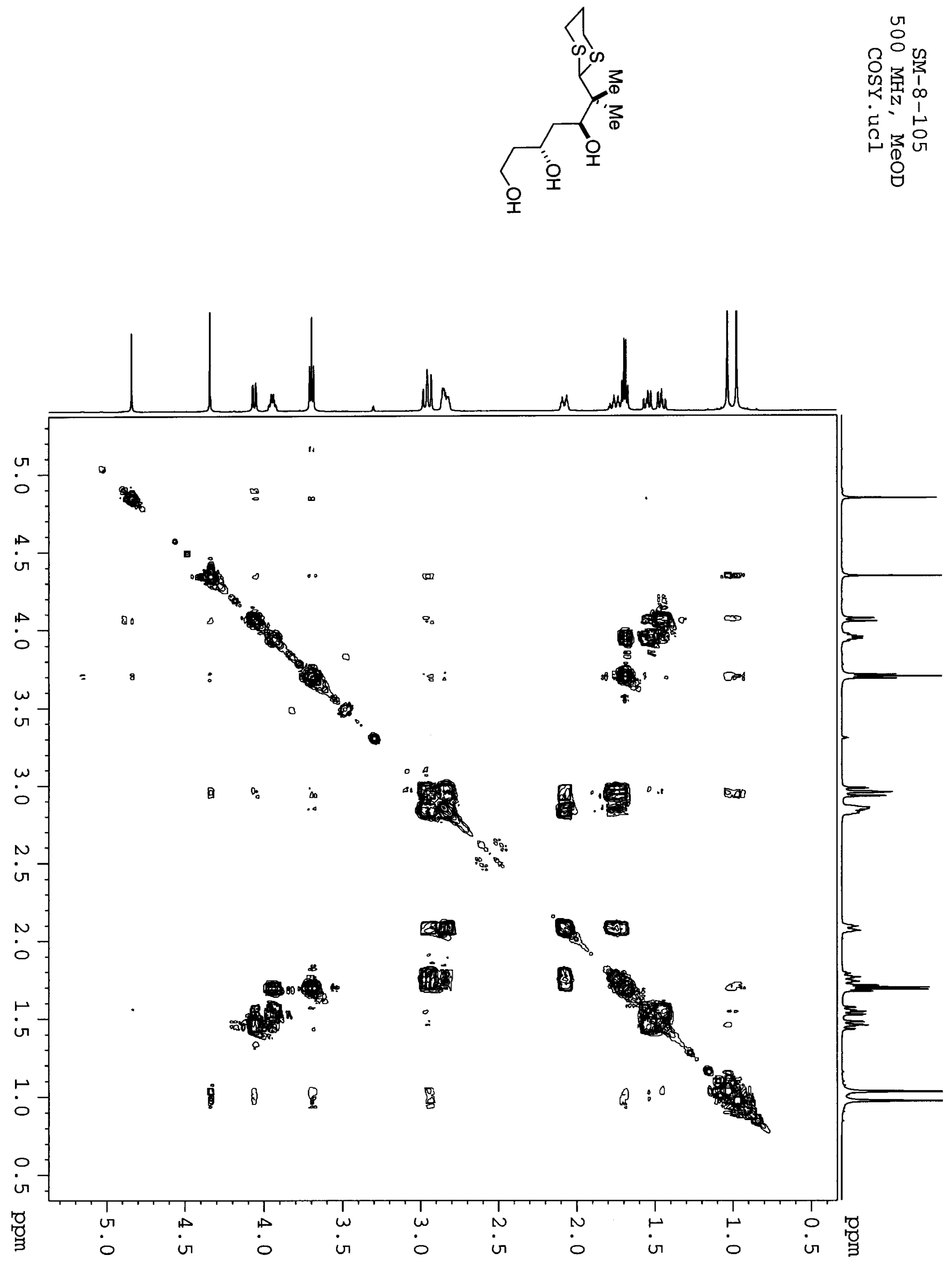


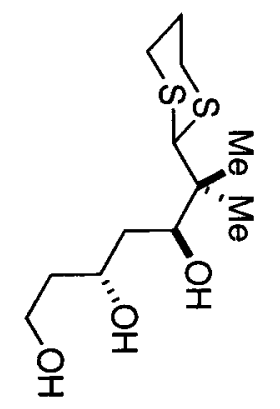

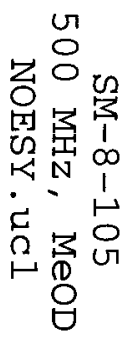

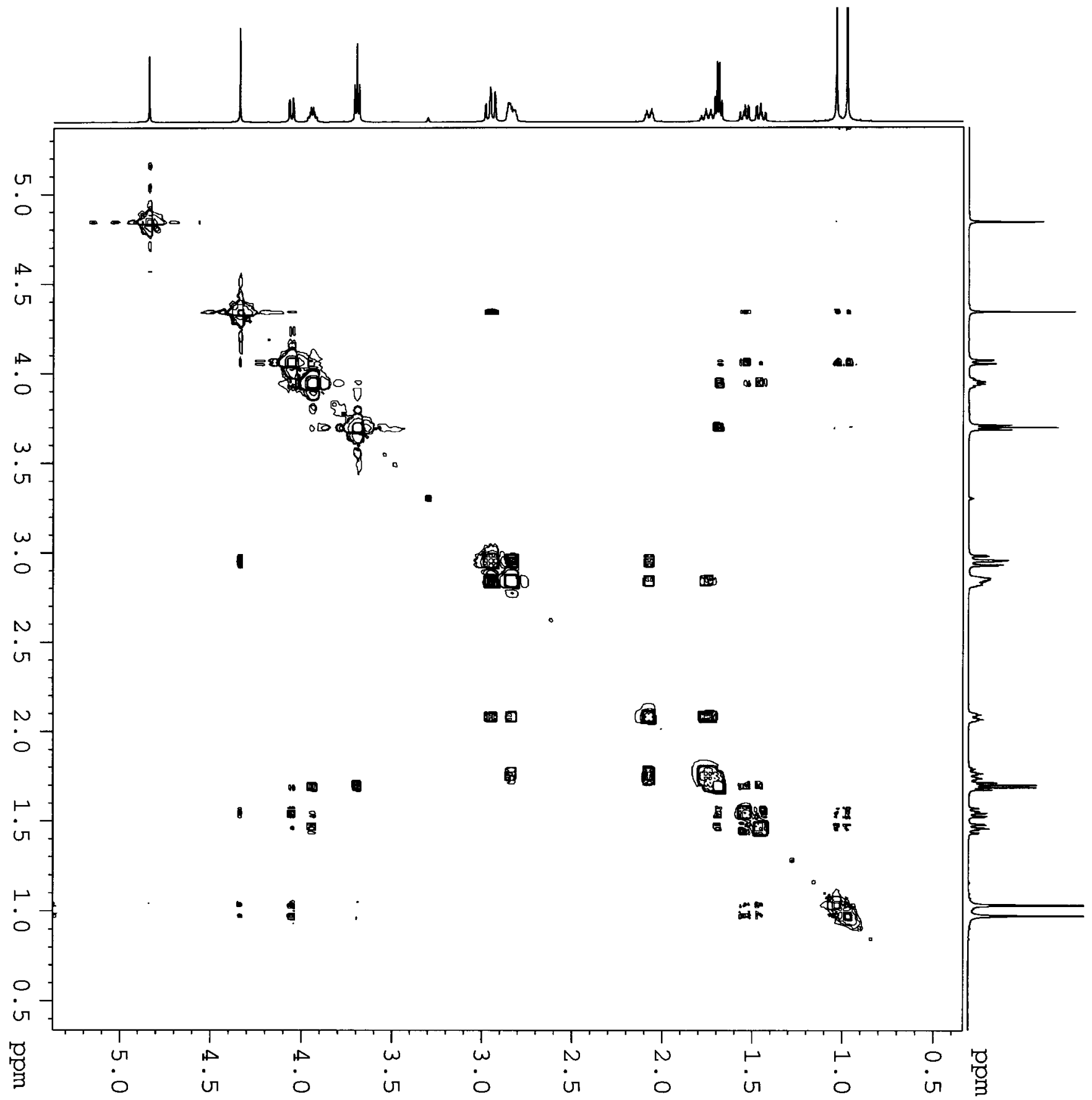



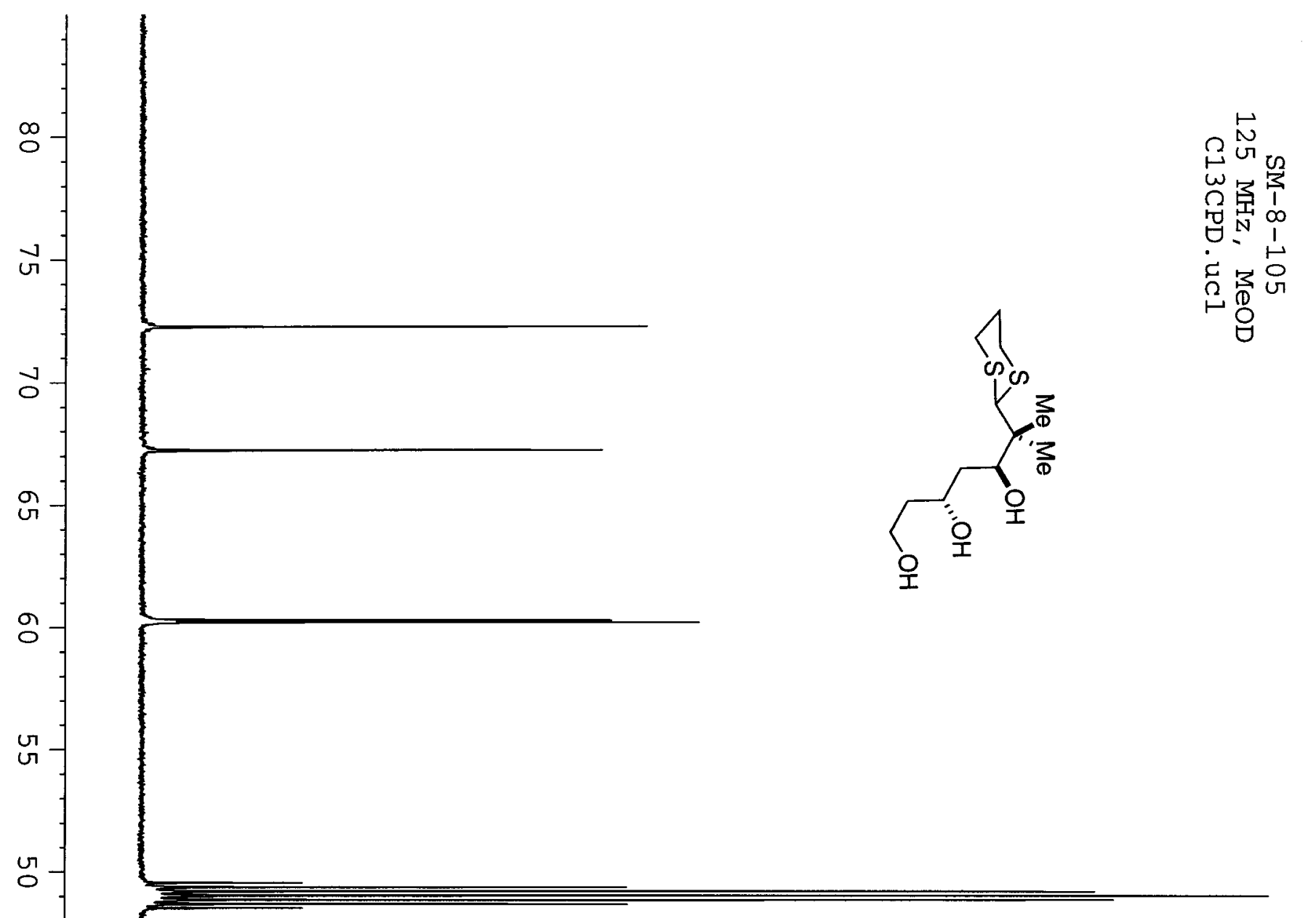

G

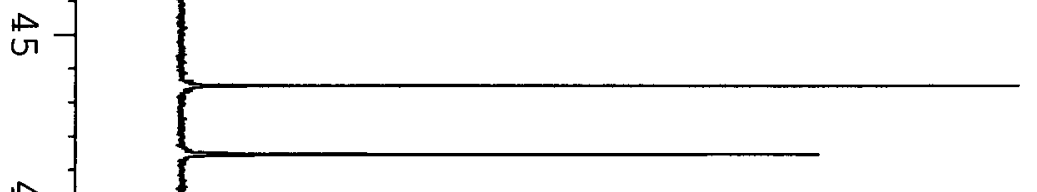

$\stackrel{10}{0}$

$\omega$

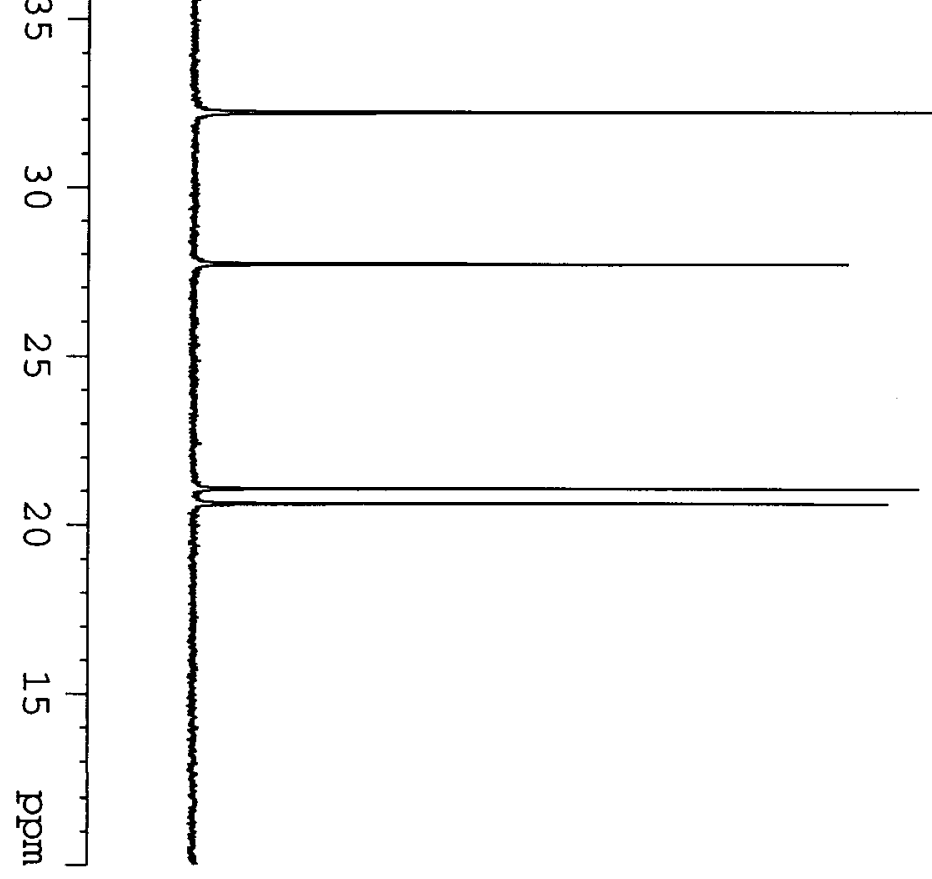




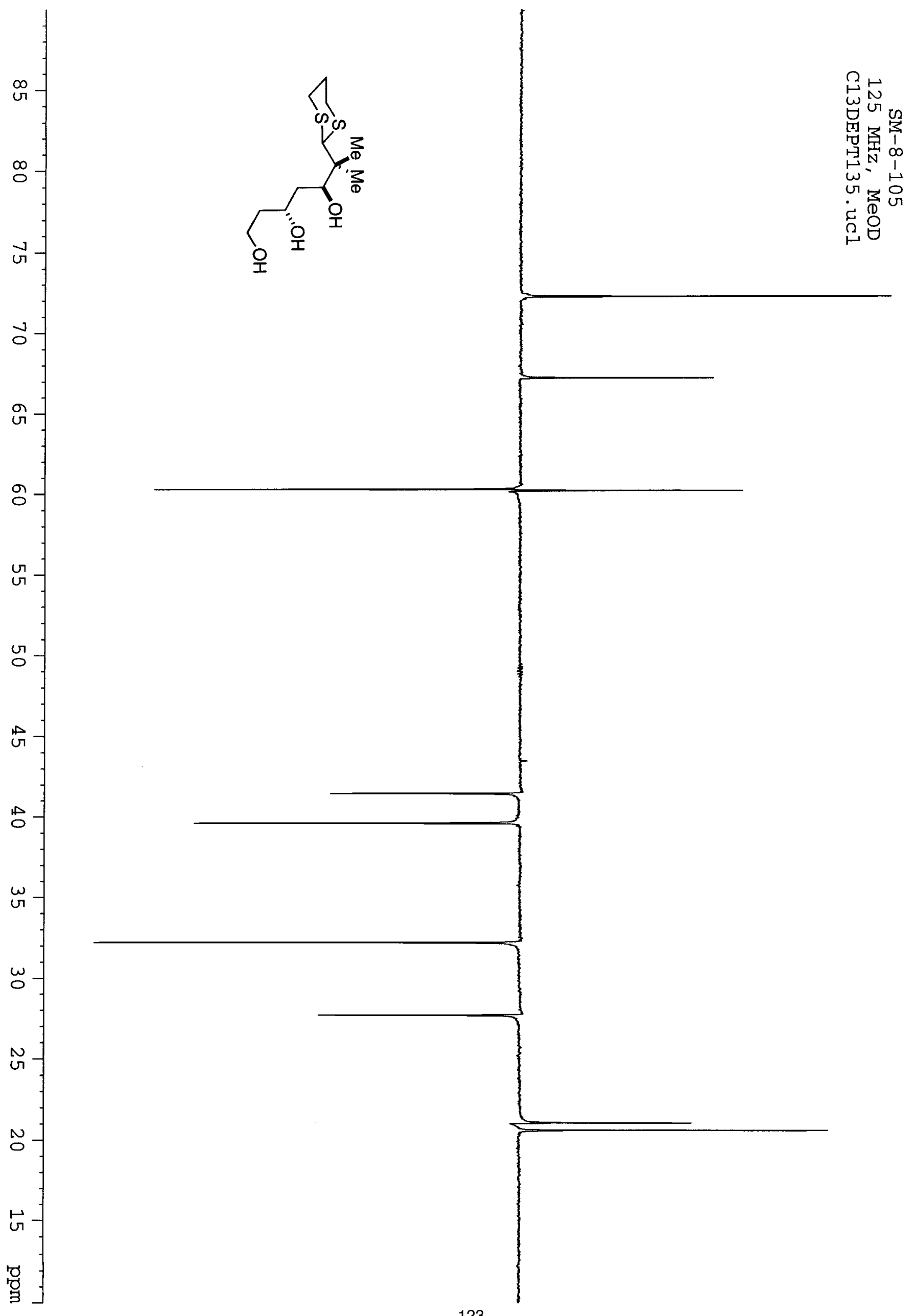



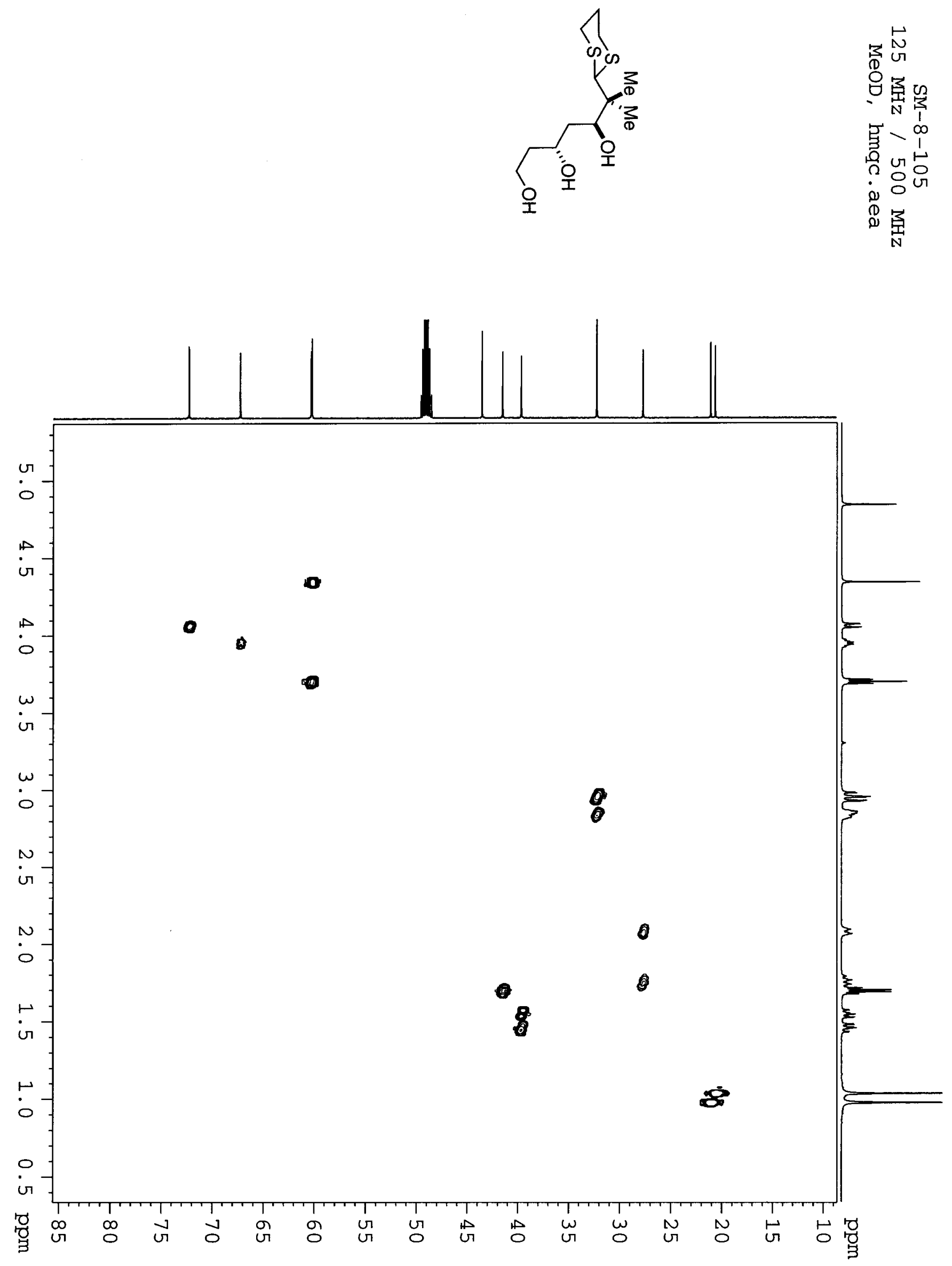

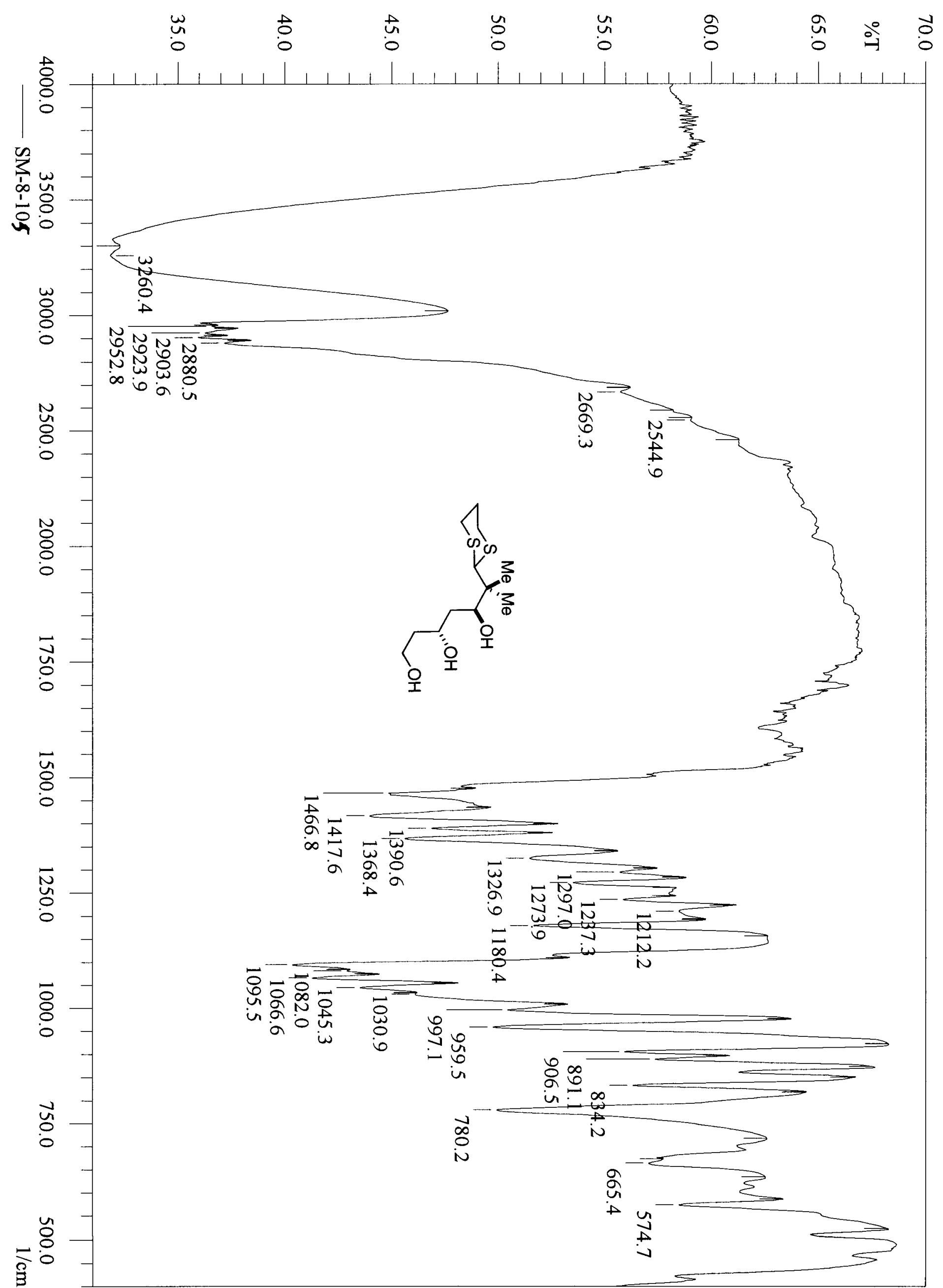


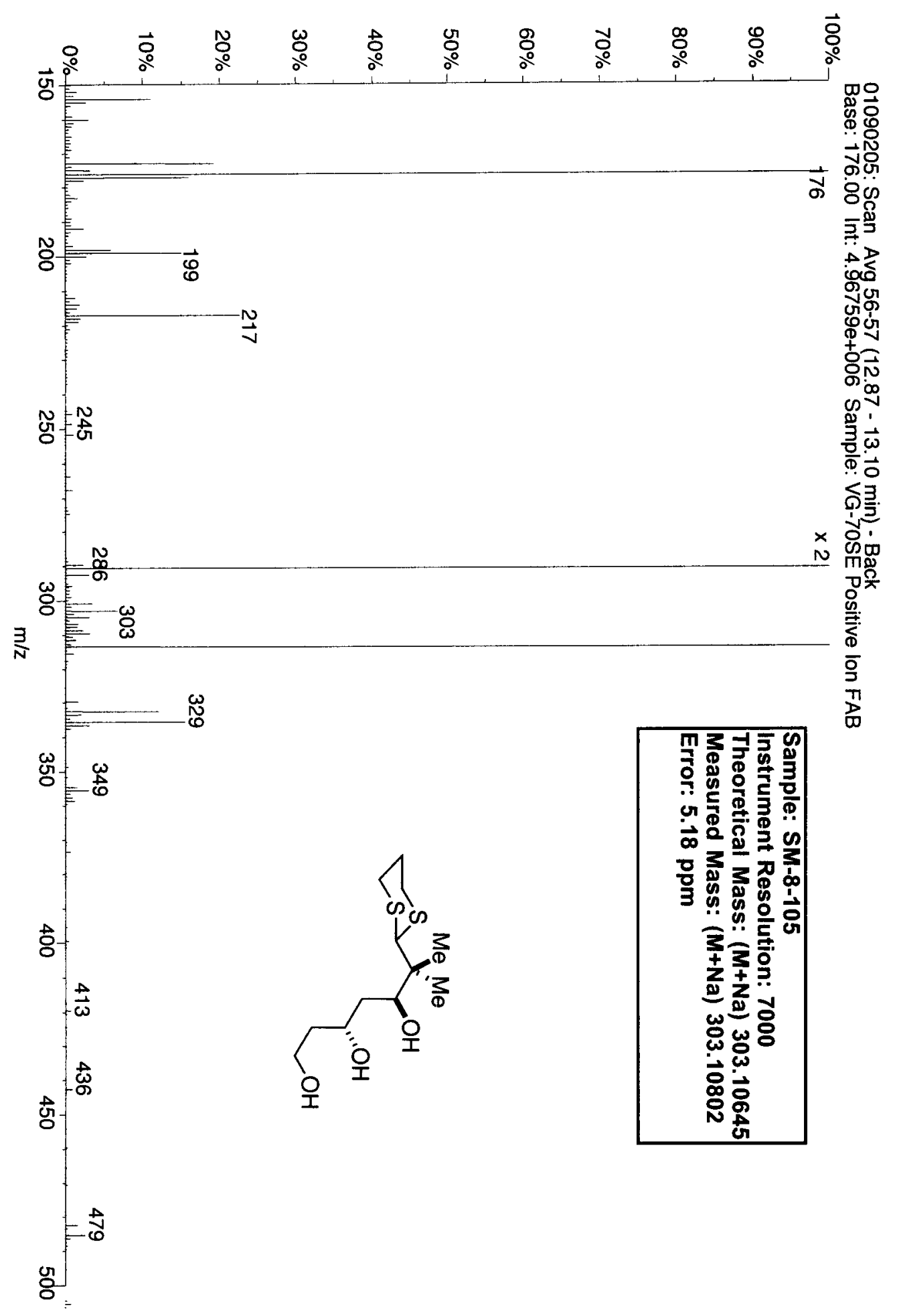




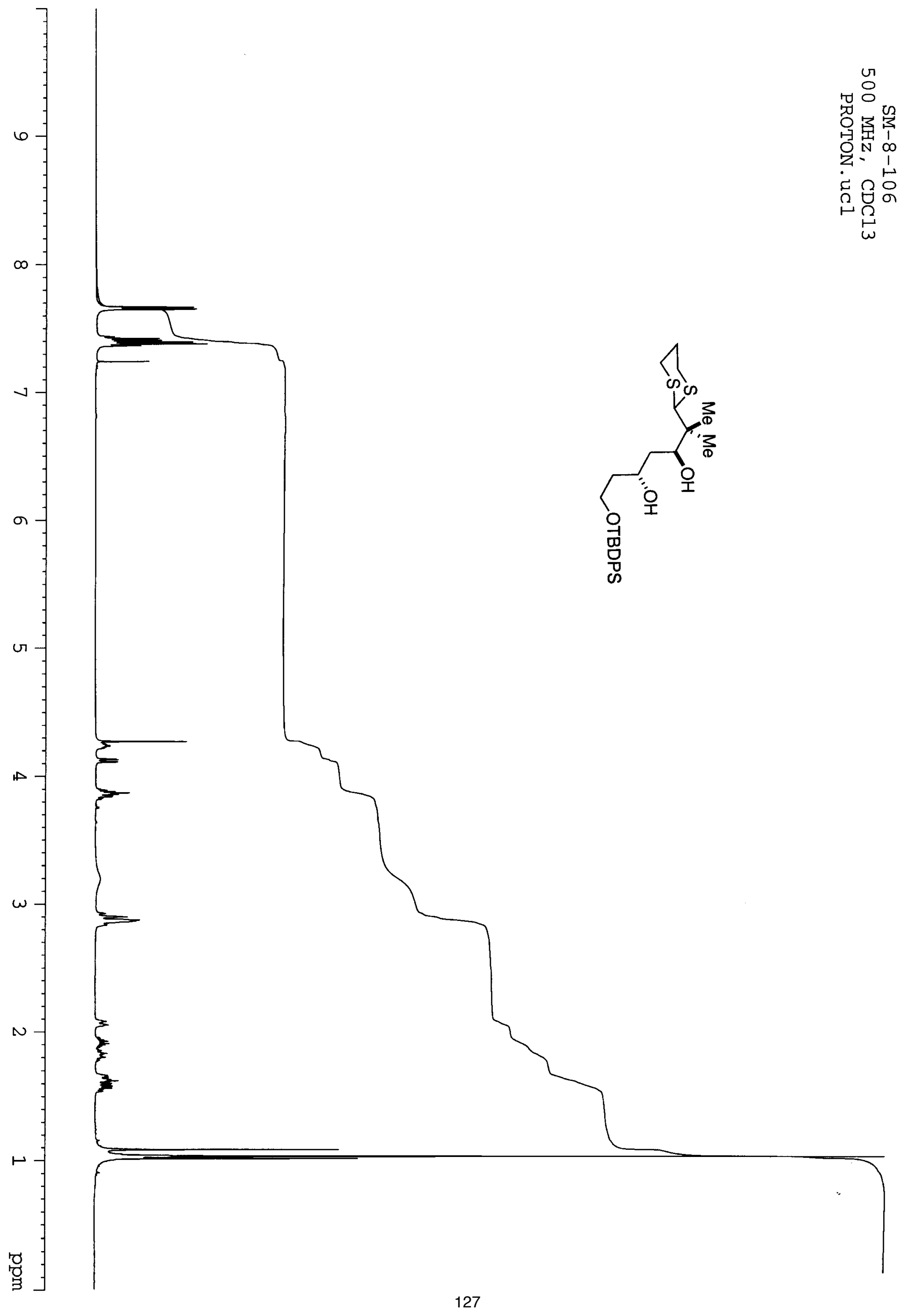



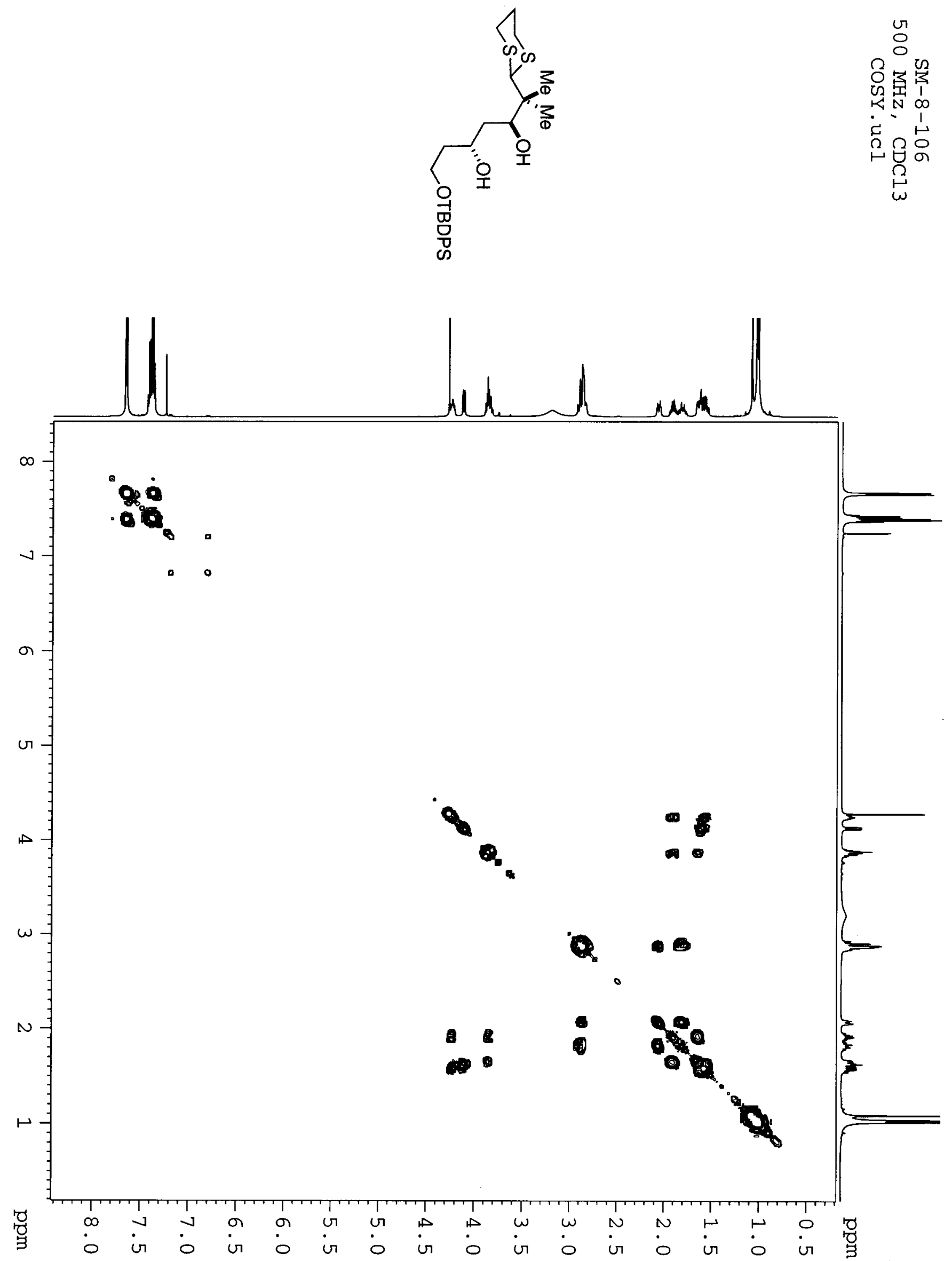

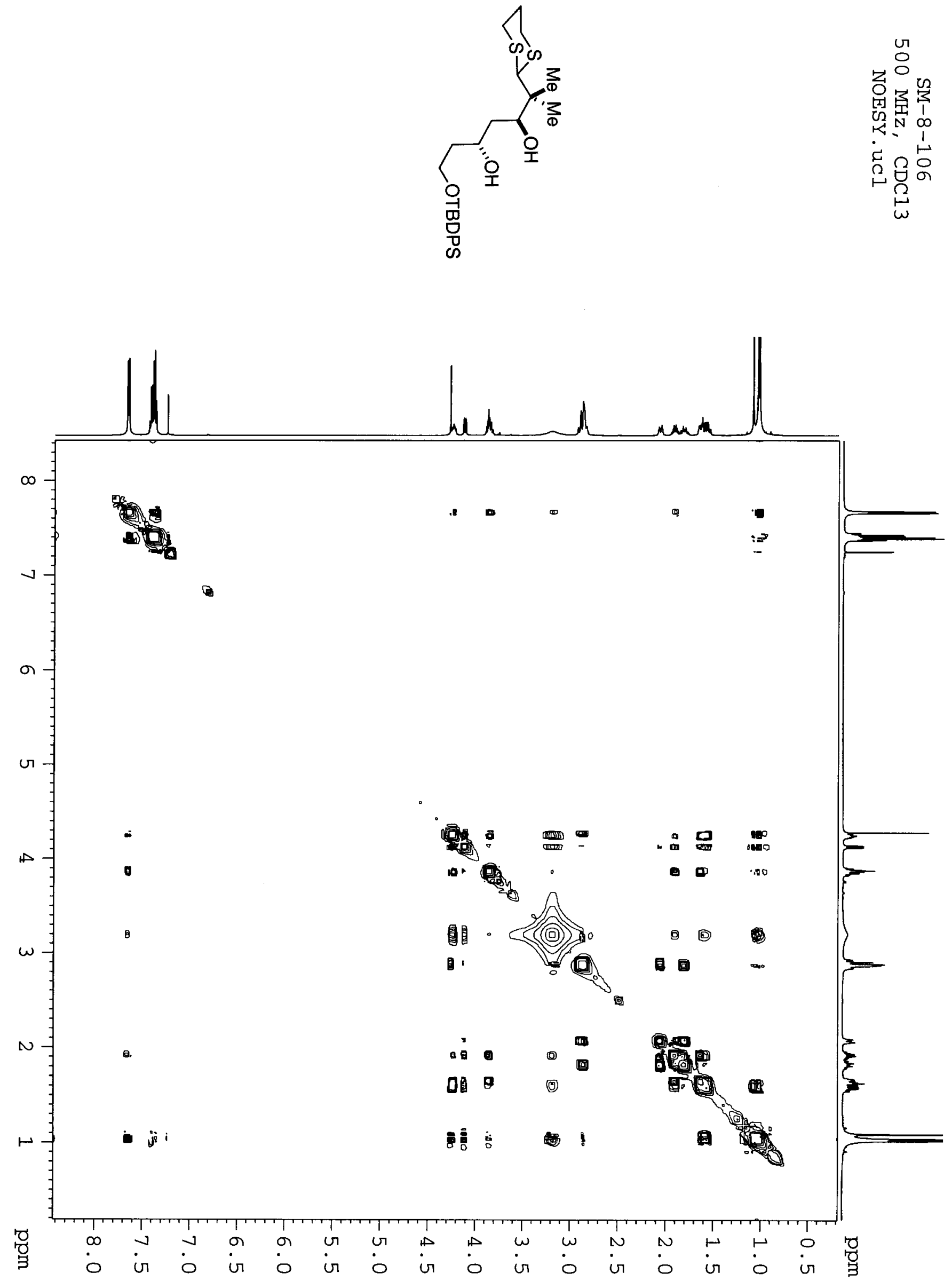

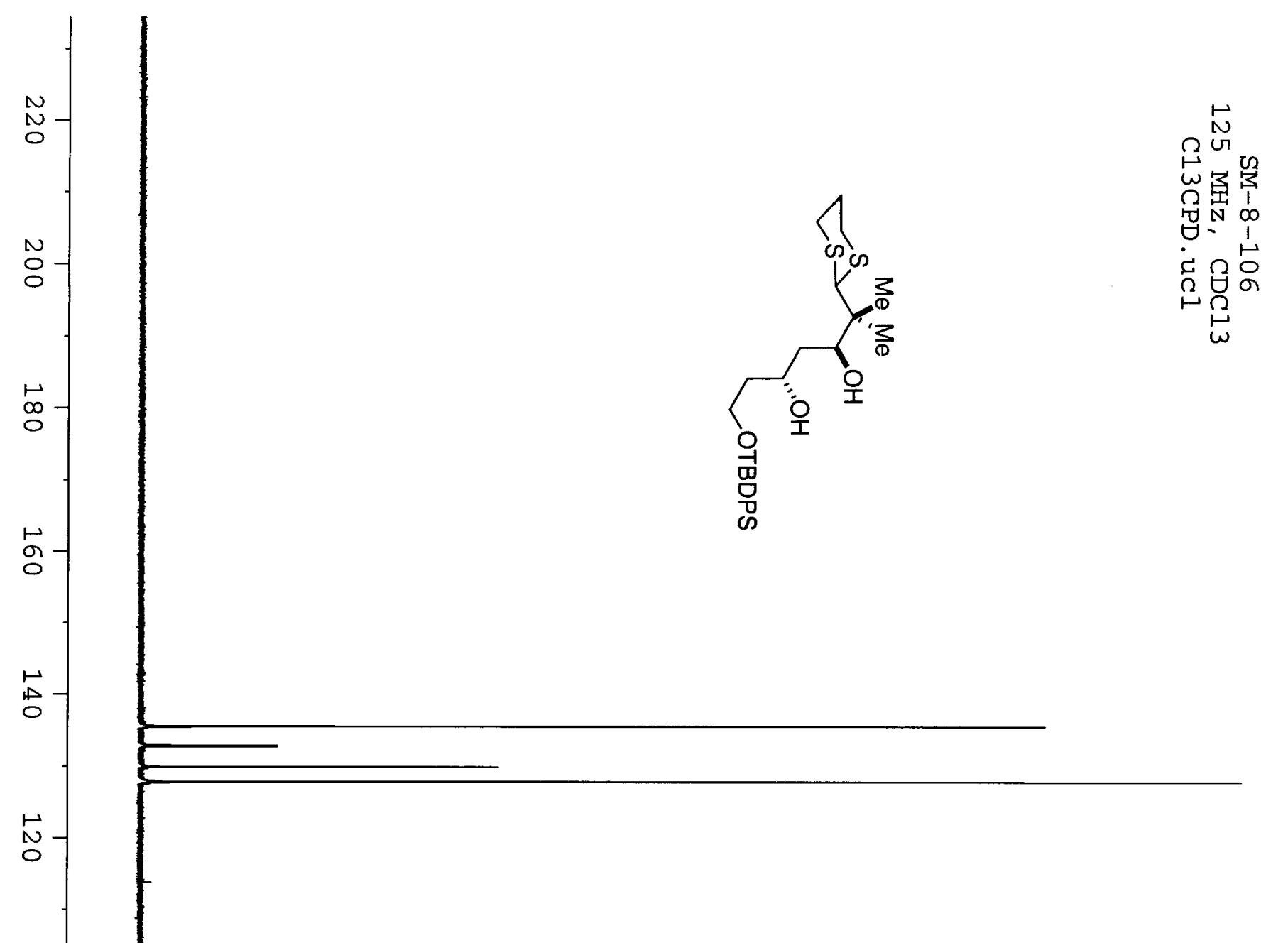

옹

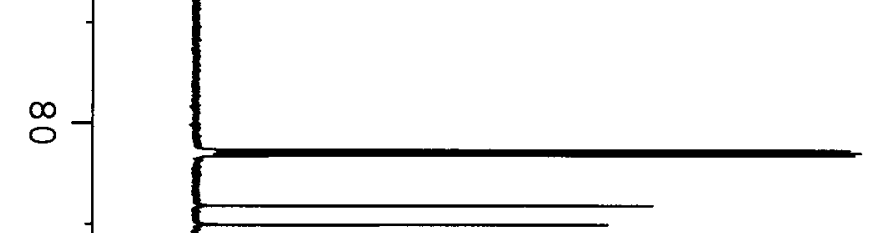

(2) 


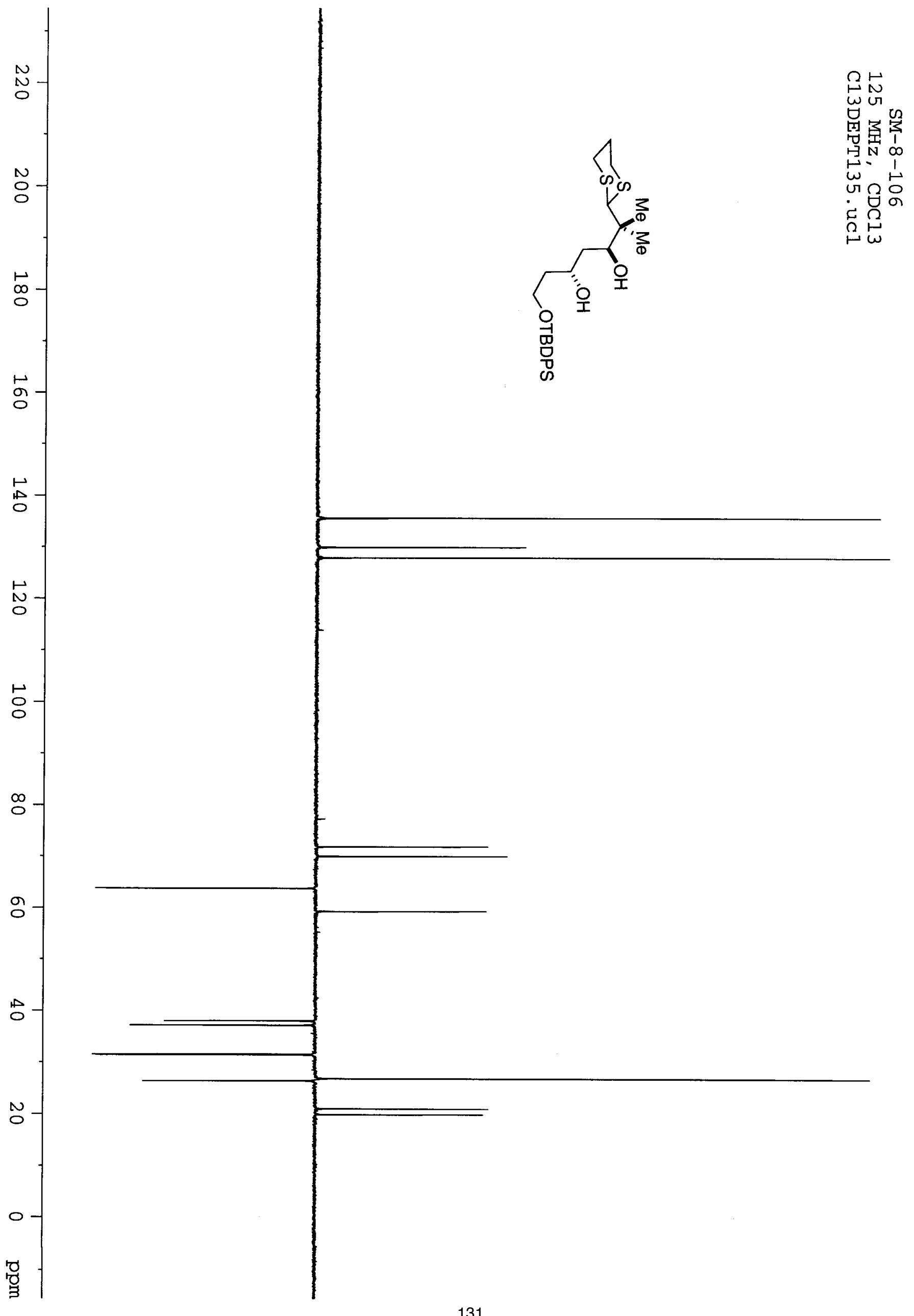



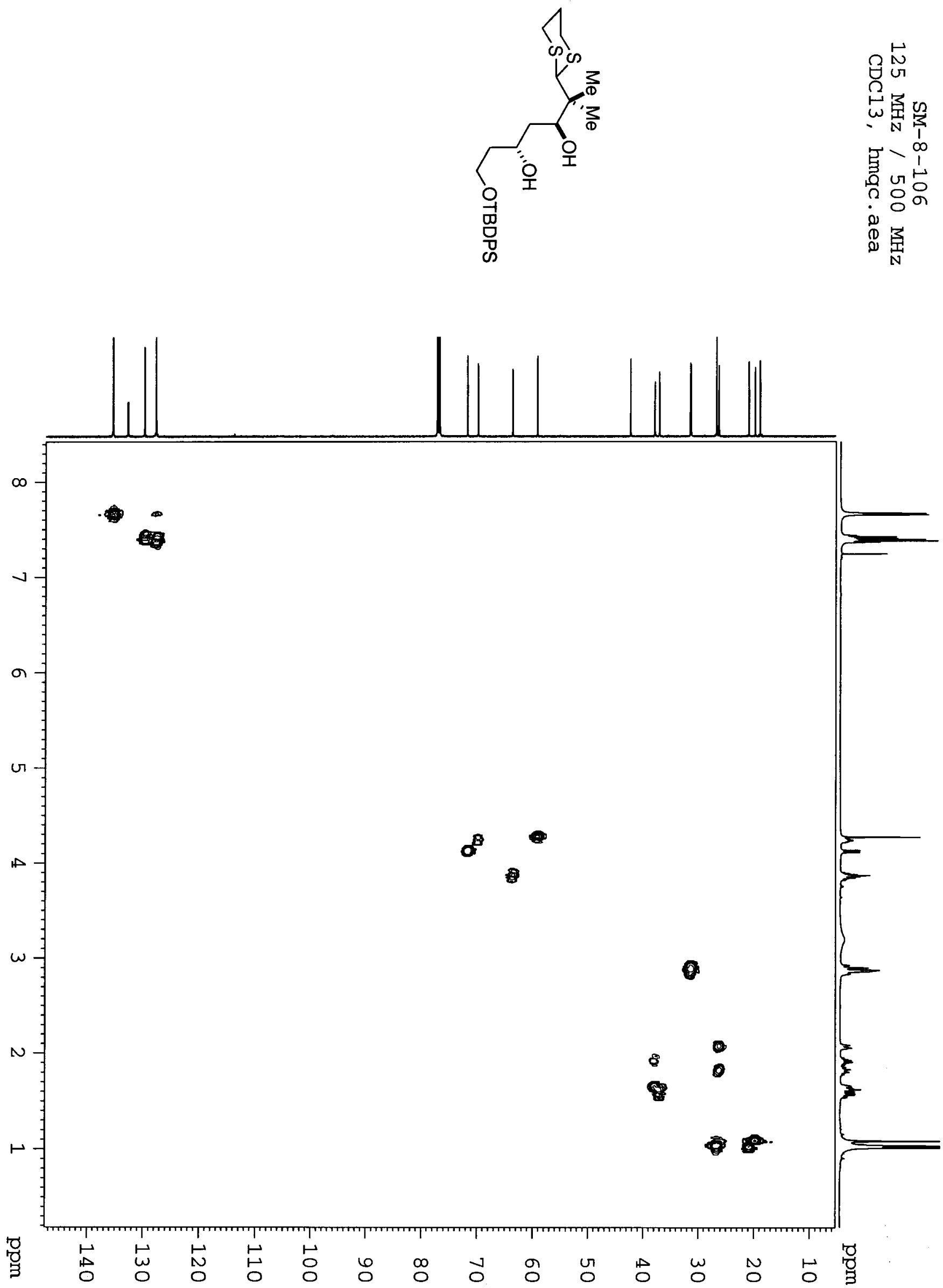


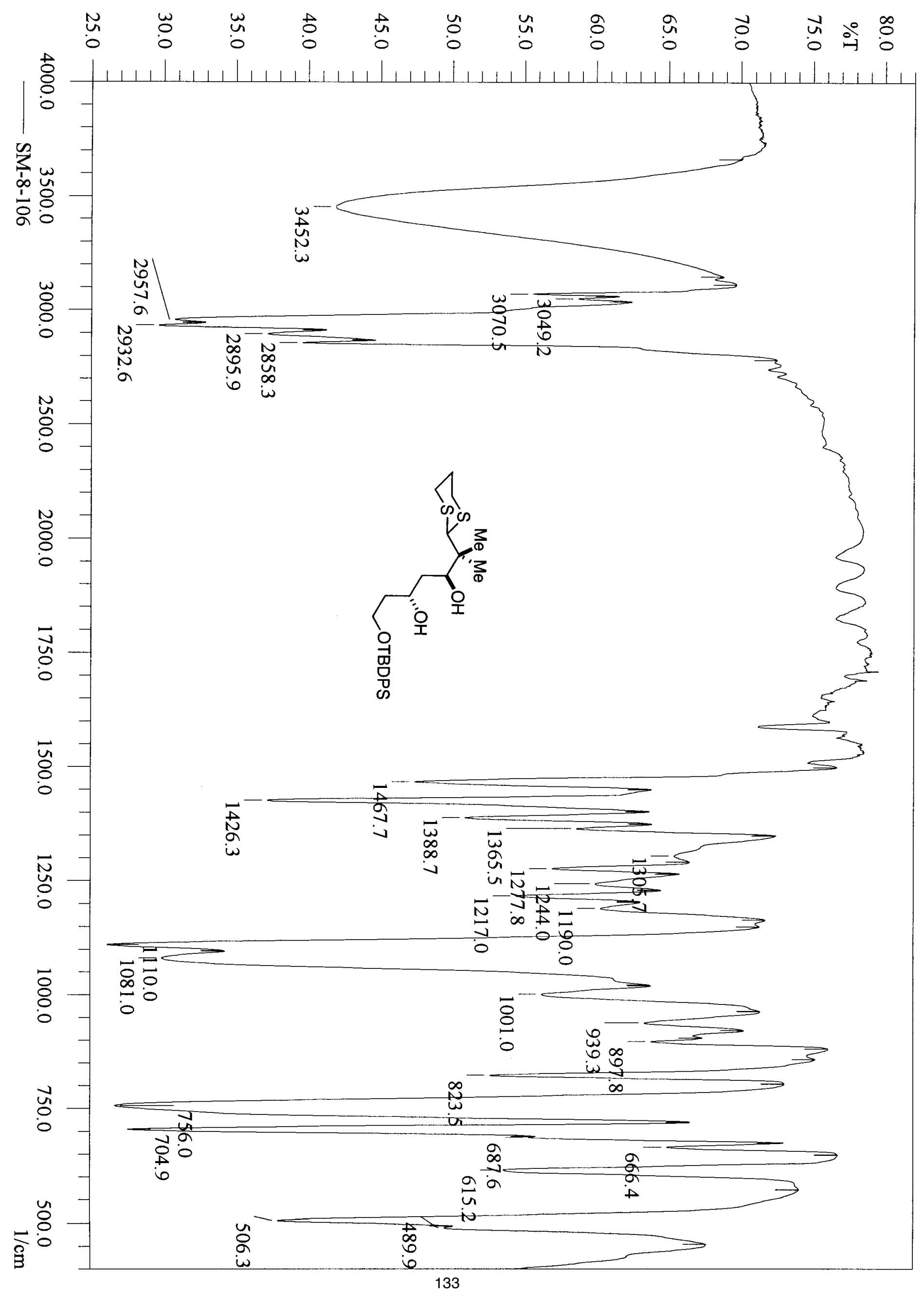




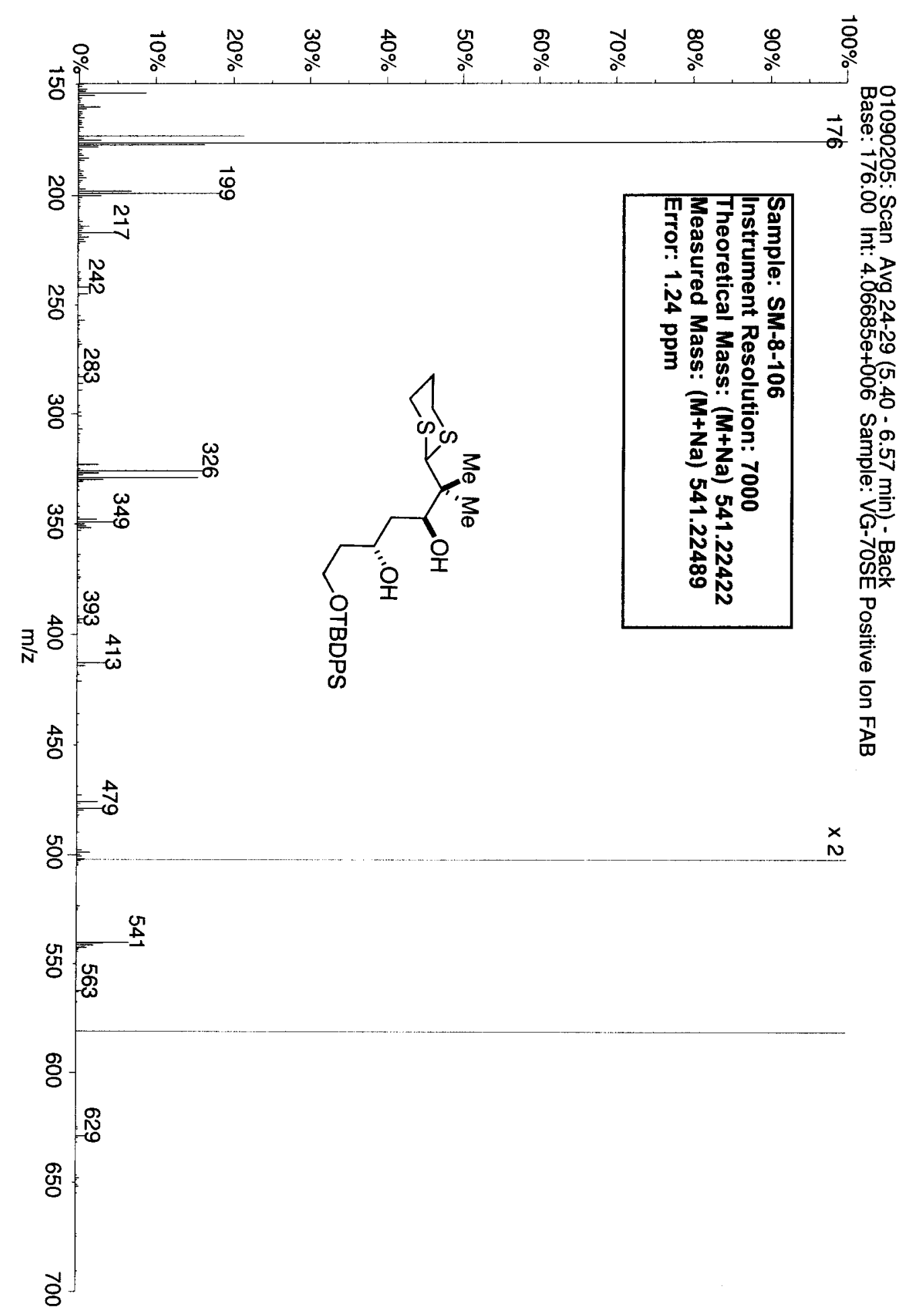




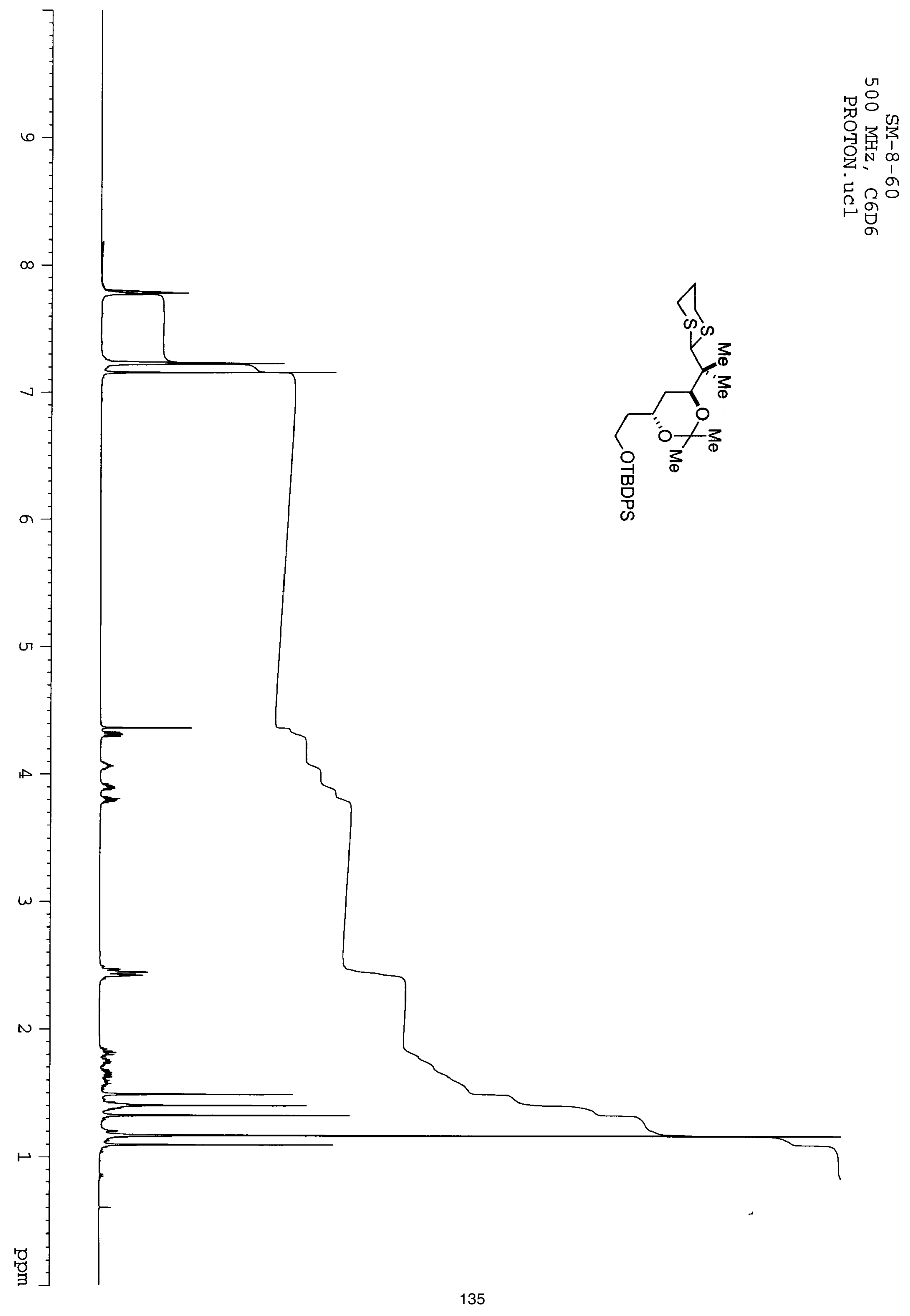



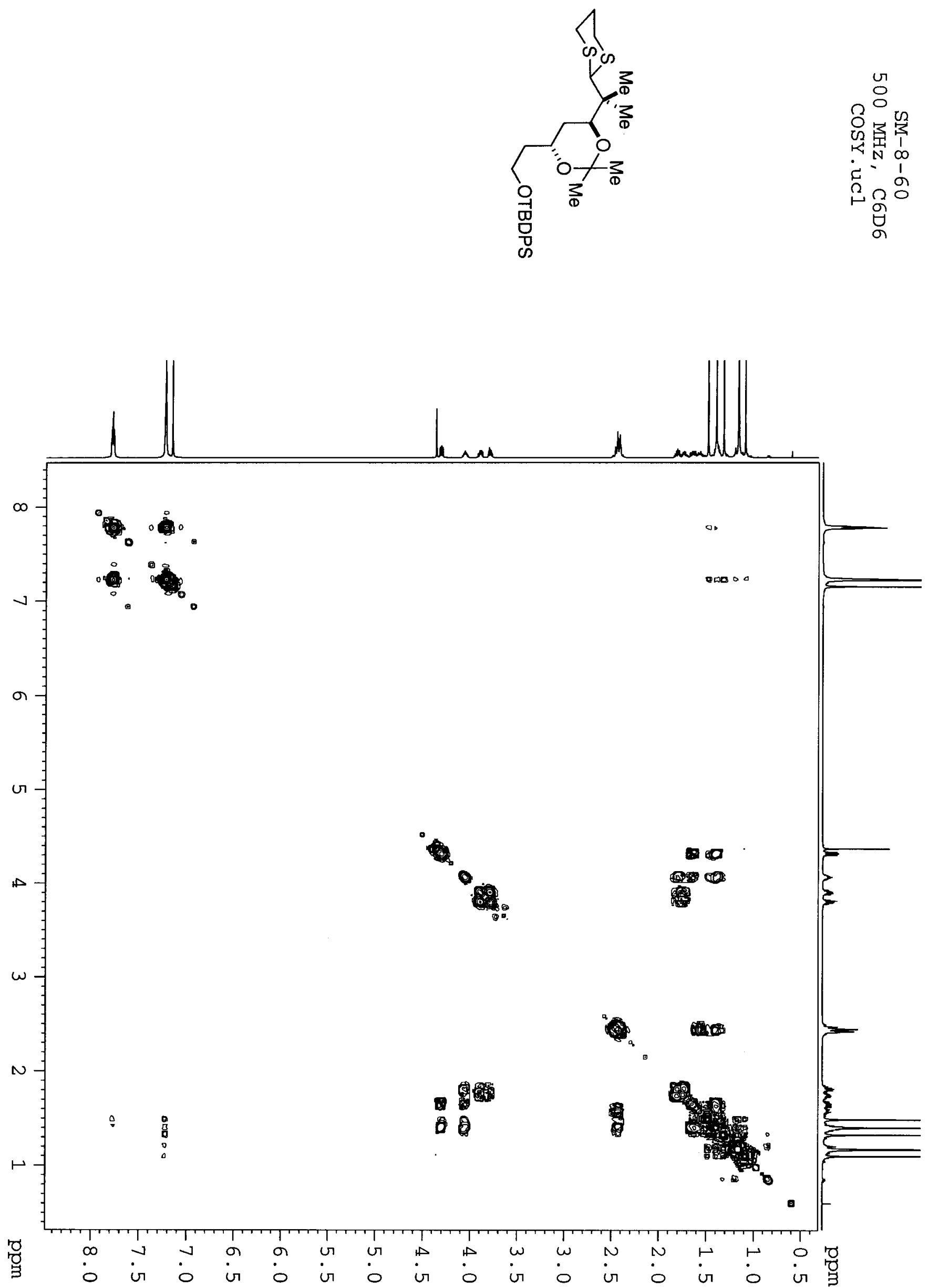

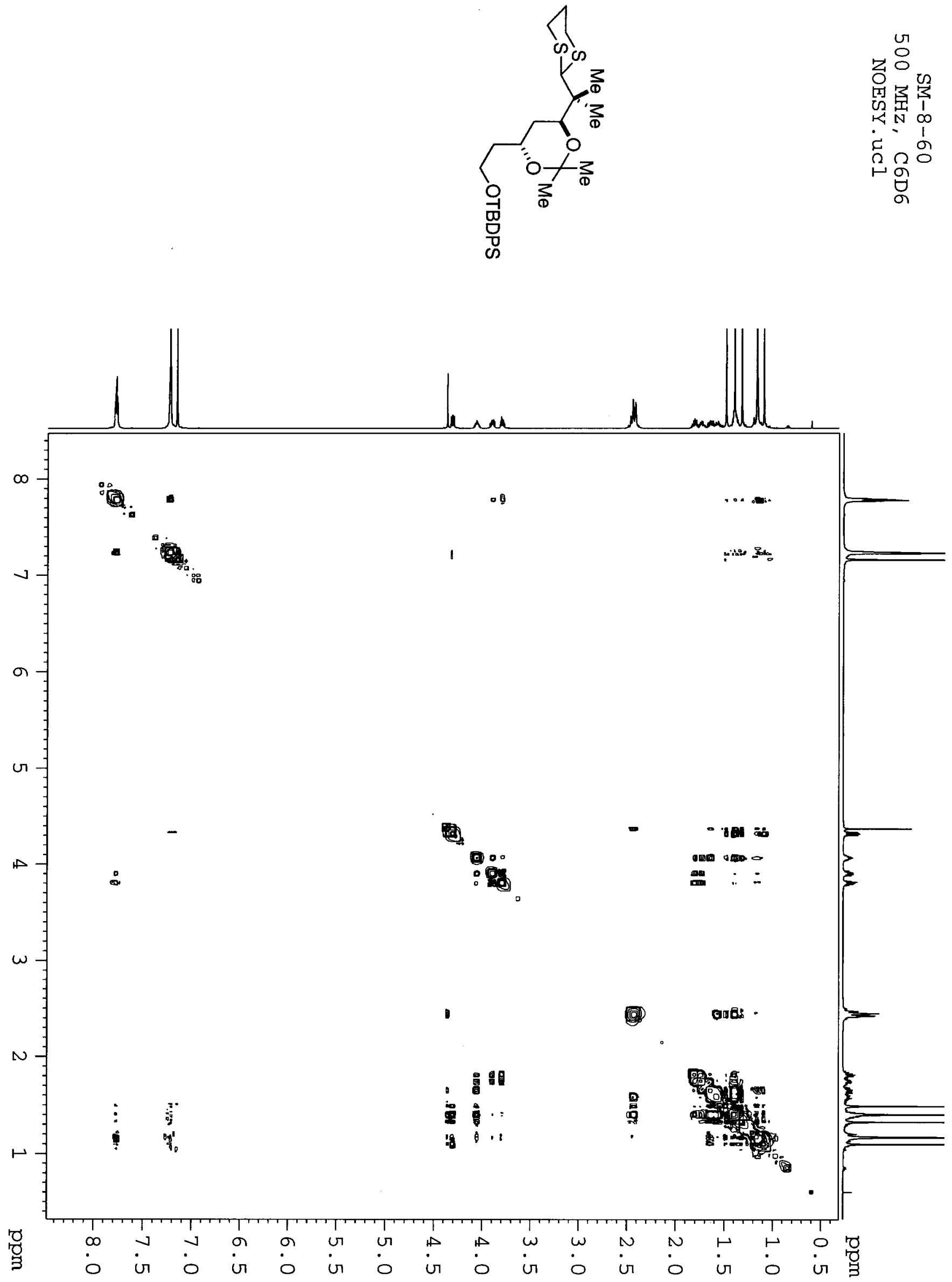


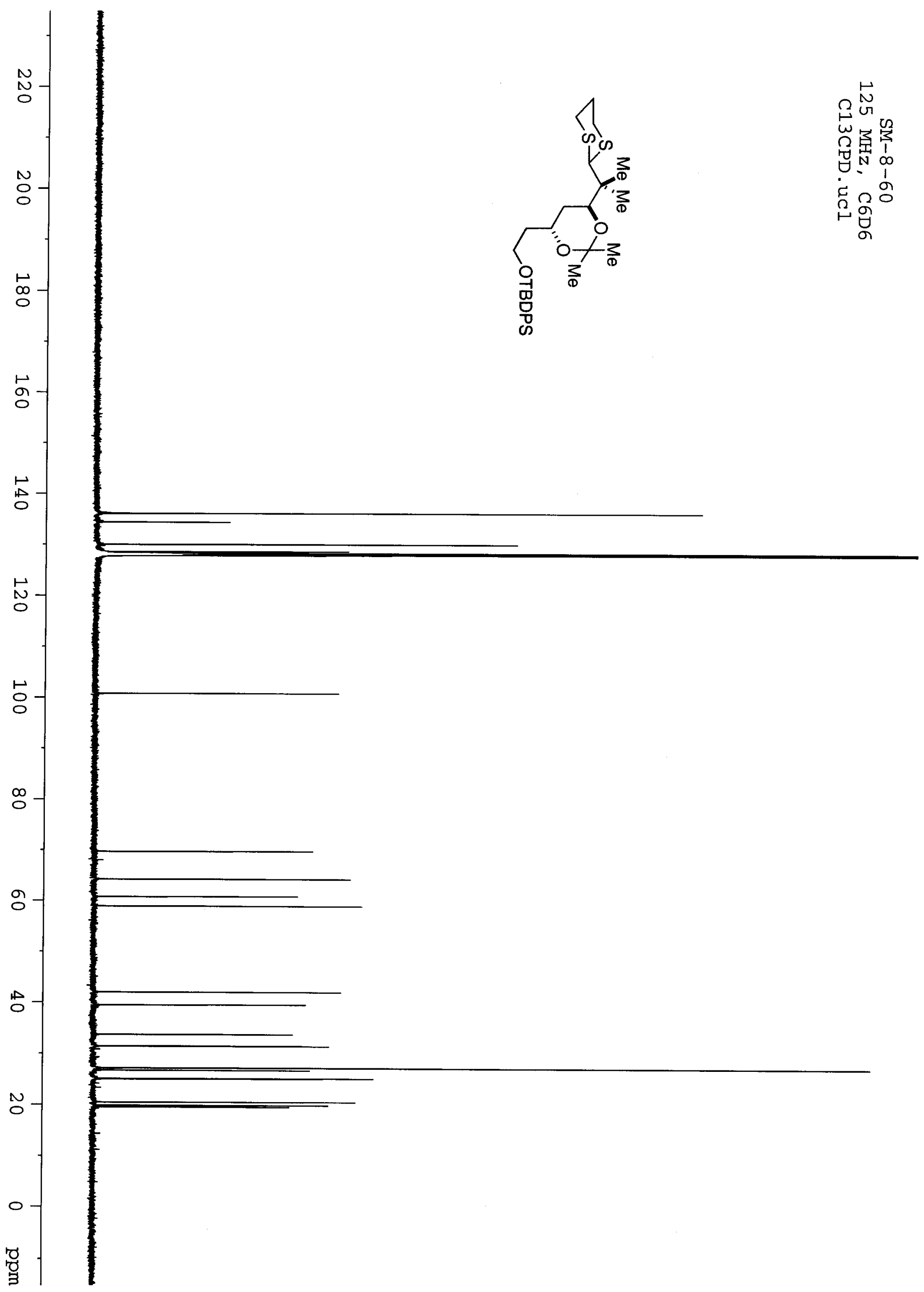




$$
\frac{1}{=}
$$



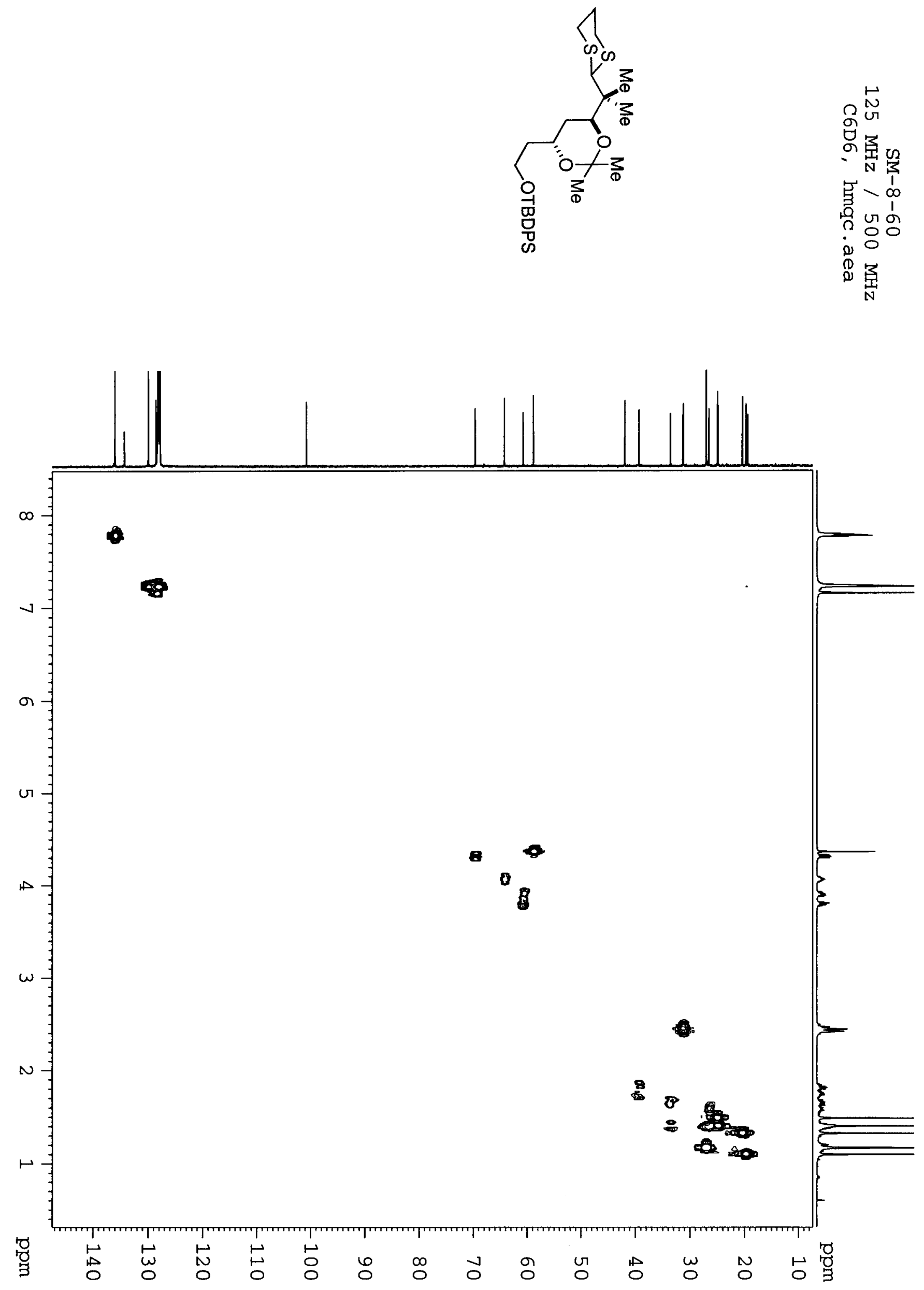


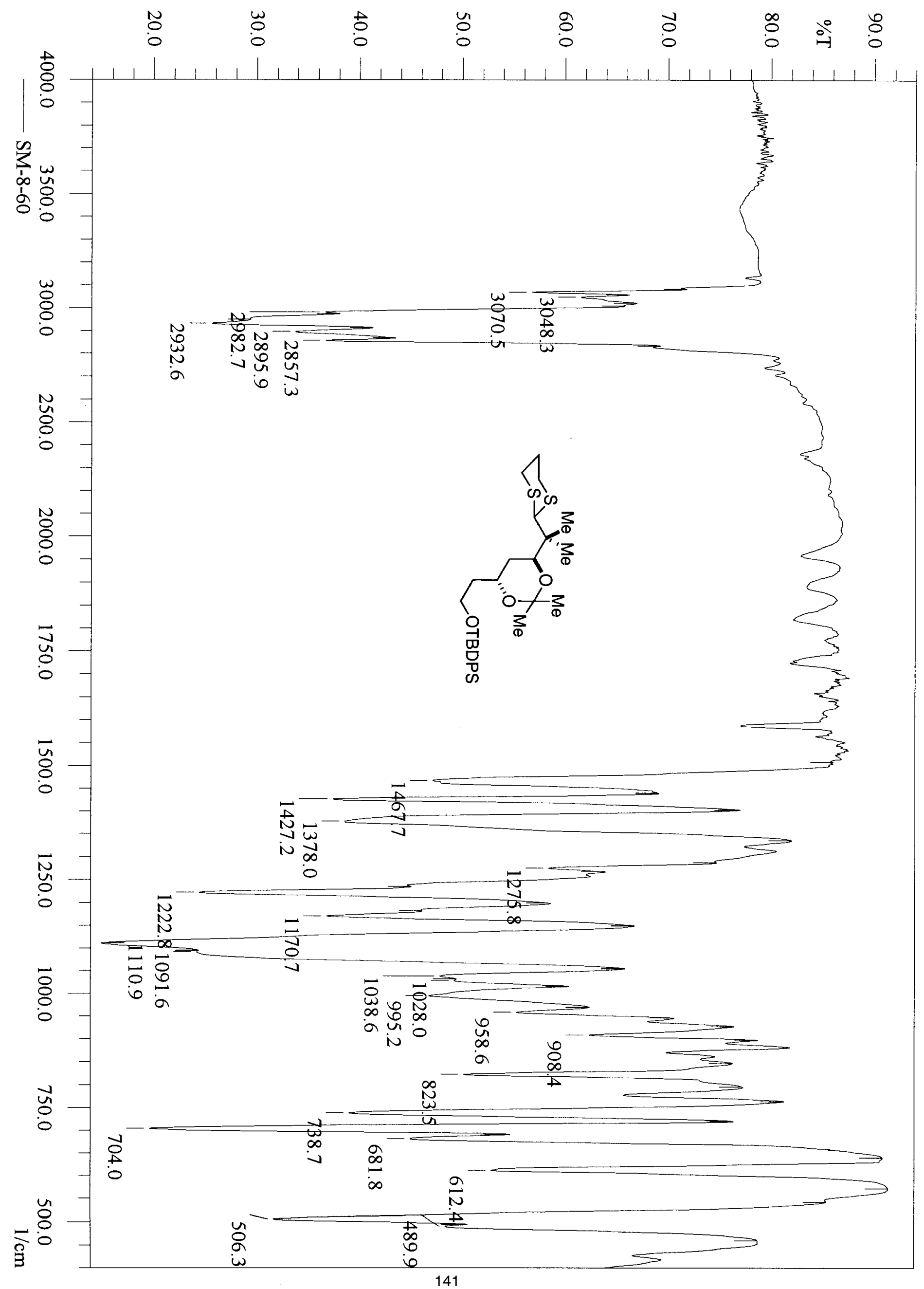




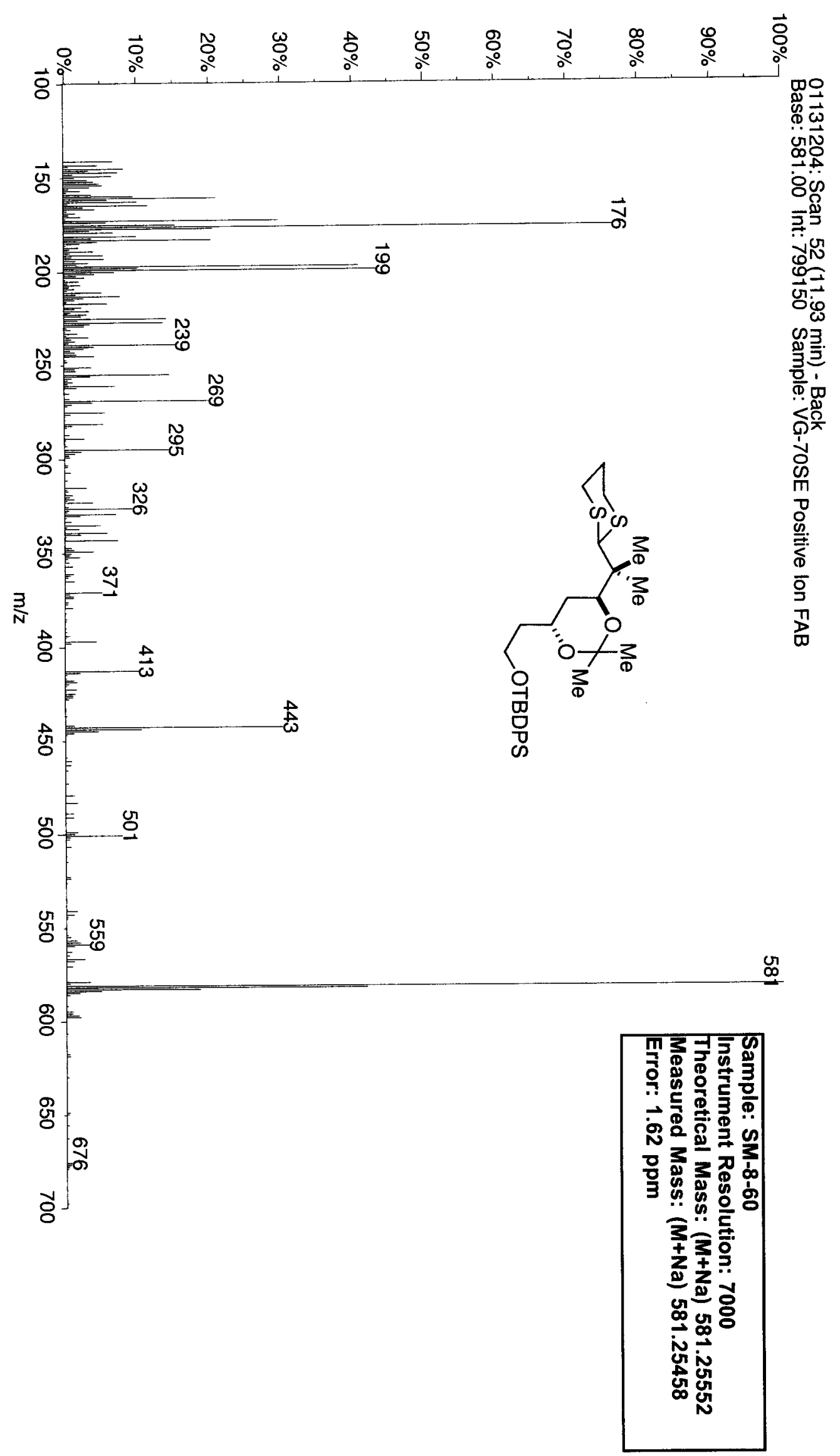




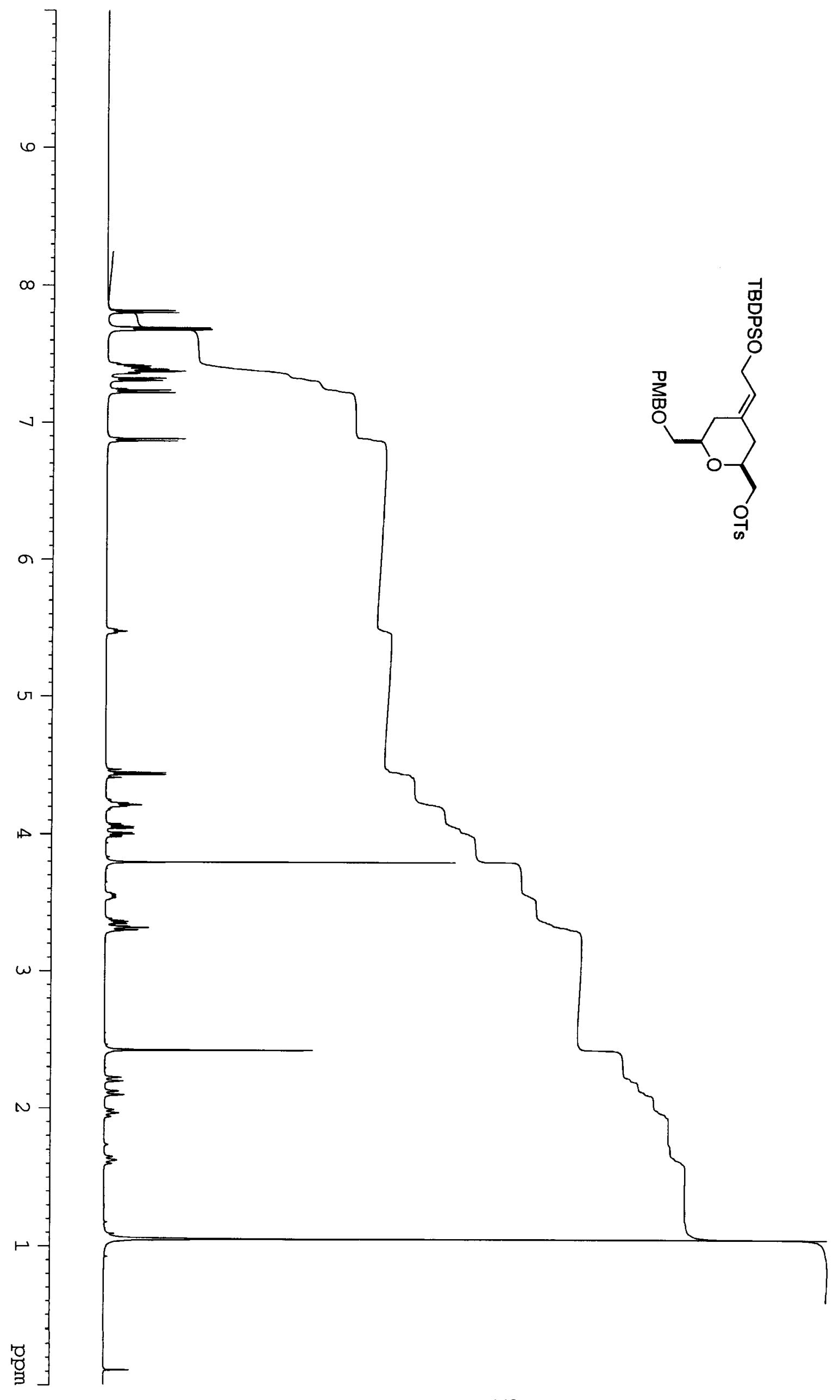

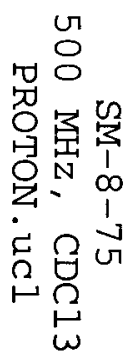



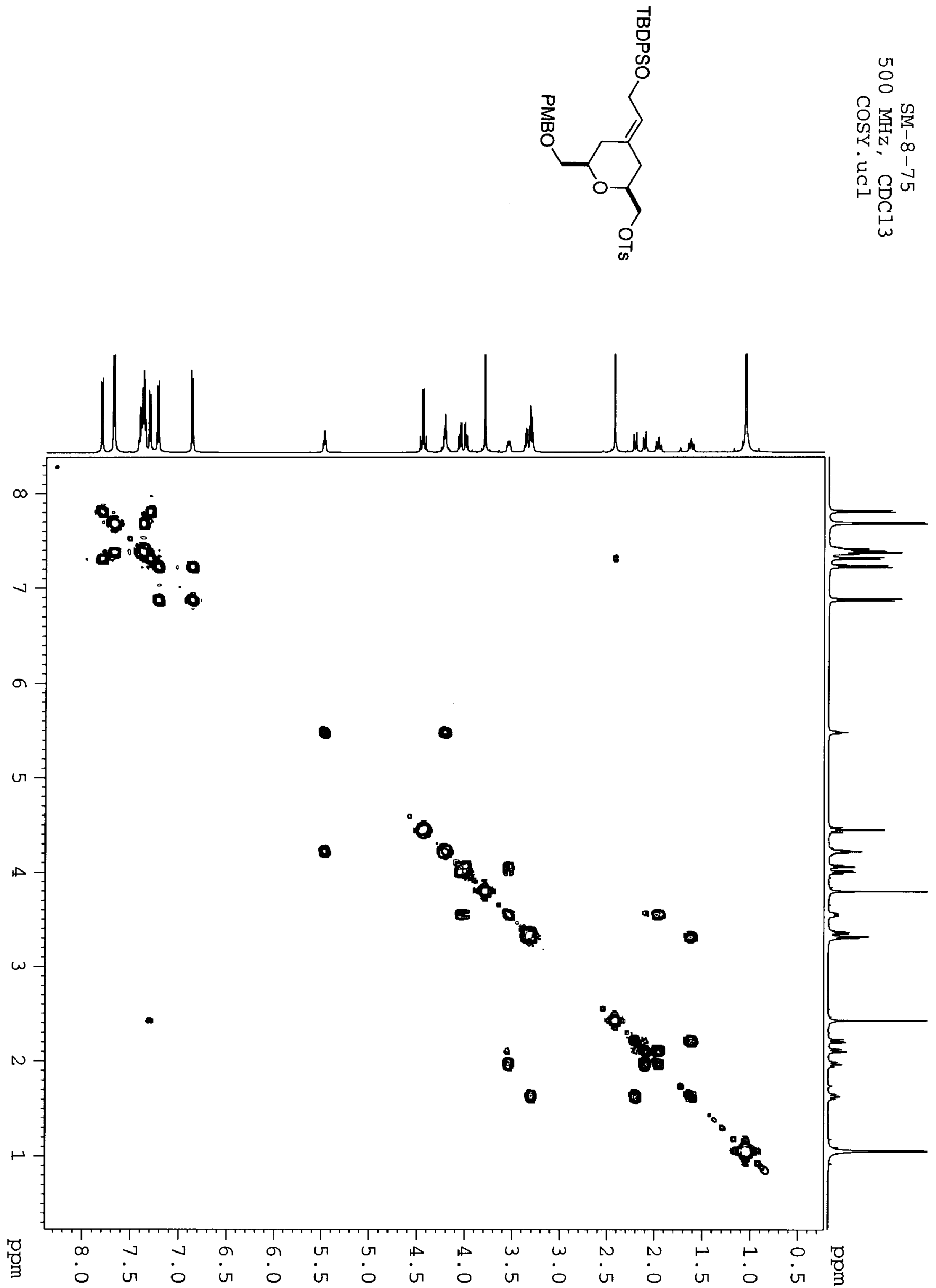


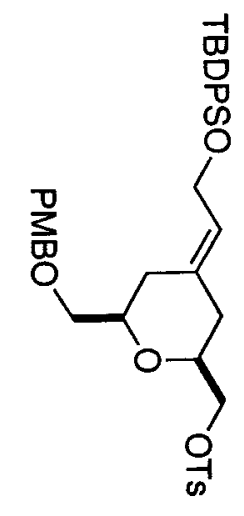

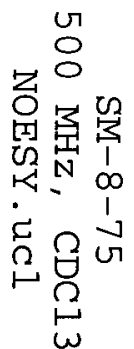

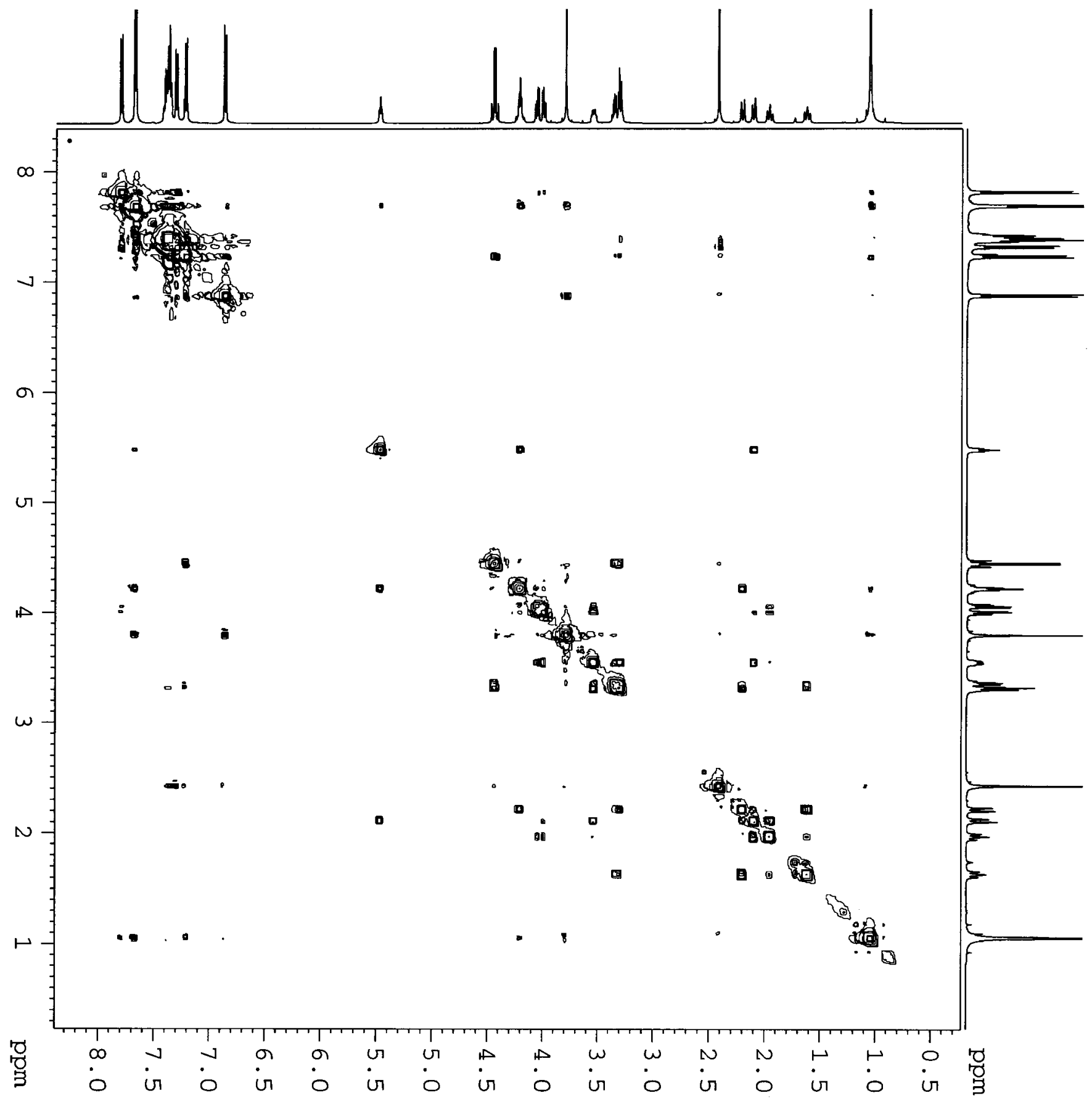



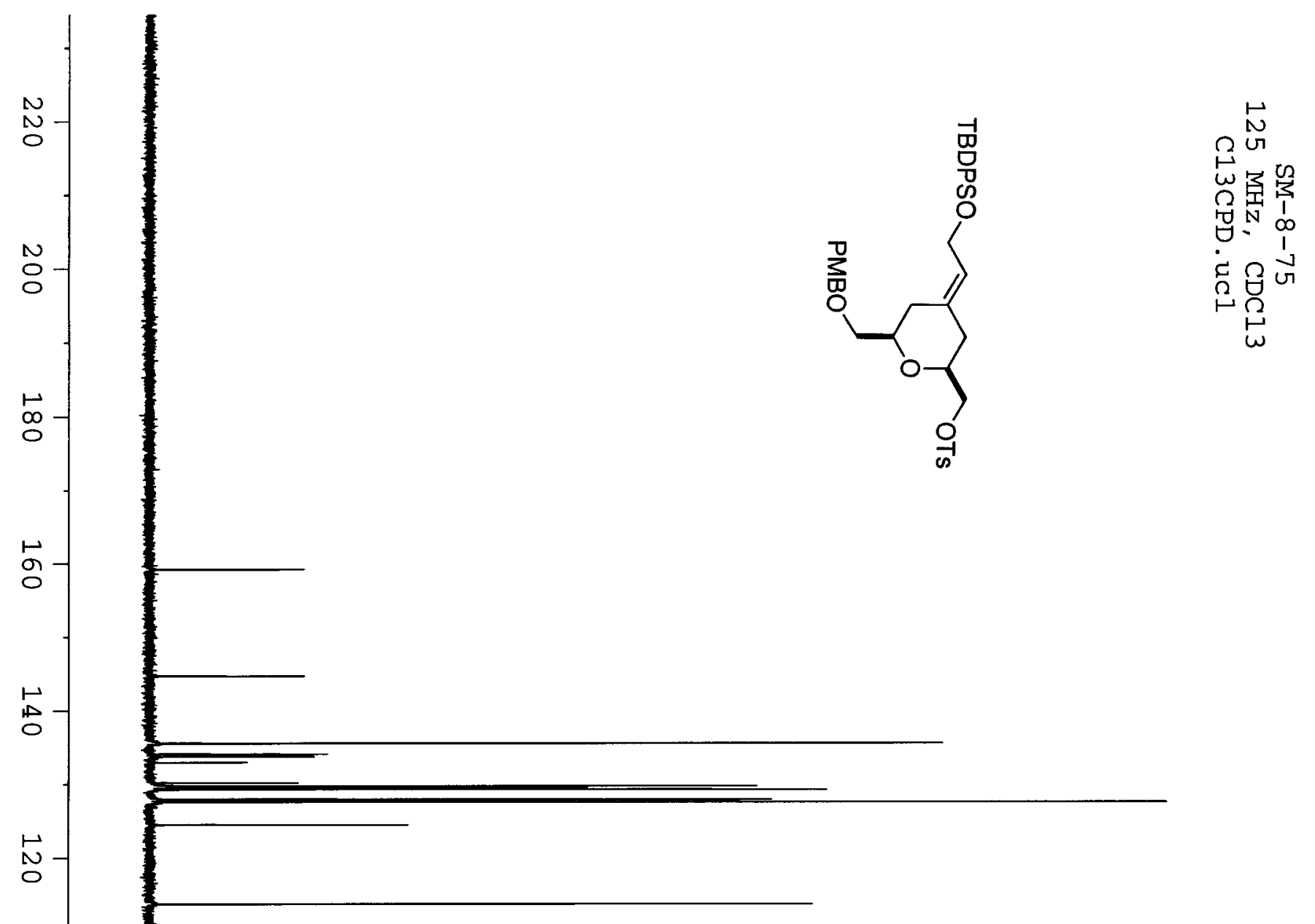

点

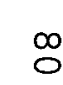




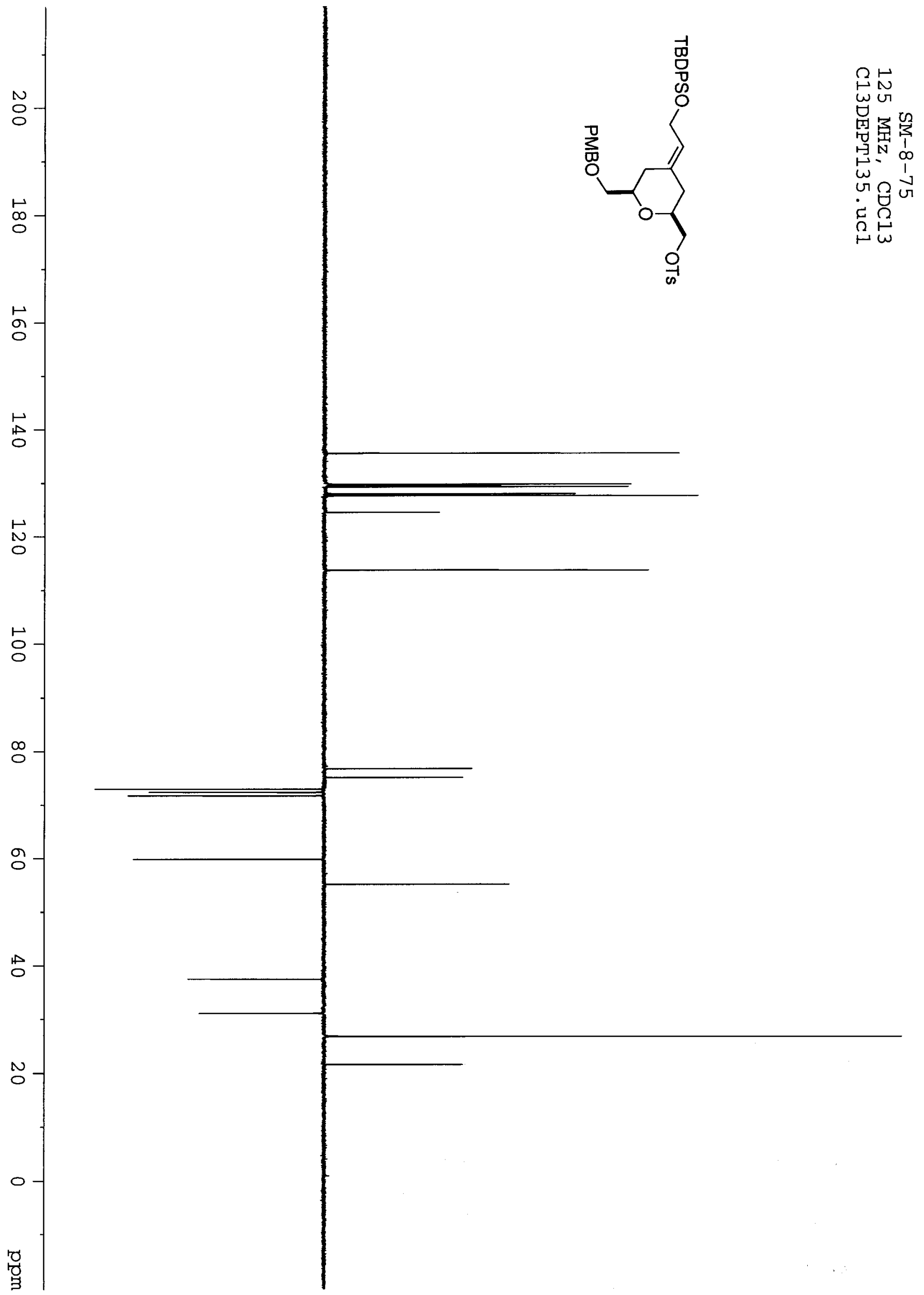




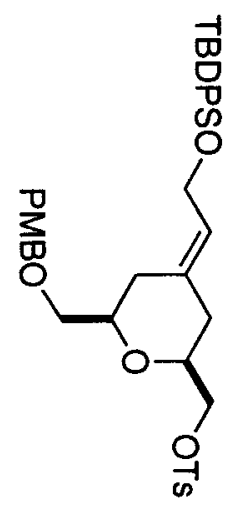

원

当

옹

- $\infty$

总

? O

ข

強

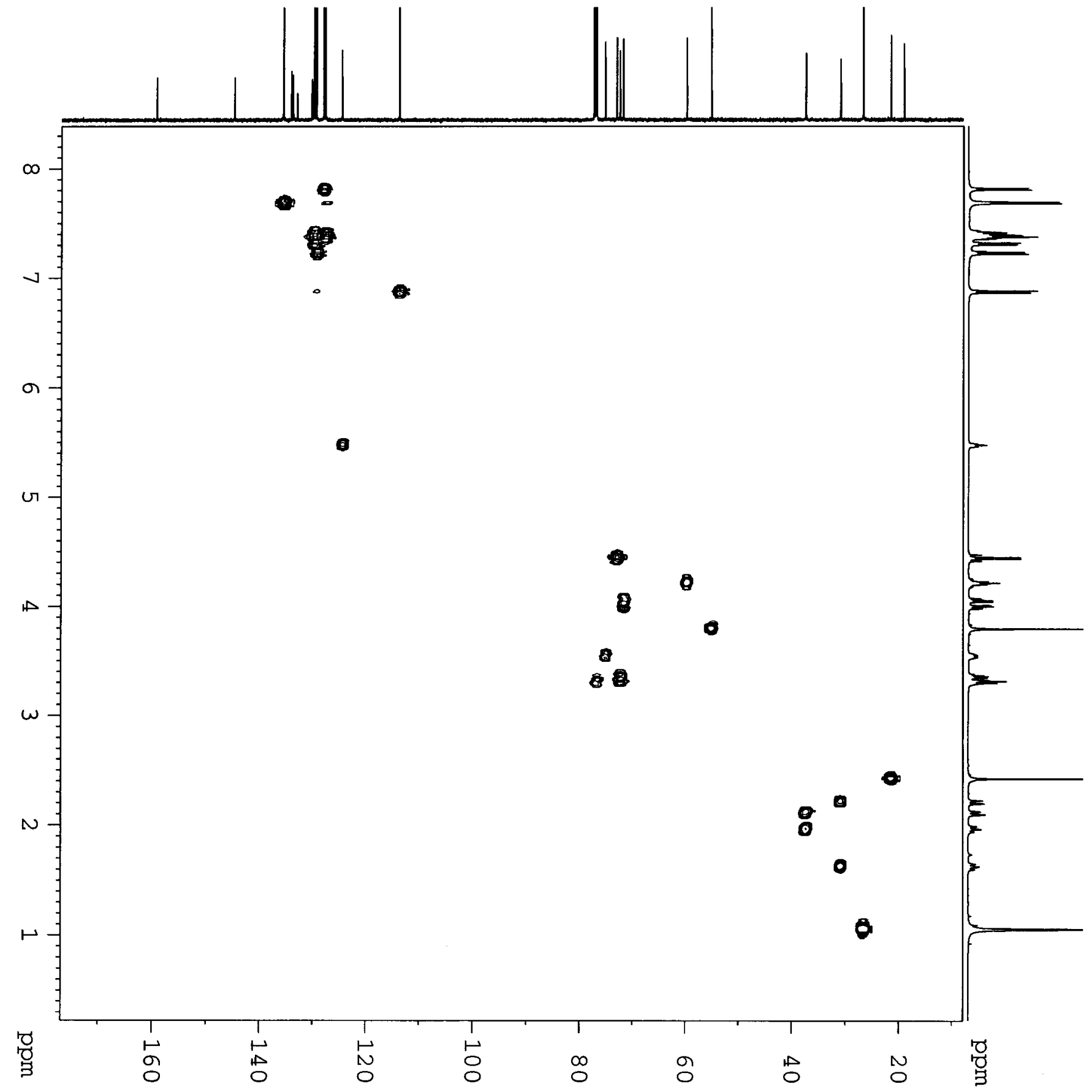




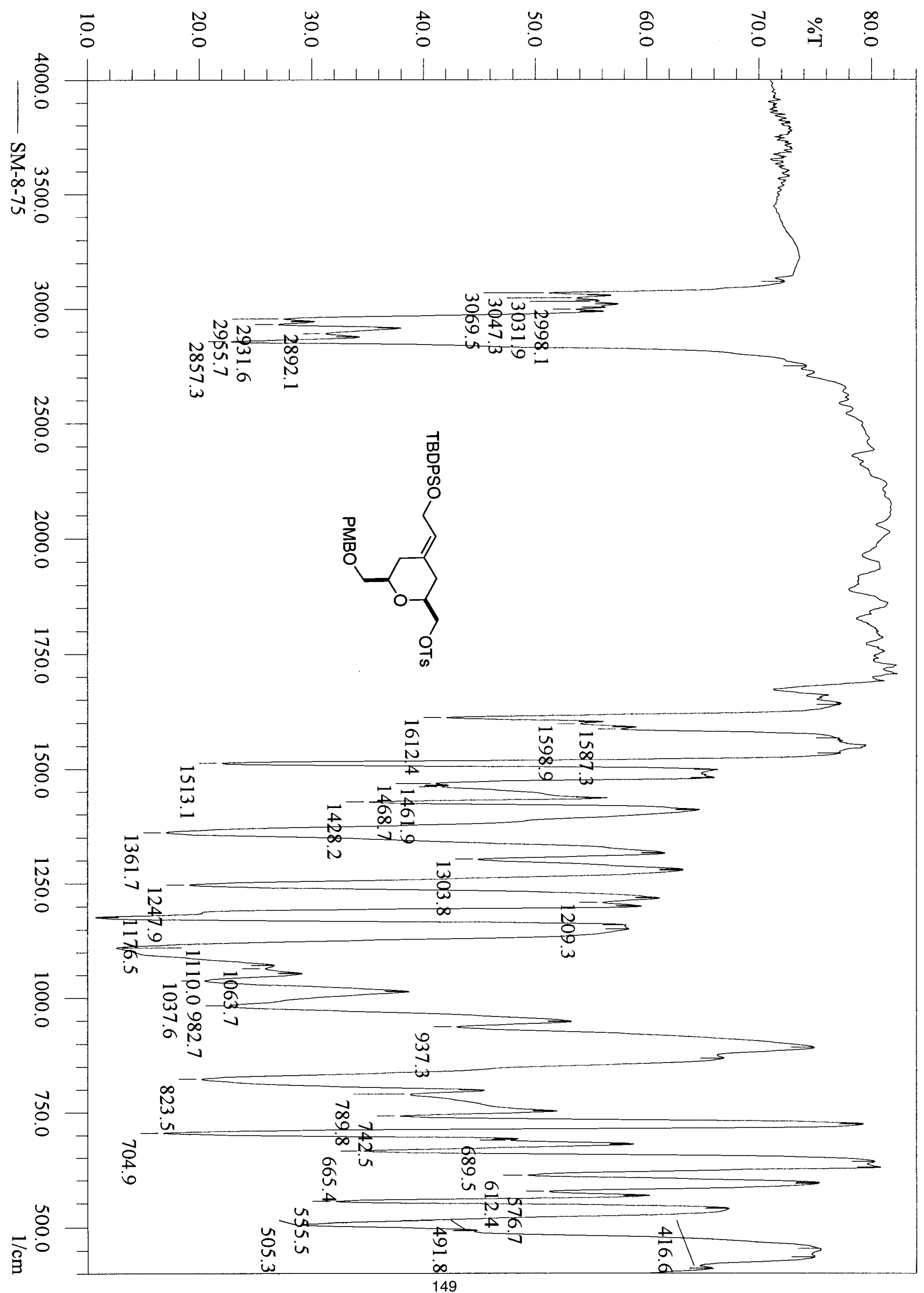




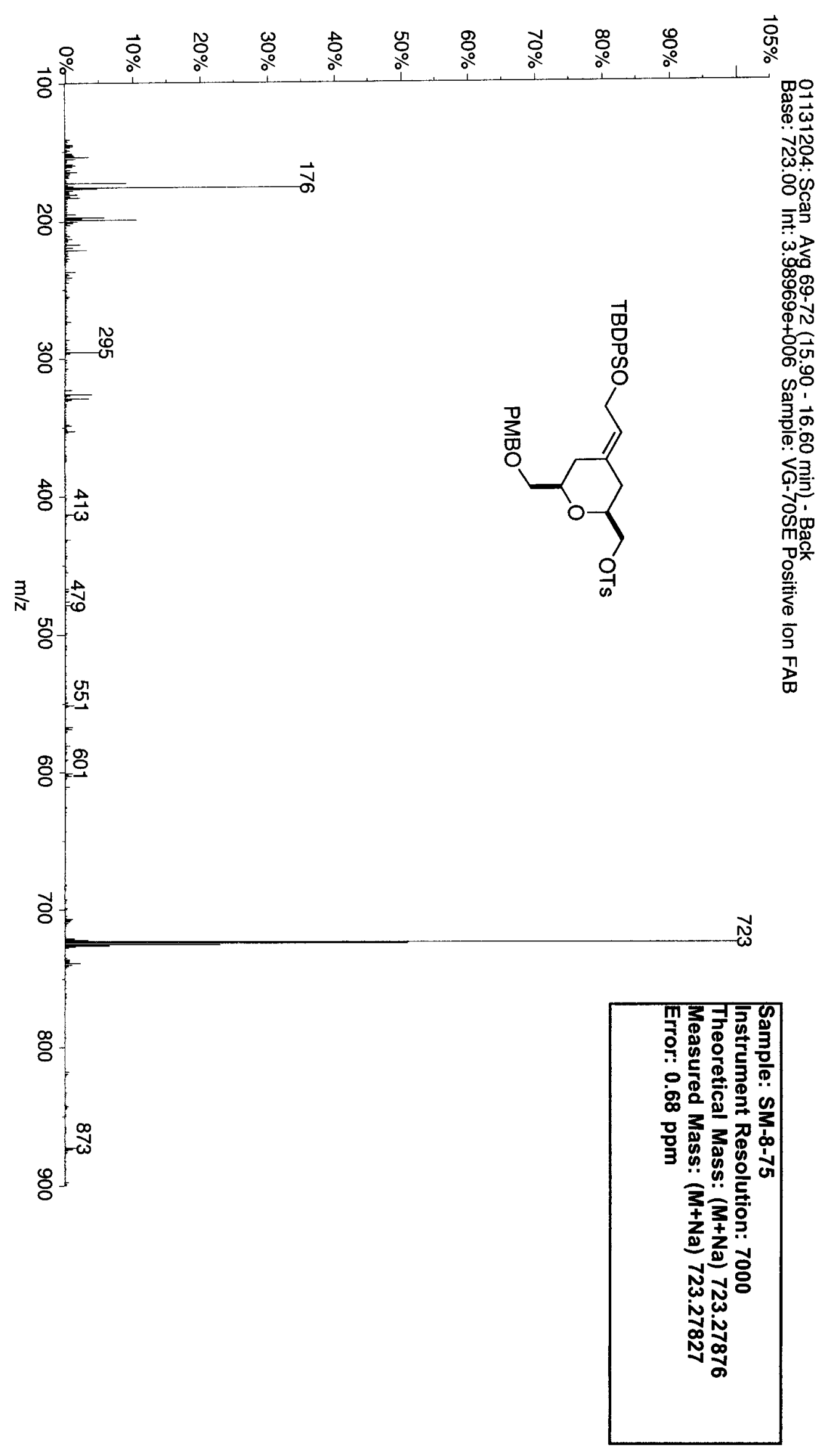



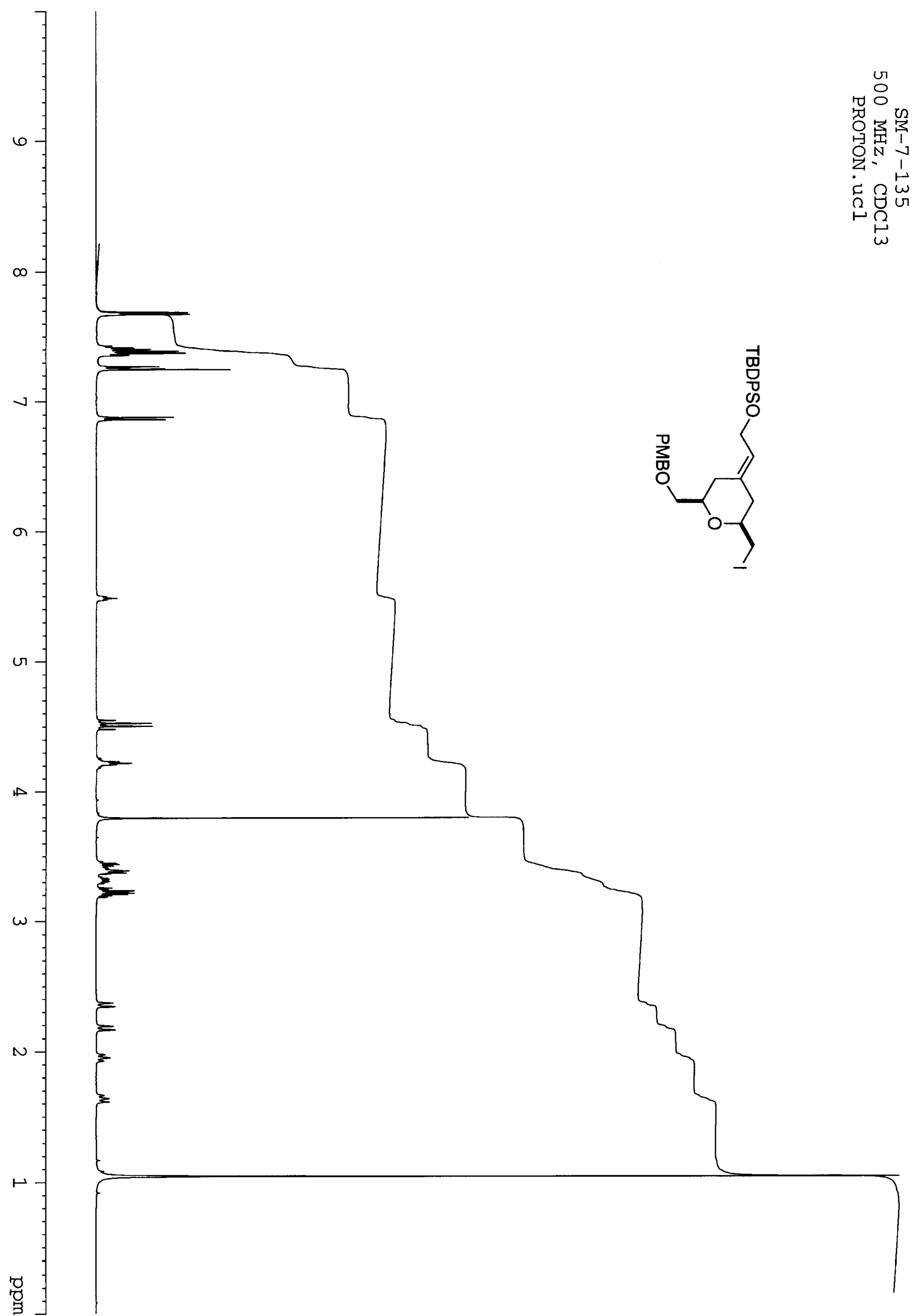

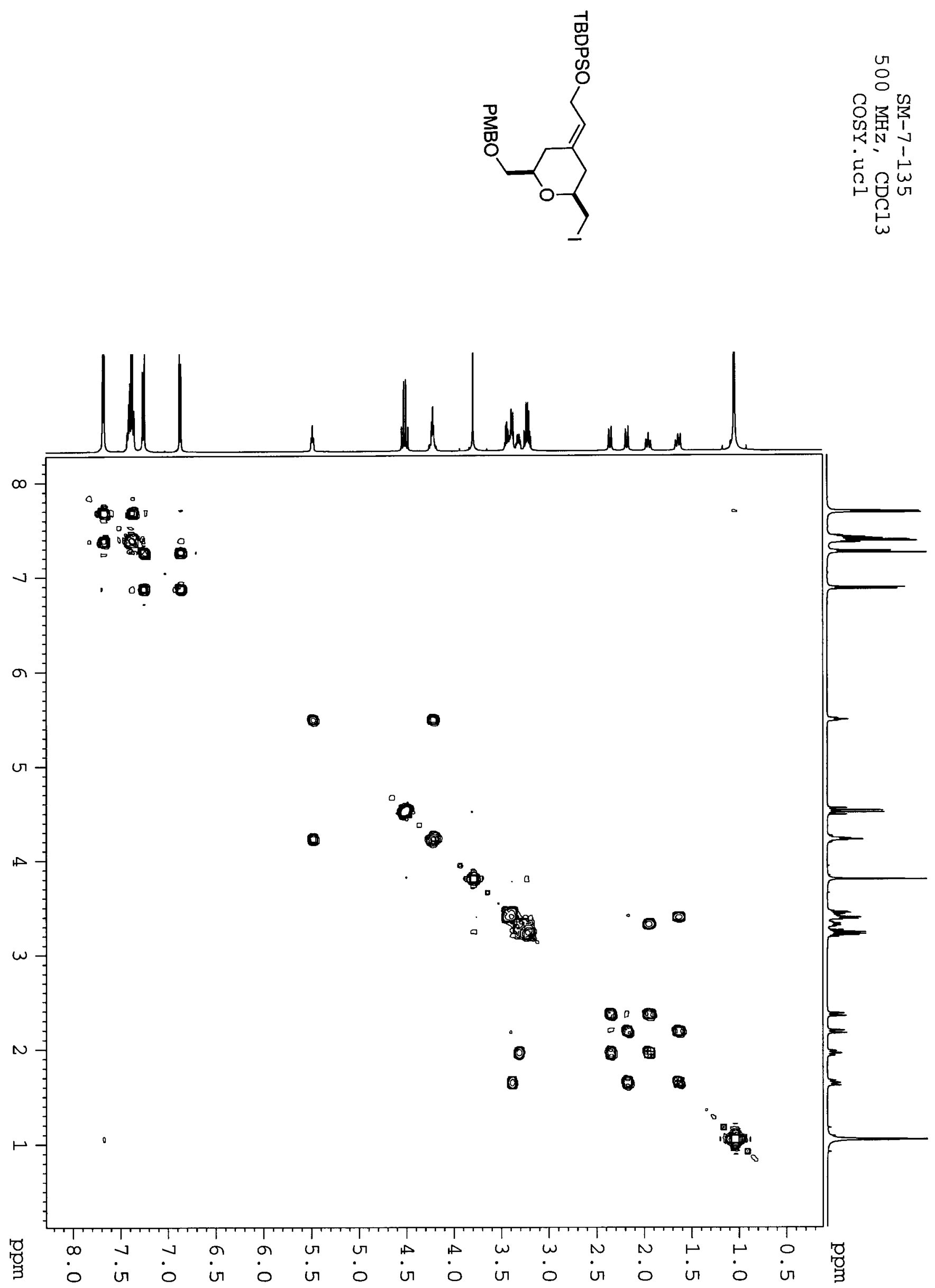

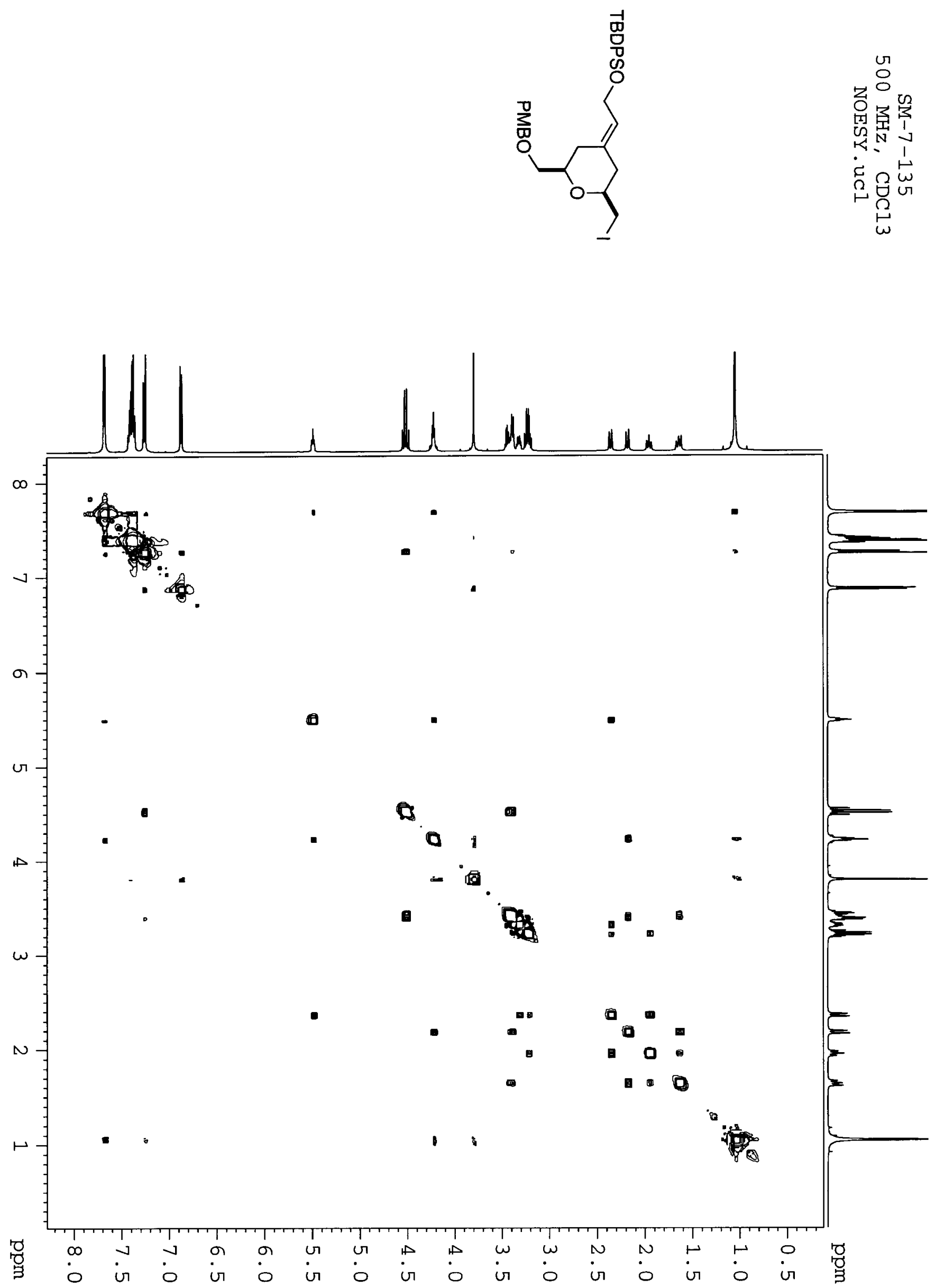


$$
E^{\prime \prime}
$$




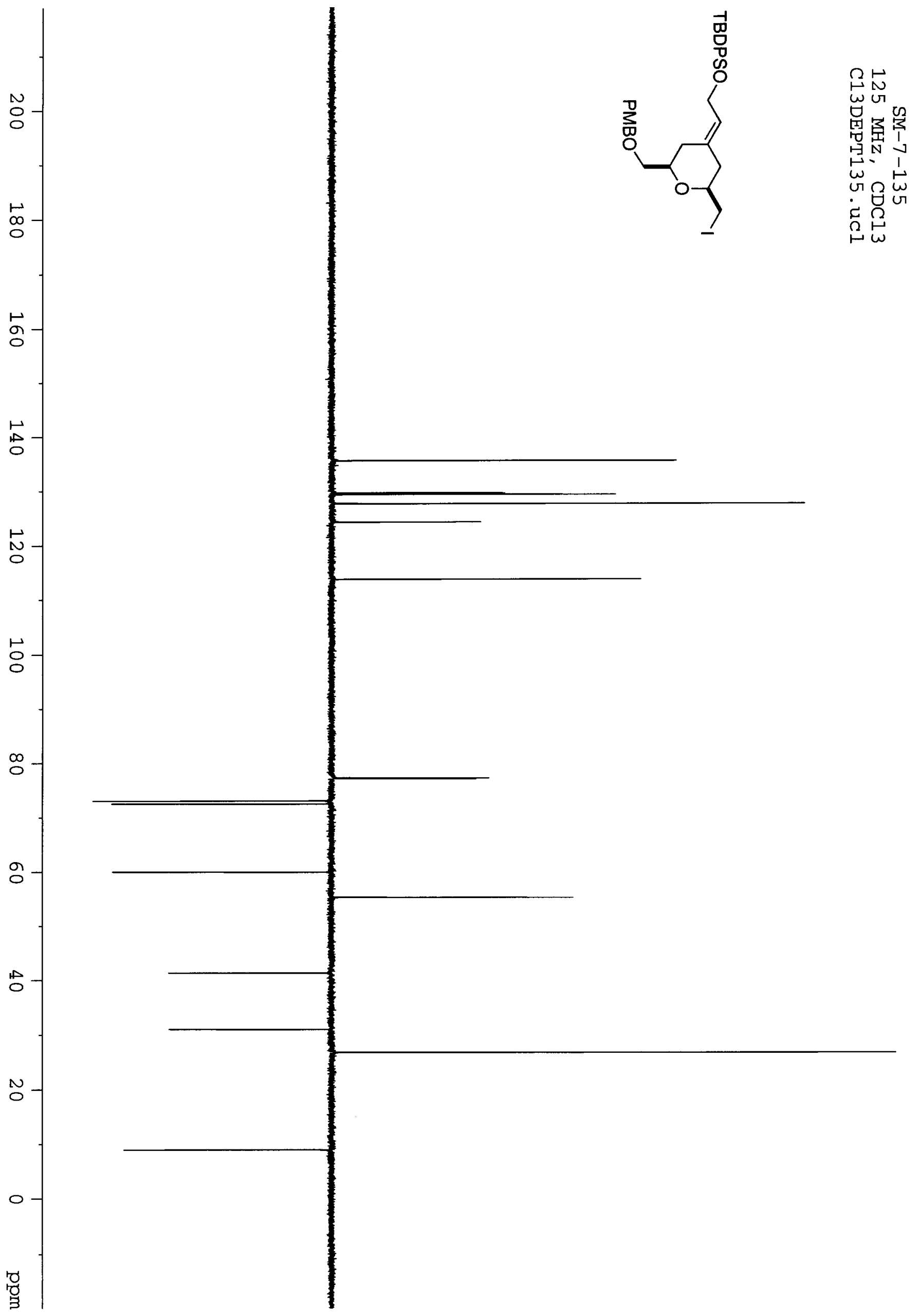




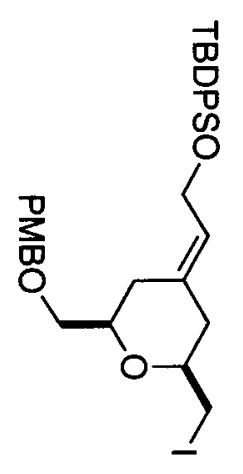

오ํ

宫䍃

- N

T)

产 ज岕

으

各

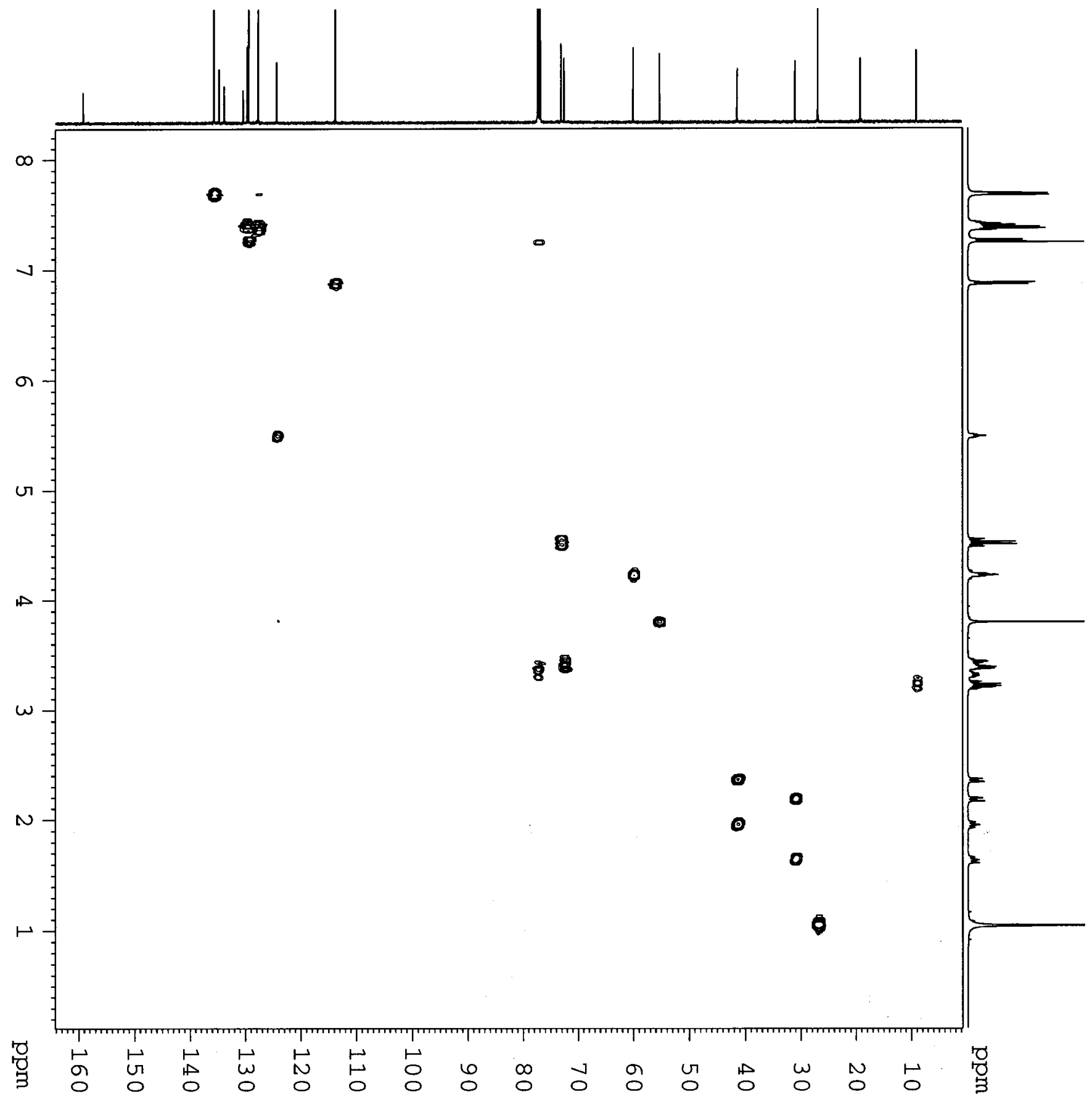




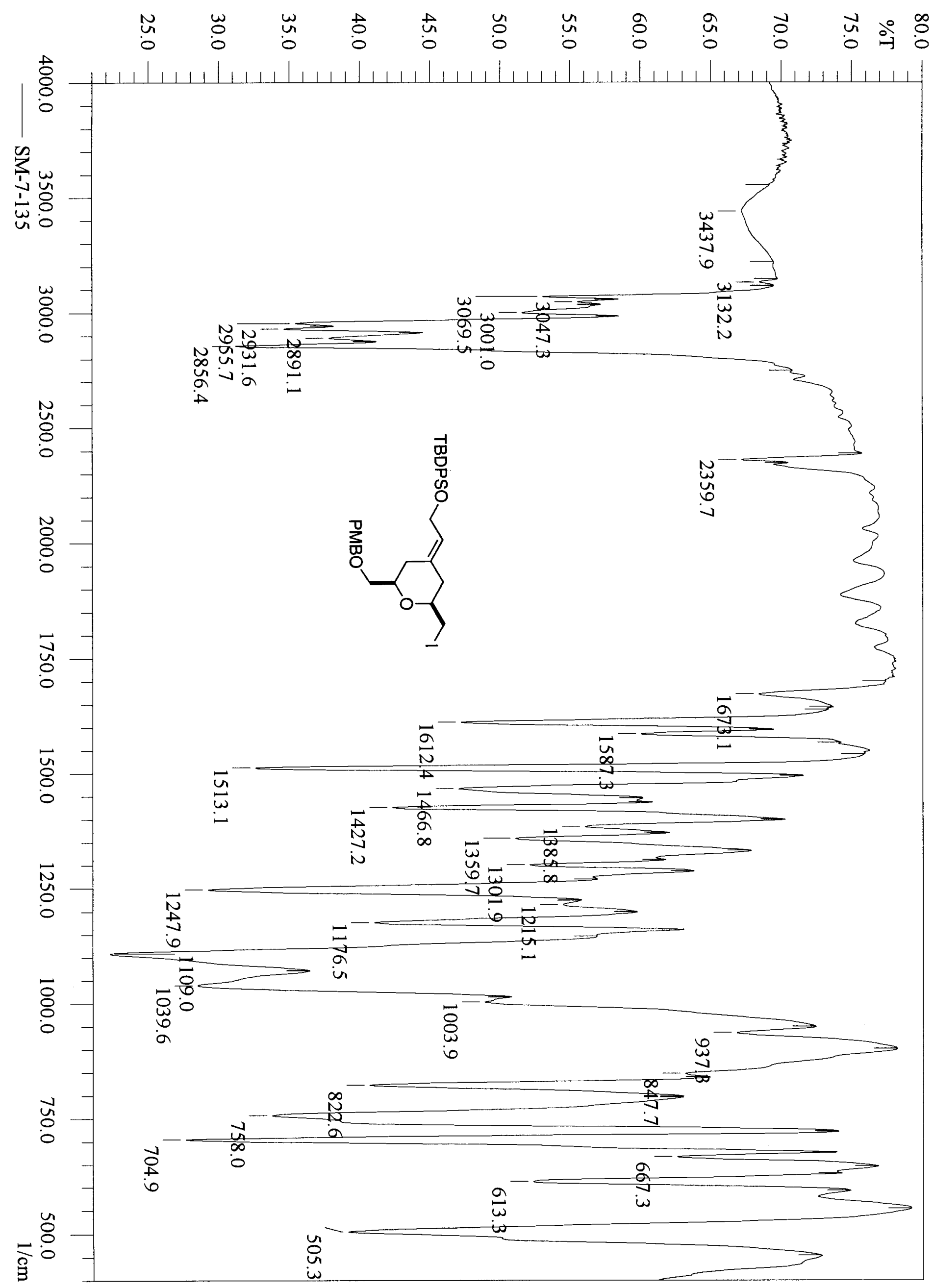




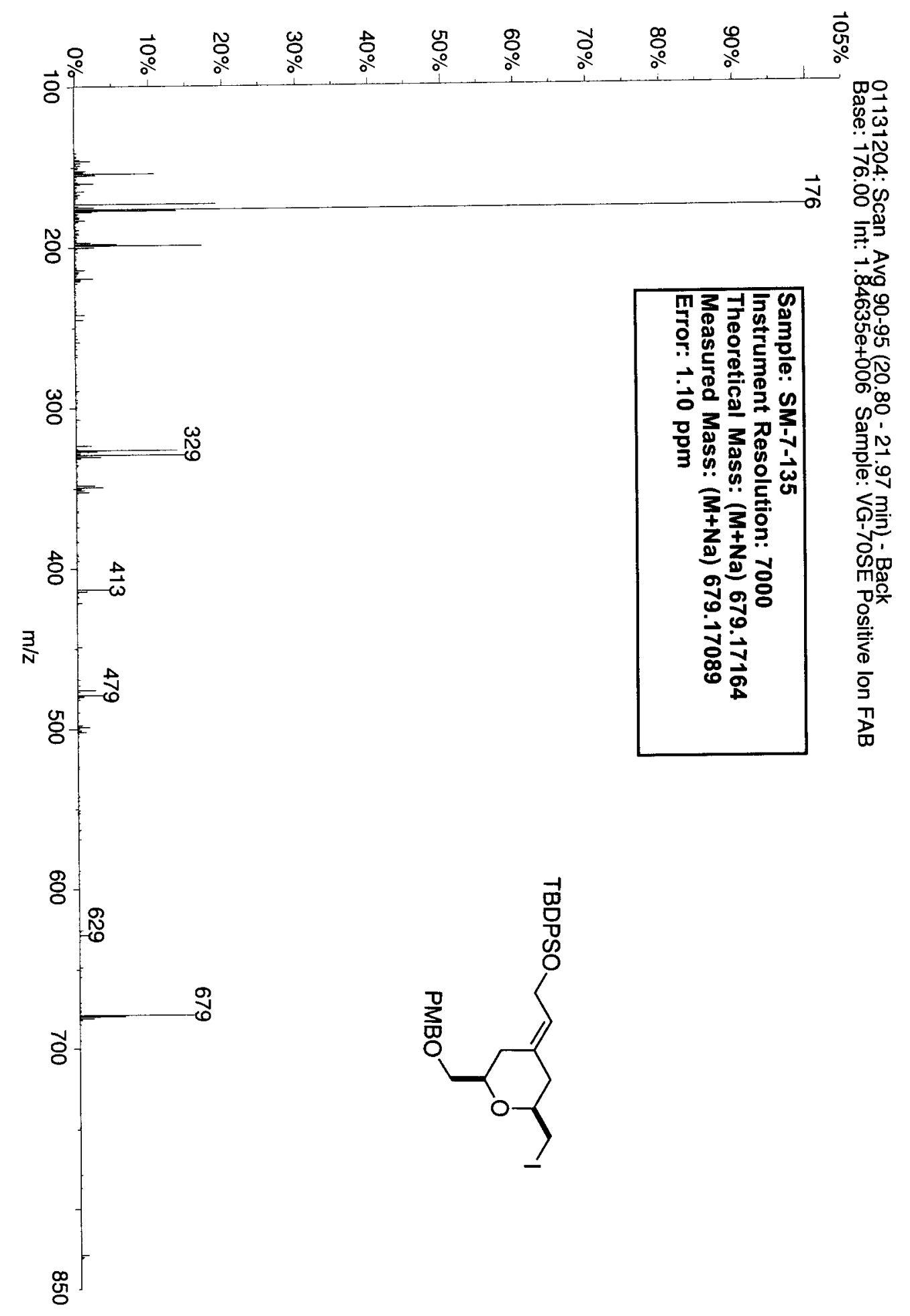




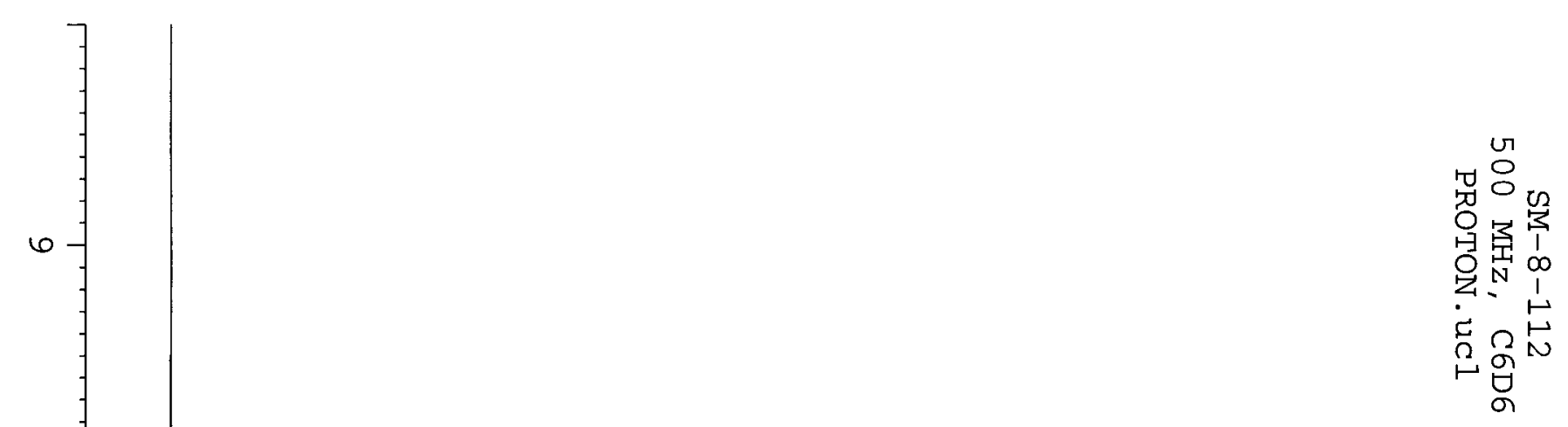

$\infty$

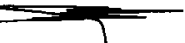

$\checkmark$

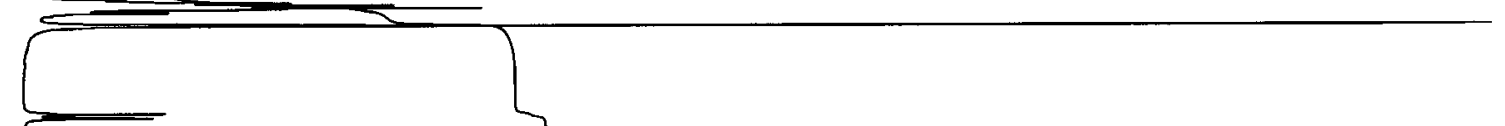

1

$\Delta-1\}$

?

N

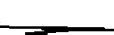

3
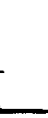

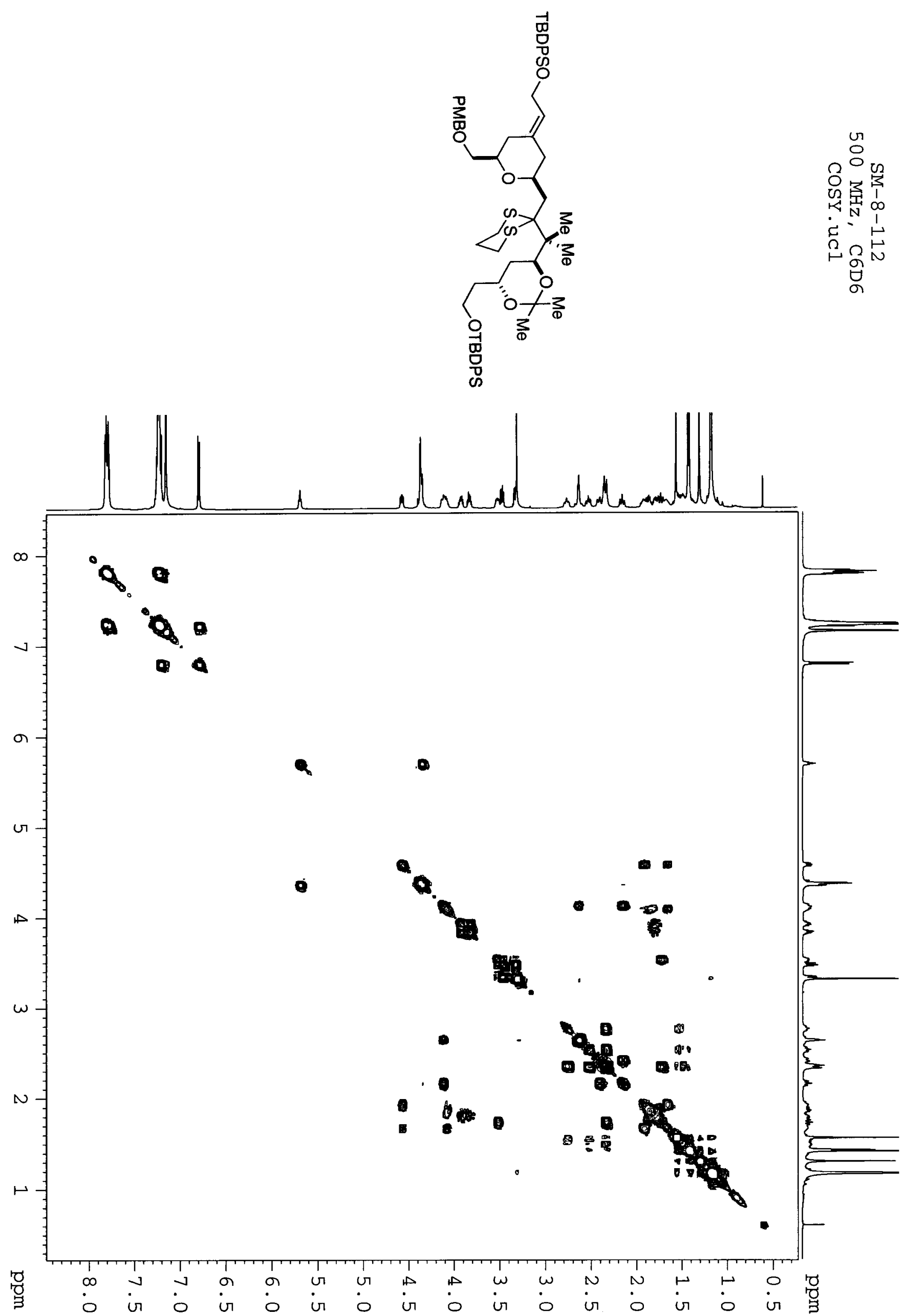

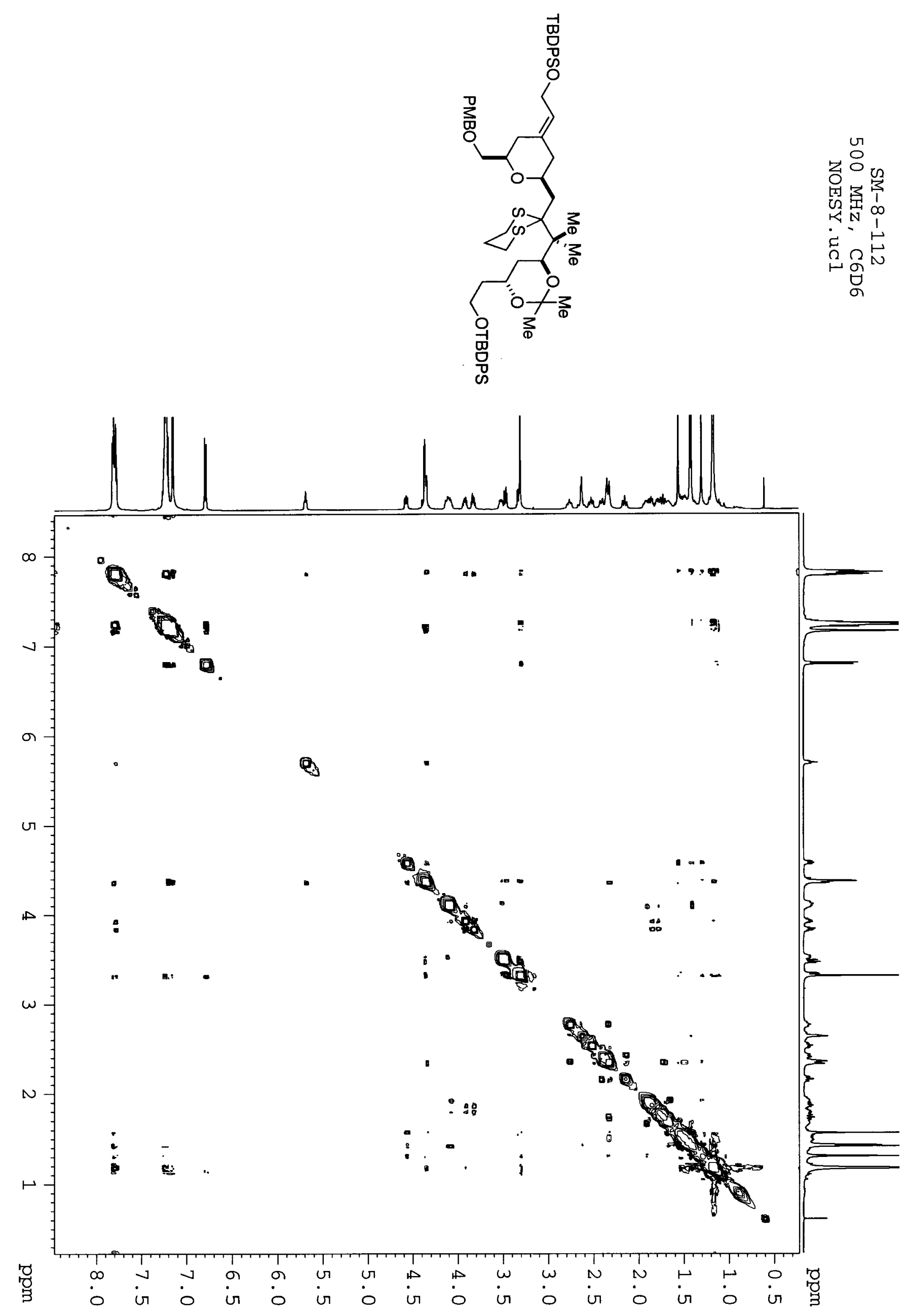


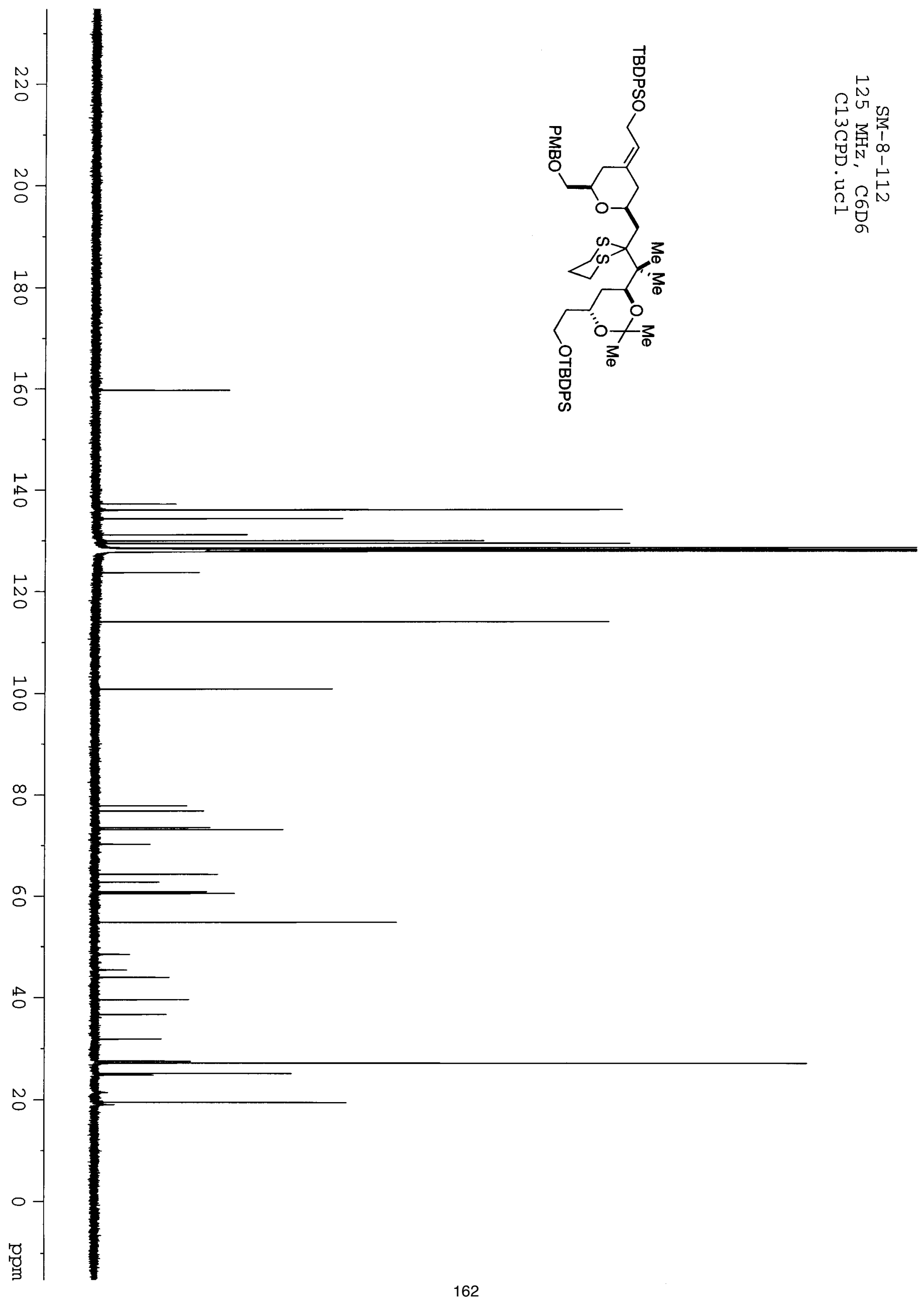




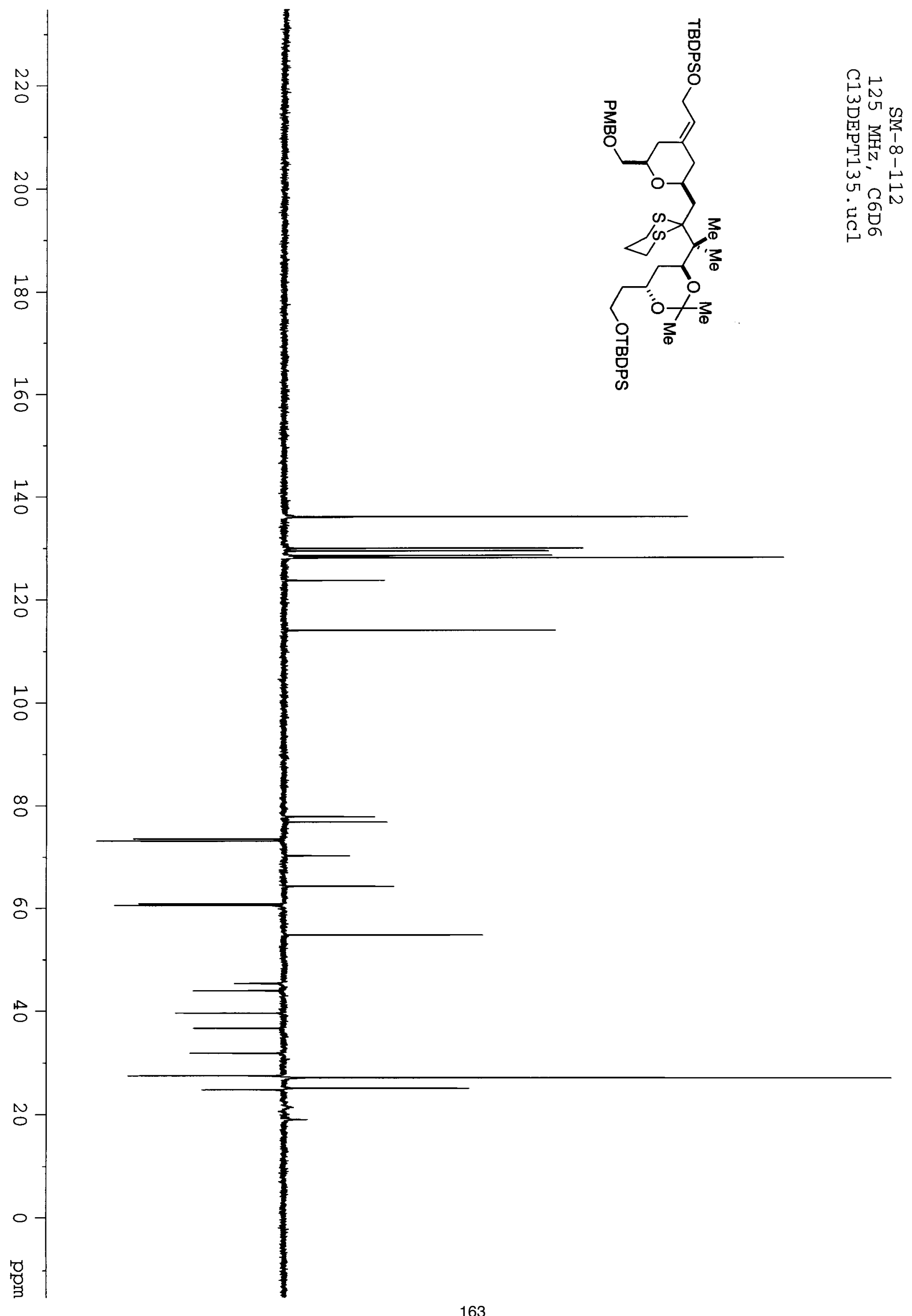




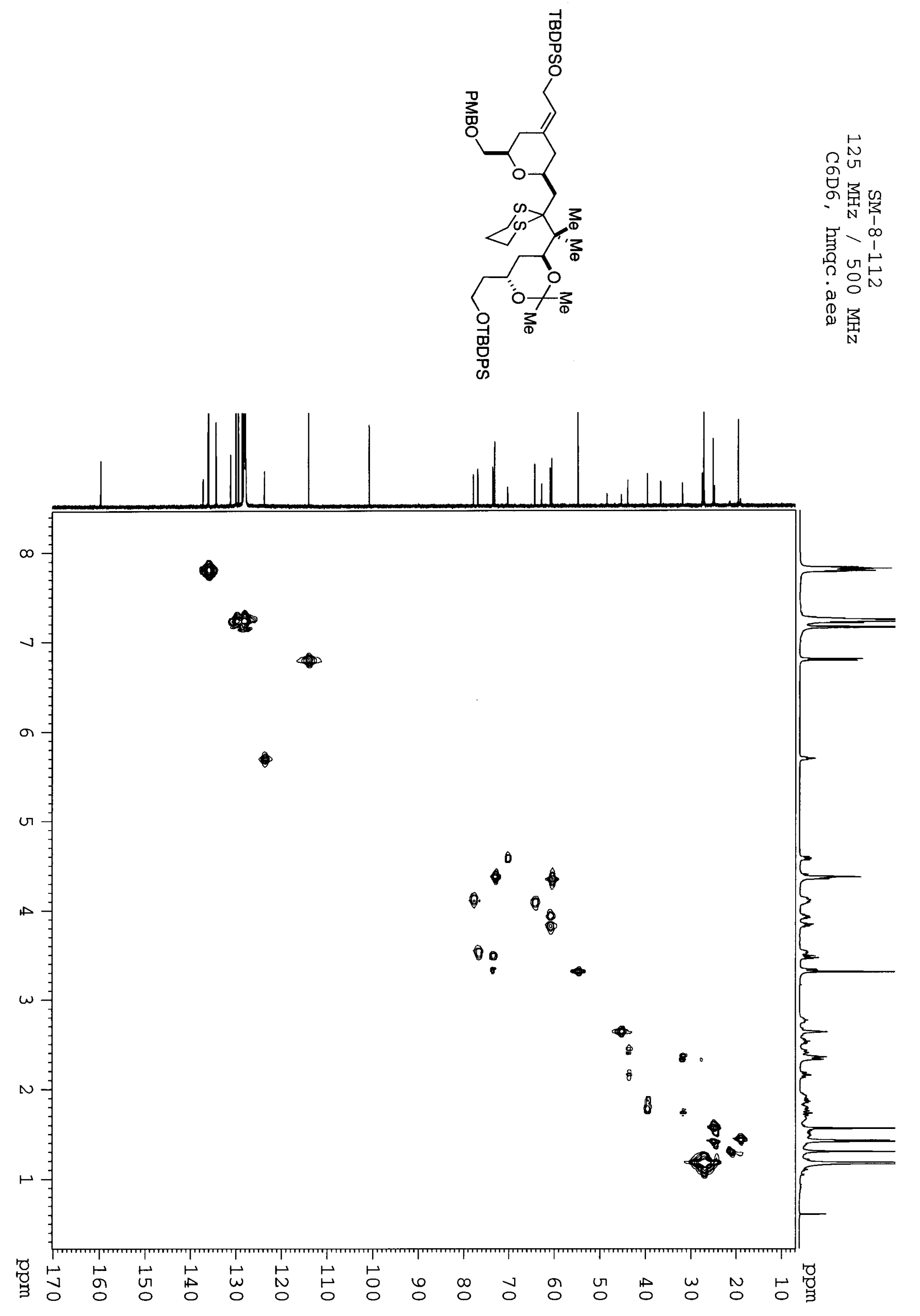




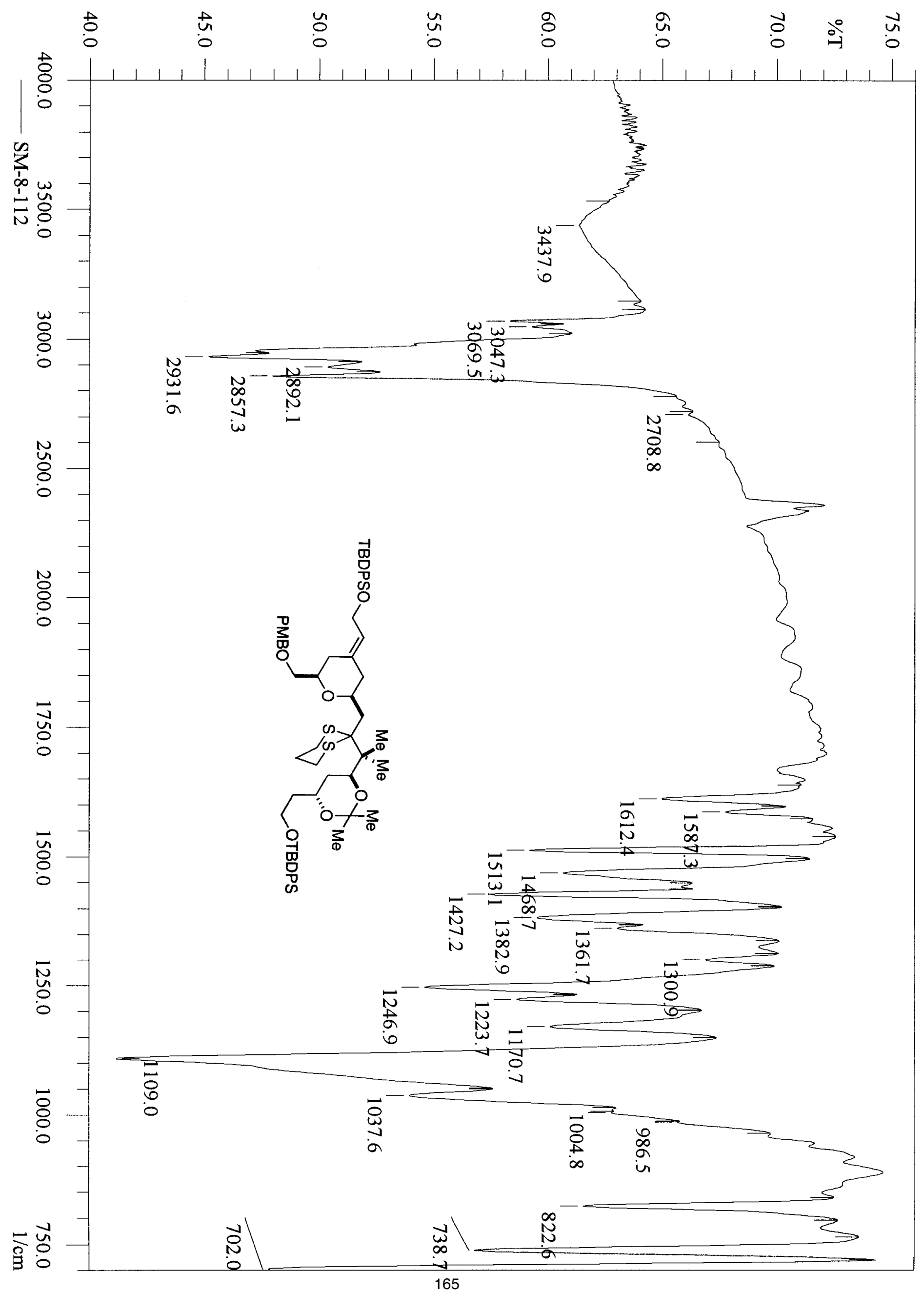




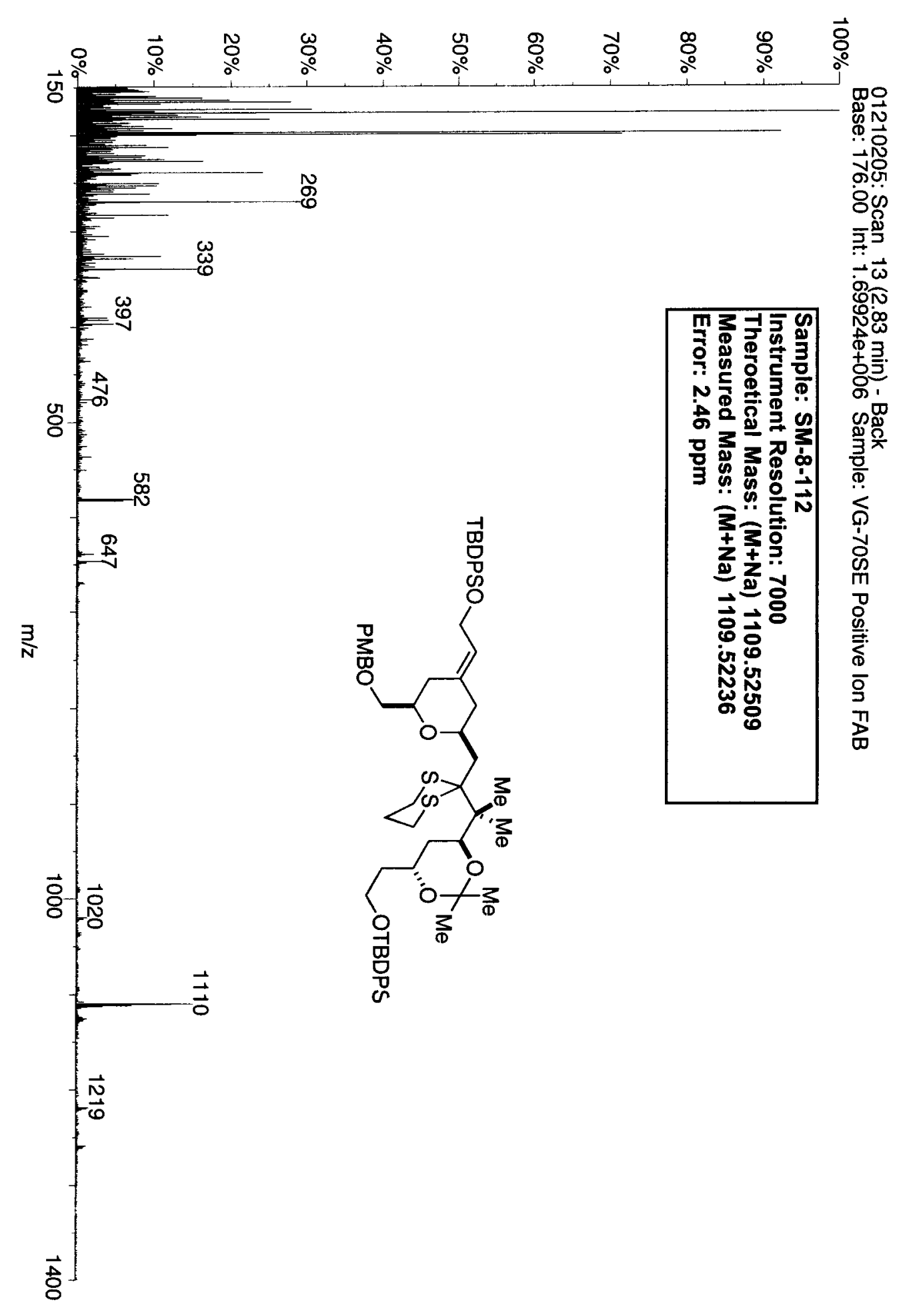




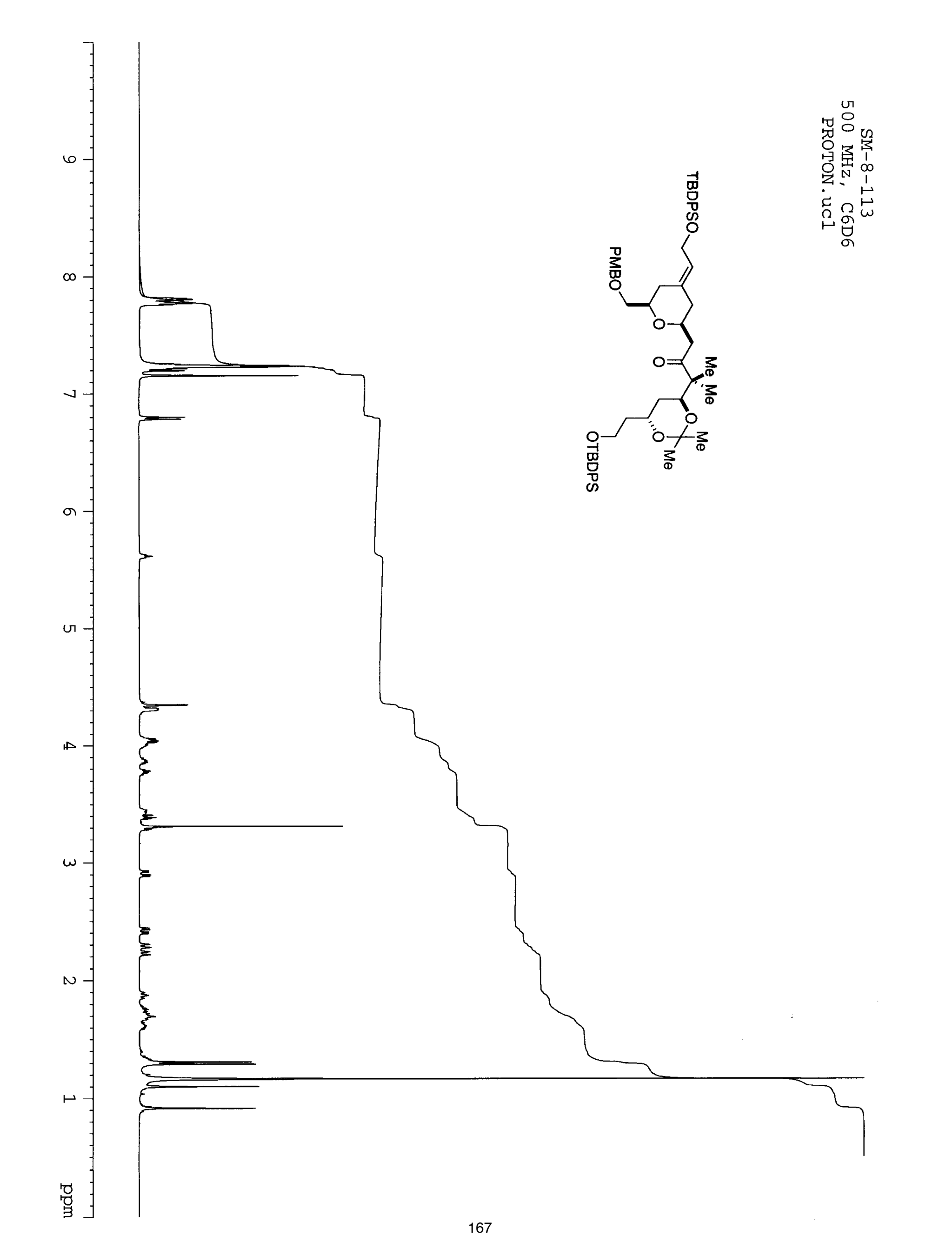



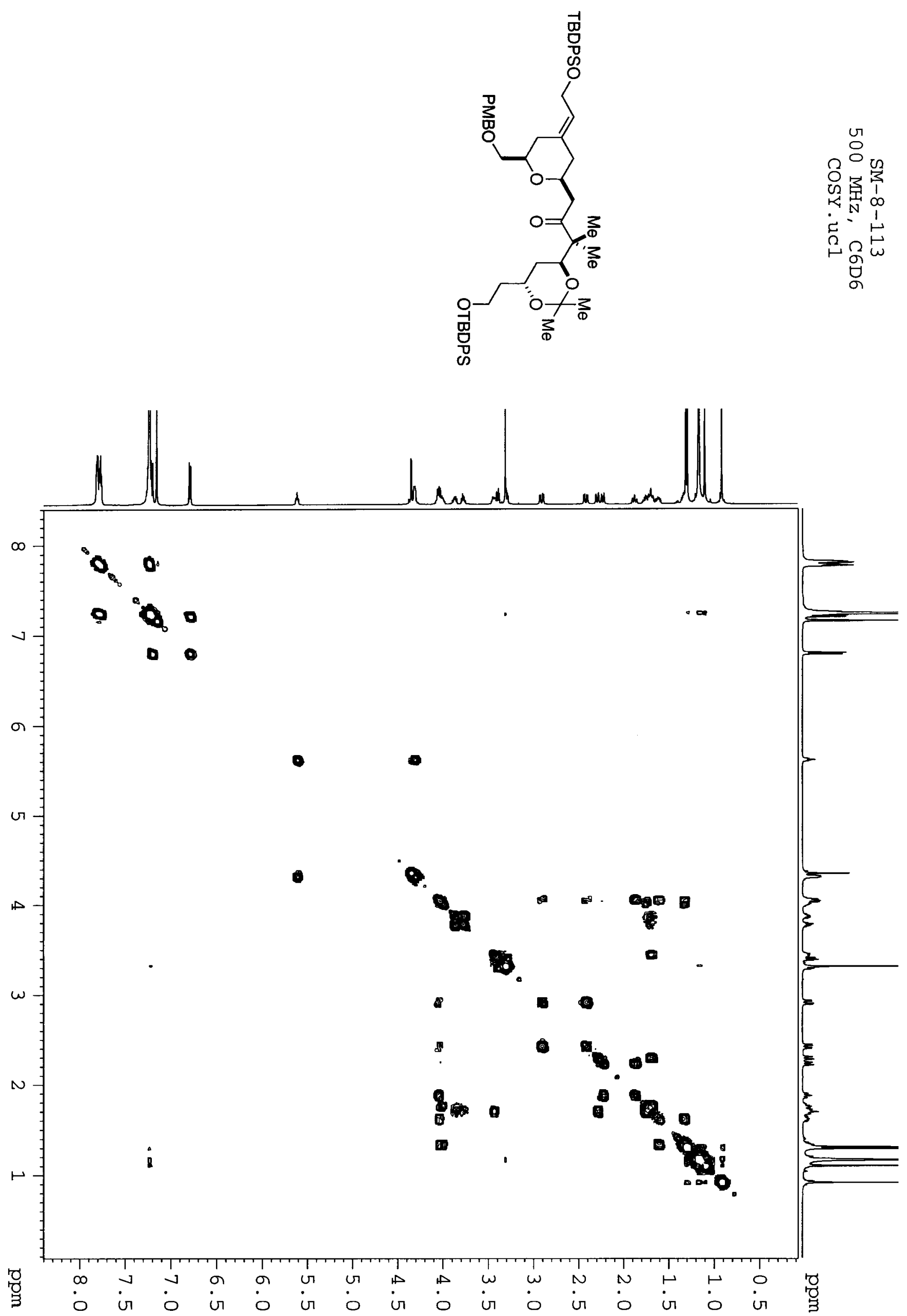

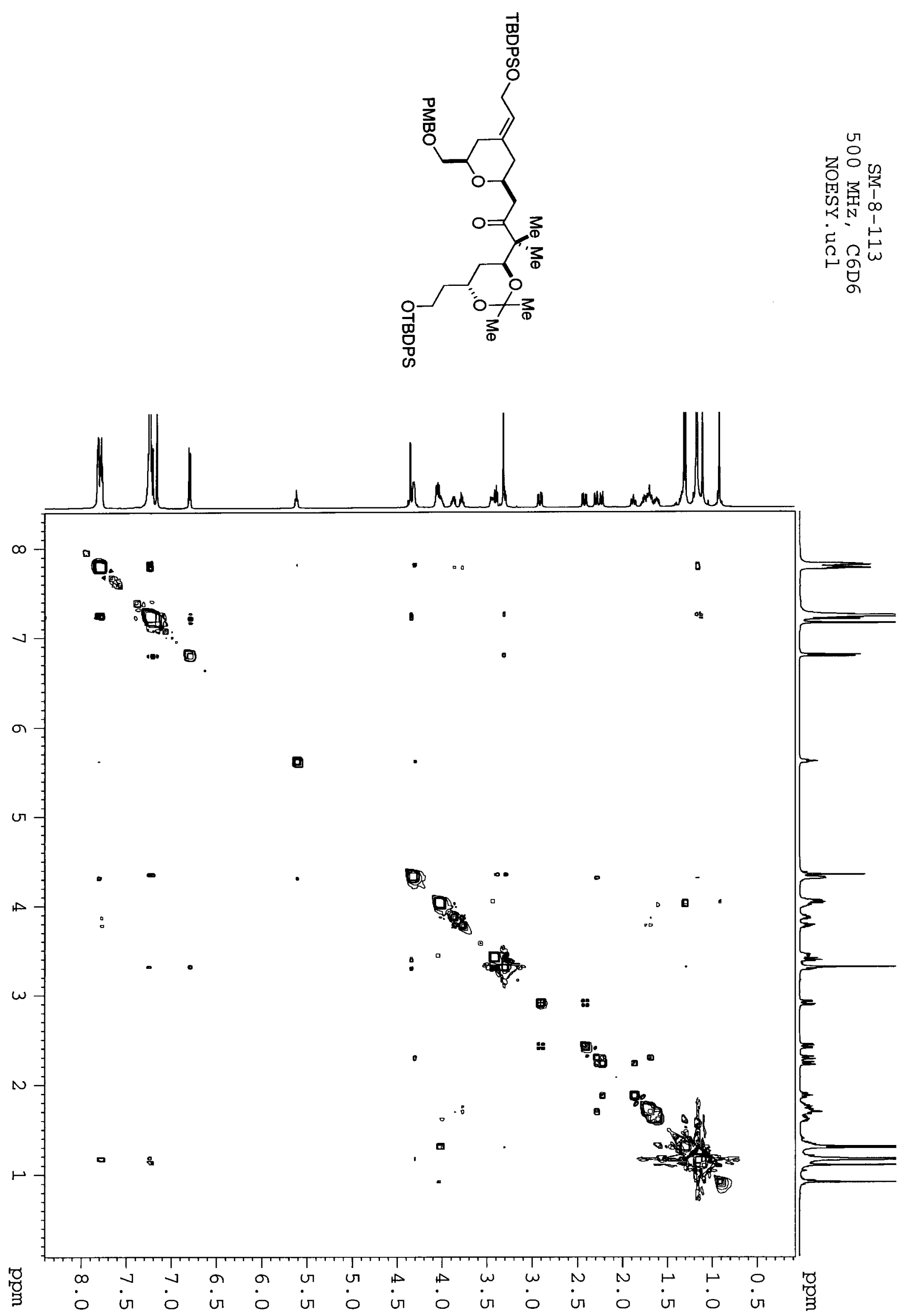


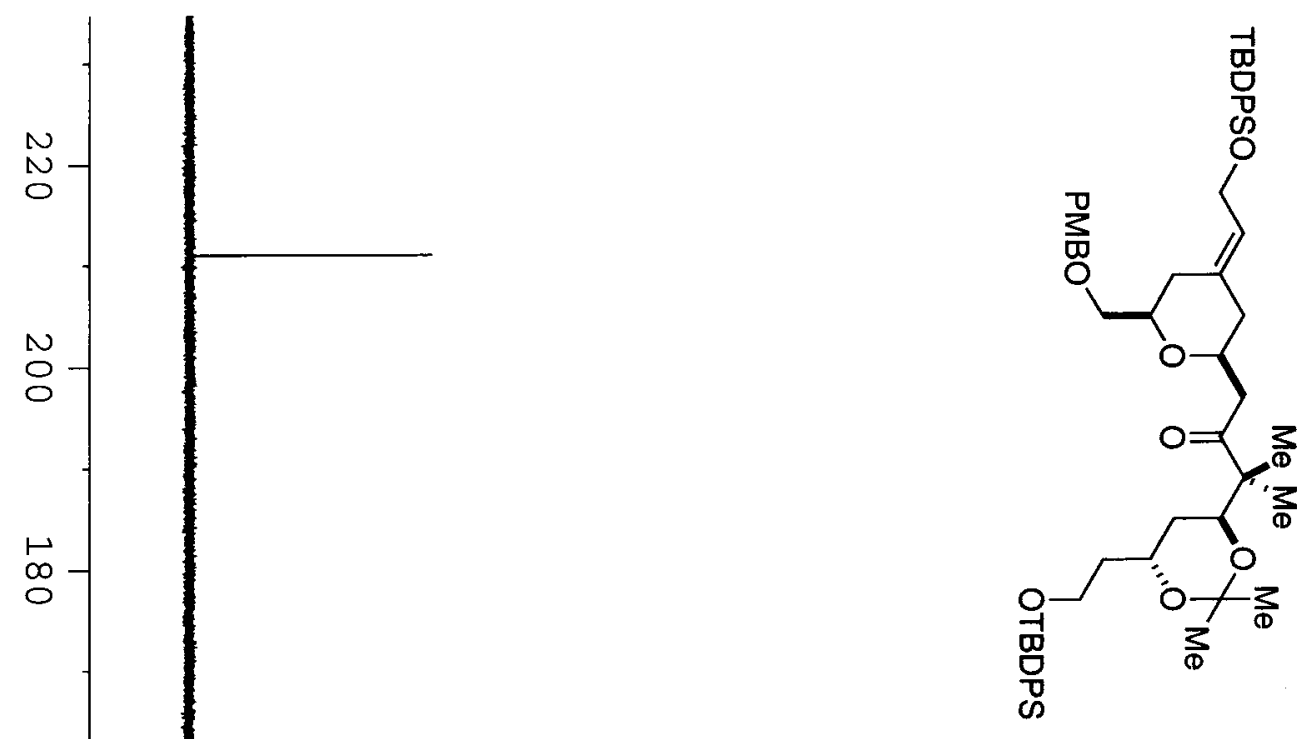

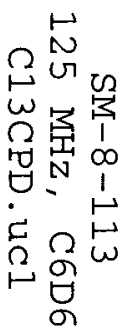

응

品

$\stackrel{p}{0}$

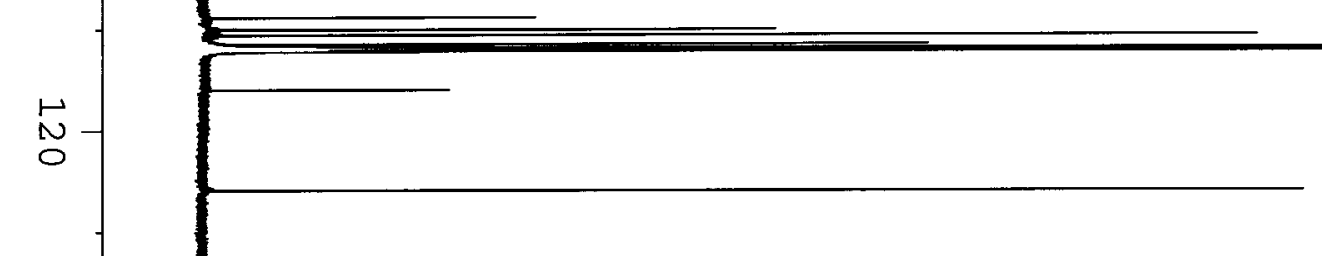

:

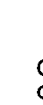

$\infty$

。

o

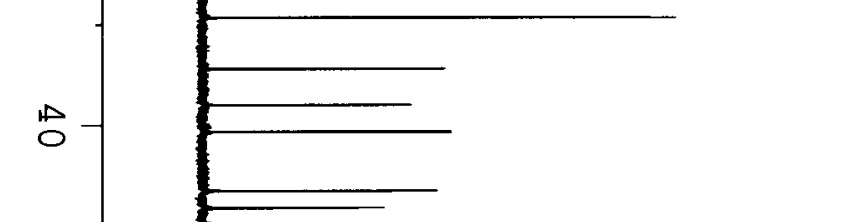

N

照

. 


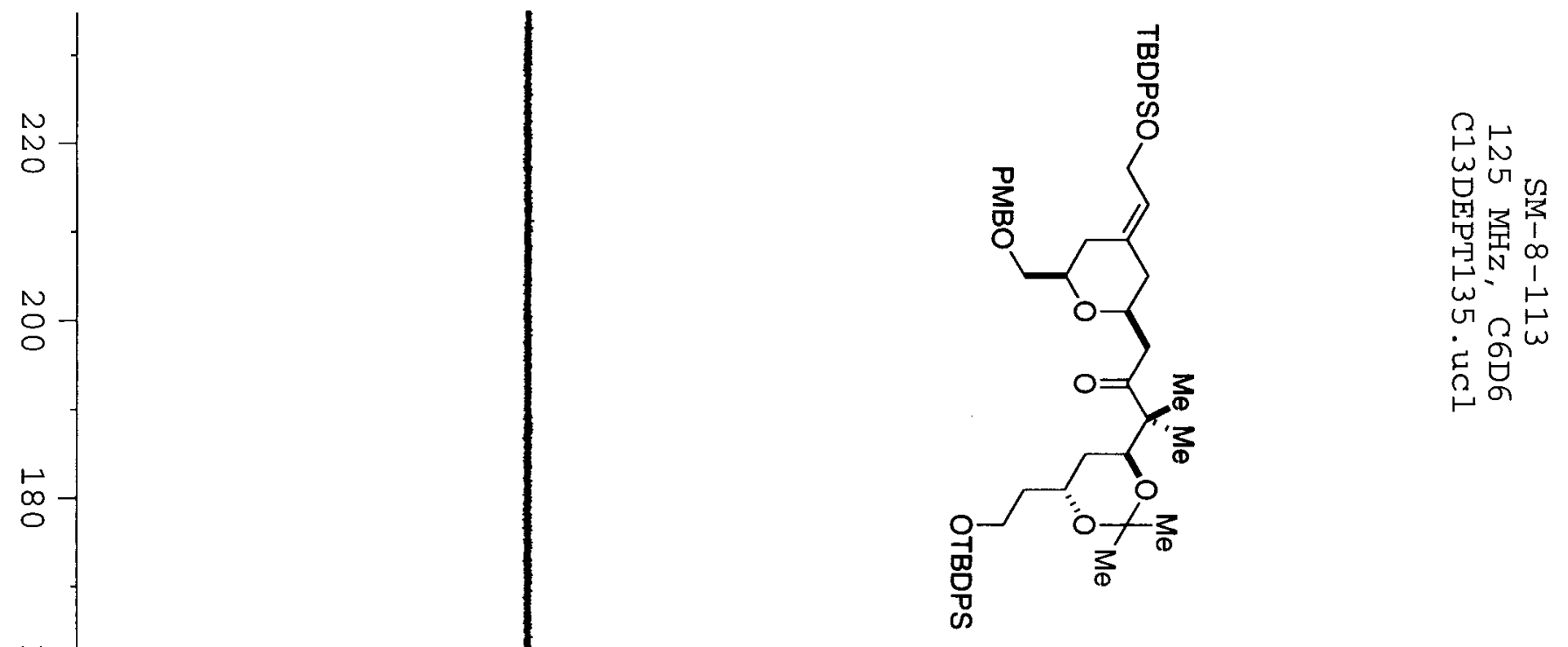

م

$\stackrel{D}{0}$

N

•
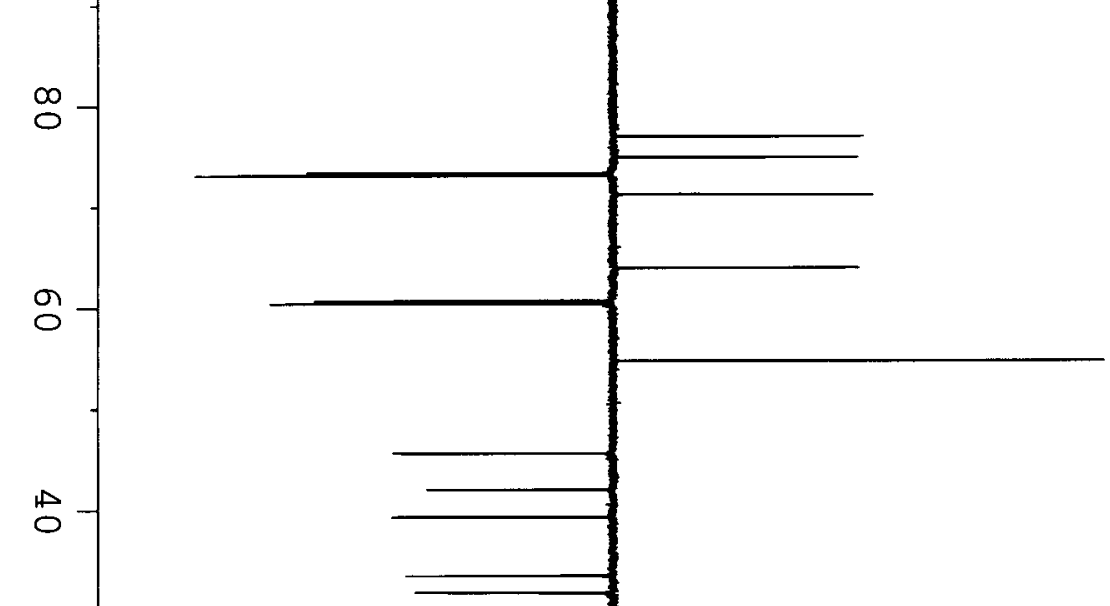

N

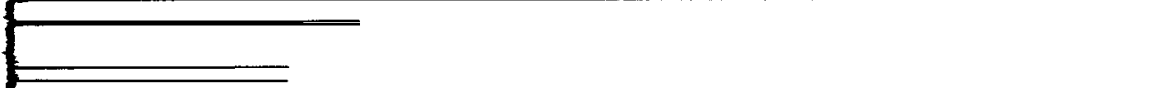

- 

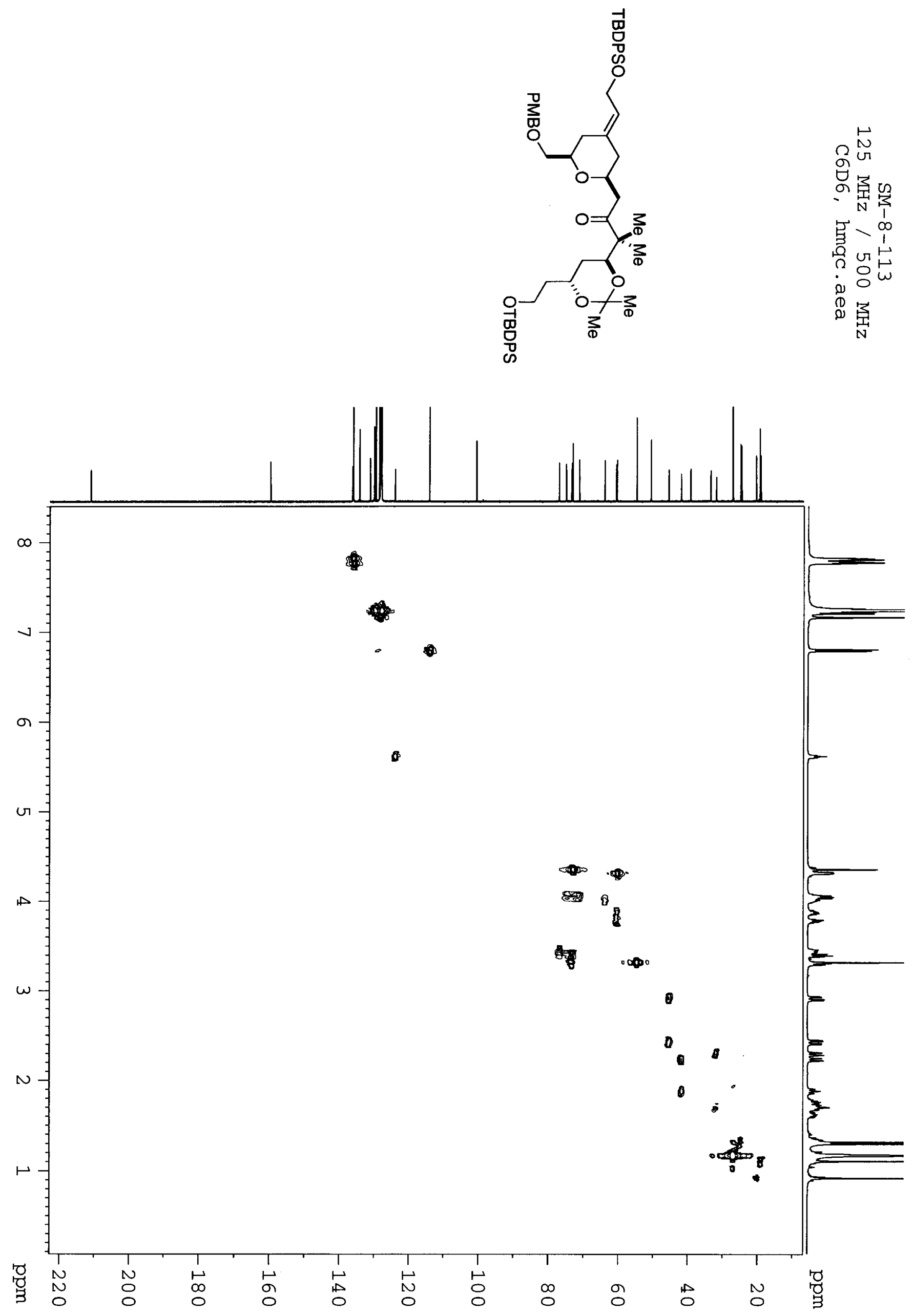


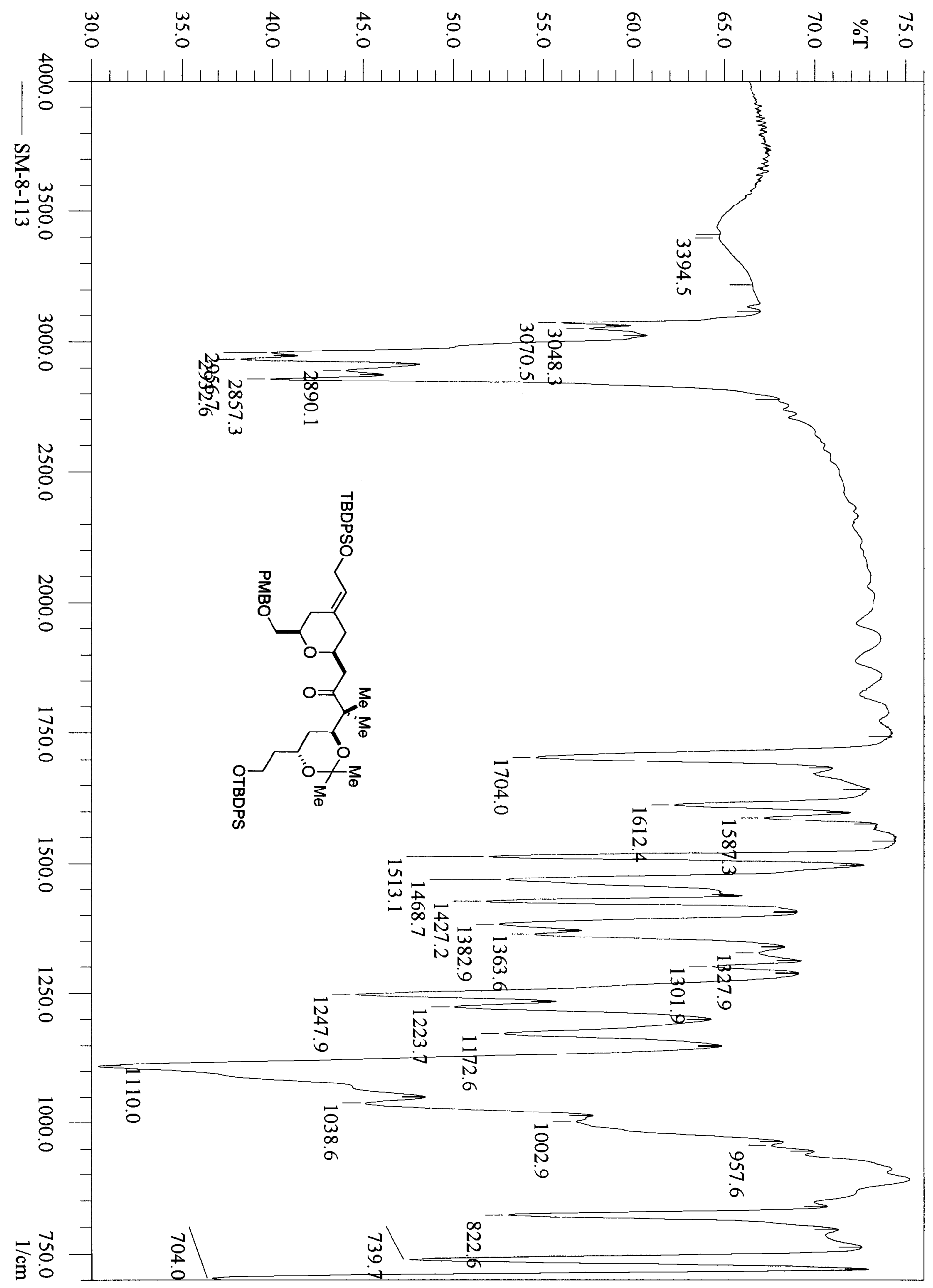




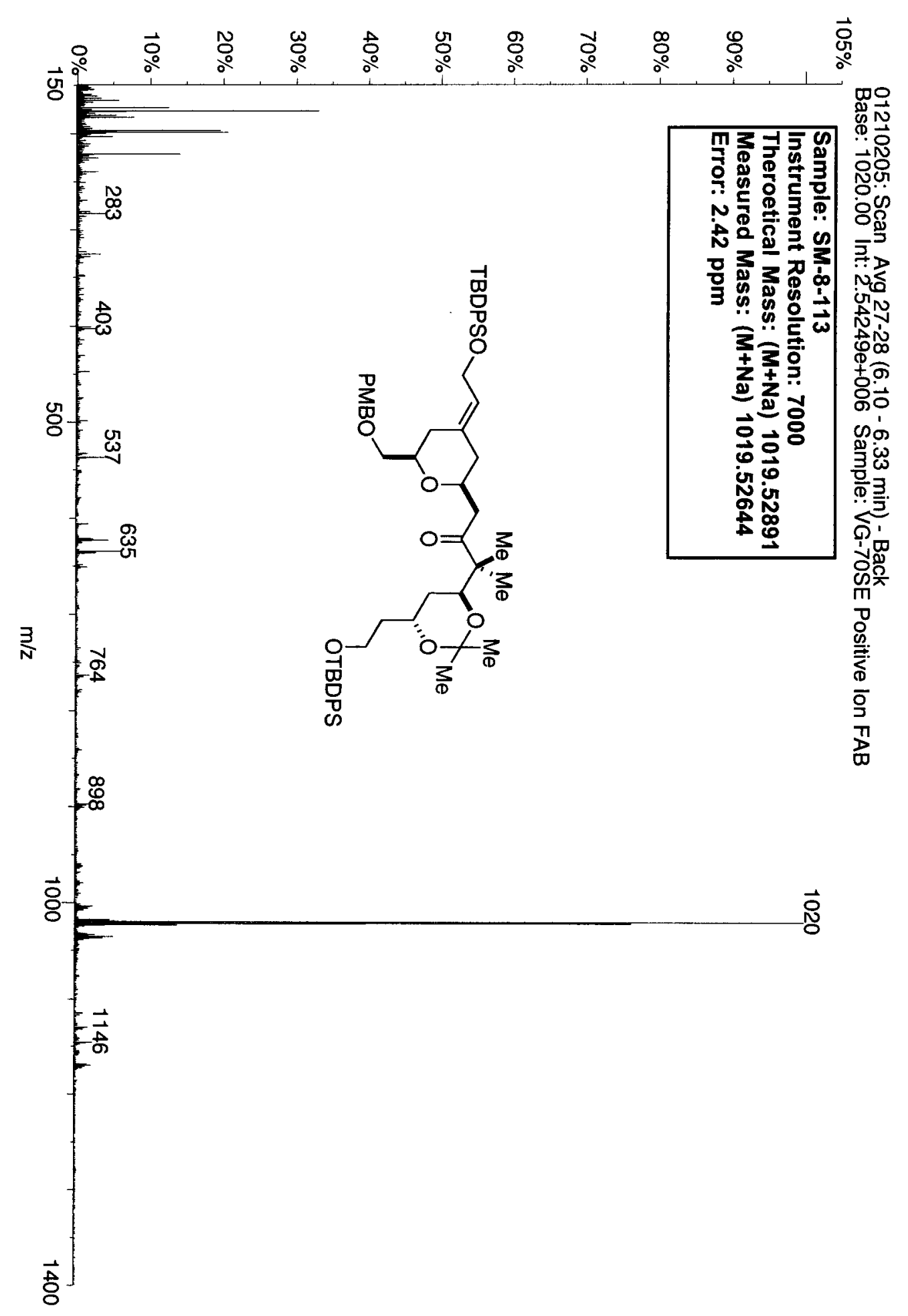




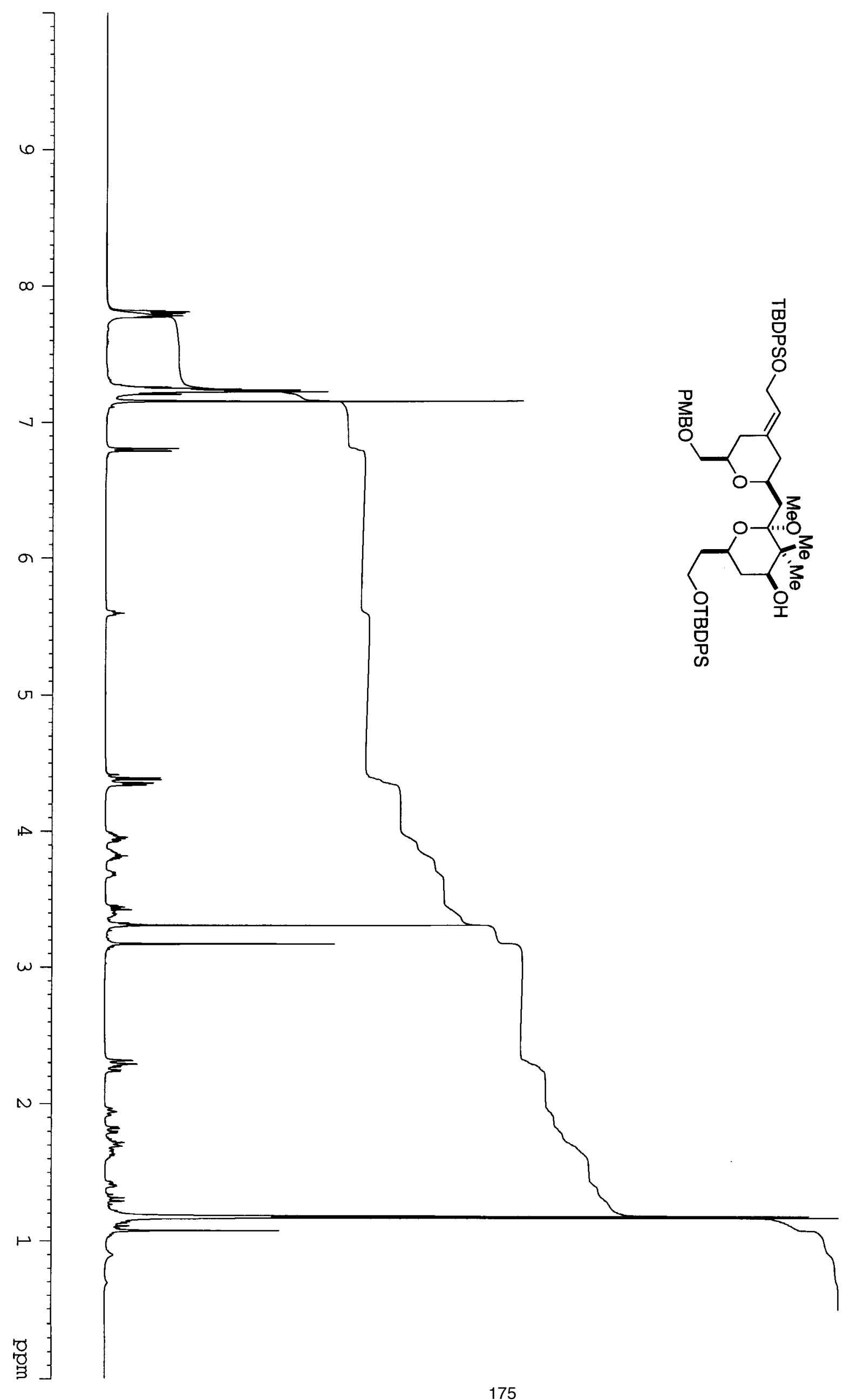

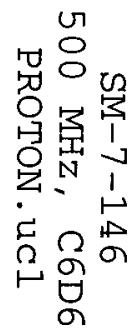



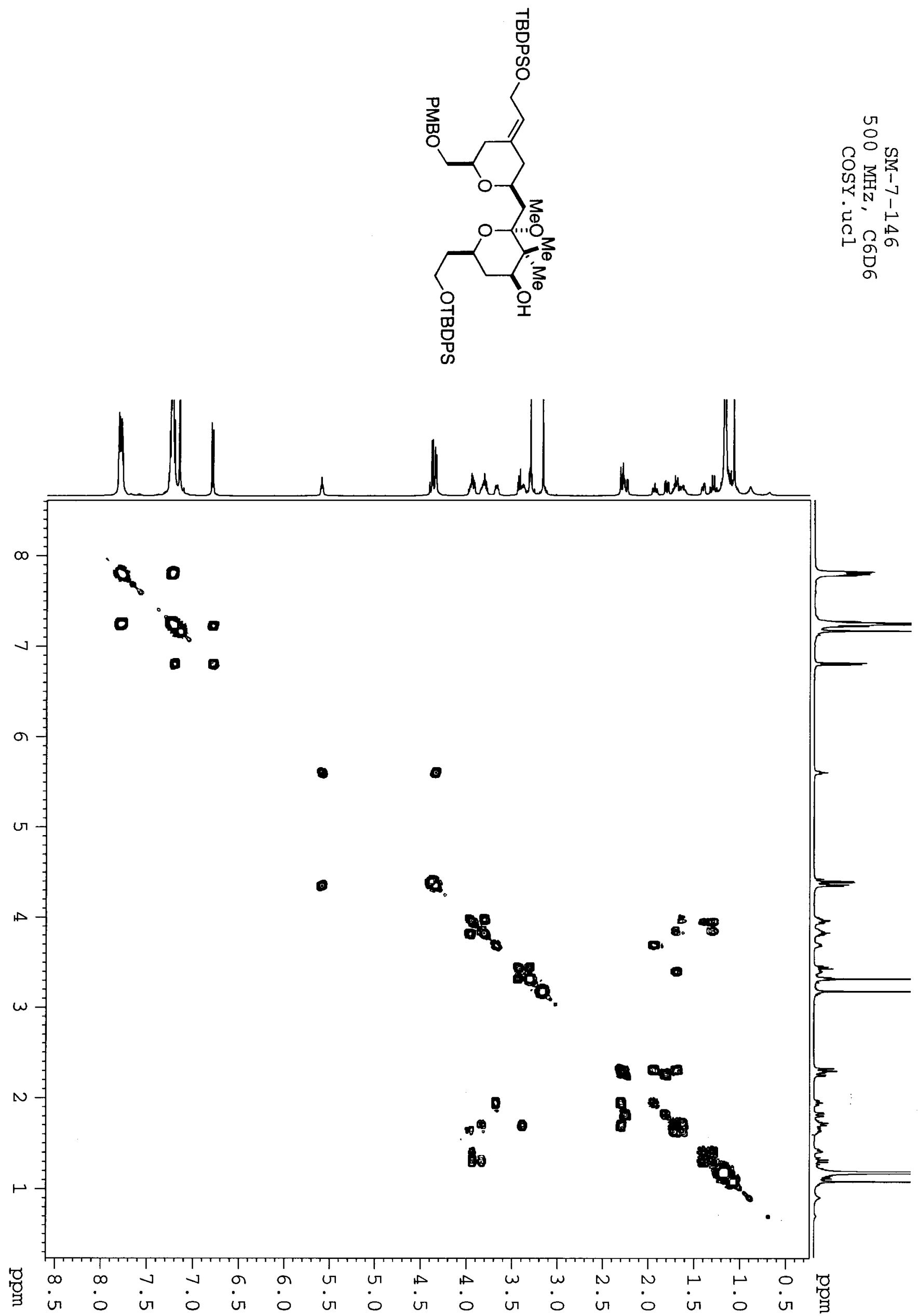

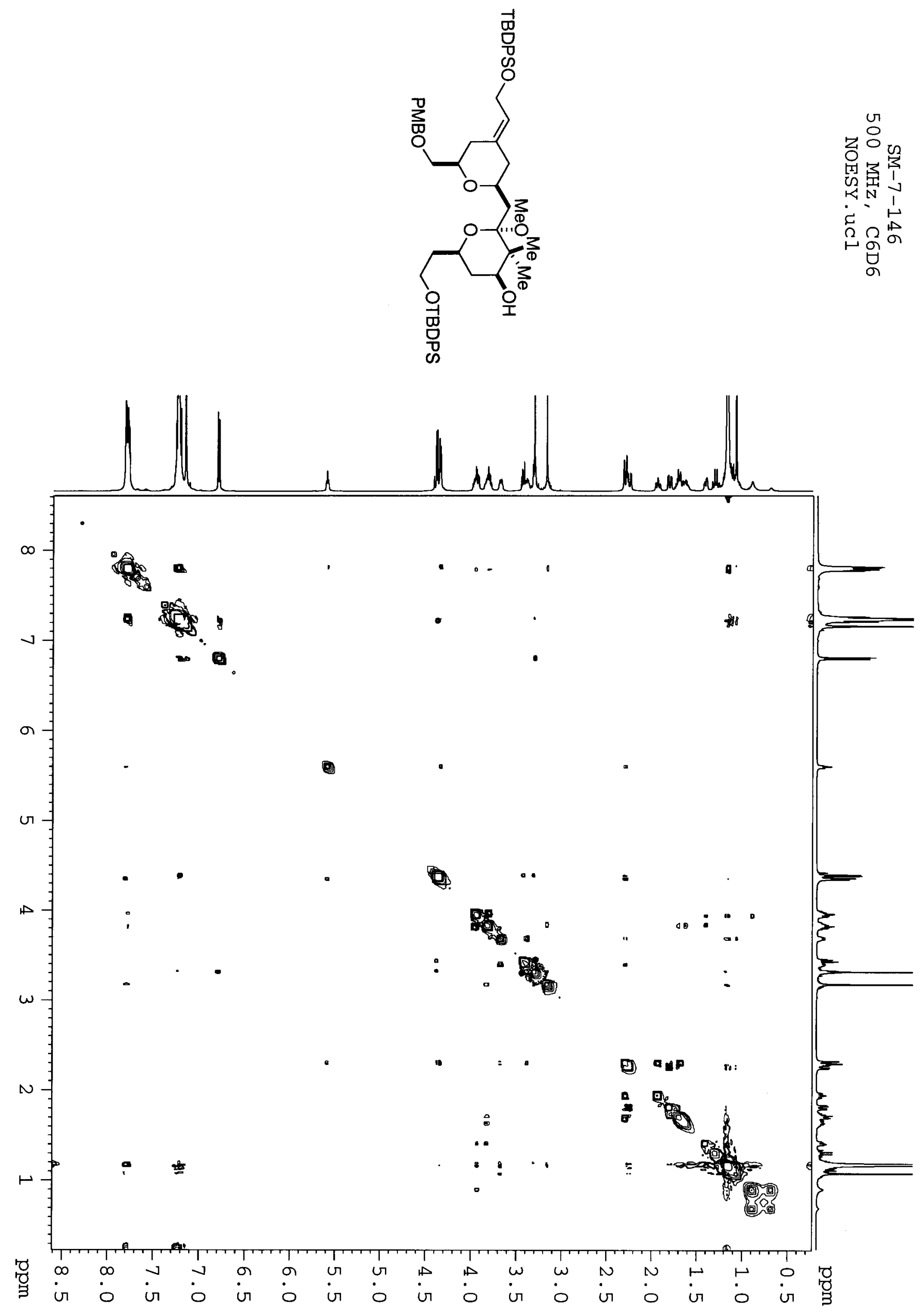

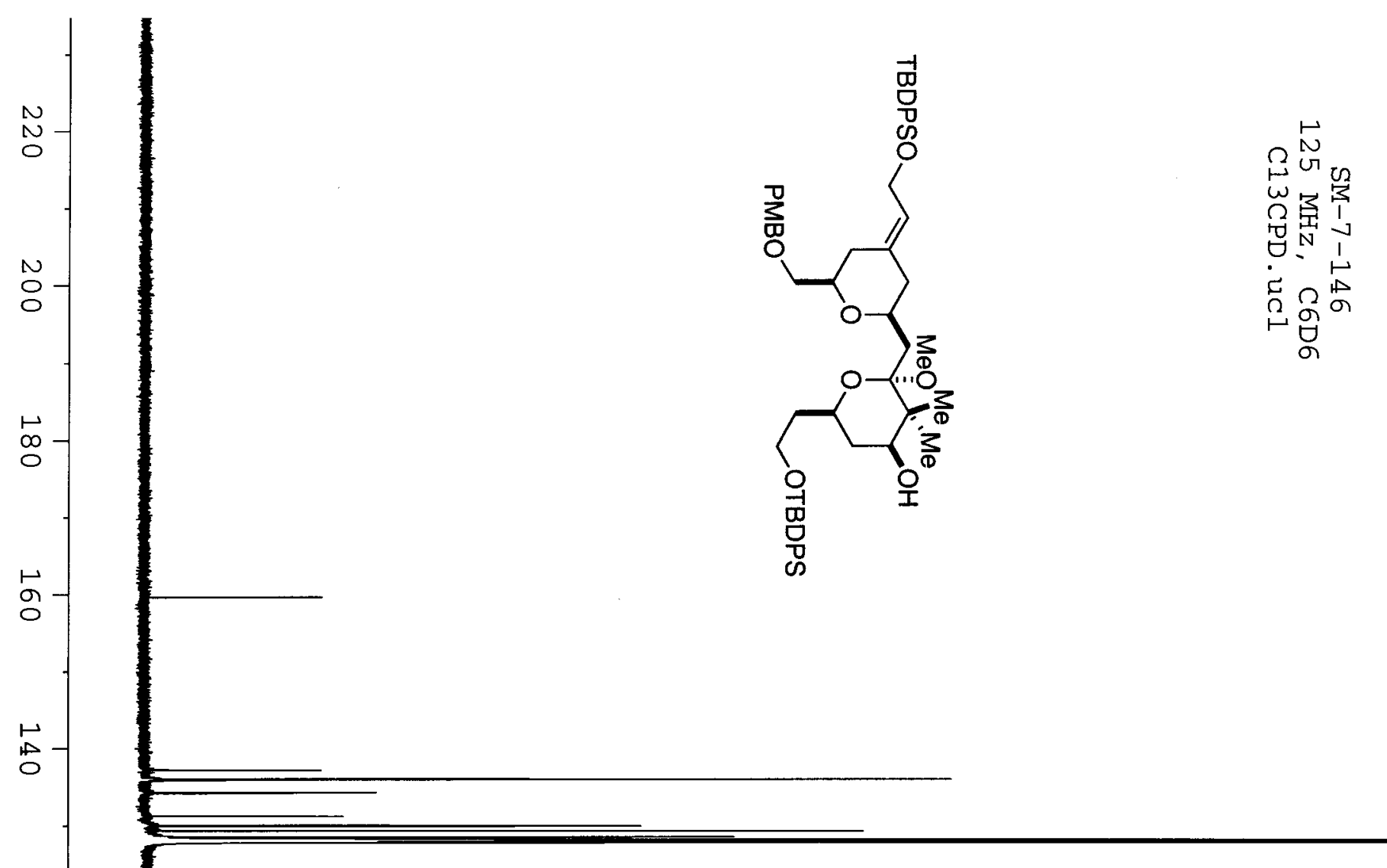

心 .

:

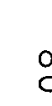

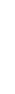

응

$\stackrel{B}{0}$

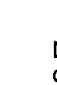

N

署

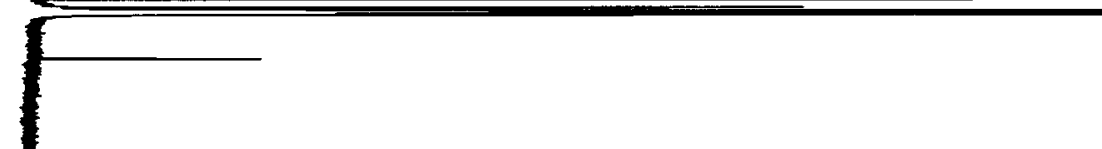




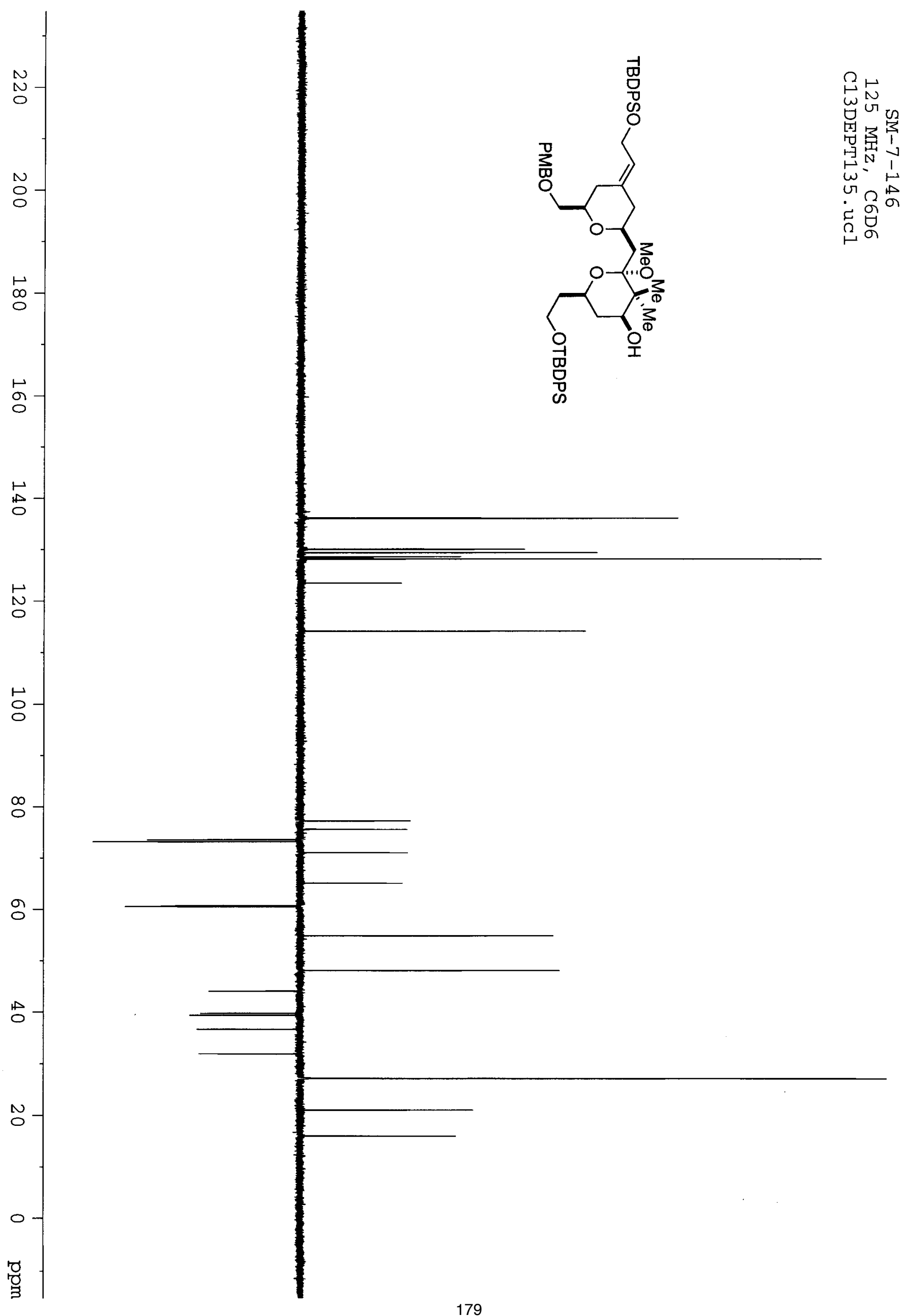



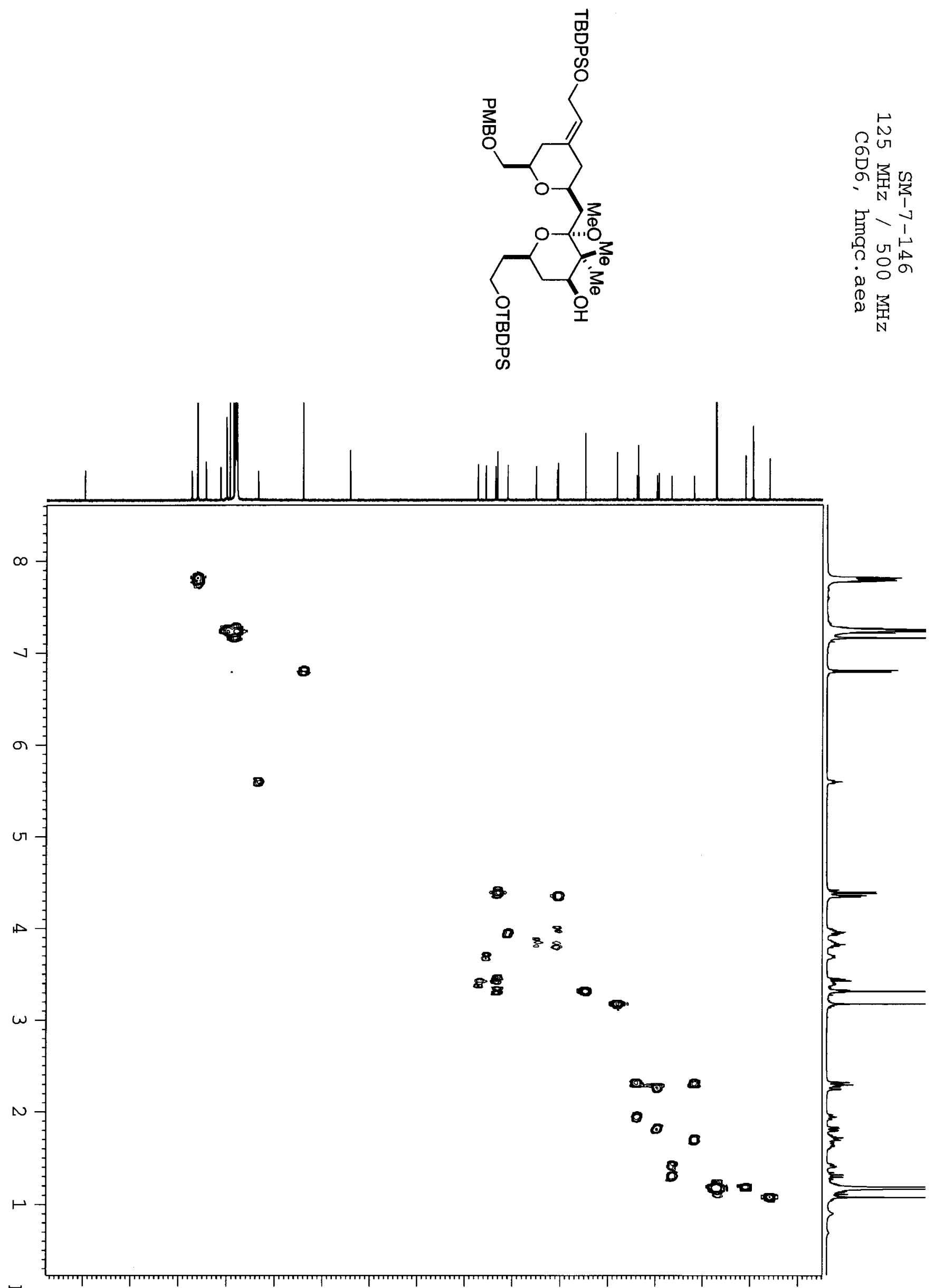

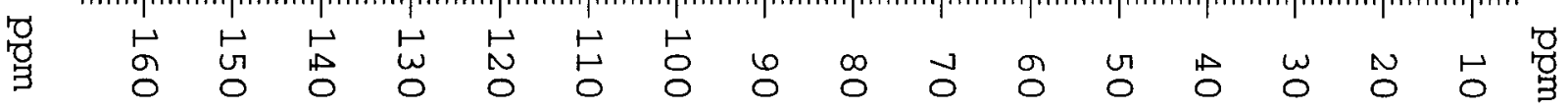




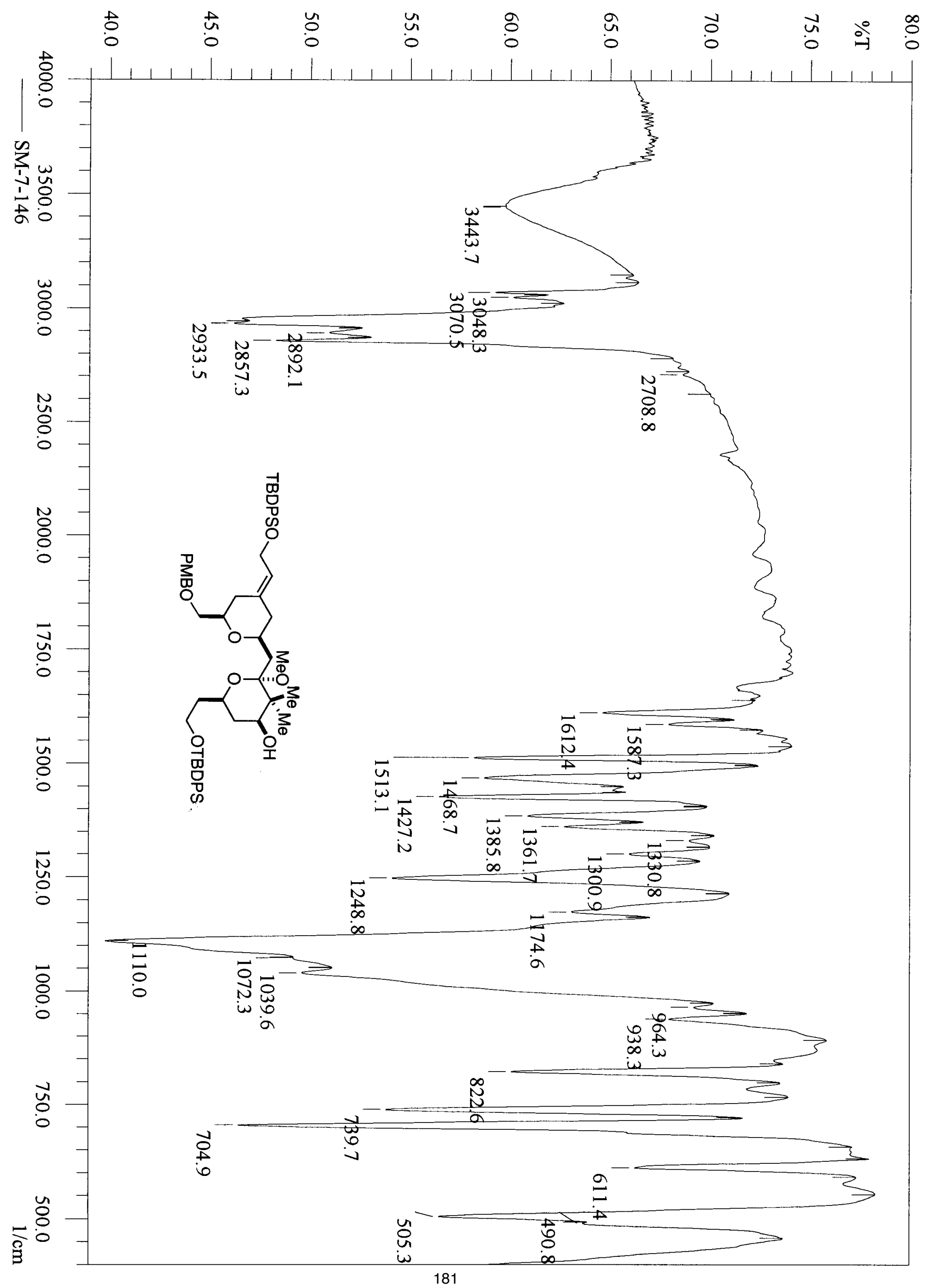




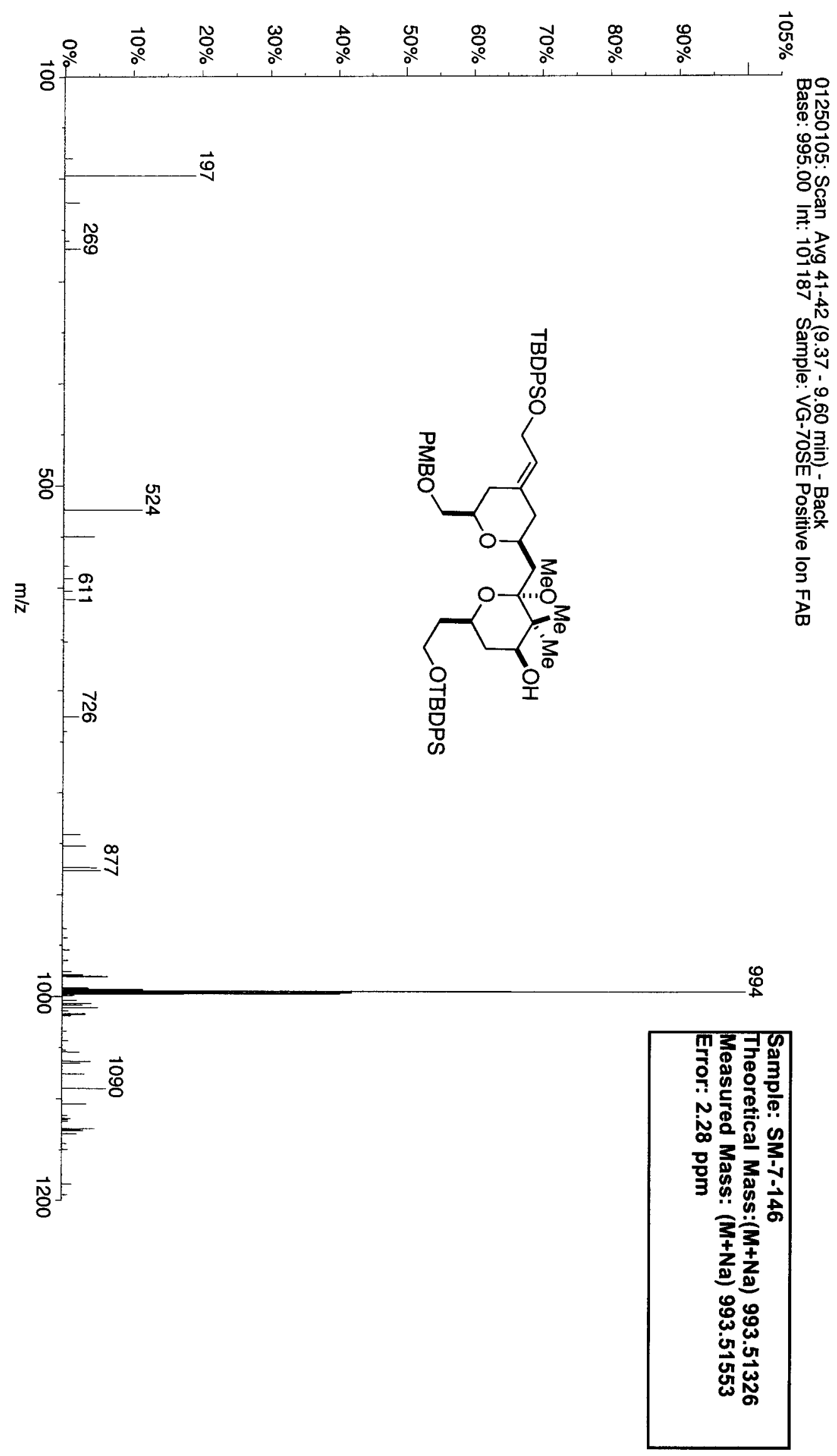




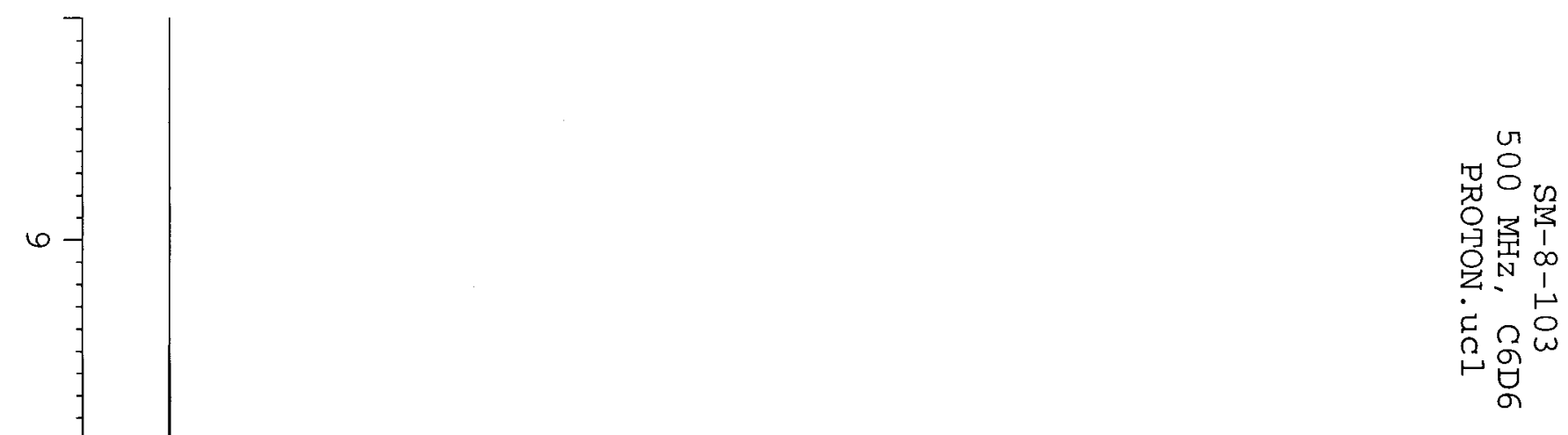

$\infty$

.

o

a

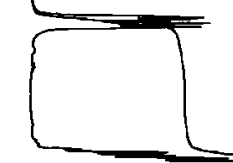

G

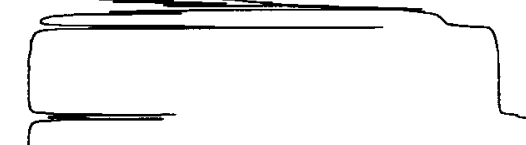

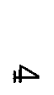

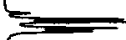

\}

$\omega$

$\sum$



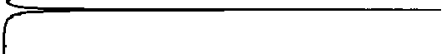

产

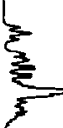

$\xi$

-

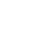




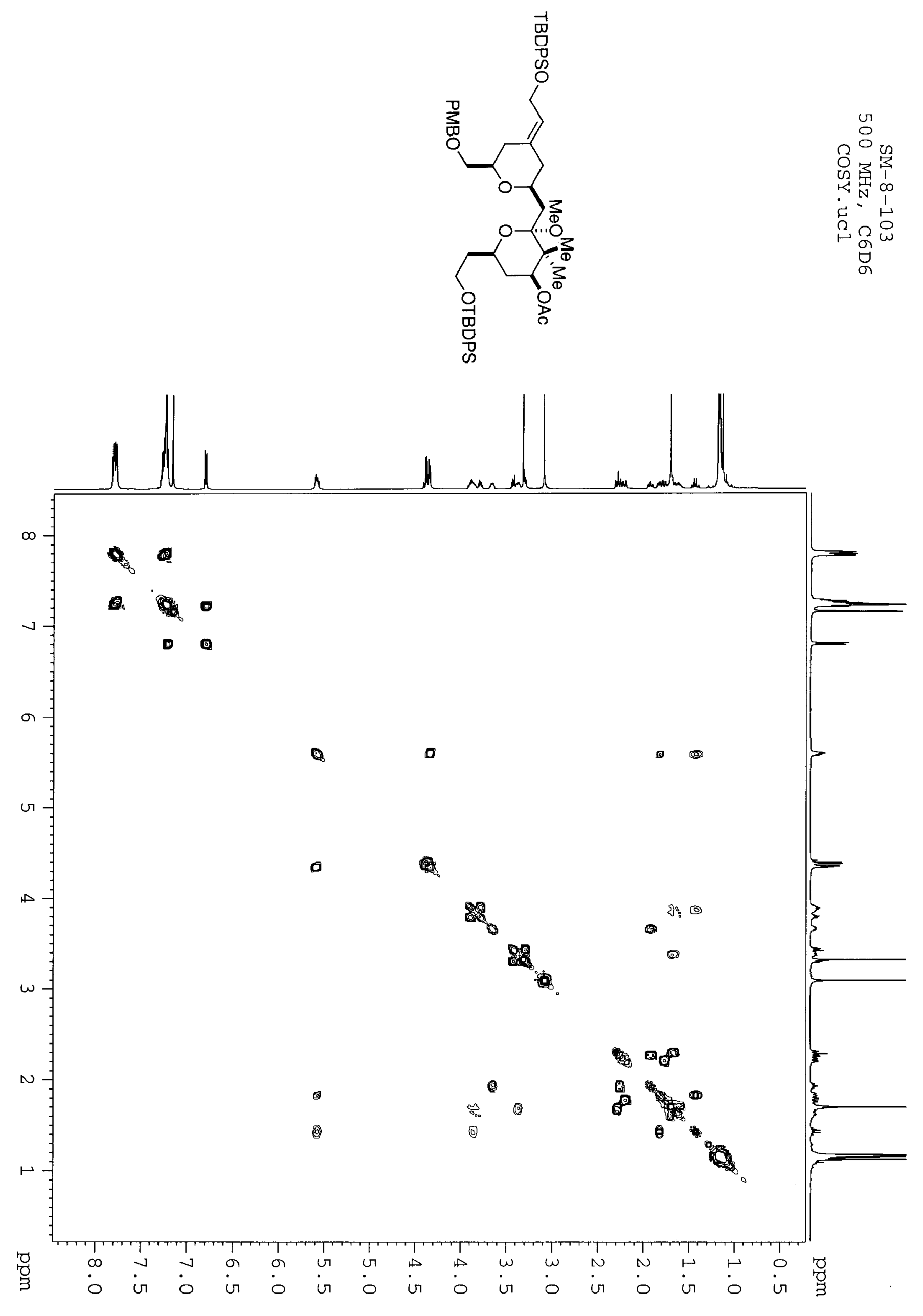



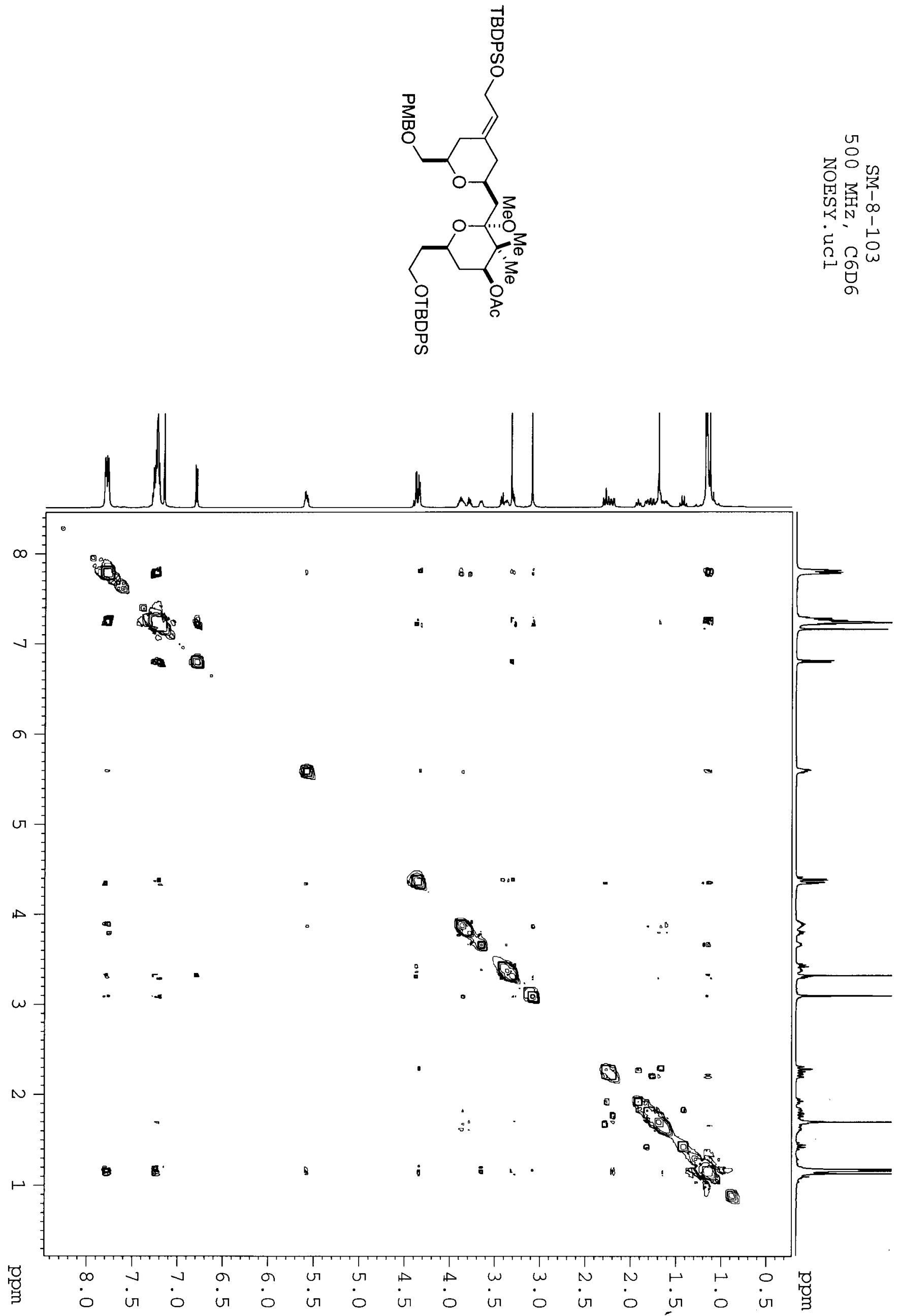


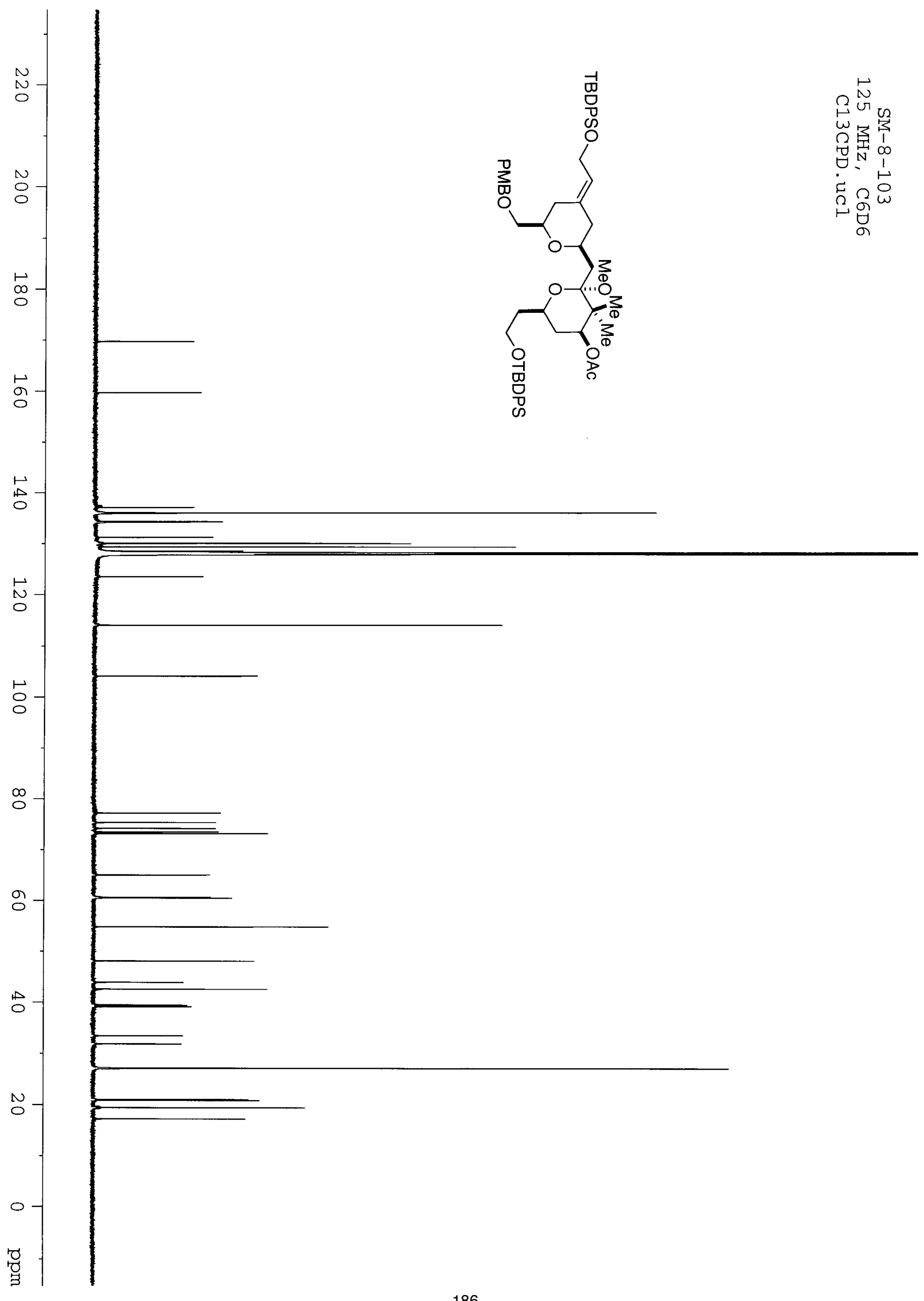




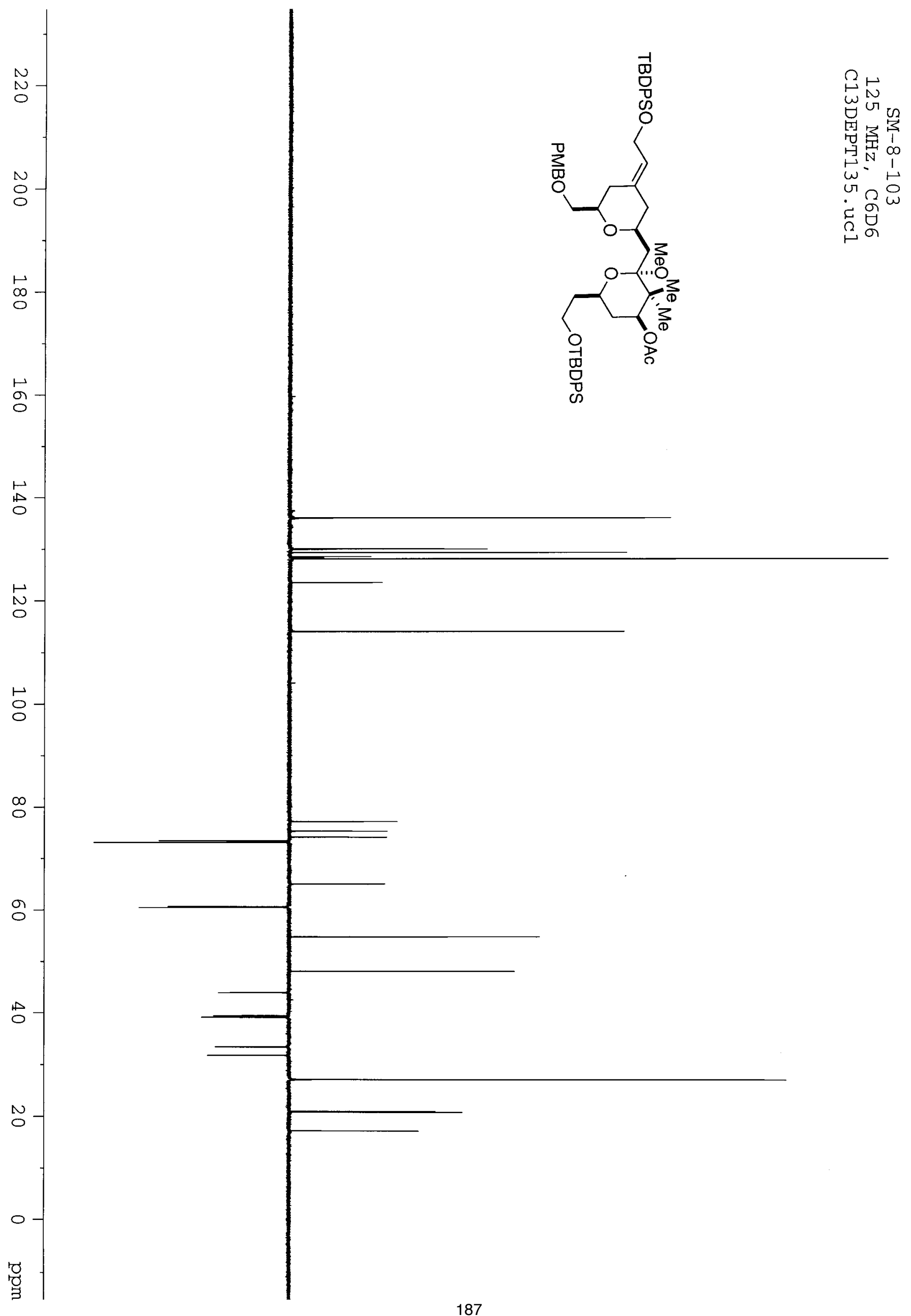



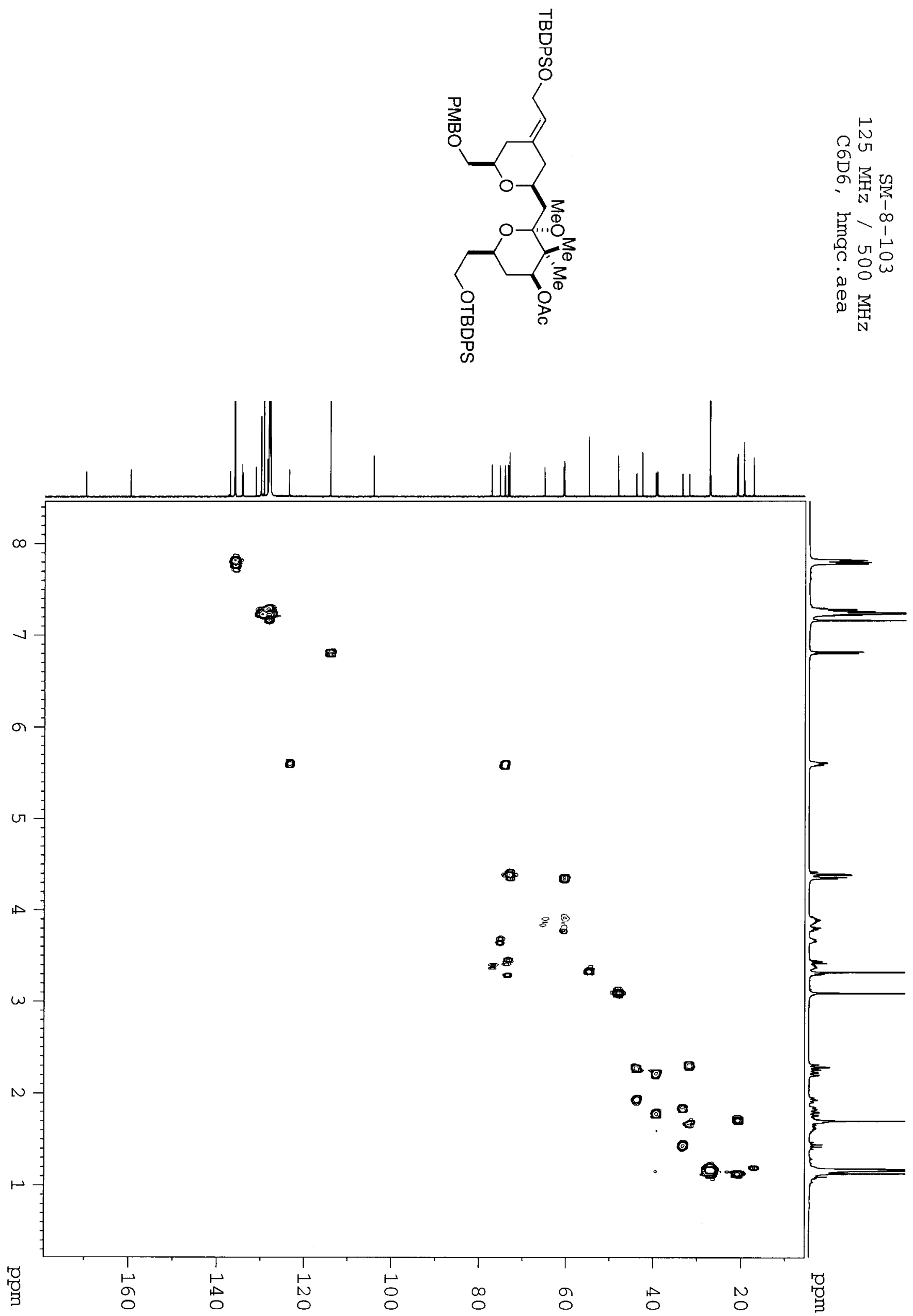


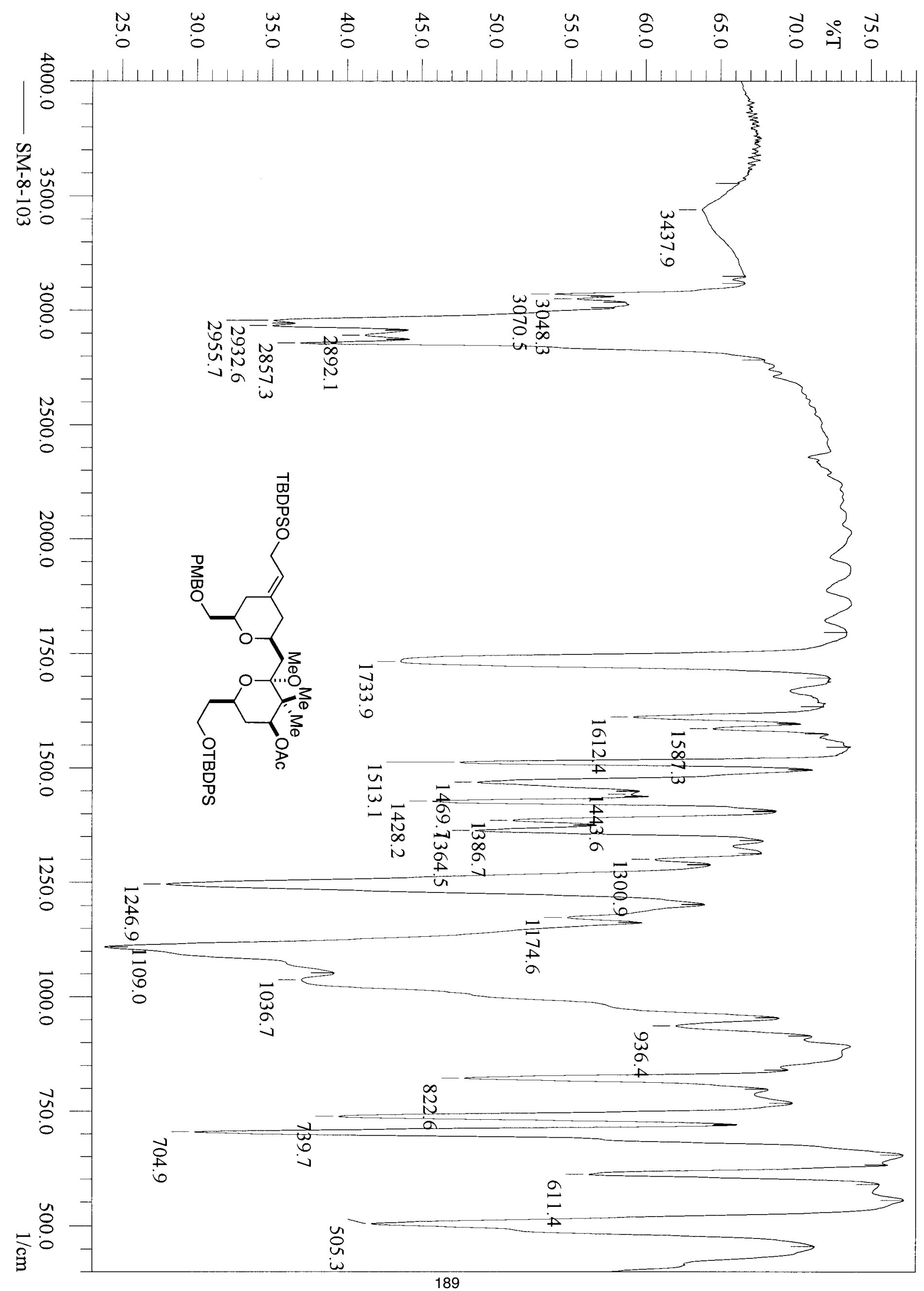




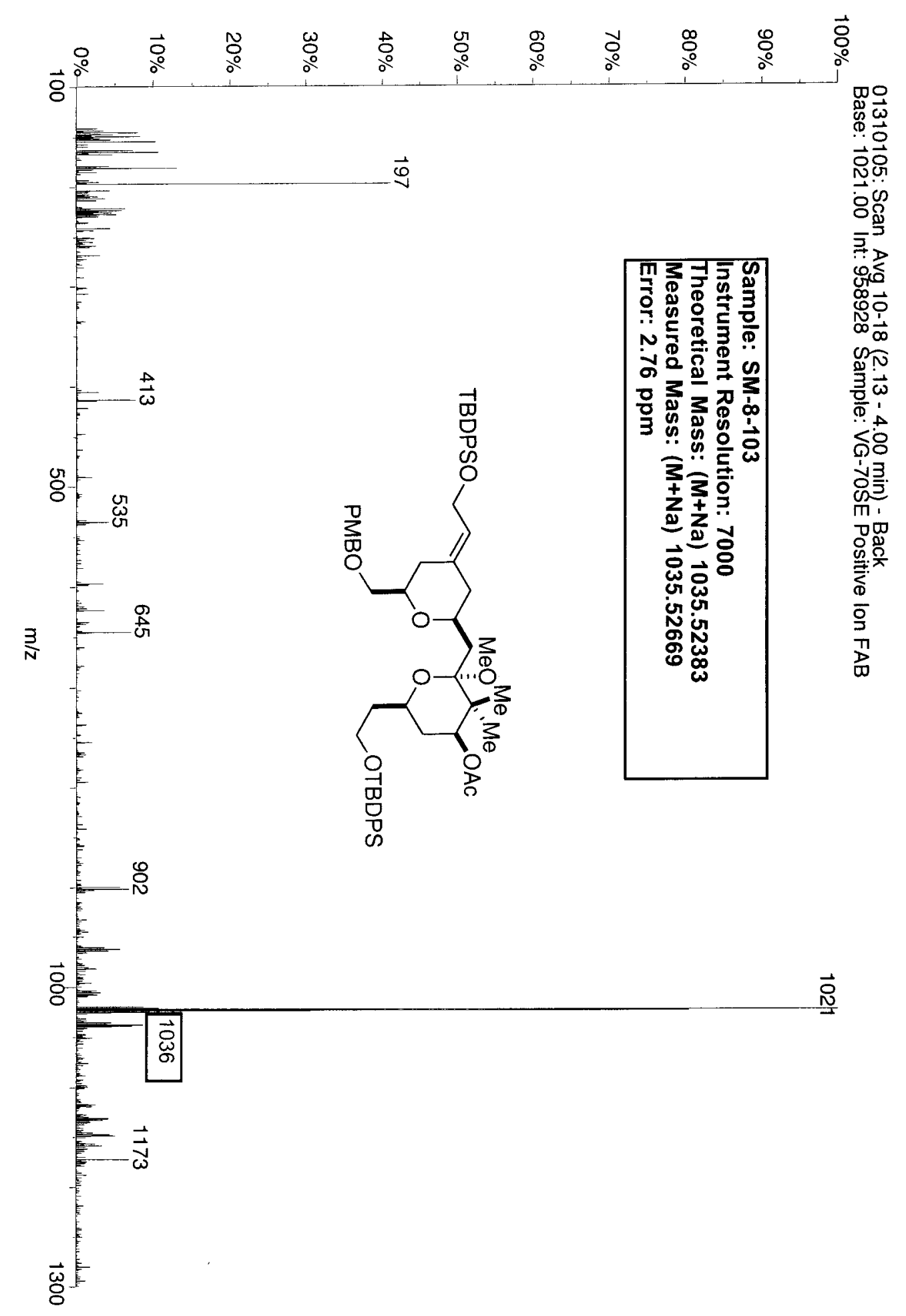




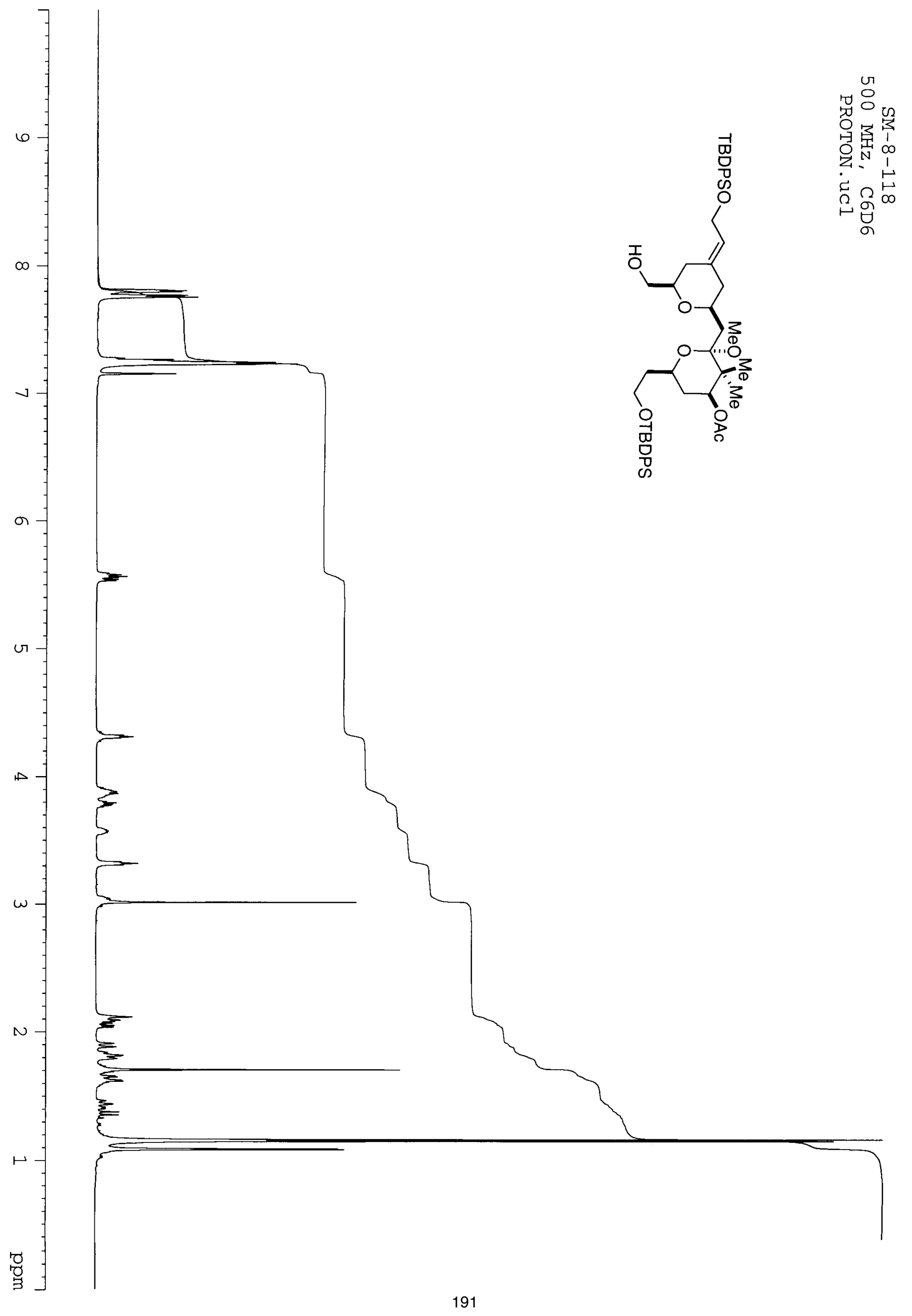



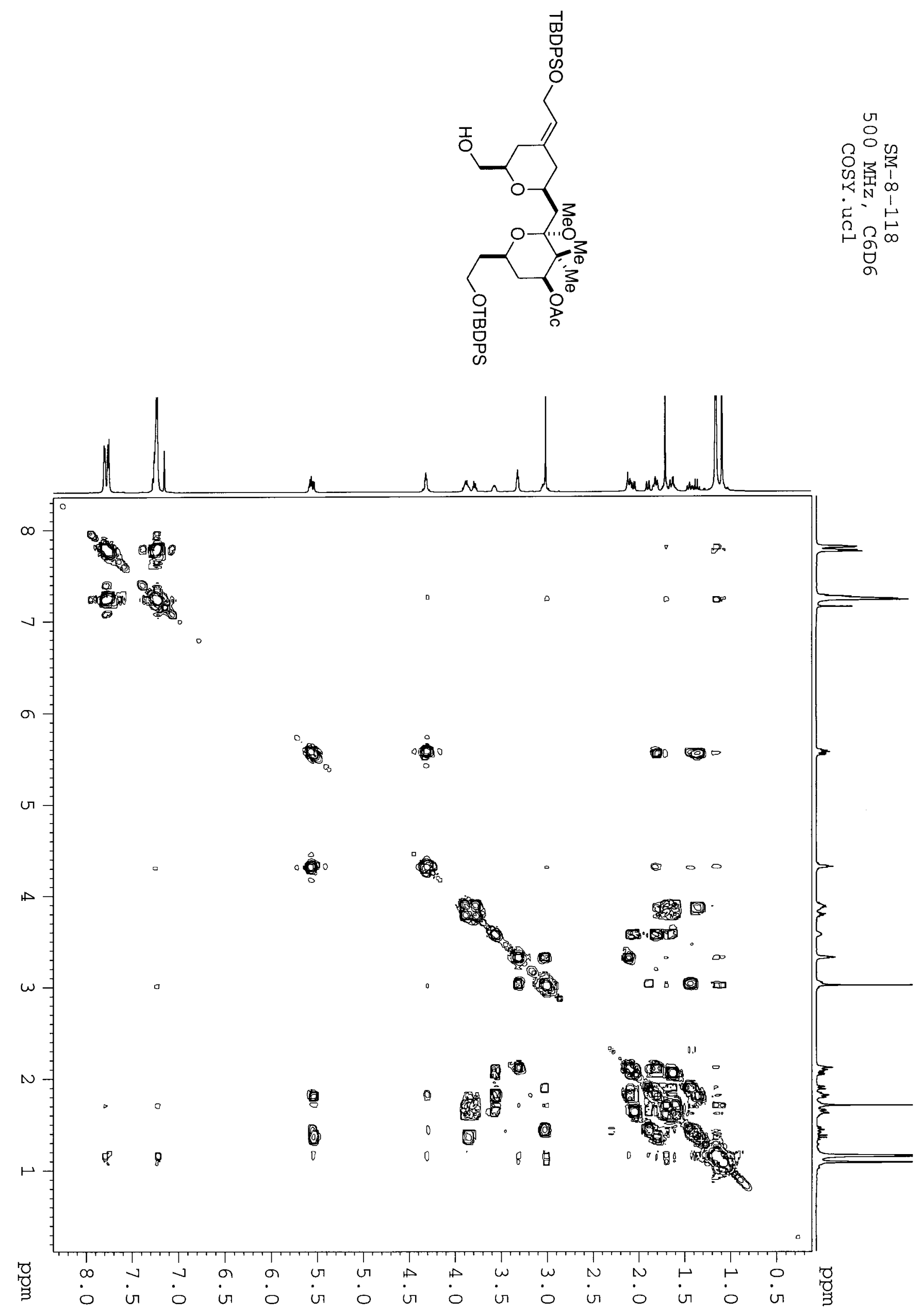

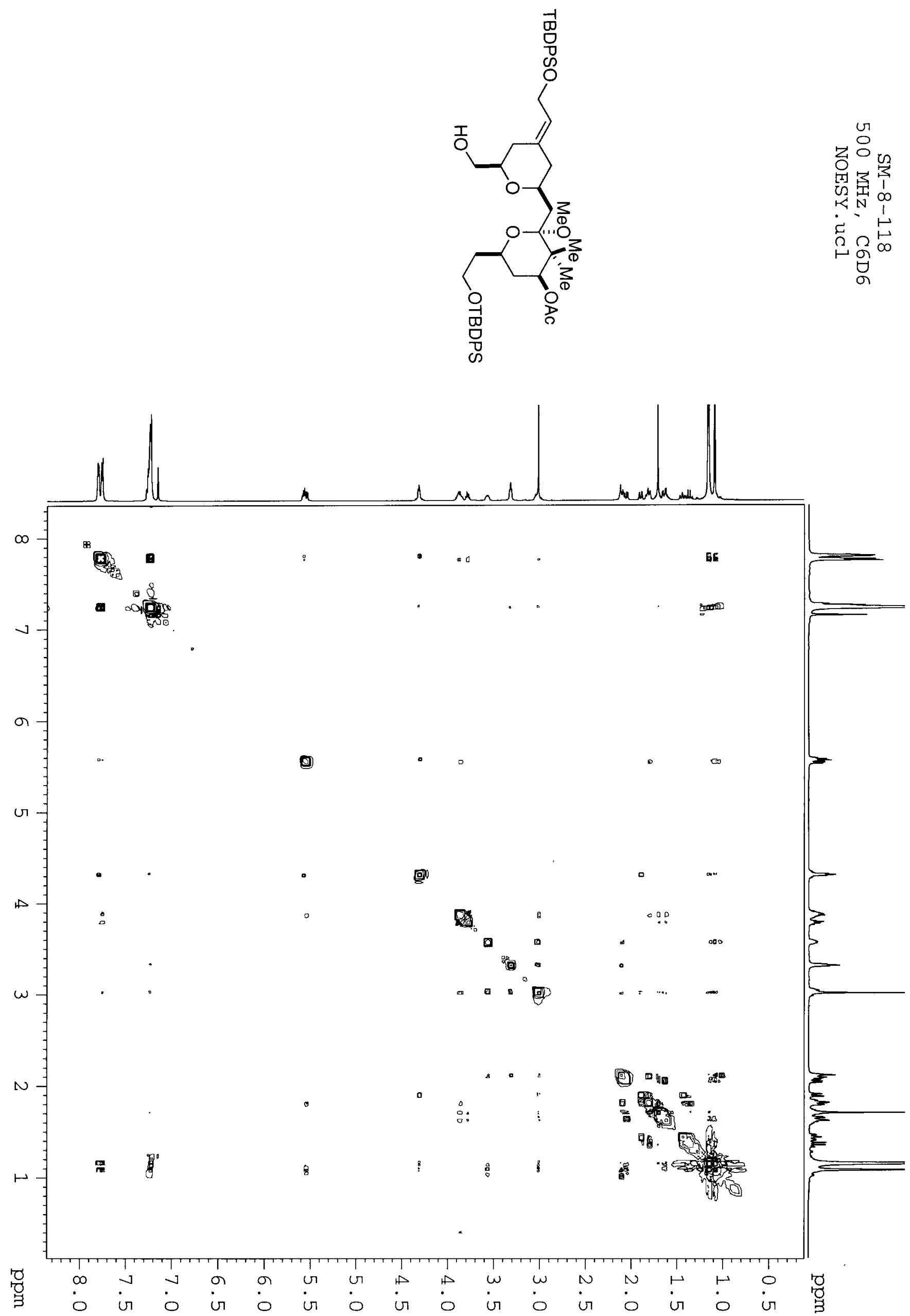


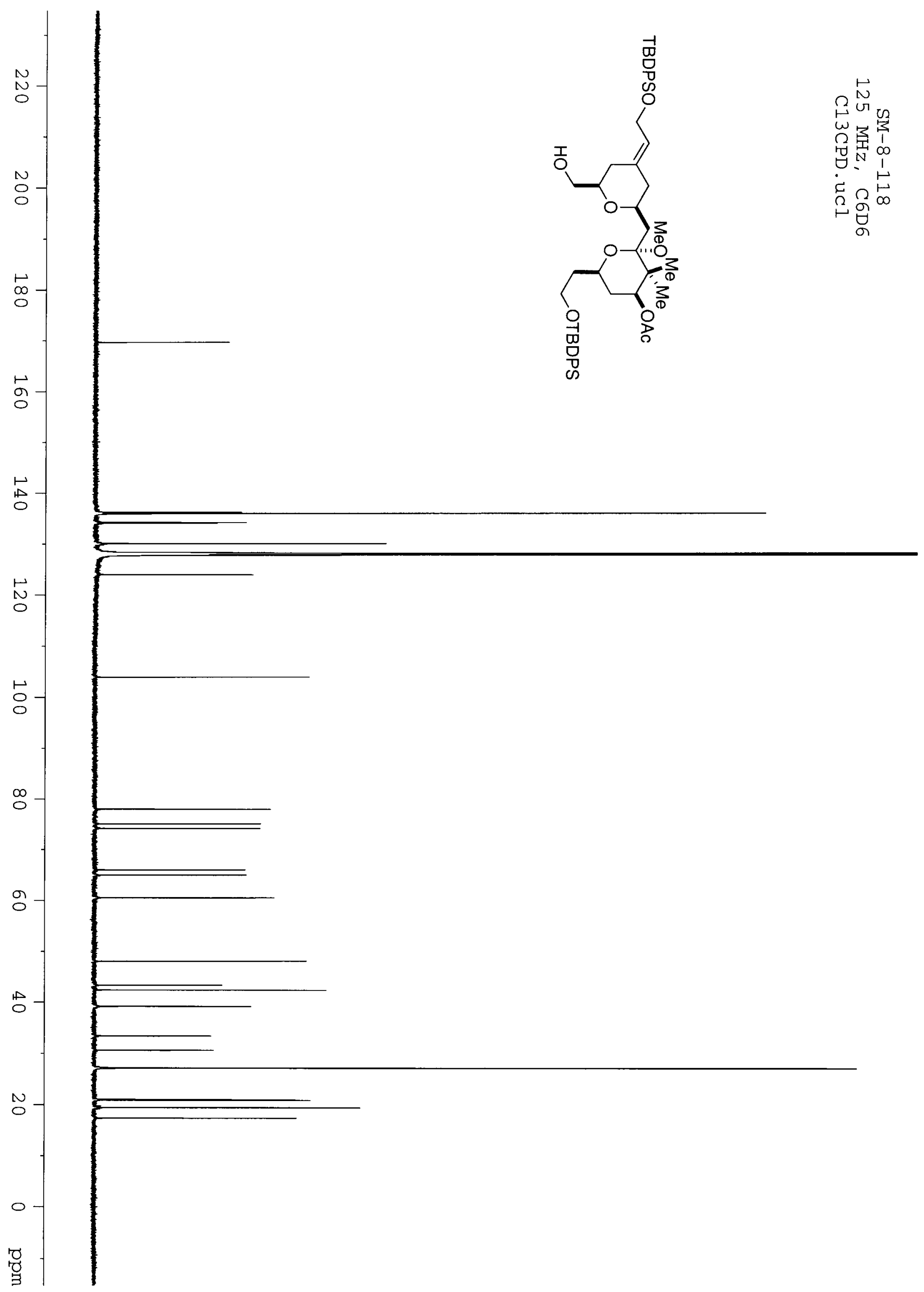




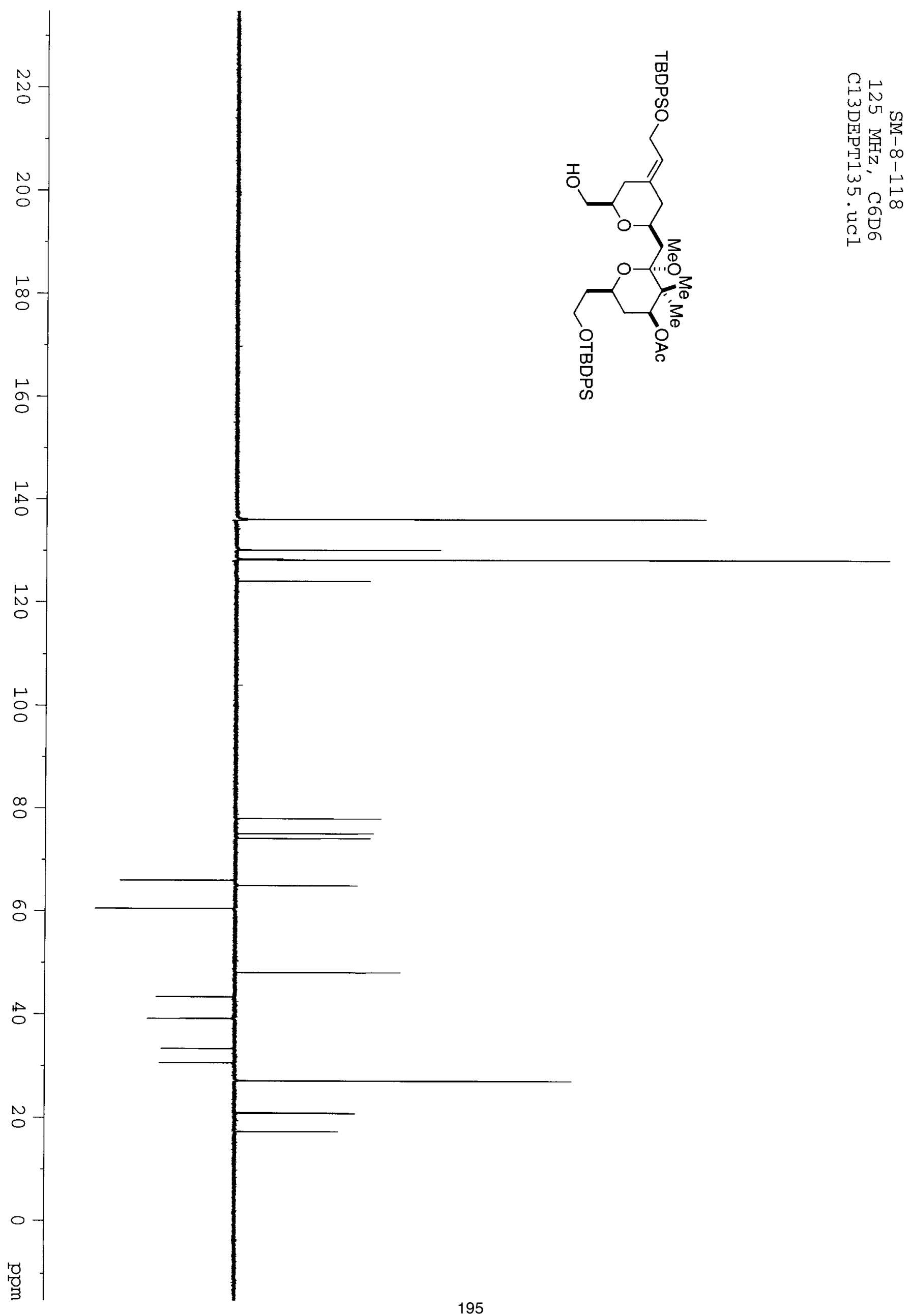




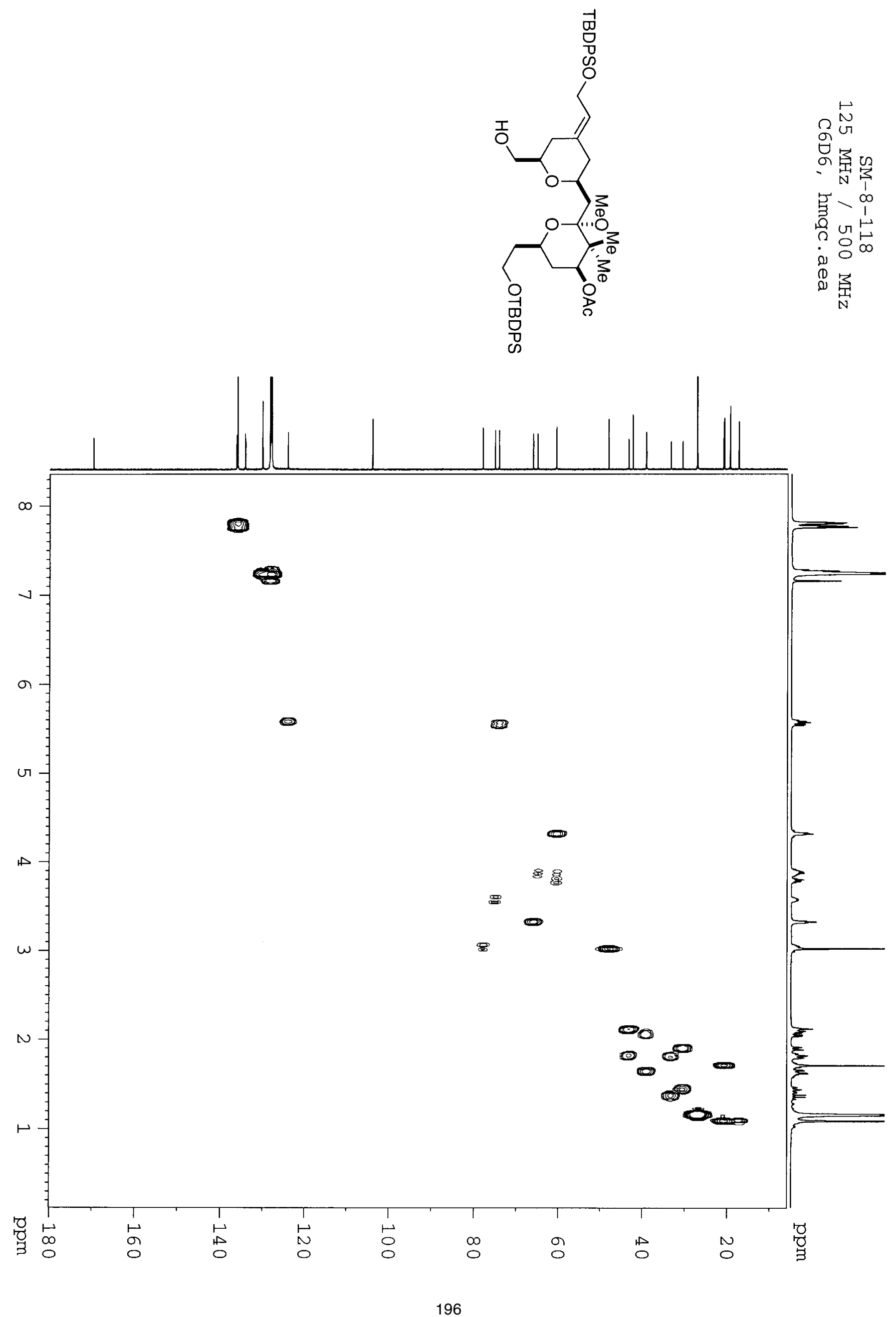




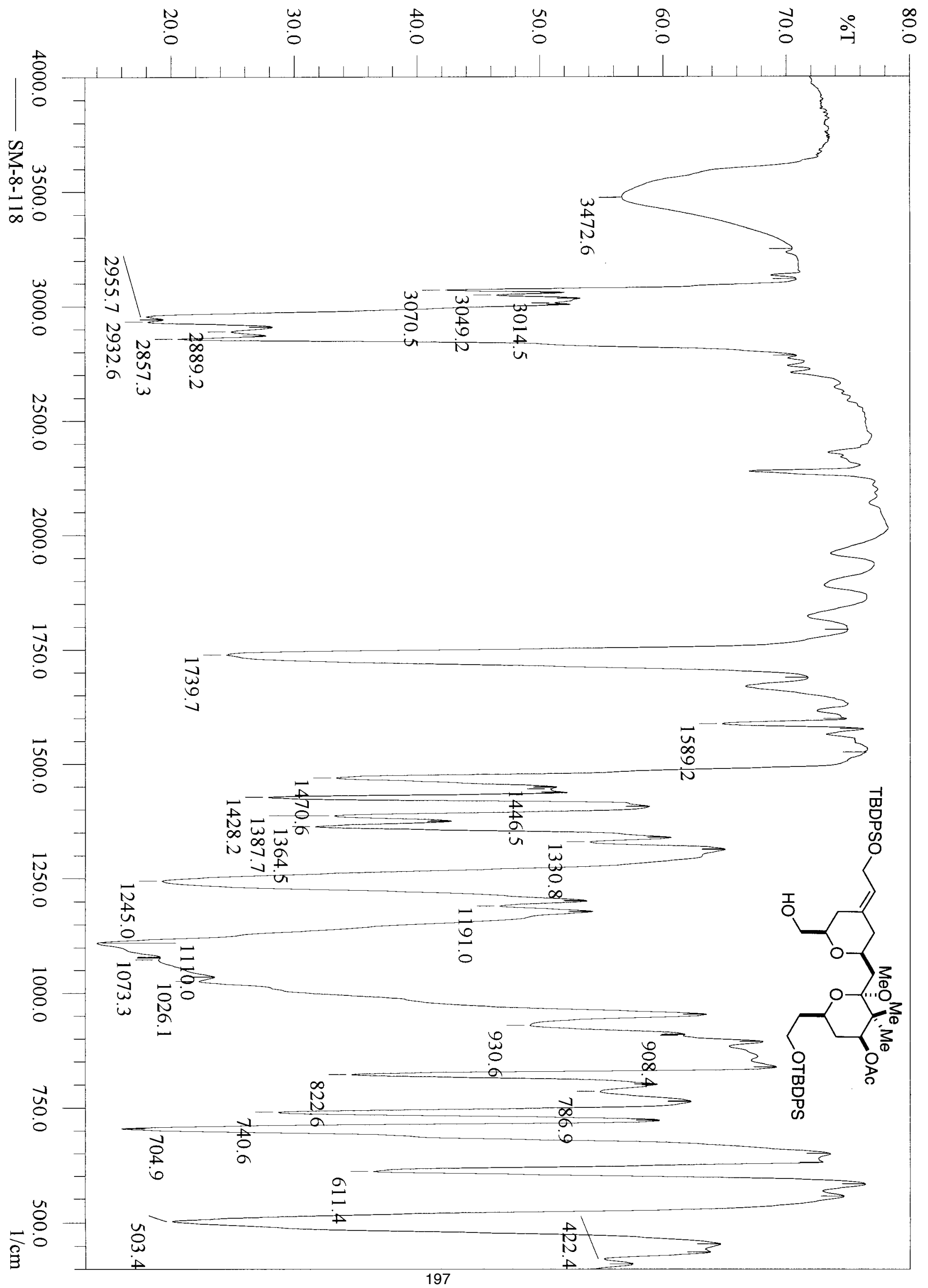




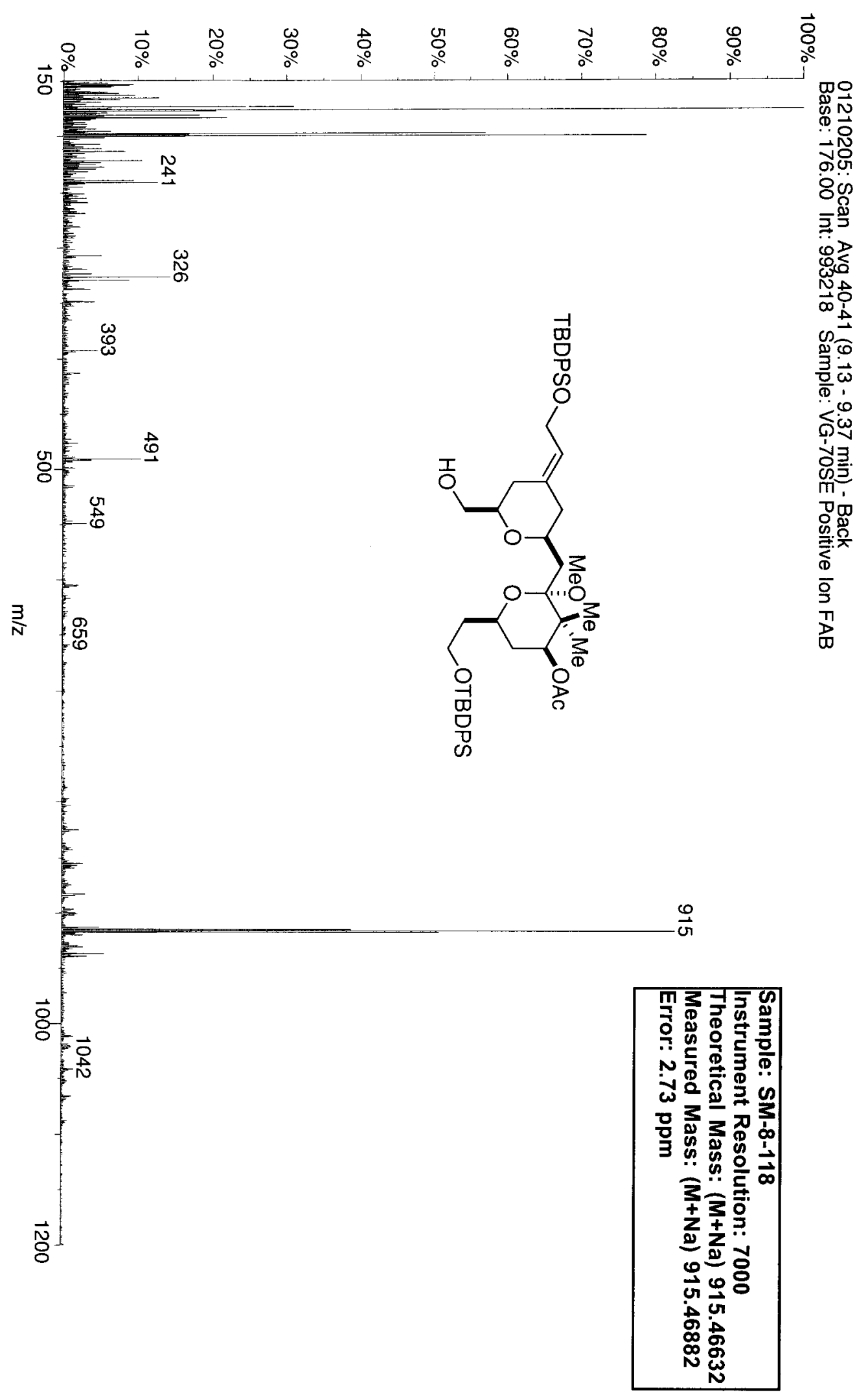




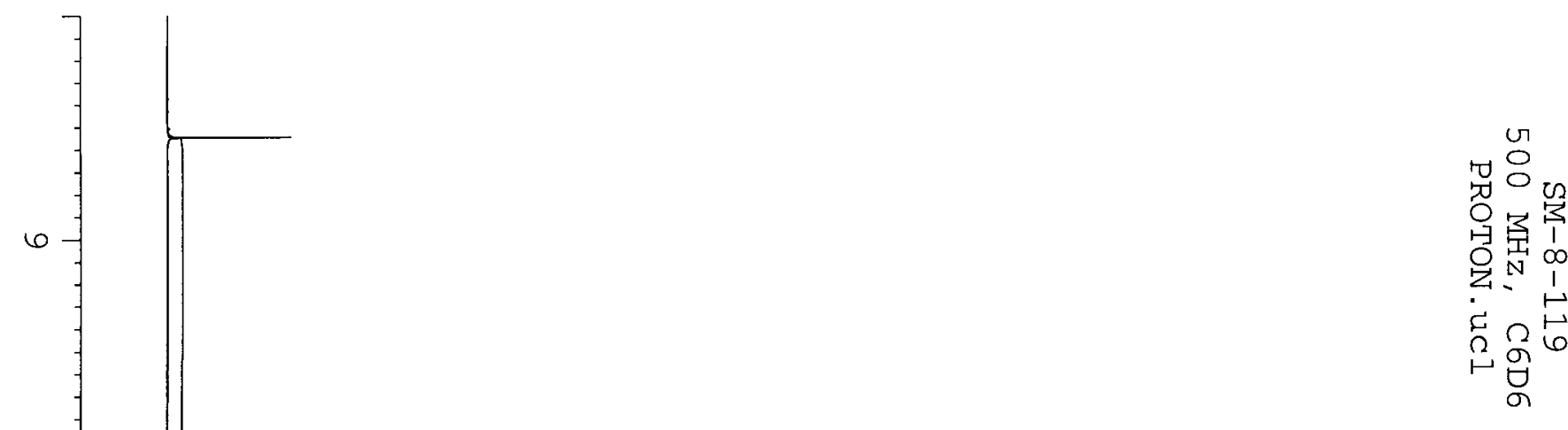

$\infty$

$\checkmark$

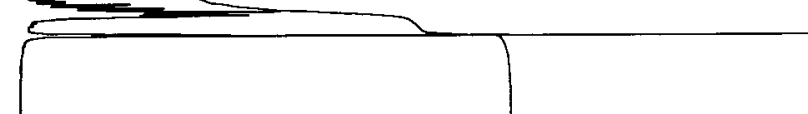

$a$

1

$\omega$

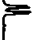
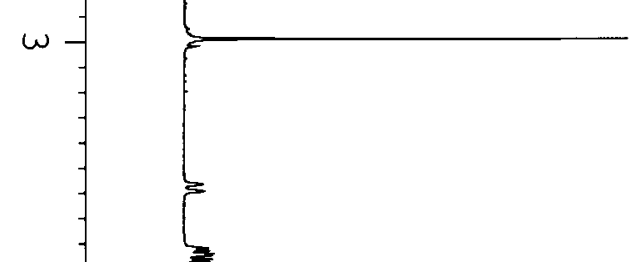

N

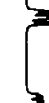

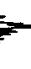

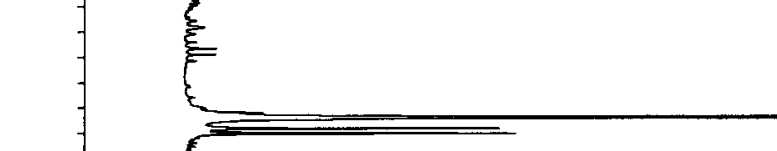

$\bullet$

8

8

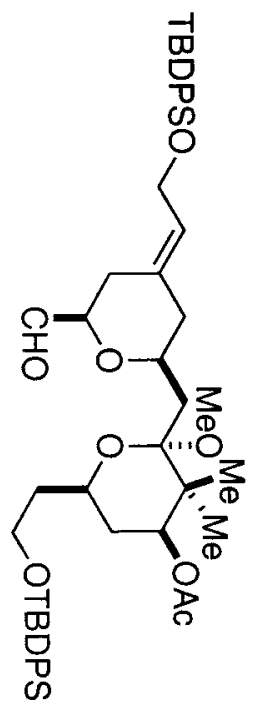



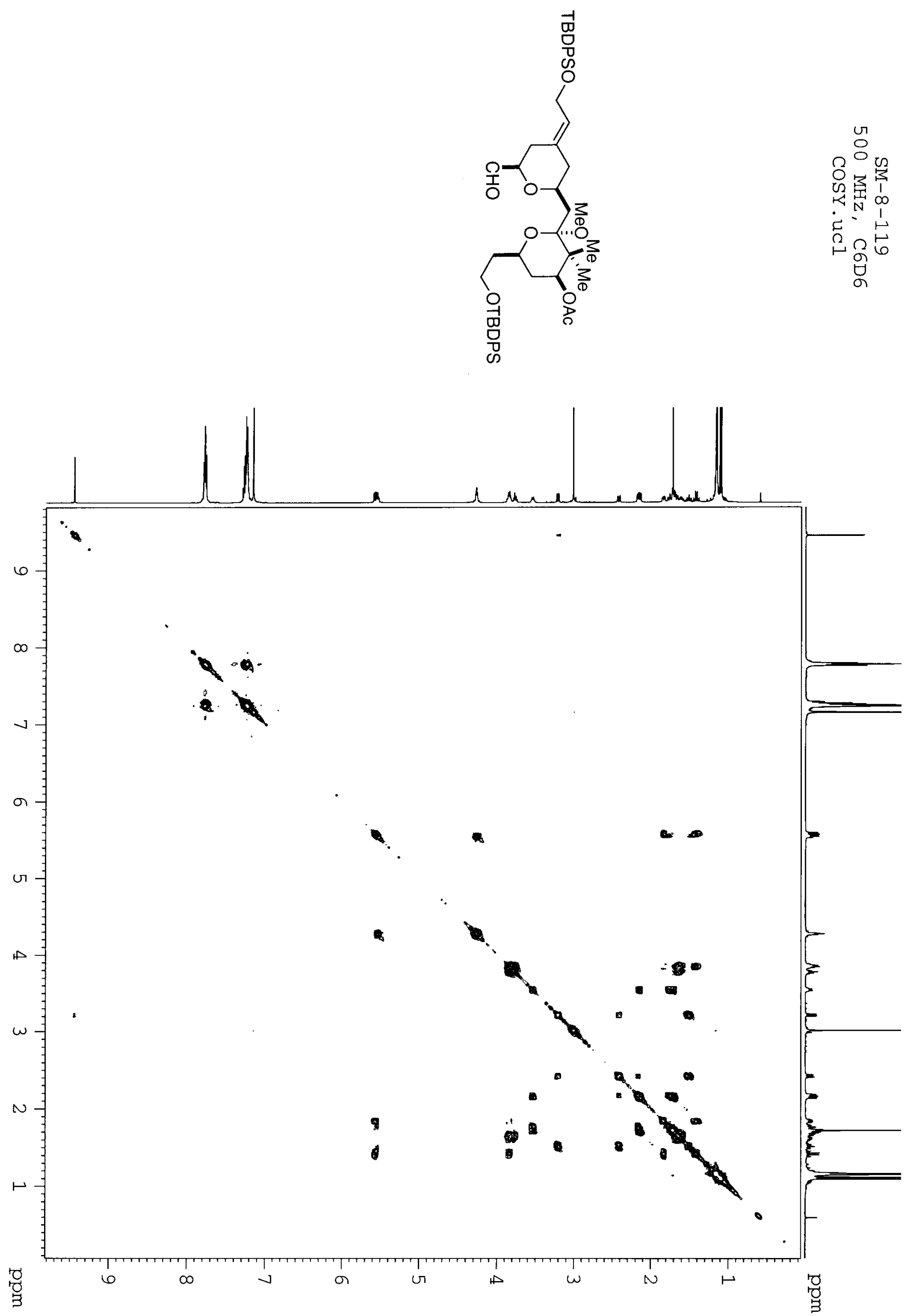

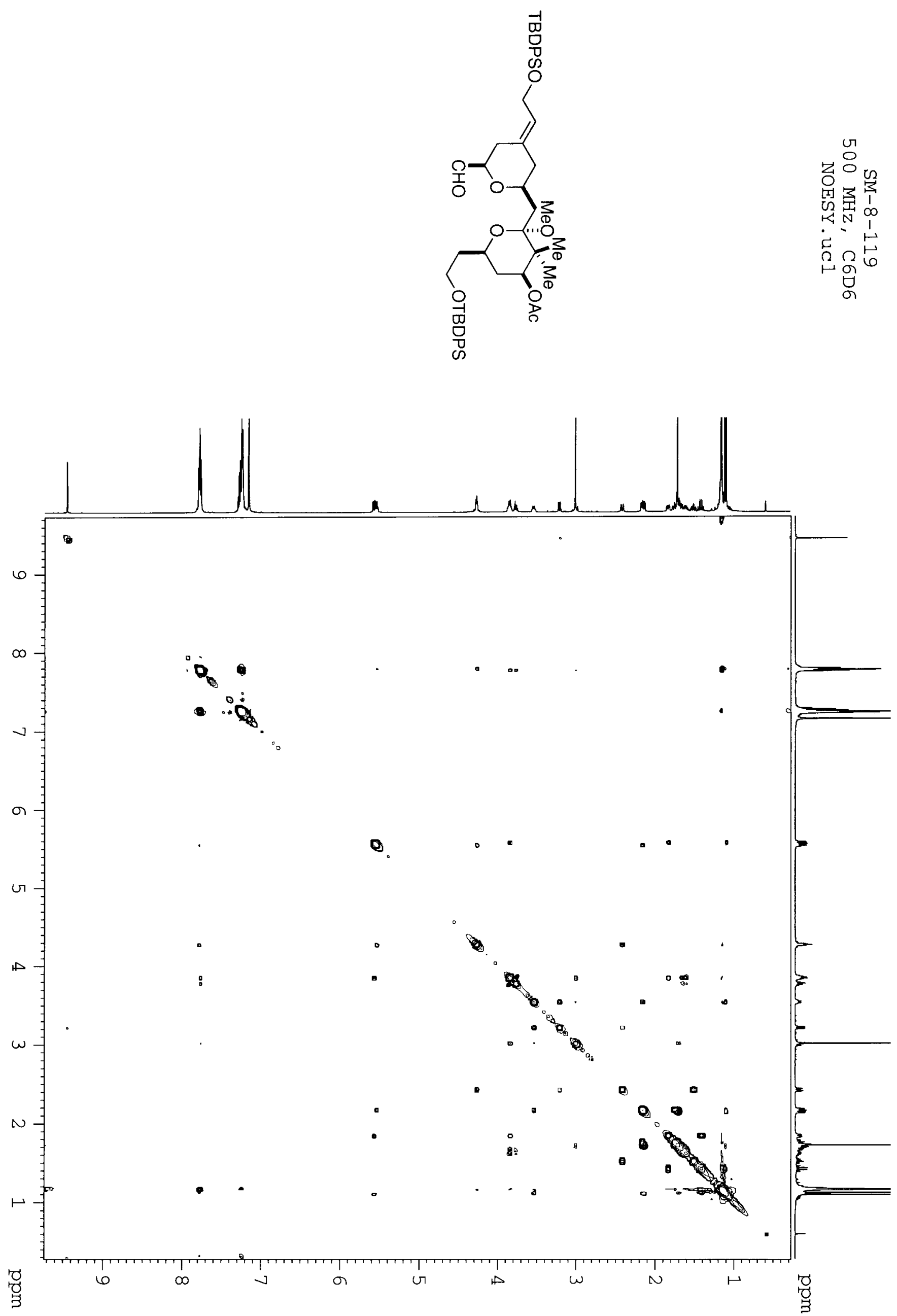


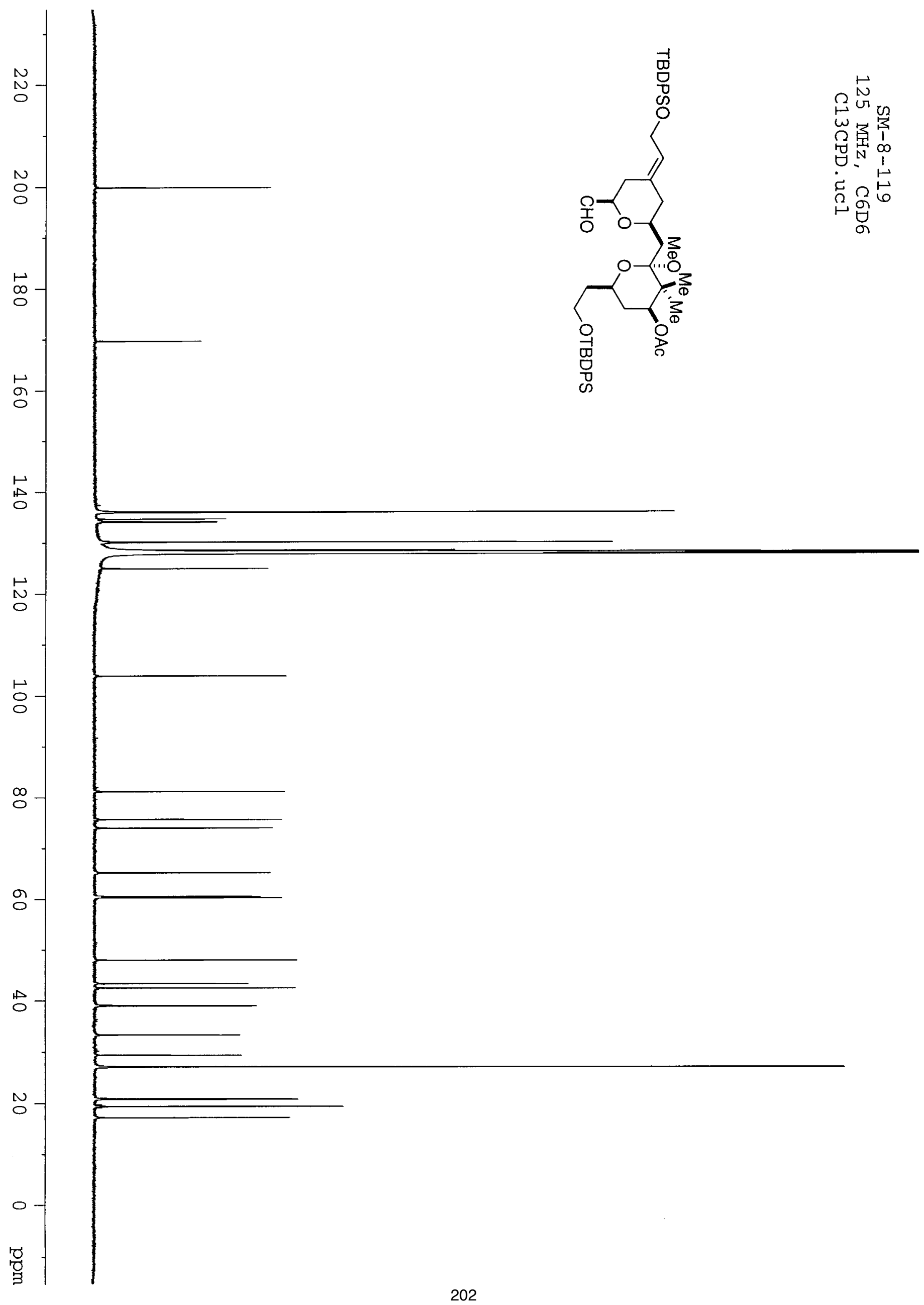




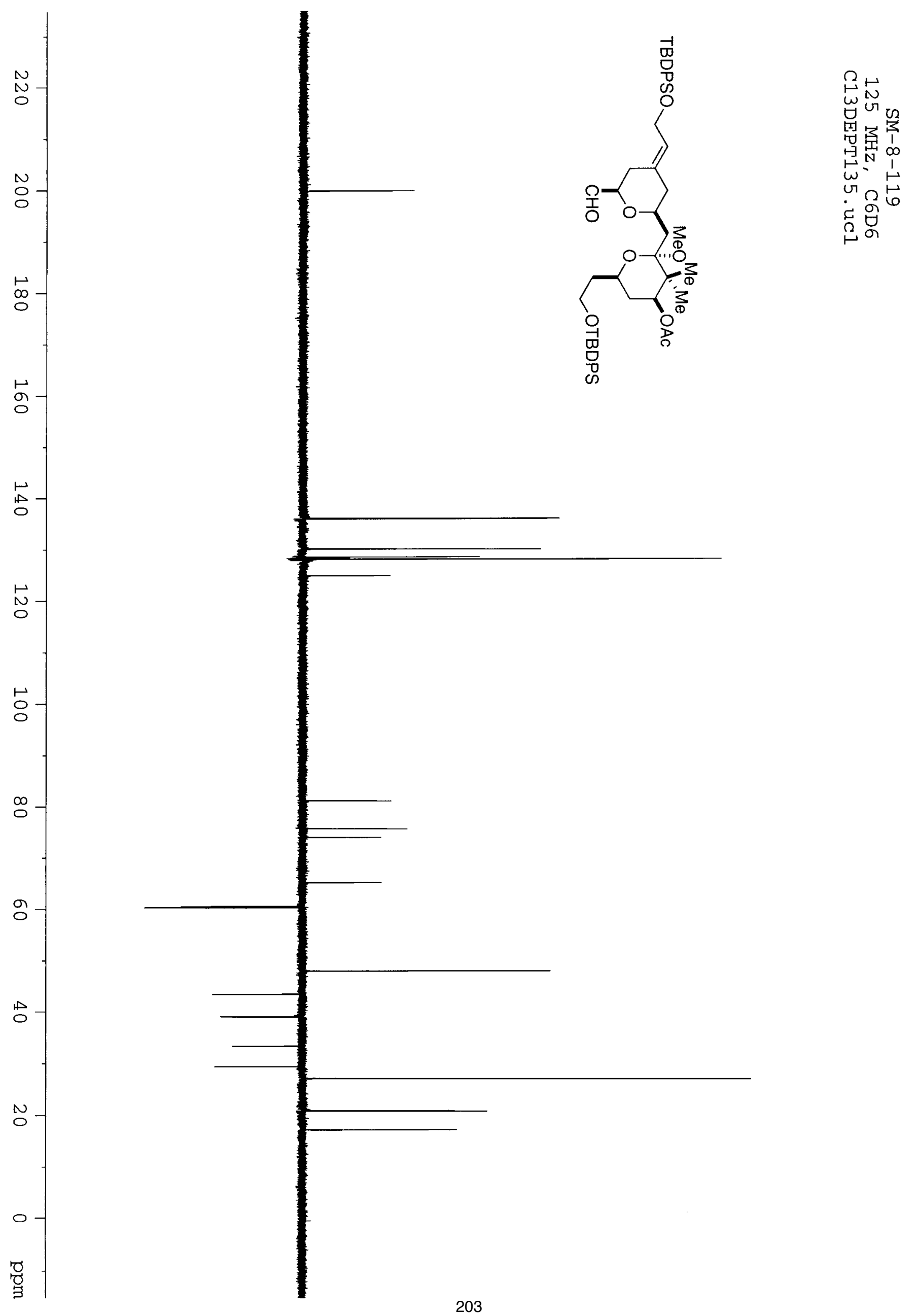




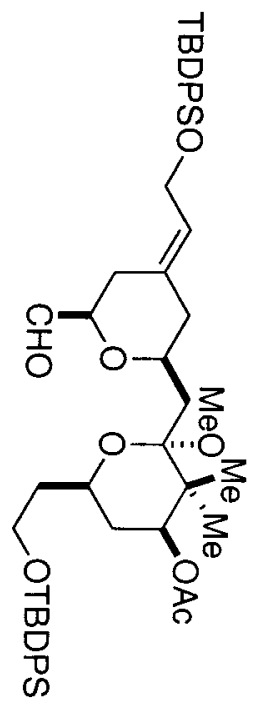

م芯

它军

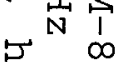

完 1

암

- 96

(D)

风

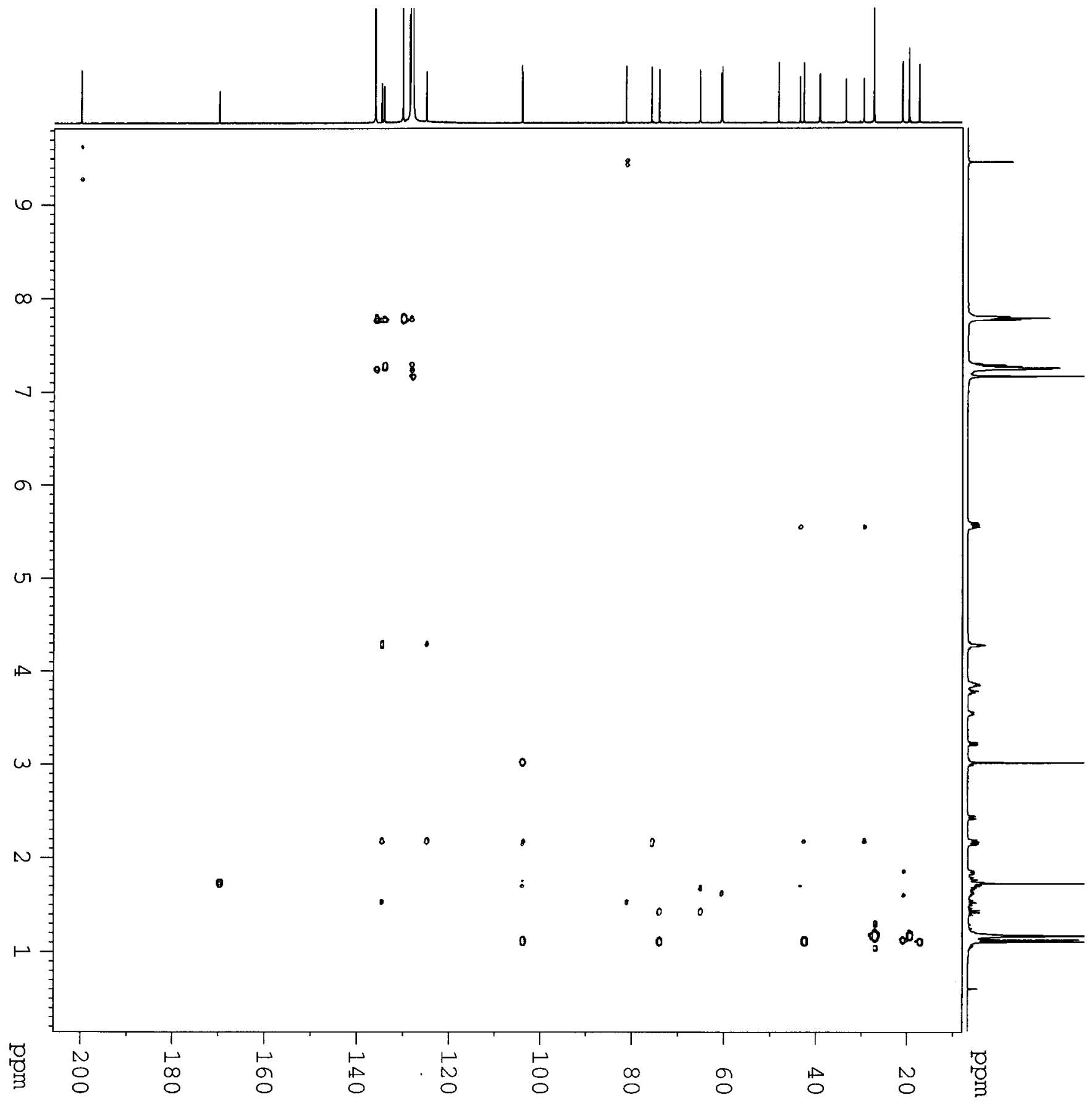




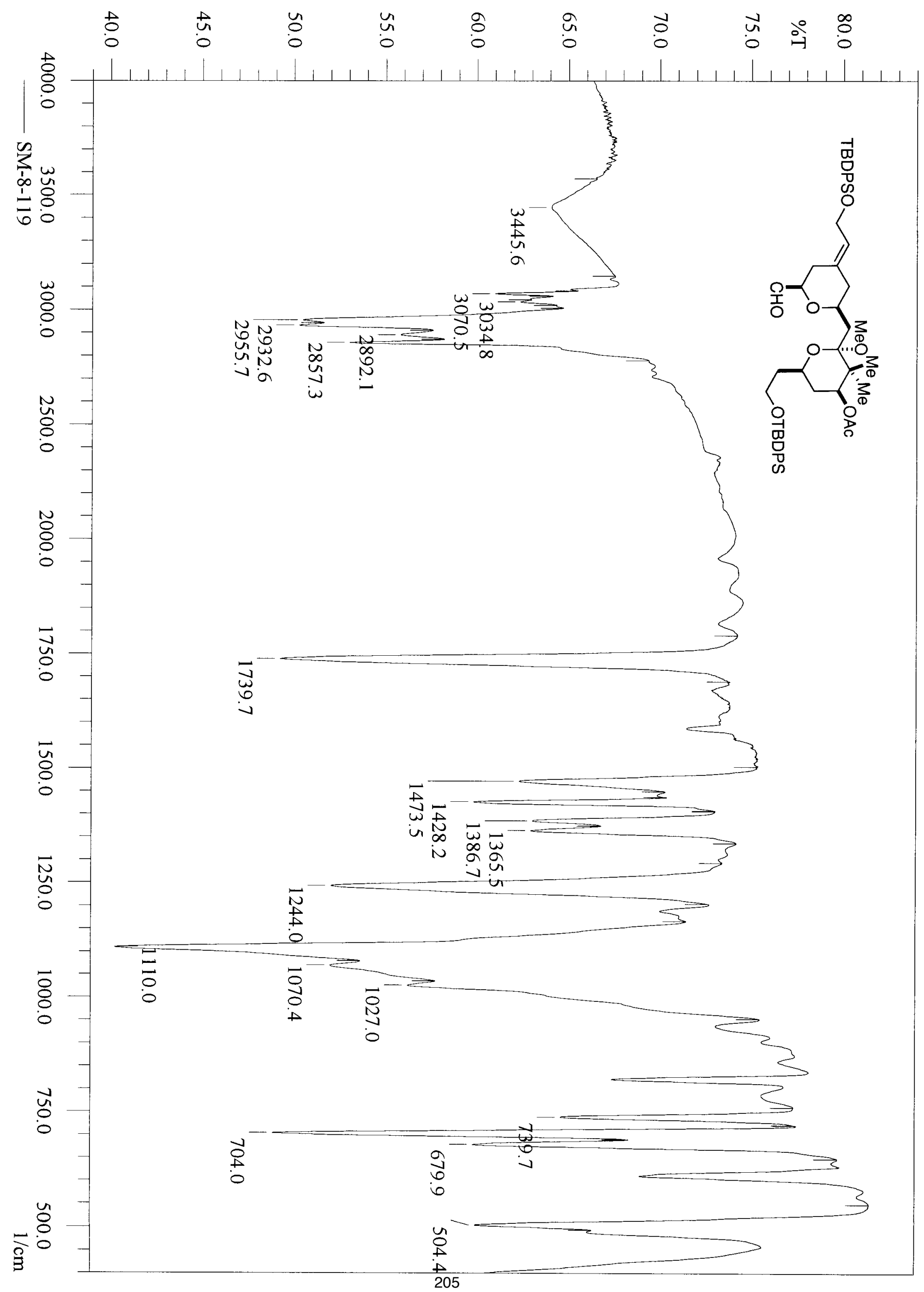




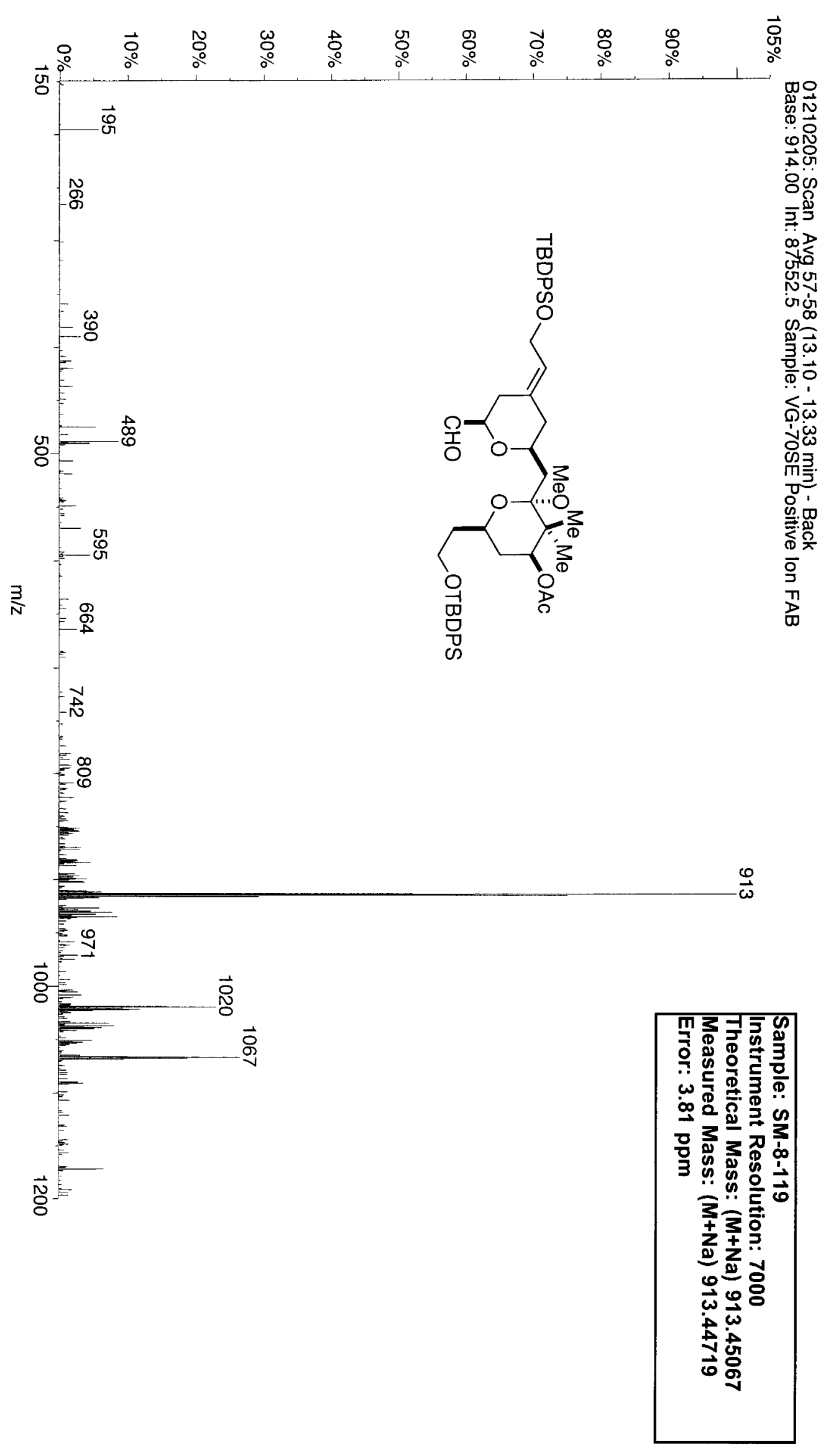




\title{
Enantioselective Formal Total Synthesis of the Antitumor Macrolide Bryostatin 7
}

\author{
Soraya Manaviazar," Mark Frigerio, Gurpreet S. Bhatia, \\ Marc G. Hummersone, Abil E. Aliev, and Karl J. Hale* \\ The Christopher Ingold Laboratories, \\ The Chemistry Department, \\ University College London, \\ 20 Gordon Street, \\ London WC1H OAJ, UK.
}

Supporting Information. Part 3.

\section{Proof of Structure of the UCL Version of the Masamune Bryostatin 7 AB Intermediate 1}

(Please Note: The numbering system used for the literature references in Part 3 of this $\mathrm{SI}$ is simply a continuation of that used in our main OL communication paper) 
In the main communication part of this Organic Letter, we documented our inability to correlate the $500 \mathrm{MHz}$ ${ }^{1} \mathrm{H}$ NMR spectrum of our synthetic 1 in $\mathrm{CDCl}_{3}$ with the ${ }^{1} \mathrm{H}$ NMR data that had previously been reported for 1 in $\mathrm{CDCl}_{3}$ by Masamune and coworkers. ${ }^{7 \mathrm{a}}$ In order to help clarify the origins of these differences, and to unambiguously guarantee the authenticity of our version of $\mathbf{1}$, we decided to undertake an independent chemical and NMR proof of structure of the ABfragment 1 that we had synthesised. The results of these efforts are now presented.

We began our proof of structure of the UCL bryostatin $7 \mathrm{AB}$-intermediate 1 by resynthesizing its B-ring precursors, namely, alcohol $\mathbf{1 0}^{10 \mathrm{~d}}$ and iodide 5, from commercially available (S)-(-)-glycidol 29 (Scheme 5), having carefully evaluated the $[\alpha]_{D}$ of the starting epoxide 29 before beginning each run of the synthetic sequence. Significantly, the spectral and optical rotational data that we obtained for the

Scheme 5. Correlation of our Bryostatin 7 B-Ring Alcohol $10^{10 d}$ with H.M.R Hoffmann's Bryostatin B-Ring Intermediate $34 .^{13 a}$

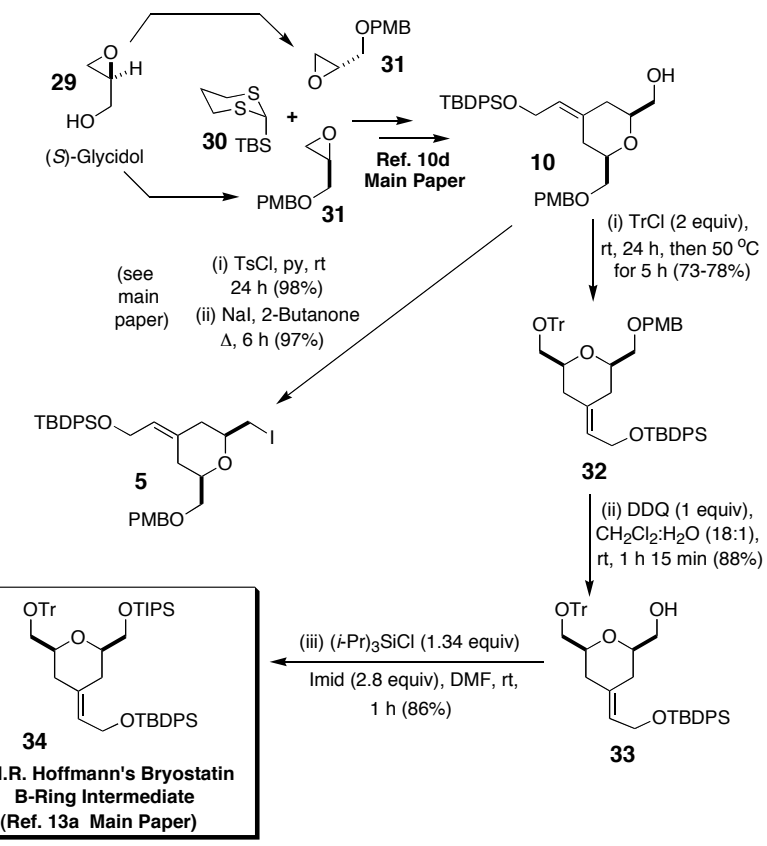

resynthesized fragments $10^{10 \mathrm{~d}}$ and $\mathbf{5}$ matched those that we had recorded previously (see Pt 1 of SI).

To further confirm the structural integrity of our Bring iodide 5 , we converted its alcohol precursor $10^{10 \mathrm{~d}}$ into the bryostatin B-ring intermediate 34 of H.M.R. Hoffmann and coworkers, ${ }^{13 a}$ for which high quality ${ }^{1} \mathrm{H}$ and ${ }^{13} \mathrm{C}$ NMR spectral and optical rotation data existed. Significantly, the spectral and optical rotational data that we obtained for 34 , prepared via the route shown in Scheme 5, correlated fully with the values reported by the Hoffmann group (see this $\mathrm{SI}$ Parts 4 and 5). ${ }^{13 a, 23}$ In light of this agreement, and our own independent NMR analyses of $\mathbf{1 0}$ and $\mathbf{5}$, we have concluded that the B-ring absolute stereochemistry in our version of $\mathbf{1}$ is totally secure, as Hoffmann et al. had previously confirmed their B-ring absolute stereochemistry for $34^{13 a}$ by single crystal X-ray analysis on an earlier progenitor. ${ }^{23}$

Having unambiguously defined the B-ring absolute stereochemistry that was present in our version of $\mathbf{1}$, we next set about confirming the absolute stereochemistry of our A-ring dithiane precursor 6 (see Scheme 6 of this SI). The synthesis of 6 is described in Schemes 2 and 3 of the main communication paper and in Part 1 of this SI.

First, we used Rychnovsky's ${ }^{13} \mathrm{C}$ NMR acetonide analysis method ${ }^{23}$ to confirm the relative stereochemistry between the two stereocentres in $\mathbf{6}$; we also applied the Rychnovsky method ${ }^{24}$ to the thioacetal hydrolysis product, aldehyde 35 (see Scheme 6 for structures). Significantly, the two $O$ isopropylidene Me singlets for dithiane 6 resonated at $\delta 25.03$ and 24.97 in $\mathrm{C}_{6} \mathrm{D}_{6}$, so confirming a 1,3-anti-

(23) (a) Lampe, T.F.J.; Hoffmann, H.M.R. Tetrahedron Lett. 1996 37, 7695. (c) Lampe, T.F.J.; Hoffmann, H.M.R.; Bornscheuer, U.T. Tetrahedron: Asymmetry. 1996, 7, 2889.

(24) (a) Rychnovsky, S.D.; Richardson, T.I.; Rogers, B.N. J. Org. Chem. 1997, 62, 2925. (b) Rychnovsky, S.D.; Skalitzky, D.J. Tetrahedron Lett. 1990, 31, 945. (c) Rychnovsky, S.D.; Rogers, B.; Yang, G. J. Org. Chem. 1993, 58, 3511 . 
Scheme 6. Correlation of our Bryostatin A-Ring Intermediates 20 and 6 with the Masamune A-Ring Aldehyde 35 and Methyl Ketone 36.
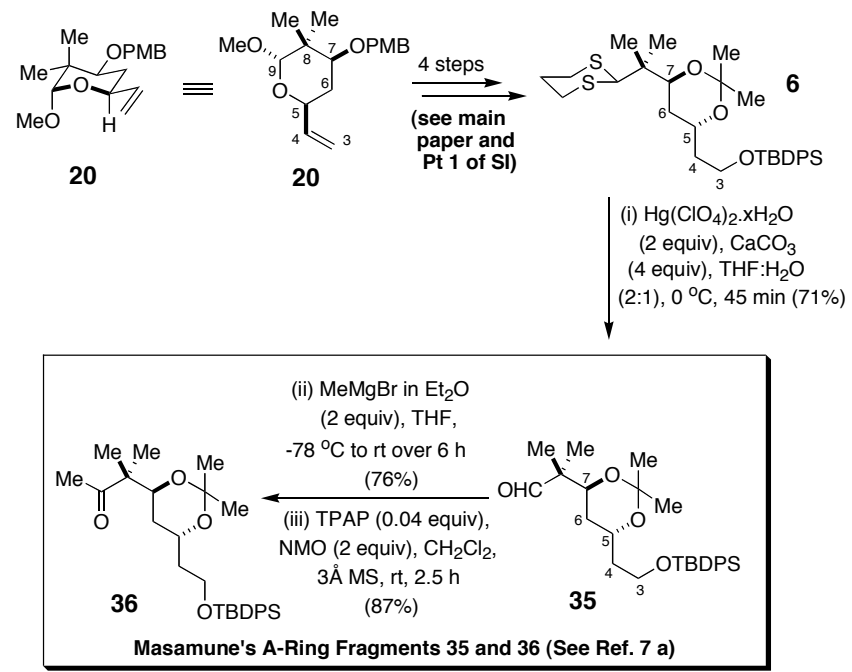

relationship between the C-5 and C-7-stereocenters. Likewise, for aldehyde 35 , its two $O$-isopropylidene Me groups resonated at $\delta 24.35$ and $24.56 \mathrm{ppm}$ in $\mathrm{C}_{6} \mathrm{D}_{6}$, and at $\delta 24.42$ and $24.18 \mathrm{ppm}$ in $\mathrm{CDCl}_{3}$, so proving that an anti-relationship existed between its C-5 and C-7 stereocenters and corroborating our previous assignment for 6 .

Further proof of the 1,3-anti-relationship between the $0-5$ and $0-7$ substituents in 6 was provided by its $500 \mathrm{MHz}{ }^{1} \mathrm{H}$ NMR spectra in $\mathrm{C}_{6} \mathrm{D}_{6}$ and $\mathrm{CDCl}_{3}$ which revealed that 6 was adopting the twist-boat conformation $^{24}$ expected for this stereochemical arrangement. In this regard, $\mathrm{H}-6 \mathrm{a}$ and $\mathrm{H}-6 \mathrm{~b}$ both showed large vicinal couplings of ca. $10 \mathrm{~Hz}$ to $\mathrm{H}-5$ and $\mathrm{H}-7$ respectively, and there were significant NOEs between $\mathrm{H}-5$ and the syn-Me of the $\mathrm{O}$ isopropylidene group, and $\mathrm{H}-7$ and its syn-oriented Me group.

Given that these methods had only allowed the relative stereochemistry of the $\mathrm{C}-5$ and $\mathrm{C}-7$ stereocentres in 6 and 35 to be assigned, we performed a detailed $500 \mathrm{MHz}{ }^{1} \mathrm{H}$ NMR study on an earlier precursor in our route, where one of the absolute stereocentres had always remained fixed. The compound that we selected for analysis was alkene 20 (see Scheme 6), since its anomeric C-9OMe group had remained unperturbed throughout our synthesis. A secure knowledge of the absolute stereochemistry at C-9 would allow confident assignment of the absolute stereochemistry at the nearby C-5 and C-7 stereocentres.

The absolute stereochemistry of alkene 20 was readily determined from the $500 \mathrm{MHz}{ }^{1} \mathrm{H} /{ }^{1} \mathrm{H}$ COSY and NOESY spectra that were acquired in $\mathrm{CDCl}_{3}$ (see Parts 1 and 2 of this $\mathrm{SI}$ ). Of particular significance were the $J$-couplings for $\mathrm{H}-7_{\mathrm{ax}}$ at $\delta 3.62\left(J_{6 \mathrm{ax}, 7 \mathrm{ax}}=11.5\right.$ $\left.\mathrm{Hz}, J_{6 \mathrm{eq}, 7 \mathrm{ax}}=4.8 \mathrm{~Hz}\right)$ and for $\mathrm{H}-6_{\mathrm{ax}}$ at $\delta 1.51\left(J_{6 \mathrm{ax}, 6 \mathrm{eq}}=\right.$ $J_{5 a x, 6 a x}=12.0 \mathrm{~Hz}$ ). The large $J$ values of $c a .12 \mathrm{~Hz}$ for $\mathrm{H}-6_{\mathrm{ax}}$ confirmed its antiperiplanar relationship with $\mathrm{H}-5$ and $\mathrm{H}-7$. The presence of strong positive mutual NOEs between $\mathrm{H}-5, \mathrm{H}-7$, and the $\mathrm{C}-9-\mathrm{OMe}$ which resonated at $\delta 3.30$ further supported our assignment. Evidence for the axial orientation of the C-9-methoxy was further provided by the two strong positive NOEs that were found between the two C-8-Me groups and $\mathrm{H}-9_{\text {eq }}$; significantly, if $\mathrm{H}(9)$ had been axial, only one NOE would have been observed. Other evidence in favor of the proposed absolute stereostructure for $\mathbf{2 0}$ was provided by the strong NOEs that existed between the axial $\mathrm{C}-8-\mathrm{Me}$ at $\delta 1.00$ and the $\mathrm{H}-6_{\mathrm{ax}}$ proton, and between $\mathrm{H}-5_{\mathrm{ax}}$ and $\mathrm{H}-6_{\text {eq }}$ (the latter resonance appeared at $\delta$ 1.90). Together these combined experimental observations guaranteed that the absolute stereochemistry in our A-ring dithiane coupling partner 6 had to be as depicted (Scheme 6).

Having completely secured the absolute stereochemistry of our A- and B-ring coupling partners 5 and $\mathbf{6}$, we next set about completely assigning all of the resonances in the $500 \mathrm{MHz}{ }^{1} \mathrm{H}$ spectrum of 1 in $\mathrm{CDCl}_{3}$ via a combination of COSY, NOESY, and HMQC 2D NMR techniques. Our 
assignments are presented in full in Table 1, along with refined $J$ values as determined by the resolutionenhancement technique. ${ }^{25}$

For purposes of this discussion, Table 1 also presents Masamune's previously reported ${ }^{1} \mathrm{H}$ NMR chemical shift data for 1 in $\mathrm{CDCl}_{3}$. A quick survey of both sets of data soon reveals substantial differences between the tabulated chemical shifts. Particularly striking are the significant deviations observed for the OAc resonance in our version of 1 (where the OAc appears at $\delta 2.04)$ and that reported previously ${ }^{7 a}$ (where the OAc resonates at $\delta 1.73 \mathrm{ppm}$ ). In light of this, we considered it essential that we try to intersect with other intermediates in the Masamune synthesis to confirm their absolute stereochemistry.

It transpired that aldehyde 35 (see this $\mathrm{SI}$ Pt 3, Scheme 6) had previously featured in Masamune's route to $1 .^{7 a}$ In light of this, we therefore decided to compare its $500 \mathrm{MHz}{ }^{1} \mathrm{H}$ NMR spectrum in $\mathrm{CDCl}_{3}$ with the $300 \mathrm{MHz}{ }^{1} \mathrm{H}$ NMR data for 35 (in $\mathrm{CDCl}_{3}$ ) that had previously been reported. ${ }^{7 a}$ Unfortunately, our spectral comparison did not allow us to be confident that we were dealing with identical materials.

However, following our successful conversion of aldehyde 35 into Masamune's methyl ketone $36,{ }^{7 a}$ by the route shown in Scheme 6, an excellent agreement was obtained between our $500 \mathrm{MHz}{ }^{1} \mathrm{H}$ NMR data for 36 in $\mathrm{CDCl}_{3}$ and the $300 \mathrm{MHz}{ }^{1} \mathrm{H}$ NMR data that had been reported for $\mathbf{3 6}$ by Masamune (see Part 5 of the current SI).

Not long after we had performed these detailed chemical and NMR correlations, and whilst we were still endeavoring to gather high quality NMR spectra on our analytical samples of $\mathbf{2 8}$ and $\mathbf{2 4}$, we observed that both these molecules were unstable to storage in $\mathrm{CDCl}_{3}$, and this led us to record their spectra in $\mathrm{C}_{6} \mathrm{D}_{6}$. It transpired that this simple change in NMR

(25) In the spectral data reported in Part 1 of this SI, the $J$ values for 1 were determined from the spectrum without the aid of resolution enhancement.
Table 1. Comparison of Our New $500 \mathrm{MHz}{ }^{1} \mathrm{H}$ NMR Data with that Previously Published for 1 in $\mathrm{CDCl}_{3}$ in Ref. $7 \mathrm{a}$.

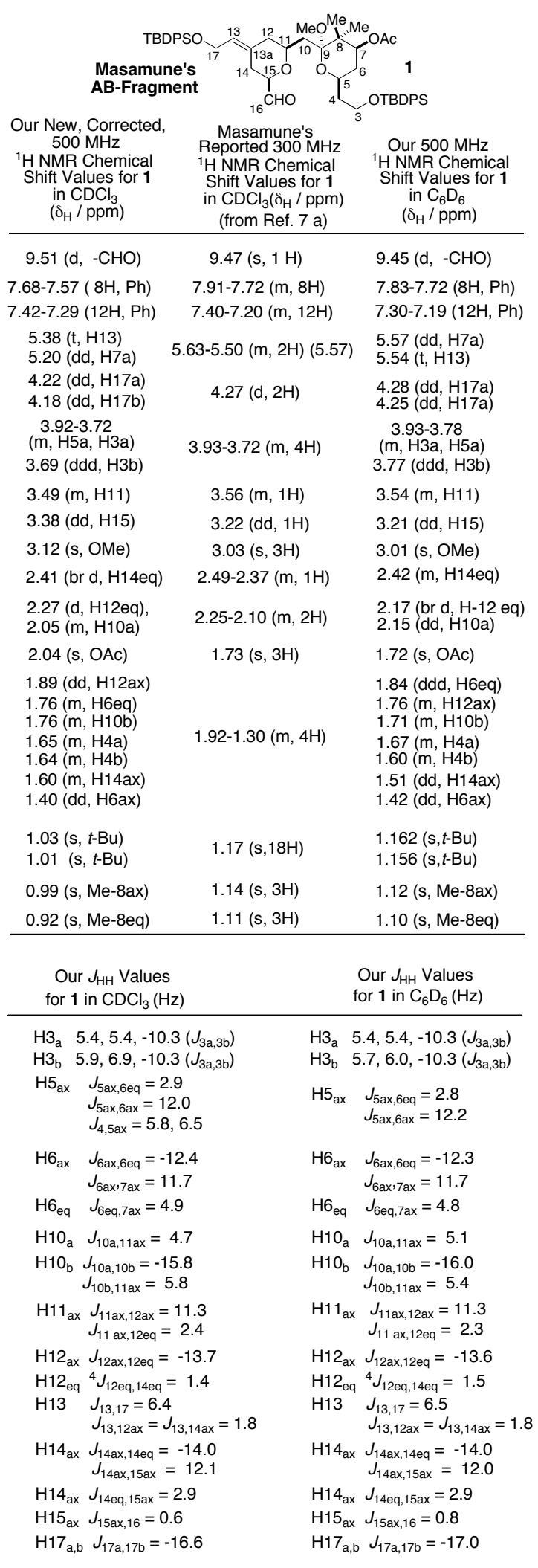


solvent proved critical to us elucidating the source of the spectral disagreement for $\mathbf{1}$. In this regard, when we recorded the $500 \mathrm{MHz}{ }^{1} \mathrm{H}$ NMR spectra of compounds 28 and 24 in $\mathrm{C}_{6} \mathrm{D}_{6}$, we found that the OAc-Me resonances both appeared at $\delta 1.70$ respectively (see Part 1 of the SI for the NMR spectra these two molecules, and Fig. 1 below). This observation proved highly significant for we were acutely aware that the OAc-Me resonance for the ABfragment 1 had previously been reported to have appeared at $\delta 1.73$ in $\mathrm{CDCl}_{3}$, yet we observed it to resonate at $\delta 2.04$ in the same solvent.

Fig 1. O-Acetate Resonance Positions for 28, 24 and 1 in their respective $500 \mathrm{MHz}{ }^{1} \mathrm{H}$ NMR Spectra in $\mathrm{C}_{6} \mathrm{D}_{6}$.

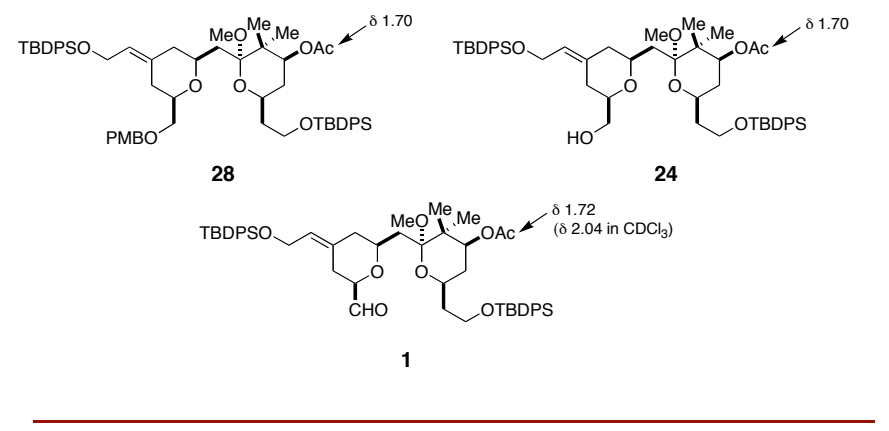

This immediately led to us running the $500 \mathrm{MHz}{ }^{1} \mathrm{H}$ NMR spectrum of our version of 1 in $\mathrm{C}_{6} \mathrm{D}_{6}$ and, at once, we noticed that there was now very good agreement between the chemical shifts that we were observing, and those that had originally been stated to be for 1 in $\mathrm{CDCl}_{3}$ by Masamune (see Table 1). ${ }^{7 \mathrm{a}}$

In light of this excellent spectral correlation, and all of the other chemical evidence that we have presented, we have concluded that the originally quoted $300 \mathrm{MHz}{ }^{1} \mathrm{H}$ NMR spectrum for 1 in $\mathrm{CDCl}_{3}{ }^{7 \mathrm{a}}$ was actually recorded in $\mathrm{C}_{6} \mathrm{D}_{6}$. Accordingly we are now revising Masamune's published ${ }^{1} \mathrm{H}$ NMR spectral data $^{7 \mathrm{a}}$ on 1 to accommodate our new findings.

Given our successful ${ }^{1} \mathrm{H}$ NMR spectral correlation of 1 in $\mathrm{C}_{6} \mathrm{D}_{6}$ with the data of Masamune, ${ }^{7 \mathrm{a}}$ and all of the other chemical and NMR correlations that we have reported herein, we now believe that the correctness of our own structure for $\mathbf{1}$ has been successfully demonstrated beyond any doubt. We have thus confirmed our intersection with Masamune's AB-aldehyde fragment $\mathbf{1}$ and the integrity of our own enantioselective formal total synthesis of bryostatin 7 . 


\title{
Enantioselective Formal Total Synthesis of the Antitumor Macrolide Bryostatin 7
}

\author{
Soraya Manaviazar, ${ }^{*}$ Mark Frigerio, Gurpreet S. Bhatia, \\ Marc G. Hummersone, Abil E. Aliev, and Karl J. Hale* \\ The Christopher Ingold Laboratories, \\ The Chemistry Department, \\ University College London, \\ 20 Gordon Street, \\ London WC1H OAJ, UK.
}

Supporting Information. Part 4.

Experimental Procedures for the Synthesis of Compounds 32, 33, 34, 35, and 36 and their Characterisation Data. 


\section{Trityl Ether 32}

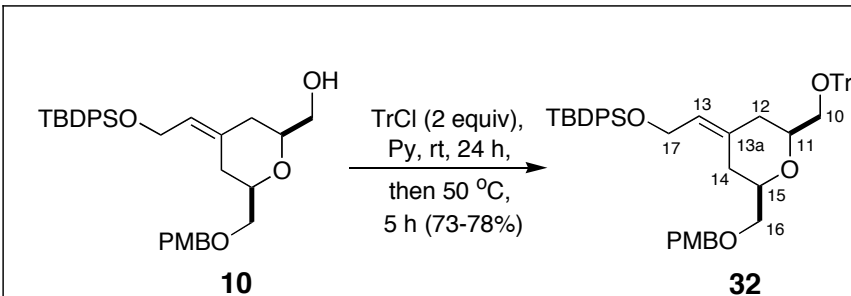

The UCL B-Ring Alcohol 10 was prepared from (S)-(-)-glycidol according to: Hale, K.J.; Hummersone, M.G.; Bhatia, G.S. Org. Lett. 2000, 2, 2189.

To a stirred solution of alcohol $10^{10 \mathrm{~d}}(0.4 \mathrm{~g}, 0.732 \mathrm{mmol})$ in anhydrous pyridine $(5 \mathrm{~mL})$ under $\mathrm{N}_{2}$ was added triphenylmethyl chloride $(0.41 \mathrm{~g}, 1.463 \mathrm{mmol}, 2.0$ equiv) in one portion at rt. The reactants were stirred at rt for $24 \mathrm{~h}$ and then heated at $50{ }^{\circ} \mathrm{C}$ for $5 \mathrm{~h}$. The reaction mixture was then allowed to cool to rt, quenched with solid $\mathrm{NaHCO}_{3}(500 \mathrm{mg})$, and diluted with $\mathrm{CH}_{2} \mathrm{Cl}_{2}(80 \mathrm{~mL})$ and saturated aqueous $\mathrm{NaHCO}_{3}(30 \mathrm{~mL})$. The organic layer was separated, and the aqueous layer extracted with $\mathrm{CH}_{2} \mathrm{Cl}_{2}(3 \times 30 \mathrm{~mL})$. The combined organic extracts were washed with $\mathrm{H}_{2} \mathrm{O}(2 \times 10 \mathrm{~mL})$, dried over $\mathrm{MgSO}_{4}$, filtered, and concentrated in vacuo. The residue was coevaporated with PhMe $(2 \times 20 \mathrm{~mL})$, and multiply purified by $\mathrm{SiO}_{2}$ flash chromatography with petrol-EtOAc (10:1) as eluent to give "technical grade" trityl ether $32\left(0.51 \mathrm{~g}\right.$, ca. 73-78\%) as a colorless oil. ${ }^{1} \mathrm{H}$ NMR analysis of this trityl ether 32 revealed that it was only $85-90 \%$ pure (see spectrum of 32 in Part 6 of this SI), it typically being contaminated with $10-15 \%$ of $\mathrm{Ph}_{3} \mathrm{COH}$; the latter contaminant has virtually identical TLC mobility with 32 and is extremely difficult to remove completely. However, the presence of $\mathrm{Ph}_{3} \mathrm{COH}$ did not have a detrimental effect on the subsequent chemistry that took place and this "technical grade" material could be used for the next step without any problems being encountered.

Data for 32: $[\alpha]_{D}+17.12^{\circ}\left(c\right.$ 0.438, $\left.\mathrm{CH}_{2} \mathrm{Cl}_{2}\right)$; IR (neat film) $3061(\mathrm{w}), 3029(\mathrm{w}), 2932(\mathrm{~m}), 2857(\mathrm{~m}), 1612(\mathrm{w})$, $1513(\mathrm{~m}), 1490$ (w), 1467 (w), 1427 (w), 1248 (m), 1109 (s), 1077 (s), 1036 (s), 908 (m), 823 (w), 764 (m), 735 (s), 703 (s), 505 (w) cm ${ }^{-1} .{ }^{1} \mathrm{H}$ NMR (500 MHz, $\left.\mathrm{CDCl}_{3}, 298 \mathrm{~K}\right) \delta$ 7.78-7.70 (m, 5H, Ph), 7.57-7.18 (m, 22H, Ph), $6.87(\mathrm{~m}, 2 \mathrm{H}, \mathrm{PMP}), 5.56(\mathrm{dd}, 1 \mathrm{H}, J=6.4 \mathrm{~Hz}, \underline{\mathrm{H}}-13), 4.57\left(\mathrm{~d}, 1 \mathrm{H}, J=-11.8, \mathrm{PMPC}_{2}-\right), 4.51(\mathrm{~d}, 1 \mathrm{H}, J=-11.8$, PMPC至 $2^{-}$), 4.30 (dd, $\left.1 \mathrm{H}, J=6.8,-12.6 \mathrm{~Hz}, \underline{\mathrm{H}}-17 \mathrm{a}\right), 4.26$ (dd, $\left.1 \mathrm{H}, J=6.6,-12.6 \mathrm{~Hz}, \underline{\mathrm{H}}-17 \mathrm{~b}\right), 3.80$ (s, 3H, PMP$\left.\mathrm{OC}_{3}\right), 3.63(\mathrm{~m}, 1 \mathrm{H}, \underline{\mathrm{H}}-11)$, 3.52-3.37 (m, 3H, $\left.\underline{\mathrm{H}}-15, \underline{\mathrm{H}}-16 \mathrm{a}, \underline{\mathrm{H}}-16 \mathrm{~b}\right), 3.34$ (dd, 1H, J= 5.2, -9.1 Hz, 브-10a), 3.07 (dd, $1 \mathrm{H}, J=5.9,-9.1 \mathrm{~Hz}, \underline{\mathrm{H}}-10 \mathrm{~b}), 2.34$ (d, 1H, J = -13.3 Hz, $\underline{\mathrm{H}}-12_{\text {eq }}$ ), 2.27 (d, 1H, J = -13.6 Hz, $\underline{\mathrm{H}}-14_{\text {eq }}$ ), 2.06 (dd, $\left.1 \mathrm{H}, J=12.2,-12.4 \mathrm{~Hz}, \underline{\mathrm{H}}-12_{\mathrm{ax}}\right), 1.70\left(\mathrm{dd}, 1 \mathrm{H}, J=11.8,-12.5 \mathrm{~Hz}, \underline{\mathrm{H}}-14_{\mathrm{ax}}\right), 1.11$ (s, 9H, $\left.t-\underline{B u}\right) \mathrm{ppm} ;{ }^{13} \mathrm{C}$ NMR $(125$ $\left.\mathrm{MHz}, \mathrm{CDCl}_{3}, 298 \mathrm{~K}\right) \delta 159.08$ (PMP q $\underline{\mathrm{C}}$ bearing OMe), $146.82(\mathrm{Ph}), 144.05$ (ㄷ-13a), $135.61(\mathrm{Ph}), 133.80(\mathrm{Ph})$, 130.31 (Ph), $129.56(\mathrm{Ph}), 129.55(\mathrm{Ph}), 129.32(\mathrm{Ph}), 128.73(\mathrm{Ph}), 127.87(\mathrm{Ph}), 127.86(\mathrm{Ph}), 127.70(\mathrm{Ph}), 127.60$ $(\mathrm{Ph}), 127.19(\mathrm{Ph}), 126.86(\mathrm{Ph}), 123.53(\mathrm{Ph}), 113.69(\mathrm{Ph}), 86.32$ (q $\underline{\mathrm{C}}-\mathrm{Tr}), 81.93\left(\mathrm{Ph}_{3} \mathrm{COH}\right.$ contaminent), 77.29

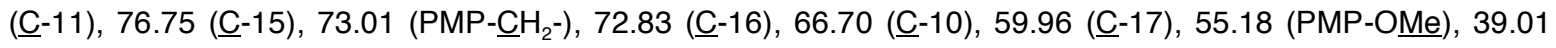

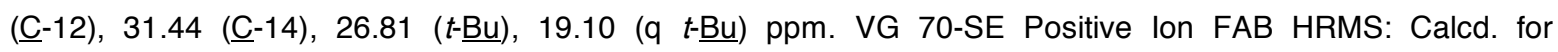
$\mathrm{C}_{52} \mathrm{H}_{56} \mathrm{O}_{5} \mathrm{SiNa}(\mathrm{M}+\mathrm{Na})^{+}:$: 811.37945 Found: 811.37749 . 


\section{Alcohol 33}

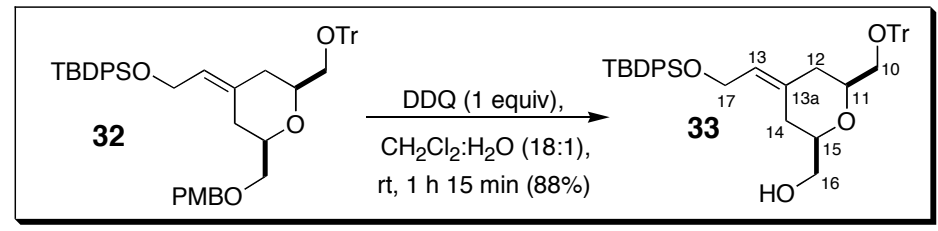

To a solution of trityl ether $32(0.4 \mathrm{~g}, 0.507 \mathrm{mmol})$ in a mixture of $\mathrm{CH}_{2} \mathrm{Cl}_{2}(18 \mathrm{~mL})$ and $\mathrm{H}_{2} \mathrm{O}(1 \mathrm{~mL})$ at $\mathrm{rt}$ was added 2,3-dichloro-5,6-dicyanobenzoquinone (DDQ) $(0.12 \mathrm{~g}, 0.507 \mathrm{mmol}, 1$ equiv) in one portion. The reactants were stirred for $1 \mathrm{~h} 15 \mathrm{~min}$ and then quenched with solid $\mathrm{NaHCO}_{3}(500 \mathrm{mg})$ followed by saturated aqueous $\mathrm{NaHCO}_{3}(20 \mathrm{~mL})$. The resulting mixture was diluted with $\mathrm{CH}_{2} \mathrm{Cl}_{2}(20 \mathrm{~mL})$. The organic layer was separated, and the aqueous phase extracted with $\mathrm{CH}_{2} \mathrm{Cl}_{2}(3 \times 20 \mathrm{~mL})$. The combined extracts were washed with sat. aq. $\mathrm{NaHCO}_{3}(2 \times 50 \mathrm{~mL})$. After drying over $\mathrm{MgSO}_{4}$, the solution was filtered, and concentrated in vacuo. The crude residue was purified by $\mathrm{SiO}_{2}$ flash chromatography (gradient elution with 10:1 then 8:1 petrolEtOAc as eluent) to give alcohol 33 (297 $\mathrm{mg}, 88 \%)$ as a white foam.

Data for 33: $[\alpha]_{D}+6.15^{\circ}\left(c 0.39, \mathrm{CH}_{2} \mathrm{Cl}_{2}\right)$; IR (neat film) $3449(\mathrm{br} \mathrm{m}), 3057(\mathrm{~m}), 3021(\mathrm{~m}), 2958(\mathrm{~s}), 2931(\mathrm{~s})$, $2858(\mathrm{~s}), 1490(\mathrm{~m}), 1472(\mathrm{~m}), 1448(\mathrm{~m}), 1427(\mathrm{~m}), 1388(\mathrm{w}), 1362(\mathrm{w}), 1218(\mathrm{~m}), 1110(\mathrm{~s}), 1074(\mathrm{~s}), 1042(\mathrm{~s})$, 760 (s), 704 (s), 505 (m) cm ${ }^{-1} .{ }^{1} \mathrm{H}$ NMR (500 MHz, $\left.\mathrm{CDCl}_{3}, 298 \mathrm{~K}\right) \delta$ 7.78-7.67 (m, 4H, Ph), 7.54-7.20 (m, 21H, $\mathrm{Ph}), 5.54(\mathrm{t}, 1 \mathrm{H}, J=6.4 \mathrm{~Hz}, \underline{\mathrm{H}}-13), 4.28(\mathrm{dd}, 1 \mathrm{H}, J=6.8,-12.5 \mathrm{~Hz}, \underline{\mathrm{H}}-17 \mathrm{a}), 4.24$ (dd, $1 \mathrm{H}, J=6.4,-12.5 \mathrm{~Hz}, \underline{\mathrm{H}}-$ 17b), 3.59 (m, 1H, $\underline{\mathrm{H}}-11$ ), 3.55 (dd, 1H, J=2.8, -11.5 Hz, 브-16a), 3.45 (dd, 1H, J = 6.8, -11.5 Hz, 브-16b), 3.29 (m, 1H, ㅂ-15), 3.26 (dd, 1H, J=5.4, $-9.4 \mathrm{~Hz}, \underline{\mathrm{H}}-10 \mathrm{a}), 3.07$ (dd, $1 \mathrm{H}, J=5.3,-9.4 \mathrm{~Hz}, \underline{\mathrm{H}}-10 \mathrm{~b}), 2.26(\mathrm{~d}, 1 \mathrm{H}, J=-$ $13.4 \mathrm{~Hz}, \underline{\mathrm{H}}-12_{\text {eq }}$ ), 2.14 (d, $1 \mathrm{H}, J=-13.5 \mathrm{~Hz}, \underline{\mathrm{H}}-14_{\text {eq }}$ ), 2.10 (very br s, $1 \mathrm{H},-\mathrm{O} \underline{\mathrm{H}}$ ), 2.04 (dd, $1 \mathrm{H}, J=12.2,-12.6 \mathrm{~Hz}$, $\left.\underline{\mathrm{H}}-12_{\mathrm{ax}}\right), 1.65$ (dd, $\left.1 \mathrm{H}, J=12.5,-12.7 \mathrm{~Hz}, \underline{\mathrm{H}}-14_{\mathrm{ax}}\right), 1.08$ (s, 9H, $t$ - $\underline{\mathrm{Bu}}$ ) ppm; ${ }^{13} \mathrm{C}$ NMR $\left(125 \mathrm{MHz}, \mathrm{CDCl}_{3}, 298 \mathrm{~K}\right) \delta$ 143.96 (ㅁ-13a), $135.59(\mathrm{Ph}), 135.56(\mathrm{Ph}), 135.21(\mathrm{Ph}), 133.80(\mathrm{Ph}), 133.76(\mathrm{Ph}), 129.61(\mathrm{Ph}), 129.60(\mathrm{Ph})$,

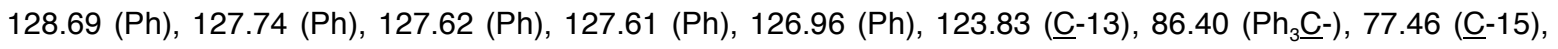

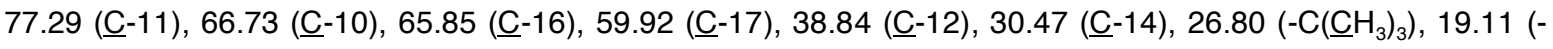
$\left.\underline{\mathrm{C}}\left(\mathrm{CH}_{3}\right)_{3}\right)$ ppm. VG 70-SE Positive Ion FAB HRMS: Calcd. for $\mathrm{C}_{44} \mathrm{H}_{48} \mathrm{O}_{4} \mathrm{SiNa}(\mathrm{M}+\mathrm{Na})^{+}: 691.32194$ Found: 691.32364 .

\section{Our Correlation with H.M.R Hoffmann's Pyran 34 and Alcohol 37}

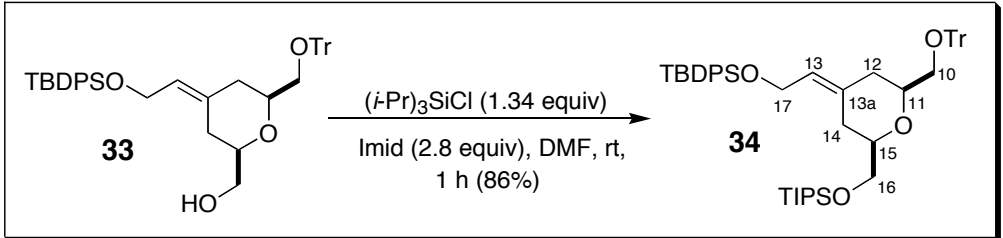

To a stirred rt solution of alcohol $33(197 \mathrm{mg}, 0.295 \mathrm{mmol})$ and imidazole $(56.14 \mathrm{mg}, 0.825 \mathrm{mmol}, 2.8$ equiv) in dry DMF (1 mL) under $\mathrm{N}_{2}$ was added triisopropylsilyl chloride $(0.085 \mathrm{~mL}, 0.395 \mathrm{mmol}, 1.34$ equiv) in one portion. After stirring at rt for $1 \mathrm{~h}$, the reactants were quenched by successively adding solid $\mathrm{NaHCO}_{3}(200 \mathrm{mg}), \mathrm{Et}_{2} \mathrm{O}$ 
$(20 \mathrm{~mL})$, and saturated aqueous $\mathrm{NaHCO}_{3}(20 \mathrm{~mL})$. The organic layer was separated, and the aqueous fraction was extracted with $\mathrm{Et}_{2} \mathrm{O}(3 \times 10 \mathrm{~mL})$. The combined organic extracts were washed with $\mathrm{H}_{2} \mathrm{O}(2 \times 20 \mathrm{~mL})$, dried over $\mathrm{MgSO}_{4}$, filtered, and concentrated in vacuo. Purification of the organic residue by $\mathrm{SiO}_{2} \mathrm{flash}$ chromatography using petrol-EtOAc $(40: 1)$ as eluent provided TIPS-ether $34(0.21 \mathrm{~g}, 86 \%)$ in pure condition (see accompanying spectra) as a white foam.

Data for 34: $[\alpha]_{\mathrm{D}}+6.7^{\circ}\left(c 0.12, \mathrm{CHCl}_{3}\right)\left\{\right.$ Lit. $\left.[\alpha]_{\mathrm{D}}+1.8^{\circ}\left(c 1, \mathrm{CHCl}_{3}\right)\right\}$; IR (neat film) $3061(\mathrm{w}), 3028(\mathrm{w}), 2937(\mathrm{~s})$, 2862 (s), 1458 (m), 1439 (m), 1385 (w), 1107 (s), 1072 (s), 1040 (m), 885 (w), 773 (w), 748 (w), 702 (s), 503 (m) $\mathrm{cm}^{-1} .{ }^{1} \mathrm{H}$ NMR $\left(500 \mathrm{MHz}, \mathrm{CDCl}_{3}, 298 \mathrm{~K}\right) \delta$ 7.75-7.64 (m, 4H, Ph), 7.52-7.18 (m, 21H, Ph), $5.50(\mathrm{t}, 1 \mathrm{H}, J=6.4$ $\mathrm{Hz}, \underline{\mathrm{H}}-13), 4.28(\mathrm{~m}, 1 \mathrm{H}, \underline{\mathrm{H}}-17 \mathrm{a}), 4.26(\mathrm{~m}, 1 \mathrm{H}, \underline{\mathrm{H}}-17 \mathrm{~b}), 3.74$ (dd, 1H, J=5.0, -10.0 Hz, ㅂ-16a), 3.55 (dd, $1 \mathrm{H}, J=$ 6.0, $-10.0 \mathrm{~Hz}, \underline{\mathrm{H}}-16 \mathrm{~b})$ superimposed upon $3.53(\mathrm{~m}, 1 \mathrm{H}, \underline{\mathrm{H}}-11), 3.27(\mathrm{~m}, 1 \mathrm{H}, \underline{\mathrm{H}}-15), 3.22$ (dd, $1 \mathrm{H}, J=5.2,-9.2$ $\mathrm{Hz}, \underline{\mathrm{H}}-10 \mathrm{a}), 2.99$ (dd, 1H, J=5.7, $-9.2 \mathrm{~Hz}, \underline{\mathrm{H}}-10 \mathrm{~b}), 2.44$ (d, $\left.1 \mathrm{H}, J=-13.5 \mathrm{~Hz}, \underline{\mathrm{H}}-14_{\text {eq }}\right), 2.25(\mathrm{~d}, 1 \mathrm{H}, J=-13.2 \mathrm{~Hz}$, $\underline{\mathrm{H}}-12_{\text {eq }}$ ), 2.01 (dd, $1 \mathrm{H}, J=12.1,-12.4 \mathrm{~Hz}, \underline{\mathrm{H}}-12_{\mathrm{ax}}$ ), 1.62 (apparent t, $\left.1 \mathrm{H}, J=12.4 \mathrm{~Hz}, \underline{\mathrm{H}}-14_{\mathrm{ax}}\right), 1.06(\mathrm{~s}, 9 \mathrm{H}, t$ - $\underline{\mathrm{Bu}})$, 1.02 and $1.01\left(2 \times \mathrm{s}, 18 \mathrm{H}\right.$, TIPS- $\left.\underline{\mathrm{H}}_{3}\right)$ superimposed upon 1.10-0.98 (m, 3H, TIPS-C$\left.(\mathrm{Me})_{2}\right)$ ppm; ${ }^{13} \mathrm{C} \mathrm{NMR}$ (125 MHz, $\left.\mathrm{CDCl}_{3}, 298 \mathrm{~K}\right) \delta 144.09$ (ㅡ-13a), $135.95(\mathrm{Ph}), 135.56(\mathrm{Ph}), 133.92(\mathrm{Ph}), 133.91(\mathrm{Ph}), 129.54(\mathrm{Ph})$, $128.77(\mathrm{Ph}), 127.70(\mathrm{Ph}), 127.60(\mathrm{Ph}), 127.59(\mathrm{Ph}), 126.88(\mathrm{Ph}), 123.35(\underline{\mathrm{C}}-13), 86.35\left(\mathrm{Ph}_{3} \underline{\mathrm{C}}-\right), 78.16(\underline{\mathrm{C}}-15)$,

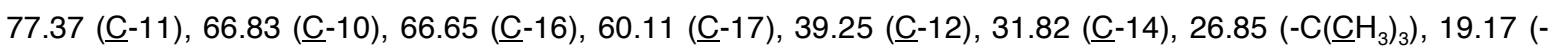
$\left.\underline{\mathrm{C}}\left(\mathrm{CH}_{3}\right)_{3}\right), 17.95$ (i-Pr), 11.91 (i- $\left.\underline{\mathrm{Pr}}\right)$ ppm. VG 70-SE Positive Ion FAB HRMS: Calcd. for $\mathrm{C}_{53} \mathrm{H}_{68} \mathrm{O}_{4} \mathrm{Si}_{2} \mathrm{Na}(\mathrm{M}+\mathrm{Na})$ : 847.45536 Found: 847.45804.

Following the procedure reported by H.M.R. Hoffmann et al. (see the SI in Org. Lett. 2001, 3, 929), compound 34 was also converted into alcohol 37. The ${ }^{13} \mathrm{C}$ NMR spectral data for our synthetic 37 is reported in Part 5 of the present SI along with the ${ }^{13} \mathrm{C}$ NMR data published by H.M.R. Hoffmann et al. for comparison purposes. See Part 6 of our SI for copies of the actual spectra that we obtained for $\mathbf{3 7}$. Unfortunately, we were unable to completely purify our sample of $\mathbf{3 7}$. Nevertheless, the sample of $\mathbf{3 7}$ that was synthesized was of sufficient purity to allow us to make a second definitive structural correlation with the Hoffmann synthesis of the same compound.

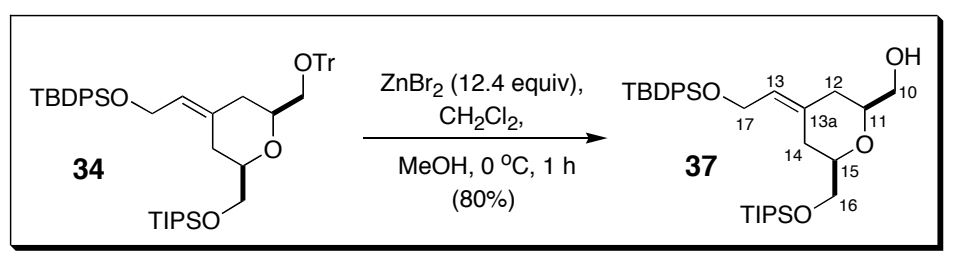




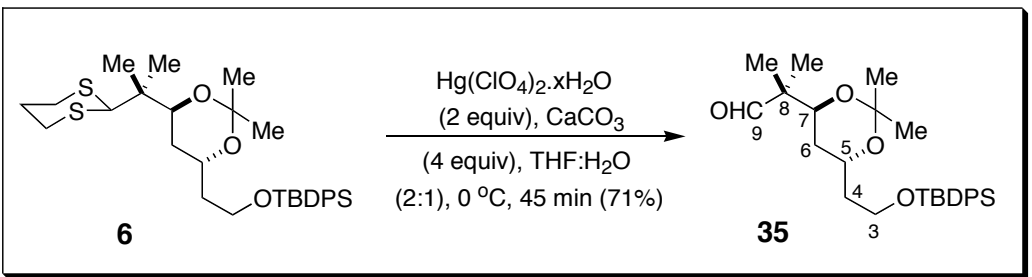

To a stirred solution of dithiane $6(1.12 \mathrm{~g}, 2.00 \mathrm{mmol})$ in THF $(20 \mathrm{~mL})$ and $\mathrm{H}_{2} \mathrm{O}(10 \mathrm{~mL})$ at $0{ }^{\circ} \mathrm{C}$ was added calcium carbonate $(0.81 \mathrm{~g}, 8.02 \mathrm{mmol}, 4.0$ equiv) and mercury (II) perchlorate hydrate (1.6 g, $4.0 \mathrm{mmol}, 2.0$ equiv). After $45 \mathrm{~min}$ at $0{ }^{\circ} \mathrm{C}$, the reaction mixture was diluted with EtOAc $(50 \mathrm{~mL})$ and saturated aqueous $\mathrm{NaHCO}_{3}(30 \mathrm{~mL})$. The resulting mixture was transferred to a separatory funnel. The organic layer was separated and the aqueous phase was extracted with EtOAc $(3 \times 100 \mathrm{~mL})$. The combined organic extracts were dried over $\mathrm{MgSO}_{4}$, filtered, and concentrated in vacuo. The crude residue was purified by $\mathrm{SiO}_{2} \mathrm{flash}$ chromatography with petrol-EtOAc $(50: 1)$ as eluent to provide aldehyde $35(0.67 \mathrm{~g}, 71 \%)$ as a colorless oil.

Data for 35: $[\alpha]_{D}-13.0^{\circ}\left(c 0.46, \mathrm{CH}_{2} \mathrm{Cl}_{2}\right)$; IR (neat film) $3072(\mathrm{w}), 3049(\mathrm{w}), 2986(\mathrm{~m}), 2934(\mathrm{~s}), 2858(\mathrm{~m}), 1728$ (s), $1472(\mathrm{~m}), 1427$ (s), 1379 (s), 1223 (s), 1171 (w), 1113 (s), 1092 (s), 1028 (w), 824 (m), 737 (m), 702 (s), $689(\mathrm{w}), 615(\mathrm{~m}), 505(\mathrm{~s}), 490(\mathrm{w}) \mathrm{cm}^{-1}$.

${ }^{1} \mathrm{H}$ NMR (500 MHz, $\left.\mathrm{C}_{6} \mathrm{D}_{6}, 298 \mathrm{~K}\right) \delta 9.49$ (s, 1H, C-9-C트), 7.82-7.70 (m, 4H, Ph), 7.29-7.18 (m, 6H, Ph), 3.97 (m, $1 \mathrm{H}, \underline{\mathrm{H}}-5), 3.84$ (ddd, $1 \mathrm{H}, J=5.4,8.0,-10.2 \mathrm{~Hz}, \underline{\mathrm{H}}-3 \mathrm{a}), 3.76$ (dd, $1 \mathrm{H}, J=6.3,10.0 \mathrm{~Hz}, \underline{\mathrm{H}}-7$ ), 3.75 (ddd, $1 \mathrm{H}, J$ $=5.1,6.1,-10.5 \mathrm{~Hz}, \underline{\mathrm{H}}-3 \mathrm{~b}), 1.73(\mathrm{~m}, 1 \mathrm{H}, \underline{\mathrm{H}}-4 \mathrm{a}), 1.65(\mathrm{~m}, 1 \mathrm{H}, \underline{\mathrm{H}}-4 \mathrm{~b}), 1.55$ (ddd, $1 \mathrm{H}, J=6.0,10.0,-12.7 \mathrm{~Hz}, \underline{\mathrm{H}}-$ 6a), $1.26\left(\mathrm{~s}, 3 \mathrm{H}\right.$, Isopropylidene- $\left.\mathrm{C}_{3}\right), 1.23\left(\mathrm{~s}, 3 \mathrm{H}\right.$, Isopropylidene- $\left.\underline{\mathrm{H}}_{3}\right), 1.22(\mathrm{~m}, 1 \mathrm{H}, \underline{\mathrm{H}}-6 \mathrm{~b}), 1.16(\mathrm{~s}, 9 \mathrm{H},-$ $\left.\mathrm{C}\left(\mathrm{CH}_{3}\right)_{3}\right), 0.95\left(\mathrm{~s}, 3 \mathrm{H}, \mathrm{C}-8-\mathrm{CH}_{3}\right), 0.75\left(\mathrm{~s}, 3 \mathrm{H}, \mathrm{C}-8-\mathrm{CH}_{3}\right)$ ppm; ${ }^{13} \mathrm{C}$ NMR $\left(125 \mathrm{MHz}, \mathrm{C}_{6} \mathrm{D}_{6}, 298 \mathrm{~K}\right) \delta 204.39(\underline{\mathrm{C}}-9$ $\mathrm{C}=\mathrm{O}), 135.93$ (o-Ph), 135.92 (o-Ph), 134.18 (q Ph), 129.98 (p-Ph), 128.07 (m-Ph), 128.05 (m-Ph), 100.59

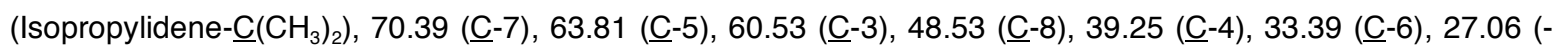
$\left.\mathrm{C}\left(\underline{\mathrm{CH}}_{3}\right)_{3}\right), 24.56$ (Isopropylidene- $\left.\underline{\mathrm{CH}}_{3}\right), 24.35$ (Isopropylidene- $\left.\underline{\mathrm{C}} \mathrm{H}_{3}\right), 19.39\left(-\underline{\mathrm{C}}\left(\mathrm{CH}_{3}\right)_{3}\right), 18.55\left(\mathrm{C}-8-\underline{\mathrm{C}} \mathrm{H}_{3}\right), 16.47$ $\left(\mathrm{C}-8-\underline{\mathrm{CH}}_{3}\right) \mathrm{ppm}$.

${ }^{1} \mathrm{H}$ NMR (500 MHz, $\left.\mathrm{CDCl}_{3}, 298 \mathrm{~K}\right) \delta 9.56$ (s, 1H, C-9-C브), 7.70-7.57 (m, 4H, Ph), 7.45-7.28 (m, 6H, Ph), 4.00 $(\mathrm{m}, 1 \mathrm{H}, \underline{\mathrm{H}}-5), 3.90$ (dd, $1 \mathrm{H}, J=6.4,9.9 \mathrm{~Hz}, \underline{\mathrm{H}}-7$ ), 3.76 (ddd, $1 \mathrm{H}, J=5.4,7.8,-10.3 \mathrm{~Hz}, \underline{\mathrm{H}}-3 \mathrm{a}), 3.68$ (ddd, $1 \mathrm{H}, J=$ 5.5, 5.5, -10.5 Hz, 브-3b), 1.76 (m, 1H, $\underline{\mathrm{H}}-6 \mathrm{a}), 1.74$ (m, 1H, $\underline{\mathrm{H}}-4 \mathrm{a}), 1.70$ (m, 1H, $\underline{\mathrm{H}}-4 \mathrm{~b}), 1.48$ (m, $1 \mathrm{H}, \underline{\mathrm{H}}-6 \mathrm{~b}), 1.29$ (s, 3H, Isopropylidene- $\left.\underline{\mathrm{H}}_{3}\right), 1.27\left(\mathrm{~s}, 3 \mathrm{H}\right.$, Isopropylidene- $\left.\underline{\mathrm{C}}_{3}\right), 1.04\left(\mathrm{~s}, 3 \mathrm{H}, \mathrm{C}-8-\mathrm{C}_{3}\right), 1.03\left(\mathrm{~s}, 9 \mathrm{H},-\mathrm{C}\left(\mathrm{C}_{3}\right)_{3}\right)$,

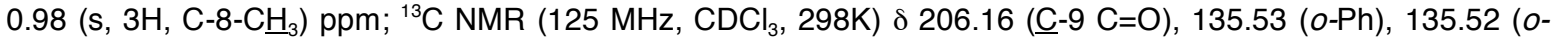
$\mathrm{Ph}$ ), 133.85 (q Ph), 133.80 (q Ph), 129.59 (p-Ph), 129.58 (p-Ph), 127.63 (m-Ph), 127.61 (m-Ph), 100.54

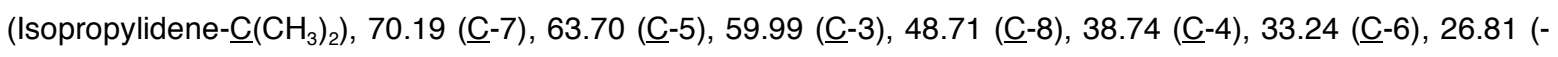
$\left.\mathrm{C}\left(\underline{\mathrm{C}}_{3}\right)_{3}\right), 24.42$ (Isopropylidene- $\left.\underline{\mathrm{C}} \mathrm{H}_{3}\right), 24.18$ (Isopropylidene- $\left.\underline{\mathrm{C}} \mathrm{H}_{3}\right), 19.17\left(-\underline{\mathrm{C}}\left(\mathrm{CH}_{3}\right)_{3}\right), 18.68\left(\mathrm{C}-8-\underline{\mathrm{C}} \mathrm{H}_{3}\right), 16.21(\mathrm{C}-$ 8- $\mathrm{CH}_{3}$ ) ppm. VG 70-SE Positive Ion FAB HRMS: Calcd for $\mathrm{C}_{28} \mathrm{H}_{40} \mathrm{O}_{4} \mathrm{SiNa}(\mathrm{M}+\mathrm{Na})$ : 491.25934. Found: 491.26070 . 


\section{Our Conversion of 35 into Masamune's Methyl Ketone 36 and the Subsequent Successful Correlation with Masamune's Reported ${ }^{1} \mathrm{H}$ NMR Data for 36}

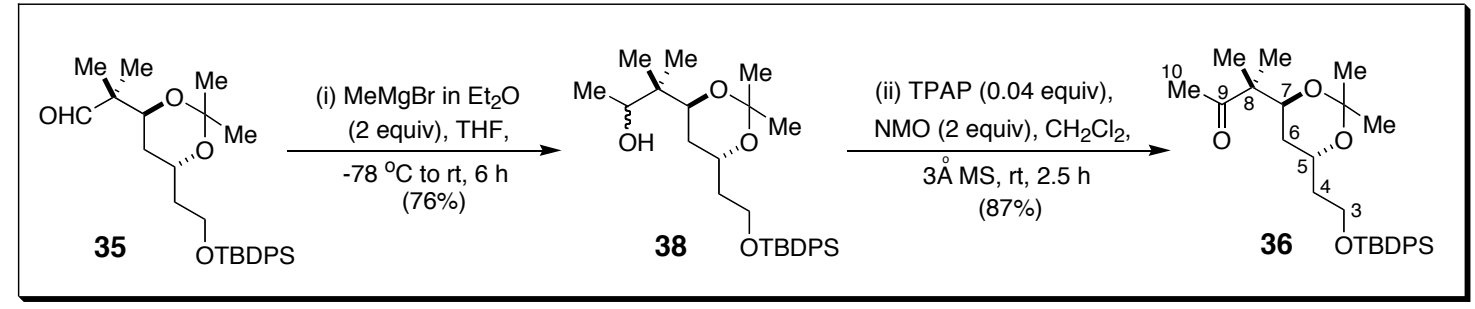

(i) To a $-78{ }^{\circ} \mathrm{C}$ solution of aldehyde $35(470 \mathrm{mg}, 1.0 \mathrm{mmol})$ in dry THF $(5 \mathrm{~mL})$ under $\mathrm{N}_{2}$ was added methyl magnesium bromide ( $3.0 \mathrm{M}$ solution in $\mathrm{Et}_{2} \mathrm{O}, 0.67 \mathrm{~mL}, 2.01 \mathrm{mmol}, 2.0$ equiv) dropwise over $1 \mathrm{~min}$. The reaction mixture was gradually warmed to rt over $6 \mathrm{~h}$. When TLC analysis indicated the reaction was complete, the resulting mixture was diluted with EtOAc $(50 \mathrm{~mL})$ and saturated aqueous $\mathrm{NaHCO}_{3}(50 \mathrm{~mL})$. The organic layer was separated, and the aqueous layer extracted with EtOAc $(3 \times 100 \mathrm{~mL})$. The combined organic extracts were dried over $\mathrm{MgSO}_{4}$, filtered and concentrated in vacuo. The residue was purified by $\mathrm{SiO}_{2}$ flash chromatography with petrol-EtOAc (15:1) as eluent to give the epimeric mixture of alcohols $\mathbf{3 8}(0.37 \mathrm{~g}, 76 \%)$ as a colorless oil

(ii) To a stirred solution of alcohols $38(370 \mathrm{mg}, 0.76 \mathrm{mmol})$, powdered $3 \AA ̊$ molecular sieves (freshly flame dried under high vacuum, $0.5 \mathrm{~g}$ ) and $\mathrm{N}$-methylmorpholine $\mathrm{N}$-oxide (180 mg, $1.53 \mathrm{mmol}, 2.0$ equiv) in dry $\mathrm{CH}_{2} \mathrm{Cl}_{2}(5$ $\mathrm{mL}$ ) was added tetra- $n$-propylammonium perruthenate (TPAP) $(10.7 \mathrm{mg}, 0.031 \mathrm{mmol}, 0.04$ equiv) in one portion. After $2.5 \mathrm{~h}$ stirring at $\mathrm{rt}$, the reaction mixture was filtered through a pad of Celite and the filtrate concentrated in vacuo. The black residue was purified by $\mathrm{SiO}_{2}$ flash chromatography with petrol-EtOAc (20:1) as eluent to afford ketone $\mathbf{3 6}$ ( $320 \mathrm{mg}, \mathbf{8 7 \%}$ ) as a colorless oil.

Data for 36: $[\alpha]_{D}-15.6^{\circ}\left(c\right.$ 0.66, $\left.\mathrm{CH}_{2} \mathrm{Cl}_{2}\right)$; $[\alpha]_{D}-15.1^{\circ}\left(c\right.$ 0.744, $\left.\mathrm{CHCl}_{3}\right)\left\{\mathrm{Lit}:[\alpha]_{D}-6.4^{\circ}\left(c\right.\right.$ 1.74, $\left.\mathrm{CHCl}_{3}\right)$; IR (neat film) 3071 (w), 3049 (w), 2933 (s), 2857 (s), 1705 (s), 1589 (w), 1473 (s), 1466 (m), 1428 (s), 1380 (s), 1362 (s), $1224(\mathrm{~s}), 1173(\mathrm{~m}), 1112(\mathrm{~s}), 1091(\mathrm{~s}), 1029(\mathrm{~m}), 989(\mathrm{w}), 960(\mathrm{w}), 824(\mathrm{~m}), 738(\mathrm{~m}), 702(\mathrm{~s}), 688(\mathrm{~m}), 614(\mathrm{~m})$, $506(\mathrm{~s}), 491(\mathrm{~m}) \mathrm{cm}^{-1}$.

${ }^{1} \mathrm{H}$ NMR (500 MHz, $\left.\mathrm{C}_{6} \mathrm{D}_{6}, 298 \mathrm{~K}\right)$ ठ 7.83-7.71 (m, 4H, Ph), 7.29-7.18 (m, $\left.6 \mathrm{H}, \mathrm{Ph}\right), 3.99(\mathrm{~m}, 1 \mathrm{H}, \underline{\mathrm{H}}-5), 3.93(\mathrm{dd}$, $1 \mathrm{H}, J=6.4,9.7 \mathrm{~Hz}, \underline{\mathrm{H}}-7$ ), 3.85 (ddd, $1 \mathrm{H}, J=5.4,8.0,-10.1 \mathrm{~Hz}, \underline{\mathrm{H}}-3 \mathrm{a}$ ), 3.75 (dd, $1 \mathrm{H}, J=5.6,5.5,-10.3 \mathrm{~Hz}, \underline{\mathrm{H}}-$ 3b), $1.92\left(\mathrm{~s}, 3 \mathrm{H}, \mathrm{C}-10-\mathrm{C}_{3}\right), 1.75(\mathrm{~m}, 1 \mathrm{H}, \underline{\mathrm{H}}-4 \mathrm{a}), 1.67(\mathrm{~m}, 1 \mathrm{H}, \underline{\mathrm{H}}-4 \mathrm{~b}), 1.60(\mathrm{~m}, 1 \mathrm{H}, \underline{\mathrm{H}}-6 \mathrm{a}), 1.31(\mathrm{~m}, 1 \mathrm{H}, \underline{\mathrm{H}}-6 \mathrm{~b})$, $1.28\left(\mathrm{~s}, 3 \mathrm{H}\right.$, Isopropylidene- $\left.\mathrm{CH}_{3}\right), 1.26\left(\mathrm{~s}, 3 \mathrm{H}\right.$, Isopropylidene- $\left.\mathrm{CH}_{3}\right), 1.16\left(\mathrm{~s}, 9 \mathrm{H},-\mathrm{C}\left(\mathrm{CH}_{3}\right)_{3}\right), 1.05(\mathrm{~s}, 3 \mathrm{H}, \mathrm{C}-8-$ $\left.\mathrm{C}_{3}\right), 0.86$ (s, 3H, C-8- $\underline{\mathrm{H}}_{3}$ ) ppm; ${ }^{13} \mathrm{C}$ NMR (125 MHz, $\left.\mathrm{C}_{6} \mathrm{D}_{6}, 298 \mathrm{~K}\right)$ \& $210.42(\underline{\mathrm{C}}-9), 135.94(o-\mathrm{Ph}), 135.93$ (o$\mathrm{Ph}$ ), 134.22 (q Ph), 129.96 (p-Ph), 128.07 (m-Ph), 128.04 (m-Ph), 100.48 (Isopropylidene- $\left.\underline{\mathrm{C}}\left(\mathrm{CH}_{3}\right)_{2}\right), 71.21$ (ㄸ-

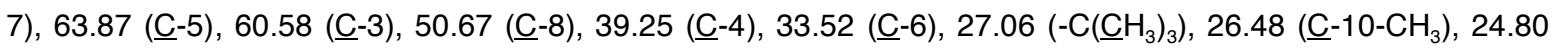
(Isopropylidene- $\left.\mathrm{C}\left(\mathrm{CH}_{3}\right)_{2}\right), 24.49$ (Isopropylidene- $\left.\mathrm{C}\left(\underline{\mathrm{C}} \mathrm{H}_{3}\right)_{2}\right), 20.78\left(\mathrm{C}-8-\underline{\mathrm{C}} \mathrm{H}_{3}\right), 19.40\left(\underline{\mathrm{C}}\left(\mathrm{CH}_{3}\right)_{3}\right), 19.33\left(\mathrm{C}-8-\underline{\mathrm{C}} \mathrm{H}_{3}\right)$ ppm. 
${ }^{1} \mathrm{H}$ NMR (500 MHz, $\left.\mathrm{CDCl}_{3}, 298 \mathrm{~K}\right) \delta$ 7.71-7.55 (m, 4H, Ph), 7.45-7.28 (m, 6H, Ph), 3.97 (m, 1H, $\left.\underline{\mathrm{H}}-5\right), 3.94$ (dd, $1 \mathrm{H}, J=6.4,9.7 \mathrm{~Hz}, \underline{\mathrm{H}}-7$ ), 3.75 (ddd, $1 \mathrm{H}, J=5.4,7.8,-10.2 \mathrm{~Hz}, \underline{\mathrm{H}}-3 \mathrm{a}), 3.67$ (ddd, $1 \mathrm{H}, J=5.5,5.5,-10.5 \mathrm{~Hz}, \underline{\mathrm{H}}-$ 3b), 2.15 (s, 3H, C-10-ㄹe), 1.75-1.64 (m, 3H, $\underline{\mathrm{H}}-4 \mathrm{a}, \underline{\mathrm{H}}-4 \mathrm{~b}, \underline{\mathrm{H}}-6 \mathrm{a}), 1.46$ (ddd, $1 \mathrm{H}, J=6.5,9.8,-9.8 \mathrm{~Hz}, \underline{\mathrm{H}}-6 \mathrm{~b})$, $1.28\left(\mathrm{~s}, 3 \mathrm{H}\right.$, Isopropylidene- $\left.\mathrm{C}\left(\underline{\mathrm{C}}_{3}\right)_{2}\right), 1.27\left(\mathrm{~s}, 3 \mathrm{H}\right.$, Isopropylidene- $\left.\mathrm{C}\left(\underline{\mathrm{CH}}_{3}\right)_{2}\right), 1.10\left(\mathrm{~s}, 3 \mathrm{H}, \mathrm{C}-8-\underline{\mathrm{H}}_{3}\right), 1.04(\mathrm{~s}, 3 \mathrm{H}$, $\left.\mathrm{C}-8-\underline{\mathrm{C}}_{3}\right), 1.02$ (s, 9H, $t$ - $\left.\underline{\mathrm{Bu}}\right) ;{ }^{13} \mathrm{C}$ NMR (125 MHz, CDCl, $\left.298 \mathrm{~K}\right) \delta 213.03(\underline{\mathrm{C}}-9), 135.53$ (o-Ph), 135.52 (o-Ph), 133.86 (q Ph), 133.84 (q Ph), 129.56 (p-Ph), 129.55 (p-Ph), 127.62 (m-Ph), 127.60 (m-Ph), 100.37

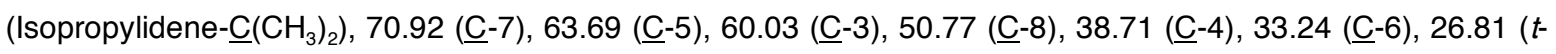
$\underline{\mathrm{Bu}}$ ), 24.64 (Isopropylidene-C$\left.\left(\underline{\mathrm{C}} \mathrm{H}_{3}\right)_{2}\right), 24.29$ (Isopropylidene-C $\left.\left(\underline{\mathrm{C}} \mathrm{H}_{3}\right)_{2}\right), 20.81\left(\mathrm{C}-8-\underline{\mathrm{CH}}_{3}\right), 19.17$ (q, $t$ - $\left.\underline{\mathrm{Bu}}\right), 19.02$ $\left(\mathrm{C}-8-\underline{-} \mathrm{H}_{3}\right)$. VG 70-SE Positive Cl-Methane HRMS: Calcd. for $\mathrm{C}_{29} \mathrm{H}_{43} \mathrm{O}_{4} \mathrm{Si}(\mathrm{M}+\mathrm{H})^{+}$: 483.29305. Found: 483.29100.

For a detailed tabular comparison of our ${ }^{1} \mathrm{H}$ NMR spectral data for 36 with the data of Masamune and coworkers in Ref. 7 a see Part 5 of this SI. 


\section{Enantioselective Formal Total Synthesis of the Antitumor Macrolide Bryostatin 7}

Soraya Manaviazar, ${ }^{*}$ Mark Frigerio, Gurpreet S. Bhatia, Marc G. Hummersone, Abil E. Aliev, and Karl J. Hale*

The Christopher Ingold Laboratories,

The Chemistry Department,

University College London,

20 Gordon Street,

London WC1H OAJ, UK.

Supporting Information. Part 5.

Detailed Tabulated Spectral Comparisons of Compounds 34, 37 and 36 Prepared Via Our Route with the Spectral Data Reported for the Same Intermediates Independently Prepared by Hoffmann and Masamune. 


\section{Detailed Tabular Comparison of Our $500 \mathrm{MHz}{ }^{1} \mathrm{H}$ NMR Data for 34 in $\mathrm{CDCl}_{3}$ with H.M.R. Hoffmann's Data}

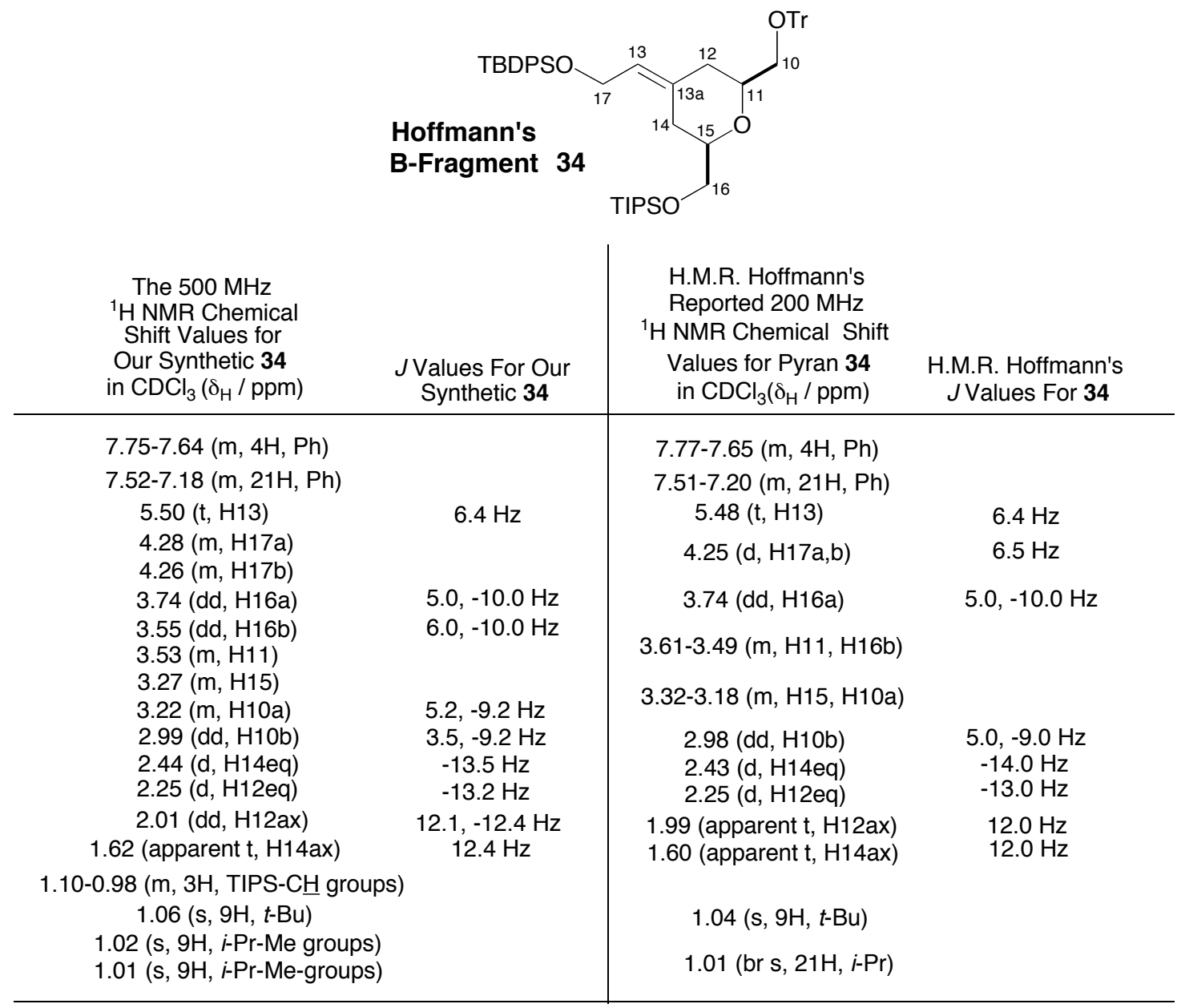


Detailed Tabular Comparison of Our $125 \mathrm{MHz}{ }^{13} \mathrm{C}$ NMR Data for 34 in $\mathrm{CDCl}_{3}$ with H.M.R. Hoffmann's Data Recorded at $50 \mathrm{MHz}$

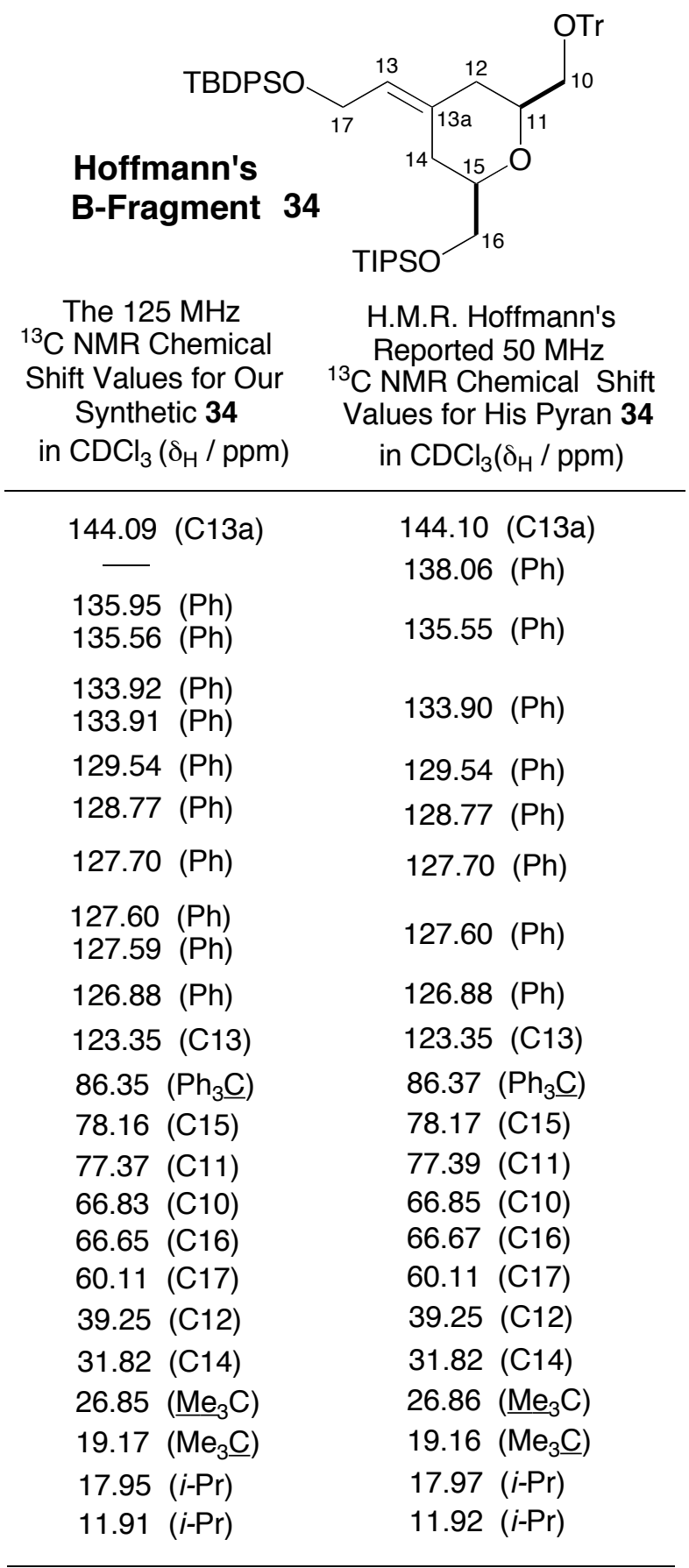


Detailed Tabular Comparison of Our $125 \mathrm{MHz}{ }^{13} \mathrm{C}$ NMR Data for 37 in $\mathrm{CDCl}_{3}$ with H.M.R. Hoffmann's Data Recorded at $50 \mathrm{MHz}$

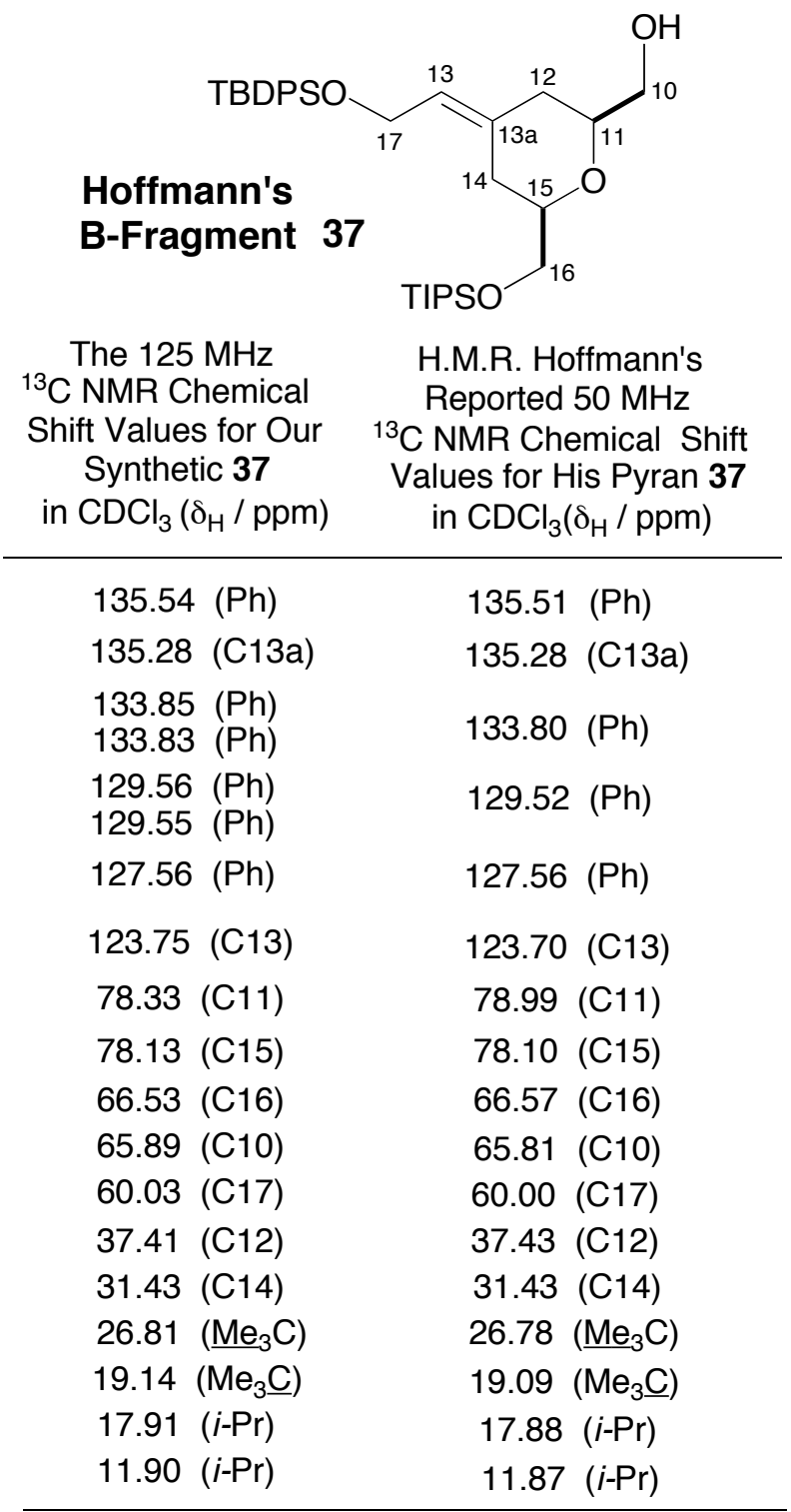




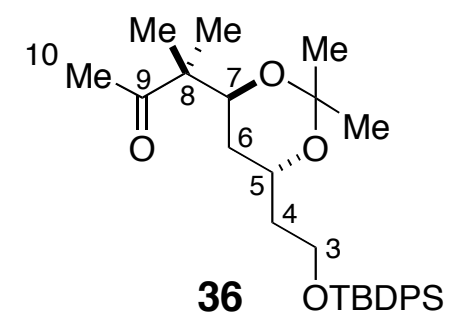

\begin{tabular}{|c|c|c|}
\hline $\begin{array}{c}500 \mathrm{MHz}{ }^{1} \mathrm{H} \text { NMR } \\
\text { Chemical Shifts for } \\
\text { Our Synthetic } 36 \\
\text { in } \mathrm{CDCl}_{3}(\delta / \mathrm{ppm})\end{array}$ & Our $J$ Values & $\begin{array}{c}\text { Masamune's } \\
300 \mathrm{MHz}^{1} \mathrm{H} \text { NMR } \\
\text { Chemical Shifts for } 36 \\
\text { in } \mathrm{CDCl}_{3}(\delta / \mathrm{ppm})\end{array}$ \\
\hline $\begin{array}{l}7.71-7.55(\mathrm{~m}, 4 \mathrm{H}, \mathrm{Ph}) \\
7.45-7.28(\mathrm{~m}, 6 \mathrm{H}, \mathrm{Ph})\end{array}$ & & $\begin{array}{l}\text { 7.71-7.54 (m, 4H, Ph) } \\
7.48-7.28(\mathrm{~m}, 6 \mathrm{H}, \mathrm{Ph})\end{array}$ \\
\hline $\begin{array}{l}3.97(\mathrm{~m}, \mathrm{H} 5) \\
3.94(\mathrm{dd}, \mathrm{H} 7)\end{array}$ & $6.4,9.7 \mathrm{~Hz}$ & $4.02-3.86(\mathrm{~m}, 2 \mathrm{H})$ \\
\hline $\begin{array}{l}3.75 \text { (ddd, H3a) } \\
3.67 \text { (ddd, H3b), }\end{array}$ & $\begin{array}{l}5.4,7.8,-10.2 \mathrm{~Hz} \\
5.5,5.5,-10.5 \mathrm{~Hz}\end{array}$ & $3.81-3.62(m, 2 H)$ \\
\hline $2.15(\mathrm{~s}, 3 \mathrm{H}, \mathrm{C} 10-\mathrm{Me})$ & & $2.16(\mathrm{~s}, 3 \mathrm{H})$ \\
\hline $1.75-1.64(\mathrm{~m}, 3 \mathrm{H}, \mathrm{H} 4 \mathrm{a}, \mathrm{H} 4 \mathrm{~b}, \mathrm{H} 6 \mathrm{a})$ & & $1.69(\mathrm{~m}, 3 \mathrm{H})$ \\
\hline 1.46 (ddd, $1 \mathrm{H}, \mathrm{H} 6 \mathrm{~b})$ & $6.5,9.8,-9.8 \mathrm{~Hz}$ & $1.52-1.38(\mathrm{~m}, 1 \mathrm{H})$ \\
\hline $1.28\left(\mathrm{~s}, 3 \mathrm{H},-\mathrm{C}(\underline{\mathrm{Me}})_{2}\right)$ & & $1.28(\mathrm{~s}, 3 \mathrm{H})$ \\
\hline $1.27\left(\mathrm{~s}, 3 \mathrm{H},-\mathrm{C}(\underline{\mathrm{Me}})_{2}\right)$ & & $1.27(\mathrm{~s}, 3 \mathrm{H})$ \\
\hline $1.10(\mathrm{~s}, \mathrm{C} 8-\underline{\mathrm{Me}})$ & & $1.10(\mathrm{~s}, 3 \mathrm{H})$ \\
\hline $\begin{array}{l}1.04(\mathrm{~s}, 3 \mathrm{H}, \mathrm{C} 8-\mathrm{Me}) \\
1.02(\mathrm{~s}, 9 \mathrm{H}, t-\mathrm{Bu} \mathrm{Me})\end{array}$ & & $1.02(\mathrm{~s}, 12 \mathrm{H})$ \\
\hline
\end{tabular}




\title{
Enantioselective Formal Total Synthesis of the Antitumor Macrolide Bryostatin 7
}

\author{
Soraya Manaviazar, ${ }^{*}$ Mark Frigerio, Gurpreet S. Bhatia, \\ Marc G. Hummersone, Abil E. Aliev, and Karl J. Hale* \\ The Christopher Ingold Laboratories, \\ The Chemistry Department, \\ University College London, \\ 20 Gordon Street, \\ London WC1H OAJ, UK.
}

\section{Supporting Information. Part 6}

Spectra of the Intermediates Used in the Various Correlations Performed with the Hoffmann and Masamune Bryostatin Intermediates 


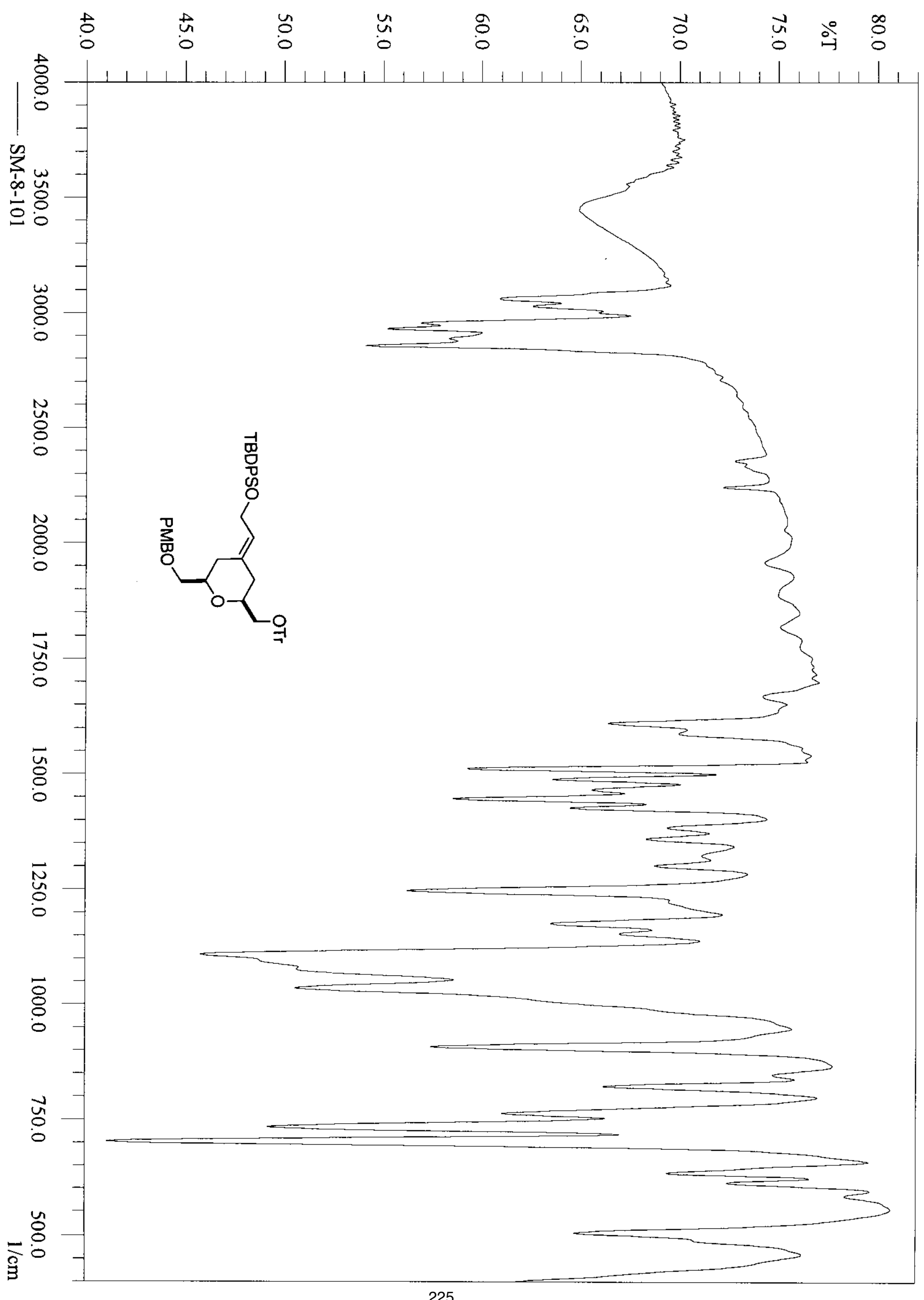




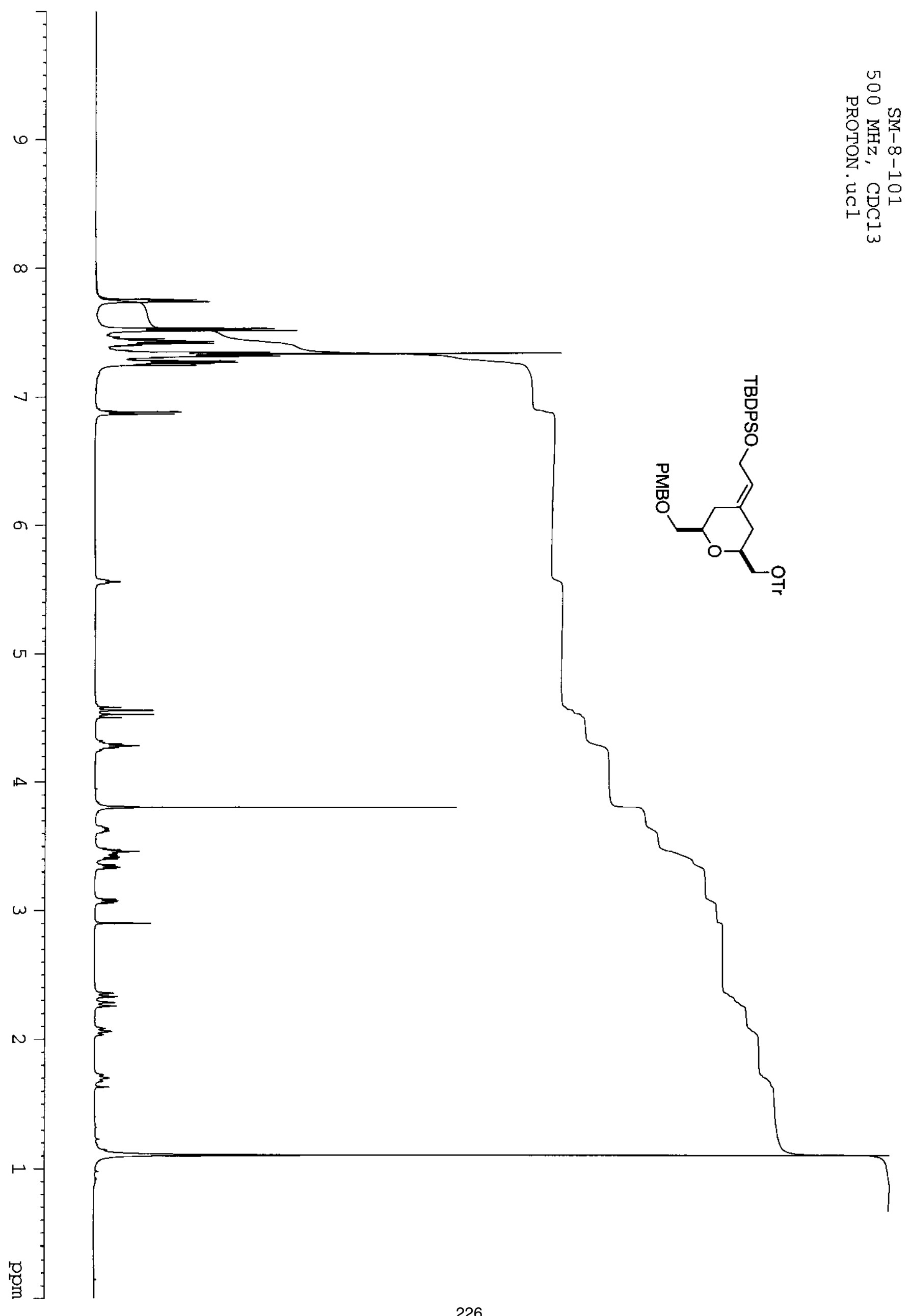



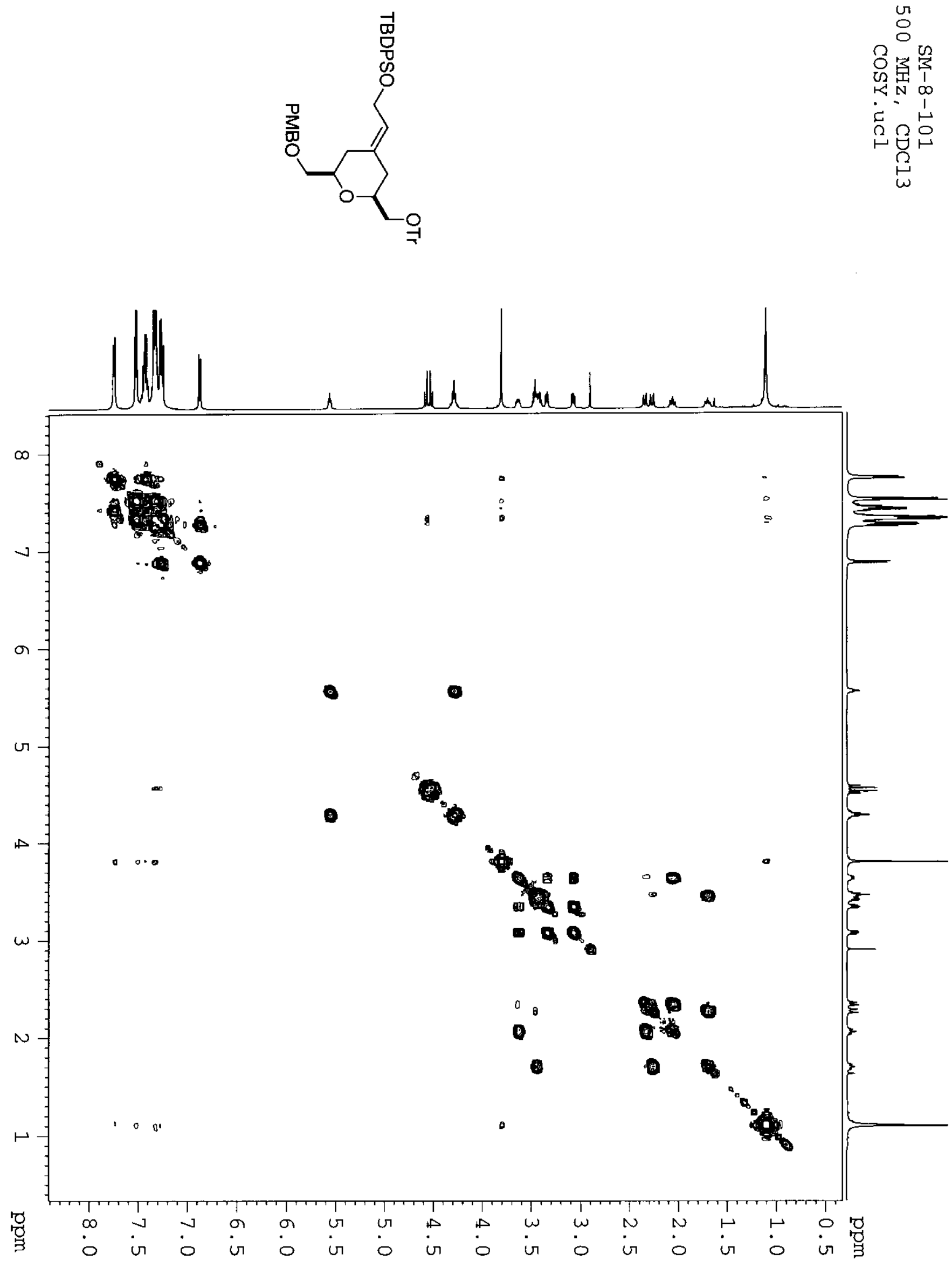

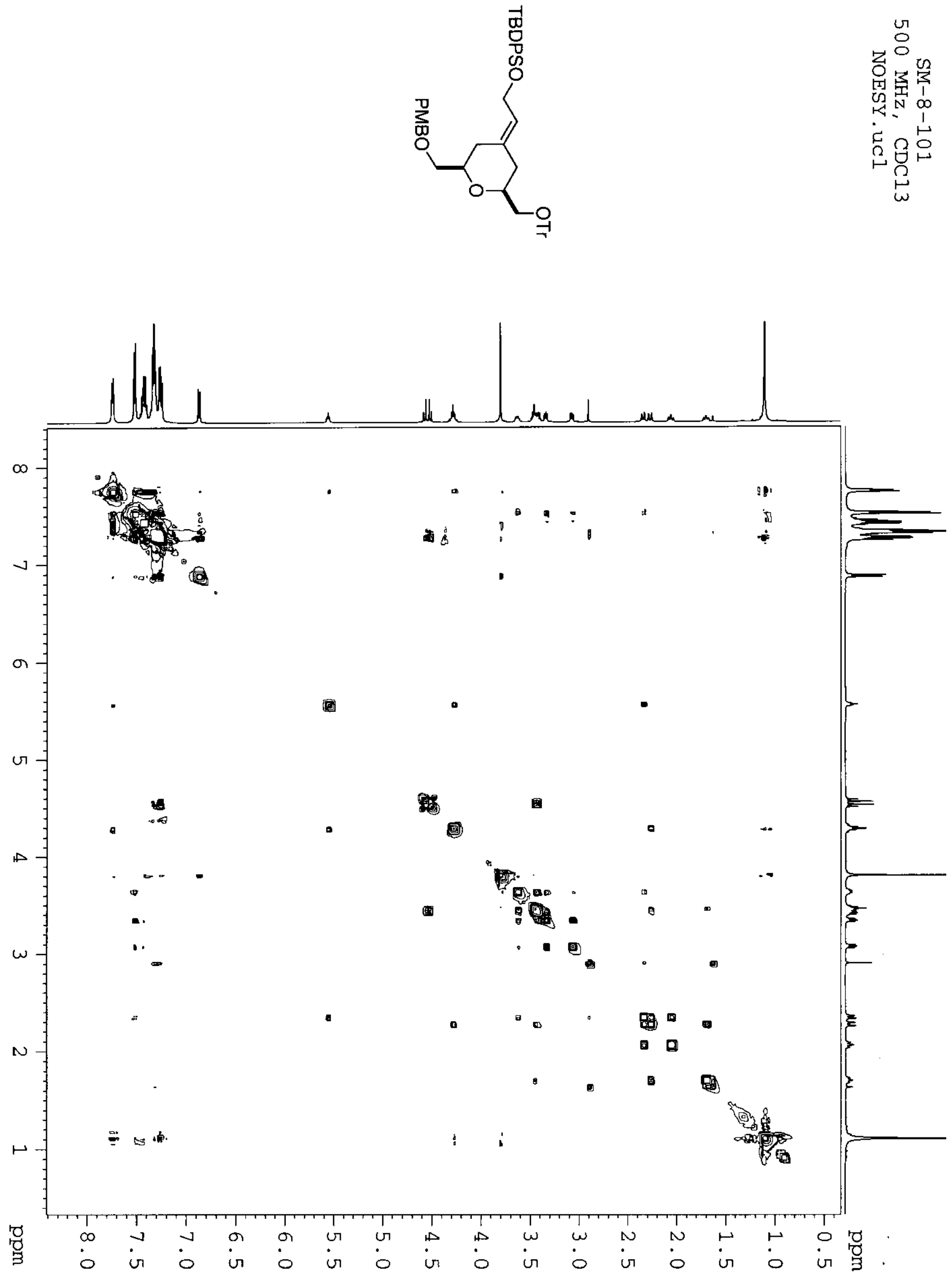

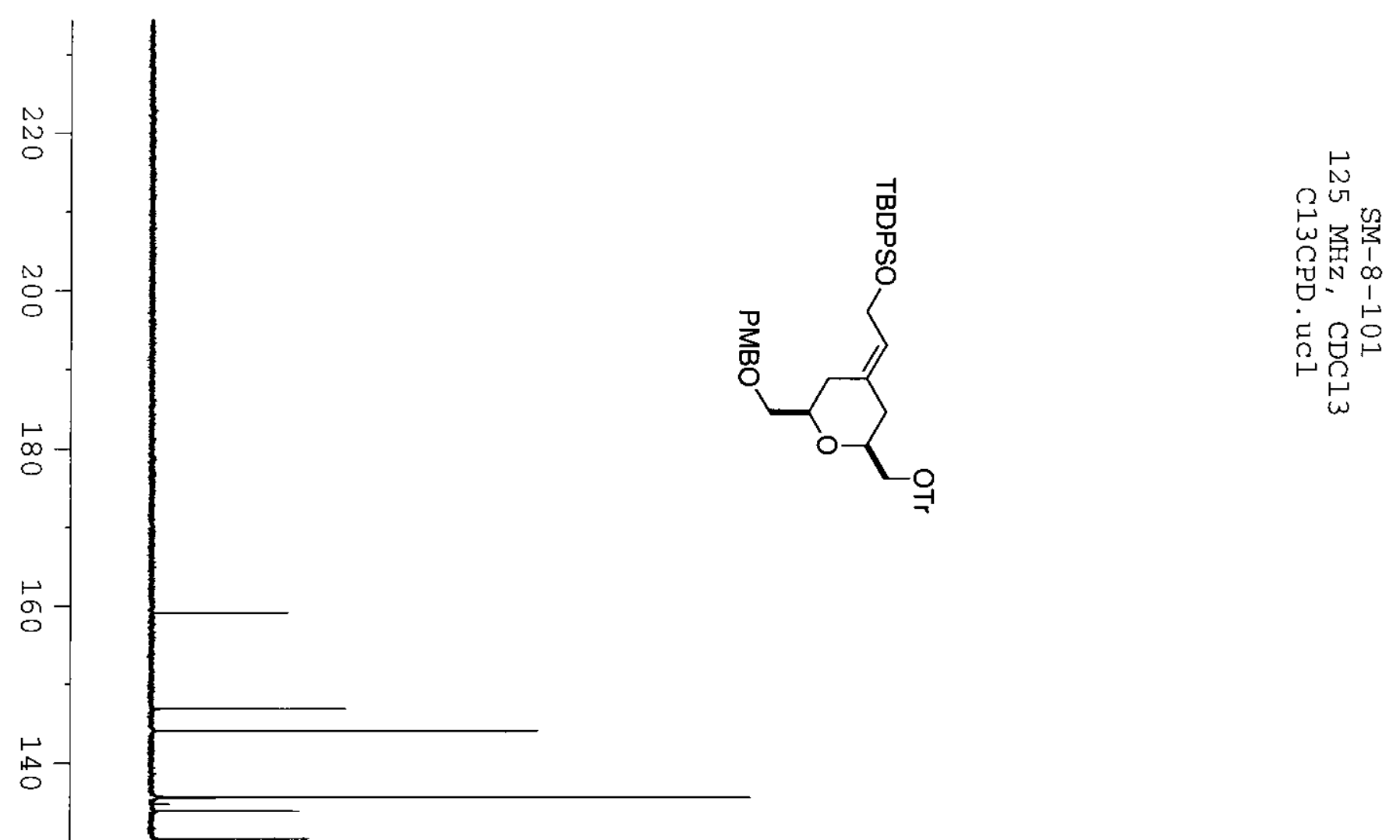

N

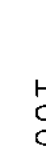

$\infty$

$\stackrel{\circ}{0}$

$\infty$

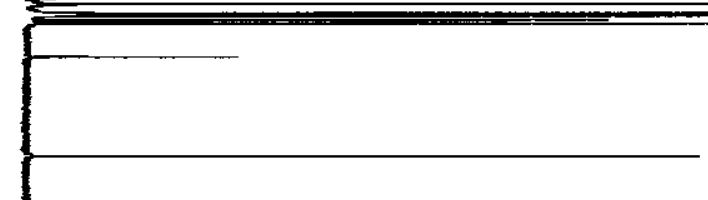

o

$\stackrel{10}{0}$

$N$

$-1$ 


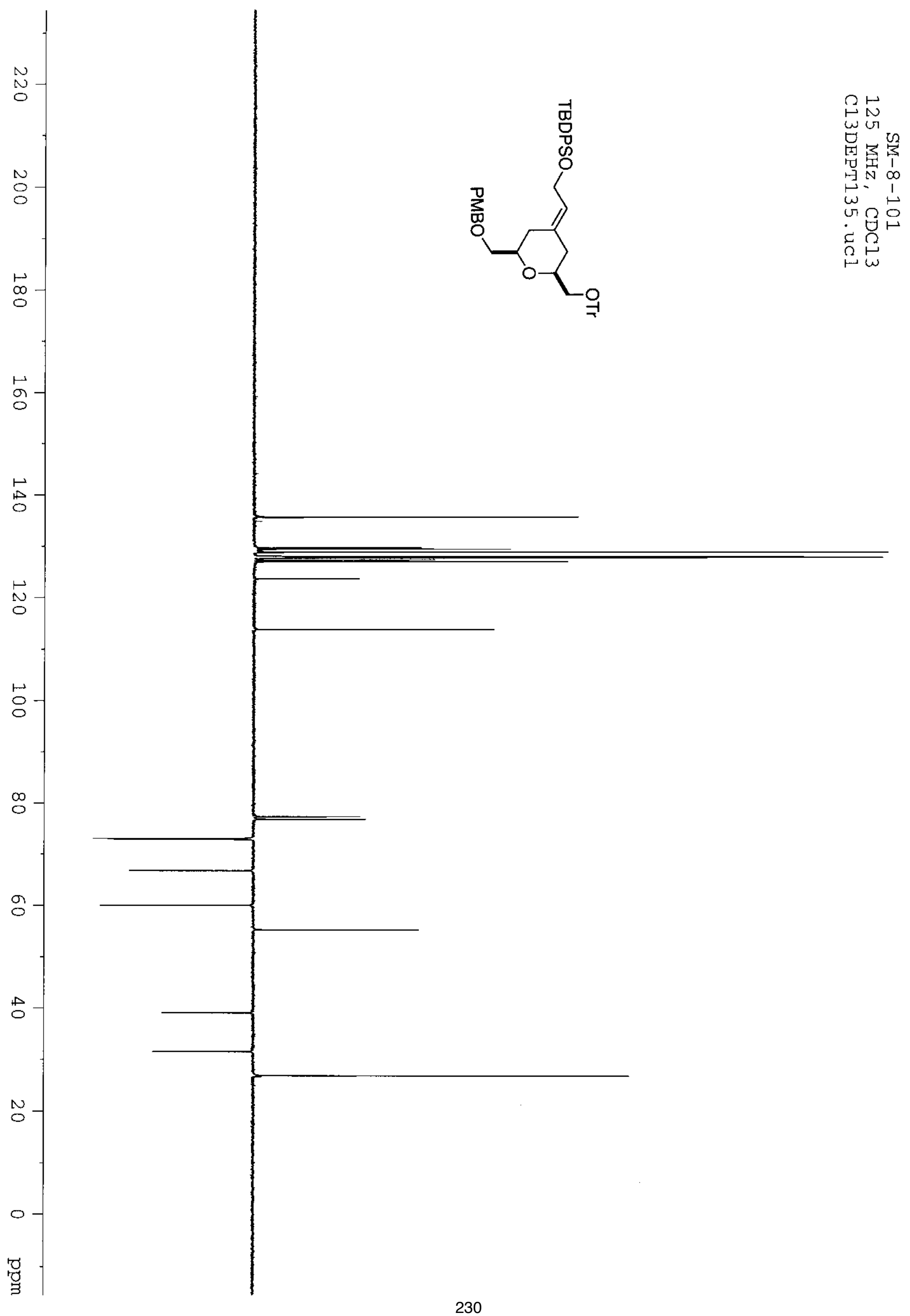




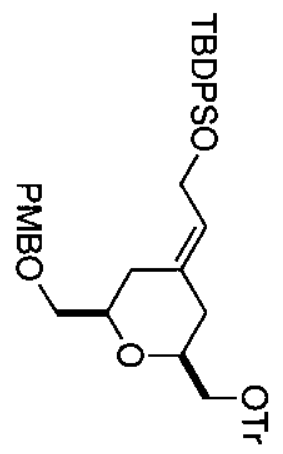

$\theta^{\stackrel{\bullet}{*}}$

卢案管

官一

옹

$\therefore 8$

俩军

$\mathrm{N}$

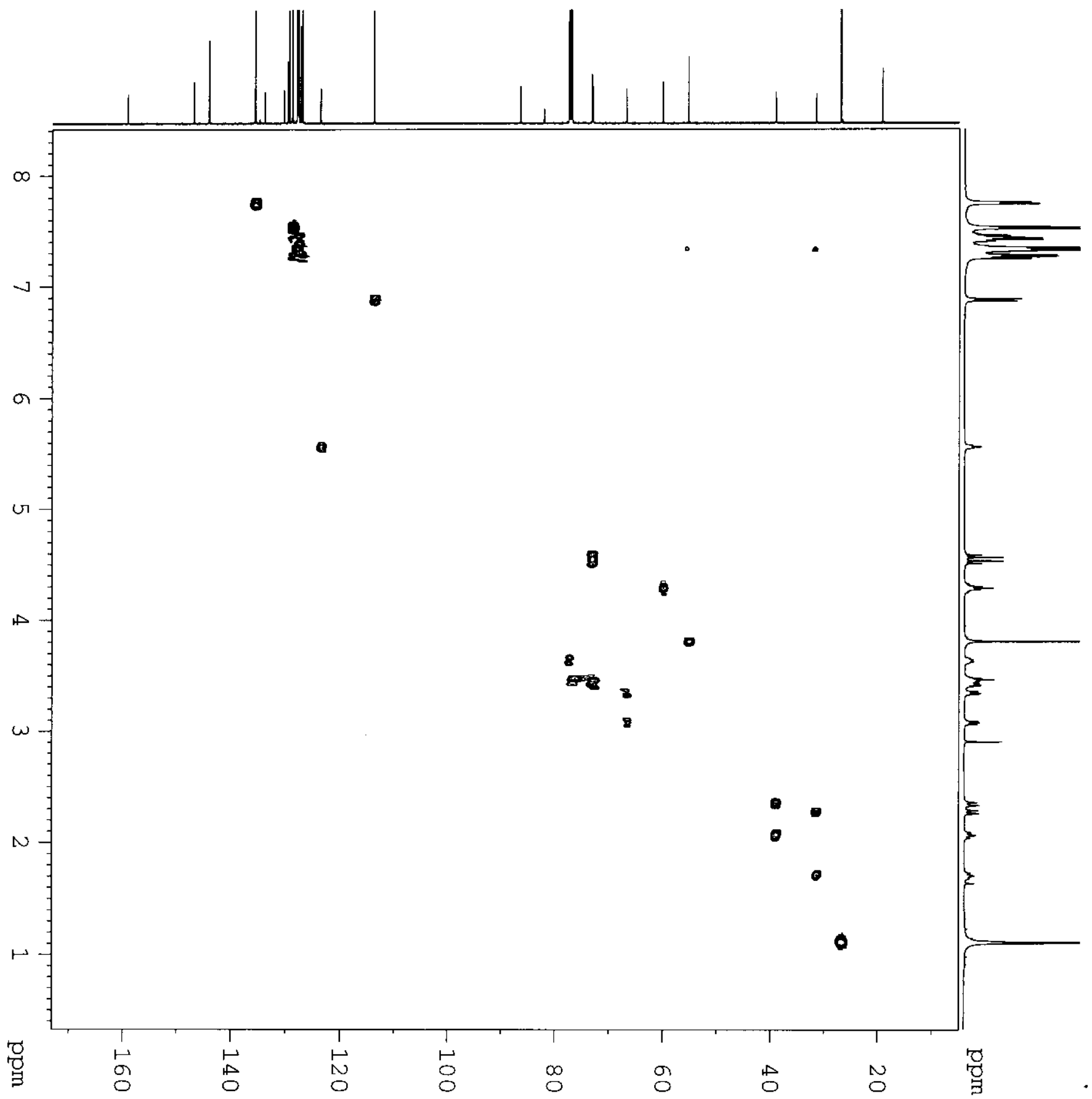




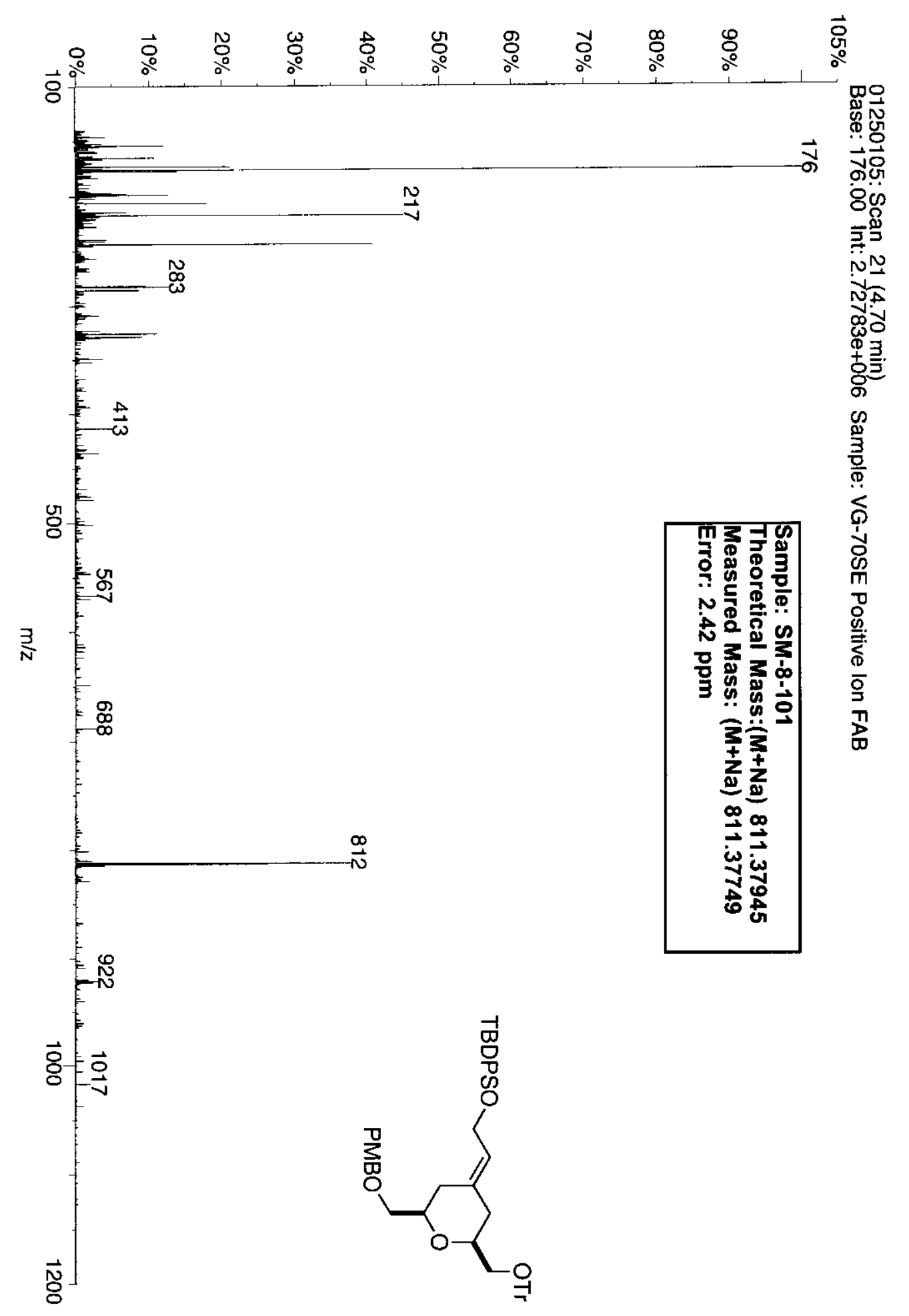




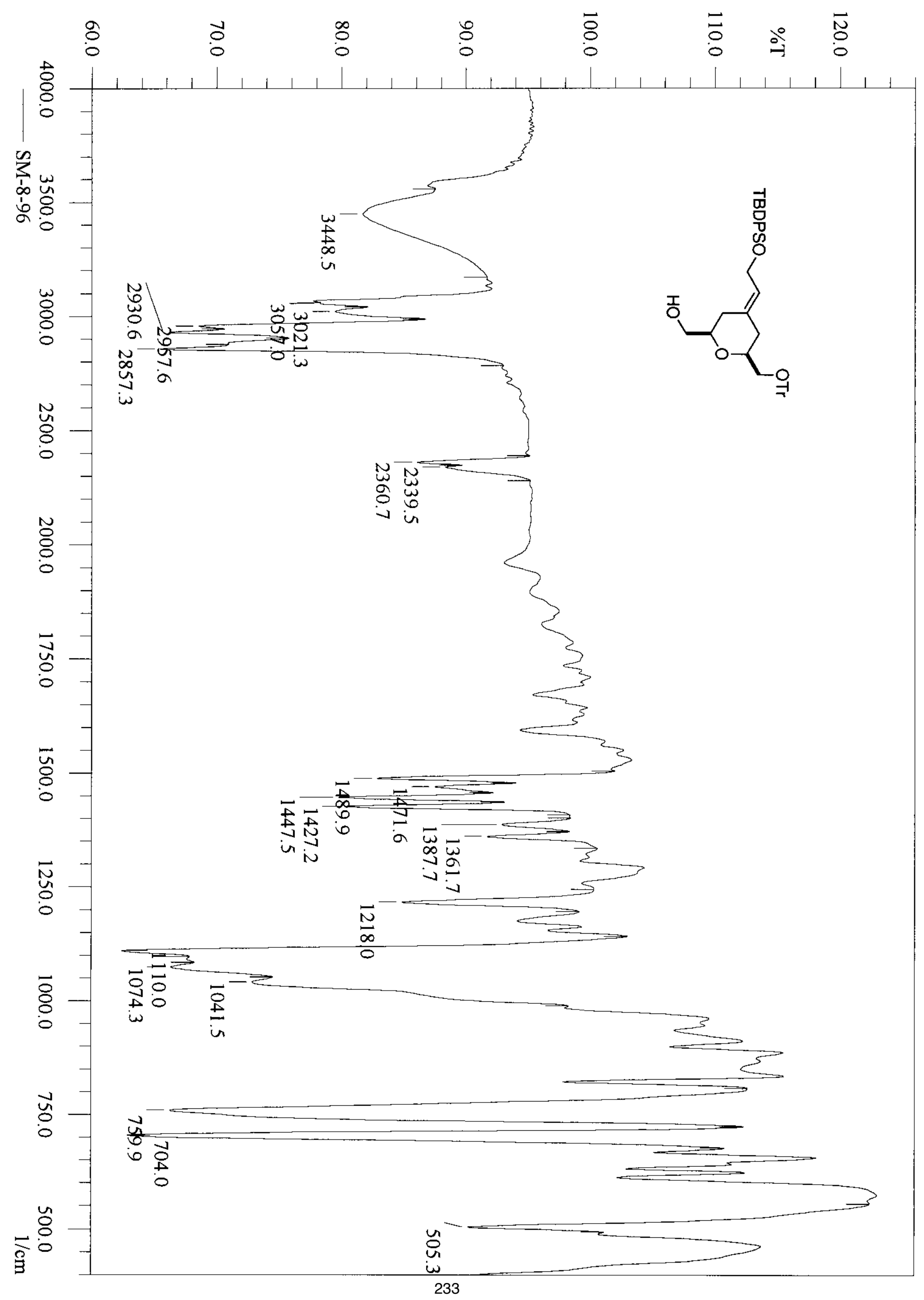




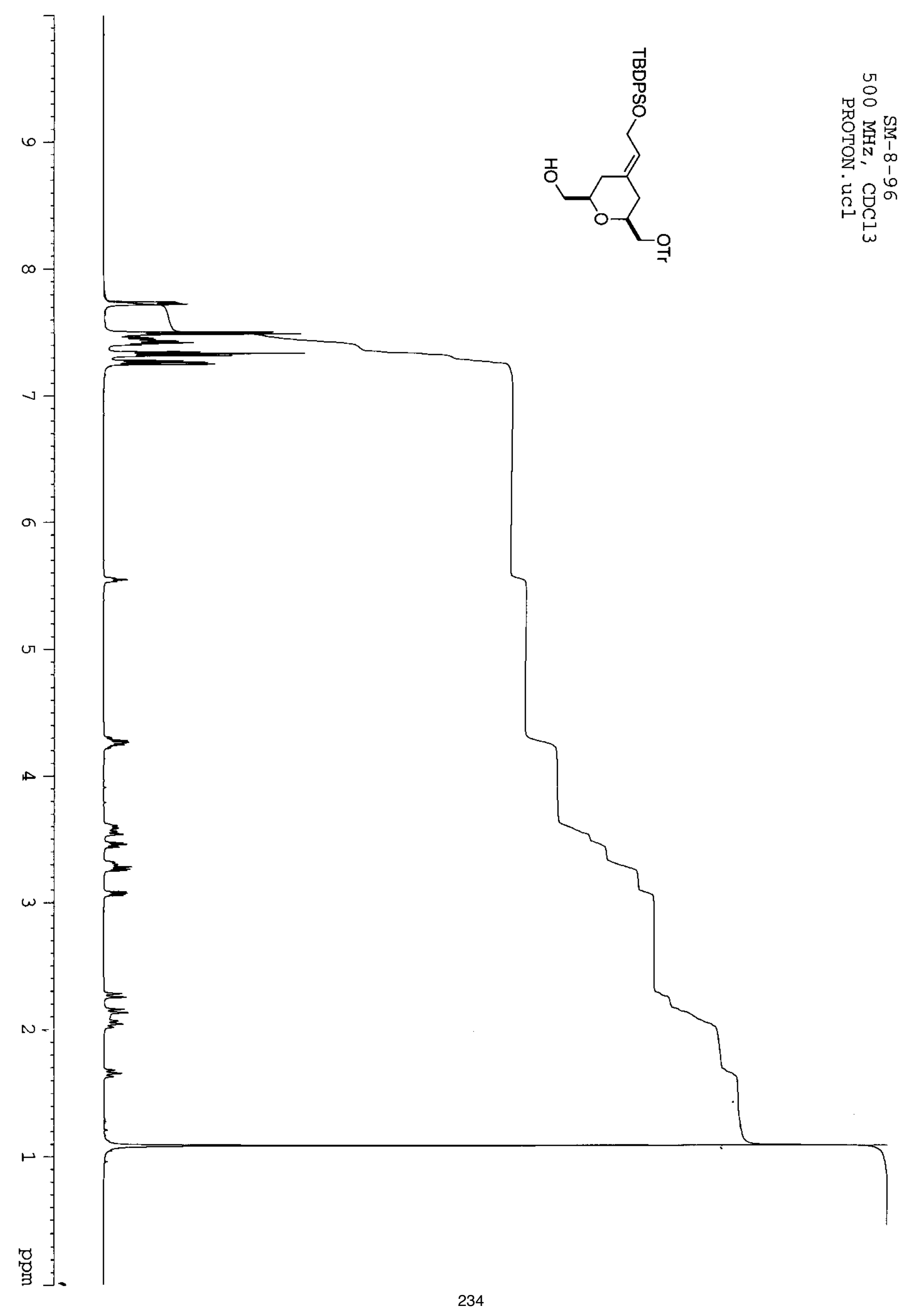



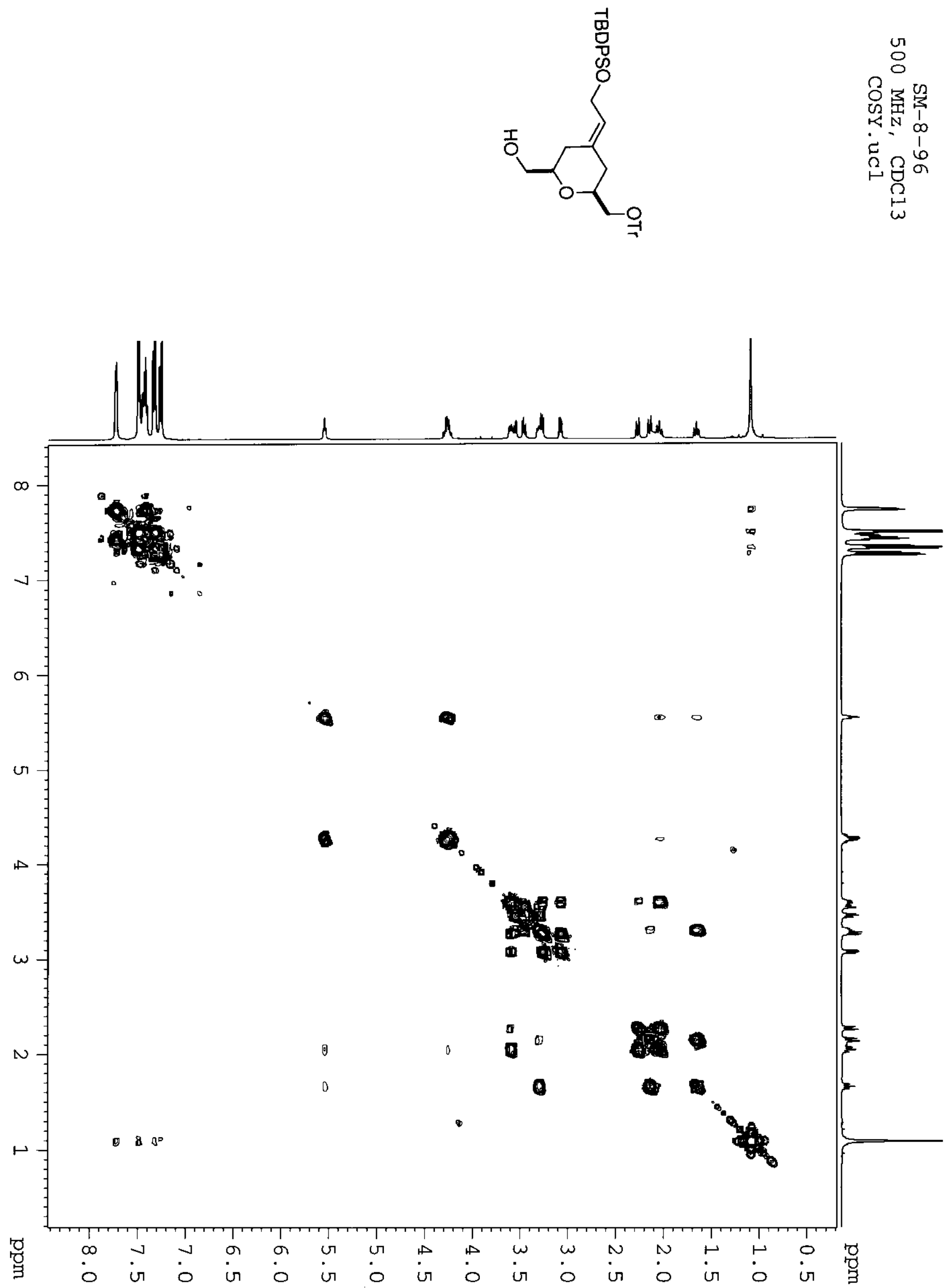

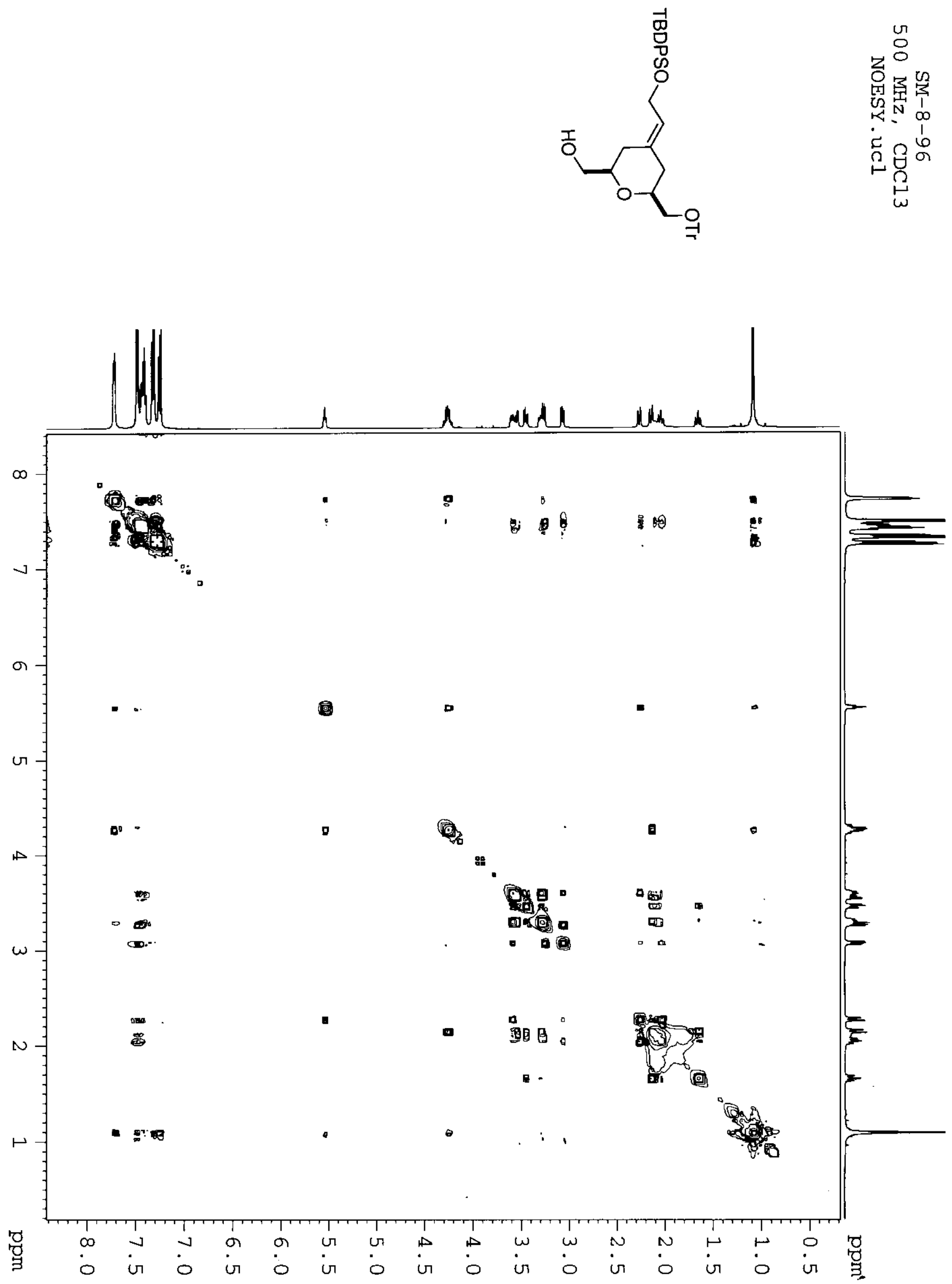


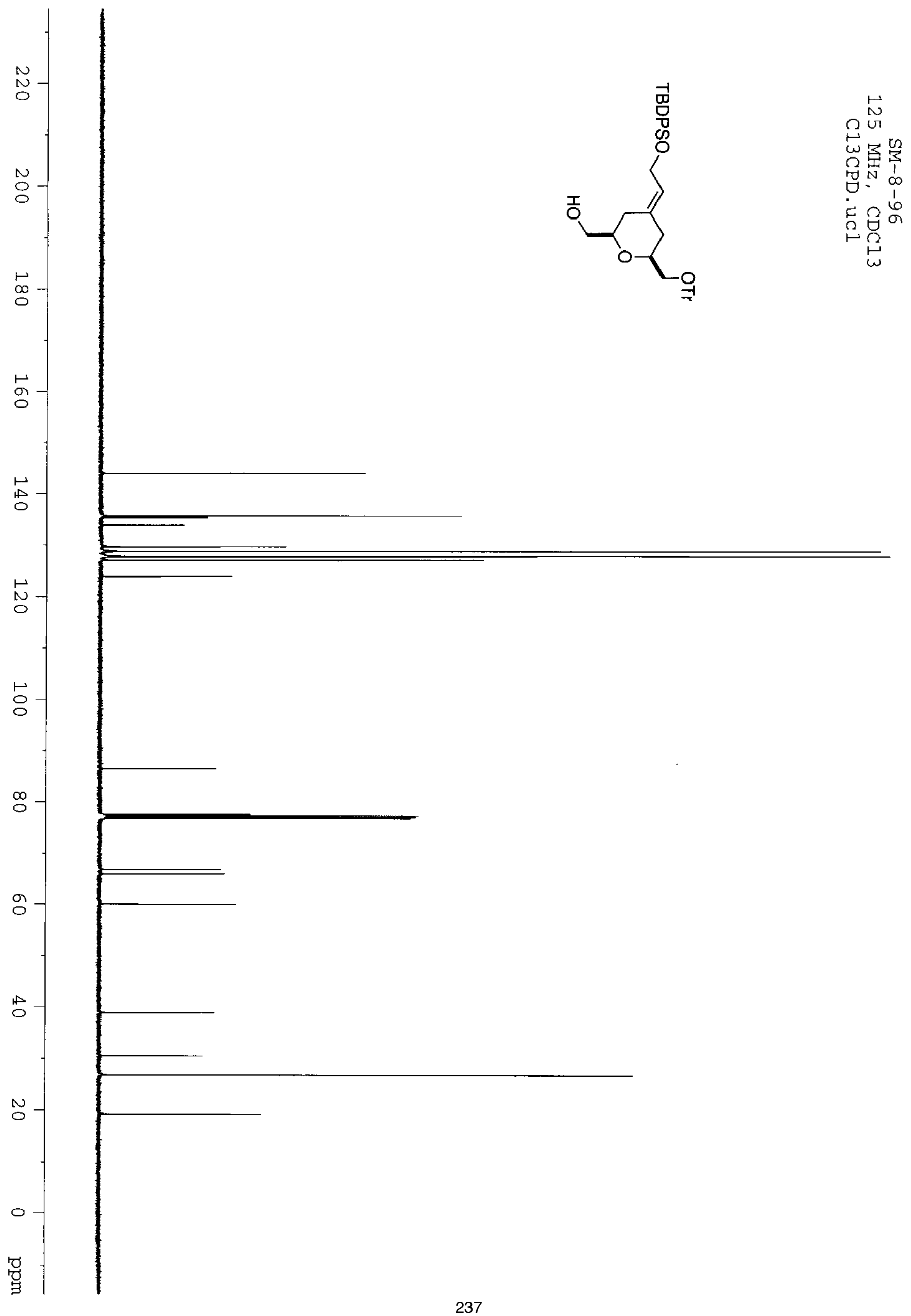




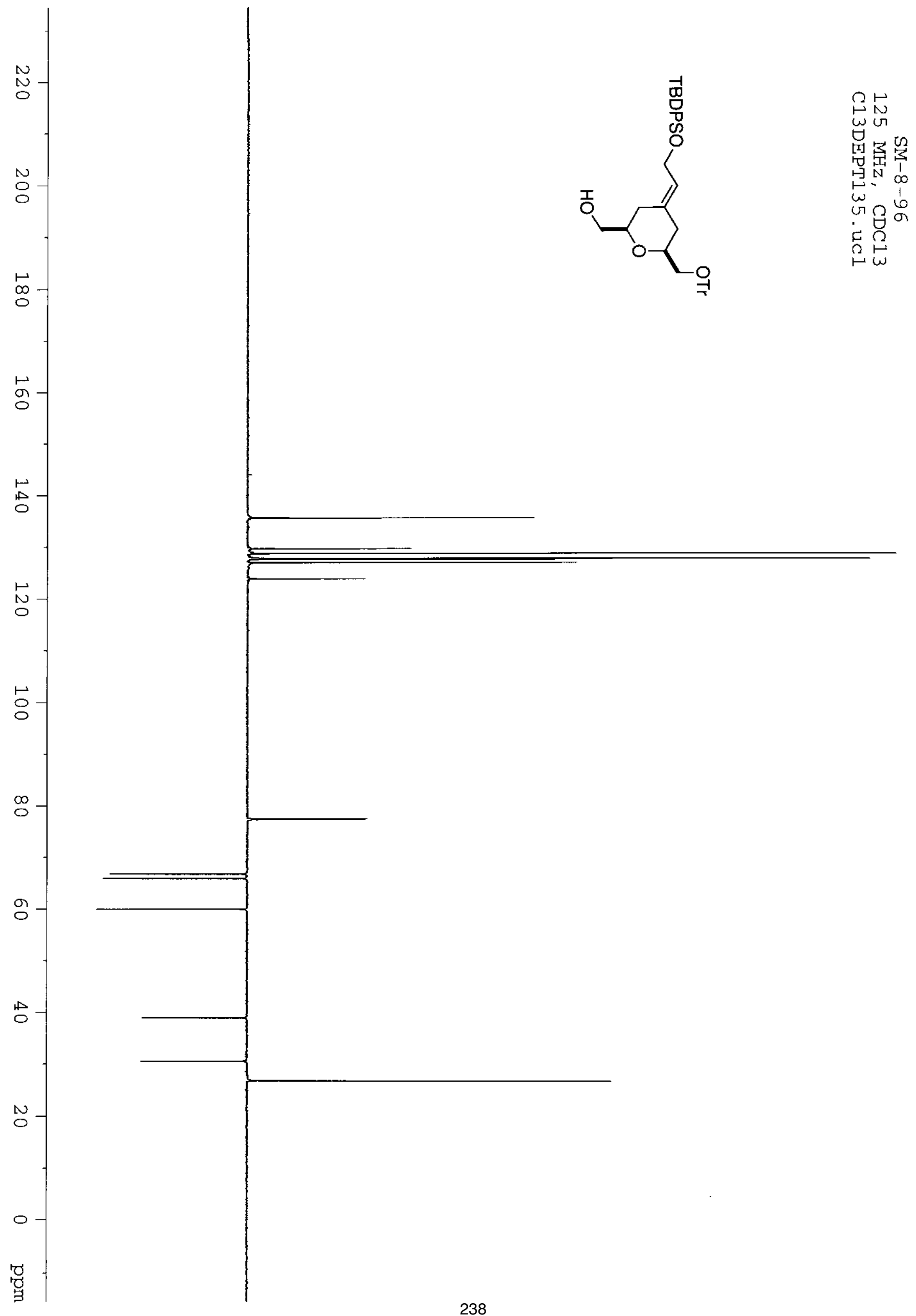




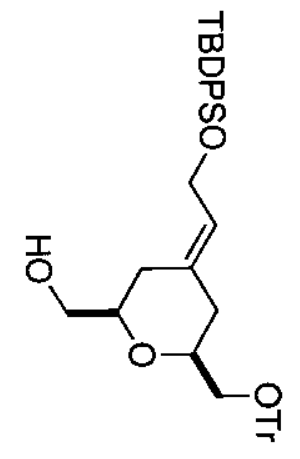

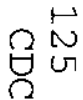

占军

的虽资

- $\infty$

ง

? 8 \%

i)

点

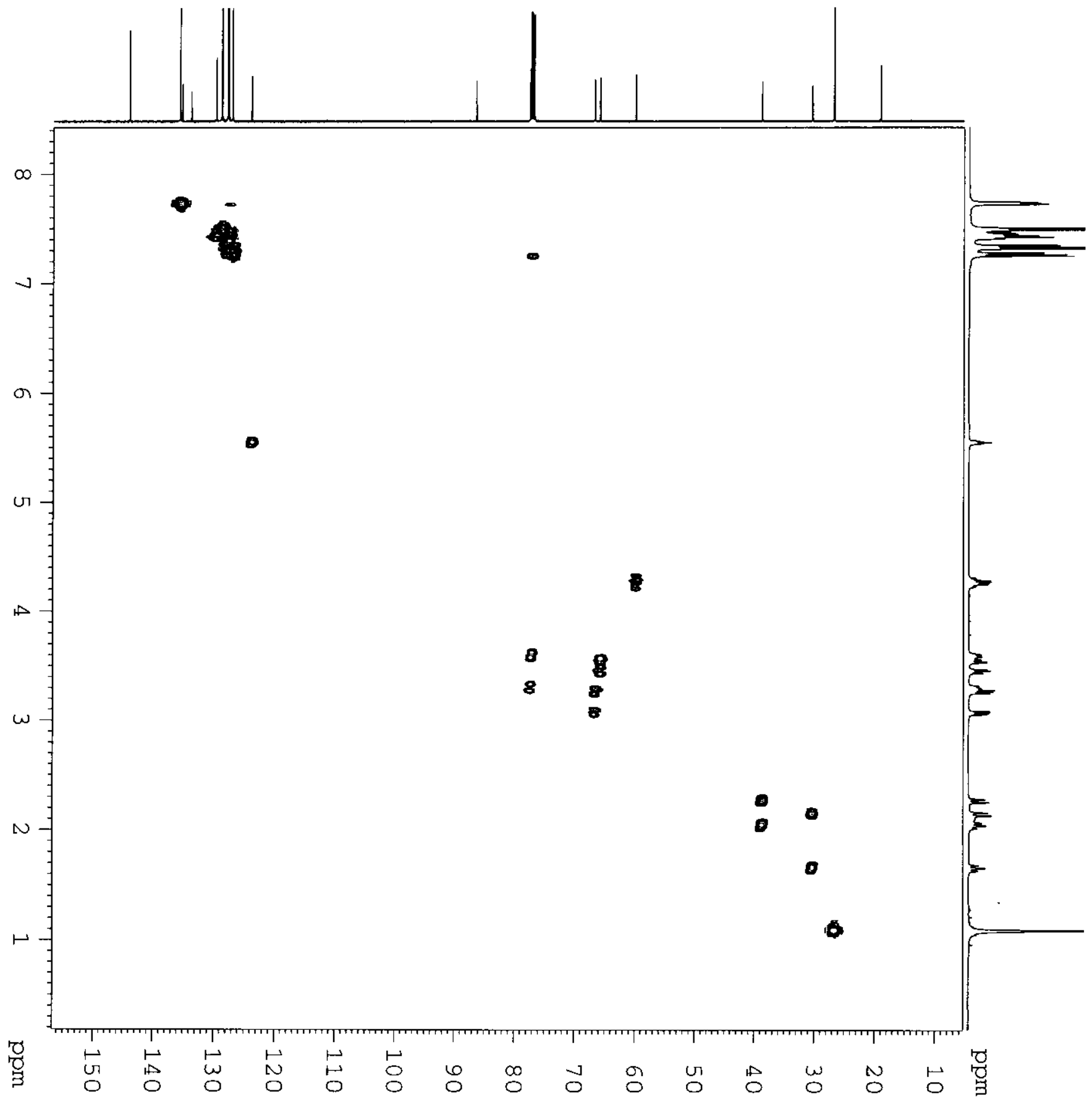




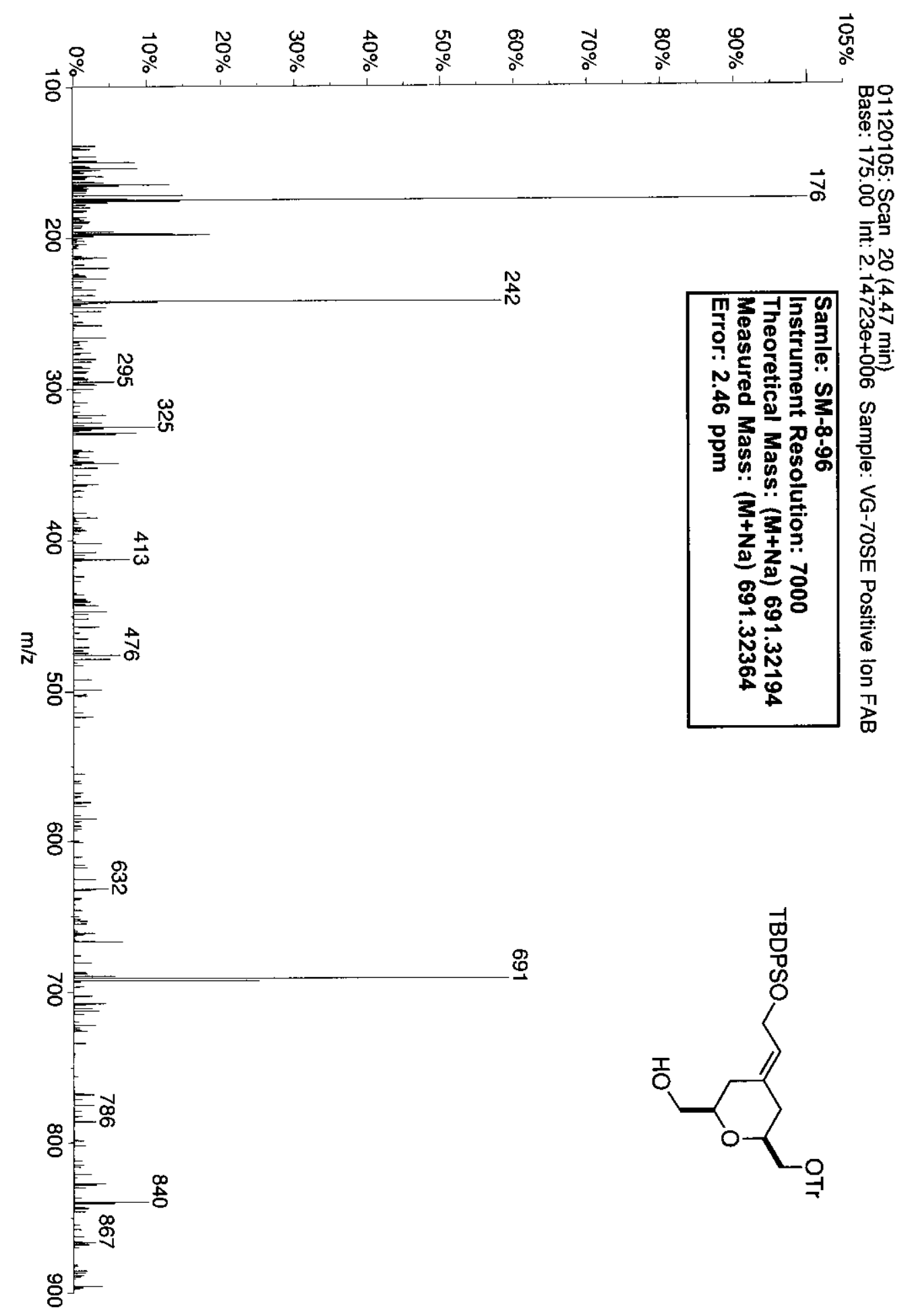




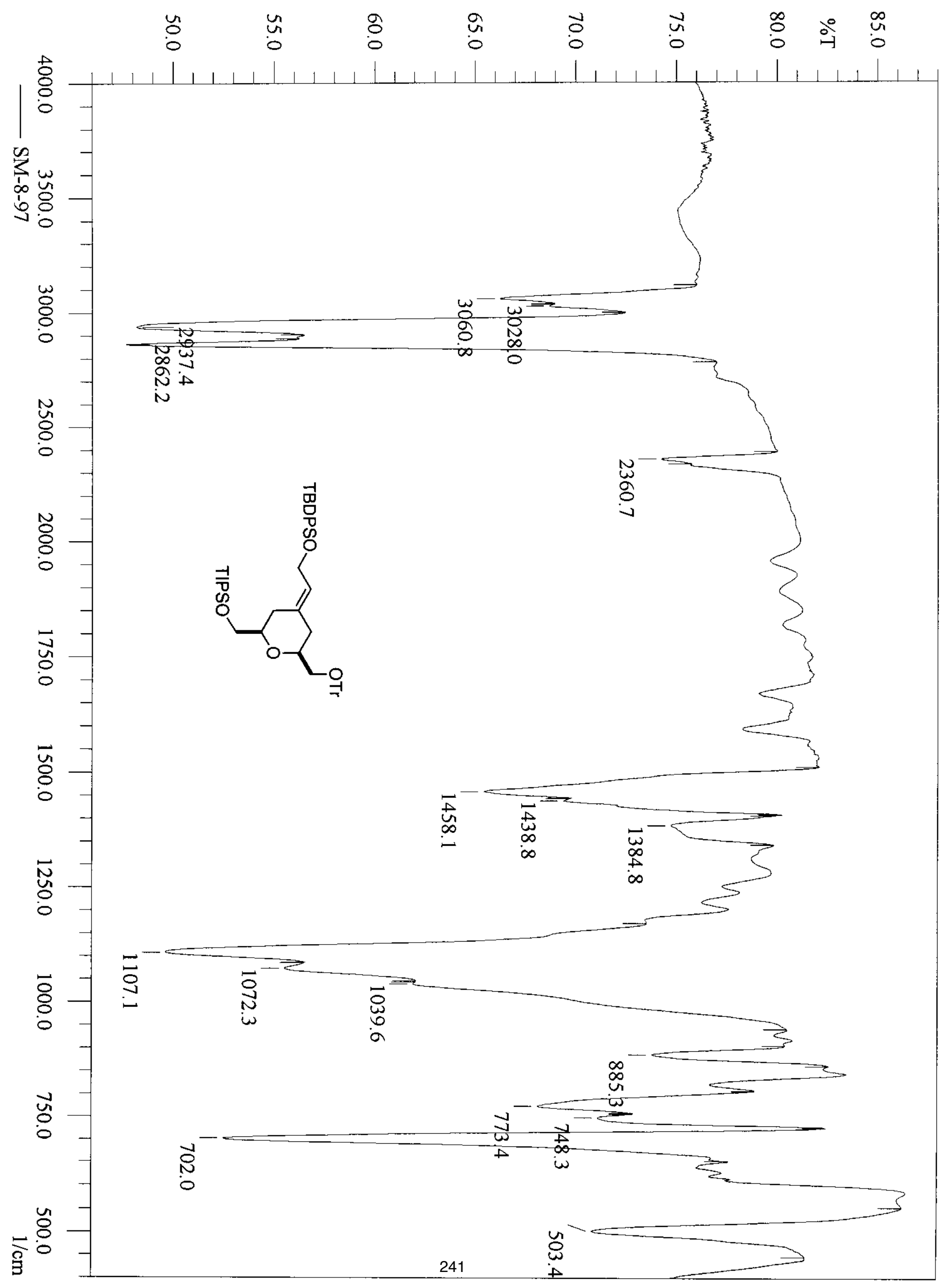




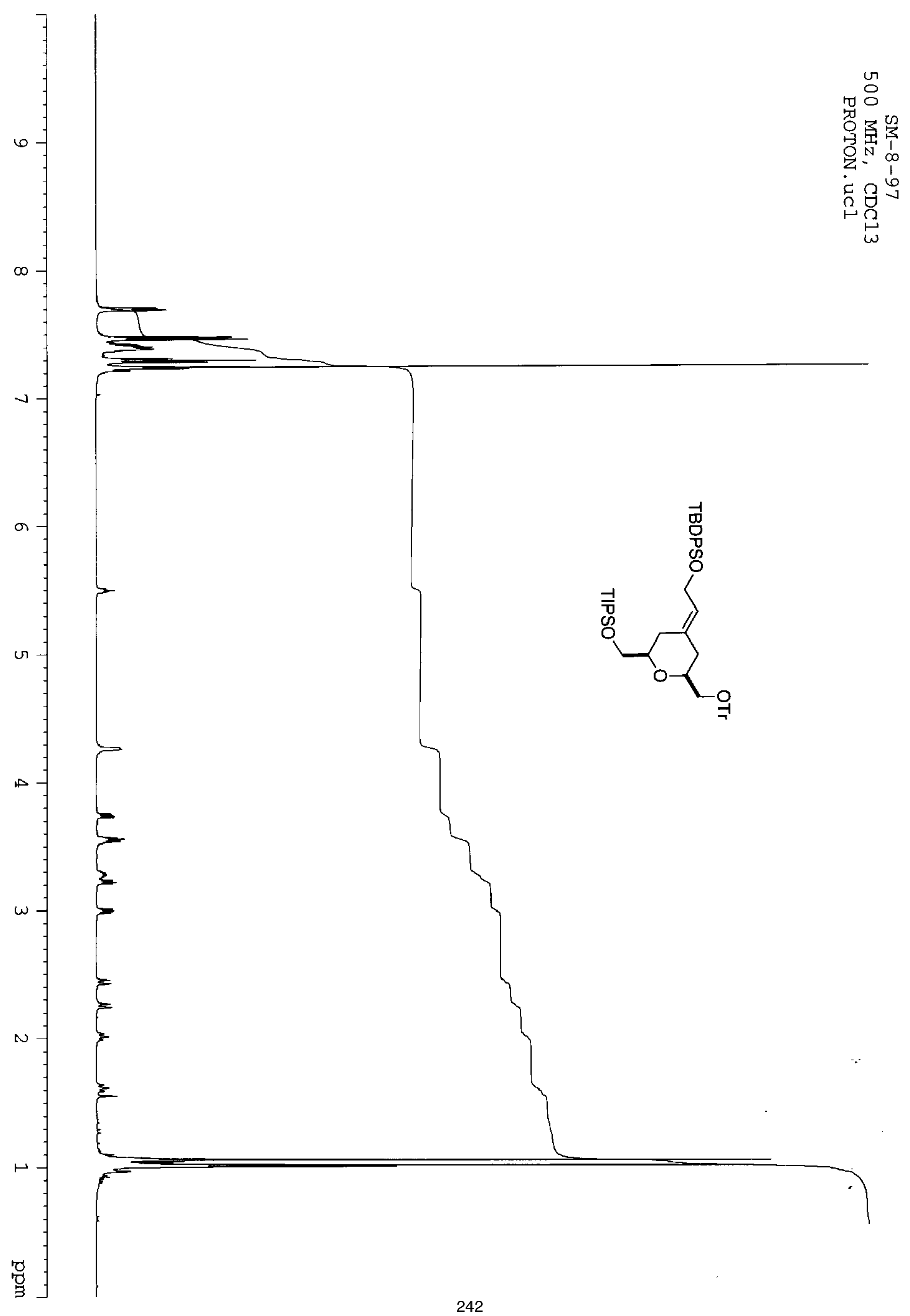



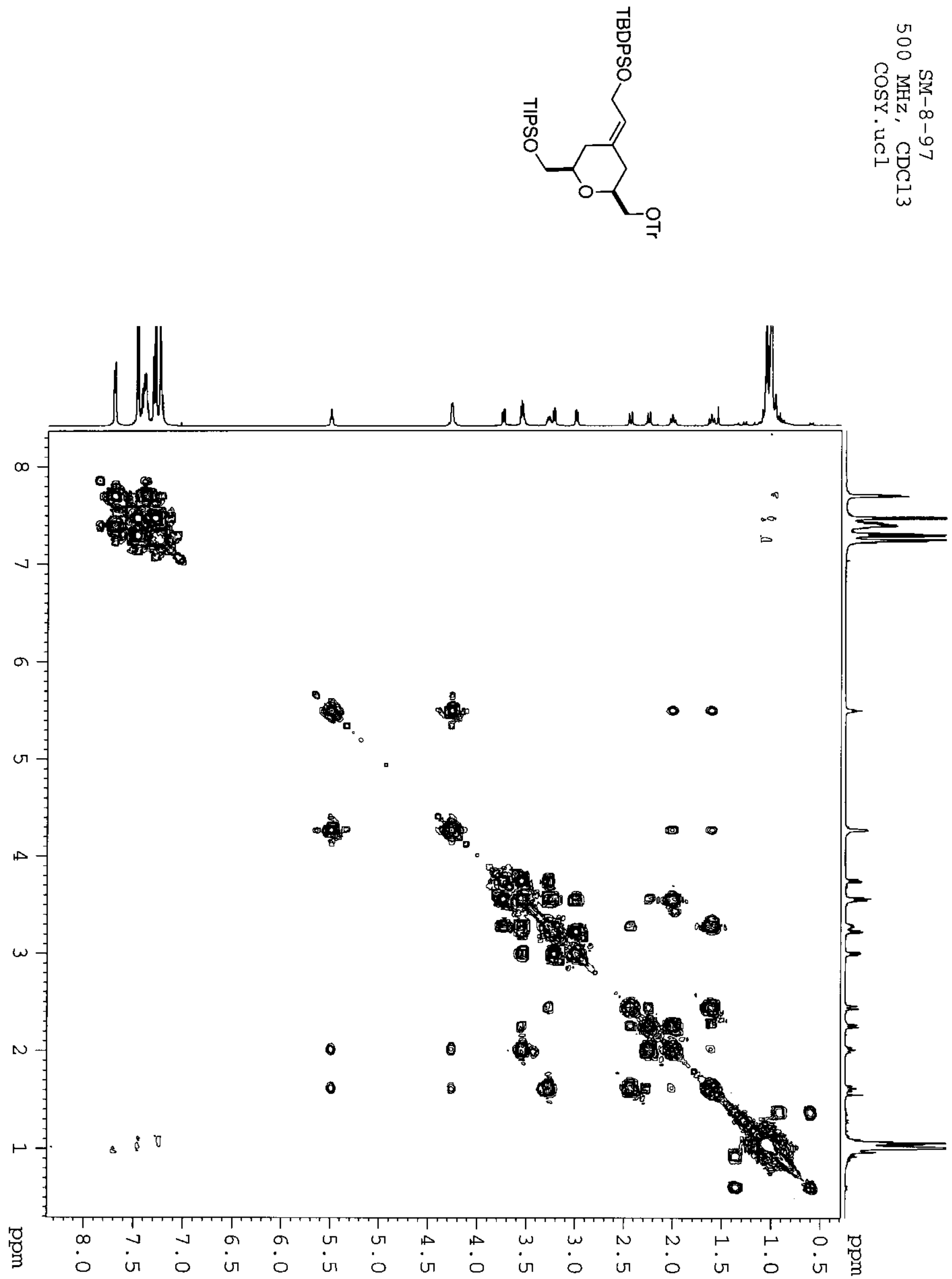

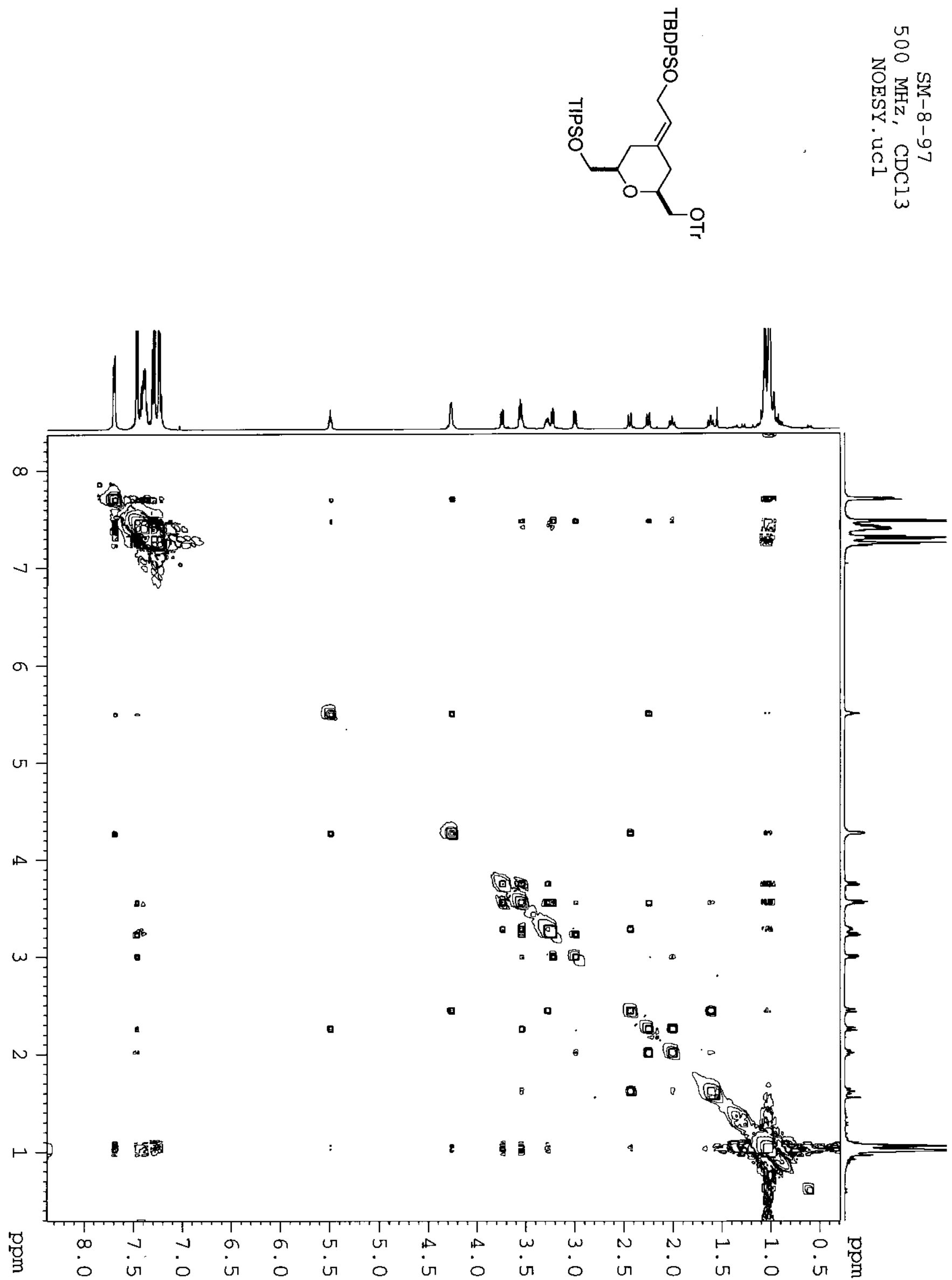


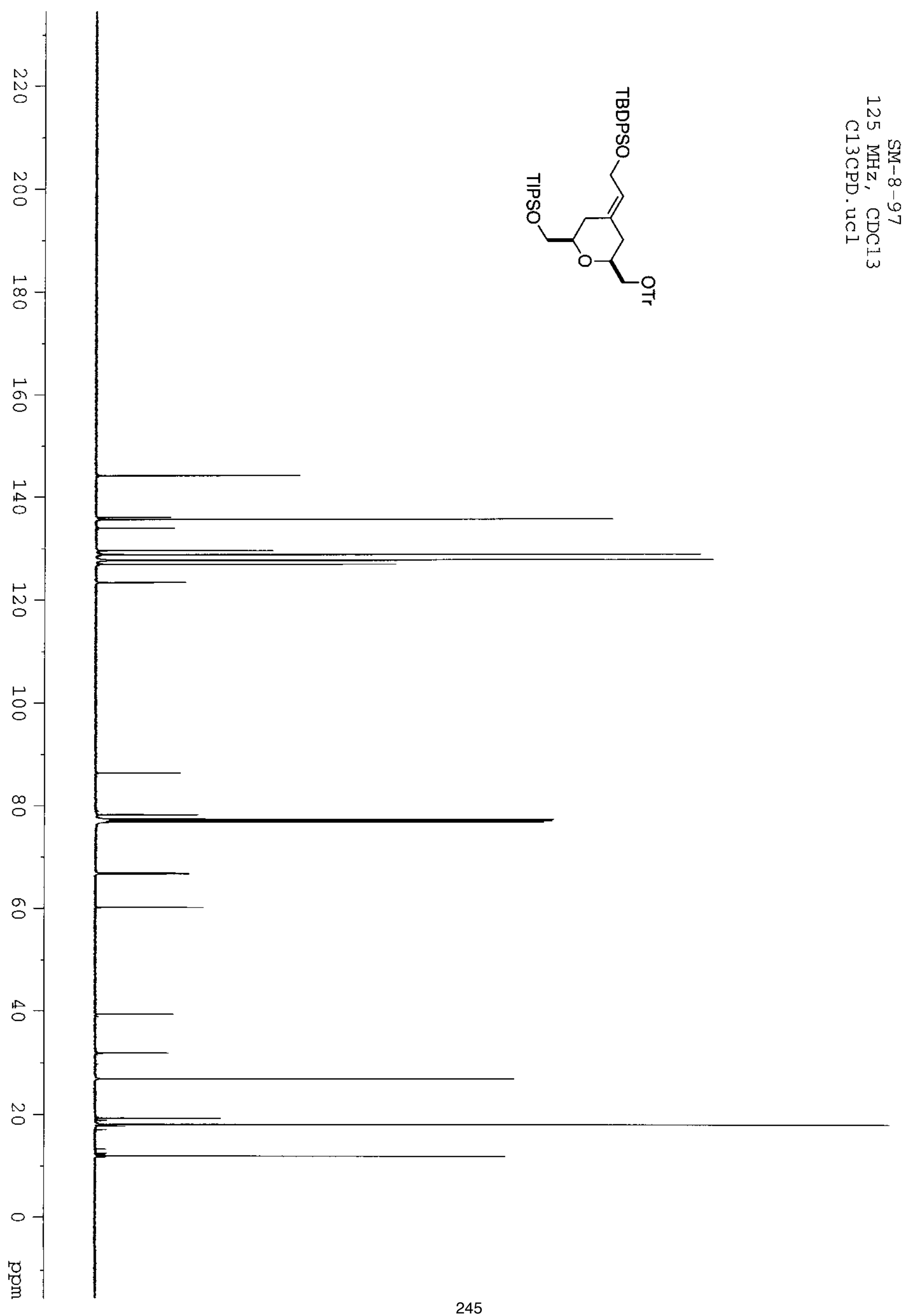




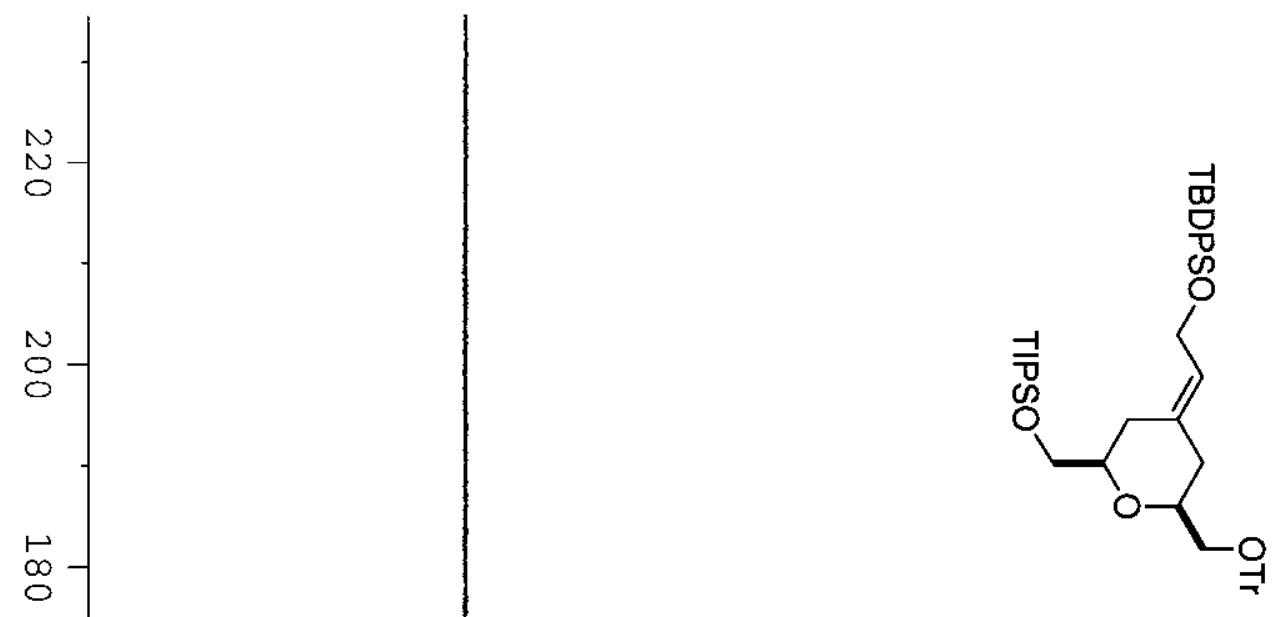

P忩

$\omega$ u

国家

㝵采

光 1

ज 20

ڤڤ

مै

$\stackrel{\bullet}{\stackrel{5}{0}}$

$\stackrel{\bullet}{\circ}$

붕

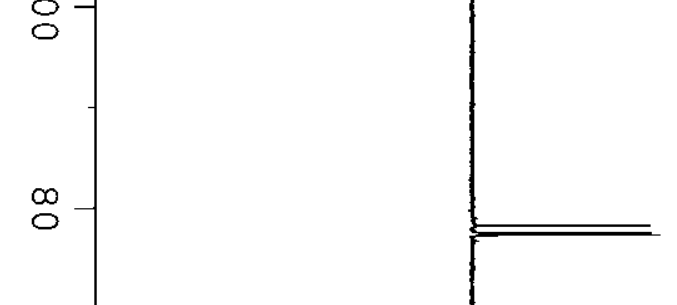

o

$\stackrel{\circ}{\circ}$

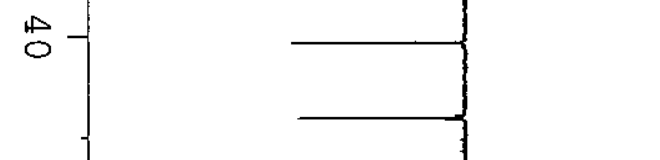

N

o

稟 

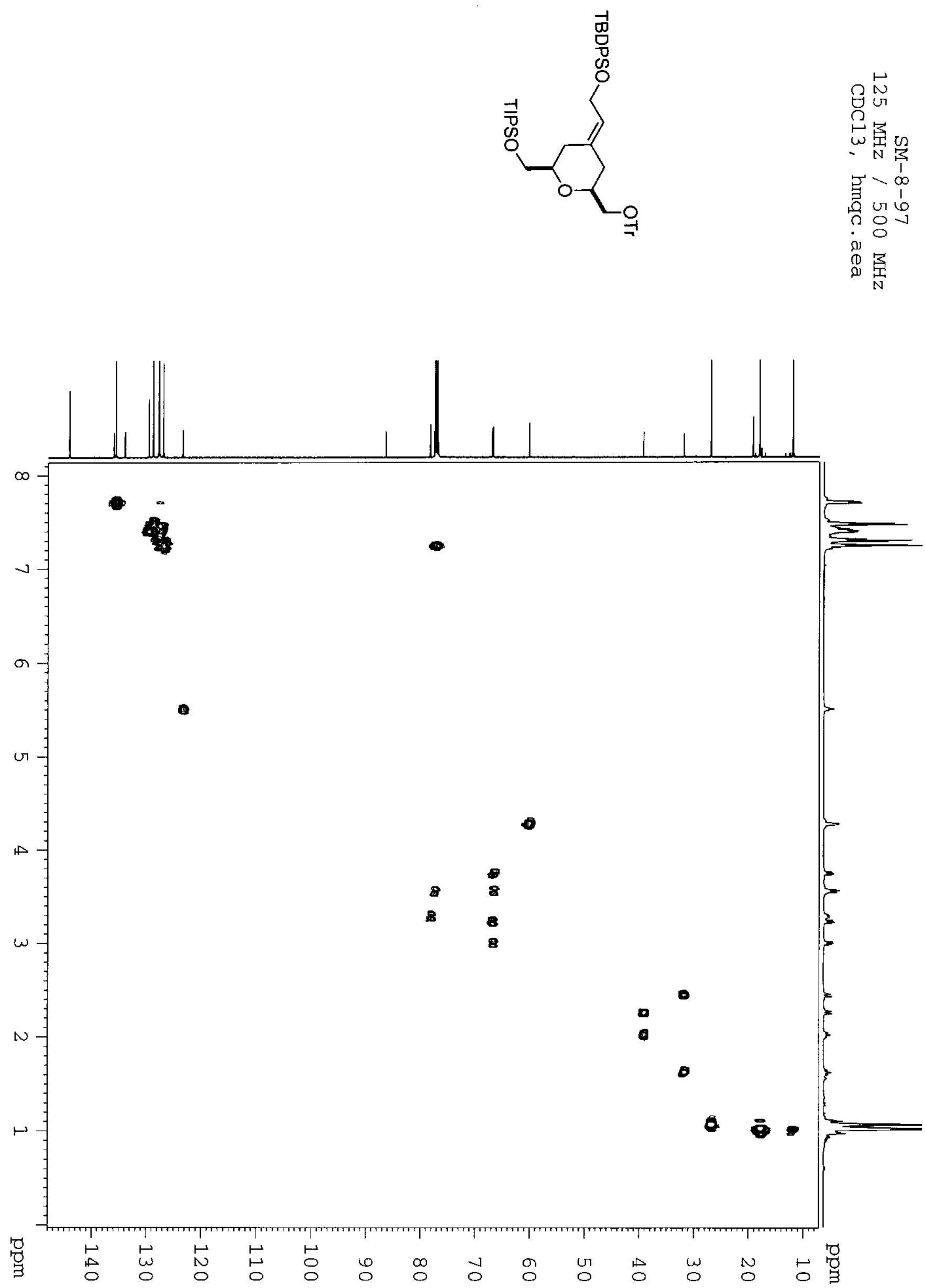


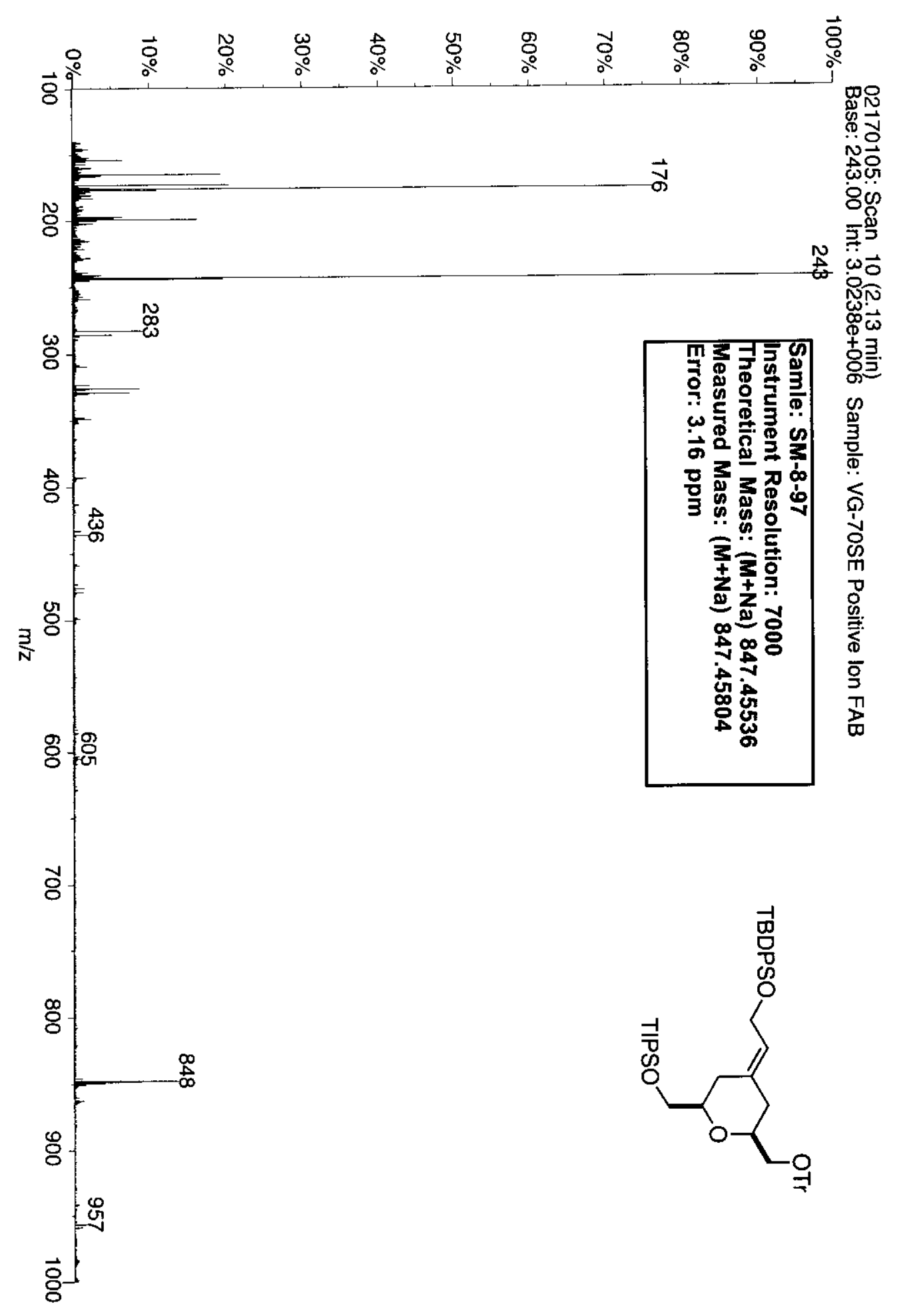




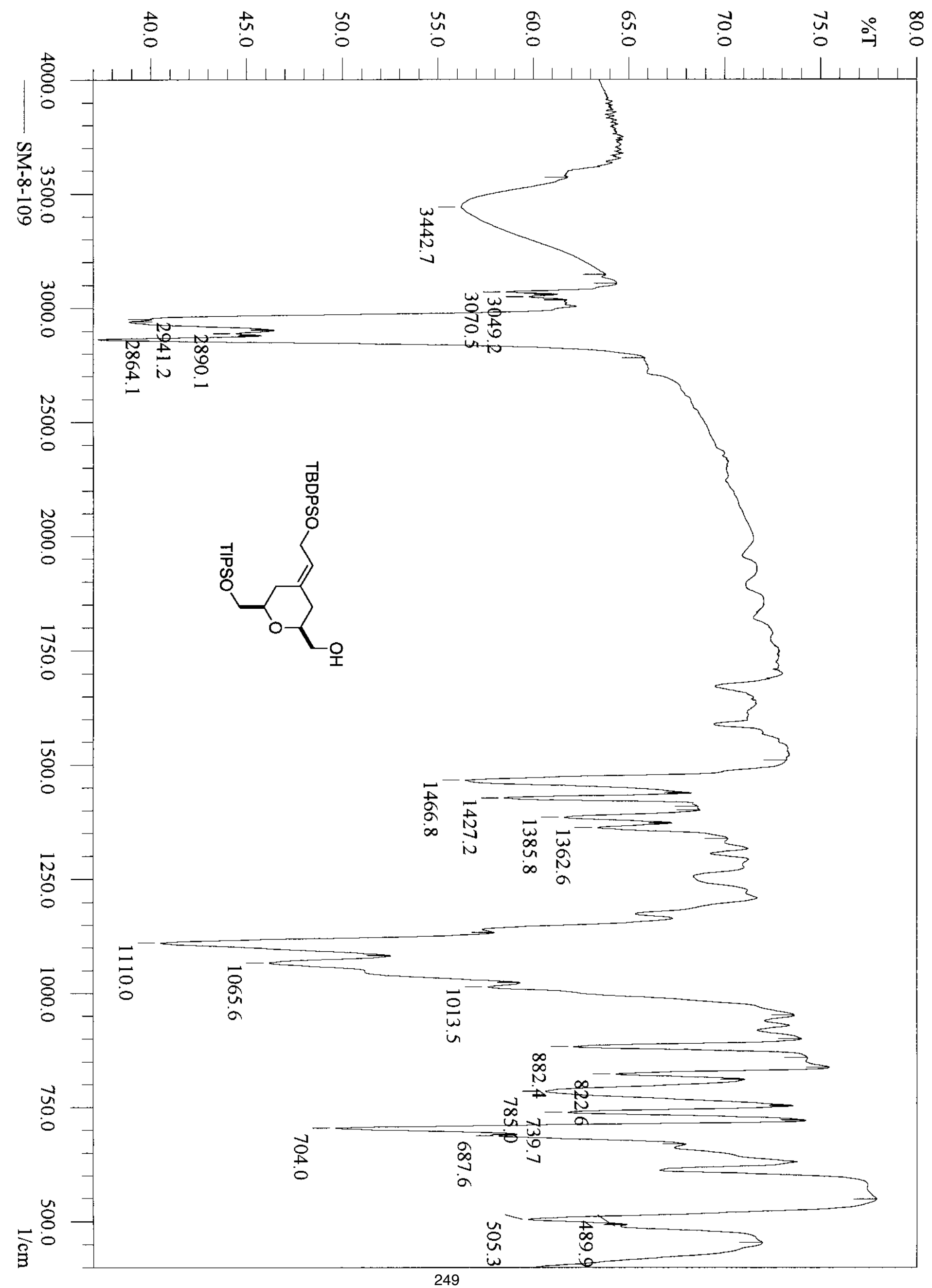




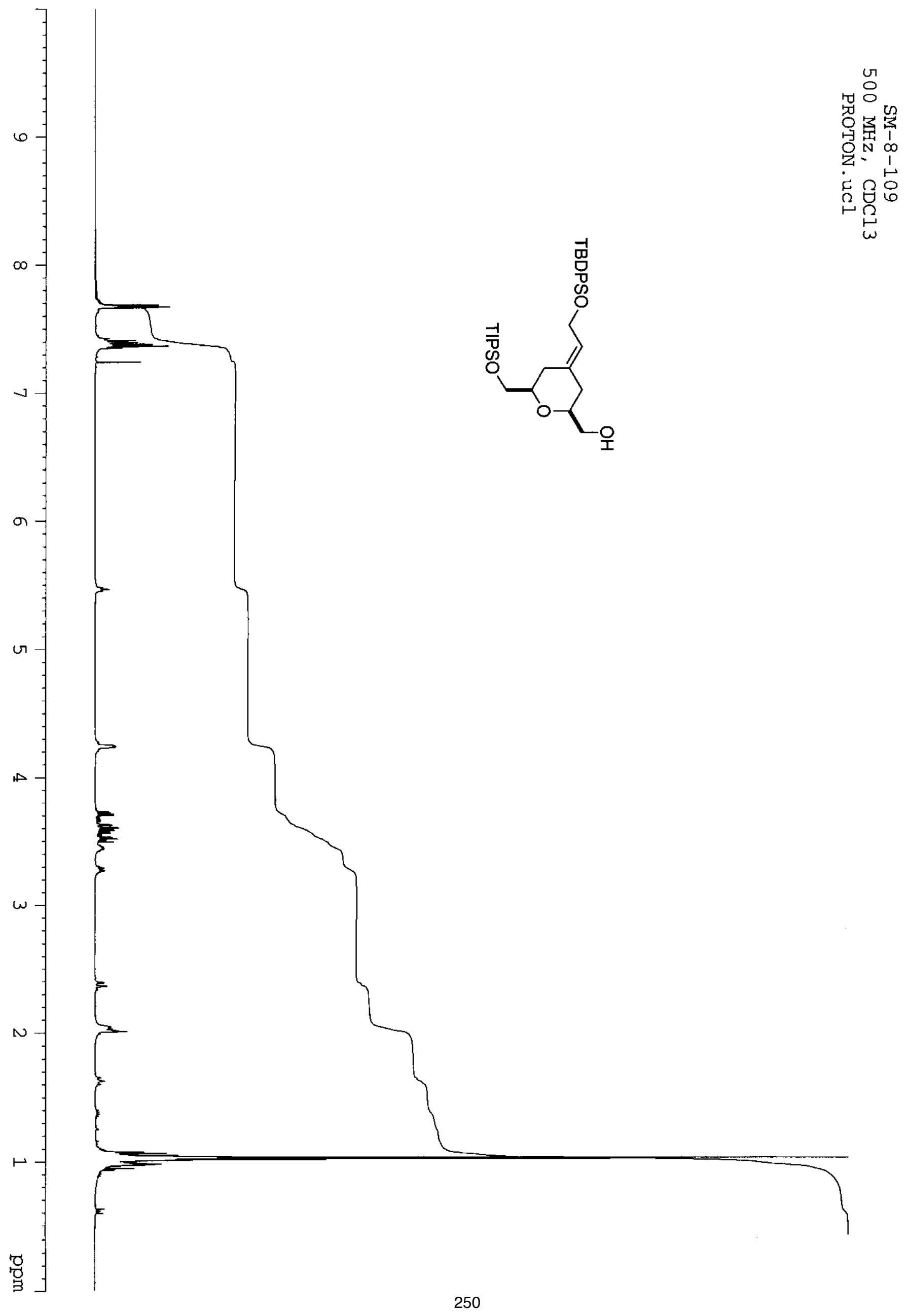



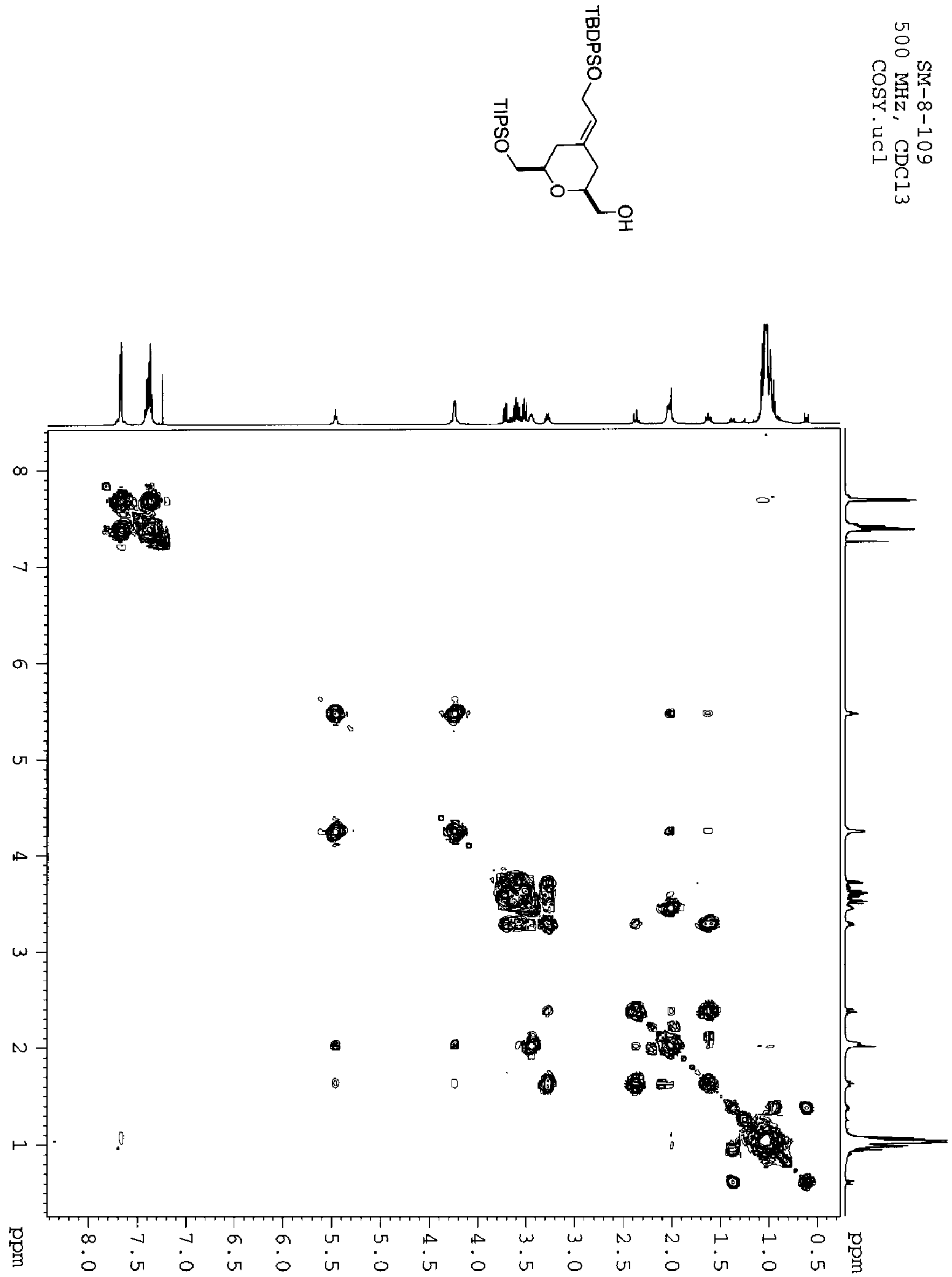

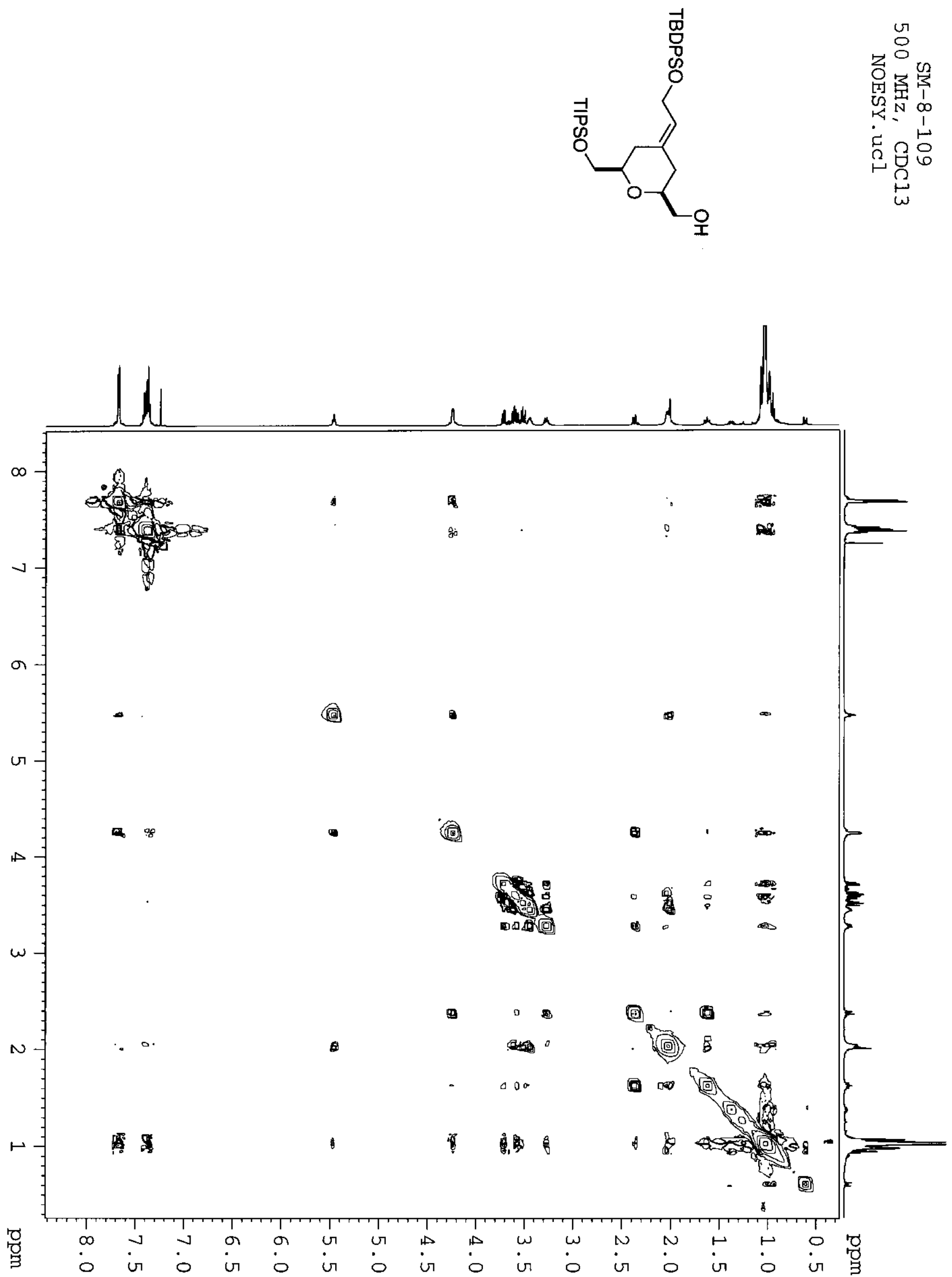

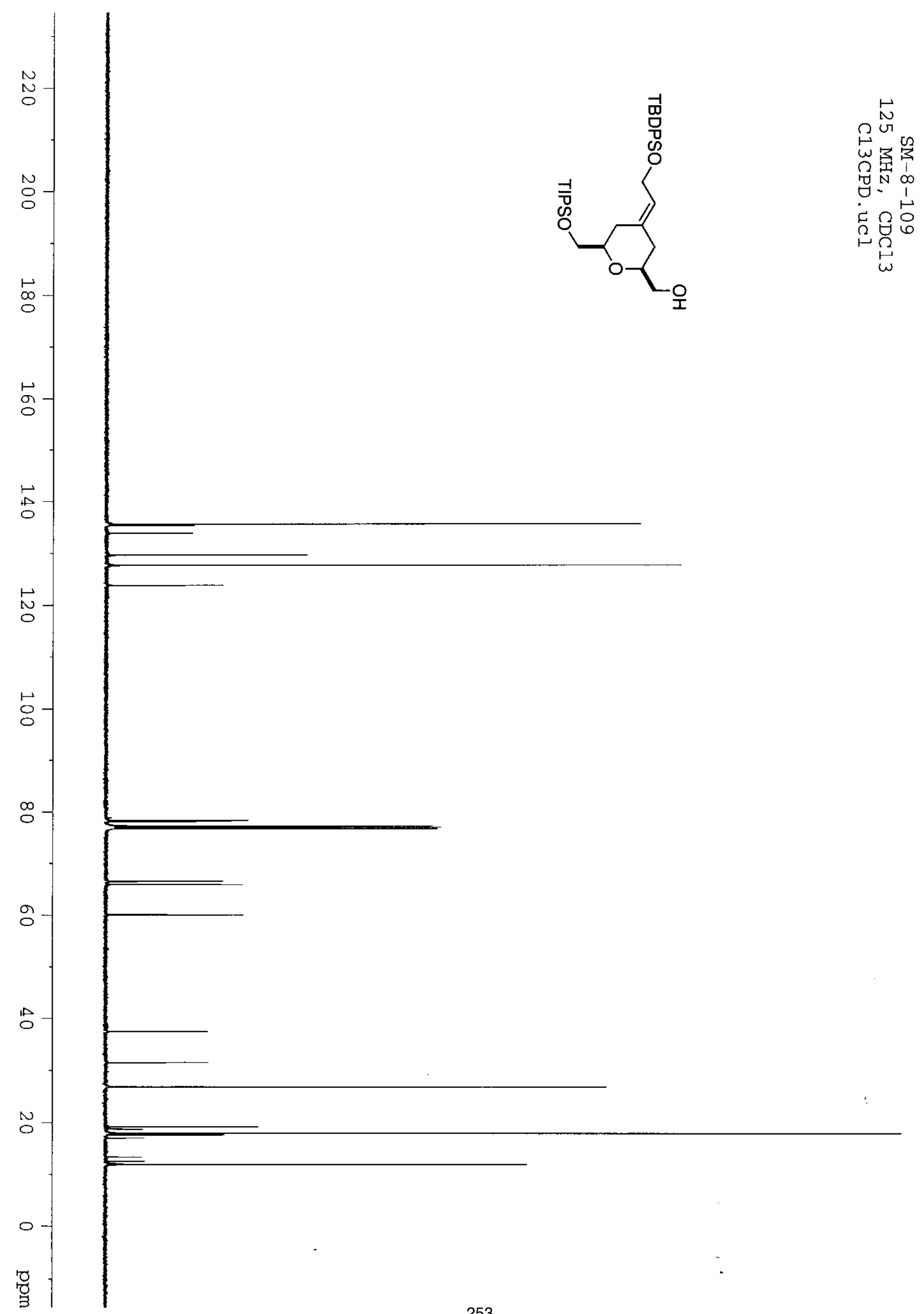


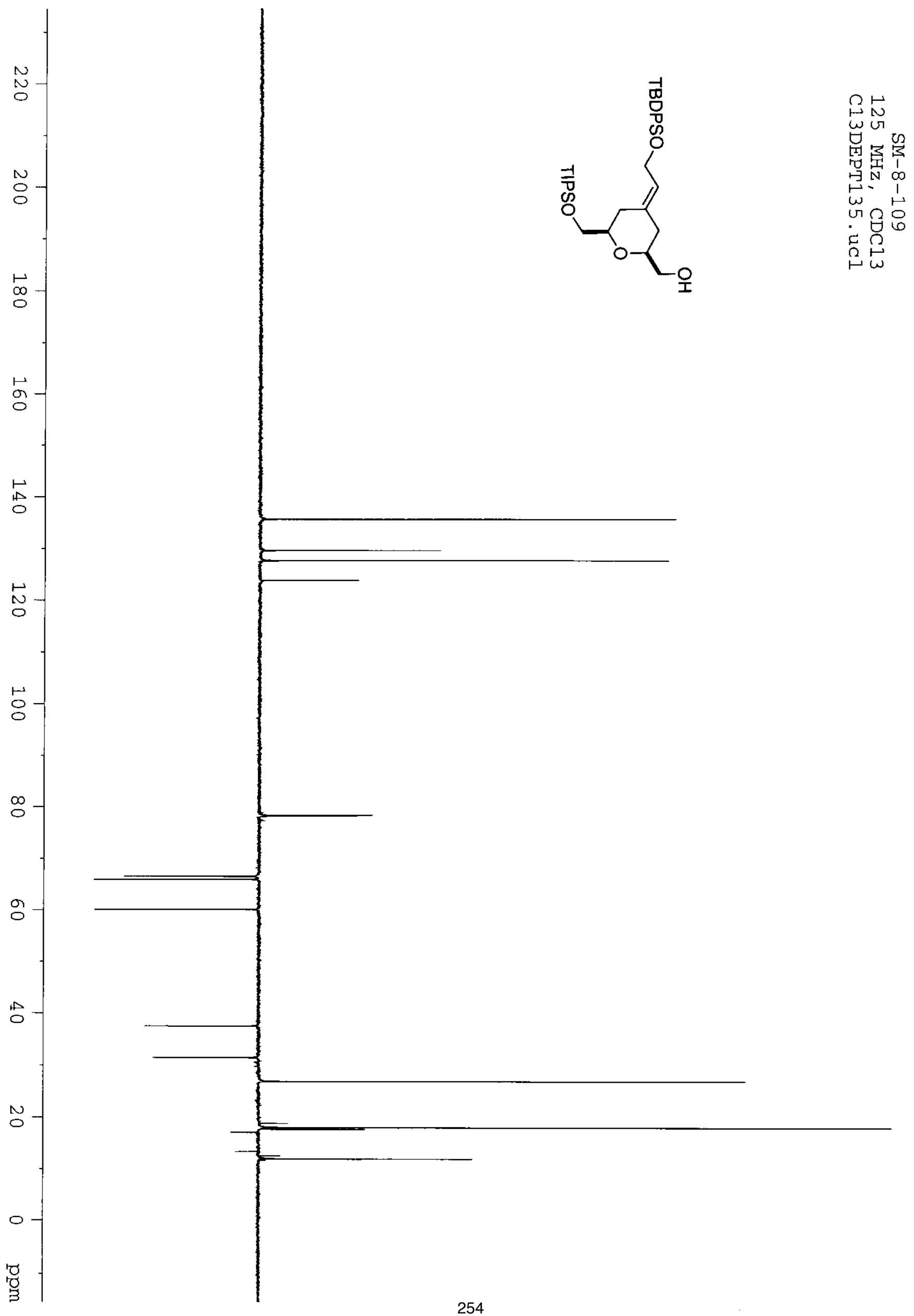




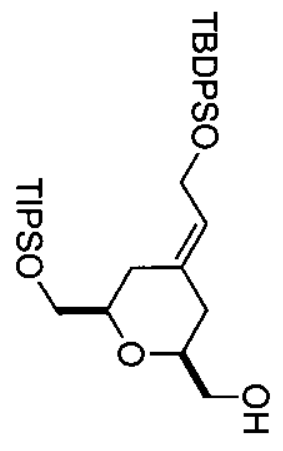

콘

点䁷

- N

- 1

옹 당응

i

造写

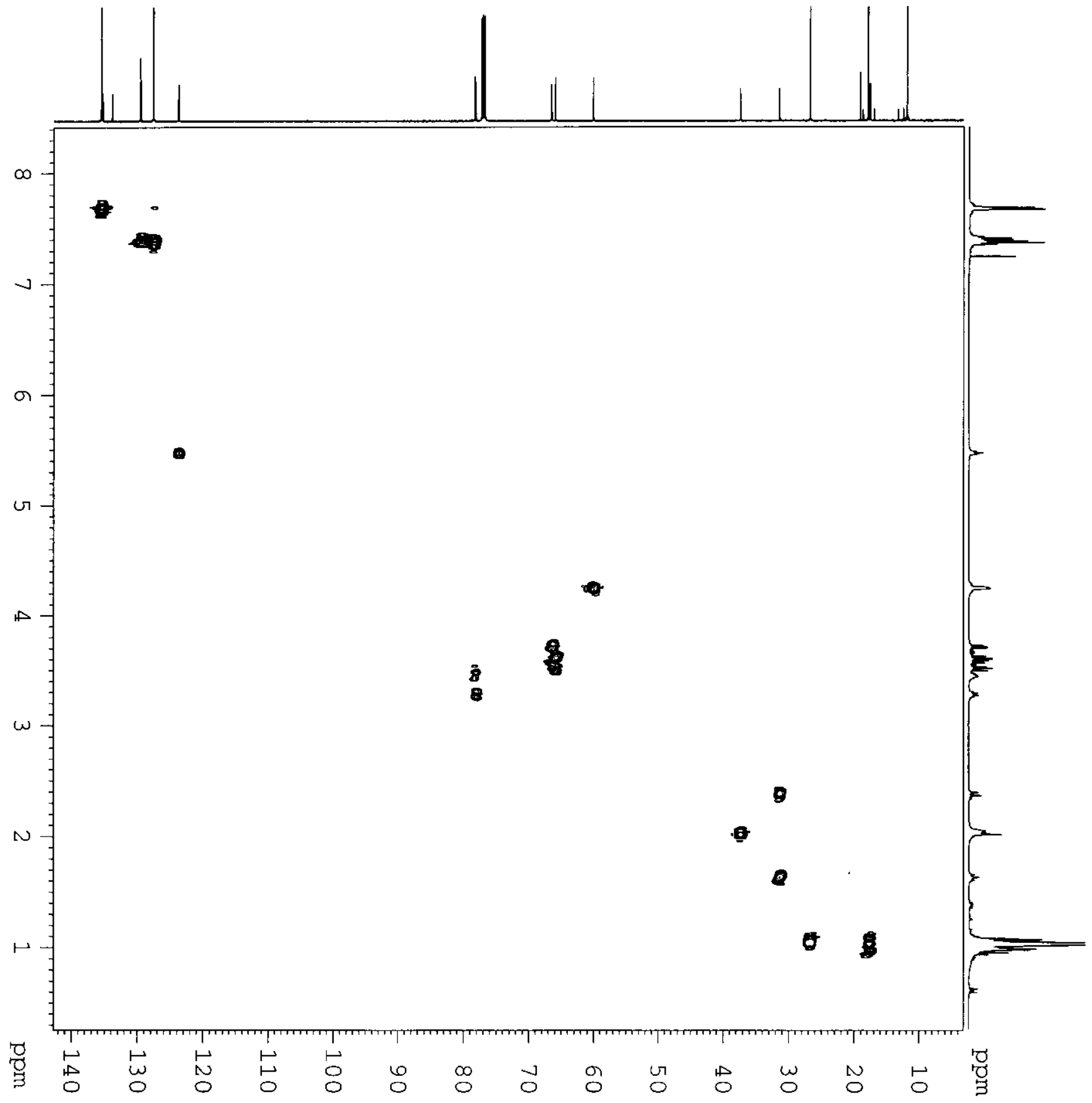




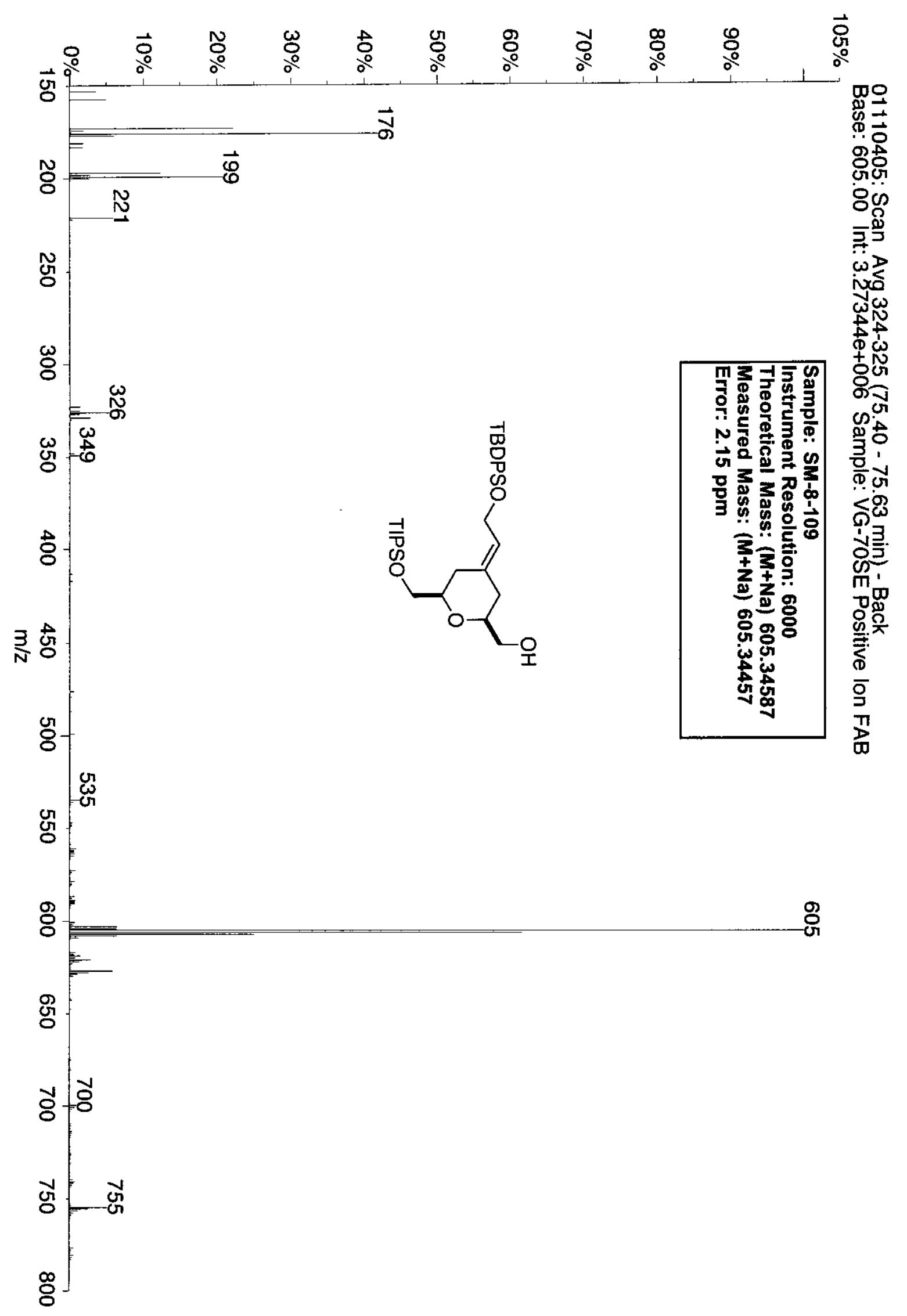




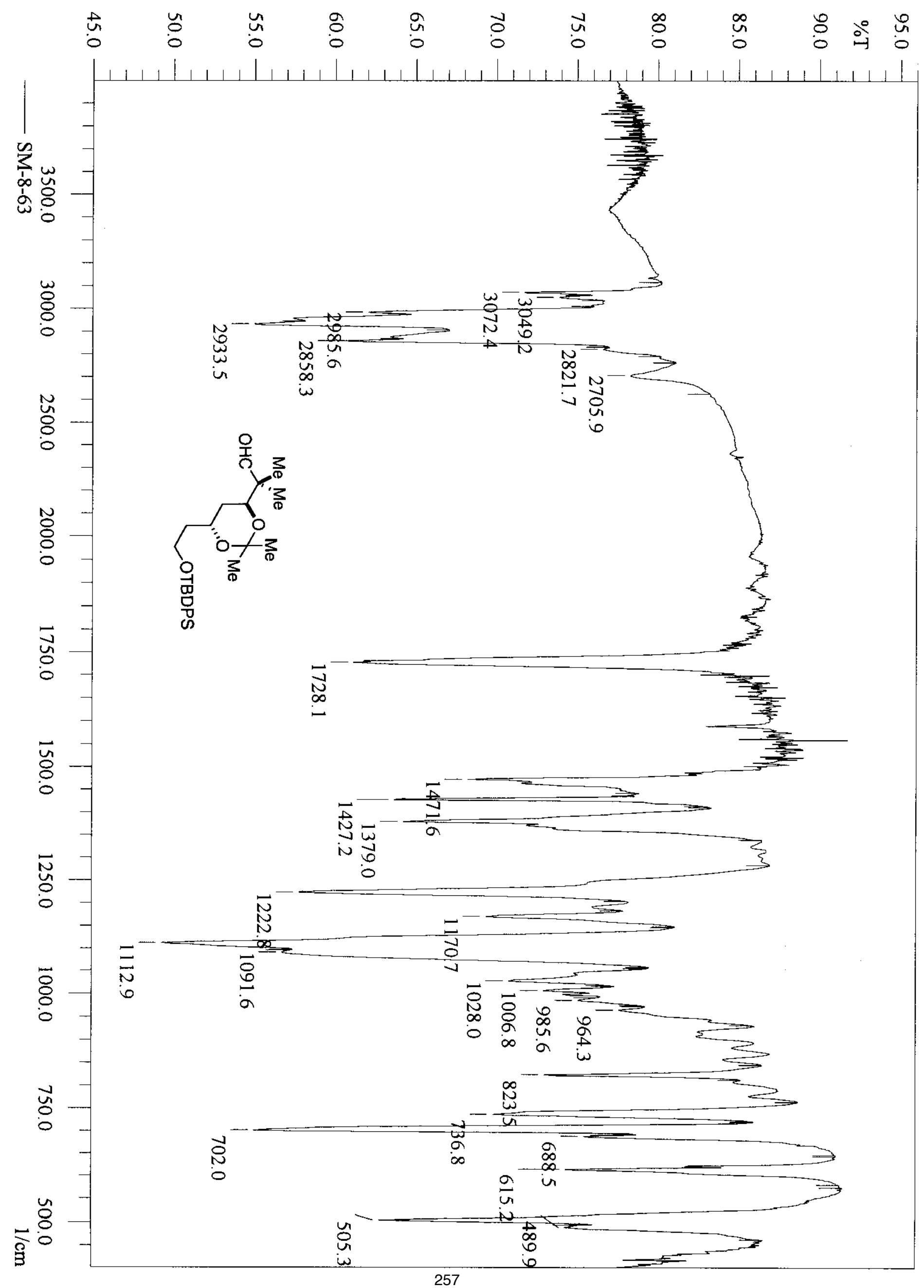




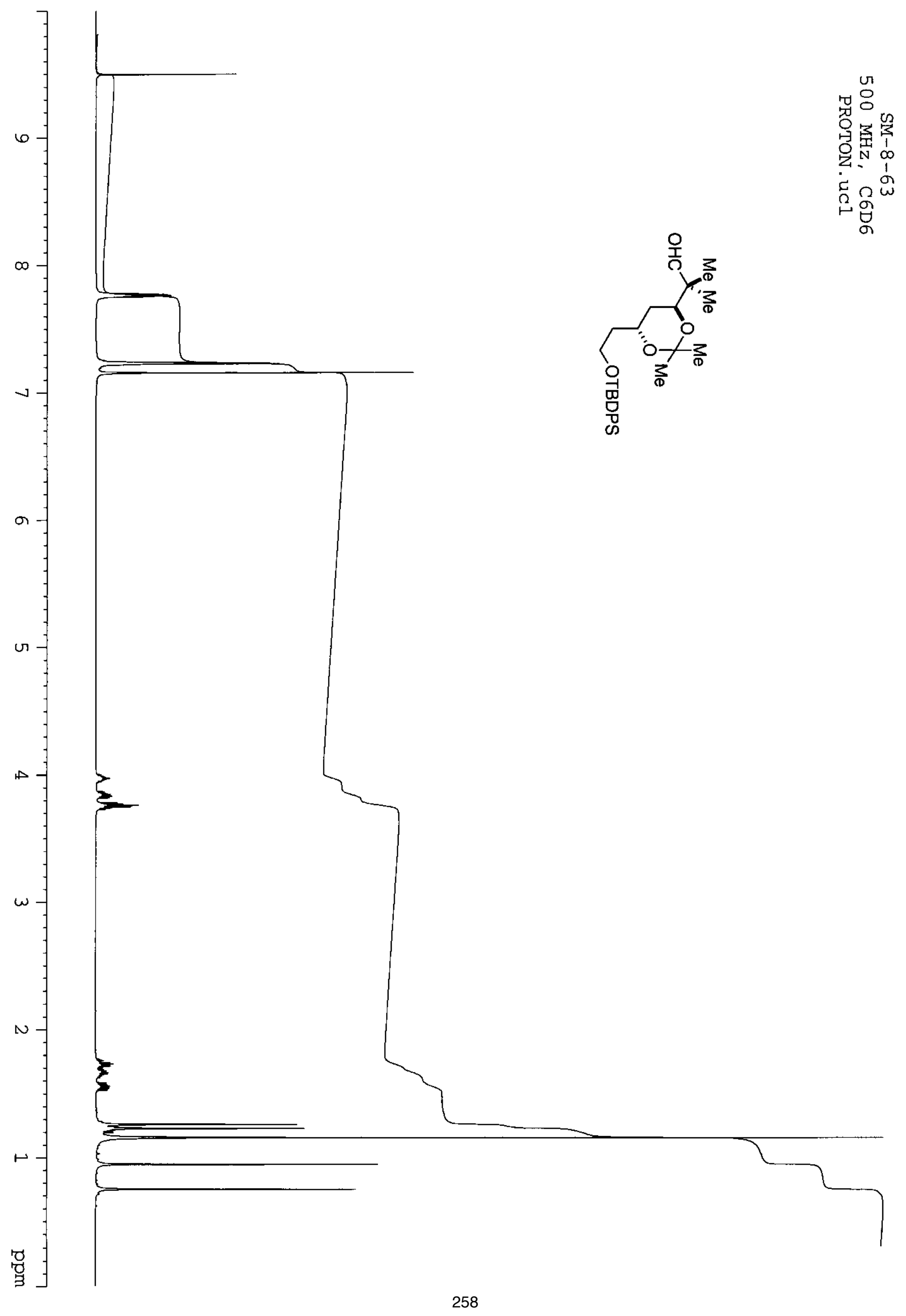



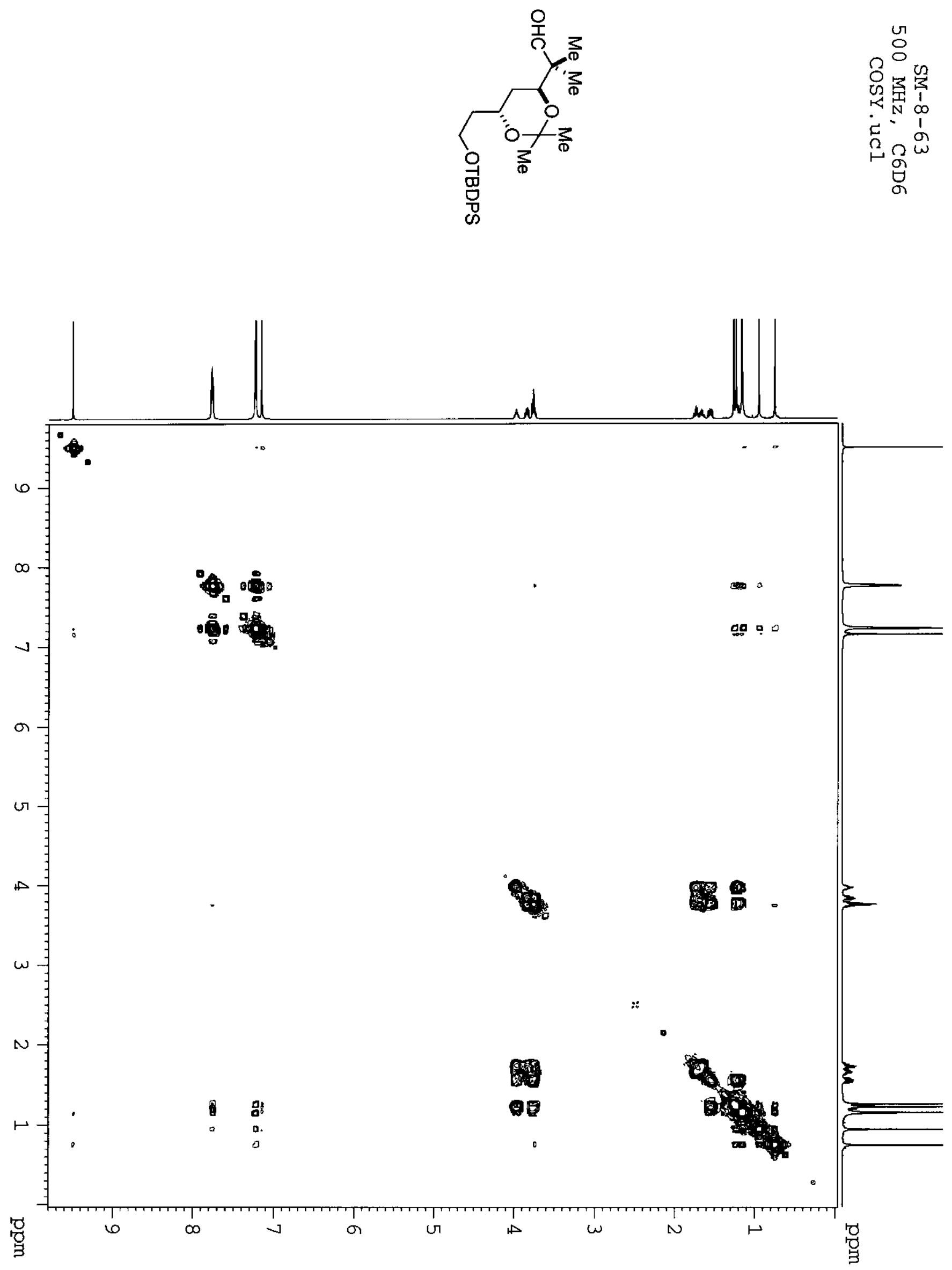

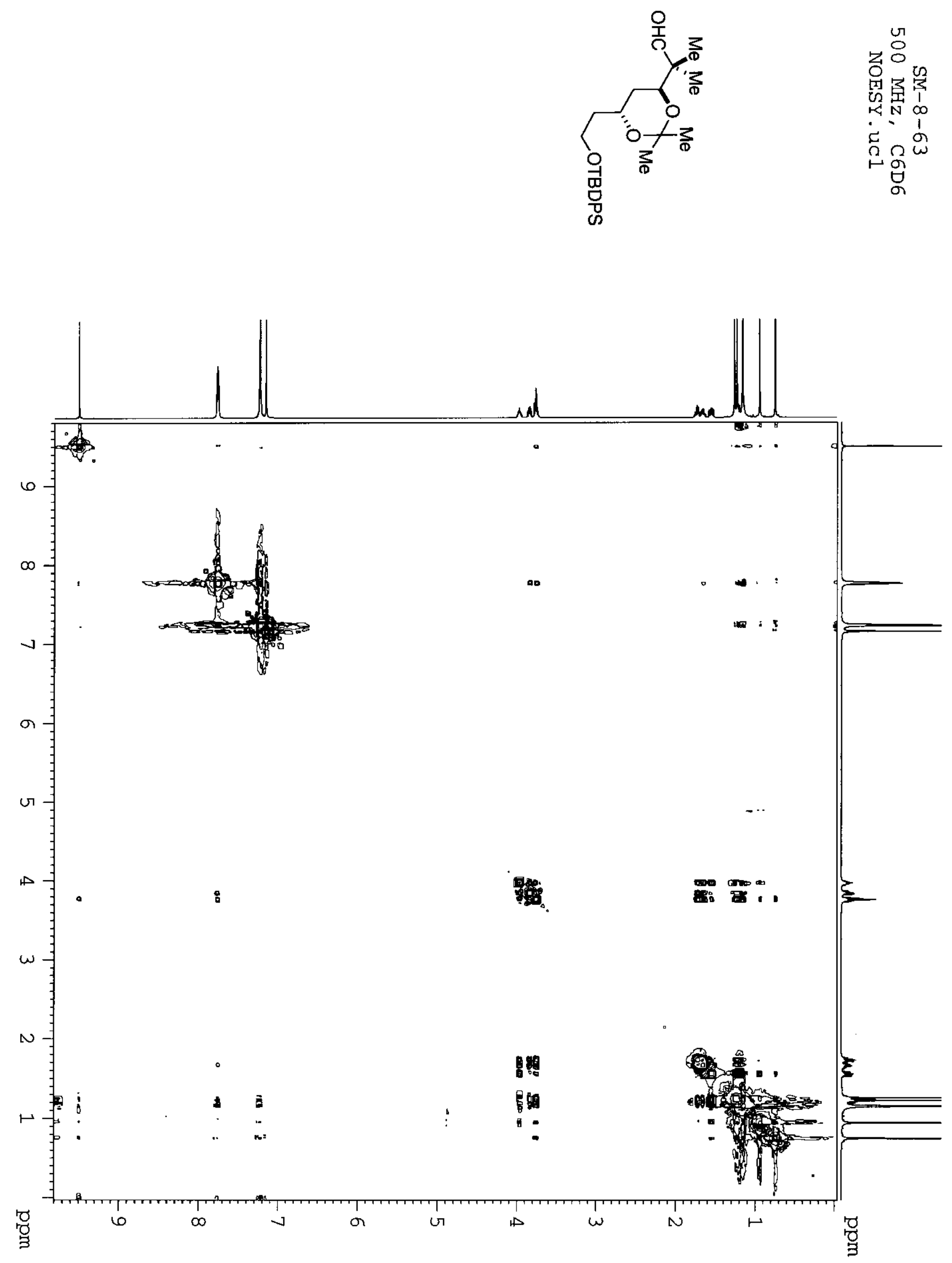


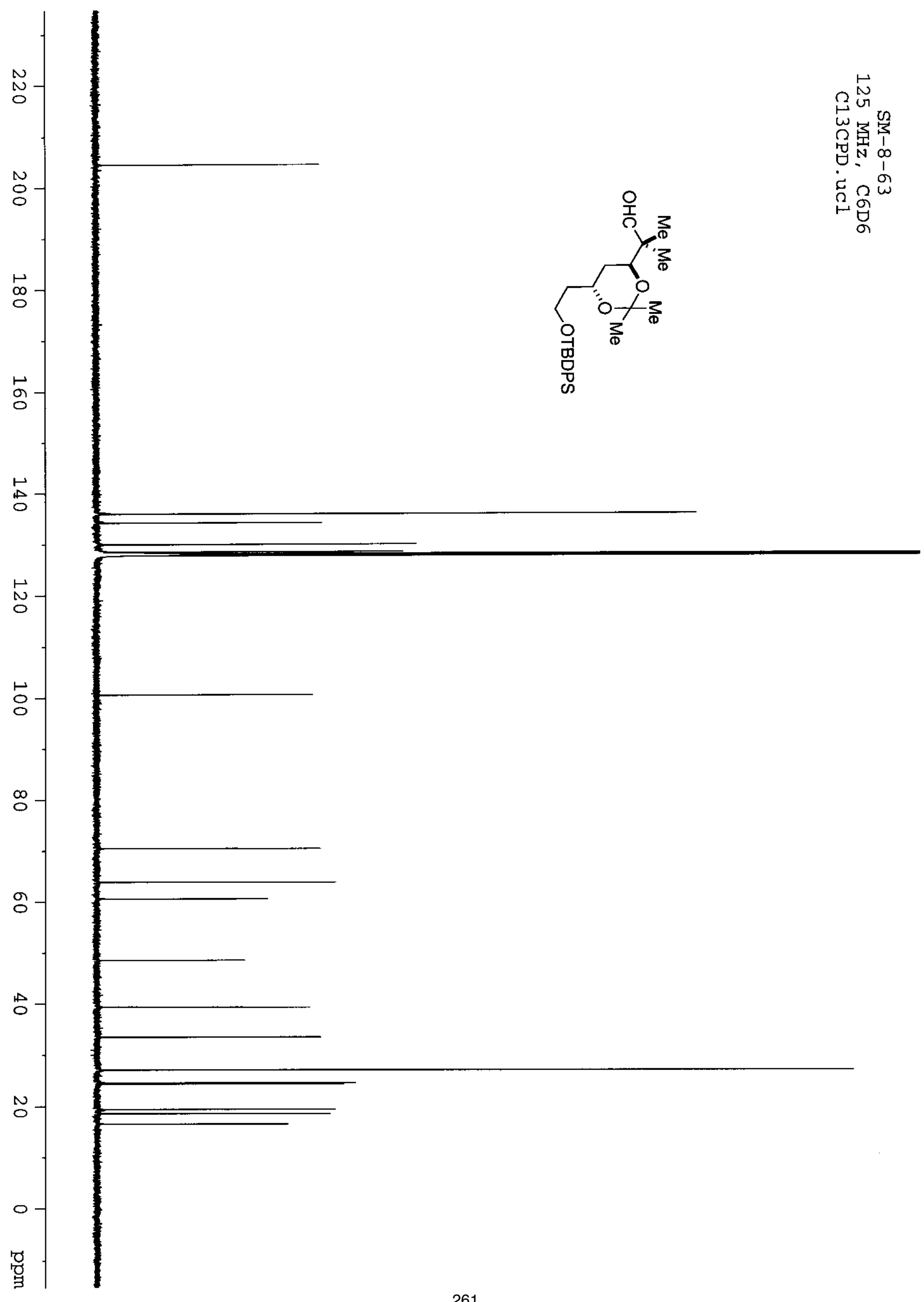




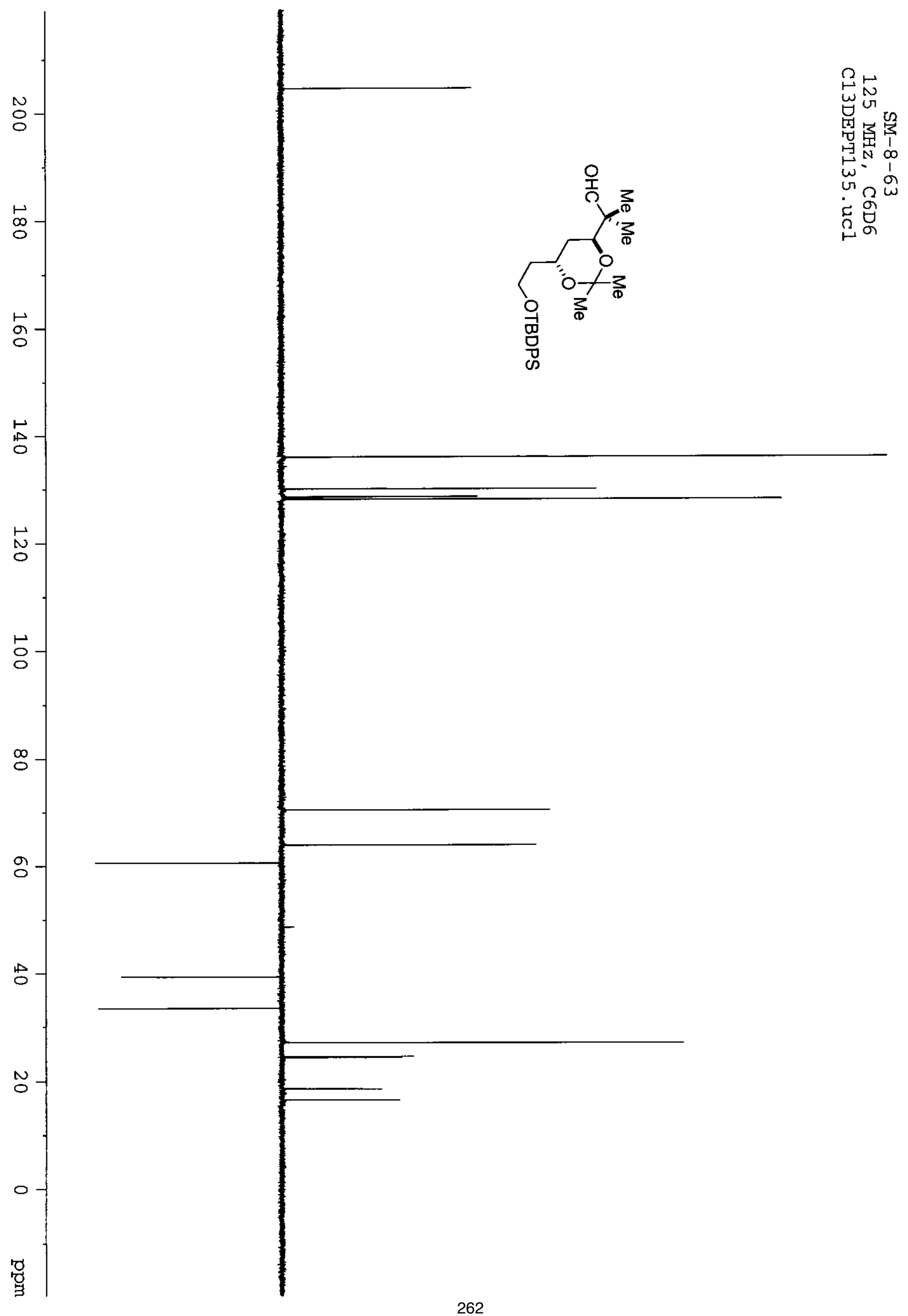



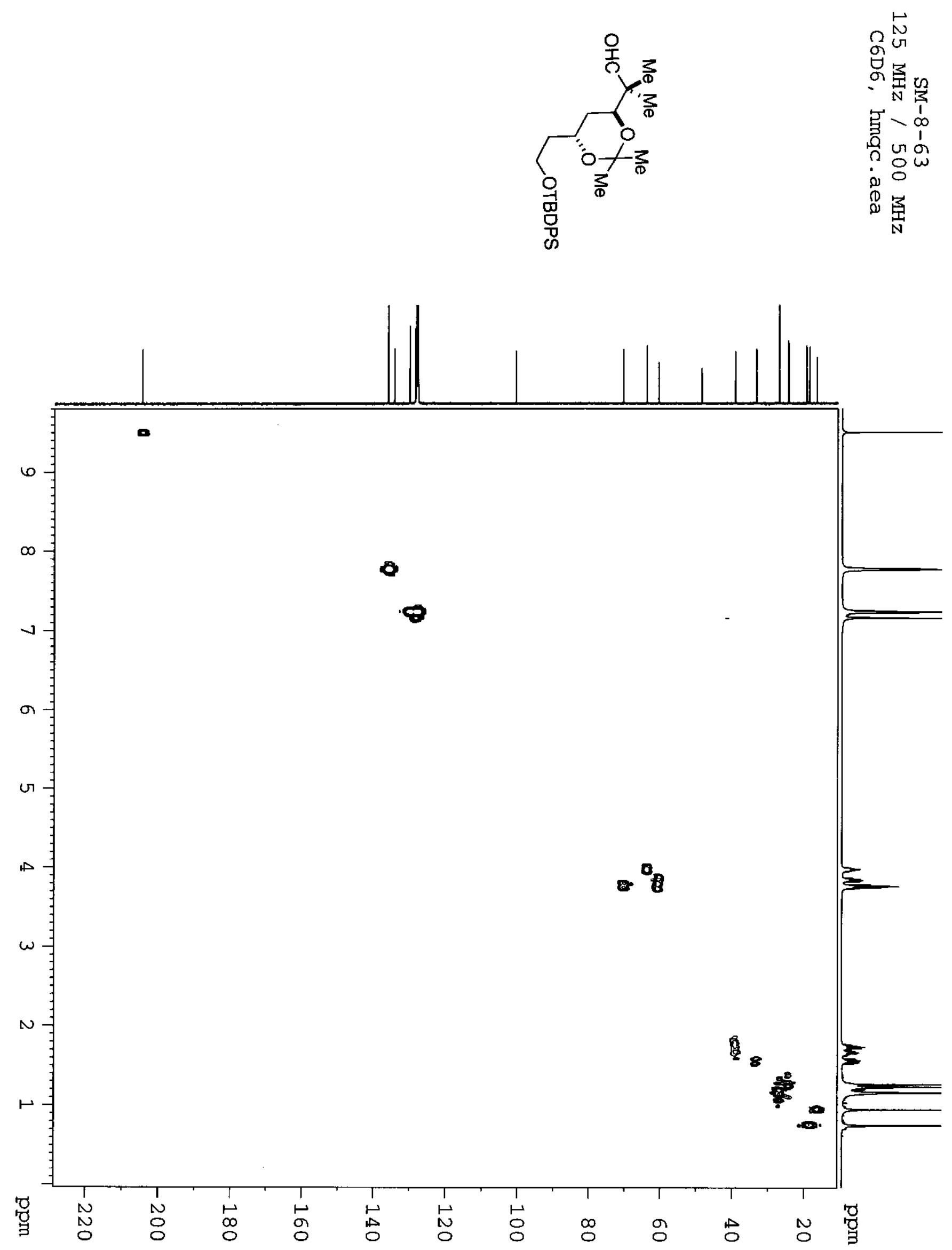


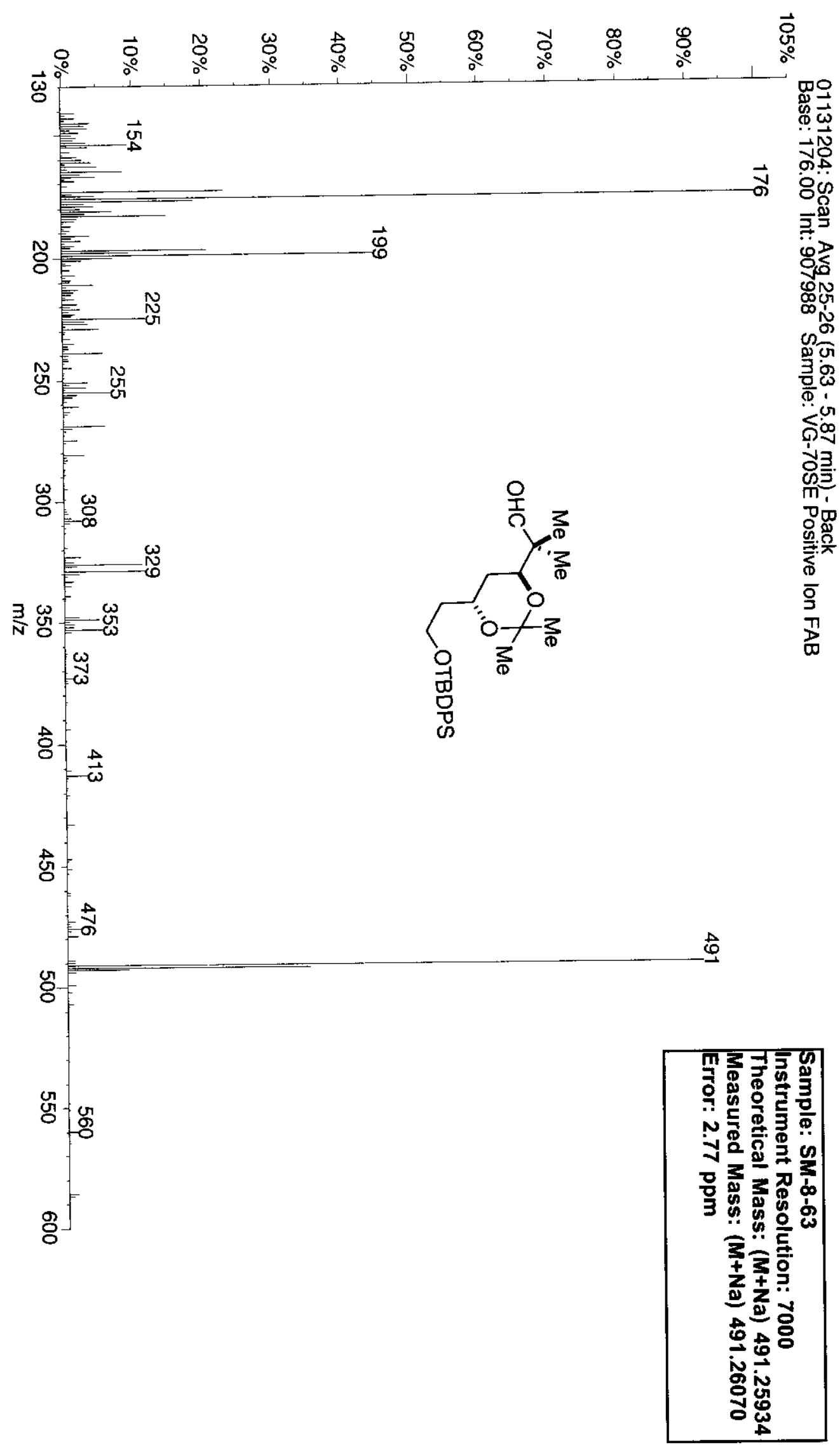




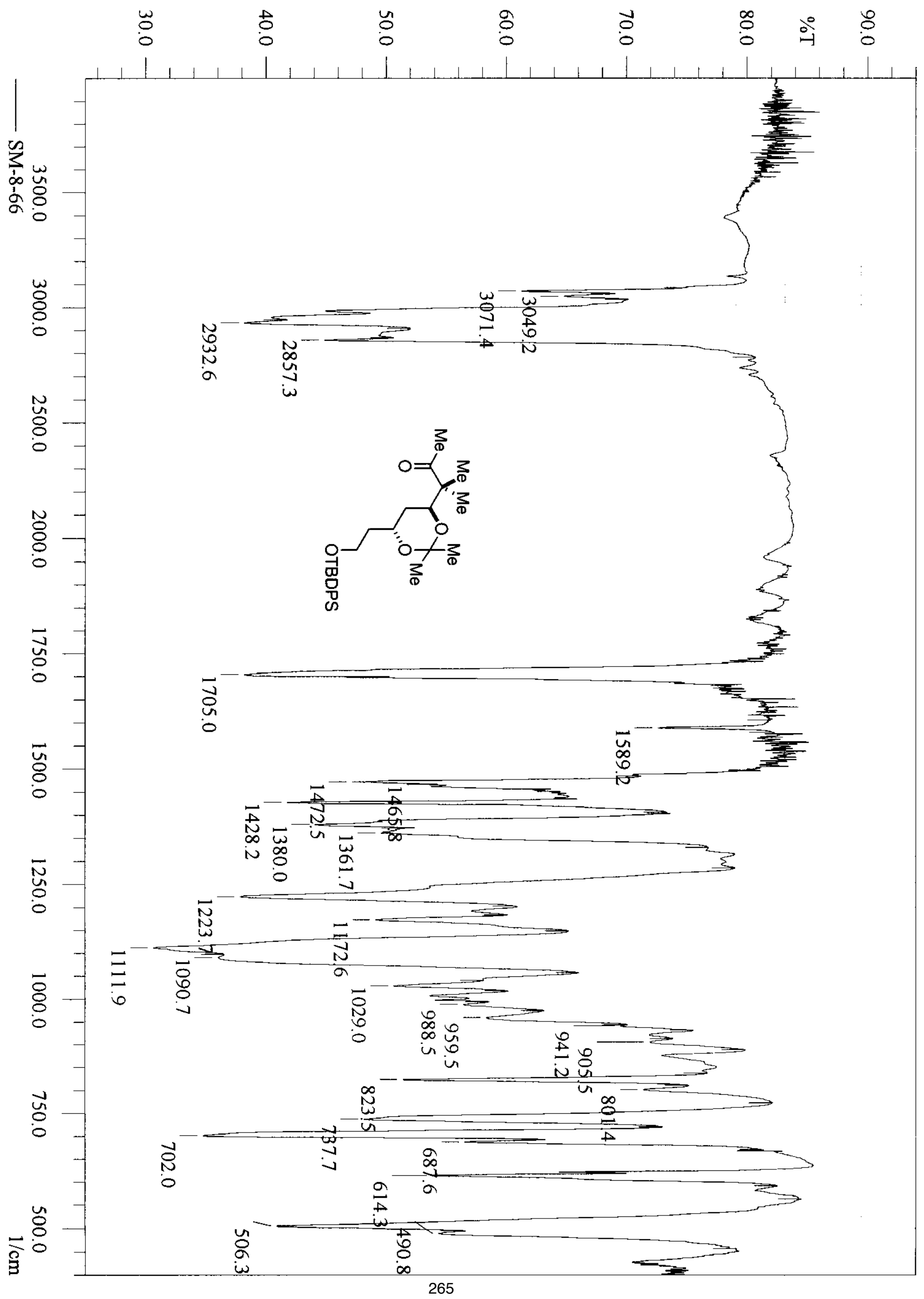




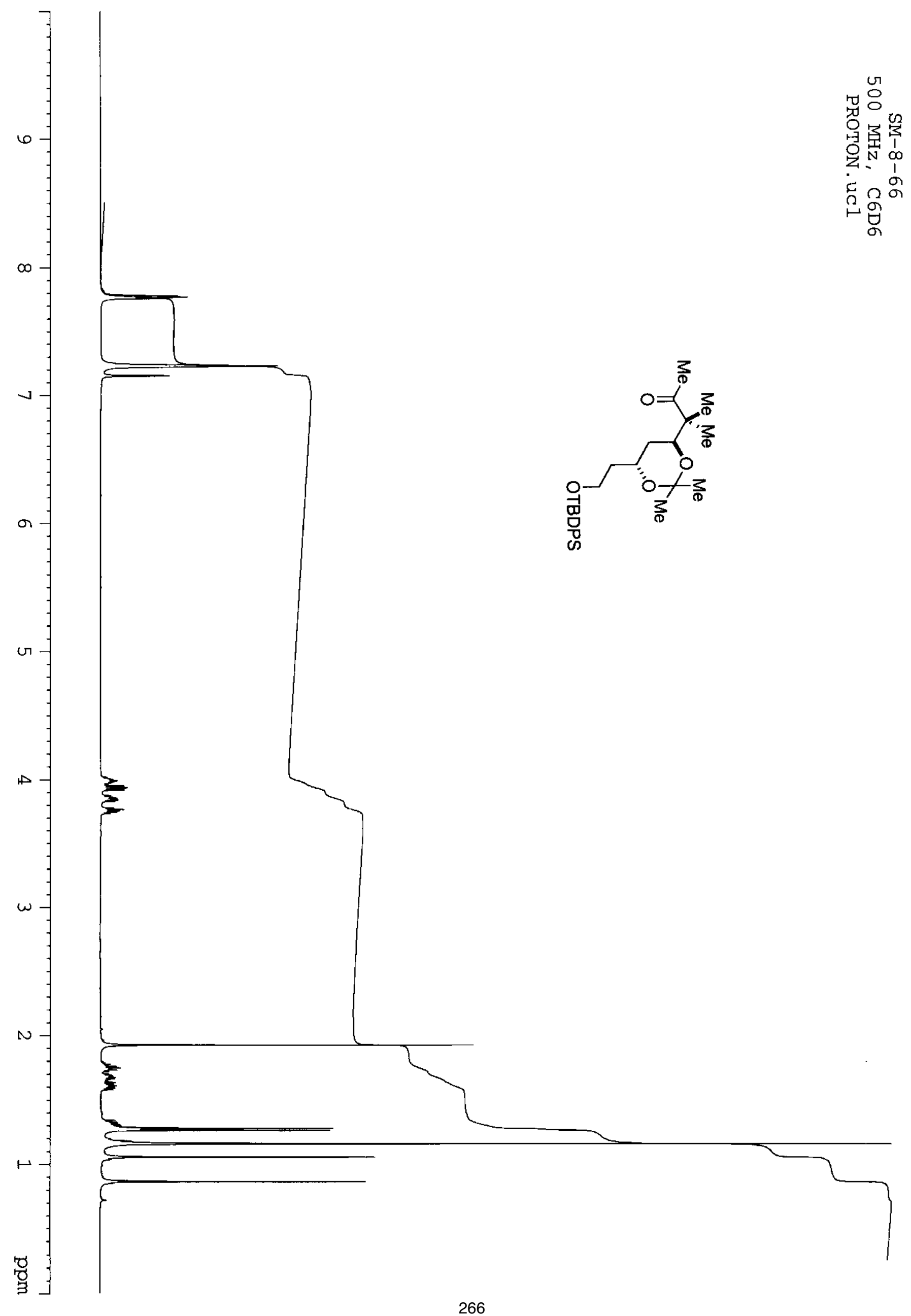



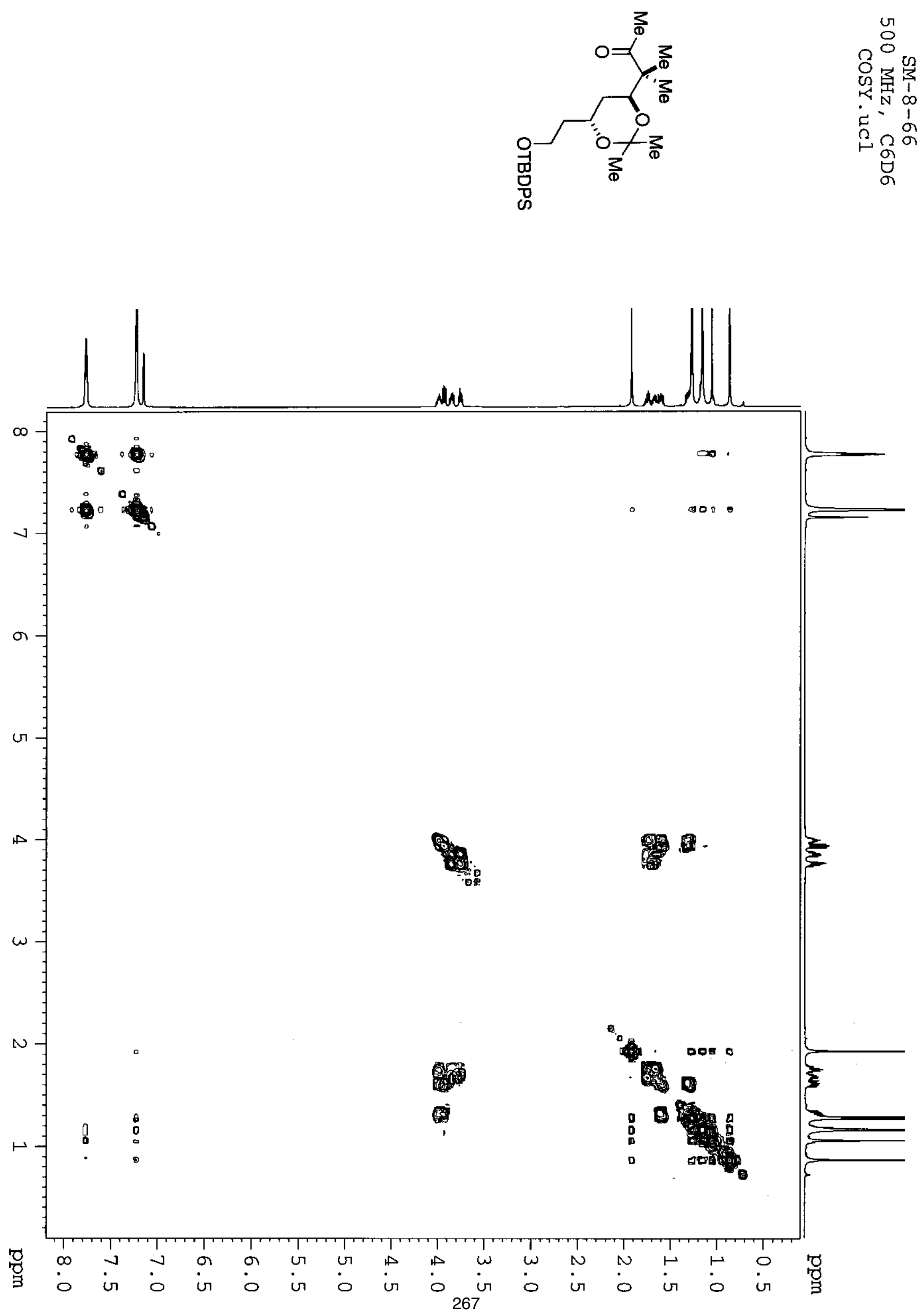

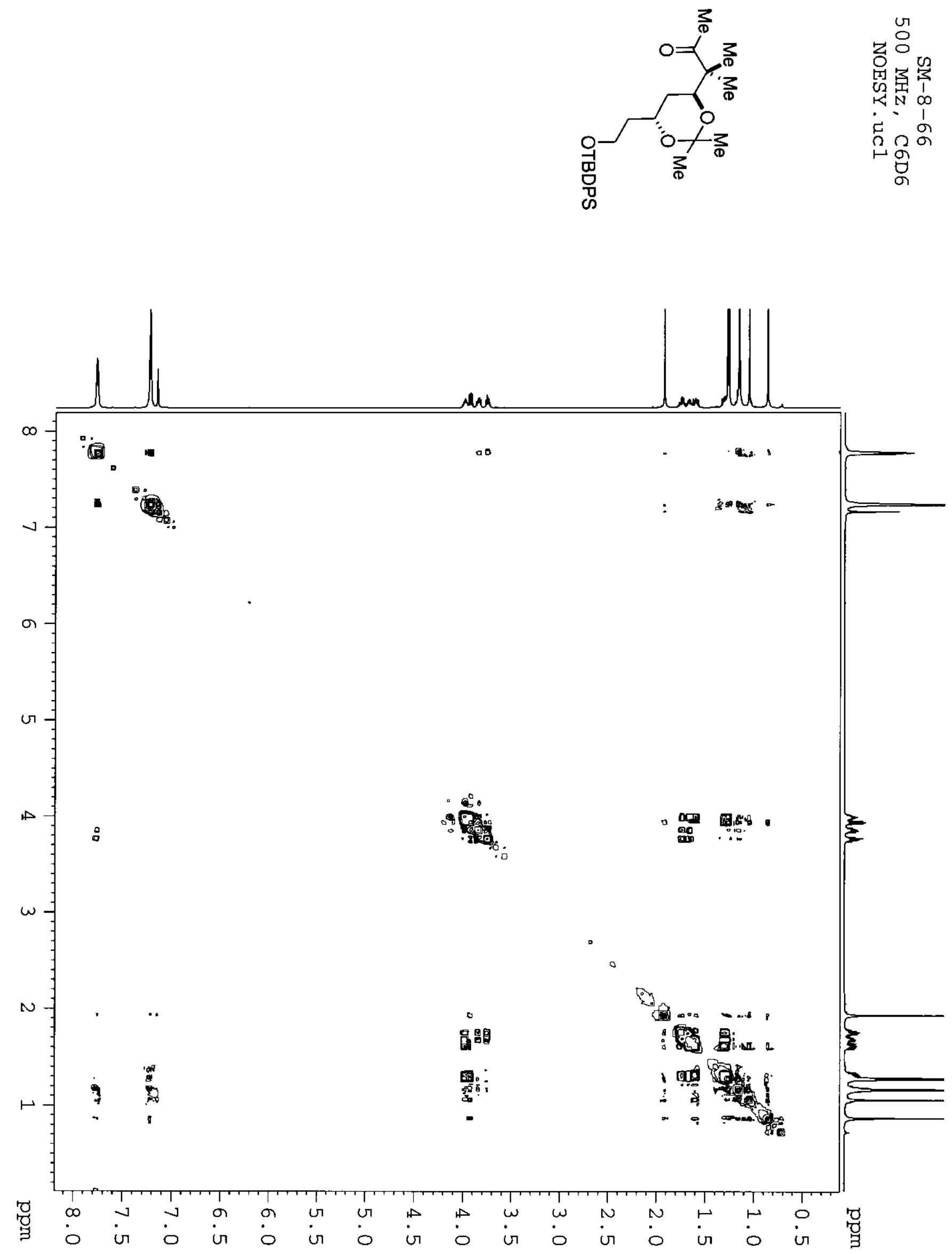


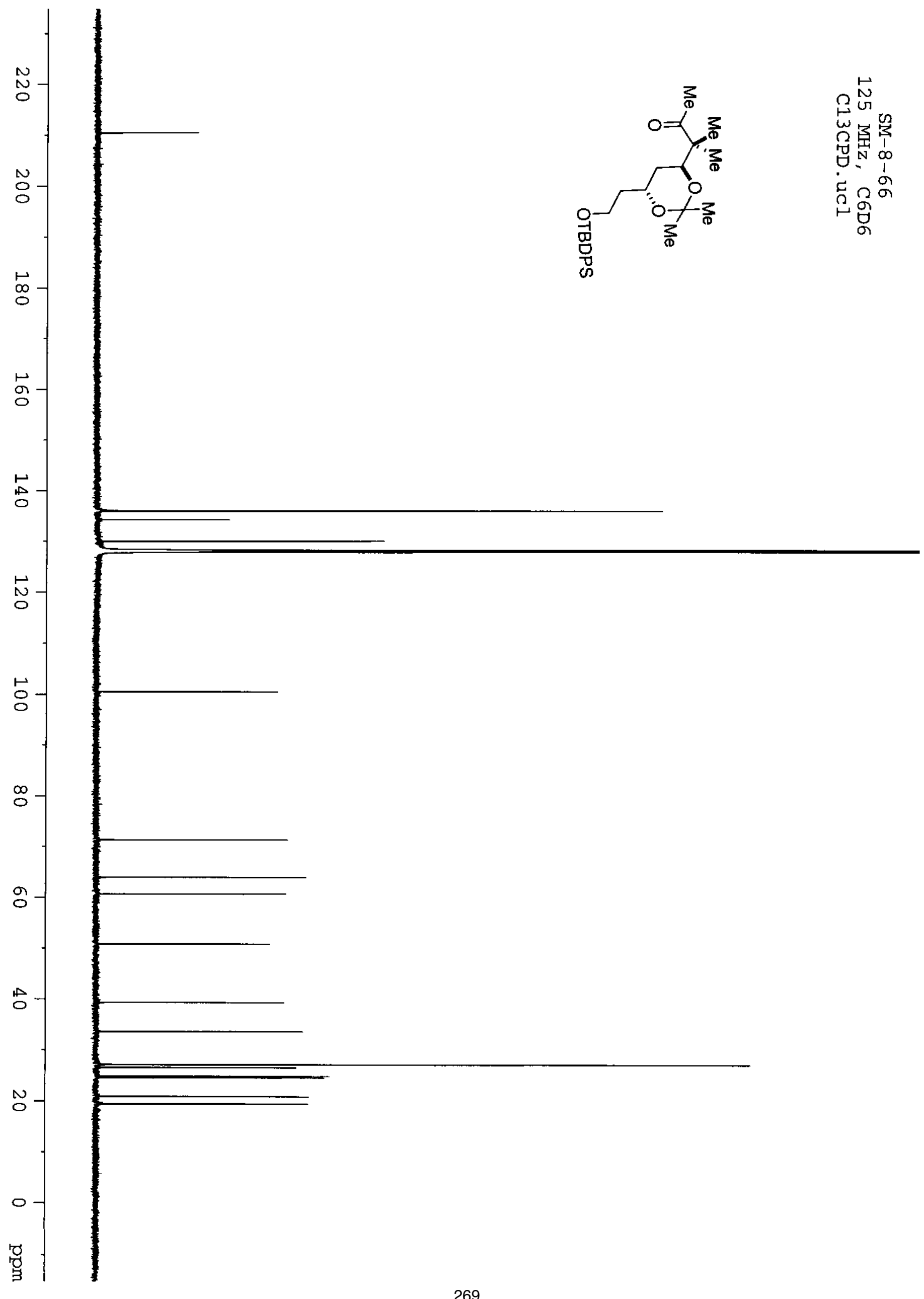




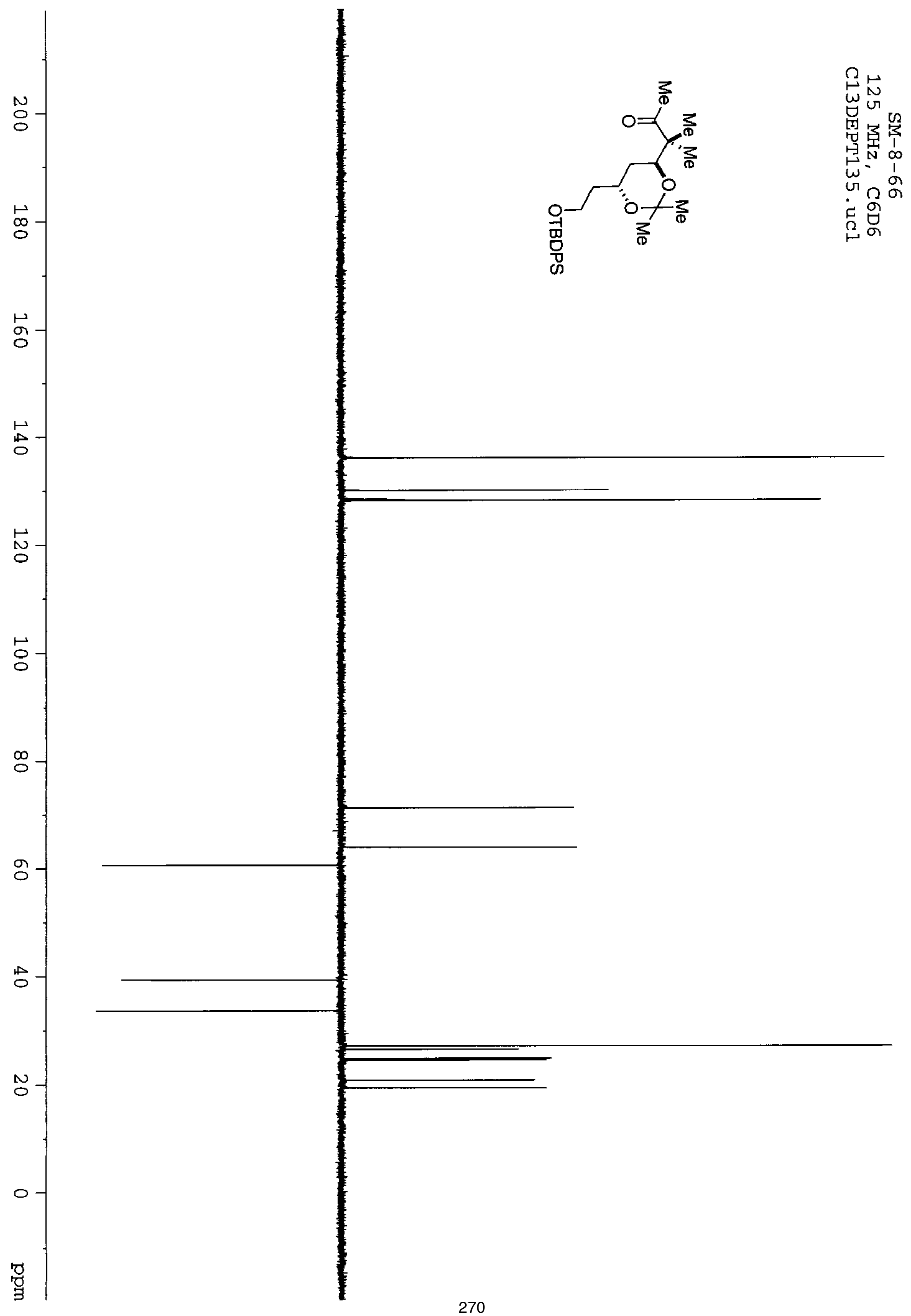



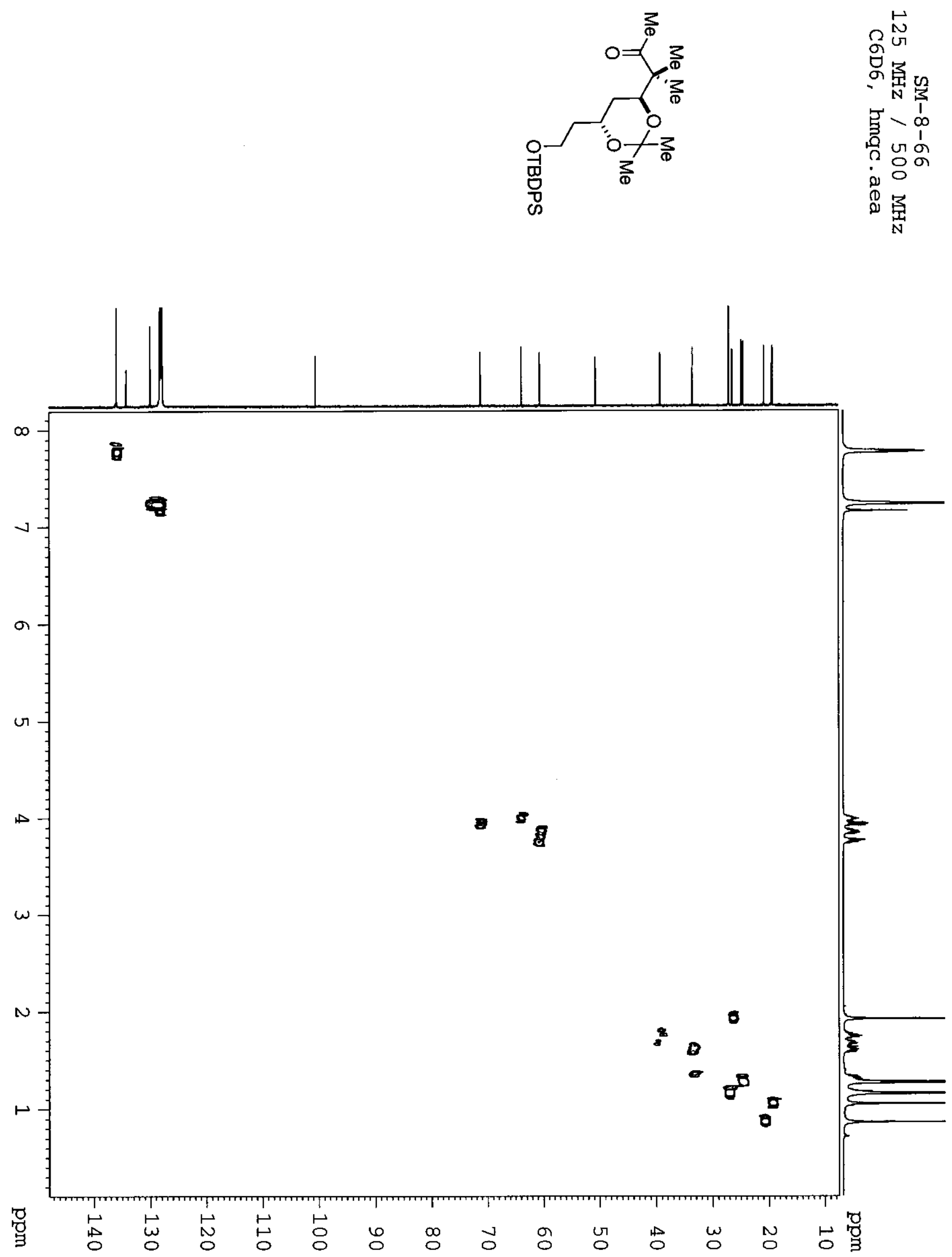


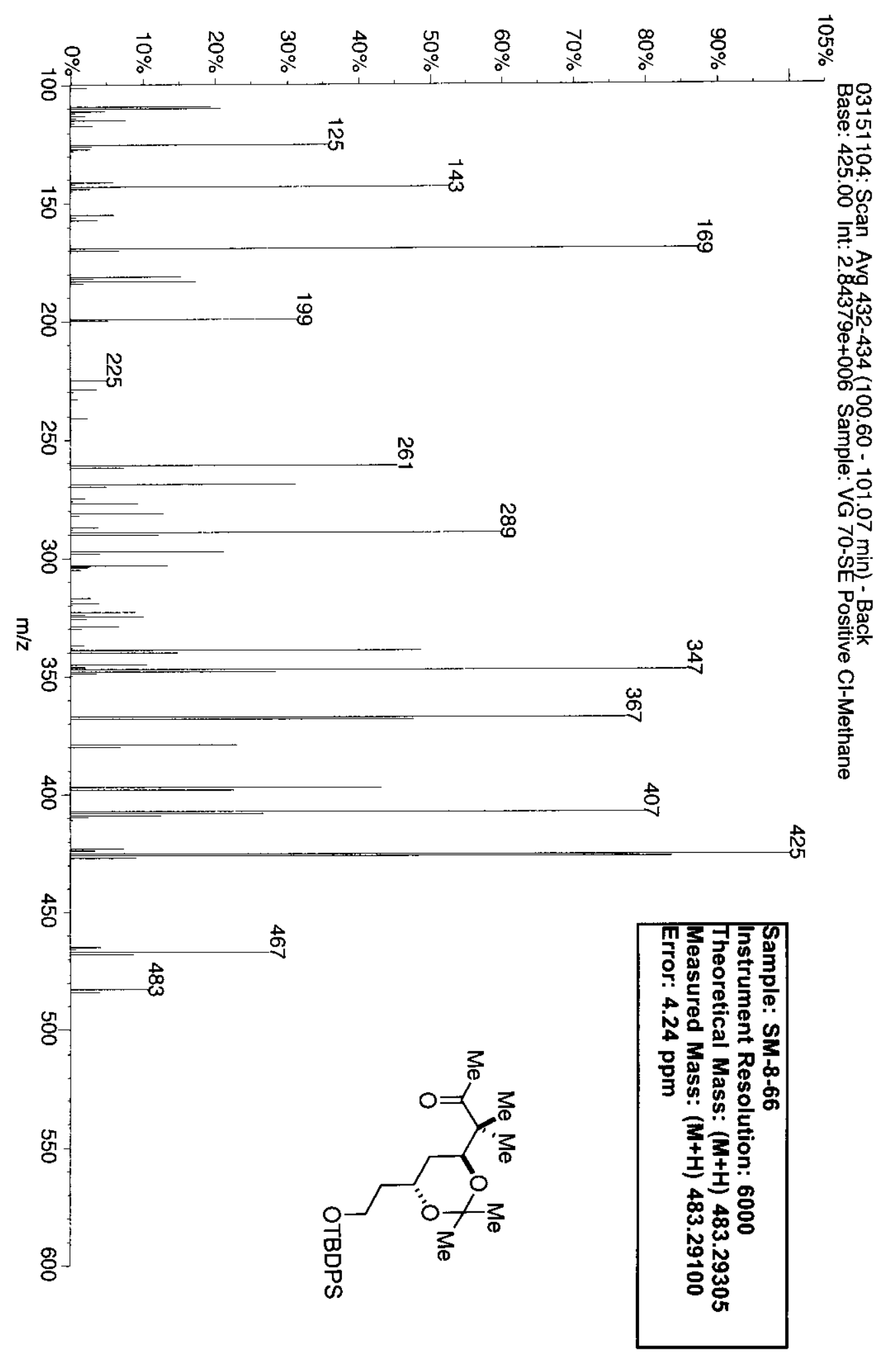




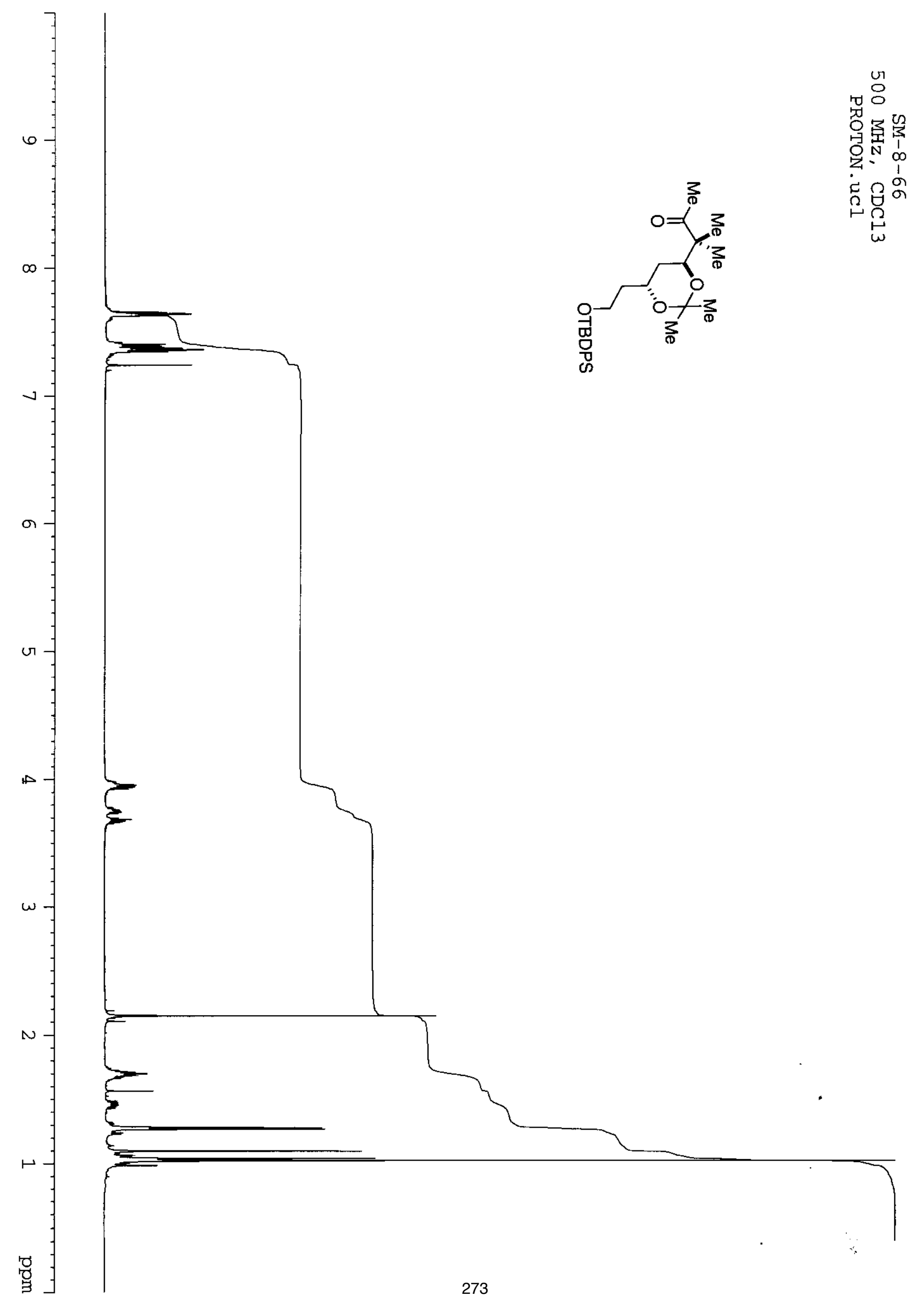



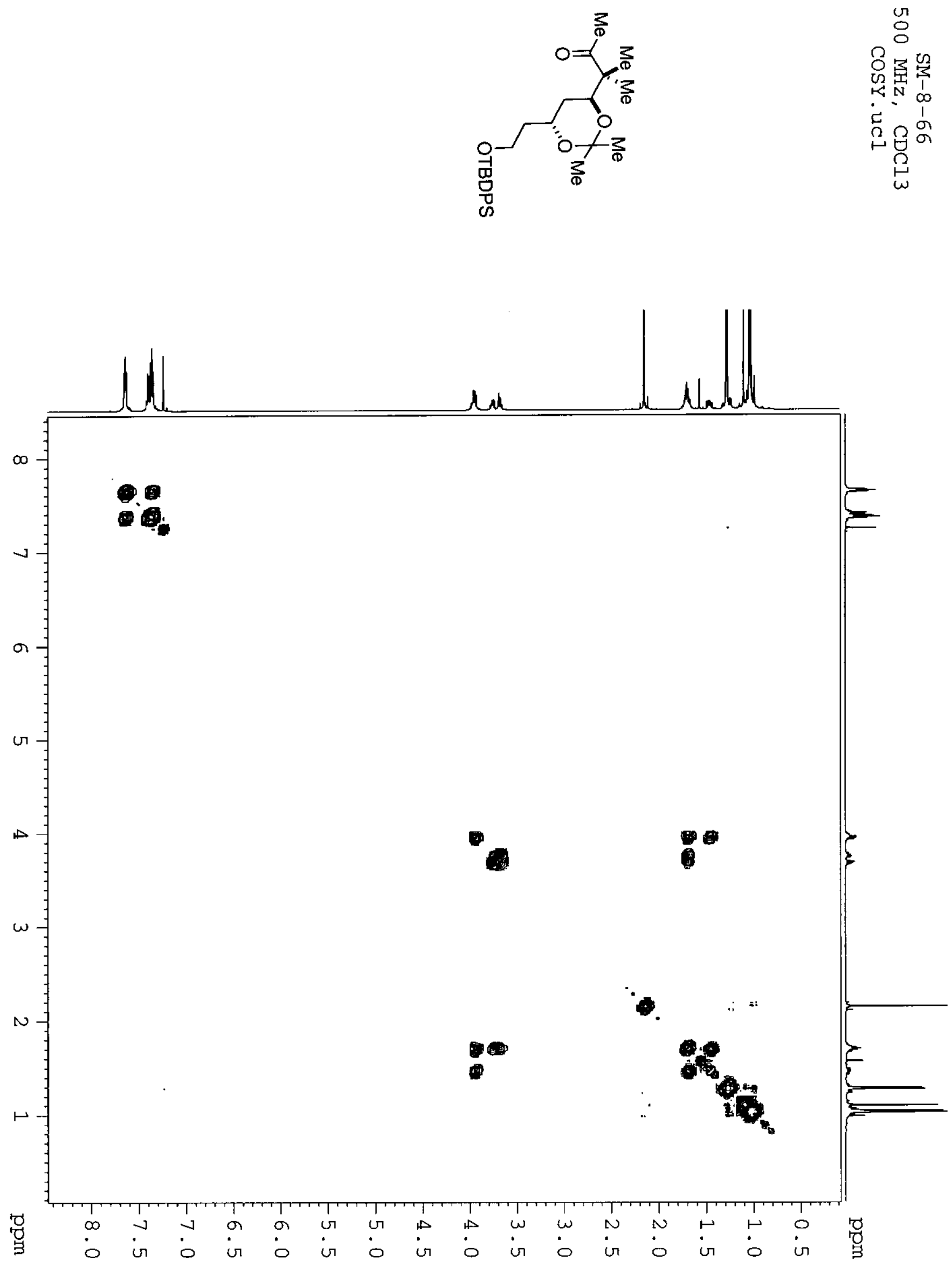


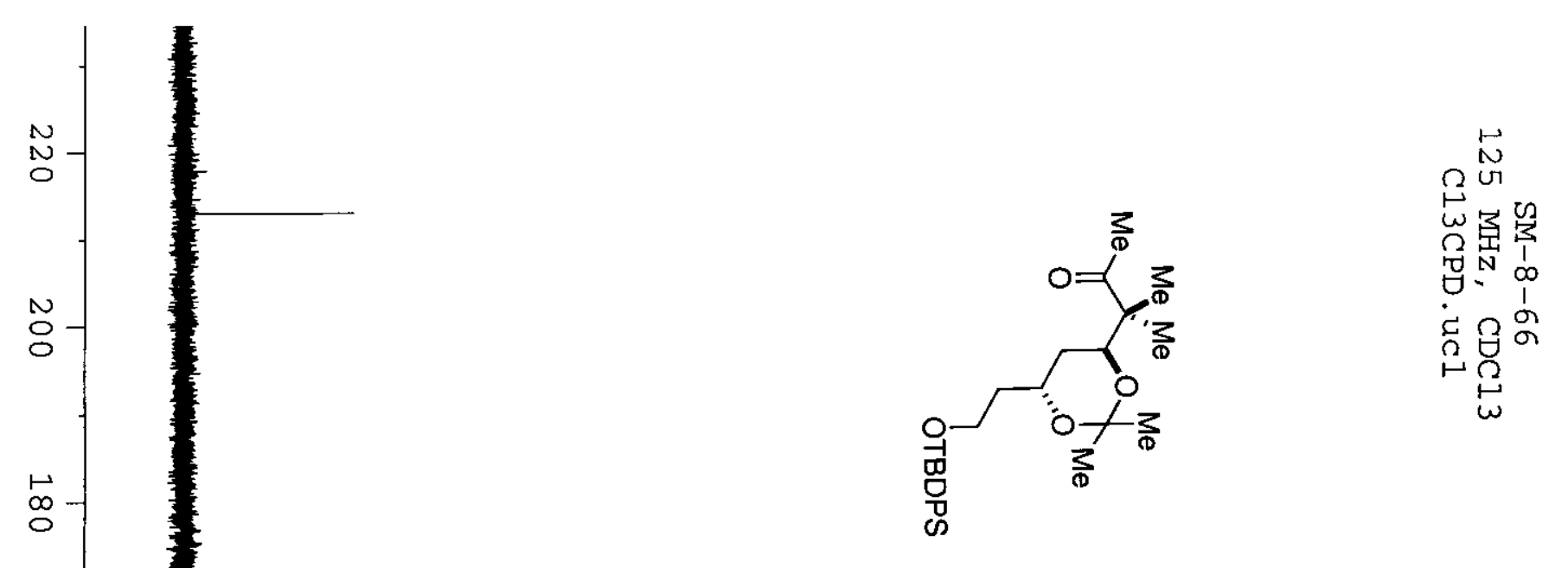

哭

点

茄

号

$\infty$

a

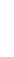

吕

. 

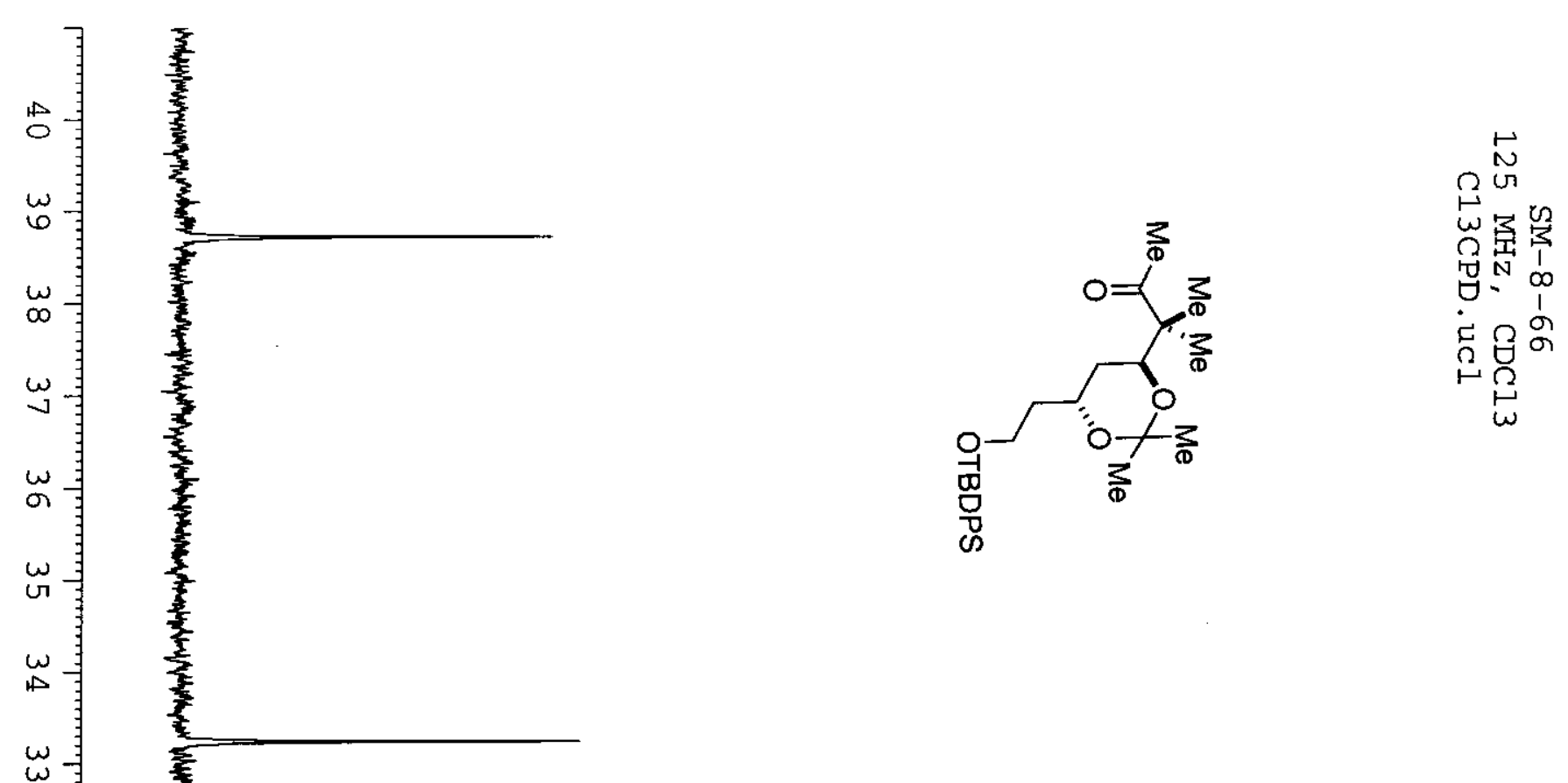

$\omega$

$\stackrel{\omega}{\bullet}$

$\omega$

$\checkmark$

6

$\infty$

N

(

U

$N$

$\omega$

$N$

$N$

$N$

0

6

曋

集

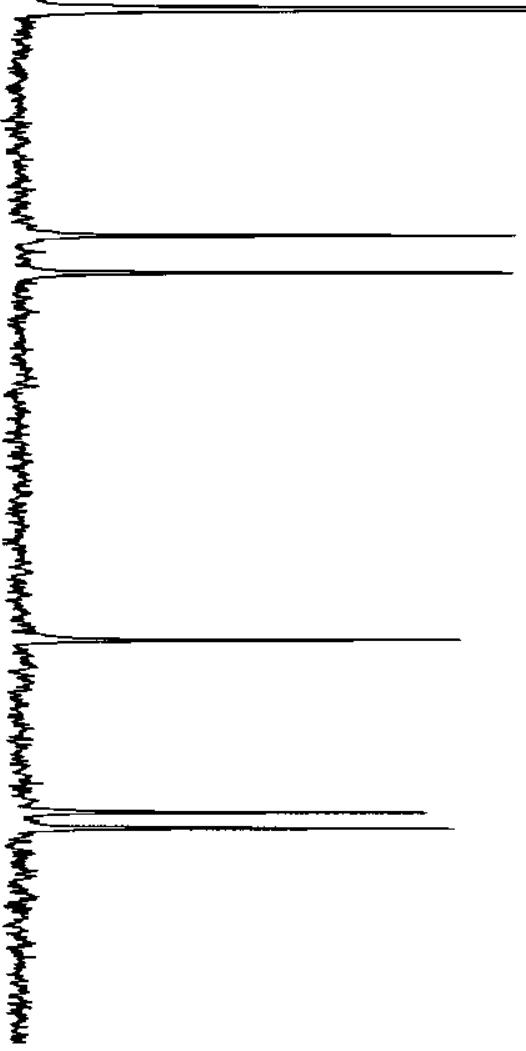




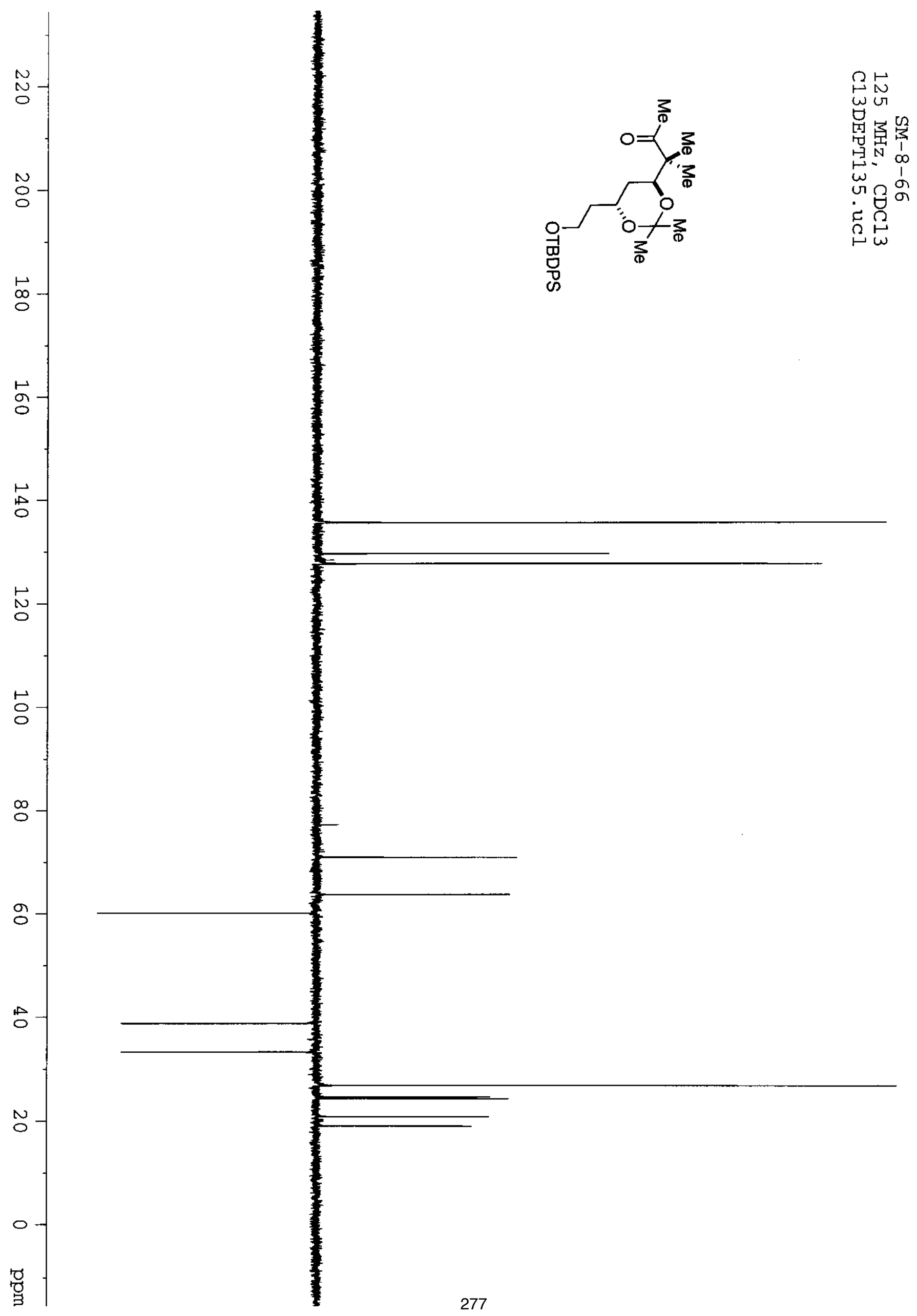




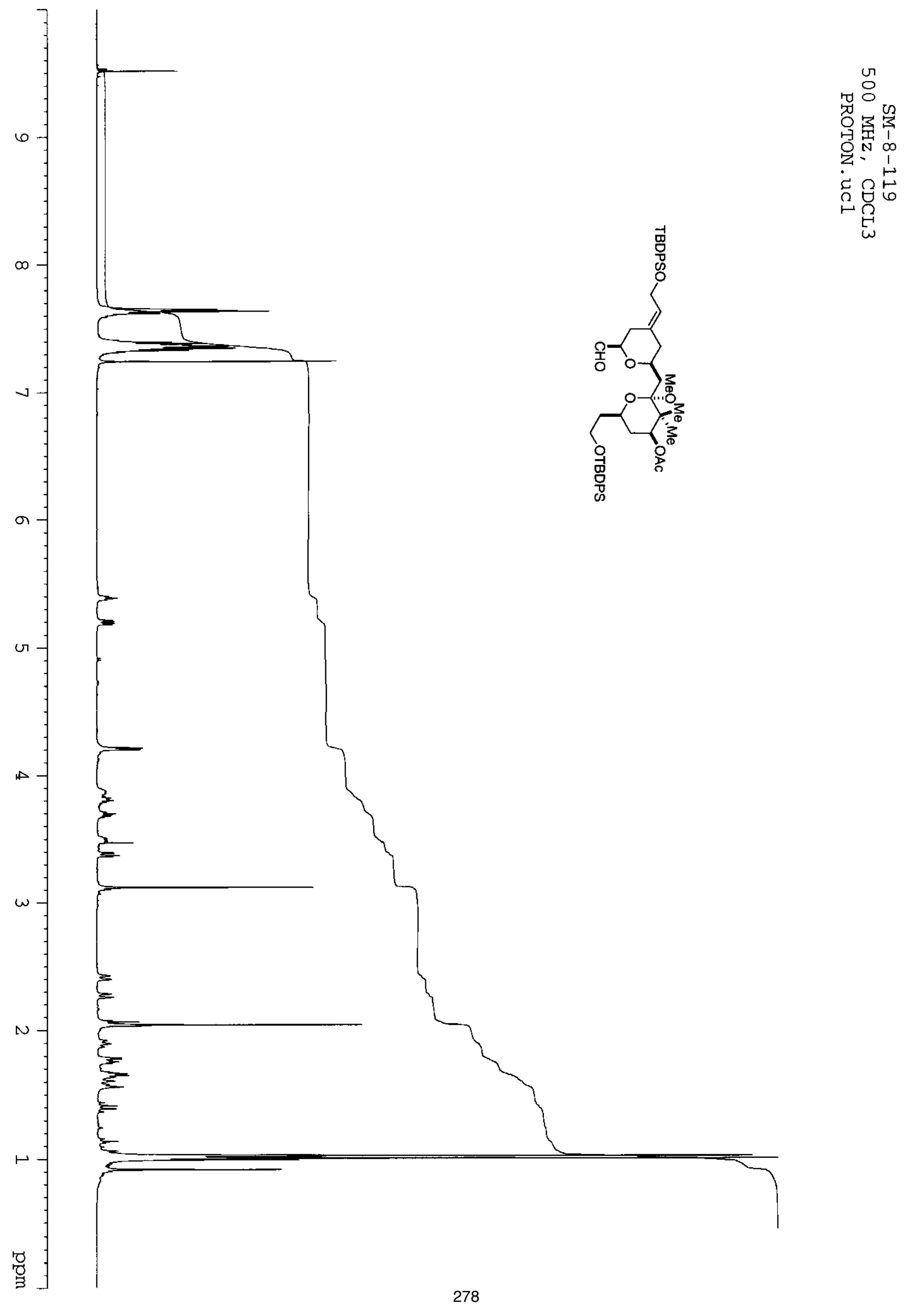



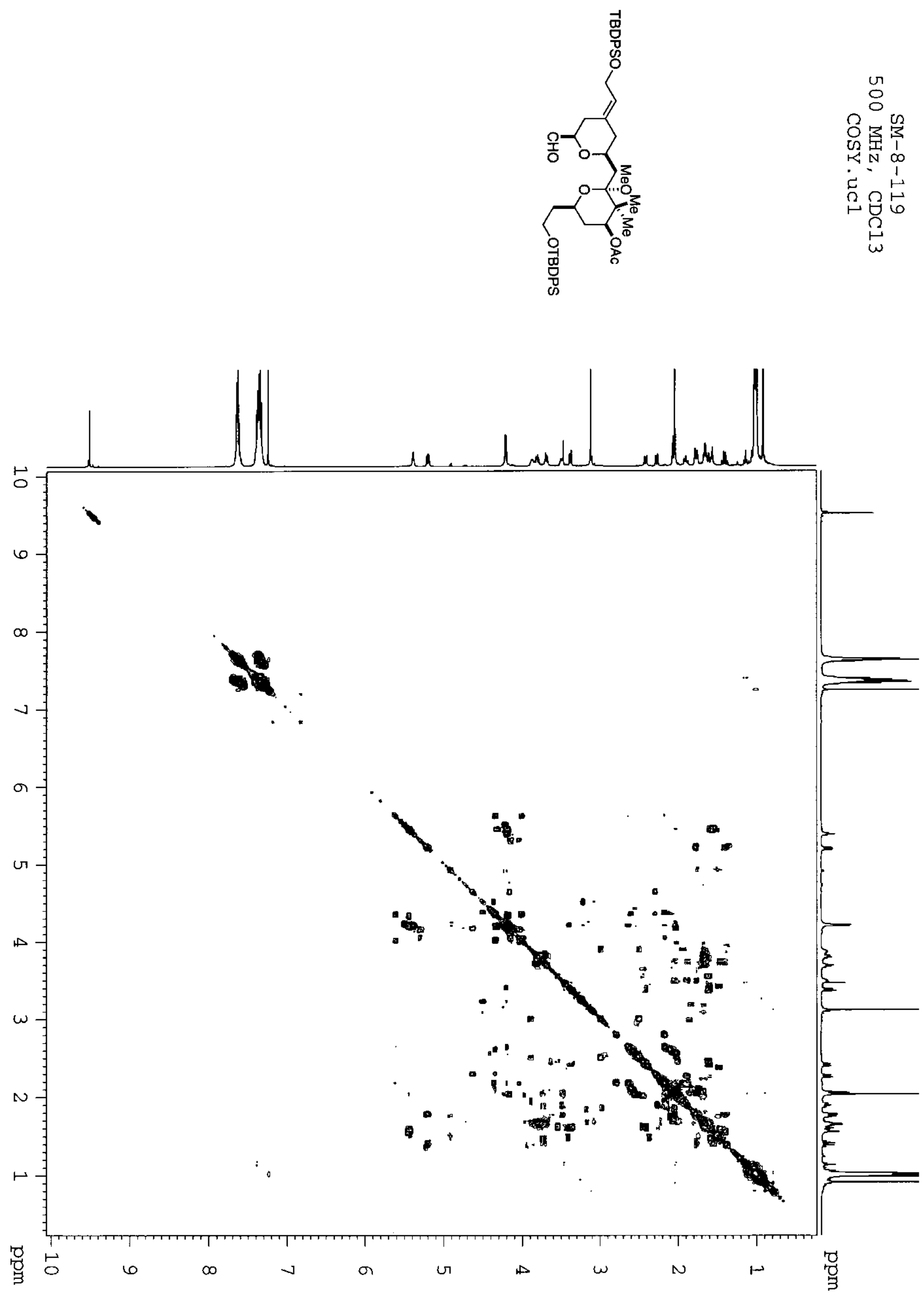

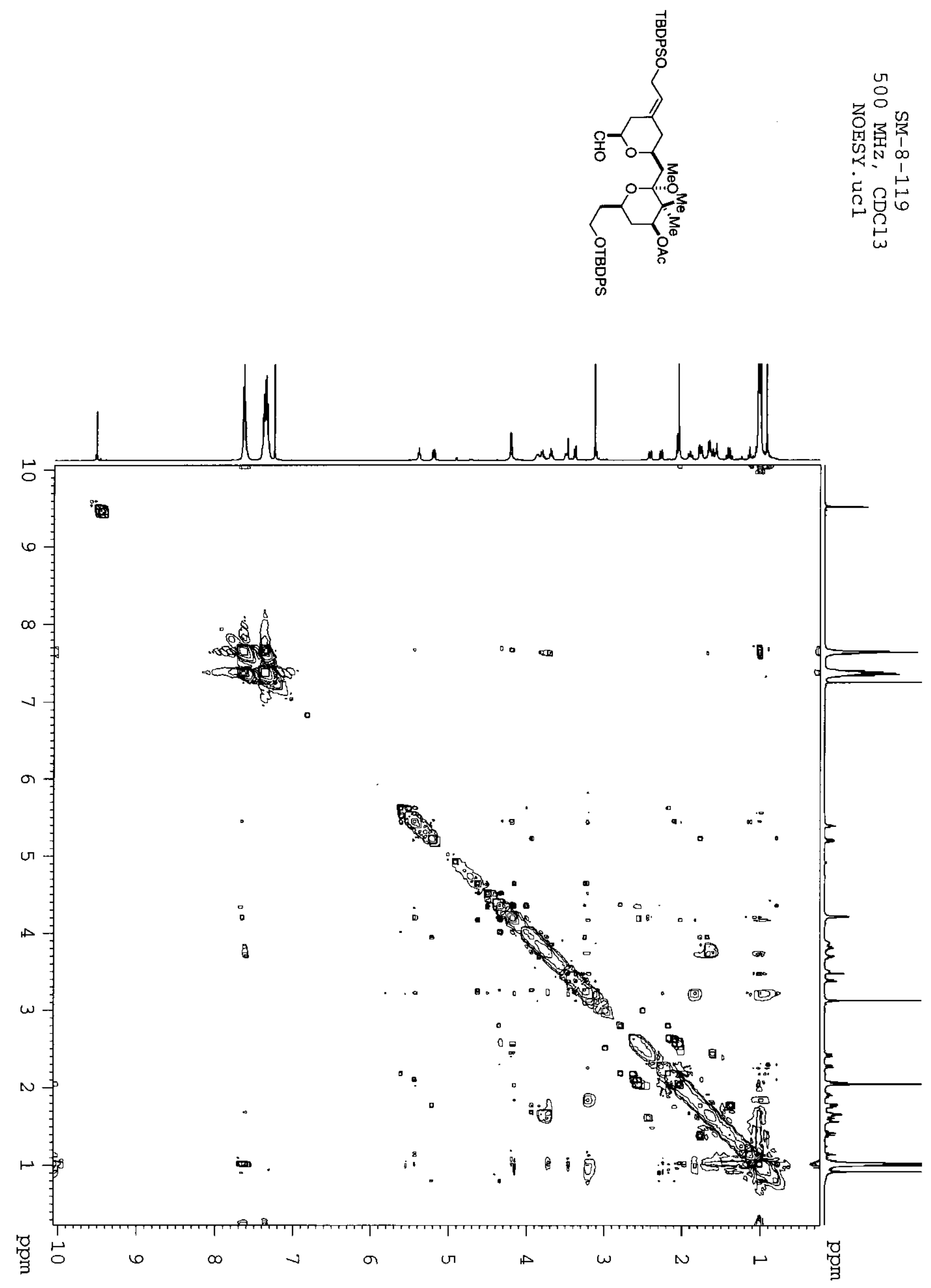


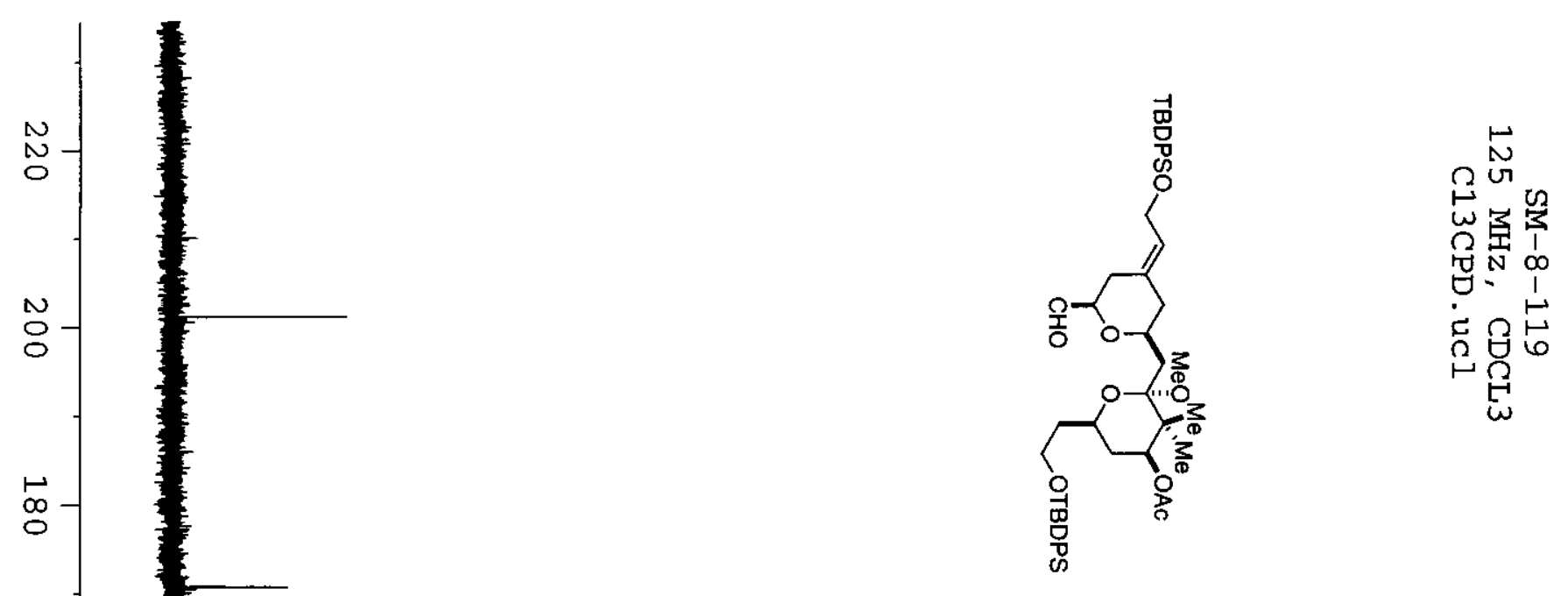

.

尚

$\stackrel{\bullet}{\circ}$

$\stackrel{\bullet}{\circ}$

•

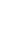

$\infty$

o

$\stackrel{\bullet}{\circ}$

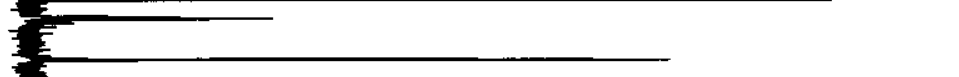

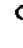

$-$

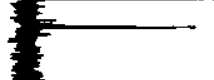



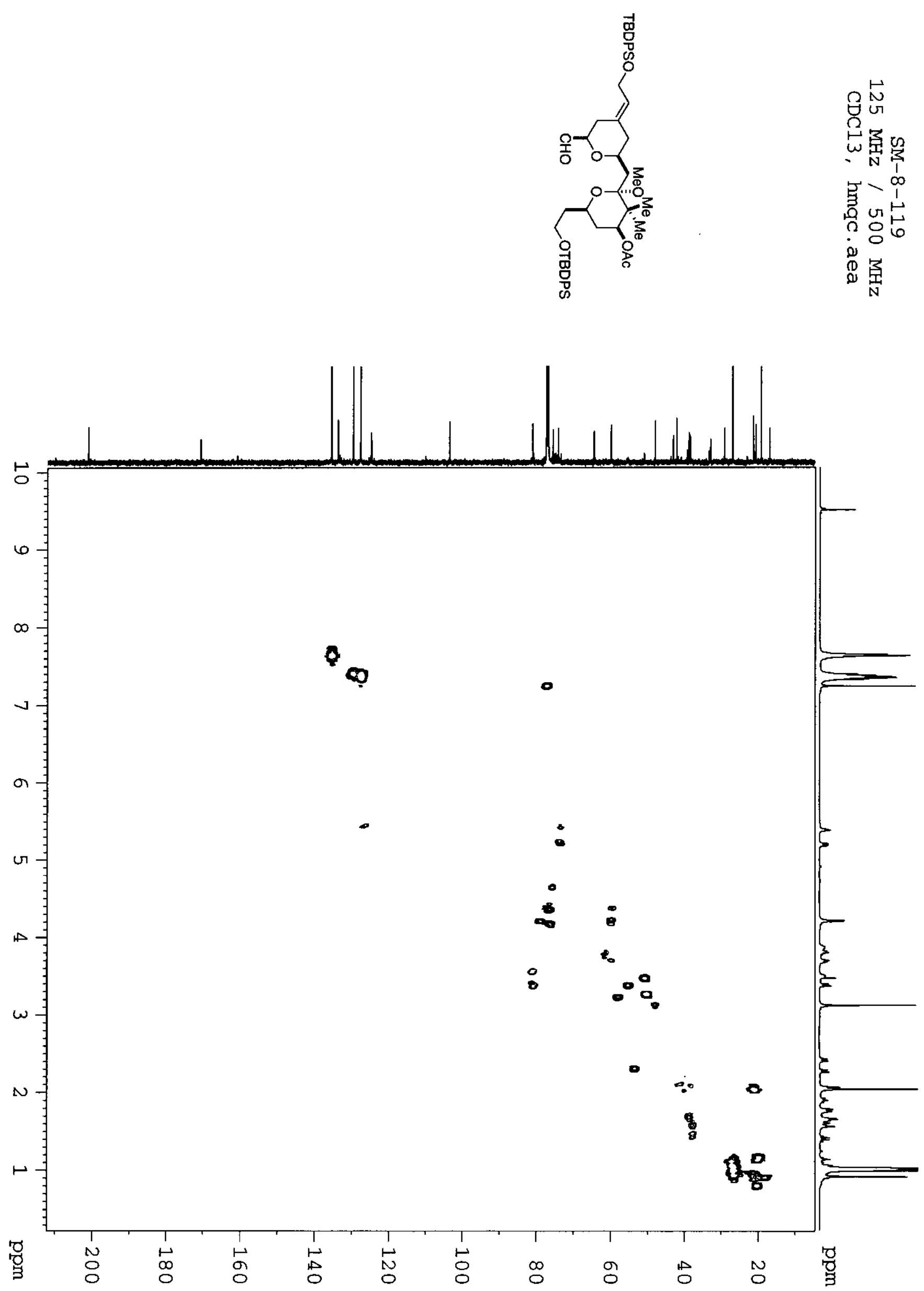